\title{
Palladium-Catalyzed Long-Range Deconjugative Isomerization of Highly Substituted $\alpha, \beta$-Unsaturated Carbonyl Compounds
}

\author{
Luqing Lin ${ }^{\ddagger}$, Ciro Romano ${ }^{\ddagger}$, Clément Mazet* \\ ${ }^{\ddagger}$ These authors contributed equally \\ Department of Organic Chemistry, University of Geneva \\ Quai Ernest Ansermet 30, 1211 Geneva 4, Switzerland \\ e-mail: clement.mazet@unige.ch
}

\section{Table of content}

$1 \quad$ General methods $\quad$ S2

2 Experimental procedures $\quad$ S3

2.1 General procedure for deconjugative isomerization S3

2.2 Substrates synthesis $\quad$ S3

2.3 Characterization of isomerization products $\quad$ S57

$\begin{array}{lll}2.4 & \text { Mechanistic studies } & \text { S69 }\end{array}$

2.4.1 Evidence for de-coordinative process $\quad \mathbf{S 6 9}$

2.4.1.1 Synthesis of $2 \mathrm{~m} \quad \mathbf{S 6 9}$

$\begin{array}{lll}2.4 .1 .2 & \text { Isomerization of } 2 \mathrm{~m} & \mathbf{S 7 0}\end{array}$

2.4.2 Assessment of the bidirectional nature of the process $\quad \mathbf{S 7 3}$

2.4.2.1 Synthesis of $\mathbf{2 0} \quad \mathbf{S 7 3}$

2.4.2.2 Isotopic labelling experiments $\quad \mathbf{S 7 4}$

2.4.3 Assessment of site-selectivity of $[\mathrm{Pd}-\mathrm{H}]$ insertion $\quad \mathbf{S 7 8}$

2.4.3.1 Synthesis of $2 p \quad S 78$

2.4.3.2 Isomerization of $2 p \quad S 80$

2.4.3.3 Independent synthesis of $2 q$

2.4.4 Control experiment using $3 \mathbf{g} \quad \mathbf{S 8 5}$

2.5 Optimization of the deconjugative isomerization under microwave irradiation $\mathbf{S 8 6}$

$\begin{array}{lll}2.5 .1 & \text { Chiral ligand screening } & \text { S87 }\end{array}$

2.6 General procedure for enantioselective deconjugative isomerization $\quad$ S88

3 References $\quad$ S92

$4 \quad$ NMR spectra $\quad$ S94 


\section{General Methods}

All reactions were carried out under an inert atmosphere of nitrogen using either twomanifold vacuum/inert gas lines or a M.Braun glove-box, unless otherwise noted. Solvents were dried over activated alumina columns and further degassed by three successive "freeze-pump-thaw" cycles if necessary. Commercial reagents were purchased from Aldrich, Acros or Strem and used without further purification, unless otherwise noted. Liquid reagents were transferred with stainless steel syringes or cannula. Thin layer chromatography (TLC) was performed on plates of silica precoated with $0.25 \mathrm{~mm}$ Kieselgel 60 F254. Flash chromatography was performed using silica gel 60 (230-400 mesh ASTM) from Aldrich. $\mathrm{NaBAr}_{F}$ were prepared according to literature procedures ${ }^{1}$. NMR spectra were recorded on AMX-400 and AM-500 Bruker Avance spectrometers. ${ }^{1} \mathrm{H}$ and ${ }^{13} \mathrm{C}\left\{{ }^{1} \mathrm{H}\right\}$-NMR chemical shifts are given in ppm relative to $\mathrm{SiMe}_{4}$, with the solvent resonance used as internal reference. ${ }^{31} \mathrm{P}\left\{{ }^{1} \mathrm{H}\right\}-\mathrm{NMR}$ chemical shifts are reported in ppm relative to $\mathrm{H}_{3} \mathrm{PO}_{4}$. Infrared spectra were obtained on a Perkin-Elmer 1650 FT-IR spectrometer using neat samples on a diamond ATR Golden Gate sampler. HPLC analyses were performed on a Shimadzu CTO-20AA using chiral column. Retention times $\left(t_{R}\right)$ are given in minutes. Chiral GC analyses were performed on an Agilent HP6850 gas chromatograph. 


\section{Experimental procedures}

\subsection{General procedure for deconjugative isomerization (GP1)}

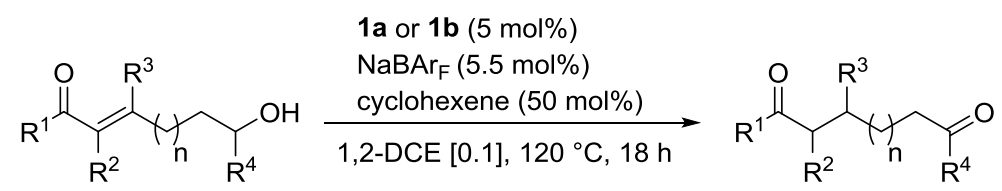

Inside the glovebox, a $5 \mathrm{~mL}$ Young valve Schlenk was charged with the appropriate palladium precatalyst $1 \mathrm{a}$ or $\mathbf{1 b}(0.0125 \mathrm{mmol})$ and $\operatorname{NaBAr}_{\mathrm{F}}(12.2 \mathrm{mg}, 0.0138 \mathrm{mmol})$, dissolved in 1,2-dichloroethane (2.5 mL) and cyclohexene was added (13 $\mu \mathrm{L}, 0.125 \mathrm{mmol})$. The reaction mixture was stirred for 5 minutes at room temperature. Next, the appropriate substrate $(0.25 \mathrm{mmol})$ was added to the mixture, the Schlenk sealed and placed in an oil bath pre-heated at $120{ }^{\circ} \mathrm{C}$. After $18 \mathrm{~h}$, the conversion was assessed by ${ }^{1} \mathrm{H}-\mathrm{NMR}$ analysis of the crude mixture using an internal standard, and the crude mixture was purified by flash chromatography on silica gel to afford the analytically pure dicarbonyl product.

\subsection{Substrates synthesis}

\section{General procedure for Horner-Wadsworth-Emmons (HWE) reaction (GP2)}

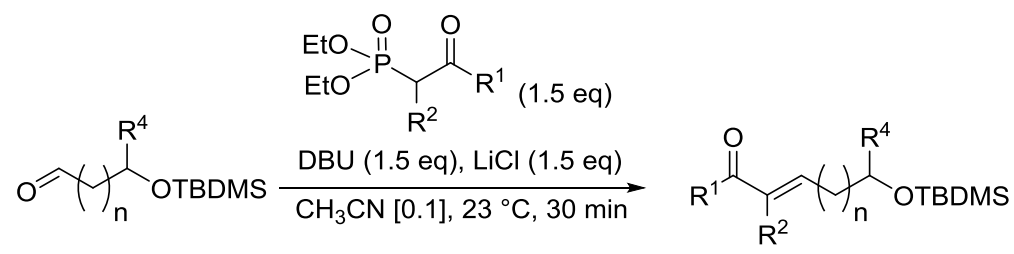

A round bottomed flask was charged with the appropriate phosphonate (1.5 eq), $\mathrm{CH}_{3} \mathrm{CN}(0.1$ M) and $\mathrm{LiCl}$ (1.5 eq). To this, 1,8-Diaza-bicyclo[5.4.0]undec-7-ene (DBU) (1.5 eq) was added via syringe. The reaction mixture was stirred at room temperature for 15 minutes then cooled to $0{ }^{\circ} \mathrm{C}$. The appropriate aldehyde $(1 \mathrm{eq})$ in $\mathrm{CH}_{3} \mathrm{CN}(1 \mathrm{M})$ was added dropwise via cannula and the reaction mixture was monitored by TLC (reaction complete within 30 minutes) then quenched with cold water, extracted with diethyl ether (3 times), dried over anhydrous $\mathrm{Na}_{2} \mathrm{SO}_{4}$ and concentrated in vacuo. 


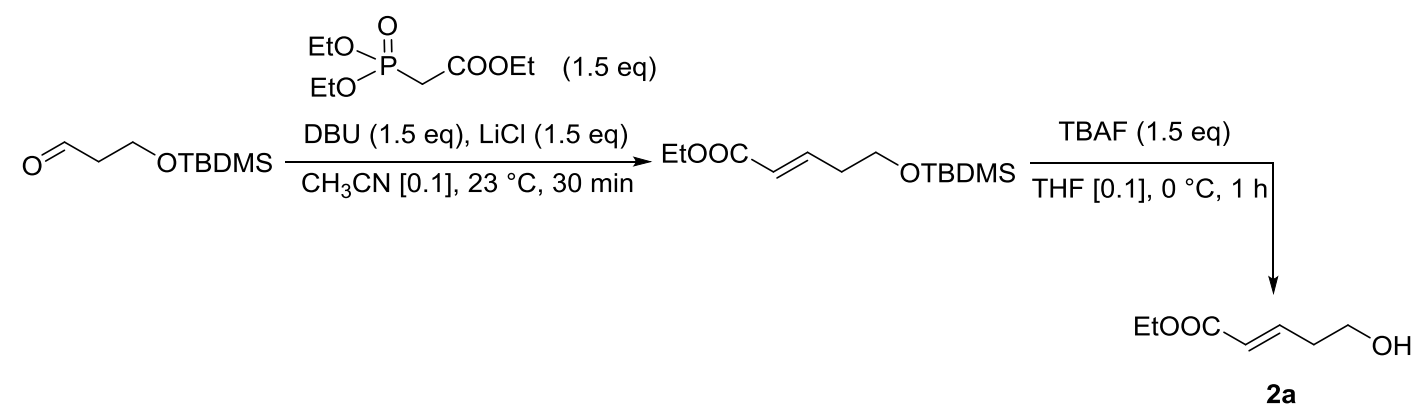

Figure S1: Synthesis of Ethyl $(E)$-5-hydroxypent-2-enoate (2a)

Etooc

Ethyl (E)-5-((tert-butyldimethylsilyl)oxy)pent-2-enoate: The reaction was run according to GP2 starting from 3-((tertbutyldimethylsilyl)oxy)propanal ${ }^{2}(11.7 \mathrm{mmol})$. Flash chromatography (Pentane/Diethyl ether $=10: 1)$ afforded the product as a colourless oil ( $2.9 \mathrm{~g}, 94 \%$ yield). All spectroscopic analyses were in agreement with those reported in the literature. ${ }^{3}$

General procedure for tert-butyldimethylsilyl (TBDMS) deprotection with tetrabutylammonium fluoride (TBAF) (GP3)

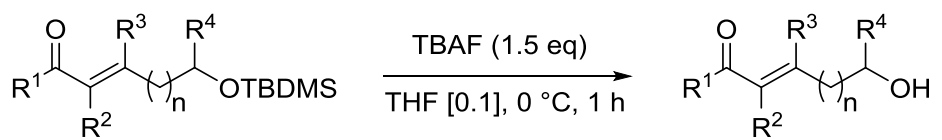

The appropriate protected alcohol (1 eq) was dissolved in THF (0.1 M) and TBAF (1.0 M in THF, $1.5 \mathrm{eq}$ ) was added slowly at $0{ }^{\circ} \mathrm{C}$. After stirring for $1 \mathrm{~h}$ at $0{ }^{\circ} \mathrm{C}$, the mixture was quenched with saturated aqueous $\mathrm{NH}_{4} \mathrm{Cl}$ solution, extracted with diethyl ether (3 times), dried over anhydrous $\mathrm{Na}_{2} \mathrm{SO}_{4}$ and concentrated in vacuo.

Ethyl (E)-5-hydroxypent-2-enoate (2a): The reaction was run according
to $\mathrm{GP3}$ starting from ethyl $(E)-5$-((tert-butyldimethylsilyl)oxy)pent-2enoate $(2.0 \mathrm{~g}, 7.8 \mathrm{mmol})$. Purification by flash chromatography on silica gel (Pentane/Diethyl ether $=1: 1$ ) afforded $2 \mathbf{a}$ in $68 \%$ yield $(763 \mathrm{mg}$ ). All spectroscopic analyses were in agreement with those reported in the literature. ${ }^{4}$ 


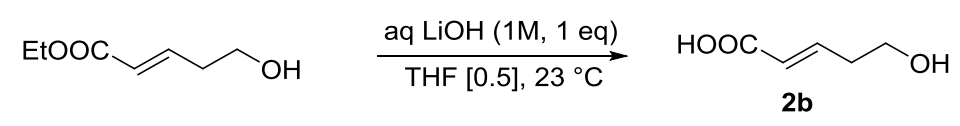

Figure S2: Synthesis of $(E)$-5-hydroxypent-2-enoic acid (2b)

$\mathrm{HOOC}_{\mathrm{OH}}$

(E)-5-hydroxypent-2-enoic acid (2b): To a solution of ethyl $(E)-5$ hydroxypent-2-enoate $(210 \mathrm{mg}, 1.5 \mathrm{mmol})$ in THF $(3 \mathrm{~mL})$, an aqueous solution of $\mathrm{LiOH}(1 \mathrm{M}, 1.5 \mathrm{~mL})$ was added at $0^{\circ} \mathrm{C}$ and stirred $16 \mathrm{~h}$ at room temperature. The reaction was quenched with an aqueous $\mathrm{HCl}$ solution $(2 \mathrm{M}, 2 \mathrm{~mL})$, extracted with diethyl ether $(3 \times 5 \mathrm{~mL})$, dried over $\mathrm{Na}_{2} \mathrm{SO}_{4}$ and concentrated. The residue was purified by flash chromatography on silica gel (EtOAc/Diethyl ether $=10: 1)$ to give $\mathbf{2 b}$ as colourless oil in $67 \%$ yield (120 mg). $\mathbf{R}_{\mathbf{f}}=0.1$ (Diethyl ether). IR (neat): $v\left(\mathrm{~cm}^{-1}\right)=3259,2949,1691,1653,1411$, 1041, 975. ${ }^{1} \mathrm{H}$ NMR (400 MHz, $\left.\mathrm{CD}_{2} \mathrm{Cl}_{2}\right) \delta(\mathrm{ppm}) 2.41-2.59\left(\mathrm{~m}, 2 \mathrm{H}, \mathrm{CH}_{2} \mathrm{CH}_{2} \mathrm{OH}\right), 3.81$ (t, $\left.{ }^{3} J_{\mathrm{HH}}=6.3 \mathrm{~Hz}, 2 \mathrm{H}, \mathrm{CH}_{2} \mathrm{OH}\right), 5.95\left(\mathrm{dt},{ }^{3} \mathrm{~J}_{\mathrm{HH}}=15.7 \mathrm{~Hz},{ }^{4} \mathrm{~J}_{\mathrm{HH}}=1.4 \mathrm{~Hz}, 1 \mathrm{H}, \mathrm{CH}=\mathrm{CHCH}_{2}\right), 7.08$ $\left(\mathrm{dt},{ }^{3} \mathrm{~J}_{\mathrm{HH}}=15.8,{ }^{3} \mathrm{~J}_{\mathrm{HH}}=7.1 \mathrm{~Hz}, 1 \mathrm{H}, \mathrm{CH}=\mathrm{CHCH}_{2}\right) .{ }^{13} \mathrm{C}\left\{{ }^{1} \mathrm{H}\right\} \mathbf{N M R}\left(101 \mathrm{MHz}, \mathrm{CD}_{2} \mathrm{Cl}_{2}\right) \delta(\mathrm{ppm})$ $35.6\left(\mathrm{CH}_{2} \mathrm{CH}_{2} \mathrm{OH}\right), 61.0\left(\mathrm{CH}_{2} \mathrm{OH}\right), 122.8\left(\mathrm{CH}=\mathrm{CHCH}_{2}\right), 148.1\left(\mathrm{CH}=\mathrm{CHCH}_{3}\right), 170.1(\mathrm{C}=\mathrm{O})$. LRMS (IC) found $[\mathrm{M}+\mathrm{H}]^{+}=134$.

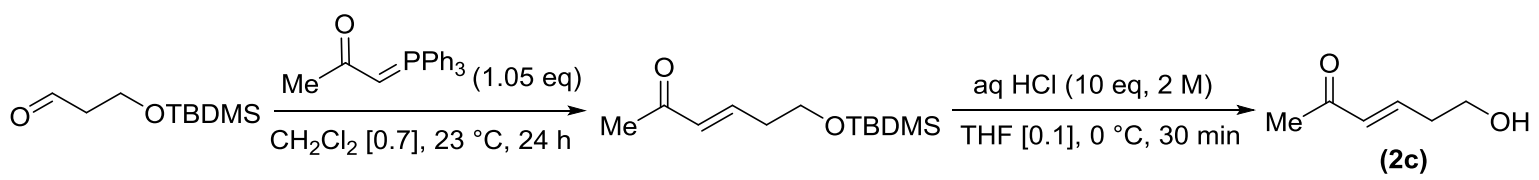

Figure S3: Synthesis of $(E)$-6-hydroxyhex-3-en-2-one (2c)

(E)-6-((tert-butyldimethylsilyl)oxy)hex-3-en-2-one: 3-((tert-
OTBDMS $\mathrm{CH}_{2} \mathrm{Cl}_{2}(14 \mathrm{~mL}$ ) and 1-(triphenylphosphoranylidene)-2-propanone (3.35 g, $10 \mathrm{mmol}, 1.05 \mathrm{eq})$ was added at $0{ }^{\circ} \mathrm{C}$. The reaction mixture was stirred at room temperature for $24 \mathrm{~h}$. Silica gel $(5 \mathrm{~g})$ was added and the solvent was removed under reduced pressure. The residue was purified by flash chromatography (Pentane/Diethyl ether $=10: 1$ ) to afford the desired compound in $86 \%$ yield $(2.0 \mathrm{~g})$. All spectroscopic analyses were in agreement with those reported in the literature. ${ }^{5}$ 
General procedure for tert-butyldimethylsilyl (TBDMS) deprotection with aqueous $\mathrm{HCl}$ (GP4)

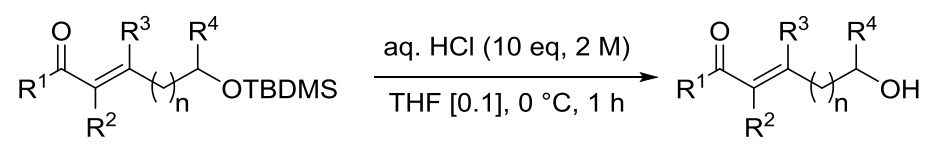

To a solution of the appropriate protected alcohol $(1 \mathrm{eq})$ in THF $(0.1 \mathrm{M})$, aqueous $\mathrm{HCl}(2 \mathrm{M}$, 10 eq) was added at $0^{\circ} \mathrm{C}$ and stirred for 30 minutes then quenched with water, extracted with EtOAc (3 times), dried over anhydrous $\mathrm{Na}_{2} \mathrm{SO}_{4}$ and concentrated in vacuo.

$(E)$-6-hydroxyhex-3-en-2-one (2c): The reaction was run according to
$\mathrm{OH}$ GP4 starting from $(E)-6$-((tert-butyldimethylsilyl)oxy)hex-3-en-2-one (1.2 $\mathrm{g}, 5.2 \mathrm{mmol})$. The residue was purified by flash chromatography on silica gel (Pentane/Diethyl ether $=1: 4)$ to give $\mathbf{2 c}$ in $65 \%$ yield $(430 \mathrm{mg})$. All spectroscopic analyses were in agreement with those reported in the literature. ${ }^{6}$

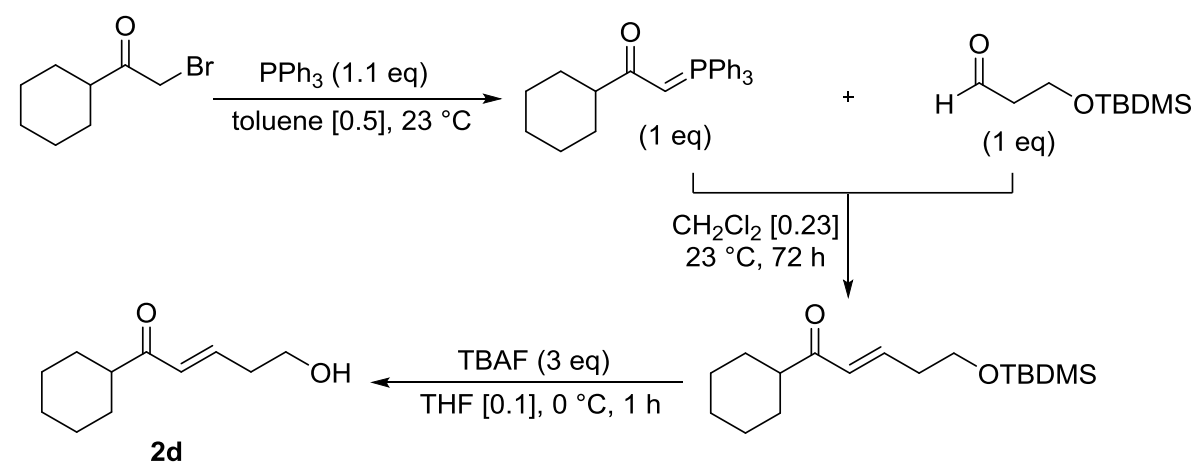

Figure S4: Synthesis of $(E)$-1-cyclohexyl-5-hydroxypent-2-en-1-one (2d)

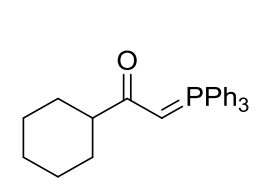

1-cyclohexyl-2-(triphenyl- $\lambda^{5}$-phosphanylidene)ethan-1-one: To a

solution of 2-bromo-1-cyclohexylethan-1-one $(6.8 \mathrm{~g}, 33 \mathrm{mmol})$ in toluene $(30 \mathrm{~mL})$, a second solution of triphenylphosphine $(9.6 \mathrm{~g}, 36 \mathrm{mmol})$ in toluene $(40 \mathrm{~mL})$ was added slowly and stirred vigorously at room temperature. After $17 \mathrm{~h}$, the solid obtained was filtered and redissolved in $\mathrm{CH}_{2} \mathrm{Cl}_{2}(182 \mathrm{~mL})$. An aqueous $\mathrm{NaOH}$ solution $(2 \mathrm{~N}, 60 \mathrm{~mL})$ was next added and the mixture stirred at room temperature. After $1 \mathrm{~h}$, the organic phase was washed with water $(2 \times 90 \mathrm{~mL})$ and concentrated to give the desired compound in $73 \%$ yield $(9.4 \mathrm{~g})$, which was used in the next step without further purification. 
<smiles>CCCOCC/C=C/C(=O)C1CCCCC1</smiles>

(E)-5-((tert-butyldimethylsilyl)oxy)-1-cyclohexylpent-2-en-1-

one: To a solution of 3-((tert-butyldimethylsilyl)oxy)propanal (4.4 g, $23 \mathrm{mmol})$ in $\mathrm{CH}_{2} \mathrm{Cl}_{2}(100 \mathrm{~mL})$, 1-1-cyclohexyl-2-(triphenyl- $\lambda^{5}$ phosphanylidene)ethan-1-one $(8.9 \mathrm{~g}, 23 \mathrm{mmol})$ was added at $0{ }^{\circ} \mathrm{C}$. The reaction mixture was warmed to room temperature. After $72 \mathrm{~h}$, silica gel $(10 \mathrm{~g})$ was added and the solvent was removed under reduced pressure. The solid deposit was charged on column of silica gel and flushed with Pentane/Diethyl ether $=20: 1$ to afford $5.4 \mathrm{~g}$ of an oil containing the desired enone $^{7}$ and the starting aldehyde in 6.7:1 ratio. This mixture was used for next step directly.<smiles>O=C(/C=C/CCO)C1CCCCC1</smiles>

(E)-1-cyclohexyl-5-hydroxypent-2-en-1-one (2d): A part (1.6 g) of the mixture obtained from the previous step was dissolved in THF $(45 \mathrm{~mL})$ and TBAF (1.0 M in THF, $6.8 \mathrm{mmol}, 6.8 \mathrm{~mL}$ ) was added slowly at $0{ }^{\circ} \mathrm{C}$. After stirring for $1 \mathrm{~h}$ at $0{ }^{\circ} \mathrm{C}$, the mixture was quenched with a saturated aqueous $\mathrm{NH}_{4} \mathrm{Cl}$ solution $(45 \mathrm{~mL})$, extracted with diethyl ether $(3 \times 60 \mathrm{~mL})$, dried over anhydrous $\mathrm{Na}_{2} \mathrm{SO}_{4}$ and concentrated in vacuo. After purification by flash chromatography on silica gel (Pentane/Diethyl ether $=1: 1$ ), the product was obtained in $72 \%$ yield $(590 \mathrm{mg}) . \mathbf{R}_{\mathbf{f}}=0.3$ (Pentane/Diethyl ether $=1: 1$ ). IR (neat): $v\left(\mathrm{~cm}^{-1}\right)=3415,2928,2854,1688,1659,1624$, 1449, 1046. ${ }^{1} \mathrm{H}$ NMR $\left(400 \mathrm{MHz}, \mathrm{CD}_{2} \mathrm{Cl}_{2}\right) \delta(\mathrm{ppm}) 1.13-1.36\left(\mathrm{~m}, 5 \mathrm{H}, \mathrm{CH}_{2}-\mathrm{Cy}\right), 1.46\left(\mathrm{t},{ }^{3} \mathrm{~J}_{\mathrm{HH}}=\right.$ $5.5 \mathrm{~Hz}, 1 \mathrm{H}, \mathrm{OH}), 1.66(\mathrm{~m}, 1 \mathrm{H}, \mathrm{CH}-\mathrm{Cy}), 1.77$ (m, 4H, CH $2.55(\mathrm{~m}, 1 \mathrm{H}, \mathrm{CH}-\mathrm{Cy}), 3.73\left(\mathrm{~m}, 2 \mathrm{H}, \mathrm{CH}_{2} \mathrm{OH}\right), 6.22\left(\mathrm{dt},{ }^{3} \mathrm{~J}_{\mathrm{HH}}=15.8 \mathrm{~Hz},{ }^{4} J_{\mathrm{HH}}=1.5 \mathrm{~Hz}, 1 \mathrm{H}\right.$, $\mathrm{CH}=\mathrm{CHCOCy}$ ), 6.80 (dt, $\left.{ }^{3} \mathrm{~J}_{\mathrm{HH}}=15.7,{ }^{3} \mathrm{~J}_{\mathrm{HH}}=7.1 \mathrm{~Hz}, 1 \mathrm{H}, \mathrm{CH}=\mathrm{CHCOCy}\right) .{ }^{13} \mathrm{C}\left\{{ }^{1} \mathrm{H}\right\}$ NMR $(101$ $\left.\mathrm{MHz}, \mathrm{CD}_{2} \mathrm{Cl}_{2}\right) \delta$ (ppm) $26.1\left(\mathrm{CH}_{2}-\mathrm{Cy}\right), 26.3\left(\mathrm{CH}_{2}-\mathrm{Cy}\right), 29.1\left(\mathrm{CH}_{2}-\mathrm{Cy}\right), 36.1\left(\mathrm{CH}_{2} \mathrm{CH}_{2} \mathrm{OH}\right), 48.9$ (CH-Cy), $61.4\left(\mathrm{CH}_{2} \mathrm{OH}\right), 130.9$ ( $\left.\mathrm{CH}=\mathrm{CHCOCy}\right), 143.1(\mathrm{CH}=\mathrm{CHCOCy}), 203.1(\mathrm{C}=\mathrm{O})$. LRMS (IC) found $[\mathrm{M}+\mathrm{H}]^{+}=183 ;\left[\mathrm{M}+\mathrm{NH}_{4}\right]^{+}=200$.

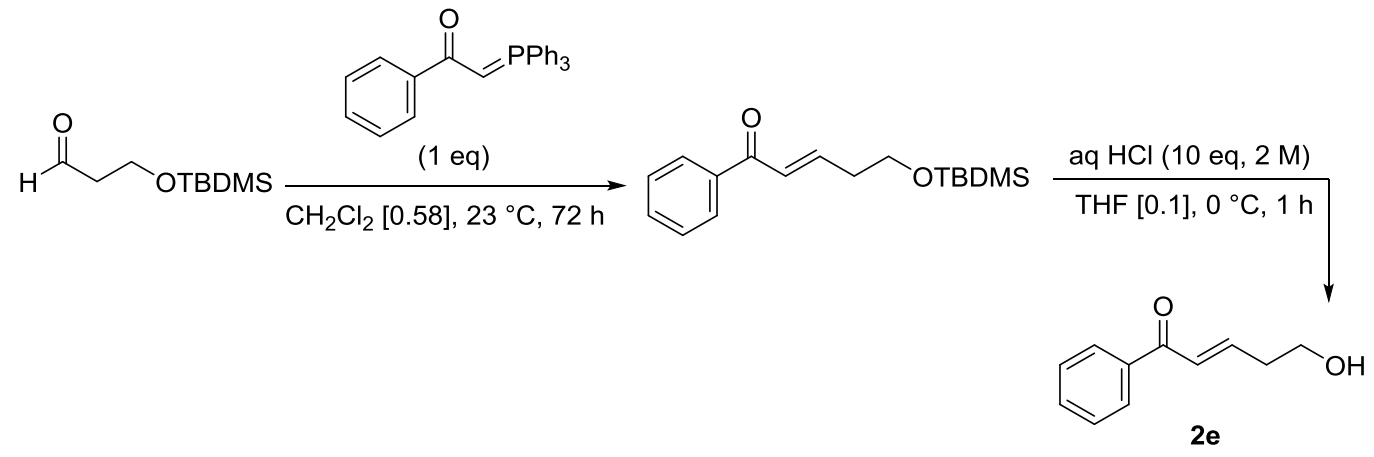

Figure S5: Synthesis of $(E)$-5-hydroxy-1-phenylpent-2-en-1-one (2e) 


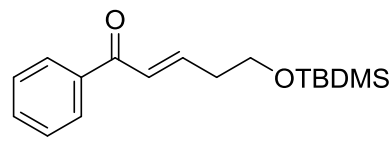

(E)-5-((tert-butyldimethylsilyl)oxy)-1-phenylpent-2-en-1-one:

The product was prepared in $72 \%$ yield $(2.5 \mathrm{~g})$ starting from $3-$ ((tert-butyldimethylsilyl)oxy)propanal $(2.2 \mathrm{~g}, 11.8 \mathrm{mmol})$ in $\mathrm{CH}_{2} \mathrm{Cl}_{2}$ $(20 \mathrm{~mL})$ and 1-phenyl-2-(triphenyl- $\lambda^{5}$-phosphanylidene)ethan-1-one ${ }^{8}$ (3.62 g, $\left.11.7 \mathrm{mmol}\right)$ according to a procedure reported in the literature ${ }^{9}$.<smiles>O=C(/C=C/CCO)c1ccccc1</smiles>

(E)-5-hydroxy-1-phenylpent-2-en-1-one (2e): To a solution of $(E)$-5((tert-butyldimethylsilyl)oxy)-1-phenylpent-2-en-1-one $\quad(600 \quad \mathrm{~g}, \quad 3.4$ $\mathrm{mmol})$ in THF (34 $\mathrm{mL})$, aqueous $\mathrm{HCl}(2 \mathrm{M}, 17 \mathrm{~mL})$ was added and stirred for 30 minutes at $0{ }^{\circ} \mathrm{C}$. The reaction was quenched with water $(30 \mathrm{~mL})$, extracted with diethyl ether $(3 \times 30 \mathrm{~mL})$, dried over anhydrous $\mathrm{Na}_{2} \mathrm{SO}_{4}$ and concentrated in vacuo. The residue was purified by flash chromatography on silica gel (Pentane/Diethyl ether $=1: 4$ ) to give $2 \mathrm{e}$ in $90 \%$ yield (330 $\mathrm{mg}$ ). The spectroscopic analyses were in agreement with those reported in the literature. ${ }^{10}$

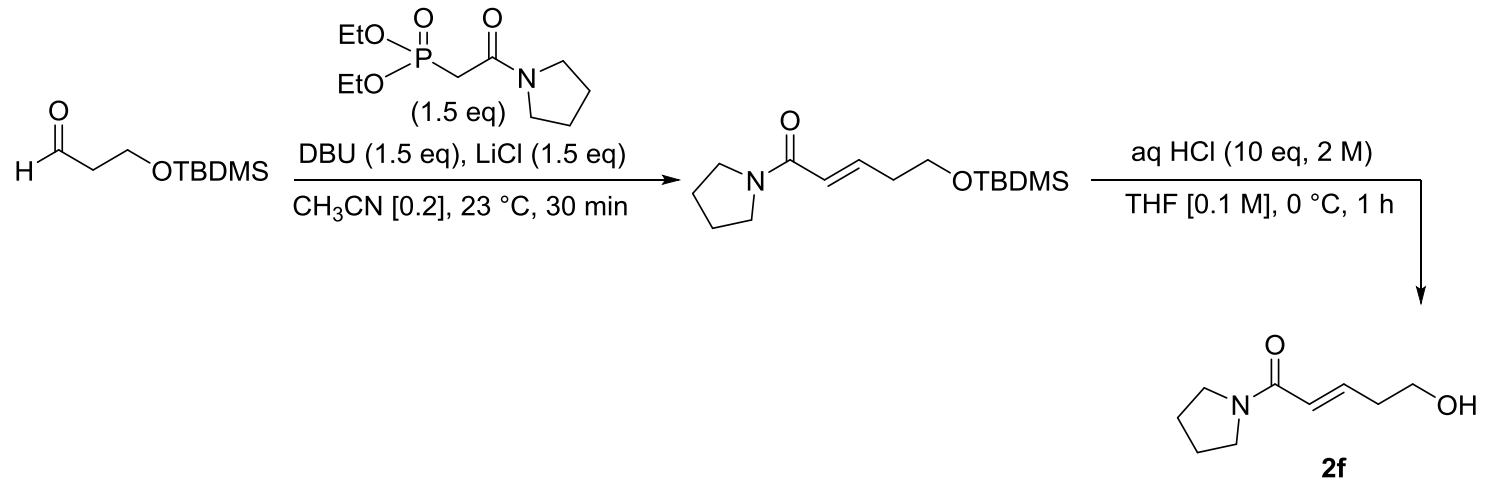

Figure S6: Synthesis of $(E)$-5-hydroxy-1-(pyrrolidin-1-yl)pent-2-en-1-one (2f)

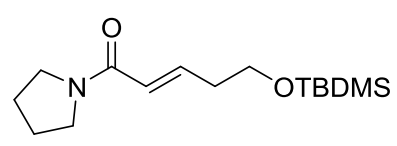

(E)-5-((tert-butyldimethylsilyl)oxy)-1-(pyrrolidin-1-yl)pent-2en-1-one: The product was synthetized according to GP2 starting from the 3-((tert-butyldimethylsilyl)oxy)propanal (398 mg, 2.12 mmol) and diethyl 2-oxo-2-(pyrrolidin-1-yl)ethylphosphonate (791 mg, $3.18 \mathrm{mmol}$ ). Purification by flash chromatography (Pentane/EtOAc $=1: 1$ ) gave the product in $50 \%$ yield $\left(370 \mathrm{mg}\right.$ ) as light yellow oil. $\mathbf{R}_{\mathbf{f}}=0.5$ (Pentane/EtOAc $\left.=1: 2\right)$. IR (neat): $v\left(\mathrm{~cm}^{-1}\right)=2929,2858$, 1661, 1606, 1431, 833. ${ }^{1} \mathrm{H}$ NMR $\left(400 \mathrm{MHz}, \mathrm{CDCl}_{3}\right) \delta(\mathrm{ppm}) 0.04\left(\mathrm{~s}, 6 \mathrm{H},\left(\mathrm{CH}_{3}\right)_{2} \mathrm{Si}\right), 0.88$ (s, $\left.9 \mathrm{H},\left(\mathrm{CH}_{3}\right)_{3} \mathrm{CSi}\right), 1.80-1.98\left(\mathrm{~m}, 4 \mathrm{H}, \mathrm{NCH}_{2} \mathrm{CH}_{2} \mathrm{CH}_{2}\right), 2.41\left(\mathrm{qd},{ }^{3} J_{H H}=6.9,1.5 \mathrm{~Hz}, 2 \mathrm{H}\right.$, $\mathrm{CH}_{2} \mathrm{CH}_{2} \mathrm{OTBDMS}$ ), 3.52 (d, $\left.{ }^{3} \mathrm{~J}_{\mathrm{HH}}=6.6 \mathrm{~Hz}, 4 \mathrm{H}, \mathrm{N}\left(\mathrm{CH}_{2}\right)_{2}\right), 3.71$ (t, ${ }^{3} J_{\mathrm{HH}}=6.7 \mathrm{~Hz}, 2 \mathrm{H}$, $\mathrm{CH}_{2} \mathrm{OTBDMS}$ ), $6.16\left(\mathrm{dt},{ }^{3} \mathrm{~J}_{\mathrm{HH}}=15.1,{ }^{3} J_{\mathrm{HH}}=1.5 \mathrm{~Hz}, 1 \mathrm{H}, \mathrm{CH}=\mathrm{CHCH}_{2}\right), 6.88\left(\mathrm{dt},{ }^{3} J_{\mathrm{HH}}=14.7\right.$, $\left.{ }^{4} J_{\mathrm{HH}}=7.1 \mathrm{~Hz}, 1 \mathrm{H}, \quad \mathrm{CH}=\mathrm{CHCH}{ }_{2}\right) \cdot{ }^{13} \mathrm{C}\left\{{ }^{1} \mathrm{H}\right\} \operatorname{NMR}\left(101 \mathrm{MHz}, \mathrm{CDCl}_{3}\right) \delta(\mathrm{ppm})-5.2\left(\mathrm{CH}_{3}\right)_{2} \mathrm{Si}$, 
$18.4\left(\left(\mathrm{CH}_{3}\right)_{3} \mathrm{CSi}\right), 24.5\left(\mathrm{NCH}_{2} \mathrm{CH}_{2} \mathrm{CH}_{2}\right), 26.0\left(\left(\mathrm{CH}_{3}\right)_{3} \mathrm{CSi}\right), 36.1\left(\mathrm{CH}_{2} \mathrm{CH}_{2} \mathrm{OTBDMS}\right), 46.0$ $\left(\mathrm{CH}_{2} \mathrm{NCH}_{2}\right), \quad 46.6 \quad\left(\mathrm{CH}_{2} \mathrm{NCH}_{2}\right), \quad 62.0 \quad\left(\mathrm{CH}_{2} \mathrm{OTBDMS}\right), \quad 123.5 \quad\left(\mathrm{CH}=\mathrm{CHCH}_{2}\right), \quad 142.1$ $\left(\mathrm{CH}=\mathrm{CHCH}_{2}\right), 164.8(\mathrm{C}=\mathrm{O})$. LRMS (IC) found $[\mathrm{M}+\mathrm{H}]^{+}=284$.

(E)-5-hydroxy-1-(pyrrolidin-1-yl)pent-2-en-1-one (2f): The product<smiles>O=C(/C=C/CCO)N1CCCC1</smiles>
was prepared according to GP4 starting from the $(E)-5-(($ tertbutyldimethylsilyl)oxy)-1-(pyrrolidin-1-yl)pent-2-en-1-one (370 mg, 1.35 $\mathrm{mmol}$ ). The residue was purified by flash chromatography (Pentane/EtOAc $=1: 2$ ) to give the product in $73 \%$ yield $\left(160 \mathrm{mg}\right.$ ) as a colourless oil. $\mathbf{R}_{\mathbf{f}}=0.1$ (Pentane/EtOAc $\left.=1: 2\right)$. IR (neat): $v\left(\mathrm{~cm}^{-1}\right)=3398,2950,2875,1658,1590,1440,1045 .{ }^{1} \mathbf{H}$ NMR $\left(400 \mathrm{MHz}, \mathrm{CD}_{2} \mathrm{Cl}_{2}\right) \delta(\mathrm{ppm})$ $\left.1.80-1.89\left(\mathrm{~m}, 2 \mathrm{H},\left(\mathrm{CH}_{2} \mathrm{CH}_{2}\right)_{2} \mathrm{~N}\right), 1.91-2.00\left(\mathrm{~m}, 2 \mathrm{H}, \mathrm{CH}_{2} \mathrm{CH}_{2}\right)_{2} \mathrm{~N}\right), 2.04(\mathrm{~s}, 1 \mathrm{H}, \mathrm{OH}), 2.44$ (dtd, $\left.{ }^{3} \mathrm{~J}_{\mathrm{HH}}=7.6,6.2 \mathrm{~Hz},{ }^{4} \mathrm{~J}_{\mathrm{HH}}=1.5 \mathrm{~Hz}, 2 \mathrm{H}, \mathrm{CH}_{2} \mathrm{CH}_{2} \mathrm{OH}\right), 3.48\left(\mathrm{~m}, 4 \mathrm{H},\left(\mathrm{CH}_{2} \mathrm{CH}_{2}\right)_{2} \mathrm{~N}\right), 3.72(\mathrm{t}, J$ $\left.=6.2 \mathrm{~Hz}, 2 \mathrm{H}, \mathrm{CH} \mathrm{H}_{2} \mathrm{OH}\right), 6.23\left(\mathrm{dt},{ }^{3} \mathrm{~J}_{\mathrm{HH}}=15.2,{ }^{4} \mathrm{~J}_{\mathrm{HH}}=1.5 \mathrm{~Hz}, 1 \mathrm{H}, \mathrm{CH}=\mathrm{CHCH}_{2}\right), 6.82\left(\mathrm{dt},{ }^{3} \mathrm{~J}_{\mathrm{HH}}=\right.$ 14.8, 7.3 Hz, $\left.\left.1 \mathrm{H}, \mathrm{CH}=\mathrm{CHCH}_{2}\right) \cdot{ }^{13} \mathrm{C}\left\{{ }^{1} \mathrm{H}\right\} \operatorname{NMR}\left(101 \mathrm{MHz}, \mathrm{CD}_{2} \mathrm{Cl}_{2}\right) \delta(\mathrm{ppm}) 24.3\left(\mathrm{CH}_{2} \mathrm{CH}_{2}\right)_{2} \mathrm{~N}\right)$, $\left.26.0\left(\mathrm{CH}_{2} \mathrm{CH}_{2}\right)_{2} \mathrm{~N}\right), 35.8\left(\mathrm{CH}_{2} \mathrm{CH}_{2} \mathrm{OH}\right), 45.8\left(\left(\mathrm{CH}_{2} \mathrm{CH}_{2}\right)_{2} \mathrm{~N}\right), 46.6\left(\left(\mathrm{CH}_{2} \mathrm{CH}_{2}\right)_{2} \mathrm{~N}\right), 61.1\left(\mathrm{CH}_{2} \mathrm{OH}\right)$, $124.1\left(\mathrm{CH}=\mathrm{CHCH}_{2}\right), 141.6\left(\mathrm{CH}=\mathrm{CHCH}_{2}\right), 164.4(\mathrm{C}=\mathrm{O})$. LRMS (IC) found $[\mathrm{M}+\mathrm{H}]^{+}=170$.

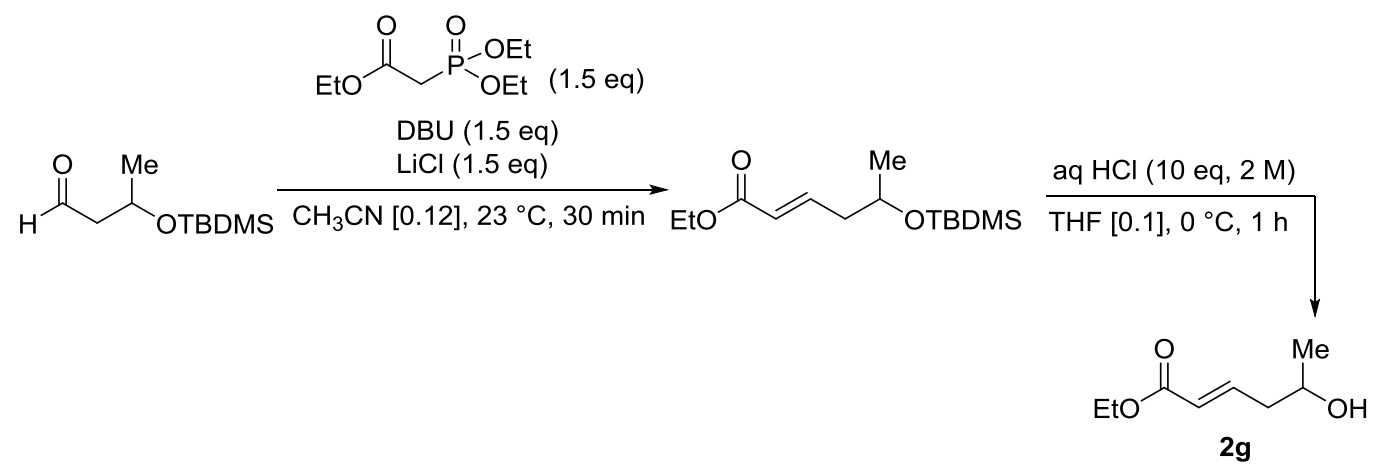

Figure S7: Synthesis of $(E)$-5-hydroxyhex-2-enoate $\mathbf{( 2 g})$<smiles>CCOC(=O)C=CCC(C)OCC</smiles>

Ethyl (E)-5-((tert-butyldimethylsilyl)oxy)hex-2-enoate: The product was synthetized according to GP2 starting from 3-((tertbutyldimethylsilyl)oxy)butanal $^{10}(856 \mathrm{mg}, 4.24 \mathrm{mmol})$ and triethyl phosphonacetate $(1.4 \mathrm{~g}$, $6.36 \mathrm{mmol}$ ). The residue was purified by flash chromatography on silica gel (Pentane/Diethyl ether $=5: 1)$ to afford the desired product in $83 \%$ yield $(960 \mathrm{mg})$ as a colourless oil. The spectroscopic analyses were in agreement with those reported in the literature. ${ }^{11}$ 
Me Ethyl (E)-5-hydroxyhex-2-enoate (2g): The product was prepared butyldimethylsilyl)oxy)hex-2-enoate $(960 \mathrm{mg}, 3.5 \mathrm{mmol})$. The crude mixture was purified by flash chromatography on silica gel (Pentane/Diethyl ether $=1: 2$ ) to give $\mathbf{2} \mathbf{g}$ as a colorless oil in $77 \%$ yield $(430 \mathrm{mg}$ ). The spectroscopic analyses were in agreement with those reported in the literature. ${ }^{12}$

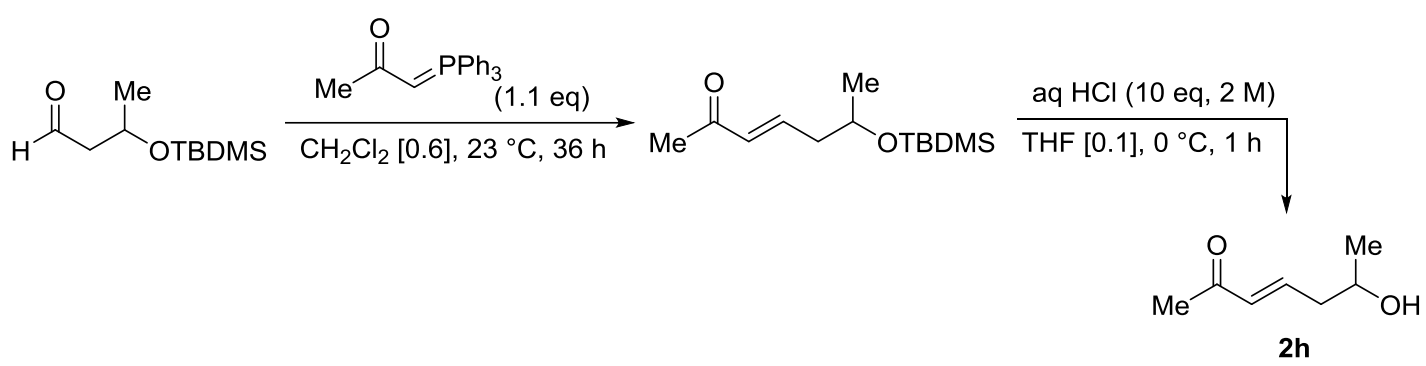

Figure S8: Synthesis of $(E)-6$-hydroxyhept-3-en-2-one (2h)<smiles>[Y4]C(C/C=C/C(C)=O)O[13CH3]</smiles>

(E)-6-((tert-butyldimethylsilyl)oxy)hept-3-en-2-one: To a solution of 3-((tert-butyldimethylsilyl)oxy)butanal (8 mmol) in $\mathrm{CH}_{2} \mathrm{Cl}_{2}(14 \mathrm{~mL})$ was added 1-(triphenylphosphoranylidene)-2-propanone $(2.8 \mathrm{~g}, 8.8 \mathrm{mmol})$ at $0{ }^{\circ} \mathrm{C}$. The reaction mixture was warmed to room temperature. After $36 \mathrm{~h}$, silica gel was added $(3 \mathrm{~g})$ and the solvent was removed under reduced pressure. The solid deposit was charged on a column of silica gel and flushed with Pentane/Diethyl ether $=10: 1$ to afford $1.1 \mathrm{~g}$ of a mixture of the corresponding $(E)$ - $\alpha, \beta$-enone and starting aldehyde in 7:1 ratio. It was used in the next step directly.

(E)-6-hydroxyhept-3-en-2-one (2h): The product was prepared
$\mathrm{Me}$ according to GP4 starting from (E)-6-((tert-butyldimethylsilyl)oxy)hept-3en-2-one $(1.1 \mathrm{~g}, 4.0 \mathrm{mmol})$. The crude was purified by flash chromatography on silica gel (Pentane/Diethyl ether $=2: 1$ ) to afford $\mathbf{2 h}$ in $68 \%$ yield $(350 \mathrm{mg}$ ) as a colourless oil. All spectroscopic analyses were in agreement with those reported in the literature. ${ }^{13}$ 


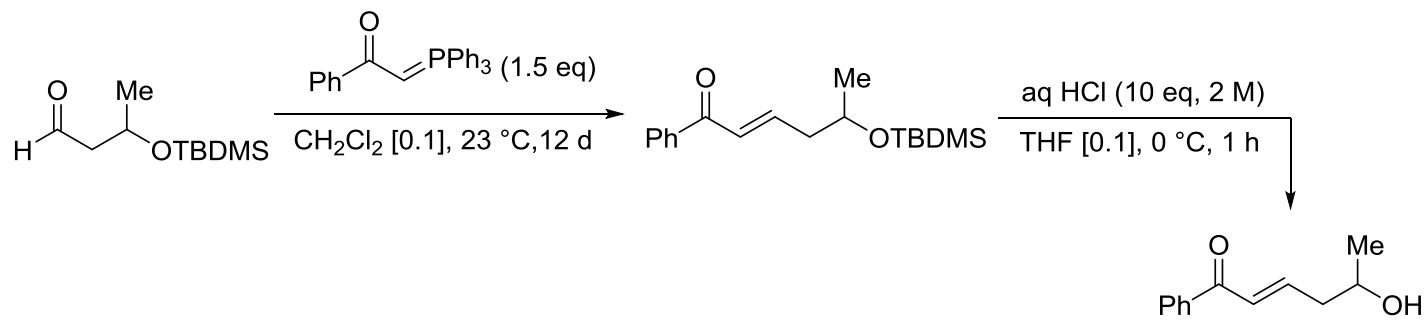

(2i)

Figure S9: Synthesis of $(E)$-5-hydroxy-1-phenylhex-2-en-1-one (2i)<smiles>CCOC(C)CCC=CC(=O)c1ccccc1</smiles>

(E)-5-((tert-butyldimethylsilyl)oxy)-1-phenylhex-2-en-1-one: 1phenyl-2-(triphenyl- $\lambda^{5}$-phosphanylidene)ethan-1-one $(1.91 \mathrm{~g}, 5.2$ mmol) was added to a solution of 3-((tert-butyldimethylsilyl)oxy)butanal (700 mg, $3.5 \mathrm{mmol})$ in $\mathrm{CH}_{2} \mathrm{Cl}_{2}(35 \mathrm{~mL})$ at $0{ }^{\circ} \mathrm{C}$ and warmed to room temperature. After 12 days, water $(40 \mathrm{~mL})$ was added and the organic phase was extracted with $\mathrm{CH}_{2} \mathrm{Cl}_{2}(3 \times 30 \mathrm{~mL})$, dried over anhydrous $\mathrm{Na}_{2} \mathrm{SO}_{4}$ and concentrated in vacuo. The crude mixture was passed through a pad of silica gel and concentrated to give an oil which was used in the next step without further purification.

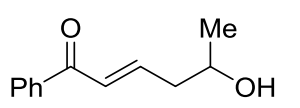

(E)-5-hydroxy-1-phenylhex-2-en-1-one (2i): The mixture obtained in the The crude mixture was purified by flash chromatography on silica gel (Pentane/EtOAc =1:2) to afford $\mathbf{2} \mathbf{i}$ in $71 \%$ yield $\left(230 \mathrm{mg}\right.$ ) as a colourless oil. $\mathbf{R}_{\mathbf{f}}=0.2$ ( $n$-Pentane/Diethyl ether $=$ 1:2). IR (neat): $v\left(\mathrm{~cm}^{-1}\right)=3416,2970,1666,1617,977 .{ }^{1} \mathbf{H} \mathbf{~ N M R}\left(400 \mathrm{MHz}, \mathrm{CD}_{2} \mathrm{Cl}_{2}\right) \delta$ (ppm) $1.24\left(\mathrm{~d},{ }^{3} \mathrm{~J}_{\mathrm{HH}}=6.3 \mathrm{~Hz}, 3 \mathrm{H}, \mathrm{CH}_{3} \mathrm{CH}\right), 1.58-1.65(\mathrm{~m}, 1 \mathrm{H}, \mathrm{CHOH}), 2.40-2.54(\mathrm{~m}, 2 \mathrm{H}$, $\left.\mathrm{CH}_{2} \mathrm{CHOH}\right), 4.00(\mathrm{~m}, 1 \mathrm{H}, \mathrm{CHOH}), 6.89-7.14(\mathrm{~m}, 2 \mathrm{H}, \mathrm{CH}=\mathrm{CH}), 7.47(\mathrm{~m}, 2 \mathrm{H}, \mathrm{CH}-\mathrm{Ar}), 7.53-$ $7.62(\mathrm{~m}, 1 \mathrm{H}, \mathrm{CH}-\mathrm{Ar}), 7.84-8.05(\mathrm{~m}, 2 \mathrm{H}, \mathrm{CH}-\mathrm{Ar}) .{ }^{13} \mathrm{C}\left\{{ }^{1} \mathrm{H}\right\} \mathrm{NMR}\left(101 \mathrm{MHz}, \mathrm{CD}_{2} \mathrm{Cl}_{2}\right) \delta(\mathrm{ppm})$ $23.1\left(\mathrm{CH}_{3} \mathrm{CH}\right), 42.3\left(\mathrm{CH}_{2} \mathrm{CHOH}\right), 66.7(\mathrm{CHOH}), 128.1(\mathrm{PhCOCH}=\mathrm{CH}), 128.4(\mathrm{CH}-\mathrm{Ar}), 128.5$ ( $\mathrm{CH}-\mathrm{Ar}), 132.6(\mathrm{CH}-\mathrm{Ar}), 137.8\left(\mathrm{C}_{\text {quat }}\right), 145.4\left(\mathrm{CH}=\mathrm{CHCH}_{2}\right), 190.1(\mathrm{C}=\mathrm{O})$. LRMS (IC) found $[\mathrm{M}+\mathrm{H}]^{+}=191 ;\left[\mathrm{M}+\mathrm{NH}_{4}\right]^{+}=208$. 


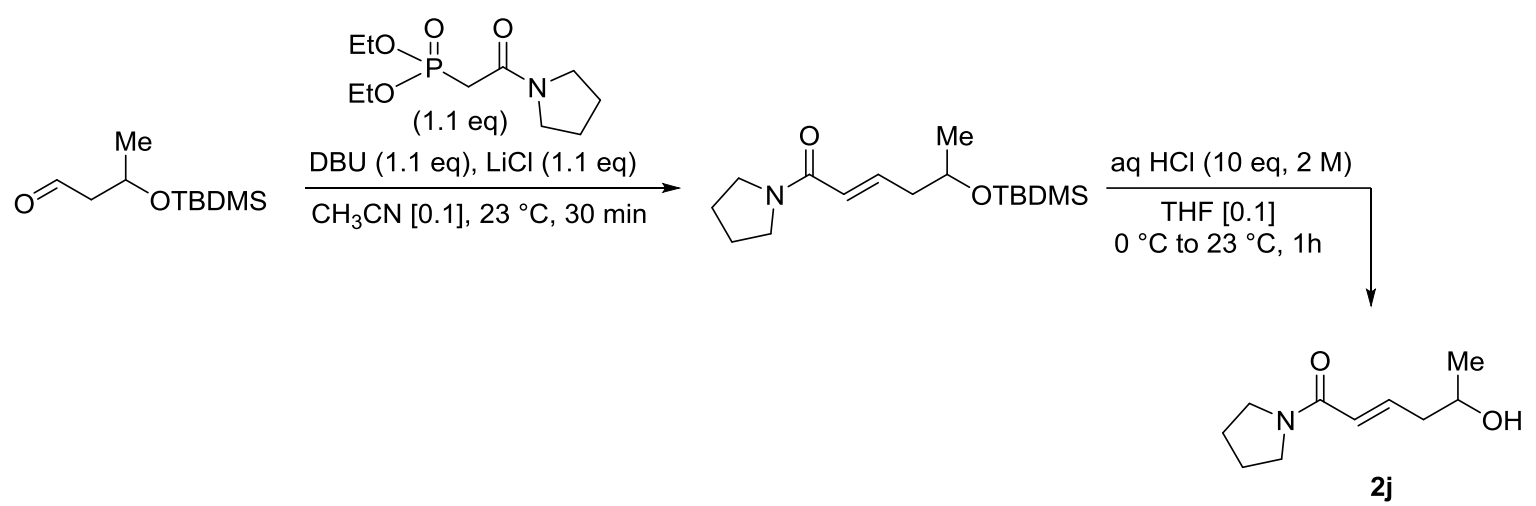

Figure S10: Synthesis of (E)-5-hydroxy-1-(pyrrolidin-1-yl)hex-2-en-1-one (2j)

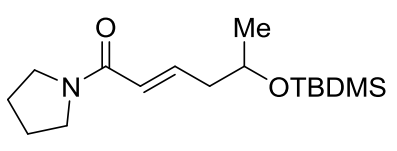

(E)-5-((tert-butyldimethylsilyl)oxy)-1-(pyrrolidin-1-yl)hex-2-en-

1-one: The reaction was run according to GP2 starting from 3((tert-butyldimethylsilyl)oxy)butanal (728 mg, $3.6 \mathrm{mmol})$. The crude mixture was purified by flash chromatography on silica gel (Pentane/Diethyl ether $=1: 2$ ) to afford the desired product in $77 \%$ yield $(820 \mathrm{mg})$ as a colourless oil. $\mathbf{R}_{\mathbf{f}}=0.2$ (Pentane/Diethyl ether = 1:2). IR (neat): $v\left(\mathrm{~cm}^{-1}\right)=2956,2929,1664,1620,1427,835 .{ }^{1} \mathbf{H}$ NMR $\left(400 \mathrm{MHz}, \mathrm{CDCl}_{3}\right) \delta(\mathrm{ppm}) 0.05$ (s, 6H, $\left.\left(\mathrm{CH}_{3}\right)_{2} \mathrm{Si}\right), 0.88$ (s, 9H, $\left.\left(\mathrm{CH}_{3}\right)_{3} \mathrm{CSi}\right), 1.15$ (d, ${ }^{3} \mathrm{~J}_{\mathrm{HH}}$ $\left.=6.1 \mathrm{~Hz}, 3 \mathrm{H}, \mathrm{CH}_{3} \mathrm{CHOTBDMS}\right), 1.74-2.01\left(\mathrm{~m}, 4 \mathrm{H},\left(\mathrm{CH}_{2} \mathrm{CH}_{2}\right)_{2} \mathrm{~N}\right), 2.19-2.46(\mathrm{~m}, 2 \mathrm{H}$, $\mathrm{CH}_{2} \mathrm{CHOTBDMS}$ ), 3.52 (t, $\left.{ }^{3} \mathrm{~J}_{\mathrm{HH}}=6.6 \mathrm{~Hz}, 4 \mathrm{H},\left(\mathrm{CH}_{2} \mathrm{CH}_{2}\right)_{2} \mathrm{~N}\right), 3.92(\mathrm{~m}, 1 \mathrm{H}, \mathrm{CHOTBDMS}), 6.12$ $\left(\mathrm{dt},{ }^{3} J_{\mathrm{HH}}=15.1,{ }^{4} \mathrm{~J}_{\mathrm{HH}}=1.4 \mathrm{~Hz}, 1 \mathrm{H}, \mathrm{CH}=\mathrm{CHCH}_{2}\right), 6.87\left(\mathrm{dt},{ }^{3} J_{\mathrm{HH}}=15.1,7.6 \mathrm{~Hz}, 1 \mathrm{H}\right.$, $\left.\mathrm{CH}=\mathrm{CHCH}_{2}\right) .{ }^{13} \mathrm{C}\left\{{ }^{1} \mathrm{H}\right\}$ NMR $\left(101 \mathrm{MHz}, \mathrm{CDCl}_{3}\right) \delta(\mathrm{ppm})-4.6\left(\left(\mathrm{CH}_{3}\right)_{2} \mathrm{Si}\right),-4.4\left(\left(\mathrm{CH}_{3}\right)_{2} \mathrm{Si}\right), 18.3$ $\left(\left(\mathrm{CH}_{3}\right)_{3} \mathrm{CSi}\right), 23.8\left(\mathrm{CH}_{3} \mathrm{CH}\right), 24.4\left(\left(\mathrm{CH}_{2} \mathrm{CH}_{2}\right)_{2} \mathrm{~N}\right), 26.0\left(\left(\mathrm{CH}_{3}\right)_{3} \mathrm{CSi}\right), 43.0\left(\mathrm{CH}_{2} \mathrm{CHOTBDMS}\right)$, $46.0\left(\left(\mathrm{CH}_{2} \mathrm{CH}_{2}\right)_{2} \mathrm{~N}\right), 46.6\left(\left(\mathrm{CH}_{2} \mathrm{CH}_{2}\right)_{2} \mathrm{~N}\right), 67.9(\mathrm{CHOTBDMS}), 123.8\left(\mathrm{CH}=\mathrm{CHCH}_{2}\right), 142.3$ $\left(\mathrm{CH}=\mathrm{CHCH}_{2}\right), 164.8(\mathrm{C}=\mathrm{O})$. LRMS (IC) found $[\mathrm{M}+\mathrm{H}]^{+}=299$.

(E)-5-hydroxy-1-(pyrrolidin-1-yl)hex-2-en-1-one (2j): The reaction was run according to GP4 starting from (E)-5-((tert$\mathrm{mmol})$. The crude mixture was purified by flash chromatography on silica gel (EtOAc/MeOH $=10: 1)$ to give $\mathbf{2} \mathbf{j}$ in $55 \%$ yield $(150 \mathrm{mg})$ as a light yellow oil. $\mathbf{R}_{\mathbf{f}}=0.2(\mathrm{EtOAc} / \mathrm{MeOH}=10: 1)$. IR (neat): $v\left(\mathrm{~cm}^{-1}\right)=3396,2972,2876,1661,1601,1442,1126,980 .{ }^{1} \mathbf{H}$ NMR $(400 \mathrm{MHz}$, $\left.\mathrm{CDCl}_{3}\right) \delta(\mathrm{ppm}) 1.10\left(\mathrm{~d},{ }^{3} \mathrm{~J}_{\mathrm{HH}}=6.2 \mathrm{~Hz}, 3 \mathrm{H}, \mathrm{CH}_{3} \mathrm{CHOH}\right), 1.54$ (bs, $\left.1 \mathrm{H}, \mathrm{OH}\right), 1.67-1.89(\mathrm{~m}$, $\left.4 \mathrm{H},\left(\mathrm{CH}_{2} \mathrm{CH}_{2}\right)_{2} \mathrm{~N}\right), 2.17-2.32\left(\mathrm{~m}, 2 \mathrm{H}, \mathrm{CH}_{2} \mathrm{CHOH}\right), 3.34\left(\mathrm{t},{ }^{3} \mathrm{~J}_{\mathrm{HH}}=6.9 \mathrm{~Hz}, 2 \mathrm{H},\left(\mathrm{CH}_{2} \mathrm{CH}_{2}\right)_{2} \mathrm{~N}\right)$, $3.40\left(\mathrm{t},{ }^{3} \mathrm{~J}_{\mathrm{HH}}=6.8 \mathrm{~Hz}, 3 \mathrm{H},\left(\mathrm{CH}_{2} \mathrm{CH}_{2}\right)_{2} \mathrm{~N}\right), 3.76-3.91(\mathrm{~m}, 1 \mathrm{H}, \mathrm{CHOH}), 6.10\left(\mathrm{dt},{ }^{3} J_{\mathrm{HH}}=15.1\right.$, $\left.{ }^{4} J_{\mathrm{HH}}=1.5 \mathrm{~Hz}, 1 \mathrm{H}, \mathrm{CH}=\mathrm{CHCH}_{2}\right), 6.70\left(\mathrm{dt},{ }^{3} \mathrm{~J}_{\mathrm{HH}}=15.1,7.5 \mathrm{~Hz}, 1 \mathrm{H}, \mathrm{CH}=\mathrm{CHCH}_{2}\right) \cdot{ }^{13} \mathrm{C}\left\{{ }^{1} \mathrm{H}\right\} \mathrm{NMR}$ $\left(101 \mathrm{MHz}, \mathrm{CDCl}_{3}\right) \delta(\mathrm{ppm}) 23.4\left(\mathrm{CH}_{3} \mathrm{CH}\right), 24.7\left(\left(\mathrm{CH}_{2} \mathrm{CH}_{2}\right)_{2} \mathrm{~N}\right), 26.5\left(\left(\mathrm{CH}_{2} \mathrm{CH}_{2}\right)_{2} \mathrm{~N}\right), 42.5$ 
$\left(\mathrm{CH}_{2} \mathrm{CHOH}\right), 46.1\left(\left(\mathrm{CH}_{2} \mathrm{CH}_{2}\right)_{2} \mathrm{~N}\right), 46.9\left(\left(\mathrm{CH}_{2} \mathrm{CH}_{2}\right)_{2} \mathrm{~N}\right), 67.2(\mathrm{CHOH}), 125.0\left(\mathrm{CH}=\mathrm{CHCH}_{2}\right)$, $141.4\left(\mathrm{CH}=\mathrm{CHCH}_{2}\right), 164.7(\mathrm{C}=\mathrm{O})$. LRMS (IC) found $[\mathrm{M}+\mathrm{H}]^{+}=184$.

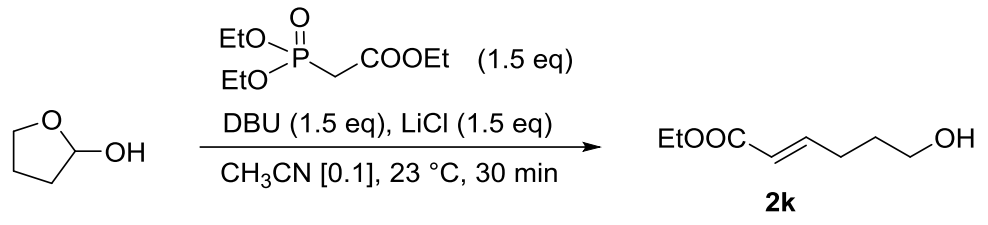

Figure S11: Synthesis of Ethyl (E)-6-hydroxyhex-2-enoate (2k)

EtooC $2 \mathrm{OH}$ Ethyl (E)-6-hydroxyhex-2-enoate (2k): The reaction was run according to GP2 starting from tetrahydrofuran-2-ol (15.6 mmol, $1.4 \mathrm{~g}$ ). The crude mixture was purified by flash chromatography on silica gel (Pentane/Diethyl ether $=1: 2$ ) affording $\mathbf{2 k}$ in $80 \%$ yield $(1.9 \mathrm{~g})$. All spectroscopic analyses were in agreement with those reported in the literature. ${ }^{14}$

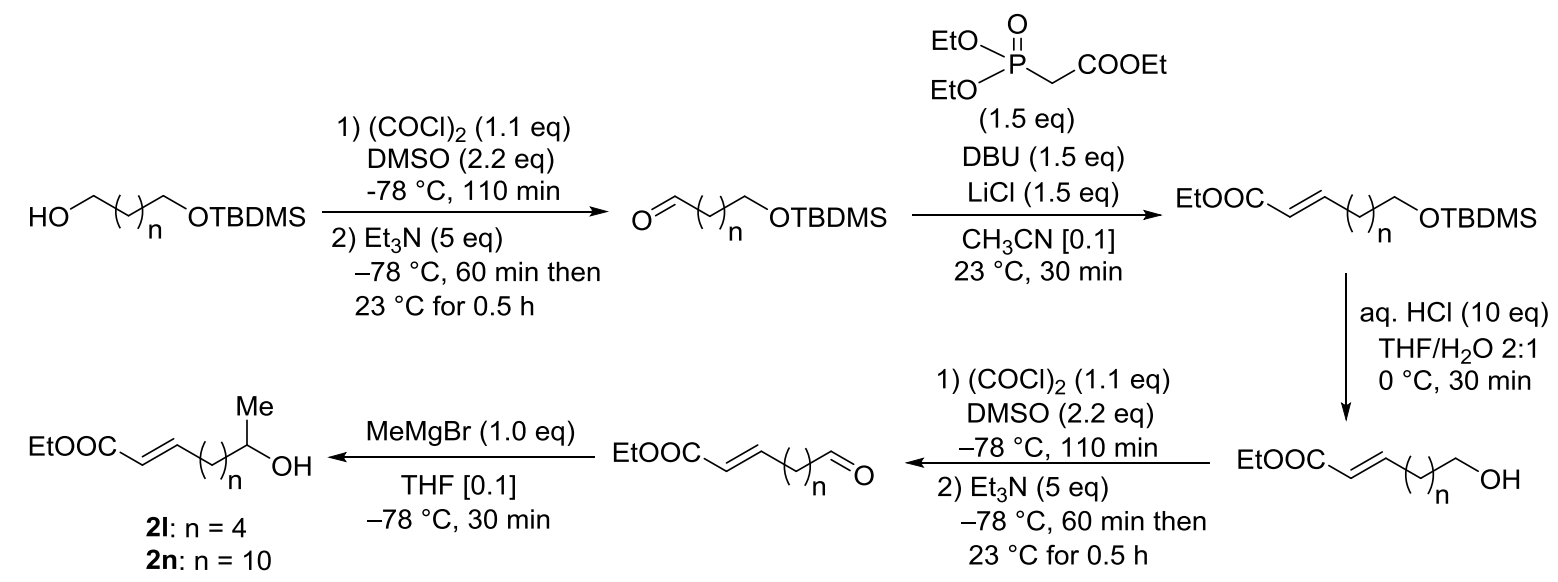

Figure S12: Synthesis of Ethyl $(E)-8$-hydroxynon-2-enoate (2I) and Ethyl (E)-14-

hydroxypentadec-2-enoate (2n)

General procedure for Swern oxidation (GP5): To a solution of oxalyl chloride (1.1 eq) in $\mathrm{CH}_{2} \mathrm{Cl}_{2}(0.3 \mathrm{M})$ at $-78^{\circ} \mathrm{C}$, a solution of DMSO (2.2 eq) was added dropwise and stirred for 50 minutes at the same temperature. A solution of the appropriate alcohol (1.0 eq) in $\mathrm{CH}_{2} \mathrm{Cl}_{2}$ $(1.0 \mathrm{M})$ was slowly added and stirred at the same temperature for 60 minutes. $\mathrm{Next}, \mathrm{Et}_{3} \mathrm{~N}$ (5 eq) was added at $-78^{\circ} \mathrm{C}$ and stirred for $1 \mathrm{~h}$, then warmed to room temperature and stirred for an additional 30 minutes. The reaction mixture was quenched with water, extracted with $\mathrm{CH}_{2} \mathrm{Cl}_{2}$ (3 times), dried over $\mathrm{Na}_{2} \mathrm{SO}_{4}$ and concentrated in vacuo. The crude mixture was filtered through a small pad of silica gel (Pentane/EtOAc $=10: 1)$ to give the desired product which was used in the next step without any further purification. 
Eto

Ethyl (E)-8-((tert-butyldimethylsilyl)oxy)oct-2-enoate: 6-

(tert-Butyldimethylsiloxy)-1-hexanol ${ }^{15}(6.5 \mathrm{mmol})$ was oxidized to the corresponding aldehyde according to GP5. The following olefination was performed according to GP2. The crude mixture was purified by flash chromatography on silica gel (Pentane/Diethyl ether $=10: 1$ ) to give the desired product as a colourless oil in $81 \%$ yield $(1.65 \mathrm{~g})$ over 2 steps. All spectroscopic analyses were in agreement with those reported in the literature. ${ }^{16}$

Ethyl (E)-8-hydroxyoct-2-enoate: The reaction was run
according to GP4 starting from ethyl (E)-8-((tertbutyldimethylsilyl)oxy)oct-2-enoate $(1.65 \mathrm{~g}, 5.5 \mathrm{mmol})$. The crude mixture was purified by flash chromatography (Pentane/Diethyl ether $=1: 2$ ) to give the free alcohol in $78 \%$ yield $(800$ $\mathrm{mg}$ ) as a colourless oil. All spectroscopic analyses were in agreement with those reported in the literature. ${ }^{17}$

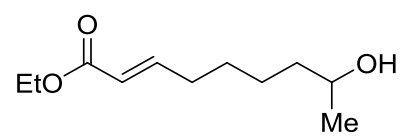

Ethyl (E)-8-hydroxynon-2-enoate (2l): Ethyl $(E)$-8-hydroxyoct-2enoate (276 mg, $1.5 \mathrm{mmol}$ ) was oxidized according to GP5. A THF solution $(10 \mathrm{~mL})$ of the resulting aldehyde was cooled to $-78^{\circ} \mathrm{C}$ and a solution of $\mathrm{MeMgBr}(1.5 \mathrm{mmol})$ was added dropwise and stirred at the same temperature for 30 minutes. The reaction was quenched with saturated aqueous $\mathrm{NH}_{4} \mathrm{Cl}$ solution $(10 \mathrm{~mL})$, extracted with diethyl ether $(3 \times 10 \mathrm{~mL})$, dried over anhydrous $\mathrm{Na}_{2} \mathrm{SO}_{4}$, and concentrated in vacuo. The crude mixture was purified by flash chromatography on silica gel (Pentane/Diethyl ether $=1: 2)$ to give 21 in $40 \%$ yield $(120 \mathrm{mg}$ ) over 2 steps. All spectroscopic analyses were in agreement with those reported in the literature. ${ }^{18}$

Eto ${ }^{\text {Ethyl }} \begin{aligned} & (E)-14-((t e r t- \\ & \text { butyldimethylsilyl)oxy)tetradec-2-enoate: } 12-\end{aligned}$ tert-Butyldimethylsilyloxydodecan-1-ol $(2.8 \mathrm{mmol})$ was oxidized to the corresponding aldehyde ${ }^{19}$ according to GP5. The following olefination was performed according to GP2. The crude mixture was purified by flash chromatography on silica gel to give the desired product as a colourless oil in $86 \%$ yield $\left(920 \mathrm{mg}\right.$ ) over 2 steps. $\mathbf{R}_{\mathbf{f}}=0.3$ ( $n$-Pentane/Diethyl ether = 10:1). IR (neat): $v(\mathrm{~cm}-1)=2927,2855,1723,1097,834,775 .{ }^{1} \mathbf{H}$ NMR $(400 \mathrm{MHz}$, $\left.\mathrm{CDCl}_{3}\right) \delta(\mathrm{ppm}) 0.04\left(\mathrm{~s}, 6 \mathrm{H},\left(\mathrm{CH}_{3}\right)_{2} \mathrm{Si}\right), 0.89\left(\mathrm{~s}, 9 \mathrm{H},\left(\mathrm{CH}_{3}\right)_{3} \mathrm{CSi}\right), 1.22-1.37(\mathrm{~m}, 17 \mathrm{H}$, $\left.\mathrm{CH}_{3} \mathrm{CH}_{2} \mathrm{O} ; \quad\left(\mathrm{CH}_{2}\right)_{7} \mathrm{CH}_{2} \mathrm{CH}_{2} \mathrm{OTBDMS}\right), \quad 1.41-1.52 \quad\left(\mathrm{~m}, \quad 4 \mathrm{H}, \quad \mathrm{CH}=\mathrm{CHCH}_{2} \mathrm{CH}_{2}\right.$; $\mathrm{CH}_{2} \mathrm{CH}_{2} \mathrm{OTBDMS}$ ), 2.19 (qd, ${ }^{3} \mathrm{~J}_{\mathrm{HH}}=7.1,{ }^{4} \mathrm{~J}_{\mathrm{HH}}=1.5 \mathrm{~Hz}, 2 \mathrm{H}, \mathrm{CH}=\mathrm{CHCH}_{2}$ ), $3.59\left(\mathrm{t},{ }^{3} \mathrm{~J}_{\mathrm{HH}}=6.6\right.$ 
$\mathrm{Hz}, 2 \mathrm{H}, \mathrm{CH}_{2} \mathrm{OTBDMS}$ ), 4.18 (q, ${ }^{3} \mathrm{~J}_{\mathrm{HH}}=7.1 \mathrm{~Hz}, 2 \mathrm{H}, \mathrm{CH}_{3} \mathrm{CH}_{2} \mathrm{O}$ ), 5.81 (dt, ${ }^{3} \mathrm{~J}_{\mathrm{HH}}=15.6,{ }^{4} J_{\mathrm{HH}}=$ $\left.1.5 \mathrm{~Hz}, 1 \mathrm{H}, \mathrm{CH}=\mathrm{CHCH}_{2}\right), 6.96\left(\mathrm{dt},{ }^{3} J_{\mathrm{HH}}=15.6,6.9 \mathrm{~Hz}, 1 \mathrm{H}, \mathrm{CH}=\mathrm{CHCH}_{2}\right) .{ }^{13} \mathrm{C}\left\{{ }^{1} \mathrm{H}\right\} \mathrm{NMR}(101$ $\left.\left.\mathrm{MHz}, \mathrm{CDCl}_{3}\right) \delta(\mathrm{ppm})-5.1\left(\mathrm{CH}_{3}\right)_{2} \mathrm{Si}\right), 14.4\left(\mathrm{CH}_{3} \mathrm{CH}_{2} \mathrm{O}\right), 18.5\left(C_{\text {quat }}\right), 25.9\left(\mathrm{CH}_{2}\right), 26.1$ $\left.\left(\mathrm{CH}_{3}\right)_{3} \mathrm{CSi}\right), 28.2\left(\mathrm{CH}=\mathrm{CHCH}_{2} \mathrm{CH}_{2}\right), 29.31\left(\mathrm{CH}_{2}\right), 29.5\left(\mathrm{CH}_{2}\right), 29.6\left(\mathrm{CH}_{2}\right), 29.7\left(\mathrm{CH}_{2}\right), 29.7$ $\left(\mathrm{CH}_{2}\right), 29.8\left(\mathrm{CH}_{2}\right), 32.4\left(\mathrm{CH}=\mathrm{CHCH}_{2}\right), 33.0\left(\mathrm{CH}_{2} \mathrm{CH}_{2} \mathrm{OTBDMS}\right), \quad 60.3\left(\mathrm{CH}_{3} \mathrm{CH}_{2} \mathrm{OCO}\right) 63.5$ ( $\left.\mathrm{CH}_{2} \mathrm{OTBDMS}\right), 121.3\left(\mathrm{CH}=\mathrm{CHCH}_{2}\right), 149.7\left(\mathrm{CH}=\mathrm{CHCH}_{2}\right), 167.0(\mathrm{C}=\mathrm{O})$. LRMS (IC) found $[\mathrm{M}+\mathrm{H}]^{+}=386$.

Eto $\begin{aligned} & \text { Ethyl }(E) \text {-14-hydroxytetradec-2-enoate: The } \\ & \text { reaction was run according to GP4 starting from ethyl }\end{aligned}$ (E)-14-((tert-butyldimethylsilyl)oxy)tetradec-2-enoate (920 mg, $2.4 \mathrm{mmol})$. The crude mixture was purified by flash chromatography on silica gel (Pentane/Diethyl ether $=2: 1$ ) to give the free alcohol in $99 \%$ yield $\left(660 \mathrm{mg}\right.$ ) as a colourless oil. $\mathbf{R}_{\mathbf{f}}=0.5$ (Pentane/Diethyl ether = 1:2). IR (neat): $v\left(\mathrm{~cm}^{-1}\right)=3419,2925,2854,1720,1266,1179,1042 .{ }^{1} \mathbf{H}$ NMR $\left(400 \mathrm{MHz}, \mathrm{CDCl}_{3}\right)$ $\delta$ (ppm) $1.17-1.40\left(\mathrm{~m}, 17 \mathrm{H},\left(\mathrm{CH}_{2}\right)_{7} \mathrm{CH}_{2} \mathrm{CH}_{2} \mathrm{OH} ; \mathrm{CH}_{3} \mathrm{CH}_{2} \mathrm{O}\right), 1.40-1.50\left(\mathrm{CH}=\mathrm{CHCH}_{2} \mathrm{CH}_{2}\right)$, $1.50-1.66\left(\mathrm{CH}_{2} \mathrm{CH}_{2} \mathrm{OH}\right), 2.16-2.22\left(\mathrm{~m}, 2 \mathrm{H}, \mathrm{CH}=\mathrm{CHCH}_{2}\right), 3.64\left(\mathrm{t},{ }^{3} J_{\mathrm{HH}}=6.6 \mathrm{~Hz}, 2 \mathrm{H}\right.$, $\mathrm{CH}_{2} \mathrm{OH}$ ), 4.18 (q, ${ }^{3} J_{\mathrm{HH}}=7.1 \mathrm{~Hz}, 2 \mathrm{H}, \mathrm{CH}_{3} \mathrm{CH}_{2} \mathrm{O}$ ), 5.81 (dt, ${ }^{3} J_{\mathrm{HH}}=15.6,{ }^{4} J_{\mathrm{HH}}=1.6 \mathrm{~Hz}, 1 \mathrm{H}$, $\left.\mathrm{CH}=\mathrm{CHCH}_{2}\right), 6.96\left(\mathrm{dt},{ }^{3} \mathrm{JHH}_{\mathrm{HH}}=15.6,6.9 \mathrm{~Hz}, 1 \mathrm{H}, \mathrm{CH}=\mathrm{CHCH}_{2}\right) \cdot{ }^{13} \mathrm{C}\left\{{ }^{1} \mathrm{H}\right\} \mathbf{N M R}\left(101 \mathrm{MHz}, \mathrm{CDCl}_{3}\right)$ $\delta$ (ppm) $14.4\left(\mathrm{CH}_{3} \mathrm{CH}_{2} \mathrm{O}\right), 25.9\left(\mathrm{CH}_{2} \mathrm{CH}_{2} \mathrm{CH}_{2} \mathrm{OH}\right), 28.2\left(\mathrm{CH}_{2} \mathrm{CH}_{2} \mathrm{CH}=\mathrm{CH}_{2}\right), 29.3\left(\mathrm{CH}_{2}\right), 29.5$ $\left(\mathrm{CH}_{2}\right)$, $29.6\left(\mathrm{CH}_{2}\right)$, $29.6\left(\mathrm{CH}_{2}\right), 29.7\left(\mathrm{CH}_{2}\right), 29.7\left(\mathrm{CH}_{2}\right)$, $32.3(\mathrm{CH}=\mathrm{CHCH}), 33.0\left(\mathrm{CH}_{2} \mathrm{CH}_{2} \mathrm{OH}\right)$, $60.3\left(\mathrm{CH}_{3} \mathrm{CH}_{2} \mathrm{O}\right), 63.3\left(\mathrm{CH}_{2} \mathrm{OH}\right), 121.4\left(\mathrm{CH}=\mathrm{CHCH}_{2}\right), 149.7\left(\mathrm{CH}=\mathrm{CHCH}_{2}\right), 167.0(\mathrm{C}=\mathrm{O})$. LRMS (IC) found $[\mathrm{M}+\mathrm{H}]^{+}=271$.

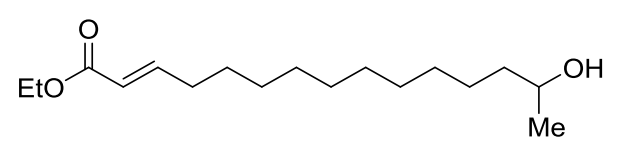

Ethyl (E)-14-hydroxypentadec-2-enoate (2n): The product was oxidized according to GP5 starting from ethyl (E)-14-hydroxytetradec-2-enoate (268 mg, 1.0 $\mathrm{mmol})$. A THF solution $\left(10 \mathrm{~mL}\right.$ ) of the resulting aldehyde was cooled to $-78^{\circ} \mathrm{C}$ and a solution of $\mathrm{MeMgBr}(1.0 \mathrm{mmol})$ was added dropwise. The mixture was stirred at the same temperature for 30 minutes then quenched with a saturated aqueous $\mathrm{NH}_{4} \mathrm{Cl}$ solution (10 $\mathrm{mL})$, extracted with diethyl ether $(3 \times 10 \mathrm{~mL})$, dried over anhydrous $\mathrm{Na}_{2} \mathrm{SO}_{4}$ and concentrated in vacuo. The residue was purified by column chromatography on silica gel (Pentane/Diethyl ether from 2:1 to $1: 1$ ) to give $2 \mathrm{n}$ as a colourless oil in $45 \%$ yield $(130 \mathrm{mg}$ ) over 2 steps. $\mathbf{R}_{\mathbf{f}}=0.5$ (Pentane/Diethyl ether $=1: 2$ ). IR (neat): $v\left(\mathrm{~cm}^{-1}\right)=3473,3436,2925$, 2854, 1720, 1180, 1042, 980. ${ }^{1} \mathrm{H}$ NMR $\left(400 \mathrm{MHz}, \mathrm{CDCl}_{3}\right) \delta(\mathrm{ppm}) 1.19\left(\mathrm{~d},{ }^{3} \mathrm{~J}_{\mathrm{HH}}=6.2 \mathrm{~Hz}, 3 \mathrm{H}\right.$, $\left.\mathrm{CH}_{3} \mathrm{CHOH}\right), 1.24-1.50\left(\mathrm{~m}, 22 \mathrm{H},(\mathrm{CH})_{9} \mathrm{CHOH} ; \mathrm{CH}_{3} \mathrm{CH}_{2} \mathrm{O} ; \mathrm{OH}\right), 2.16-2.22(\mathrm{~m}, 2 \mathrm{H}$, 
$\mathrm{CH}=\mathrm{CHCH}_{2}$ ), $3.75-3.83(\mathrm{~m}, 1 \mathrm{H}, \mathrm{CHOH}), 4.18\left(\mathrm{q},{ }^{3} \mathrm{JHH}_{\mathrm{HH}}=7.1 \mathrm{~Hz}, 2 \mathrm{H}, \mathrm{CH}_{3} \mathrm{CH}_{2} \mathrm{O}\right), 5.81$ (dt, $\left.{ }^{3} J_{\mathrm{HH}}=15.6,{ }^{4} J_{\mathrm{HH}}=1.6 \mathrm{~Hz}, 1 \mathrm{H}, \mathrm{CH}=\mathrm{CHCH}_{2}\right), 6.96\left(\mathrm{dt},{ }^{3} \mathrm{~J}_{\mathrm{HH}}=15.6,6.9 \mathrm{~Hz}, 1 \mathrm{H}, \mathrm{CH}=\mathrm{CHCH}_{2}\right)$. ${ }^{13} \mathrm{C}\left\{{ }^{1} \mathrm{H}\right\}$ NMR $\left(101 \mathrm{MHz}, \mathrm{CDCl}_{3}\right) \delta(\mathrm{ppm}) 14.4\left(\mathrm{CH}_{3} \mathrm{CH}_{2} \mathrm{O}\right), 23.7\left(\mathrm{CH}_{3} \mathrm{CHOH}\right), 25.9$ $\left(\mathrm{CH}_{2} \mathrm{CH}_{2} \mathrm{CHOH}\right), 28.2\left(\mathrm{CH}_{2} \mathrm{CH}_{2} \mathrm{CH}=\mathrm{CH}_{2}\right), 29.3\left(\mathrm{CH}_{2}\right), 29.5\left(\mathrm{CH}_{2}\right), 29.6\left(\mathrm{CH}_{2}\right), 29.7\left(\mathrm{CH}_{2}\right)$, $29.7\left(\mathrm{CH}_{2}\right), 29.8\left(\mathrm{CH}_{2}\right), 32.3\left(\mathrm{CH}=\mathrm{CHCH}_{2}\right), 39.5\left(\mathrm{CH}_{2} \mathrm{CHOH}\right), 60.3\left(\mathrm{CH}_{3} \mathrm{CH}_{2} \mathrm{O}\right), 68.3$ $(\mathrm{CHOH}), 121.4\left(\mathrm{CH}=\mathrm{CHCH}_{2}\right), 149.6\left(\mathrm{CH}=\mathrm{CHCH}_{2}\right), 166.96(\mathrm{C}=\mathrm{O})$. LRMS (IC) found $[\mathrm{M}+\mathrm{H}]^{+}$ $=285$.

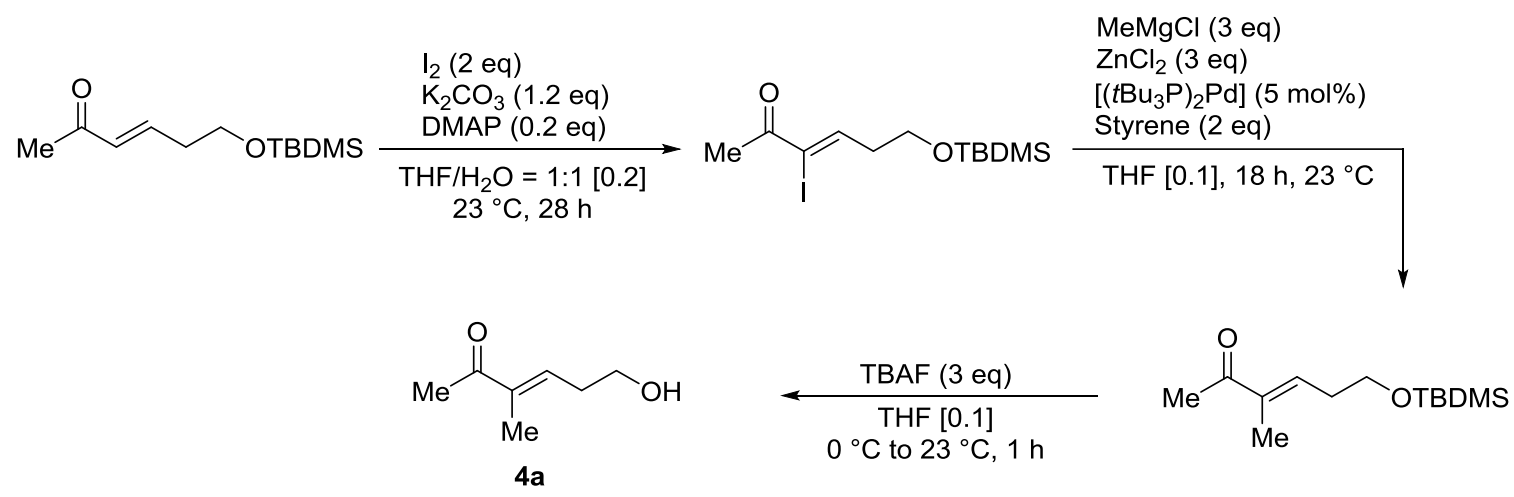

Figure S13: Synthesis of $(E)$-6-hydroxy-3-methylhex-3-en-2-one (4a)<smiles>CCOCC/C=C(\I)C(C)=O</smiles>

(Z)-6-((tert-butyldimethylsilyl)oxy)-3-iodohex-3-en-2-one: To a solution of $(E)-6$-((tert-butyldimethylsilyl)oxy)hex-3-en-2-one ${ }^{5}(5.9 \mathrm{~g}$, $25.9 \mathrm{mmol})$ in $\mathrm{THF} / \mathrm{H}_{2} \mathrm{O} 1: 1(130 \mathrm{~mL})$ were sequentially added $\mathrm{K}_{2} \mathrm{CO}_{3}(4.3 \mathrm{~g}, 1.2 \mathrm{mmol}), \mathrm{I}_{2}(13.1 \mathrm{~g}, 51.8 \mathrm{mmol}$ ) and 4-dimethylaminopyridine (DMAP) (630 $\mathrm{mg}, 5.2 \mathrm{mmol})$. After $28 \mathrm{~h}$, the reaction mixture was diluted with diethyl ether $(300 \mathrm{~mL})$ and washed with a saturated aqueous $\mathrm{Na}_{2} \mathrm{~S}_{2} \mathrm{O}_{3}$ solution $(600 \mathrm{~mL})$. The mixture was dried over $\mathrm{Na}_{2} \mathrm{SO}_{4}$, filtered on a pad of silical gel and concentrated in vacuo. The crude mixture was purified by flash chromatography (Pentane/Diethyl ether $=30: 1$ ) to afford the corresponding $\alpha$-iodoenone in $44 \%$ yield $(4.0 \mathrm{~g})$. Note: the remaining starting material $(25 \%, 1.5 \mathrm{~g})$ could be recovered. $\mathbf{R}_{\mathrm{f}}=0.3$ (Pentane/Diethyl ether $\left.=10: 1\right)$. IR (neat): $v\left(\mathrm{~cm}^{-1}\right)=2953,2929,2857$, 2130, 1681, 1092, 832, 774, 714. ${ }^{1} \mathrm{H}$ NMR $\left(400 \mathrm{MHz}, \mathrm{CDCl}_{3}\right) \delta(\mathrm{ppm}) 0.08$ (s, 6H, $\left.\left(\mathrm{CH}_{3}\right)_{2} \mathrm{Si}\right)$, 0.90 (s, 9H, $\left(\mathrm{CH}_{3}\right)_{3} \mathrm{C}$ ), 2.51 (s, 3H, $\mathrm{CH}_{3} \mathrm{CO}$ ), 2.64 (td, ${ }^{3} \mathrm{~J}_{\mathrm{HH}}=6.2 \mathrm{~Hz}, 6.5 \mathrm{~Hz}, 2 \mathrm{H}, \mathrm{CH}_{2}$ ), 3.80 (t, $\left.{ }^{3} J_{\mathrm{HH}}=6.2 \mathrm{~Hz}, 2 \mathrm{H}, \mathrm{CH}_{2} \mathrm{OTBDMS}\right), 7.16\left(\mathrm{t},{ }^{3} \mathrm{~J}_{\mathrm{HH}}=6.5 \mathrm{~Hz}, 1 \mathrm{H}, \mathrm{CH}\right) \cdot{ }^{13} \mathrm{C}\left\{{ }^{1} \mathrm{H}\right\} \mathrm{NMR}(101 \mathrm{MHz}$, $\left.\mathrm{CDCl}_{3}\right) \delta(\mathrm{ppm})$-5.2 $\left(\left(\mathrm{CH}_{3}\right)_{2} \mathrm{Si}\right), 18.4\left(\left(\mathrm{CH}_{3}\right)_{3} \mathrm{CSi}\right), 25.3\left(\mathrm{CH}_{3}\right), 26.0\left(\left(\mathrm{CH}_{3}\right)_{3} \mathrm{CSi}\right), 41.6$ $\left(\mathrm{CH}_{2} \mathrm{CH}_{2}\right), 60.7\left(\mathrm{CH}_{2} \mathrm{CH}_{2} \mathrm{OTBDMS}\right), 113.6(\mathrm{C}=\mathrm{CH}), 150.9(\mathrm{C}=\mathrm{CH}), 192.5(\mathrm{C}=\mathrm{O})$. LRMS (IC) found $[\mathrm{M}+\mathrm{H}]^{+}=355$. 
<smiles>CCCCOCCC=C(C)C(C)=O</smiles>

(E)-6-((tert-butyldimethylsilyl)oxy)-3-methylhex-3-en-2-one: In a Young valve Schlenk, the electrophilic alkylzinc reagent was prepared in situ by mixing $\mathrm{MeMgCl}(7.2 \mathrm{~mL}$ from $1.0 \mathrm{M}$ in diethyl ether) and $\mathrm{ZnCl}_{2}(7.2 \mathrm{~mL}$ from a $1.0 \mathrm{M}$ in THF). A white precipitate formed rapidly. In a separate Schlenk, $\left[\left(t \mathrm{Bu}_{3} \mathrm{P}\right){ }_{2} \mathrm{Pd}\right](5 \mathrm{~mol} \%)$ was dissolved in THF $(60 \mathrm{~mL})$ and $(Z)-6-(($ tertbutyldimethylsilyl)oxy)-3-iodohex-3-en-2-one $(2.12 \mathrm{~g}, 6.0 \mathrm{mmol})$ was added. The latter solution was transferred by cannula to the suspension of the alkylzinc reagent and stirred at room temperature. After $18 \mathrm{~h}$, the mixture was quenched with a saturated aqueous $\mathrm{NH}_{4} \mathrm{Cl}$ solution $(10 \mathrm{~mL})$, extracted with diethyl ether $(3 \times 50 \mathrm{~mL})$, dried over anhydrous $\mathrm{Na}_{2} \mathrm{SO}_{4}$ and concentrated in vacuo. The crude mixture was purified by flash chromatography on silica gel (Pentane/Diethyl ether $=10: 1)$ to afford the desired cross-coupling product in $52 \%$ yield $(760$ $\mathrm{mg}$ ) as a colourless oil. $\mathbf{R}_{\mathrm{f}}=0.25$ (Pentane/Diethyl ether $\left.=10: 1\right)$. IR (neat): $v\left(\mathrm{~cm}^{-1}\right)=2954$, 2930, 1670, 1253, 1096, 937, 833, 775. '1H NMR (400 MHz, $\left.\mathrm{CDCl}_{3}\right) \delta$ (ppm) 0.06 (s, $\left.6 \mathrm{H},\left(\mathrm{CH}_{3}\right)_{2} \mathrm{Si}\right), 0.89$ (s, 9H, $\left.\left(\mathrm{CH}_{3}\right)_{3} \mathrm{CSi}\right), 1.78$ (q, $\left.{ }^{4} \mathrm{~J}_{\mathrm{HH}}=1.0 \mathrm{~Hz}, 3 \mathrm{H}, \mathrm{CH}_{3}\right), 2.31(\mathrm{~s}, 3 \mathrm{H}$, $\left.\mathrm{CH}_{3} \mathrm{CO}\right), 2.37-2.70\left(\mathrm{~m}, 2 \mathrm{H}, \quad \mathrm{CH}_{2} \mathrm{CH}_{2} \mathrm{OTBDMS}\right), 3.74\left(\mathrm{t},{ }^{3} J_{\mathrm{HH}}=6.4 \mathrm{~Hz}, 2 \mathrm{H}\right.$, $\left.\mathrm{CH}_{2} \mathrm{CH}_{2} \mathrm{OTBDMS}\right), 6.65-6.73(\mathrm{~m}, 1 \mathrm{H}, \mathrm{C}=\mathrm{CH}) \cdot{ }^{13} \mathrm{C}\left\{{ }^{1} \mathrm{H}\right\} \mathrm{NMR}\left(101 \mathrm{MHz}, \mathrm{CDCl}_{3}\right) \delta(\mathrm{ppm})-5.2$ $\left(\left(\mathrm{CH}_{3}\right)_{2} \mathrm{Si}\right), 11.5\left(\mathrm{CH}_{3} \mathrm{C}=\mathrm{CH}\right), 18.4\left(\left(\mathrm{CH}_{3}\right)_{3} \mathrm{CSi}\right), 25.6\left(\mathrm{CH}_{3} \mathrm{C}=\mathrm{O}\right), 26.0 \quad\left(\left(\mathrm{CH}_{3}\right)_{3} \mathrm{CSi}\right), 32.9$ $\left(\mathrm{CH}_{2} \mathrm{CH}_{2} \mathrm{OTBDMS}\right), 61.8\left(\mathrm{CH}_{2} \mathrm{CH}_{2} \mathrm{OTBDMS}\right), 139.0(\mathrm{C}=\mathrm{O}), 140.4(\mathrm{C}=\mathrm{CH}), 200.0(\mathrm{C}=\mathrm{O})$. LRMS (IC) found $[\mathrm{M}+\mathrm{H}]^{+}=243$.<smiles>CC(=O)C(C)=CCCO</smiles>

(E)-6-hydroxy-3-methylhex-3-en-2-one (4a): The reaction was run according to GP3 starting from (E)-6-((tert-butyldimethylsilyl)oxy)-3methylhex-3-en-2-one $(760 \mathrm{mg}, 3.1 \mathrm{mmol})$. The crude mixture was purified by flash chromatography (Pentane/Diethyl ether $=1: 3$ ) on silica gel to give $4 a$ in $51 \%$ yield $(230 \mathrm{mg})$ as a colourless oil. $\mathbf{R}_{\mathbf{f}}=0.1$ (Pentane/Diethyl ether $\left.=1: 2\right)$. IR (neat): $v\left(\mathrm{~cm}^{-1}\right)=3339,2930,2876,1706,1664,1460,1368,1280,1160 .{ }^{1} \mathbf{H}$ NMR $(400 \mathrm{MHz}$, $\left.\mathrm{CDCl}_{3}\right) \delta(\mathrm{ppm}) 1.64(\mathrm{~s}, 1 \mathrm{H}, \mathrm{OH}), 1.80\left(\mathrm{~d},{ }^{4} \mathrm{~J}_{\mathrm{HH}}=1.2 \mathrm{~Hz}, 3 \mathrm{H}, \mathrm{CH}_{3} \mathrm{C}=\mathrm{CH}\right), 2.32(\mathrm{~s}, 3 \mathrm{H}$, $\mathrm{CH}_{3} \mathrm{CO}$ ), $2.46-2.61$ (m, 2H, $\mathrm{CH}_{2} \mathrm{CH}_{2} \mathrm{OH}$ ), 3.80 (t, ${ }^{3} \mathrm{JHH}_{\mathrm{HH}}=6.4 \mathrm{~Hz}, 2 \mathrm{H}, \mathrm{CH}_{2} \mathrm{CH}_{2} \mathrm{OH}$ ), 6.68 (tq, $\left.{ }^{3} J_{\mathrm{HH}}=7.2,{ }^{4} J_{\mathrm{HH}}=1.3 \mathrm{~Hz}, 1 \mathrm{H}, \mathrm{C}=\mathrm{CH}\right) \cdot{ }^{13} \mathrm{C}\left\{{ }^{1} \mathrm{H}\right\}$ NMR $\left(101 \mathrm{MHz}, \mathrm{CDCl}_{3}\right) \delta(\mathrm{ppm}) 11.6$ $\left(\mathrm{CH}_{3} \mathrm{C}=\mathrm{CH}\right), 25.7\left(\mathrm{CH}_{3} \mathrm{CO}\right), 32.6\left(\mathrm{CH}_{2} \mathrm{CH}_{2} \mathrm{OH}\right), 61.6\left(\mathrm{CH}_{2} \mathrm{CH}_{2} \mathrm{OH}\right), 139.3(\mathrm{C}=\mathrm{CH}), 139.7$ $(C=\mathrm{CH}), 199.9(\mathrm{C}=\mathrm{O})$. LRMS (IC) found $[\mathrm{M}-\mathrm{OH}]^{+}=111$. 


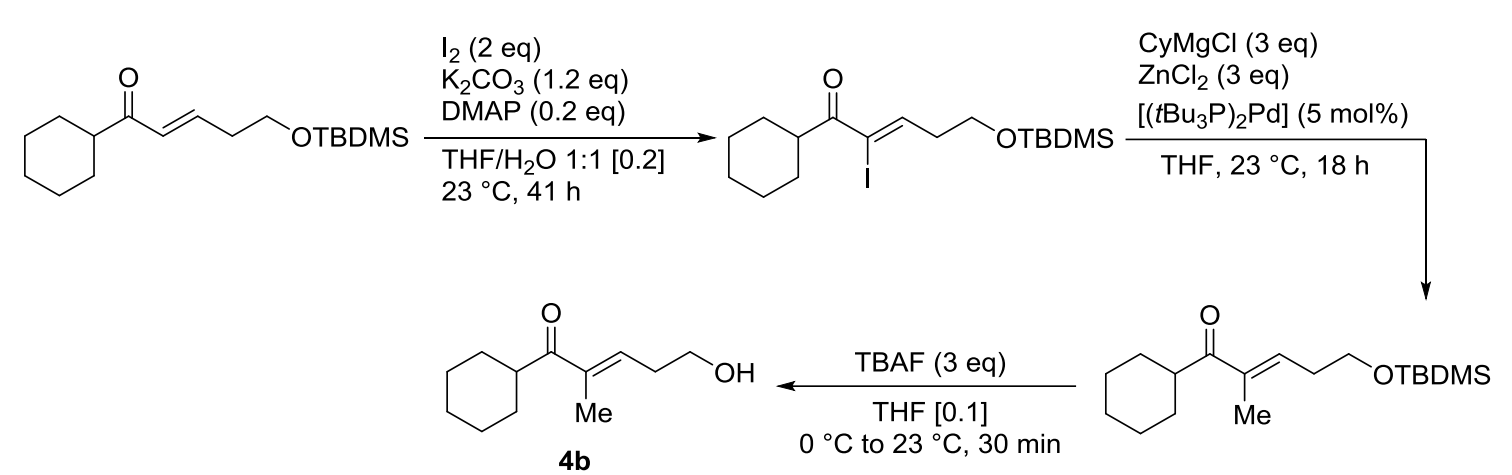

Figure S14: Synthesis of $(E)$-1-cyclohexyl-5-hydroxy-2-methylpent-2-en-1-one (4b)

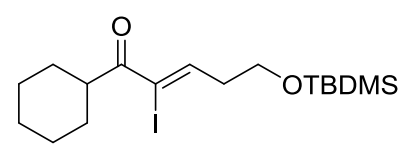

(Z)-5-((tert-butyldimethylsilyl)oxy)-1-cyclohexyl-2-iodopent-2-

en-1-one: To a solution of (E)-5-((tert-butyldimethylsilyl)oxy)-1cyclohexylpent-2-en-1-one $(2.4 \mathrm{~g}, 8.1 \mathrm{mmol})$ in $\mathrm{THF} / \mathrm{H}_{2} \mathrm{O} 1: 1$ (40 $\mathrm{mL}$ ) were sequentially added $\mathrm{K}_{2} \mathrm{CO}_{3}(1.4 \mathrm{~g}, 1.0 \mathrm{mmol}), \mathrm{I}_{2}(4.1 \mathrm{~g}, 16.2 \mathrm{mmol})$ and DMAP (196 $\mathrm{mg}, 1.6 \mathrm{mmol})$. After $41 \mathrm{~h}$, the reaction mixture was diluted with diethyl ether $(300 \mathrm{~mL})$, washed with a saturated aqueous $\mathrm{Na}_{2} \mathrm{~S}_{2} \mathrm{O}_{3}$ solution $(600 \mathrm{~mL})$, dried over $\mathrm{Na}_{2} \mathrm{SO}_{4}$, filtered on a pad of silica gel and concentrated in vacuo. The crude mixture was purified by flash chromatography on silica gel to afford the title compound in $47 \%$ yield $(1.6 \mathrm{~g})$ as a light yellow oil. Note: the remaining starting material $(23 \%, 560 \mathrm{mg})$ could be recovered. $\mathbf{R}_{\mathbf{f}}=0.5$ (Pentane/Diethyl ether $=10: 1)$. IR (neat): $v\left(\mathrm{~cm}^{-1}\right)=2930,2856,1678,1450,1253 .{ }^{1} \mathbf{H}$ NMR (400 MHz, $\left.\mathrm{CDCl}_{3}\right) \delta(\mathrm{ppm}) 0.08\left(\mathrm{~s}, 6 \mathrm{H},\left(\mathrm{CH}_{3}\right)_{2} \mathrm{Si}\right), 0.91$ (s, 9H, $\left.\left(\mathrm{CH}_{3}\right)_{3} \mathrm{CSi}\right), 1.18-1.52$ (m, $\left.5 \mathrm{H}, \mathrm{CH}_{2}-\mathrm{Cy}\right), 1.66-1.90\left(\mathrm{~m}, 5 \mathrm{H}, \mathrm{CH}_{2}-\mathrm{Cy}\right), 2.63$ (td, ${ }^{3} \mathrm{~J}_{\mathrm{HH}}=6.2 \mathrm{~Hz}, 6.2 \mathrm{~Hz}, 2 \mathrm{H}$, $\mathrm{CH}_{2} \mathrm{CH}_{2} \mathrm{OTBDMS}$ ), $3.14\left(\mathrm{tt},{ }^{3} J_{\mathrm{HH}}=11.6,3.1 \mathrm{~Hz}, 1 \mathrm{H}, \mathrm{CH}-\mathrm{Cy}\right), 3.80\left(\mathrm{t},{ }^{3} J_{\mathrm{HH}}=6.1 \mathrm{~Hz}, 2 \mathrm{H}\right.$, $\left.\mathrm{CH}_{2} \mathrm{CH}_{2} \mathrm{OTBDMS}\right), 7.14\left(\mathrm{t},{ }^{3} \mathrm{~J}_{\mathrm{HH}}=6.5 \mathrm{~Hz}, 1 \mathrm{H}, \mathrm{CH}\right) \cdot{ }^{13} \mathrm{C}\left\{{ }^{1} \mathrm{H}\right\} \mathbf{N M R}\left(101 \mathrm{MHz}, \mathrm{CDCl}_{3}\right) \delta(\mathrm{ppm})-$ $\left.5.3\left(\left(\mathrm{CH}_{3}\right)_{2} \mathrm{Si}\right), 18.2\left(\mathrm{CH}_{3}\right)_{3} \mathrm{CSi}\right), 25.8\left(\mathrm{CH}_{2}-\mathrm{Cy}\right), 30.0$ ( $\left.\mathrm{CH}-\mathrm{Cy}\right), 41.4\left(\mathrm{CH}_{2} \mathrm{CH}_{2} \mathrm{OTBDMS}\right), 45.3$ $\left(\mathrm{CH}_{2}\right.$-Cy), $60.6\left(\mathrm{CH}_{2} \mathrm{CH}_{2} \mathrm{OTBDMS}\right), 113.2\left(\mathrm{C}=\mathrm{CH}_{t}\right), 148.7(\mathrm{C}=\mathrm{CH}), 198.2(\mathrm{C}=\mathrm{O})$. LRMS (IC) found $[\mathrm{M}+\mathrm{H}]^{+}=423$. 


\section{Procedure for Negishi cross-coupling (GP6A)}

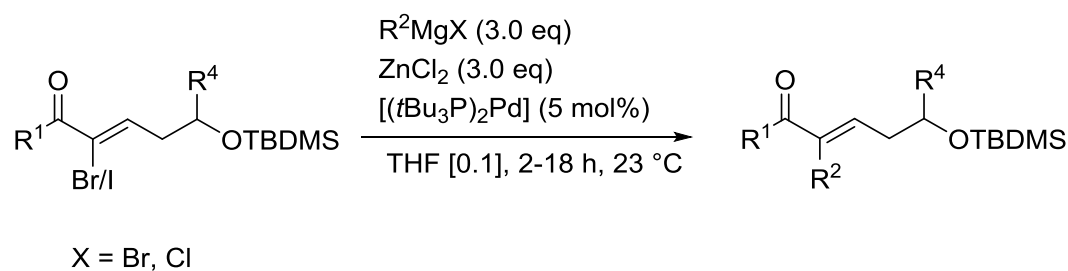

In a Young valve Schlenk, the electrophilic alkylzinc reagent was in situ prepared by mixing the appropriate Grignard (3.0 eq) and $\mathrm{ZnCl}_{2}(3.0 \mathrm{eq}, 1.0 \mathrm{M}$ in THF) for 10 to 30 minutes until a white precipitate formed. In a separate Schlenk, $\left[\left(t B u_{3} \mathrm{P}\right)_{2} \mathrm{Pd}\right](5 \mathrm{~mol} \%)$ was dissolved in THF $(0.1 \mathrm{M})$ and the appropriate bromo- or iodo-derivative (1 eq) was added. The latter solution was transferred by cannula to the suspension of the alkylzinc reagent and stirred at room temperature. After 2-18 h, the mixture was quenched with saturated aqueous $\mathrm{NH}_{4} \mathrm{Cl}$ solution, extracted with diethyl ether (3 times), dried over anhydrous $\mathrm{Na}_{2} \mathrm{SO}_{4}$ and concentrated in vacuo.

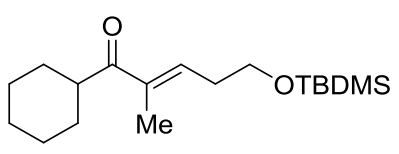

(E)-5-((tert-butyldimethylsilyl)oxy)-1-cyclohexyl-2-methylpent2-en-1-one: The reaction was performed according to GP6A starting from (Z)-5-((tert-butyldimethylsilyl)oxy)-1-cyclohexyl-2iodopent-2-en-1-one $(1.39 \mathrm{~g}, 3.2 \mathrm{mmol})$. After $2 \mathrm{~h}$, the reaction was complete. The crude mixture was purified by flash chromatography on silica gel (Pentane/Diethyl ether $=20: 1$ ) to afford the desired product in $81 \%$ yield $(800 \mathrm{mg}) . \mathbf{R}_{\mathbf{f}}=0.5$ (Pentane/Diethyl ether $\left.=10: 1\right)$. IR (neat): $v(\mathrm{~cm}-1)=2929,2856,1664,1253,1099 .{ }^{1} \mathbf{H}$ NMR $\left(400 \mathrm{MHz}, \mathrm{CDCl}_{3}\right) \delta$ (ppm) 0.07 (s, 6H, $\left.\left(\mathrm{CH}_{3}\right)_{2} \mathrm{Si}\right), 0.90$ (s, 9H, $\left.\left.\left(\mathrm{CH}_{3}\right)_{3} \mathrm{CSi}\right)\right), 1.20-1.83\left(\mathrm{~m}, 10 \mathrm{H}, \mathrm{CH}_{2} \mathrm{Cy}\right), 1.77$ (s, 3H, $\mathrm{CH}_{3} \mathrm{C}=\mathrm{CH}$ ), $2.46\left(\mathrm{~m}, 2 \mathrm{H}, \mathrm{CH}_{2} \mathrm{CH}_{2} \mathrm{OTBDMS}\right), 3.02$ (tt, $\left.{ }^{3} \mathrm{JHH}_{\mathrm{HH}}=11.4,3.3 \mathrm{~Hz}, 1 \mathrm{H}, \mathrm{CH}-\mathrm{Cy}\right), 3.74$ $\left(\mathrm{t},{ }^{3} \mathrm{~J}_{\mathrm{HH}}=6.4 \mathrm{~Hz}, 2 \mathrm{H}, \mathrm{CH}_{2} \mathrm{CH}_{2} \mathrm{OTBDMS}\right), 6.69\left(\mathrm{t},{ }^{3} \mathrm{~J}_{\mathrm{HH}}=7.0,1 \mathrm{H}, \mathrm{C}=\mathrm{CH}\right) .{ }^{13} \mathrm{C}\left\{{ }^{1} \mathrm{H}\right\}$ NMR $(101$

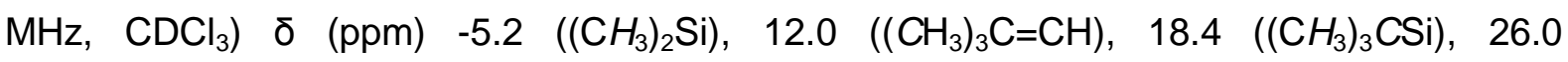
(( $\left.\left.\mathrm{CH}_{3}\right)_{3} \mathrm{CSi}\right), 26.1$ ( $\left.\mathrm{CH}_{2}-\mathrm{Cy}\right), 30.0$ ( $\left.\mathrm{CH}-\mathrm{Cy}\right), 32.7\left(\mathrm{CH}_{2} \mathrm{CH}_{2} \mathrm{OTBDMS}\right), 44.4\left(\mathrm{CH}_{2}-\mathrm{Cy}\right), 61.8$ $\left(\mathrm{CH}_{2} \mathrm{CH}_{2} \mathrm{OTBDMS}\right), 137.4(\mathrm{C}=\mathrm{CH}), 138.4(\mathrm{C}=\mathrm{CH}), 205.7(\mathrm{C}=\mathrm{O})$. LRMS (IC) found $[\mathrm{M}+\mathrm{H}]^{+}=$ 311.

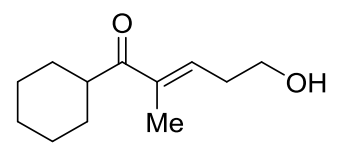

(E)-1-cyclohexyl-5-hydroxy-2-methylpent-2-en-1-one (4b): The reaction was run according to GP3 starting from $(E)-5-(($ tertbutyldimethylsilyl)oxy)-1-cyclohexyl-2-methylpent-2-en-1-one (775 mg, $2.5 \mathrm{mmol})$. The crude mixture was purified by flash chromatography on silica gel (Pentane/Diethyl ether $=1: 3$ ) to give $\mathbf{4 b}$ in $74 \%$ yield $\left(370 \mathrm{mg}\right.$ ) as a colorless oil. $\mathbf{R}_{\mathbf{f}}=0.5$ 
(Pentane/Diethyl ether = 1:2). IR (neat): $v\left(\mathrm{~cm}^{-1}\right)=2929,2856,1664,1253,1099 .{ }^{1} \mathbf{H}$ NMR $\left(400 \mathrm{MHz}, \mathrm{CDCl}_{3}\right) \delta(\mathrm{ppm}) 1.16-1.46\left(\mathrm{~m}, 6 \mathrm{H}, \mathrm{CH}_{2}-\mathrm{Cy} ; \mathrm{OH}\right), 1.65-1.85$ (m, 5H, CH $1.80\left(\mathrm{~s}, 3 \mathrm{H}, \mathrm{CH}_{3} \mathrm{C}=\mathrm{CH}\right), 2.53\left(\mathrm{td},{ }^{3} J_{\mathrm{HH}}=6.5,5.4 \mathrm{~Hz}, 2 \mathrm{H}, \mathrm{CH}_{2} \mathrm{CH}_{2} \mathrm{OH}\right), 3.02\left(\mathrm{tt},{ }^{3} J_{\mathrm{HH}}=11.4\right.$, $3.3 \mathrm{~Hz}, 1 \mathrm{H}, \mathrm{CH}-\mathrm{Cy}), 3.80$ (t, $\left.{ }^{3} \mathrm{~J}_{\mathrm{HH}}=6.4 \mathrm{~Hz}, 2 \mathrm{H}, \mathrm{CH}_{2} \mathrm{CH}_{2} \mathrm{OH}\right), 6.57-6.72(\mathrm{~m}, 1 \mathrm{H}, \mathrm{C}=\mathrm{CH})$. ${ }^{13} \mathrm{C}\left\{{ }^{1} \mathrm{H}\right\}$ NMR (101 MHz, $\left.\mathrm{CDCl}_{3}\right) \delta(\mathrm{ppm}) 12.1\left(\mathrm{CH}_{3} \mathrm{C}=\mathrm{CH}\right), 26.0\left(\mathrm{CH}_{2}-\mathrm{Cy}\right), 26.1\left(\mathrm{CH}_{2}-\mathrm{Cy}\right)$, 30.0 ( CH-Cy), $32.6\left(\mathrm{CH}_{2} \mathrm{CH}_{2} \mathrm{OH}\right), 44.5\left(\mathrm{CH}_{2}-\mathrm{Cy}\right), 61.7\left(\mathrm{CH}_{2} \mathrm{CH}_{2} \mathrm{OH}\right), 137.0(\mathrm{C}=\mathrm{CH}), 138.3$ $(\mathrm{C}=\mathrm{CH}), 205.6(\mathrm{C}=\mathrm{O})$. LRMS $(\mathrm{IC})$ found $[\mathrm{M}+\mathrm{H}]^{+}=197$.

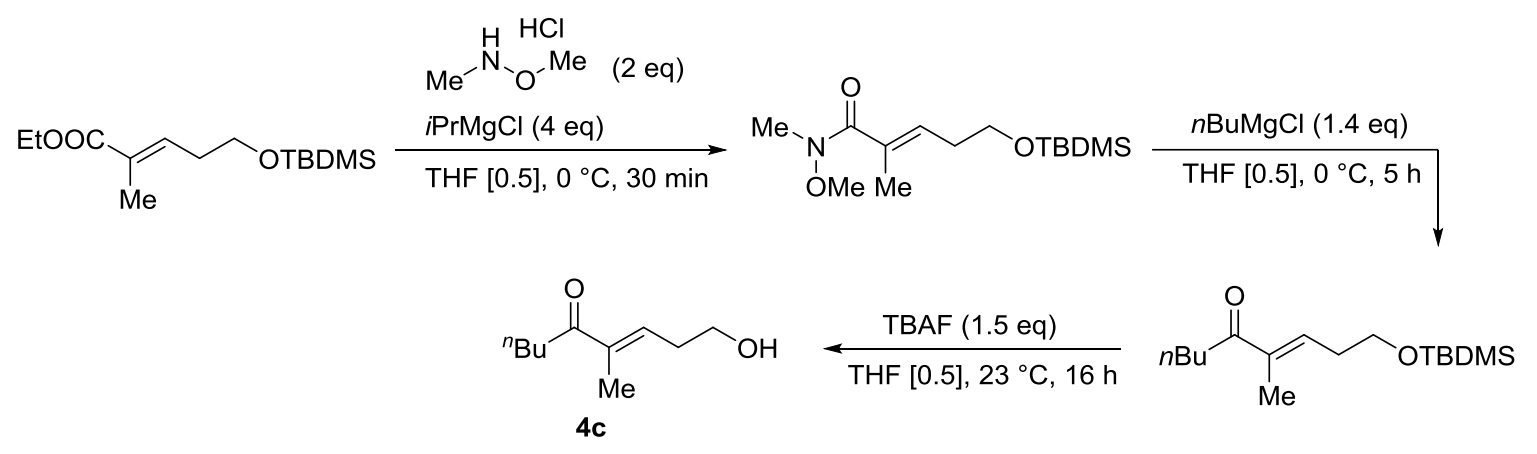

Figure S15: Synthesis of $(E)$-1-hydroxy-4-methylnon-3-en-5-one (4c)

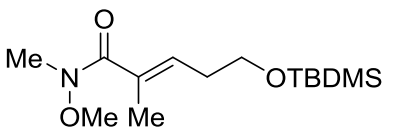

(E)-5-((tert-butyldimethylsilyl)oxy)-N-methoxy- $N, 2-$ dimethylpent-2-enamide: A suspension of ethyl $(E)-5-((t e r t-$ butyldimethylsilyl)oxy)-2-methylpent-2-enoate $\quad(470 \quad \mathrm{mg}, \quad 1.7$ $\mathrm{mmol}$ ) and $\mathrm{N}, \mathrm{O}$-dimethoxyhydroxylamine hydrochloride $(330 \mathrm{mg}, 3.4 \mathrm{mmol})$ in THF (3 $\mathrm{mL})$ was stirred for 10 minutes at $-20^{\circ} \mathrm{C}$. Isopropylmagnesium chloride $(13.65 \mathrm{mmol}, 4.55 \mathrm{~mL}, 3$ $\mathrm{M}$ in THF) was added while maintaining the temperature below $0{ }^{\circ} \mathrm{C}$. After 30 minutes at 0 ${ }^{\circ} \mathrm{C}$, the reaction was quenched with a saturated aqueous $\mathrm{NH}_{4} \mathrm{Cl}$ solution $(5 \mathrm{~mL})$, extracted with EtOAc $(2 \times 5 \mathrm{~mL})$, washed with brine $(20 \mathrm{~mL})$, dried over anhydrous $\mathrm{Na}_{2} \mathrm{SO}_{4}$ and concentrated in vacuo. The product was used in the next step without further purification.

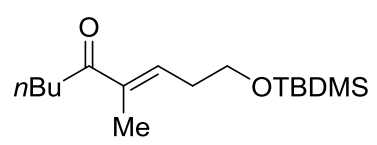
(E)-1-((tert-butyldimethylsilyl)oxy)-4-methylnon-3-en-5-one: To a solution of $(E)-5-(($ tert-butyldimethylsilyl)oxy)- $N$-methoxy- $N, 2-$ dimethylpent-2-enamide (200 $\mathrm{mg}, 0.7 \mathrm{mmol})$ in THF $(1.4 \mathrm{~mL})$, $n \mathrm{BuMgCl}\left(1.7 \mathrm{M}\right.$ in THF/toluene, $0.16 \mathrm{~mL}$ ) was slowly added at $0{ }^{\circ} \mathrm{C}$ and stirred for $5 \mathrm{~h}$. The mixture was quenched with a saturated aqueous $\mathrm{NH}_{4} \mathrm{Cl}$ solution $(2 \mathrm{~mL})$, extracted with diethyl ether $(3 \times 10 \mathrm{~mL})$, dried over anhydrous $\mathrm{Na}_{2} \mathrm{SO}_{4}$ and concentrated in vacuo. The crude mixture was purified by flash chromatography on silica gel (Pentane/Diethyl ether = $50: 1)$ to give the title compound as a colourless oil in $64 \%$ yield $\left(127 \mathrm{mg}\right.$ ) over 2 steps. $\mathbf{R}_{\mathbf{f}}=$ 0.7 (Pentane/Diethyl ether $=10: 1)$. IR (neat): $v\left(\mathrm{~cm}^{-1}\right)=2955,2930,1670,1253,1098,833$, 
775. ${ }^{1} \mathrm{H}$ NMR $\left(400 \mathrm{MHz}, \mathrm{CDCl}_{3}\right) \delta$ (ppm) $0.06\left(\mathrm{~s}, 6 \mathrm{H},\left(\mathrm{CH}_{3}\right)_{2} \mathrm{Si}\right), 0.88-0.94(\mathrm{~m}, 12 \mathrm{H}$, $\mathrm{CH}_{2} \mathrm{CH}_{2} \mathrm{CH}_{2} \mathrm{CH}_{3}$; $\left.\left(\mathrm{CH}_{3}\right)_{3} \mathrm{CSi}\right), 1.29-1.37\left(\mathrm{~m}, 2 \mathrm{H}, \mathrm{CH}_{2} \mathrm{CH}_{2} \mathrm{CH}_{2} \mathrm{CH}_{3}\right), 1.53-1.62(\mathrm{~m}, 2 \mathrm{H}$, $\left.\mathrm{CH}_{2} \mathrm{CH}_{2} \mathrm{CH}_{2} \mathrm{CH}_{3}\right), 1.78\left(\mathrm{~d},{ }^{4} \mathrm{~J}_{\mathrm{HH}}=1.5 \mathrm{~Hz}, 3 \mathrm{H}, \mathrm{CH}_{3} \mathrm{C}=\mathrm{CH}\right), 2.41-2.52(\mathrm{~m}, 2 \mathrm{H}$, $\mathrm{CH}_{2} \mathrm{CH}_{2} \mathrm{CH}_{2} \mathrm{CH}_{3}$ ), $2.59-2.72\left(\mathrm{~m}, 2 \mathrm{H}, \mathrm{CH}_{2} \mathrm{CH}_{2} \mathrm{OTBDMS}\right), 3.74$ (t, ${ }^{3} J_{\mathrm{HH}}=6.4 \mathrm{~Hz}, 2 \mathrm{H}$, $\left.\mathrm{CH}_{2} \mathrm{CH}_{2} \mathrm{OTBDMS}\right), 6.59-6.78(\mathrm{~m}, 1 \mathrm{H}, \mathrm{C}=\mathrm{CH}) .{ }^{13} \mathrm{C}\left\{{ }^{1} \mathrm{H}\right\} \mathrm{NMR}\left(101 \mathrm{MHz}, \mathrm{CDCl}_{3}\right) \delta(\mathrm{ppm})-5.2$

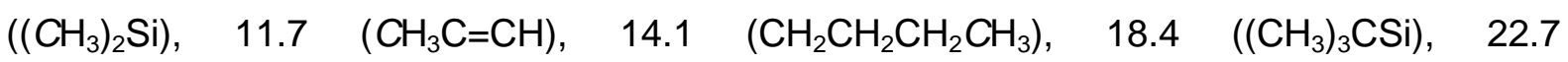
$\left(\mathrm{CH}_{2} \mathrm{CH}_{2} \mathrm{CH}_{2} \mathrm{CH}_{3}\right), 26.0\left(\left(\mathrm{CH}_{3}\right)_{3} \mathrm{CSi}\right), 27.4\left(\mathrm{CH}_{2} \mathrm{CH}_{2} \mathrm{CH}_{2} \mathrm{CH}_{3}\right), 32.8\left(\mathrm{CH}_{2} \mathrm{CH}_{2} \mathrm{CH}_{2} \mathrm{CH}_{3}\right), 37.2$ $\left(\mathrm{CH}_{2} \mathrm{CH}_{2} \mathrm{OTBDMS}\right), 61.8\left(\mathrm{CH}_{2} \mathrm{CH}_{2} \mathrm{OTBDMS}\right), 138.54(\mathrm{C}=\mathrm{CH}), 138.94 \quad(\mathrm{C}=\mathrm{CH}), 202.43$ $(C=\mathrm{O})$. LRMS (IC) found $[\mathrm{M}+\mathrm{H}]^{+}=285$.

$\mathrm{Me}_{\mathrm{OH}}^{\mathrm{O}}$

(E)-1-hydroxy-4-methylnon-3-en-5-one (4c): The reaction was run according to GP3 starting from $(E)-1-(($ tert-butyldimethylsilyl)oxy)-4methyl non-3-en-5-one (160 mg, $0.56 \mathrm{mmol})$. Purification by flash chromatography on silica gel (Pentane/Diethyl ether $=1: 3$ ) afforded $\mathbf{4 c}$ in $57 \%$ yield $(56 \mathrm{mg})$. $\mathbf{R}_{\mathbf{f}}=0.3$ (Pentane/Diethyl ether $\left.=1: 1\right)$. IR (neat): $v\left(\mathrm{~cm}^{-1}\right)=2931,1662,1046,755 .{ }^{1} \mathbf{H}$ NMR $\left(400 \mathrm{MHz}, \mathrm{CDCl}_{3}\right) \delta(\mathrm{ppm}) 0.92\left(\mathrm{t},{ }^{3} \mathrm{~J}_{\mathrm{HH}}=7.3 \mathrm{~Hz}, 3 \mathrm{H}, \mathrm{CH}_{3} \mathrm{CH}_{2} \mathrm{CH}_{2} \mathrm{CH}_{2}\right), 1.33$ (tq, ${ }^{3} \mathrm{JHH}_{\mathrm{HH}}=14.6$, $7.3 \mathrm{~Hz}, 2 \mathrm{H}, \mathrm{CH}_{3} \mathrm{CH}_{2} \mathrm{CH}_{2} \mathrm{CH}_{2}$ ), 1.59 (tt, ${ }^{3} \mathrm{JHH}_{\mathrm{HH}} 7.5 \mathrm{~Hz}, 7.5 \mathrm{~Hz}, 2 \mathrm{H}, \mathrm{CH}_{3} \mathrm{CH}_{2} \mathrm{CH}_{2} \mathrm{CH}_{2}$ ), 1.81 (s, $\left.3 \mathrm{H}, \mathrm{CH}_{3} \mathrm{C}=\mathrm{CH}\right), 2.46-2.59\left(\mathrm{~m}, 2 \mathrm{H}, \mathrm{CH}_{2} \mathrm{CH}_{2} \mathrm{OH}\right), 2.62-2.72\left(\mathrm{~m}, 2 \mathrm{H}, \mathrm{CH}_{3} \mathrm{CH}_{2} \mathrm{CH}_{2} \mathrm{CH}_{2}\right), 3.80$ $\left(\mathrm{t},{ }^{3} \mathrm{~J}_{\mathrm{HH}}=6.4 \mathrm{~Hz}, 2 \mathrm{H}, \mathrm{CH}_{2} \mathrm{CH}_{2} \mathrm{OH}\right), 6.56-6.76(\mathrm{~m}, 1 \mathrm{H}, \mathrm{C}=\mathrm{CH}) \cdot{ }^{13} \mathbf{C}\left\{{ }^{1} \mathbf{H}\right\} \mathbf{N M R}(101 \mathrm{MHz}$, $\left.\mathrm{CDCl}_{3}\right) \delta(\mathrm{ppm})-5.2\left(\left(\mathrm{CH}_{3}\right)_{2} \mathrm{Si}\right), 11.7\left(\mathrm{CH}_{3} \mathrm{C}=\mathrm{CH}\right), 14.1\left(\mathrm{CH}_{2} \mathrm{CH}_{2} \mathrm{CH}_{2} \mathrm{CH}_{3}\right), 18.44\left(\left(\mathrm{CH}_{3}\right)_{3} \mathrm{CSi}\right)$, $22.7\left(\mathrm{CH}_{2} \mathrm{CH}_{2} \mathrm{CH}_{2} \mathrm{CH}_{3}\right), 26.0\left(\left(\mathrm{CH}_{3}\right)_{3} \mathrm{CSI}\right), 27.4\left(\mathrm{CH}_{2} \mathrm{CH}_{2} \mathrm{CH}_{2} \mathrm{CH}_{3}\right), 32.8\left(\mathrm{CH}_{3} \mathrm{CH}_{2} \mathrm{CH}_{2} \mathrm{CH}_{2}\right)$, $37.1\left(\mathrm{CH}_{2} \mathrm{CH}_{2} \mathrm{OH}\right), 61.8\left(\mathrm{CH}_{2} \mathrm{CH}_{2} \mathrm{OH}\right), 138.5(\mathrm{C}=\mathrm{CH}), 138.9(\mathrm{C}=\mathrm{CH}), 202.4(\mathrm{C}=\mathrm{O})$. LRMS (IC) found $[\mathrm{M}+\mathrm{H}]^{+}=171 ;\left[\mathrm{M}+\mathrm{NH}_{4}\right]^{+}=188$. 


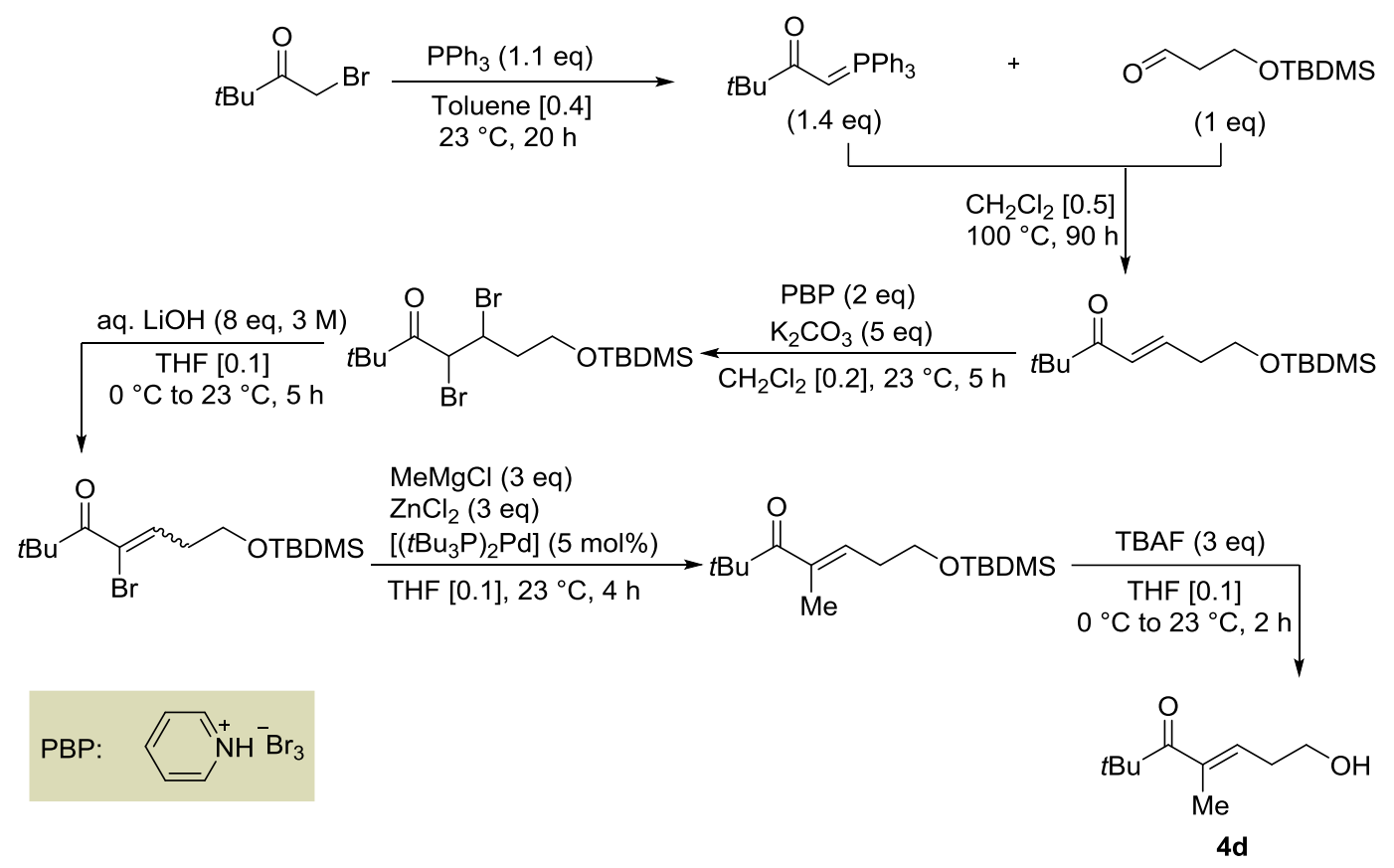

Figure S16: Synthesis of $(E)$-7-hydroxy-2,2,4-trimethylhept-4-en-3-one (4d)

$\stackrel{0}{11}=\mathrm{PPh}_{3}$ 3,3-dimethyl-1-(triphenyl- $\lambda^{5}$-phosphanylidene)butan-2-one: To a toluene slowly added a solution of triphenylphosphine (16.2 g, 61.6 mmol) in toluene (80 mL). After $20 \mathrm{~h}$ at room temperature, the white solid obtained was filtered and redissolved in $\mathrm{CH}_{2} \mathrm{Cl}_{2}$ $(182 \mathrm{~mL})$. To this solution was added an aqueous $2 \mathrm{~N} \mathrm{NaOH}$ solution $(60 \mathrm{~mL})$ which was stirred for $1 \mathrm{~h}$ at room temperature. The reaction mixture was then washed with water and concentrated to afford the title compound in $87 \%$ yield $(17.7 \mathrm{~g})$.<smiles>CCCCCC(=O)C=CCCC[Mg]C</smiles>

(E)-7-((tert-butyldimethylsilyl)oxy)-2,2-dimethylhept-4-en-3-one: $34 \mathrm{mmol})$ in THF (70 mL), 3,3-dimethyl-1-(triphenyl- $\lambda^{5}$-phosphanylidene)butan-2-one (17.7 $\mathrm{g}, 49.1 \mathrm{mmol}$ ) was added in one portion and stirred for $90 \mathrm{~h}$ at $100^{\circ} \mathrm{C}$. After cooling to room temperature, $50 \mathrm{~mL}$ of pentane/diethyl ether $(1: 1, \mathrm{v} / \mathrm{v})$ were added. The resulting white precipitate was filtered off and the remaining red solution was concentrated to give the crude product, which was purified by flash chromatography on silica gel (Pentane/Diethyl ether = $20: 1)$. The product was isolated in $33 \%$ yield $(3.0 \mathrm{~g})$ as colorless oil. $\mathbf{R}_{\mathbf{f}}=0.3$ (Pentane/Diethyl ether $=10: 1$ ). IR (neat): $v\left(\mathrm{~cm}^{-1}\right)=2956,2931,1691,1254,1099,834$, 775. ${ }^{1} \mathrm{H}$ NMR $\left(400 \mathrm{MHz}, \mathrm{CDCl}_{3}\right) \delta(\mathrm{ppm}) 0.05\left(\mathrm{~s}, 6 \mathrm{H},\left(\mathrm{CH}_{3}\right)_{2} \mathrm{Si}\right), 0.88\left(\mathrm{~s}, 9 \mathrm{H},\left(\mathrm{CH}_{3}\right)_{3} \mathrm{CSi}\right), 1.15$ (s, 9H, $\left.\left(\mathrm{CH}_{3}\right)_{3} \mathrm{CC}=\mathrm{O}\right), 2.34-2.50\left(\mathrm{~m}, 2 \mathrm{H}, \mathrm{CH}_{2} \mathrm{CH}_{2} \mathrm{OTBDMS}\right), 3.73\left(\mathrm{t},{ }^{3} J_{\mathrm{HH}}=6.4 \mathrm{~Hz}, 2 \mathrm{H}\right.$, $\mathrm{CH}_{2} \mathrm{CH}_{2} \mathrm{OTBDMS}$ ), 6.56 (dt, ${ }^{3} \mathrm{~J}_{\mathrm{HH}}=15.3,{ }^{4} \mathrm{~J}_{\mathrm{HH}}=1.5 \mathrm{~Hz}, 1 \mathrm{H}, \mathrm{COCH}=\mathrm{CH}$ ), $6.92\left(\mathrm{dt},{ }^{3} J_{\mathrm{HH}}=\right.$ 15.2, 7.1 Hz, $1 \mathrm{H}, \mathrm{COCH}=\mathrm{CH}) .{ }^{13} \mathrm{C}\left\{{ }^{1} \mathrm{H}\right\}$ NMR $\left(101 \mathrm{MHz}, \mathrm{CDCl}_{3}\right) \delta(\mathrm{ppm})-5.2\left(\left(\mathrm{CH}_{3}\right)_{2} \mathrm{Si}\right), 18.4$ 


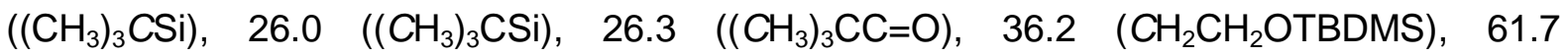
$\left(\mathrm{CH}_{2} \mathrm{CH}_{2} \mathrm{OTBDMS}\right), 126.0(\mathrm{COCH}=\mathrm{CH}), 144.2(\mathrm{COCH}=\mathrm{CH}), 204.3(\mathrm{C}=\mathrm{O})$. LRMS (IC) found $[\mathrm{M}+\mathrm{H}]^{+}=271$.

$\underbrace{\mathrm{Br}}_{\mathrm{Br}}$ OTBDMS 4,5-dibromo-7-((tert-butyldimethylsilyl)oxy)-2,2-dimethylheptan-

3-one: To a solution of $(E)-6$-((tert-butyldimethylsilyl)oxy)hex-3-en-2one (750 mg, $2.8 \mathrm{mmol})$ in $\mathrm{CH}_{2} \mathrm{Cl}_{2}(14 \mathrm{~mL}), \mathrm{K}_{2} \mathrm{CO}_{3}(1.93 \mathrm{~g}, 14 \mathrm{mmol})$ and pyridinium tribromide $(1.72 \mathrm{~g}, 5.4 \mathrm{mmol})$ were added in sequence. The mixture was stirred for $5 \mathrm{~h}$ at room temperature and then quenched with a saturated aqueous $\mathrm{NaHCO}_{3}$ solution (30 mL), extracted with $\mathrm{CH}_{2} \mathrm{Cl}_{2}(3 \times 15 \mathrm{~mL})$, dried over $\mathrm{Na}_{2} \mathrm{SO}_{4}$ and concentrated in vacuo. The crude mixture was purified by flash chromatography on silica gel (Pentane/Diethyl ether $=40: 1)$ to afford the dibrominated product in $71 \%$ yield $(850 \mathrm{mg})$ as a yellow oil. $\mathbf{R}_{\mathbf{f}}=0.3$ (Pentane/Diethyl ether $\left.=10: 1\right)$. IR (neat): $v\left(\mathrm{~cm}^{-1}\right)=2956,2931,2859$, 1714, 1472, 1255, 1100, 837. ' $\mathrm{H}$ NMR $\left(400 \mathrm{MHz}, \mathrm{CDCl}_{3}\right) \delta(\mathrm{ppm}) 0.09$ (d, J = $2.2 \mathrm{~Hz}, 6 \mathrm{H}$, $\left.\left(\mathrm{CH}_{3}\right)_{2} \mathrm{Si}\right), 0.92\left(\mathrm{~s}, 9 \mathrm{H},\left(\mathrm{CH}_{3}\right)_{3} \mathrm{CSi}\right), 1.29$ (s, 9H, $\left.\left(\mathrm{CH}_{3}\right)_{3} \mathrm{CO}\right), 1.98-2.04,2.48-2.63(\mathrm{~m}$, $\mathrm{CH}_{2} \mathrm{CH}_{2} \mathrm{OTBDMS}$ ), $3.74-3.99$ (m, 2H, $\mathrm{CH}_{2} \mathrm{CH}_{2} \mathrm{OTBDMS}$ ), 4.65 (ddd, ${ }^{3} J_{\mathrm{HH}}=11.4,9.3,2.7$ $\mathrm{Hz}, 1 \mathrm{H}, \mathrm{COCH}(\mathrm{Br}) \mathrm{CH}(\mathrm{Br})), 4.98\left(\mathrm{~d},{ }^{3} \mathrm{~J}_{\mathrm{HH}}=11.0 \mathrm{~Hz}, 1 \mathrm{H}, \mathrm{COCH}(\mathrm{Br}) \mathrm{CH}(\mathrm{Br})\right) .{ }^{13} \mathrm{C}\left\{{ }^{1} \mathrm{H}\right\} \mathbf{N M R}$ $\left(101 \mathrm{MHz}, \mathrm{CDCl}_{3}\right) \quad \delta \quad(\mathrm{ppm}) \quad-5.2 \quad\left(\left(\mathrm{CH}_{3}\right)_{2} \mathrm{Si}\right), \quad-5.2 \quad\left(\left(\mathrm{CH}_{3}\right)_{2} \mathrm{Si}\right), \quad 18.4 \quad\left(\left(\mathrm{CH}_{3}\right)_{3} \mathrm{CSi}\right), 26.1$ $\left(\left(\mathrm{CH}_{3}\right)_{3} \mathrm{CSi}\right), \quad 27.6 \quad\left(\left(\mathrm{CH}_{3}\right)_{3} \mathrm{CCO}\right), \quad 37.9 \quad\left(\mathrm{CH}_{2} \mathrm{CH}_{2} \mathrm{OTBDMS}\right), \quad 44.4 \quad\left(\left(\mathrm{CH}_{3}\right)_{3} \mathrm{CCO}\right), \quad 47.0$ $(\mathrm{COCH}(\mathrm{Br}) \mathrm{CH}(\mathrm{Br})), 49.4(\mathrm{COCH}(\mathrm{Br}) \mathrm{CH}(\mathrm{Br})), 60.4\left(\mathrm{CH}_{2} \mathrm{CH}_{2} \mathrm{OTBDMS}\right), 206.9(\mathrm{C}=\mathrm{O})$. LRMS (IC) found $[\mathrm{M}+\mathrm{H}]^{+}=431$.<smiles>COCCC=C(Br)C(=O)Br</smiles>

\section{4-bromo-7-((tert-butyldimethylsilyl)oxy)-2,2-dimethylhept-4-en-3-}

one: 4,5-dibromo-7-((tert-butyldimethylsilyl)oxy)-2,2-dimethylheptan-

3-one was dissolved in THF $(20 \mathrm{~mL})$ and an aqueous $\mathrm{LiOH}$ solution ( $3 \mathrm{M}, 5.25 \mathrm{~mL}$ ) was added dropwise. The mixture was stirred for $5 \mathrm{~h}$ at room temperature and then poured into water $(50 \mathrm{~mL})$, extracted with diethyl ether $(3 \times 50 \mathrm{~mL})$, dried over anhydrous $\mathrm{Na}_{2} \mathrm{SO}_{4}$ and concentrated in vacuo. The crude mixture was purified by flash chromatography on silica gel (Pentane/Diethyl ether $=50: 1$ ) to give the $(E)$-isomer of the product in $58 \%$ yield $(400 \mathrm{mg})$ and the $(Z)$-isomer in $32 \%$ yield $(220 \mathrm{mg})$ as light yellow oils. $(E)$-isomer: $\mathbf{R}_{\mathbf{f}}=0.4$ (Pentane/Diethyl ether $=10: 1$ ). IR (neat): $v\left(\mathrm{~cm}^{-1}\right)=2958,2859,1692$, 1101, 836. ${ }^{1} \mathrm{H}$ NMR (400 MHz, $\left.\mathrm{CDCl}_{3}\right) \delta$ (ppm) 0.05 (s, 6H, $\left.\left(\mathrm{CH}_{3}\right)_{2} \mathrm{Si}\right)$ ), 0.89 (s, 9H, $\left.\left(\mathrm{CH}_{3}\right)_{3} \mathrm{CSi}\right), 1.30$ (s, 9H, $\left(\mathrm{CH}_{3}\right)_{3} \mathrm{CCO}$ ), $2.11-2.25$ (m, 2H, $\left.\mathrm{CH}_{2} \mathrm{CH}_{2} \mathrm{OTBDMS}\right), 3.65$ (t, ${ }^{3} \mathrm{JHH}_{\mathrm{HH}}=$ $\left.6.4 \mathrm{~Hz}, 2 \mathrm{H}, \mathrm{CH}_{2} \mathrm{CH}_{2} \mathrm{OTBDMS}\right), 6.09\left(\mathrm{t},{ }^{3} \mathrm{JHH}_{\mathrm{HH}}=7.9 \mathrm{~Hz}, 1 \mathrm{H}, \mathrm{C}=\mathrm{CH}\right) \cdot{ }^{13} \mathrm{C}\left\{{ }^{1} \mathrm{H}\right\} \mathbf{N M R}(101 \mathrm{MHz}$, $\left.\mathrm{CDCl}_{3}\right) \delta(\mathrm{ppm})$-5.2 $\left(\left(\mathrm{CH}_{3}\right)_{2} \mathrm{Si}\right), 18.5\left(\left(\mathrm{CH}_{3}\right)_{3} \mathrm{CSi}\right), 26.1\left(\left(\mathrm{CH}_{3}\right)_{3} \mathrm{CSi}\right), 27.9\left(\left(\mathrm{CH}_{3}\right)_{3} \mathrm{CCO}\right), 34.8$ $\left(\mathrm{CH}_{2} \mathrm{CH}_{2} \mathrm{OTBDMS}\right), 44.0$ (( $\left.\left.\mathrm{CH}_{3}\right)_{3} \mathrm{CCO}\right), 62.0\left(\mathrm{CH}_{2} \mathrm{CH}_{2} \mathrm{OTBDMS}\right), 115.8(\mathrm{C}=\mathrm{CH}), 132.3$ 
$(\mathrm{C}=\mathrm{CH}), 207.4(\mathrm{C}=\mathrm{O})$. LRMS (IC) found $[\mathrm{M}+\mathrm{H}]^{+}=350$. (Z)-isomer: $\mathbf{R}_{\mathbf{f}}=0.35$ (Pentane/Diethyl ether $=10: 1)$. IR (neat): $v(\mathrm{~cm}-1)=2956,2931,2859,1685,1255,1097$, 837. ${ }^{1} \mathrm{H}$ NMR (400 MHz, $\left.\mathrm{CDCl}_{3}\right) \delta(\mathrm{ppm}) 0.06\left(\mathrm{~s}, 6 \mathrm{H},\left(\mathrm{CH}_{3}\right)_{2} \mathrm{Si}\right)$ ), 0.89 (s, 9H, $\left.\left(\mathrm{CH}_{3}\right)_{3} \mathrm{CSi}\right)$, 1.31 (s, 9H, $\left.\left(\mathrm{CH}_{3}\right)_{3} \mathrm{CCO}\right), 2.46-2.64\left(\mathrm{~m}, 2 \mathrm{H}, \mathrm{CH}_{2} \mathrm{CH}_{2} \mathrm{OTBDMS}\right), 3.75\left(\mathrm{t},{ }^{3} \mathrm{JHH}_{\mathrm{HH}}=6.2 \mathrm{~Hz}, 2 \mathrm{H}\right.$, $\left.\mathrm{CH}_{2} \mathrm{CH}_{2} \mathrm{OTBDMS}\right), 6.67\left(\mathrm{t},{ }^{3} \mathrm{~J}_{\mathrm{HH}}=6.6 \mathrm{~Hz}, 1 \mathrm{H}, \mathrm{C}=\mathrm{CH}\right) \cdot{ }^{13} \mathrm{C}\left\{{ }^{1} \mathrm{H}\right\}$ NMR $\left(101 \mathrm{MHz}, \mathrm{CDCl}_{3}\right) \delta$ (ppm) -5.2 $\quad\left(\left(\mathrm{CH}_{3}\right)_{2} \mathrm{Si}\right), \quad 18.4 \quad\left(\left(\mathrm{CH}_{3}\right)_{3} \mathrm{CSi}\right), \quad 26.0 \quad\left(\left(\mathrm{CH}_{3}\right)_{3} \mathrm{CSi}\right), \quad 28.1 \quad\left(\left(\mathrm{CH}_{3}\right)_{3} \mathrm{CCO}\right), \quad 35.6$ $\left(\mathrm{CH}_{2} \mathrm{CH}_{2} \mathrm{OTBDMS}\right), 44.2\left(\left(\mathrm{CH}_{3}\right)_{3} \mathrm{CCO}\right), 61.0\left(\mathrm{CH}_{2} \mathrm{CH}_{2} \mathrm{OTBDMS}\right), 123.5(\mathrm{C}=\mathrm{CH}), 136.6$ $(\mathrm{C}=\mathrm{CH}), 202.8(\mathrm{C}=\mathrm{O})$. LRMS $(\mathrm{IC})$ found $[\mathrm{M}+\mathrm{H}]^{+}=350$.

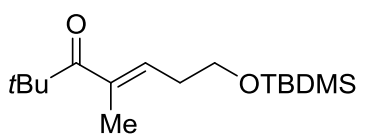

(E)-7-((tert-butyldimethylsilyl)oxy)-2,2,4-trimethylhept-4-en-3one: The reaction was run according to GP6A starting from (Z)-4bromo-7-((tert-butyldimethylsilyl)oxy)-2,2-dimethylhept-4-en-3-one (310 $\mathrm{mg}, 0.89 \mathrm{mmol}$ ). After $4 \mathrm{~h}$, the reaction was complete and purification by flash chromatography on silica gel (Pentane/Diethyl ether $=20: 1$ ) afforded the product in $93 \%$ yield $\left(235 \mathrm{mg}\right.$ ) as a light yellow oil. $\mathbf{R}_{\mathbf{f}}=0.6$ (Pentane/Diethyl ether $\left.=10: 1\right)$. IR (neat): $v\left(\mathrm{~cm}^{-1}\right)$ = 2956, 2931, 2859, 1688, 1468, 1254, 1099, 836. ${ }^{1} \mathbf{H}$ NMR $\left(400 \mathrm{MHz}, \mathrm{CDCl}_{3}\right) \delta$ (ppm) 0.06 (s, 6H, $\left.\left(\mathrm{CH}_{3}\right)_{2} \mathrm{Si}\right), 0.89$ (s, 9H, $\left.\left(\mathrm{CH}_{3}\right)_{3} \mathrm{CSi}\right), 1.24$ (s, 9H, $\left.\left(\mathrm{CH}_{3}\right)_{3} \mathrm{CCO}\right), 1.80$ (q, ${ }^{4} J_{\mathrm{HH}}=1.1 \mathrm{~Hz}$, $\left.3 \mathrm{H}, \mathrm{CH}_{3} \mathrm{C}=\mathrm{CH}\right), 2.31-2.46\left(\mathrm{~m}, 2 \mathrm{H}, \mathrm{CH}_{2} \mathrm{CH}_{2} \mathrm{OTBDMS}\right), 3.70$ (t, ${ }^{3} \mathrm{~J}_{\mathrm{HH}}=6.5 \mathrm{~Hz}, 2 \mathrm{H}$, $\mathrm{CH}_{2} \mathrm{CH}_{2} \mathrm{OTBS}$ ), 6.11 (tq, $\left.{ }^{3} \mathrm{~J}_{\mathrm{HH}}=7.1,{ }^{4} \mathrm{~J}_{\mathrm{HH}}=1.4 \mathrm{~Hz}, 1 \mathrm{H}, \mathrm{C}=\mathrm{CH}\right) \cdot{ }^{13} \mathrm{C}\left\{{ }^{1} \mathrm{H}\right\} \mathrm{NMR}(101 \mathrm{MHz}$, $\left.\mathrm{CDCl}_{3}\right) \delta(\mathrm{ppm})$-5.2 (( $\left.\left.\mathrm{CH}_{3}\right)_{2} \mathrm{Si}\right), 14.5\left(\mathrm{CH}_{3} \mathrm{C}=\mathrm{CH}\right), 18.4\left(\left(\mathrm{CH}_{3}\right)_{3} \mathrm{CSi}\right), 26.0\left(\left(\mathrm{CH}_{3}\right)_{3} \mathrm{CSi}\right), 28.4$ (( $\left.\left.\mathrm{CH}_{3}\right)_{3} \mathrm{CCO}\right), 32.0\left(\mathrm{CH}_{2} \mathrm{CH}_{2} \mathrm{OTBDMS}\right), 44.0\left(\left(\mathrm{CH}_{3}\right)_{3} \mathrm{CCO}\right), 62.0\left(\mathrm{CH}_{2} \mathrm{CH}_{2} \mathrm{OTBDMS}\right), 132.3$ $(\mathrm{C}=\mathrm{CH}), 137.3(\mathrm{C}=\mathrm{CH}), 211.5(\mathrm{C}=\mathrm{O}) \cdot$ LRMS $(\mathrm{IC})$ found $[\mathrm{M}+\mathrm{H}]^{+}=285$.

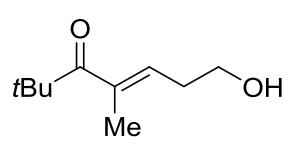

(E)-7-hydroxy-2,2,4-trimethylhept-4-en-3-one (4d): The reaction was run according to GP3 starting from (E)-7-((tert-butyldimethylsilyl)oxy)2,2,4-trimethylhept-4-en-3-one (225 mg, $0.79 \mathrm{mmol})$. The crude mixture was purified by flash chromatography on silica gel (Pentane/Diethyl ether $=1: 1$ ) to give $\mathbf{4 d}$ in $84 \%$ yield (113 mg). $\mathbf{R}_{\mathbf{f}}=0.2$ (Pentane/Diethyl ether $=2: 1$ ). IR (neat): $v\left(\mathrm{~cm}^{-1}\right)=3412,2960$, 2874, 1658, 1462, 1367, 1048, 998. ${ }^{1} \mathrm{H}$ NMR (400 MHz, $\left.\mathrm{CDCl}_{3}\right) \delta(\mathrm{ppm}) 1.23$ (s, 9H, $\left.\left(\mathrm{CH}_{3}\right)_{3} \mathrm{CCO}\right), 1.78-1.90\left(\mathrm{~m}, 3 \mathrm{H}, \mathrm{CH}_{3} \mathrm{C}=\mathrm{CH}\right), 2.31-2.46\left(\mathrm{~m}, 2 \mathrm{H}, \mathrm{CH}_{2} \mathrm{CH}_{2} \mathrm{OH}\right), 3.74\left(\mathrm{t},{ }^{3} \mathrm{~J}_{\mathrm{HH}}=\right.$ $\left.6.5 \mathrm{~Hz}, 2 \mathrm{H}, \mathrm{CH}_{2} \mathrm{CH}_{2} \mathrm{OH}\right), 5.92-6.05(\mathrm{~m}, 1 \mathrm{H}, \mathrm{C}=\mathrm{CH}) \cdot{ }^{13} \mathrm{C}\left\{{ }^{1} \mathrm{H}\right\} \mathrm{NMR}\left(101 \mathrm{MHz}, \mathrm{CDCl}_{3}\right) \delta$ (ppm) $14.7\left(\mathrm{CH}_{3} \mathrm{C}=\mathrm{CH}\right), 28.2\left(\left(\mathrm{CH}_{3}\right)_{3} \mathrm{CCO}\right), 31.7\left(\mathrm{CH}_{2} \mathrm{CH}_{2} \mathrm{OH}\right), 44.1\left(\left(\mathrm{CH}_{3}\right)_{3} \mathrm{CCO}\right), 61.9$ $\left(\mathrm{CH}_{2} \mathrm{CH}_{2} \mathrm{OH}\right), 130.4(\mathrm{C}=\mathrm{CH}), 138.7(\mathrm{C}=\mathrm{CH}), 212.0(\mathrm{C}=\mathrm{O})$. LRMS (IC) found $[\mathrm{M}+\mathrm{H}]^{+}=171$. 


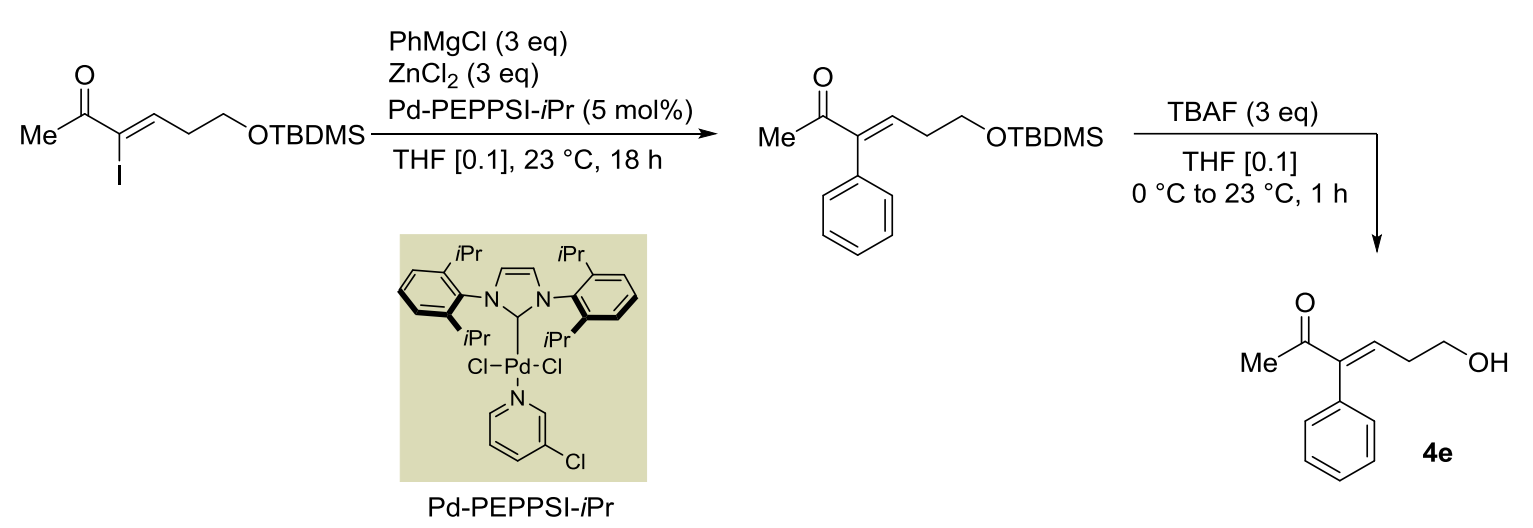

Figure S17: Synthesis of $(E)-6$-hydroxy-3-phenylhex-3-en-2-one (4e)

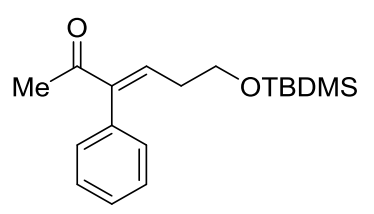

(E)-6-((tert-butyldimethylsilyl)oxy)-3-phenylhex-3-en-2-one: The reaction was run according to modified GP6A starting from (Z)-6((tert-butyldimethylsilyl)oxy)-3-iodohex-3-en-2-one $\quad(860 \quad \mathrm{mg}, \quad 2.4$ $\mathrm{mmol}$ and using [1,3-Bis(2,6-Diisopropylphenyl)imidazol-2ylidene](3-chloropyridyl)palladium(II) dichloride (Pd-PEPPSI-iPr, $81.5 \mathrm{mg}, 5 \mathrm{~mol} \%$ ) as precatalyst. The crude was purified by flash chromatography on silica gel (Pentane/Diethyl ether $=50: 1)$ to give the title compound in $60 \%$ yield $(440 \mathrm{mg}) . \mathbf{R}_{\mathbf{f}}=0.2$ (Pentane/Diethyl ether = 2:1). IR (neat): $v\left(\mathrm{~cm}^{-1}\right)=2954,2929,2858,1674,1253,1092,834 .{ }^{1} \mathbf{H}$ NMR (400 $\left.\mathrm{MHz}, \mathrm{CDCl}_{3}\right) \delta(\mathrm{ppm}) 0.03\left(\mathrm{~s}, 6 \mathrm{H},\left(\mathrm{CH}_{3}\right)_{2} \mathrm{Si}\right), 0.89$ (s, 9H, $\left.\left(\mathrm{CH}_{3}\right)_{3} \mathrm{CSi}\right), 2.25-2.36(\mathrm{~m}, 3 \mathrm{H}$, $\mathrm{CH}_{3} \mathrm{CO} ; 2 \mathrm{H} \mathrm{CH} \mathrm{CH}_{2} \mathrm{OTBDMS}$ ), 3.68 ( $\left.\mathrm{t},{ }^{3} \mathrm{JHH}_{\mathrm{HH}}=6.4 \mathrm{~Hz}, 2 \mathrm{H}, \mathrm{CH}_{2} \mathrm{CH}_{2} \mathrm{OTBDMS}\right), 6.95$ (t, ${ }^{3} \mathrm{~J}_{\mathrm{HH}}=$ $7.4 \mathrm{~Hz}, 1 \mathrm{H}, \mathrm{C}=\mathrm{CH}), 7.05-7.18(\mathrm{~m}, 2 \mathrm{H}, \mathrm{CH}-\mathrm{Ar}), 7.30-7.44(\mathrm{~m}, 3 \mathrm{H}, \mathrm{CH}-\mathrm{Ar}) .{ }^{13} \mathrm{C}\left\{{ }^{1} \mathrm{H}\right\} \mathbf{N M R}$ (101 MHz, $\left.\mathrm{CDCl}_{3}\right) \delta(\mathrm{ppm})$-5.2 $\left(\left(\mathrm{CH}_{3}\right)_{2} \mathrm{Si}\right), 26.0\left(\left(\mathrm{CH}_{3}\right)_{3} \mathrm{CSi}\right), 27.4\left(\mathrm{CH}_{2} \mathrm{CH}_{2} \mathrm{OTBDMS}\right), 33.5$ ( $\left.\mathrm{CH}_{2} \mathrm{CH}_{2} \mathrm{OTBDMS}\right), 62.0\left(\mathrm{CH}_{3} \mathrm{CO}\right), 127.6(\mathrm{CH}-\mathrm{Ar}), 128.4(\mathrm{CH}-\mathrm{Ar}), 129.7(\mathrm{CH}-\mathrm{Ar}), 136.0$ $(C=\mathrm{CH}), 141.3(\mathrm{C}=\mathrm{CH}), 144.7(\mathrm{C}-\mathrm{Ar}), 198.8(\mathrm{C}=\mathrm{O})$. LRMS $(\mathrm{IC})$ found $[\mathrm{M}+\mathrm{H}]^{+}=306$.

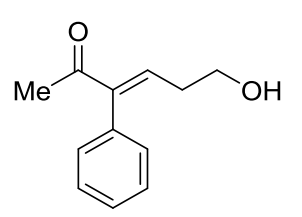

(E)-6-hydroxy-3-phenylhex-3-en-2-one (4e): The reaction was run according to GP3 starting from (E)-6-((tert-butyldimethylsilyl)oxy)-3phenylhex-3-en-2-one (440 mg, $1.2 \mathrm{mmol})$. Purification by flash chromatography on silica gel (Pentane/Diethyl ether $=1: 3$ ) afforded $4 \mathbf{e}$ in $52 \%$ yield $\left(142 \mathrm{mg}\right.$ ) as a colorless oil. $\mathbf{R}_{\mathbf{f}}=0.1$ (Pentane/Diethyl ether $\left.=1: 1\right)$. IR (neat): $v\left(\mathrm{~cm}^{-}\right.$ $\left.{ }^{1}\right)=3428,2924,1665,1353,1050,765,699 .{ }^{1} \mathbf{H}$ NMR $\left(400 \mathrm{MHz}, \mathrm{CDCl}_{3}\right) \delta(\mathrm{ppm}) 2.29$ (s, $\left.3 \mathrm{H}, \mathrm{CH}_{3} \mathrm{CO}\right), 2.35\left(\mathrm{dt},{ }^{3} J_{H H}=7.5,6.4 \mathrm{~Hz}, 2 \mathrm{H}, \mathrm{CH}_{2} \mathrm{CH}_{2} \mathrm{OH}\right), 3.73(\mathrm{t}, J=6.4 \mathrm{~Hz}$, $2 \mathrm{H}, \mathrm{CH}_{2} \mathrm{CH}_{2} \mathrm{OH}$ ), $6.94\left(\mathrm{t},{ }^{3} \mathrm{JHH}_{\mathrm{HH}}=7.4 \mathrm{~Hz}, 1 \mathrm{H}, \mathrm{C}=\mathrm{CH}\right), 7.06-7.17(\mathrm{~m}, 2 \mathrm{H}, \mathrm{CH}-\mathrm{Ar}), 7.31-7.45$ $(\mathrm{m}, 3 \mathrm{H}, \mathrm{CH}-\mathrm{Ar}) .{ }^{13} \mathrm{C}\left\{{ }^{1} \mathrm{H}\right\}$ NMR $\left(101 \mathrm{MHz}, \mathrm{CDCl}_{3}\right) \delta(\mathrm{ppm}) 27.6\left(\mathrm{CH}_{2} \mathrm{CH}_{2} \mathrm{OH}\right), 33.2$ $\left(\mathrm{CH}_{2} \mathrm{CH}_{2} \mathrm{OH}\right), 61.7\left(\mathrm{CH}_{3} \mathrm{CO}\right), 127.8(\mathrm{CH}-\mathrm{Ar}), 128.5(\mathrm{CH}-\mathrm{Ar}), 129.6(\mathrm{CH}-\mathrm{Ar}), 135.9(\mathrm{C}=\mathrm{CH})$, $140.0(\mathrm{C}=\mathrm{CH}), 145.1(\mathrm{C}-\mathrm{Ar}), 198.8(\mathrm{C}=\mathrm{O})$. LRMS $(\mathrm{IC})$ found $[\mathrm{M}+\mathrm{H}]^{+}=208$ 


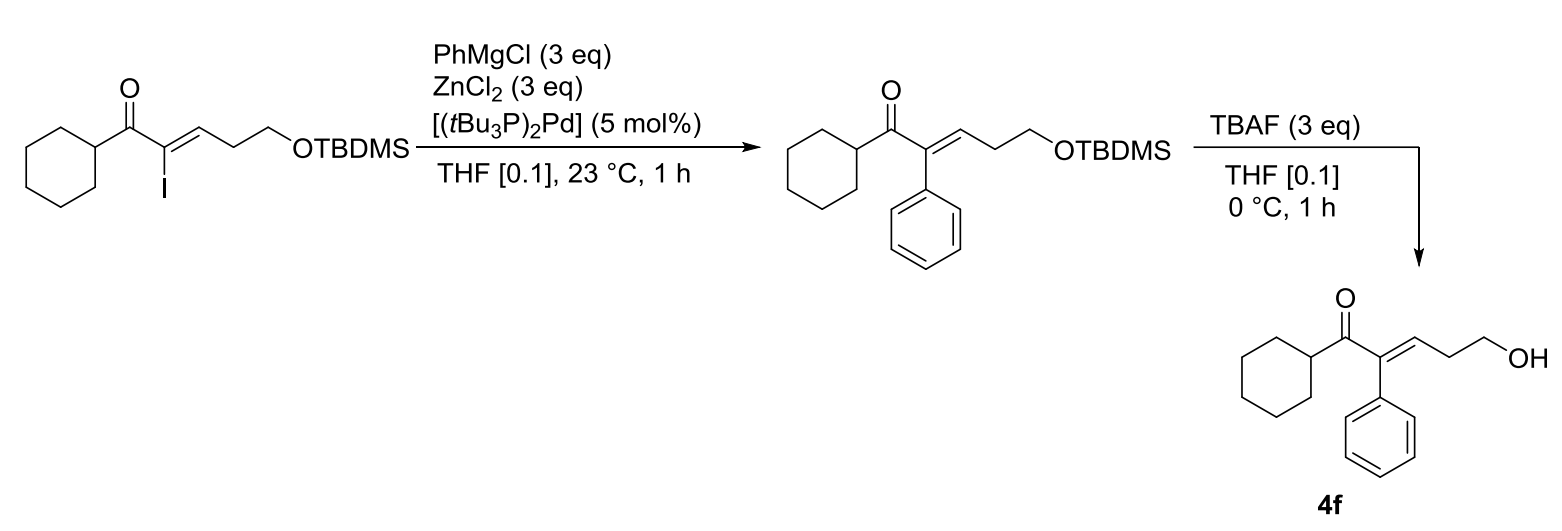

Figure S18: Synthesis of (E)-1-cyclohexyl-5-hydroxy-2-phenylpent-2-en-1-one (4f)

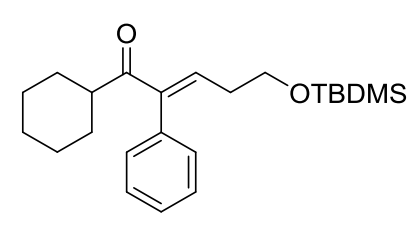

(E)-5-((tert-butyldimethylsilyl)oxy)-1-cyclohexyl-2-phenylpent-

2-en-1-one: The reaction was run according to GP6A starting from (Z)-5-((tert-butyldimethylsilyl)oxy)-1-cyclohexyl-2-iodopent-2en-1-one (569 mg, $1.3 \mathrm{mmol})$. The reaction was complete after 1 h. The crude mixture was purified by flash chromatography on silica gel (Pentane/Diethyl ether $=20: 1)$ to give the title compound in $69 \%(330 \mathrm{mg})$ as a colourless oil. $\mathbf{R}_{\mathbf{f}}=0.6$ (Pentane/Diethyl ether = 10:1). IR (neat): $v\left(\mathrm{~cm}^{-1}\right)=2930,2856,1673,1253,1098,836 .{ }^{1} \mathbf{H}$ NMR $\left(400 \mathrm{MHz}, \mathrm{CDCl}_{3}\right) \delta(\mathrm{ppm}) 0.03\left(\mathrm{~s}, 6 \mathrm{H},\left(\mathrm{CH}_{3}\right)_{2} \mathrm{Si}\right), 0.89\left(\mathrm{~s}, 9 \mathrm{H},\left(\mathrm{CH}_{3}\right)_{3} \mathrm{CSi}\right), 1.16-1.25$ (m, 3H, $\mathrm{CH}_{2}$-Cy), 1.32 - 1.44 (m, 2H, CH2-Cy), $1.59-1.69$ (m, 1H, CH $4 \mathrm{H}, \mathrm{CH}_{2}-\mathrm{Cy}$ ), $2.30\left(\mathrm{dt},{ }^{3} \mathrm{~J}_{\mathrm{HH}}=7.4,6.3 \mathrm{~Hz}, 2 \mathrm{H}, \mathrm{CH}_{2} \mathrm{CH}_{2} \mathrm{OTBDMS}\right.$ ), 2.84 (tt, ${ }^{3} J_{\mathrm{HH}}=11.6,3.2$ $\mathrm{Hz}, 1 \mathrm{H}, \mathrm{CH}-\mathrm{Cy}), 3.68\left(\mathrm{t},{ }^{3} \mathrm{~J}_{\mathrm{HH}}=6.3 \mathrm{~Hz}, 2 \mathrm{H}, \mathrm{CH}_{2} \mathrm{CH}_{2} \mathrm{OTBDMS}\right), 6.84\left(\mathrm{t},{ }^{3} \mathrm{~J}_{\mathrm{HH}}=7.4 \mathrm{~Hz}, 1 \mathrm{H}\right.$, $\mathrm{C}=\mathrm{CH}), 7.08-7.19(\mathrm{~m}, 2 \mathrm{H}, \mathrm{CH}-\mathrm{Ar}), 7.27-7.41(\mathrm{~m}, 3 \mathrm{H}, \mathrm{CH}-\mathrm{Ar}) \cdot{ }^{13} \mathbf{C}\left\{{ }^{1} \mathrm{H}\right\} \mathbf{N M R}(101 \mathrm{MHz}$, $\left.\mathrm{CDCl}_{3}\right) \delta$ (ppm) -5.22 (( $\left.\left.\mathrm{CH}_{3}\right)_{2} \mathrm{Si}\right), 18.5\left(\left(\mathrm{CH}_{3}\right)_{3} \mathrm{CSi}\right), 25.9\left(\mathrm{CH}_{2}-\mathrm{Cy}\right), 26.0\left(\mathrm{CH}_{2}-\mathrm{Cy}\right), 26.0$

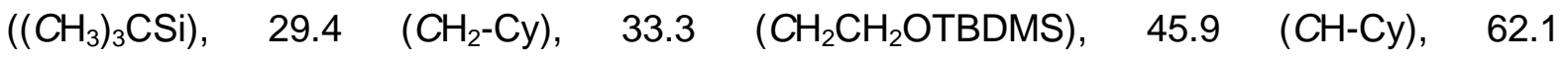
( $\left.\mathrm{CH}_{2} \mathrm{CH}_{2} \mathrm{OTBDMS}\right), 127.5$ ( $\left.\mathrm{CH}-\mathrm{Ar}\right), 128.3(\mathrm{CH}-\mathrm{Ar}), 129.7(\mathrm{CH}-\mathrm{Ar}), 136.3(\mathrm{C}-\mathrm{Ar}), 139.2$ $(\mathrm{C}=\mathrm{CH}), 143.6(\mathrm{C}=\mathrm{CH}), 204.9(\mathrm{C}=\mathrm{O})$. LRMS $(\mathrm{IC})$ found $[\mathrm{M}+\mathrm{H}]^{+}=373$.

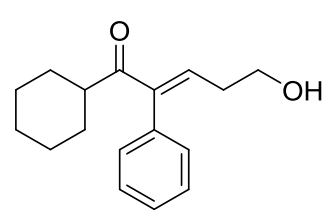

(E)-1-cyclohexyl-5-hydroxy-2-phenylpent-2-en-1-one (4f): $\quad(E)-5-$ ((tert-butyldimethylsilyl)oxy)-1-cyclohexyl-2-phenylpent-2-en-1-one (330 $\mathrm{mg}, 0.89 \mathrm{mmol})$ was dissolved in THF $(8.9 \mathrm{~mL})$ and TBAF $(1.0 \mathrm{M}$ in THF, $2.7 \mathrm{mmol}, 2.7 \mathrm{~mL}$ ) was added slowly at $0{ }^{\circ} \mathrm{C}$. After stiring at room temperature for $1 \mathrm{~h}$, the mixture was quenched with a saturated aqueous $\mathrm{NH}_{4} \mathrm{Cl}$ solution $(5 \mathrm{~mL})$, extracted with diethyl ether $(3 \times 20 \mathrm{~mL})$, dried over anhydrous $\mathrm{Na}_{2} \mathrm{SO}_{4}$ and concentrated in vacuo. Purification by flash chromatography on silica gel (Pentane/Diethyl ether $=1: 1$ ) afforded $\mathbf{4 f}$ in $65 \%$ yield $\left(150 \mathrm{mg}\right.$ ) as a light yellow oil. $\mathbf{R}_{\mathbf{f}}=0.5$ (Pentane/Diethyl ether $=1: 2)$. IR (neat): $v\left(\mathrm{~cm}^{-1}\right)=3428,2929,2855,1668,1448,1052,704 .{ }^{1} \mathbf{H}$ NMR (400 $\left.\mathrm{MHz}, \mathrm{CDCl}_{3}\right) \delta(\mathrm{ppm}) 1.10-1.25$ (m, 3H, $\left.\mathrm{CH}_{2}-\mathrm{Cy}\right), 1.30-1.44$ (m, 3H, $\left.\mathrm{CH}_{2}-\mathrm{Cy} ; \mathrm{OH}\right), 1.60$ - 
1.67 (m, 1H, CH$\left.H_{2}-\mathrm{Cy}\right), 1.69-1.82$ (m, 4H, $\left.\mathrm{CH}_{2}-\mathrm{Cy}\right), 2.30-2.41$ (m, 2H, $\mathrm{CH}_{2} \mathrm{CH}_{2} \mathrm{OTBDMS}$ ), $2.80\left(\mathrm{tt},{ }^{3} \mathrm{~J}_{\mathrm{HH}}=8.2,4.0 \mathrm{~Hz}, 1 \mathrm{H}, \mathrm{CH}-\mathrm{Cy}\right), 3.72\left(\mathrm{t},{ }^{3} \mathrm{~J}_{\mathrm{HH}}=6.4 \mathrm{~Hz}, 2 \mathrm{H}, \mathrm{CH}_{2} \mathrm{CH}_{2} \mathrm{OTBDMS}\right), 6.81$ $\left(\mathrm{t},{ }^{3} \mathrm{~J}_{\mathrm{HH}}=7.5 \mathrm{~Hz}, 1 \mathrm{H}, \mathrm{C}=\mathrm{CH}\right), 7.05-7.18(\mathrm{~m}, 2 \mathrm{H}, \mathrm{CH}-\mathrm{Ar}), 7.31-7.45(\mathrm{~m}, 3 \mathrm{H}, \mathrm{CH}-\mathrm{Ar})$. ${ }^{13} \mathrm{C}\left\{{ }^{1} \mathrm{H}\right\}$ NMR $\left(101 \mathrm{MHz}, \mathrm{CDCl}_{3}\right) \delta(\mathrm{ppm}) 25.8\left(\mathrm{CH}_{2}-\mathrm{Cy}\right), 26.0\left(\mathrm{CH}_{2}-\mathrm{Cy}\right), 29.3\left(\mathrm{CH}_{2}-\mathrm{Cy}\right), 33.2$ $\left(\mathrm{CH}_{2} \mathrm{CH}_{2} \mathrm{OH}\right), 46.1$ ( $\left.\mathrm{CH}-\mathrm{Cy}\right), 61.8\left(\mathrm{CH}_{2} \mathrm{CH}_{2} \mathrm{OH}\right), 127.6(\mathrm{CH}-\mathrm{Ar}), 128.5(\mathrm{CH}-\mathrm{Ar}), 129.6(\mathrm{CH}-$ $\mathrm{Ar}), 136.2(\mathrm{C}-\mathrm{Ar}), 138.0(\mathrm{C}=\mathrm{CH}), 144.4(\mathrm{C}=\mathrm{CH}), 205.0(\mathrm{C}=\mathrm{O})$. LRMS (IC) found $[\mathrm{M}+\mathrm{H}]^{+}=$ $259 ;\left[\mathrm{M}+\mathrm{NH}_{4}\right]^{+}=276$.

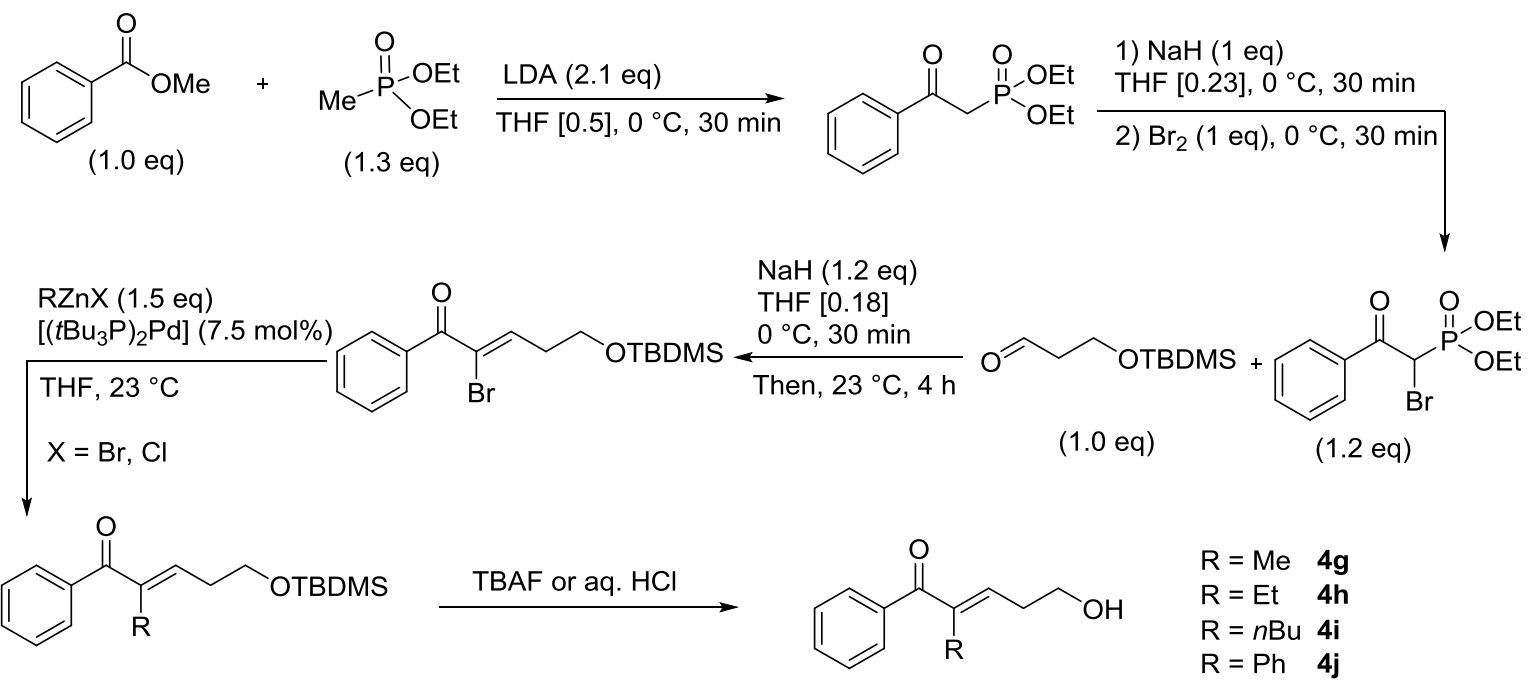

Figure S19: Synthesis of $\alpha, \beta$-unsaturated ketones $\mathbf{4 g - j}$

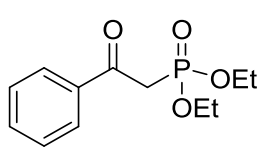

Diethyl (2-oxo-2-phenylethyl)phosphonate: In a 2-neck round bottomed flask, lithium diisopropylamide (LDA) was prepared by dropwise addition of $n \mathrm{BuLi}$ (52.22 mmol, $32.6 \mathrm{~mL}$ from a $1.6 \mathrm{M}$ sol. in hexane) to a solution of diisopropylamine $(52.22 \mathrm{mmol}, 5.28 \mathrm{~g})$ in THF $(16 \mathrm{~mL})$ at $-78{ }^{\circ} \mathrm{C}$. In a separate 3-neck flask, methyl benzoate $(23.68 \mathrm{mmol}, 3.22 \mathrm{~g})$ and methyl diethylphosphonate $(30.79 \mathrm{mmol}$, $4.68 \mathrm{~g})$ were dissolved in THF $(37.5 \mathrm{~mL})$ and cooled to $0^{\circ} \mathrm{C}$. After 30 minutes, LDA (49.73 $\mathrm{mmol}$ ) was added dropwise to the latter solution and stirred for 30 minutes at $0{ }^{\circ} \mathrm{C}$. The reaction was quenched with saturated aqueous $\mathrm{NH}_{4} \mathrm{Cl}$ solution $(40 \mathrm{~mL})$, extracted with diethyl ether $(3 \times 75 \mathrm{~mL})$, dried over $\mathrm{Na}_{2} \mathrm{SO}_{4}$ and concentrated in vacuo. Flash chromatography on silica gel (Diethyl ether) afforded the $\beta$-ketophosphonate in $96 \%$ yield $(5.93 \mathrm{~g})$ as a pale yellow oil. All spectroscopic analyses were in agreement with those reported in the literature. ${ }^{20}$

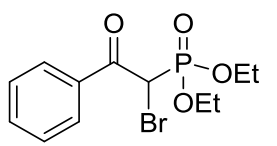

Diethyl (1-bromo-2-oxo-2-phenylethyl)phosphonate: To a solution of the $\beta$-ketophosphonate $(19 \mathrm{mmol}, 4.87 \mathrm{~g})$ in $\mathrm{THF}(83 \mathrm{~mL})$ at $0^{\circ} \mathrm{C}, \mathrm{NaH}(19$ 
$\mathrm{mmol}, 456 \mathrm{mg}$ ) was added in three portions and stirred for 30 minutes. $\mathrm{Next}, \mathrm{Br}_{2}(19 \mathrm{mmol}$, $3.04 \mathrm{~g}$ ) was carefully added at $0^{\circ} \mathrm{C}$ and stirred at the same temperature for 30 minutes. The reaction was quenched with a saturated aqueous $\mathrm{NH}_{4} \mathrm{Cl}$ solution, extracted with diethyl ether $(3 \times 100 \mathrm{~mL})$, washed with a saturated aqueous $\mathrm{Na}_{2} \mathrm{~S}_{2} \mathrm{O}_{3}$ solution, dried over $\mathrm{Na}_{2} \mathrm{SO}_{4}$ and concentrated in vacuo. The product was purified by recrystallization in diethyl ether, affording the brominated product in $60 \%$ yield $\left(3.85 \mathrm{~g}\right.$ ) as a white solid. MP: $33-35^{\circ} \mathrm{C}$. $\mathbf{R}_{\mathbf{f}}=0.5$ (Diethyl ether). IR (neat): $v\left(\mathrm{~cm}^{-1}\right)=2996,2937,1689,1594,1579,1475,1447,1392,1369,1328$, 1269, 1247, 1200, 1147, 1105, 1017, 981, 961, 846, 818, 802, 759, 740, 715, 691, 652, 623. ${ }^{1} \mathrm{H}-\mathrm{NMR}\left(\mathrm{CDCl}_{3}, 500 \mathrm{MHz}\right): \delta(\mathrm{ppm}) 1.32\left(\mathrm{dt},{ }^{3} \mathrm{~J}_{\mathrm{HH}}=6.9 \mathrm{~Hz},{ }^{4} \mathrm{~J}_{\mathrm{PH}}=22.5 \mathrm{~Hz}, 6 \mathrm{H}, \mathrm{CH}_{3} \mathrm{CH}_{2} \mathrm{O}\right)$, 4.20-4.33 (m, $\left.4 \mathrm{H}, \mathrm{CH}_{3} \mathrm{CH}_{2} \mathrm{O}\right), 5.34\left(\mathrm{~d},{ }^{2} \mathrm{~J}_{\mathrm{PH}}=13.9 \mathrm{~Hz}, 1 \mathrm{H}, \mathrm{CHP}\right), 7.47-7.51\left(\mathrm{~m}, 2 \mathrm{H}, \mathrm{CH}_{m^{-}}\right.$ Ar), $7.60-7.63\left(\mathrm{~m}, 1 \mathrm{H}, \mathrm{CH}_{p}-\mathrm{Ar}\right), 8.00-8.02(\mathrm{~m}, 2 \mathrm{H}, \mathrm{CH}-\mathrm{Ar}) \cdot{ }^{13} \mathrm{C}\left\{{ }^{1} \mathrm{H}\right\}-\mathrm{NMR}\left(\mathrm{CDCl}_{3}, 126\right.$ $\mathrm{MHz}): \delta(\mathrm{ppm}) 16.4\left(\mathrm{~m}, \mathrm{OCH}_{2} \mathrm{CH}_{3}\right), 38.6\left(\mathrm{~d},{ }^{1} J_{\mathrm{PC}}=146.1 \mathrm{~Hz}, \mathrm{CHP}\right), 64.7\left(\mathrm{t},{ }^{2} J_{\mathrm{PC}}=7.6 \mathrm{~Hz}\right.$, $\left.\mathrm{OCH}_{2} \mathrm{CH}_{3}\right), 128.9\left(\mathrm{CH}_{m}-\mathrm{Ar}\right), 129.5\left(\mathrm{CH}_{\mathrm{o}}-\mathrm{Ar}\right), 134.3\left(\mathrm{CH}_{\mathrm{p}}-\mathrm{Ar}\right), 134.7(C-\mathrm{Ar}), 189.8(C=\mathrm{O})$. ${ }^{31} \mathbf{P}\left\{{ }^{1} \mathbf{H},{ }^{13} \mathbf{C}\right\}-N M R\left(\mathrm{CDCl}_{3}, 162 \mathrm{MHz}\right): \delta(\mathrm{ppm})$ 13.4. LRMS (IC) found $[\mathrm{M}+\mathrm{H}]^{+}=336,[\mathrm{M}]=$ 335.

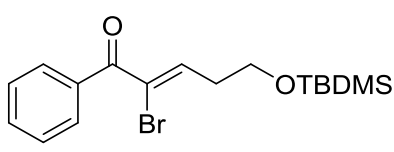

(Z)-2-bromo-5-((tert-butyldimethylsilyl)oxy)-1-phenylpent-2-en-

1-one: In a 3-neck-round-bottom flask equipped with a dropping funnel, the diethyl (1-bromo-2-oxo-2-phenylethyl)phosphonate $(6.0$ mmol, $2.01 \mathrm{~g}$ ) was dissolved in THF $(24 \mathrm{~mL})$ and cooled to $0{ }^{\circ} \mathrm{C} . \mathrm{NaH}(6.0 \mathrm{mmol}, 144 \mathrm{mg})$ was added in two portions and stirred for 30 minutes at the same temperature. 3-((tertbutyldimethylsilyl)oxy)propanal $(5.0 \mathrm{mmol}, 0.94 \mathrm{~g})$ in THF (10 mL) was added dropwise and stirred for $4 \mathrm{~h}$ at room temperature. The reaction was quenched with a saturated aqueous $\mathrm{NH}_{4} \mathrm{Cl}$ solution, extracted with diethyl ether $(3 \times 40 \mathrm{~mL})$, dried over $\mathrm{Na}_{2} \mathrm{SO}_{4}$ and concentrated in vacuo. Column chromatography on silica gel (from Pentane to Pentane/Diethyl ether = 99:1) afforded the (Z)-isomer of the product in $28 \%$ yield $(514 \mathrm{mg}$ ) as a pale yellow oil, the $(E)$-isomer in $13 \%$ yield $(243 \mathrm{mg})$ and a mixture of the two isomers in $8 \%$ yield $(136 \mathrm{mg}, E / Z$ 1:3.8). (Z)-isomer: $\mathbf{R}_{\mathbf{f}}=0.6$ (Pentane/Diethyl ether $=9: 1$ ). IR (neat): $v\left(\mathrm{~cm}^{-1}\right)=2954,2928$, 2857, 1668, 1617, 1599, 1580, 1469, 1447, 1385, 1361, 1254, 1212, 1179, 1094, 1025, 1006, 938, 834, 775, 704, 649, 570. ' $\mathrm{H}-\mathrm{NMR}\left(\mathrm{CDCl}_{3}, 500 \mathrm{MHz}\right): \delta(\mathrm{ppm}) 0.02(\mathrm{~s}, 6 \mathrm{H}$, $\left(\mathrm{CH}_{3}\right)_{2} \mathrm{Si}$ ), 0.87 (s, 9H, $\left(\mathrm{CH}_{3}\right)_{3} \mathrm{CSi}$ ), 2.25 (dt, ${ }^{3} \mathrm{~J}_{\mathrm{HH}}=7.9,6.3 \mathrm{~Hz}, 2 \mathrm{H}, \mathrm{CH}_{2} \mathrm{CH}_{2} \mathrm{OTBDMS}$ ), 3.64 (t, $\left.{ }^{3} J_{\mathrm{HH}}=6.3 \mathrm{~Hz}, 2 \mathrm{H}, \mathrm{CH}_{2} \mathrm{CH}_{2} \mathrm{OTBDMS}\right), 6.50\left(\mathrm{t},{ }^{3} \mathrm{~J}_{\mathrm{HH}}=7.9 \mathrm{~Hz}, 1 \mathrm{H}, \mathrm{C}=\mathrm{CH}\right), 7.59-7.62(\mathrm{~m}, 1 \mathrm{H}$, $\left.\mathrm{CH}_{p}-\mathrm{Ar}\right), 7.47-7.50\left(\mathrm{~m}, 2 \mathrm{H}, \mathrm{CH}_{m}-\mathrm{Ar}\right), 7.97-7.98(\mathrm{~m}, 2 \mathrm{H}, \mathrm{CH}-\mathrm{Ar}) .{ }^{13} \mathrm{C}\left\{{ }^{1} \mathrm{H}\right\}-\mathrm{NMR}\left(\mathrm{CDCl}_{3}\right.$, $126 \mathrm{MHz}): \quad \delta \quad(\mathrm{ppm}) \quad-5.2 \quad\left(\left(\mathrm{CH}_{3}\right)_{2} \mathrm{Si}\right), \quad 18.5 \quad\left(\left(\mathrm{CH}_{3}\right)_{3} \mathrm{CSi}\right), \quad 26.0 \quad\left(\left(\mathrm{CH}_{3}\right)_{3} \mathrm{CSi}\right), \quad 34.6$ $\left(\mathrm{CH}_{2} \mathrm{CH}_{2} \mathrm{OTBDMS}\right), 61.8\left(\mathrm{CH}_{2} \mathrm{CH}_{2} \mathrm{OTBDMS}\right), 116.4(\mathrm{C}=\mathrm{CH}), 128.9\left(\mathrm{CH}_{m}-\mathrm{Ar}\right), 130.1\left(\mathrm{CH}_{0^{-}}\right.$ 
Ar), $134.2\left(\mathrm{CH}_{p}-\mathrm{Ar}\right), 134.6(\mathrm{C}-\mathrm{Ar}), 135.9(\mathrm{C}=\mathrm{CH}), 191.2(\mathrm{C}=\mathrm{O})$. LRMS (IC) found $[\mathrm{M}+\mathrm{H}]^{+}=$ $370,[\mathrm{M}]=369$.

\section{Procedure for Negishi cross-coupling (GP6B)}

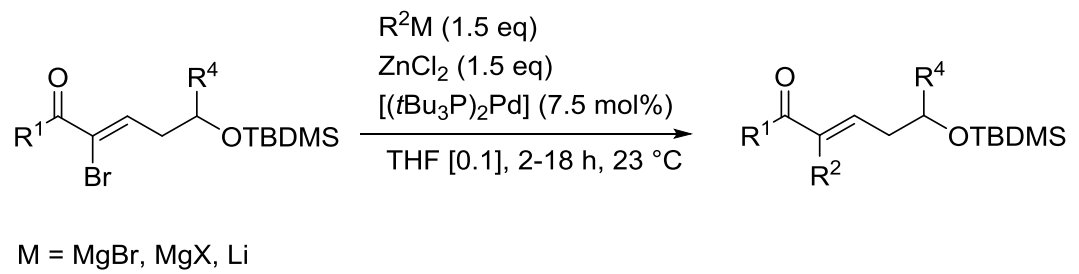

In a Young valve Schlenk, the electrophilic alkylzinc reagent was prepared by mixing the appropriate Grignard or alkylithium reagent (1.5 eq) and $\mathrm{ZnCl}_{2}(1.5 \mathrm{eq}, 1.0 \mathrm{M}$ in THF). A white precipitate formed rapidly. In separate Schlenk, $\left[\left(t \mathrm{Bu}_{3} \mathrm{P}\right)_{2} \mathrm{Pd}\right](7.55 \mathrm{~mol} \%)$ was dissolved in THF $(0.1 \mathrm{M})$ and the appropriate bromo-derivative $(1 \mathrm{eq})$ was added. The latter solution was transferred by cannula to the suspension of the alkylzinc reagent and stirred at room temperature. After 2-18 h, the mixture was quenched with saturated aqueous $\mathrm{NH}_{4} \mathrm{Cl}$ solution, extracted with diethyl ether (3 times), dried over anhydrous $\mathrm{Na}_{2} \mathrm{SO}_{4}$ and concentrated in vacuo.

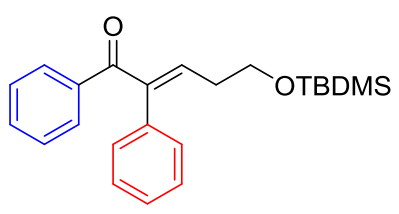

(E)-5-((tert-butyldimethylsilyl)oxy)-1,2-diphenylpent-2-en-1one: The reaction was run according to GP6B starting from (Z)-2bromo-5-((tert-butyldimethylsilyl)oxy)-1-phenylpent-2-en-1-one $(2.0 \mathrm{~g}, 5.44 \mathrm{mmol}, E / Z 1: 2.7)$. The reaction was complete after $4 \mathrm{~h}$. Purification by column chromatography on silica gel (from Pentane to Pentane/Diethyl ether = 98:2) afforded the $(E)$-isomer of the product in $19 \%$ yield $(381 \mathrm{mg})$ as a pale green oil and a mixture of isomers in $29 \%$ yield (578 $\mathrm{mg}, E / Z 2.6: 1)$ as a pale yellow oil. $(E)$-isomer: $\mathbf{R}_{\mathbf{f}}=$ 0.5 (Pentane/Diethyl ether = 9:1). IR (neat): $v\left(\mathrm{~cm}^{-1}\right)=3060,2954,2928,2856,1657,1598$, 1579, 1495, 1468, 1446, 1386, 1361, 1315, 1252, 1218, 1176, 1093, 1026, 1007, 944, 834, 810, 775, 761, 722, 697, 660, 626, 610. ' $\mathrm{H}-\mathrm{NMR}\left(\mathrm{CDCl}_{3}, 500 \mathrm{MHz}\right): \delta(\mathrm{ppm}) 0.02(\mathrm{~s}, 6 \mathrm{H}$, $\left.\left(\mathrm{CH}_{3}\right)_{2} \mathrm{Si}\right), 0.87\left(\mathrm{~s}, 9 \mathrm{H},\left(\mathrm{CH}_{3}\right)_{3} \mathrm{CSi}\right), 2.48\left(\mathrm{q},{ }^{3} \mathrm{~J}_{\mathrm{HH}}=6.2 \mathrm{~Hz}, 2 \mathrm{H}, \mathrm{CH}_{2} \mathrm{CH}_{2} \mathrm{OTBDMS}\right), 3.72$ (t, $\left.{ }^{3} J_{\mathrm{HH}}=6.2 \mathrm{~Hz}, 2 \mathrm{H}, \mathrm{CH}_{2} \mathrm{CH}_{2} \mathrm{OTBDMS}\right), 6.52\left(\mathrm{t},{ }^{3} \mathrm{~J}_{\mathrm{HH}}=7.4 \mathrm{~Hz}, 1 \mathrm{H}, \mathrm{C}=\mathrm{CH}\right), 7.30-7.32(\mathrm{~m}, 2 \mathrm{H}$, $\left.\mathrm{CH}_{m}-\mathrm{Ar}\right), 7.30-7.33\left(\mathrm{~m}, 1 \mathrm{H}, \mathrm{CH}_{p}-\mathrm{Ar}\right), 7.36-7.39\left(\mathrm{~m}, 2 \mathrm{H}, \mathrm{CH}_{m}-\mathrm{Ar}\right), 7.40-7.43\left(\mathrm{~m}, 2 \mathrm{H}, \mathrm{CH}_{0^{-}}\right.$ $\mathrm{Ar}), 7.49-7.53\left(\mathrm{~m}, 1 \mathrm{H}, \mathrm{CH}_{\mathrm{p}}-\mathrm{Ar}\right), 7.80$ - $7.82(\mathrm{~m}, 2 \mathrm{H}, \mathrm{CH}-\mathrm{Ar}) \cdot{ }^{13} \mathrm{C}\left\{{ }^{1} \mathrm{H}\right\}-\mathrm{NMR}\left(\mathrm{CDCl}_{3}, 126\right.$ $\mathrm{MHz}): \delta(\mathrm{ppm})$-5.3 $\left(\left(\mathrm{CH}_{3}\right)_{2} \mathrm{Si}\right), 18.4\left(\left(\mathrm{CH}_{3}\right)_{3} \mathrm{CSi}\right), 26.0\left(\left(\mathrm{CH}_{3}\right)_{3} \mathrm{CSi}\right), 33.2\left(\mathrm{CH}_{2} \mathrm{CH}_{2} \mathrm{OTBDMS}\right)$, $62.1\left(\mathrm{CH}_{2} \mathrm{CH}_{2} \mathrm{OTBDMS}\right), 127.7\left(\mathrm{CH}_{m}-\mathrm{Ar}\right), 128.2\left(\mathrm{CH}_{\mathrm{o}}-\mathrm{Ar}\right), 128.3\left(\mathrm{CH}_{\mathrm{m}}-\mathrm{Ar}\right), 129.7\left(\mathrm{CH}_{\mathrm{p}}-\mathrm{Ar}\right)$, 
$129.9\left(\mathrm{CH}_{o}-\mathrm{Ar}\right), 132.1\left(\mathrm{CH}_{p}-\mathrm{Ar}\right), 136.0$ (C-Ar), $138.4(\mathrm{C}=\mathrm{CH}), 141.8(\mathrm{C}=\mathrm{CH}), 143.0$ (C-Ar), $197.4(C=\mathrm{O})$. LRMS (IC) found $[\mathrm{M}+\mathrm{H}]^{+}=368$.

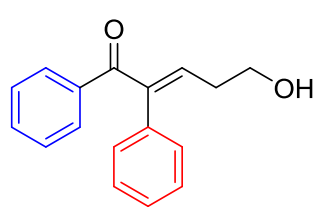

(E)-5-hydroxy-1,2-diphenylpent-2-en-1-one (4j): The reaction was run according to GP3 starting from (E)-5-((tert-butyldimethylsilyl)oxy)-1,2diphenylpent-2-en-1-one (0.56 mmol, $206 \mathrm{mg})$. Purification by column chromatography on silica gel (Pentane/Diethyl ether from 7:3 to 65:35) afforded $\mathbf{4} \mathbf{j}$ in $38 \%$ yield $\left(53 \mathrm{mg}\right.$ ) as yellow oil. $(E)$-isomer: $\mathbf{R}_{\mathbf{f}}=0.3$ (Pentane/Diethyl ether $=$ 1:1). IR (neat): $v\left(\mathrm{~cm}^{-1}\right)=3432,3058,2922,2854,1648,1596,1578,1494,1446,1377$, 1315, 1267, 1217, 1176, 1045, 1023, 920, 799, 724, 698. ${ }^{1} \mathbf{H}-\mathbf{N M R}\left(\mathrm{CDCl}_{3}, 500 \mathrm{MHz}\right): \delta$ (ppm) $1.61(\mathrm{OH}), 2.44\left(\mathrm{q}, 2 \mathrm{H},{ }^{3} \mathrm{~J}_{\mathrm{HH}}=6.4 \mathrm{~Hz}, \mathrm{CH}_{2} \mathrm{CH}_{2} \mathrm{OH}\right), 3.74$ (t, ${ }^{3} \mathrm{~J}_{\mathrm{HH}}=6.4 \mathrm{~Hz}, 2 \mathrm{H}$, $\left.\mathrm{CH}_{2} \mathrm{CH}_{2} \mathrm{OH}\right), 6.49\left(\mathrm{t},{ }^{3} \mathrm{~J}_{\mathrm{HH}}=7.4 \mathrm{~Hz}, 1 \mathrm{H}, \mathrm{C}=\mathrm{CH}\right), 7.28-7.30\left(\mathrm{~m}, 2 \mathrm{H}, \mathrm{CH} \mathrm{o}_{0}-\mathrm{Ar}\right), 7.30-7.33(\mathrm{~m}$, $\left.1 \mathrm{H}, \mathrm{CH}_{p}-\mathrm{Ar}\right), 7.36-7.39\left(\mathrm{~m}, 2 \mathrm{H}, \mathrm{CH}_{m}-\mathrm{Ar}\right), 7.40-7.43\left(\mathrm{~m}, 2 \mathrm{H}, \mathrm{CH}_{m}-\mathrm{Ar}\right), 7.49-7.53(\mathrm{~m}, 1 \mathrm{H}$, $\mathrm{CH}_{\mathrm{p}}$-Ar), $7.79-7.81$ (m, 2H, CHo $\left.-\mathrm{Ar}\right) .{ }^{13} \mathrm{C}\left\{{ }^{1} \mathrm{H}\right\}-\mathrm{NMR}\left(\mathrm{CDCl}_{3}, 126 \mathrm{MHz}\right): \delta$ (ppm) 33.1 $\left(\mathrm{CH}_{2} \mathrm{CH}_{2} \mathrm{OH}\right), 61.9\left(\mathrm{CH}_{2} \mathrm{CH}_{2} \mathrm{OH}\right), 127.8\left(\mathrm{CH}_{\mathrm{p}}-\mathrm{Ar}\right), 128.4\left(\mathrm{CH}_{\mathrm{m}}-\mathrm{Ar}\right), 128.5\left(\mathrm{CH}_{\mathrm{m}}-\mathrm{Ar}\right), 129.6$ $\left(\mathrm{CH}_{\mathrm{o}}-\mathrm{Ar}\right), 129.9\left(\mathrm{CH}_{\mathrm{o}}-\mathrm{Ar}\right), 132.3\left(\mathrm{CH}_{\mathrm{p}}-\mathrm{Ar}\right), 135.9(\mathrm{C}-\mathrm{Ar}), 138.2(\mathrm{C}=\mathrm{CH}), 140.1(\mathrm{C}=\mathrm{CH}), 143.8$ (C-Ar), $197.2(C=\mathrm{O})$. LRMS (IC) found $[\mathrm{M}+\mathrm{H}]^{+}=253$.

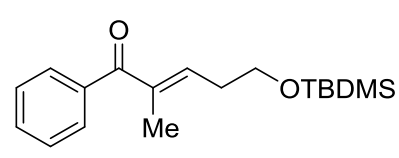

(E)-5-((tert-butyldimethylsilyl)oxy)-2-methyl-1-phenylpent-2-en-

1-one: The reaction was run according to GP6B starting from (Z)-2bromo-5-((tert-butyldimethylsilyl)oxy)-1-phenylpent-2-en-1-one (1.4 $\mathrm{mmol}$ ). After purification by flash chromatography on silica gel (Pentane/Diethyl ether $=95: 5$ ) the product was obtained in $80 \%$ yield $\left(342 \mathrm{mg}\right.$ ) as a yellow oil. $(E)$-isomer: $\mathbf{R}_{\mathbf{f}}=0.5$ (Pentane/Diethyl ether $=9: 1)$. IR (neat): $v\left(\mathrm{~cm}^{-1}\right)=2954,2930,2891,2858,1647,1598$, 1577, 1469, 1446, 1384, 1360, 1321, 1283, 1252, 1217, 1162, 1096, 1048, 1006, 939, 834, 810, 775, 701, 662, 637. ' $\mathrm{H}-\mathrm{NMR}\left(\mathrm{CDCl}_{3}, 500 \mathrm{MHz}\right): \delta(\mathrm{ppm}) 0.01\left(\mathrm{~s}, 6 \mathrm{H},\left(\mathrm{CH}_{3}\right)_{2} \mathrm{Si}\right), 0.85(\mathrm{~s}$, $\left.9 \mathrm{H},\left(\mathrm{CH}_{3}\right)_{3} \mathrm{CSi}\right), 1.98\left(\mathrm{~s}, 3 \mathrm{H}, \mathrm{CH}_{3} \mathrm{C}=\mathrm{CH}\right.$ ), 2.50 (q, ${ }^{3} J_{\mathrm{HH}}=7.2 \mathrm{~Hz}, 2 \mathrm{H}, \mathrm{CH}_{2} \mathrm{CH}_{2} \mathrm{OTBDMS}$ ), 3.70 $\left(\mathrm{t},{ }^{3} \mathrm{~J}_{\mathrm{HH}}=6.4 \mathrm{~Hz}, 2 \mathrm{H}, \mathrm{CH}_{2} \mathrm{CH}_{2} \mathrm{OTBDMS}\right), 6.33-6.36(\mathrm{~m}, 1 \mathrm{H}, \mathrm{C}=\mathrm{CH}), 7.38-7.41\left(\mathrm{~m}, 2 \mathrm{H}, \mathrm{CH}_{m^{-}}\right.$ Ar), $7.47-7.51\left(\mathrm{~m}, 1 \mathrm{H}, \mathrm{CH}_{p}-\mathrm{Ar}\right), 7.62-7.64(\mathrm{~m}, 2 \mathrm{H}, \mathrm{CH}-\mathrm{Ar}) \cdot{ }^{13} \mathbf{C}\left\{{ }^{1} \mathrm{H}\right\}-\mathrm{NMR}\left(\mathrm{CDCl}_{3}, 126\right.$ $\mathrm{MHz}): \delta$ (ppm) -5.3 $\left(\left(\mathrm{CH}_{3}\right)_{2} \mathrm{Si}\right), 12.8\left(\mathrm{CH}_{3} \mathrm{C}=\mathrm{CH}\right), 18.4\left(\left(\mathrm{CH}_{3}\right)_{3} \mathrm{CSi}\right), 26.0\left(\left(\mathrm{CH}_{3}\right)_{3} \mathrm{CSi}\right), 32.8$ ( $\left.\mathrm{CH}_{2} \mathrm{CH}_{2} \mathrm{OTBDMS}\right), 61.7\left(\mathrm{CH}_{2} \mathrm{CH}_{2} \mathrm{OTBDMS}\right), 128.2\left(\mathrm{CH}_{m}-\mathrm{Ar}\right), 129.4\left(\mathrm{CH}_{o}-\mathrm{Ar}\right), 131.5\left(\mathrm{CH}_{p^{-}}\right.$ Ar), $137.8(\mathrm{C}=\mathrm{CH}), 138.8(\mathrm{C}-\mathrm{Ar}), 143.6(\mathrm{C}=\mathrm{CH}), 199.1(\mathrm{C}=\mathrm{O})$. LRMS (IC) found $[\mathrm{M}+\mathrm{H}]^{+}=$ 306. 


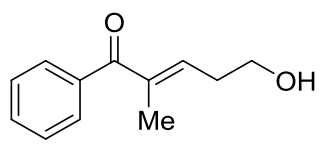

(E)-5-hydroxy-2-methyl-1-phenylpent-2-en-1-one $(\mathbf{4 g})$ : The product was synthetized according to GP3 starting from (E)-5-((tertbutyldimethylsilyl)oxy)-2-methyl-1-phenylpent-2-en-1-one $(1.12 \mathrm{mmol}$, $340 \mathrm{mg}$ ). Purification by flash chromatography on silica gel (Pentane/Diethyl ether $=1: 1$ ) afforded $\mathbf{4 g}$ in $71 \%$ yield $\left(152 \mathrm{mg}\right.$ ) as a yellow oil. $(E)$-isomer: $\mathbf{R}_{\mathbf{f}}=0.4$ (Pentane/Diethyl ether =1:1). IR (neat): $v\left(\mathrm{~cm}^{-1}\right)=3409,3059,2926,2880,1633,1597,1576,1446,1385$, 1359, 1320, 1281, 1200, 1179, 1158, 1041, 940, 901, 785, 703, 637. ${ }^{1} \mathrm{H}-\mathrm{NMR}\left(\mathrm{CDCl}_{3}, 500\right.$ $\mathrm{MHz}): \delta$ (ppm) 1.54 (bs, OH), $2.00\left(\mathrm{~s}, 3 \mathrm{H}, \mathrm{CH}_{3} \mathrm{C}=\mathrm{CH}\right), 2.57\left(\mathrm{~m}, 2 \mathrm{H}, \mathrm{CH}_{2} \mathrm{CH}_{2} \mathrm{OH}\right), 3.75\left(\mathrm{t},{ }^{3} \mathrm{JHH}_{\mathrm{HH}}\right.$ $\left.=6.5 \mathrm{~Hz}, 2 \mathrm{H}, \mathrm{CH}_{2} \mathrm{CH}_{2} \mathrm{OH}\right), 6.31(\mathrm{~m}, 1 \mathrm{H}, \mathrm{C}=\mathrm{CH}), 7.40-743\left(\mathrm{~m}, 2 \mathrm{H}, \mathrm{CH}_{m}-\mathrm{Ar}\right), 7.63-7.65(\mathrm{~m}$, $\left.2 \mathrm{H}, \mathrm{CH}_{0}-\mathrm{Ar}\right), 7.49-7.52\left(\mathrm{~m}, 1 \mathrm{H}, \mathrm{CH}_{\mathrm{p}}-\mathrm{Ar}\right) .{ }^{13} \mathrm{C}\left\{{ }^{1} \mathrm{H}\right\}-\mathrm{NMR}\left(\mathrm{CDCl}_{3}, 126 \mathrm{MHz}\right): \delta(\mathrm{ppm}) 13.0$ $\left(\mathrm{CH}_{3} \mathrm{C}=\mathrm{CH}\right), 32.6\left(\mathrm{CH}_{2} \mathrm{CH}_{2} \mathrm{OH}\right), 61.5\left(\mathrm{CH}_{2} \mathrm{CH}_{2} \mathrm{OH}\right), 141.9(\mathrm{C}=\mathrm{CH}), 128.2\left(\mathrm{CH}_{m}-\mathrm{Ar}\right), 129.5$ $\left(\mathrm{CH}_{\mathrm{o}}-\mathrm{Ar}\right), 131.7\left(\mathrm{CH}_{\mathrm{p}}-\mathrm{Ar}\right), 138.5(C-\mathrm{Ar}$ or $C=\mathrm{CH}), 138.6(C-\mathrm{Ar}$ or $C=\mathrm{CH}), 198.9(C=\mathrm{O})$. LRMS (IC) found $[\mathrm{M}+\mathrm{H}]^{+}=191$.

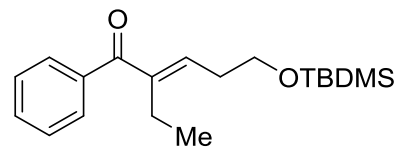

(E)-5-((tert-butyldimethylsilyl)oxy)-2-ethyl-1-phenylpent-2-en-1one: The reaction was run according to GP6B starting from (Z)-2bromo-5-((tert-butyldimethylsilyl)oxy)-1-phenylpent-2-en-1-one $(1.95 \mathrm{mmol})$. After purification by flash chromatography on silica gel (Pentane/Diethyl ether = $9: 1)$ the product was obtained in $80 \%$ yield $\left(472 \mathrm{mg}\right.$ ) as colourless oil. $(E)$-isomer: $\mathbf{R}_{\mathbf{f}}=0.1$ $\left(\mathrm{CH}_{2} \mathrm{Cl}_{2} /\right.$ Acetone $\left.=1: 1\right)$. IR (neat): $v\left(\mathrm{~cm}^{-1}\right)=2956,2930,2858,1649,1598,1578,1463$, 1447, 1385, 1362, 1289, 1251, 1217, 1162, 1094, 1053, 1025, 1006, 988, 942, 832, 775, 724, 701, 662. ${ }^{1} \mathrm{H}-\mathrm{NMR}\left(\mathrm{CDCl}_{3}, 500 \mathrm{MHz}\right): \delta(\mathrm{ppm}) 0.02\left(\mathrm{~s}, 6 \mathrm{H},\left(\mathrm{CH}_{3}\right)_{2} \mathrm{Si}\right), 0.86(\mathrm{~s}, 9 \mathrm{H}$, $\left.\left(\mathrm{CH}_{3}\right)_{3} \mathrm{CSi}\right), 1.06\left(\mathrm{t},{ }^{3} \mathrm{~J}_{\mathrm{HH}}=7.5 \mathrm{~Hz}, 3 \mathrm{H}, \mathrm{CH}_{2} \mathrm{CH}_{3}\right), 2.48-2.53\left(\mathrm{~m}, 2 \mathrm{H}, \mathrm{CH}_{2} \mathrm{CH}_{2} \mathrm{OTBDMS} ; 2 \mathrm{H}\right.$, $\mathrm{CH}_{2} \mathrm{CH}_{3}$ ), $3.70\left(\mathrm{t},{ }^{3} \mathrm{~J}_{\mathrm{HH}}=6.4 \mathrm{~Hz}, 2 \mathrm{H}, \mathrm{CH}_{2} \mathrm{CH}_{2} \mathrm{OTBDMS}\right), 6.22\left(\mathrm{t},{ }^{3} J_{\mathrm{HH}}=7.2 \mathrm{~Hz}, 1 \mathrm{H}, \mathrm{C}=\mathrm{CH}\right)$, 7.39 - $7.42\left(\mathrm{~m}, 2 \mathrm{H}, \mathrm{CH}_{m}-\mathrm{Ar}\right), 7.48-7.51\left(\mathrm{~m}, 1 \mathrm{H}, \mathrm{CH}_{p}-\mathrm{Ar}\right), 7.64-7.66\left(\mathrm{~m}, 2 \mathrm{H}, \mathrm{CH} \mathrm{H}_{0}-\mathrm{Ar}\right)$. ${ }^{13} \mathrm{C}\left\{{ }^{1} \mathrm{H}\right\}-N M R\left(\mathrm{CDCl}_{3}, 126 \mathrm{MHz}\right): \delta(\mathrm{ppm})-5.3\left(\left(\mathrm{CH}_{3}\right)_{2} \mathrm{Si}\right), 13.7\left(\mathrm{CH}_{2} \mathrm{CH}_{3}\right), 18.4\left(\left(\mathrm{CH}_{3}\right)_{3} \mathrm{CSi}\right)$, $20.4\left(\mathrm{CH}_{2} \mathrm{CH}_{3}\right), 26.0\left(\left(\mathrm{CH}_{3}\right)_{3} \mathrm{CSi}\right), 32.4\left(\mathrm{CH}_{2} \mathrm{CH}_{2} \mathrm{OTBDMS}\right), 62.0\left(\mathrm{CH}_{2} \mathrm{CH}_{2} \mathrm{OTBDMS}\right), 128.2$ $\left(\mathrm{CH}_{m}-\mathrm{Ar}\right), 129.5\left(\mathrm{CH}_{o}-\mathrm{Ar}\right), 131.6\left(\mathrm{CH}_{p}-\mathrm{Ar}\right), 139.1(\mathrm{C}-\mathrm{Ar}), 142.1(\mathrm{C}=\mathrm{CH}), 143.9(\mathrm{C}=\mathrm{CH}), 199.0$ $(C=O)$. LRMS (IC) found $[\mathrm{M}+\mathrm{H}]^{+}=320$.

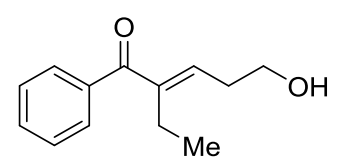

(E)-2-ethyl-5-hydroxy-1-phenylpent-2-en-1-one (4h): The reaction was run according to GP4 starting from (E)-5-((tertbutyldimethylsilyl)oxy)-2-ethyl-1-phenylpent-2-en-1-one $(1.39 \mathrm{mmol})$. The reaction was complete after $1.5 \mathrm{~h}$. The crude mixture was purified on a small pad of silica gel (Pentane/Diethyl ether $=6: 4$ ) to afford $\mathbf{4 h}$ in $52 \%$ yield $(158 \mathrm{mg}$ ) as pale yellow oil. $(E)$-isomer: $\mathbf{R}_{\mathbf{f}}=0.2$ (Pentane/Diethyl ether $=1: 1$ ). IR (neat): $v\left(\mathrm{~cm}^{-1}\right)=3420,2966,2870$, 
1635, 1596, 1577, 1446, 1380, 1313, 1288, 1253, 1046, 906, 860, 703. ${ }^{1} \mathrm{H}-\mathrm{NMR}\left(\mathrm{CDCl}_{3}, 500\right.$ $\mathrm{MHz}): \delta(\mathrm{ppm}) 1.06\left(\mathrm{td},{ }^{3} \mathrm{~J}_{\mathrm{HH}}=7.5 \mathrm{~Hz},{ }^{5} \mathrm{~J}_{\mathrm{HH}}=0.9 \mathrm{~Hz}, 3 \mathrm{H}, \mathrm{CH}_{3} \mathrm{CH}_{2}\right.$ ), 1.58 (bs, OH). 2.52 (q, ${ }^{3} J_{\mathrm{HH}}=7.5 \mathrm{~Hz}, 2 \mathrm{H}, \mathrm{CH}_{3} \mathrm{CH}_{2}$ ), 2.57 (q, ${ }^{3} \mathrm{~J}_{\mathrm{HH}}=7.4 \mathrm{~Hz}, 2 \mathrm{H}, \mathrm{CH}_{2} \mathrm{CH}_{2} \mathrm{OH}$ ), 3.74 (td, ${ }^{3} J_{\mathrm{HH}}=6.5 \mathrm{~Hz}$, $\left.{ }^{3} J_{\mathrm{HH}}=1.7 \mathrm{~Hz}, 2 \mathrm{H}, \mathrm{CH}_{2} \mathrm{CH}_{2} \mathrm{OH}\right), 6.18\left(\mathrm{t},{ }^{3} \mathrm{~J}_{\mathrm{HH}}=7.3 \mathrm{~Hz}, 1 \mathrm{H}, \mathrm{C}=\mathrm{CH}\right), 7.40-7.43\left(\mathrm{~m}, 2 \mathrm{H}, \mathrm{CH}_{m^{-}}\right.$ $\mathrm{Ar}), 7.65$ - 7.67 (m, 2H, CHo $-\mathrm{Ar}), 7.49-7.52\left(\mathrm{~m}, 1 \mathrm{H}, \mathrm{CH}_{\mathrm{p}}-\mathrm{Ar}\right) \cdot{ }^{13} \mathrm{C}\left\{{ }^{1} \mathrm{H}\right\}-\mathrm{NMR}\left(\mathrm{CDCl}_{3}, 126\right.$ $\mathrm{MHz}): \delta$ (ppm) $13.7\left(\mathrm{CH}_{3} \mathrm{CH}_{2}\right), 20.5\left(\mathrm{CH}_{3} \mathrm{CH}_{2}\right), 32.2\left(\mathrm{CH}_{2} \mathrm{CH}_{2} \mathrm{OH}\right), 61.8\left(\mathrm{CH}_{2} \mathrm{CH}_{2} \mathrm{OH}\right), 128.2$ $\left(\mathrm{CH}_{m}-\mathrm{Ar}\right), 129.5\left(\mathrm{CH}_{o}-\mathrm{Ar}\right), 131.8\left(\mathrm{CH}_{p}-\mathrm{Ar}\right), 138.9(\mathrm{C}-\mathrm{Ar}), 140.3(\mathrm{C}=\mathrm{CH}), 144.7(\mathrm{C}=\mathrm{CH}), 198.9$ $(C=\mathrm{O})$. LRMS (IC) found $[\mathrm{M}+\mathrm{H}]^{+}=205$.

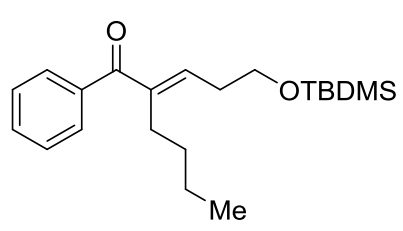

(E)-2-(3-((tert-butyldimethylsilyl)oxy)propylidene)-1phenylhexan-1-one: The reaction was run according to GP6B starting from (Z)-2-bromo-5-((tert-butyldimethylsilyl)oxy)-1phenylpent-2-en-1-one $(2.08 \mathrm{mmol})$. After purification by flash chromatography on silica gel (Pentane/Diethyl ether 98:2) the product was obtained in $50 \%$ yield (360 $\mathrm{mg}$ ) as colourless oil. $(E)$-isomer: $\mathbf{R}_{\mathbf{f}}=0.5$ (Pentane/Diethyl ether $\left.=9: 1\right)$. IR (neat): $v\left(\mathrm{~cm}^{-1}\right)=2993,2929,2854,1650,1597,1578,1463,1446,1387,1275,1253,1094,941$, 834, 775, 701. ' $\mathrm{H}-\mathrm{NMR}\left(\mathrm{CDCl}_{3}, 500 \mathrm{MHz}\right): \delta(\mathrm{ppm}) 0.02$ (s, 9H(CH$\left.)_{3} \mathrm{CSi}\right), 0.86$ (s, $6 \mathrm{H}\left(\mathrm{CH}_{3}\right)_{2} \mathrm{Si}$ ), 0.92 (t, 3H, $\mathrm{CH}_{3} \mathrm{CH}_{2} \mathrm{CH}_{2} \mathrm{CH}_{2}$ ), 1.35-1.45 (m, 4H, $\left.\mathrm{CH}_{3} \mathrm{CH}_{2} \mathrm{CH}_{2} \mathrm{CH}_{2}\right), 2.47-2.53$ (m, $4 \mathrm{H} \mathrm{CH} \mathrm{CH}_{2} \mathrm{OTBDMS}, \mathrm{CH}_{3} \mathrm{CH}_{2} \mathrm{CH}_{2} \mathrm{CH}_{2}$ ), 3.69 (t, ${ }^{3} J_{\mathrm{HH}}=6.4 \mathrm{~Hz}, 2 \mathrm{H}, \mathrm{CH}_{2} \mathrm{CH}_{2} \mathrm{OTBDMS}$ ), $6.22\left(\mathrm{t},{ }^{3} \mathrm{~J}_{\mathrm{HH}}=7.1 \mathrm{~Hz}, 1 \mathrm{H}, \mathrm{C}=\mathrm{CH}\right), 7.38-7.42\left(\mathrm{~m}, 2 \mathrm{H}, \mathrm{CH}_{m}-\mathrm{Ar}\right), 7.48-7.51\left(\mathrm{~m}, 1 \mathrm{H}, \mathrm{CH} \mathrm{H}_{p}-\mathrm{Ar}\right)$, $7.65-7.67$ (m, 2H, CHo $-\mathrm{Ar}) .{ }^{13} \mathrm{C}\left\{{ }^{1} \mathrm{H}\right\}-\mathrm{NMR}\left(\mathrm{CDCl}_{3}, 126 \mathrm{MHz}\right): \delta(\mathrm{ppm})-5.3\left(\left(\mathrm{CH}_{3}\right)_{2} \mathrm{Si}\right), 14.1$ $\left(\mathrm{CH}_{3} \mathrm{CH}_{2} \mathrm{CH}_{2} \mathrm{CH}_{2}\right), \quad 18.4 \quad\left(\left(\mathrm{CH}_{3}\right)_{3} \mathrm{CSi}\right), \quad 23.0 \quad\left(\mathrm{CH}_{3} \mathrm{CH}_{2} \mathrm{CH}_{2} \mathrm{CH}_{2}\right), \quad 26.0 \quad\left(\left(\mathrm{CH}_{3}\right)_{3} \mathrm{CSi}\right), \quad 26.8$ $\left(\mathrm{CH}_{3} \mathrm{CH}_{2} \mathrm{CH}_{2} \mathrm{CH}_{2}\right), \quad 31.3 \quad\left(\mathrm{CH}_{3} \mathrm{CH}_{2} \mathrm{CH}_{2} \mathrm{CH}_{2}\right), \quad 32.6 \quad\left(\mathrm{CH}_{2} \mathrm{CH}_{2} \mathrm{OTBDMS}\right), \quad 62.0$ $\left(\mathrm{CH}_{2} \mathrm{CH}_{2} \mathrm{OTBDMS}\right), 128.2\left(\mathrm{CH}_{m}-\mathrm{Ar}\right), 129.6\left(\mathrm{CH}_{o}-\mathrm{Ar}\right), 131.6\left(\mathrm{CH}_{p}-\mathrm{Ar}\right), 139.1(\mathrm{C}-\mathrm{Ar}), 142.2$ $(\mathrm{C}=\mathrm{CH}), 142.7(\mathrm{C}=\mathrm{CH}), 199.0(\mathrm{C}=\mathrm{O})$. LRMS $(\mathrm{IC})$ found $[\mathrm{M}+\mathrm{H}]^{+}=348$.

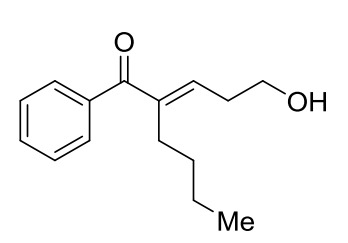

(E)-2-(3-hydroxypropylidene)-1-phenylhexan-1-one: The reaction was run according to GP4 starting from (E)-2-(3-)(tertbutyldimethylsilyl)oxy)propylidene)-1-phenylhexan-1-one (0.72 $\mathrm{mmol})$. The reaction was complete after $1.5 \mathrm{~h}$. Purification by flash chromatography on silica gel (Pentane/Diethyl ether $=6: 4$ ) afforded $\mathbf{4} \mathbf{i}$ in $73 \%$ yield $(133 \mathrm{mg})$ as a pale yellow oil. $\left(E\right.$ )-isomer: $\mathbf{R}_{\mathbf{f}}=0.2$ (Pentane/Diethyl ether $\left.=1: 1\right)$. IR (neat): $v\left(\mathrm{~cm}^{-1}\right)=$ 3420, 2968, 2867, 1636, 1596, 1580, 1444, 1387, 1311, 1285, 1250, 1038, 905, 869, 701. ${ }^{1} \mathrm{H}-\mathrm{NMR}\left(\mathrm{CDCl}_{3}, 500 \mathrm{MHz}\right): \delta(\mathrm{ppm}) 0.92\left(\mathrm{t},{ }^{3} \mathrm{~J}_{\mathrm{HH}}=7.1 \mathrm{~Hz}, 3 \mathrm{H}, \mathrm{CH}_{3} \mathrm{CH}_{2} \mathrm{CH}_{2} \mathrm{CH}_{2}\right), 1.35-1.39$ (m, 2H, $\mathrm{CH}_{3} \mathrm{CH}_{2} \mathrm{CH}_{2} \mathrm{CH}_{2}$ ), $1.41-1.46$ (m, 2H, $\mathrm{CH}_{3} \mathrm{CH}_{2} \mathrm{CH}_{2} \mathrm{CH}_{2}$ ), 1.57 (bs, OH), 2.50 - 2.53 (m, $2 \mathrm{H}, \mathrm{CH}_{3} \mathrm{CH}_{2} \mathrm{CH}_{2} \mathrm{CH}_{2}$ ), 2.58 (q, ${ }^{3} \mathrm{~J}_{\mathrm{HH}}=6.7 \mathrm{~Hz}, 2 \mathrm{H}, \mathrm{CH}_{2} \mathrm{CH}_{2} \mathrm{OH}$ ), 3.74 (t, ${ }^{3} \mathrm{JHH}_{\mathrm{HH}}=7.3 \mathrm{~Hz}, 2 \mathrm{H}$, 
$\left.\mathrm{CH}_{2} \mathrm{CH}_{2} \mathrm{OH}\right), 6.19\left(\mathrm{t},{ }^{3} \mathrm{~J}_{\mathrm{HH}}=7.3 \mathrm{~Hz}, 1 \mathrm{H}, \mathrm{C}=\mathrm{CH}\right), 7.40-7.43(\mathrm{~m}, 2 \mathrm{H}, \mathrm{CH}-\mathrm{Ar}), 7.50-7.53(\mathrm{~m}$, $\left.1 \mathrm{H}, \mathrm{CH}_{\mathrm{p}}-\mathrm{Ar}\right), 7.66-7.68\left(\mathrm{~m}, 2 \mathrm{H}, \mathrm{CH}_{0}-\mathrm{Ar}\right) \cdot{ }^{13} \mathrm{C}\left\{{ }^{1} \mathrm{H}\right\}-\mathrm{NMR}\left(\mathrm{CDCl}_{3}, 126 \mathrm{MHz}\right): \delta(\mathrm{ppm}) 14.1$ $\left(\mathrm{CH}_{3} \mathrm{CH}_{2} \mathrm{CH}_{2} \mathrm{CH}_{2}\right)$, $23.0\left(\mathrm{CH}_{3} \mathrm{CH}_{2} \mathrm{CH}_{2} \mathrm{CH}_{2}\right), 27.0\left(\mathrm{CH}_{3} \mathrm{CH}_{2} \mathrm{CH}_{2} \mathrm{CH}_{2}\right), 31.3\left(\mathrm{CH}_{3} \mathrm{CH}_{2} \mathrm{CH}_{2} \mathrm{CH}_{2}\right)$, $32.3\left(\mathrm{CH}_{2} \mathrm{CH}_{2} \mathrm{O}\right), 61.8\left(\mathrm{CH}_{2} \mathrm{CH}_{2} \mathrm{OH}\right), 128.3\left(\mathrm{CH}_{m}-\mathrm{Ar}\right), 129.6\left(\mathrm{CH}_{0}-\mathrm{Ar}\right), 131.8\left(\mathrm{CH}_{p}-\mathrm{Ar}\right), 138.8$ (C-Ar), $140.3(\mathrm{C}=\mathrm{CH}), 143.6(\mathrm{C}=\mathrm{CH}), 198.9(\mathrm{C}=\mathrm{O})$. LRMS $(\mathrm{IC})$ found $[\mathrm{M}+\mathrm{H}]^{+}=233$.

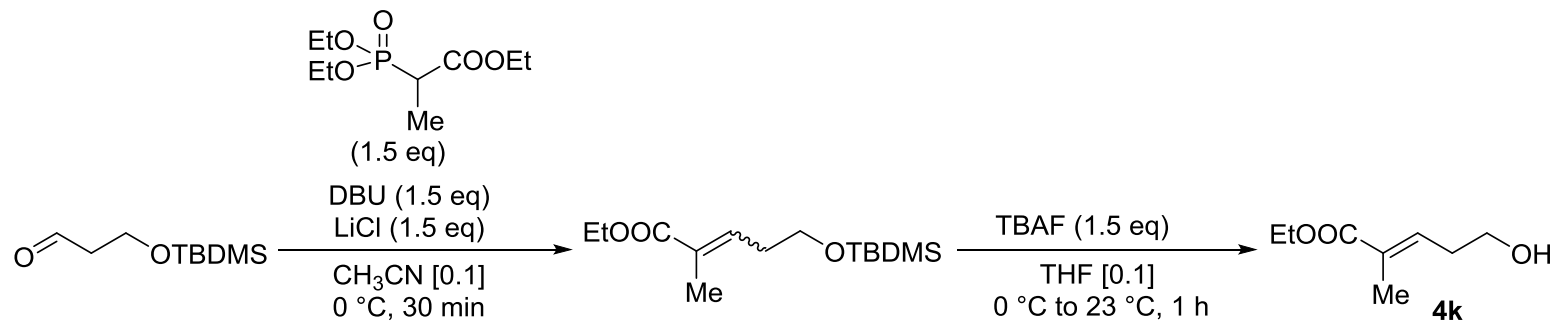

Figure S20: Synthesis of Ethyl $(E)$-5-hydroxy-2-methylpent-2-enoate (4k)

EtOOC

Ethyl (E)-5-((tert-butyldimethylsilyl)oxy)-2-methylpent-2-enoate: The reaction was run according to GP2 starting from 3-((tertbutyldimethylsilyl)oxy)propanal ( $865 \mathrm{mg}, 4.6 \mathrm{mmol}$ ). Purification by flash chromatography on silica gel (Pentane/EtOAc $=9: 1)$ afforded the product in $84 \%$ yield $(1 \mathrm{~g}, E / Z=11: 1)$ as a colorless oil, which was used for the next step without further purification.

Etooc $\mathrm{OH}_{\mathrm{OH}}$ Ethyl (E)-5-hydroxy-2-methylpent-2-enoate (4k): The product was
synthetized according to GP3 starting from 5-((tertbutyldimethylsilyl)oxy)-2-methylpent-2-enoate $(1.0 \mathrm{~g}, E / Z=11: 1,3.8 \mathrm{mmol})$. Purification by column chromatography on silica gel (Pentane/Diethyl ether from 4:1 to $2: 1$ ) afforded $\mathbf{4 k}$ in $73 \%$ yield $(440 \mathrm{mg})$ as a colorless oil. All spectroscopic analyses were in agreement with those reported in the literature. ${ }^{21}$ 


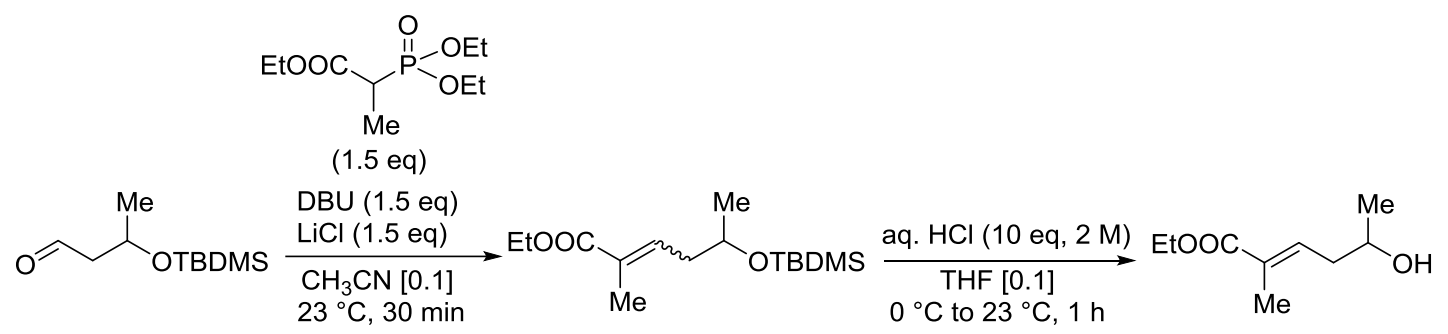

Figure S21: Synthesis of Ethyl $(E)$-5-hydroxy-2-methylhex-2-enoate (4i)<smiles>COCCOC(C)CC=CC(=O)OC</smiles>

flash chromatography (Pentane/Diethyl ether $=20: 1$ ) afforded the desired product in $89 \%$ yield (600 $\mathrm{mg}, E / Z=8: 1$ ) as a colourless oil. $(E)$-isomer: $\mathbf{R}_{\mathbf{f}}=0.2$ (Pentane/Diethyl ether $=$ 10:1). IR (neat): $v\left(\mathrm{~cm}^{-1}\right)=3675,2977,2903,1397,1251,1229,1062,894 .{ }^{1} \mathbf{H}$ NMR (400 $\left.\mathrm{MHz}, \mathrm{CDCl}_{3}\right) \delta(\mathrm{ppm}) 0.05\left(\mathrm{~s}, 6 \mathrm{H},\left(\mathrm{CH}_{3}\right)_{2} \mathrm{Si}\right), 0.88\left(\mathrm{~s}, 9 \mathrm{H},\left(\mathrm{CH}_{3}\right)_{3} \mathrm{CSi}\right), 1.17\left(\mathrm{~d},{ }^{3} \mathrm{~J}_{\mathrm{HH}}=6.1 \mathrm{~Hz}\right.$, $3 \mathrm{H}, \mathrm{CH}_{3} \mathrm{CH}$ ), $1.29\left(\mathrm{t},{ }^{3} \mathrm{~J}_{\mathrm{HH}}=7.1 \mathrm{~Hz}, 3 \mathrm{H}, \mathrm{CH}_{3} \mathrm{CH}_{2} \mathrm{O}\right), 1.83\left(\mathrm{~s}, 3 \mathrm{H}, \mathrm{CH}_{3} \mathrm{C}=\mathrm{CH}\right), 2.23-2.41(\mathrm{~m}$, $2 \mathrm{H}, \mathrm{CH}_{2} \mathrm{CHOTBDMS}$ ), $3.85-3.99$ (m, $1 \mathrm{H}, \mathrm{CH}_{2} \mathrm{CHOTBDMS}$ ), 4.18 (q, ${ }^{3} J_{\mathrm{HH}}=7.1 \mathrm{~Hz}, 2 \mathrm{H}$, $\left.\mathrm{CH}_{3} \mathrm{CH}_{2} \mathrm{O}\right), 6.80\left(\mathrm{tq},{ }^{3} \mathrm{~J}_{\mathrm{HH}}=7.5,{ }^{4} \mathrm{~J}_{\mathrm{HH}}=1.4 \mathrm{~Hz}, 1 \mathrm{H}, \mathrm{C}=\mathrm{CH}\right) \cdot{ }^{13} \mathrm{C}\left\{{ }^{1} \mathrm{H}\right\} \operatorname{NMR}\left(101 \mathrm{MHz}, \mathrm{CDCl}_{3}\right) \delta$ (ppm) -4.7 $\left(\left(\mathrm{CH}_{3}\right)_{2} \mathrm{Si}\right),-4.4\left(\left(\mathrm{CH}_{3}\right)_{2} \mathrm{Si}\right), 12.7\left(\mathrm{CH}_{3} \mathrm{C}=\mathrm{CH}\right), 14.4\left(\mathrm{CH}_{3} \mathrm{CH}_{2} \mathrm{O}\right), 18.2(\mathrm{C}=\mathrm{CH}), 24.1$ $\left.\begin{array}{lllllll}\left(\mathrm{CH}_{3} \mathrm{CH}\right. & \end{array}\right) \quad 26.0 \quad\left(\left(\mathrm{CH}_{3}\right)_{3} \mathrm{CSi}\right), \quad 39.1 \quad\left(\mathrm{CH}_{2} \mathrm{CHOTBDMS}\right), \quad 60.5 \quad\left(\mathrm{CH}_{3} \mathrm{CH}_{2} \mathrm{O}\right), \quad 68.0$ $\left(\mathrm{CH}_{2} \mathrm{CHOTBDMS}\right), 129.0(\mathrm{C}=\mathrm{CH}), 139.1(\mathrm{C}=\mathrm{CH}), 168.3(\mathrm{C}=\mathrm{O})$. LRMS $(\mathrm{IC})$ found $[\mathrm{M}+\mathrm{H}]^{+}=$ 287.<smiles>COC(=O)C(=O)CCC(O)O</smiles>

Ethyl (E)-5-hydroxy-2-methylhex-2-enoate (4i): The product was synthetized according to GP3 starting from ethyl (E)-5-((tertbutyldimethylsilyl)oxy)-2-methylhex-2-enoate (600 mg, $2.2 \mathrm{mmol}$ ). The crude mixture was purified by flash chromatography (Pentane/Diethyl ether $=1: 1$ ) to give $\mathbf{4 i}$ in $74 \%$ yield $\left(280 \mathrm{mg}\right.$ ) as a colorless oil. $\mathbf{R}_{\mathbf{f}}=0.4$ (Pentane/Diethyl ether $\left.=1: 2\right)$. IR (neat): $v\left(\mathrm{~cm}^{-1}\right)=3424,2973,1702,1368,1282,1252,1085 .{ }^{1} \mathbf{H}$ NMR $\left(400 \mathrm{MHz}, \mathrm{CDCl}_{3}\right) \delta(\mathrm{ppm})$ $1.25\left(\mathrm{~d},{ }^{3} \mathrm{~J}_{\mathrm{HH}}=6.2 \mathrm{~Hz}, 3 \mathrm{H}, \mathrm{CH}_{3} \mathrm{CHOH}\right), 1.29\left(\mathrm{t},{ }^{3} \mathrm{~J}_{\mathrm{HH}}=7.1 \mathrm{~Hz}, 3 \mathrm{H}, \mathrm{CH}_{3} \mathrm{CH}_{2} \mathrm{O}\right), 1.86(\mathrm{~s}, 3 \mathrm{H}$, $\left.\mathrm{CH}_{3} \mathrm{C}=\mathrm{CH}\right), 2.28-2.47\left(\mathrm{~m}, 2 \mathrm{H}, \mathrm{CH}_{2} \mathrm{CHOH}\right), 3.85-4.06\left(\mathrm{~m}, 1 \mathrm{H}, \mathrm{CH}_{2} \mathrm{CHOH}\right), 4.19$ (q, ${ }^{3} \mathrm{~J}_{\mathrm{HH}}=$ $7.1 \mathrm{~Hz}, 2 \mathrm{H}, \mathrm{CH}_{3} \mathrm{CH}_{2} \mathrm{O}$ ), 6.80 (tq, $\left.{ }^{3} \mathrm{~J}_{\mathrm{HH}}=7.6,{ }^{4} \mathrm{~J}_{\mathrm{HH}}=1.5 \mathrm{~Hz}, 1 \mathrm{H}, \mathrm{C}=\mathrm{CH}\right) .{ }^{13} \mathrm{C}\left\{{ }^{1} \mathrm{H}\right\}$ NMR $(101$ $\mathrm{MHz}, \mathrm{CDCl} 3) \delta(\mathrm{ppm}) 12.81\left(\mathrm{CH}_{3} \mathrm{C}=\mathrm{CH}\right), 14.42\left(\mathrm{CH}_{3} \mathrm{CH}_{2} \mathrm{O}\right), 23.43\left(\mathrm{CH}_{3} \mathrm{CHOH}\right), 38.54$ $\left(\mathrm{CH}_{2} \mathrm{CHOH}\right), 60.71\left(\mathrm{CH}_{3} \mathrm{CH}_{2} \mathrm{O}\right), 67.43\left(\mathrm{CH}_{2} \mathrm{CHOH}\right), 130.27(\mathrm{C}=\mathrm{CH}), 137.75(\mathrm{C}=\mathrm{CH}), 168.12$ $(C=\mathrm{O})$. LRMS (IC) found $\left[\mathrm{M}+\mathrm{NH}_{4}\right]^{+}=190$. 


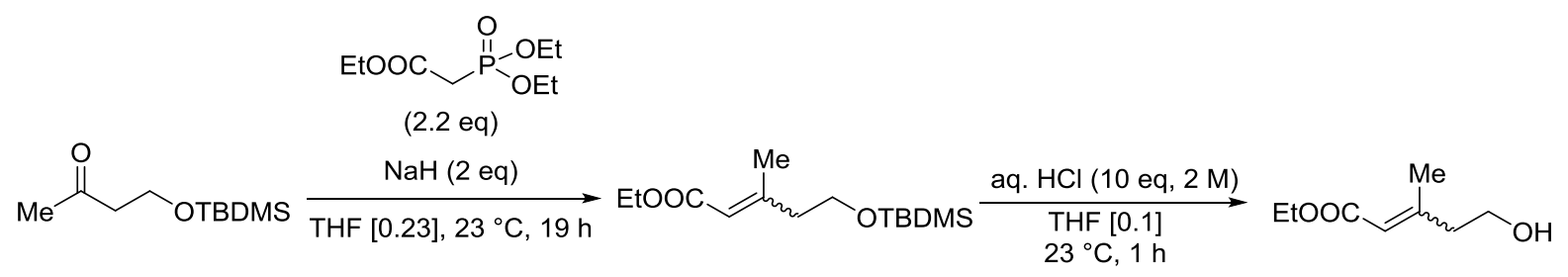

Figure S22: Synthesis of Ethyl (E)-5-hydroxyhex-2-enoate (4m)

$\begin{array}{ll}\text { Ethyl (E)-5-((tert-butyldimethylsilyl)oxy)-3-methylhex-2-enoate: } \\ \text { EtOoC }-\mathrm{N}_{\text {OTBDMS }} & \mathrm{NaH}(226 \mathrm{mg}, 9.44 \mathrm{mmol}) \text { was dissolved in } 15 \mathrm{~mL} \text { of THF and }\end{array}$ triethyl phosphonacetate $(2.0 \mathrm{~mL}, 10.4 \mathrm{mmol})$ was added dropwise at $0{ }^{\circ} \mathrm{C}$. After 30 minutes, 4-((tert-butyldimethylsilyl)oxy)butan-2-one $(0.95 \mathrm{~g}, 4.72 \mathrm{mmol})$ in THF $(5 \mathrm{~mL})$ was added at 0 ${ }^{\circ} \mathrm{C}$ and next stirred at room temperature. After $19 \mathrm{~h}$, the reaction was quenched with water $(80 \mathrm{~mL})$, extracted with EtOAc $(3 \times 15 \mathrm{~mL})$, washed with brine, dried over anhydrous $\mathrm{Na}_{2} \mathrm{SO}_{4}$ and concentrated in vacuo. Purification by flash chromatography on silica gel (Pentane/Diethyl ether $=20: 1$ ) afforded the product in $30 \%$ yield $(370 \mathrm{mg}, E / Z=3: 1$ ) as a colourless oil. $(E)$-isomer: ${ }^{1} \mathrm{H}$ NMR $\left(400 \mathrm{MHz}, \mathrm{CDCl}_{3}\right) \delta(\mathrm{ppm}) 0.04\left(\mathrm{~s}, 6 \mathrm{H},\left(\mathrm{CH}_{3}\right)_{2} \mathrm{Si}\right), 0.88(\mathrm{~s}$, $\left.9 \mathrm{H},\left(\mathrm{CH}_{3}\right)_{3} \mathrm{CSi}\right), 1.27\left(\mathrm{t},{ }^{3} \mathrm{~J}_{\mathrm{HH}}=7.1 \mathrm{~Hz}, 3 \mathrm{H}, \mathrm{CH}_{3} \mathrm{CH}_{2} \mathrm{O}\right), 2.18\left(\mathrm{~d},{ }^{3} \mathrm{~J}_{\mathrm{HH}}=1.3 \mathrm{~Hz}, 3 \mathrm{H}, \mathrm{CH}_{3} \mathrm{C}=\mathrm{CH}\right)$, $2.34\left(\mathrm{td},{ }^{3} \mathrm{~J}_{\mathrm{HH}}=6.7,1.1 \mathrm{~Hz}, 2 \mathrm{H}, \mathrm{CH}_{2} \mathrm{CH}_{2} \mathrm{OTBDMS}\right), 3.74\left(\mathrm{t},{ }^{3} \mathrm{~J}_{\mathrm{HH}}=6.7 \mathrm{~Hz}, 2 \mathrm{H}\right.$, $\mathrm{CH}_{2} \mathrm{CH}_{2} \mathrm{OTBDMS}$ ), 4.15 (q, ${ }^{3} \mathrm{~J}_{\mathrm{HH}}=7.1 \mathrm{~Hz}, 2 \mathrm{H}, \mathrm{CH}_{3} \mathrm{CH}_{2} \mathrm{O}$ ), 5.68 (q, ${ }^{3} \mathrm{~J}_{\mathrm{HH}}=1.3 \mathrm{~Hz}, 1 \mathrm{H}$, $\mathrm{CH}=\mathrm{C}$ ). All other spectroscopic analyses were in agreement with those reported in the literature. ${ }^{22}$

Me Ethyl (E)-5-hydroxyhex-2-enoate $(4 \mathrm{~m})$ : To a solution of ethyl $(E)-5-$

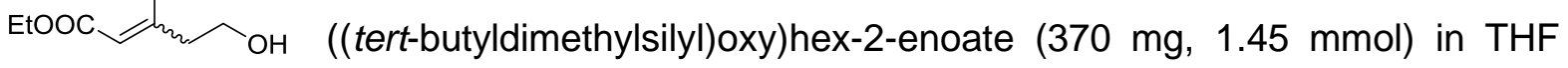
$(15 \mathrm{~mL})$, aqueous $\mathrm{HCl}(2 \mathrm{M}, 7.3 \mathrm{~mL})$ was added at $0{ }^{\circ} \mathrm{C}$ and the mixture was stirred at room temperature for $1 \mathrm{~h}$. The reaction was quenched with water $(10 \mathrm{~mL})$, extracted with EtOAc (3 $\times 30 \mathrm{~mL}$ ) and concentrated in vacuo. The crude mixture was purified by flash chromatography (Pentane/Diethyl ether $=1: 2$ ) to give $4 \mathrm{~m}$ in $83 \%$ yield $(190 \mathrm{mg}, E / Z=10: 1$ ) as a colourless oil. $(E)$-isomer: $\mathbf{R}_{\mathbf{f}}=0.2$ (Pentane/Diethyl ether $\left.=1: 2\right)$. IR (neat): $v\left(\mathrm{~cm}^{-1}\right)=$ $3404,2981,1706,1646,1220,1144,1038 .{ }^{1} \mathbf{H}$ NMR $\left(400 \mathrm{MHz}, \mathrm{CDCl}_{3}\right) \delta(\mathrm{ppm}) 1.30\left(\mathrm{t},{ }^{3} \mathrm{JHH}_{\mathrm{HH}}\right.$ $\left.=7.1 \mathrm{~Hz}, 3 \mathrm{H}, \mathrm{CH}_{3} \mathrm{CH}_{2} \mathrm{OH}\right), 2.22\left(\mathrm{~d},{ }^{3} \mathrm{~J}_{\mathrm{HH}}=1.3 \mathrm{~Hz}, 3 \mathrm{H}, \mathrm{CH}_{3} \mathrm{C}=\mathrm{CH}\right), 2.43\left(\mathrm{td},{ }^{3} \mathrm{JHH}_{\mathrm{HH}}=6.4,1.1\right.$ $\mathrm{Hz}, 2 \mathrm{H}, \mathrm{CH}_{2} \mathrm{CH}_{2} \mathrm{OH}$ ), 3.82 (t, ${ }^{3} \mathrm{~J}_{\mathrm{HH}}=6.3 \mathrm{~Hz}, 2 \mathrm{H}, \mathrm{CH}_{3} \mathrm{CH}_{2} \mathrm{OH}$ ), 4.18 (q, ${ }^{3} J_{\mathrm{HH}}=7.1 \mathrm{~Hz}, 2 \mathrm{H}$, $\left.\mathrm{CH}_{3} \mathrm{CH}_{2} \mathrm{O}\right), 5.76\left(\mathrm{q},{ }^{3} \mathrm{~J}_{\mathrm{HH}}=1.3 \mathrm{~Hz}, 1 \mathrm{H}, \mathrm{CH}=\mathrm{C}\right) .{ }^{13} \mathrm{C}\left\{{ }^{1} \mathrm{H}\right\} \mathbf{N M R}\left(101 \mathrm{MHz}, \mathrm{CDCl}_{3}\right) \delta(\mathrm{ppm}) 14.3$ $\left(\mathrm{CH}_{3} \mathrm{CH}_{2} \mathrm{O}\right), 18.7\left(\mathrm{CH}_{3} \mathrm{C}=\mathrm{CH}\right), 43.7\left(\mathrm{CH}_{2} \mathrm{CH}_{2} \mathrm{OH}\right), 59.7\left(\mathrm{CH}_{2} \mathrm{CH}_{2} \mathrm{OH}\right), 60.2\left(\mathrm{CH}_{3} \mathrm{CH}_{2} \mathrm{O}\right), 117.8$ $(\mathrm{CH}=\mathrm{C}), 155.8(\mathrm{C}=\mathrm{O}), 166.4(\mathrm{CH}=\mathrm{C})$. LRMS (IC) found $[\mathrm{M}+\mathrm{Na}]^{+}=171$. 


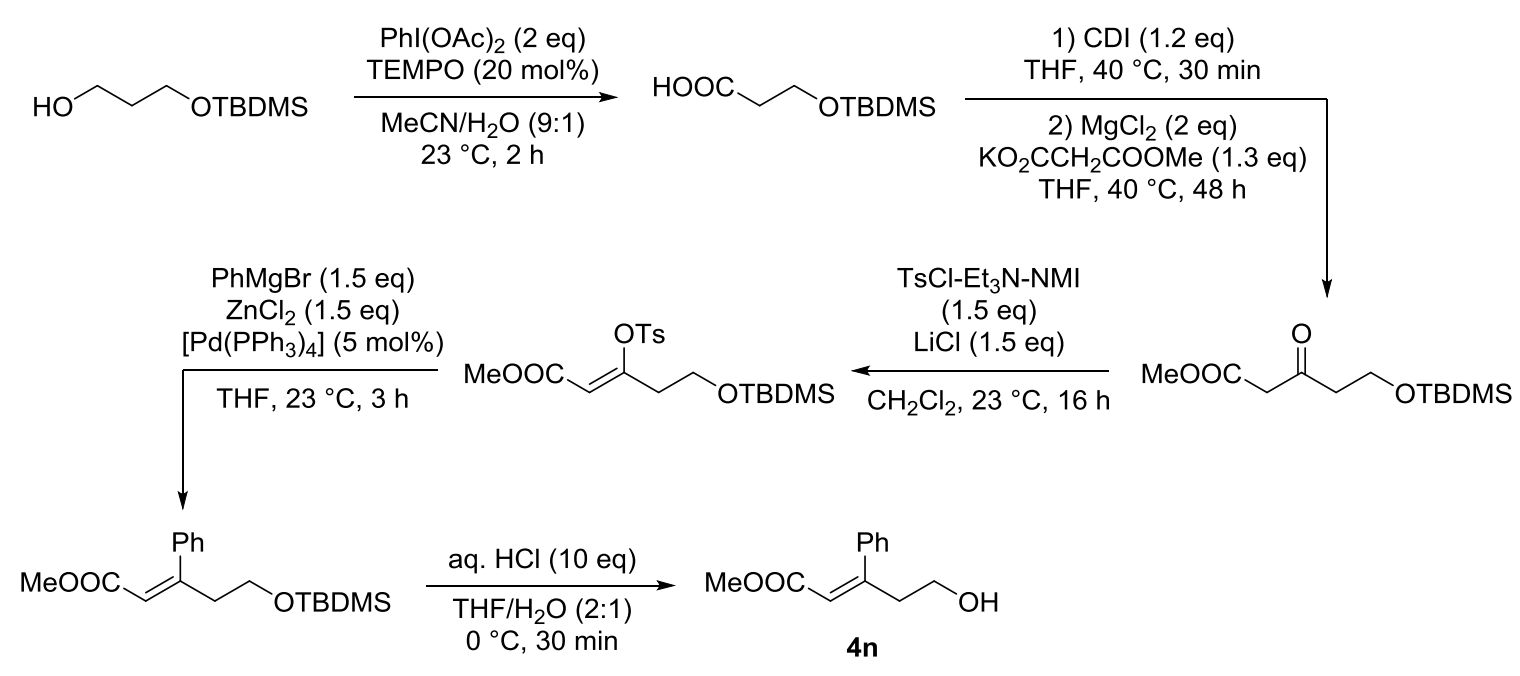

Figure S23: Synthesis of (Z)-4-hydroxy-2-phenylbut-1-en-1-yl acetate (4n)

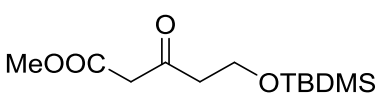

4-((tert-butyldimethylsilyl)oxy)-2-oxobutyl

acetate4-((tert-

butyldimethylsilyl)oxy)-2-oxobutyl acetate: 3-((tert-

butyldimethylsilyl)oxy)propanoic acid was prepared using a known protocol reported in the literature. ${ }^{23}$ In a Schlenk tube, $\mathrm{MgCl}_{2}(15.76 \mathrm{mmol}, 1.5 \mathrm{~g})$ and potassium monomethyl malonate $(20.49 \mathrm{mmol}, 3.2 \mathrm{~g})$ were suspended in THF $(23 \mathrm{~mL})$ and heated at $65^{\circ} \mathrm{C}$ for $3 \mathrm{~h}$. After the above reaction had proceeded for $2 \mathrm{~h}$, in a second Schlenk, 3-((tertbutyldimethylsilyl)oxy)propanoic acid (3.2 g, $15.76 \mathrm{mmol})$ was dissolved in THF (16 mL) and carbonyldiimidazole (CDI) $(3.07 \mathrm{~g}, 18.91 \mathrm{mmol})$ was added in five portions. The reaction was stirred at room temperature until $\mathrm{CO}_{2}$ releasing ceased (ca. 30 minutes). This homogeneous solution was heated at $40{ }^{\circ} \mathrm{C}$ for additional 30 minutes. The magnesium malonate suspension was cooled to $30^{\circ} \mathrm{C}$ and the other solution was added dropwise by a syringe. A white precipitate formed rapidly during this addition so vigorous stirring was necessary to avoid clamping. The resulting suspension was stirred at $40{ }^{\circ} \mathrm{C}$ for $48 \mathrm{~h}$ then cooled to $0{ }^{\circ} \mathrm{C}$ and quenched with a saturated aqueous $\mathrm{NH}_{4} \mathrm{Cl}$ solution $(15 \mathrm{~mL})$ and extracted with diethyl ether $(3 \times 50 \mathrm{~mL})$. The combined organic phases were sequentially washed with water (100 $\mathrm{mL}$ ), a saturated aqueous $\mathrm{NaHCO}_{3}$ solution $(100 \mathrm{~mL})$, brine $(100 \mathrm{~mL})$, dried over $\mathrm{Na}_{2} \mathrm{SO}_{4}$ and concentrated in vacuo. ${ }^{24}$ The product was used in the next step without further purification.

MeOOC $\overbrace{\text { OTBDMS }}^{\text {OTs }}$

(Z)-4-((tert-butyldimethylsilyl)oxy)-2-(tosyloxy)but-1-en-1-yl $5 \mathrm{mmol})$ was dissolved in $\mathrm{CH}_{2} \mathrm{Cl}_{2}(25 \mathrm{~mL})$ and $\mathrm{Et}_{3} \mathrm{~N}(7.5 \mathrm{mmol}, 1 \mathrm{~mL}), \mathrm{NMI}(7.5 \mathrm{mmol}, 0.6$ $\mathrm{mL})$ and $\mathrm{LiCl}(7.5 \mathrm{mmol}, 543 \mathrm{mg})$ were added sequentially. After 10 minutes at room temperature, $\mathrm{TsCl}(7.5 \mathrm{mmol}, 1.43 \mathrm{~g})$ was added at $0{ }^{\circ} \mathrm{C}$. The reaction was stirred at room 
temperature for $16 \mathrm{~h}$ and then quenched with a saturated aqueous $\mathrm{NH}_{4} \mathrm{Cl}$ solution $(20 \mathrm{~mL})$ and extracted with $\mathrm{Et}_{2} \mathrm{O}(3 \times 30 \mathrm{~mL})$. The organic phases were combined, dried over $\mathrm{Na}_{2} \mathrm{SO}_{4}$ and evaporated. ${ }^{25}$ The resulting crude material was purified by flash chromatography on silica gel $\left(\mathrm{Et}_{2} \mathrm{O} /\right.$ Acetone $\left.=10: 1\right)$ to afford the desired product as a pale yellow oil in $54 \%$ yield $(1.12 \mathrm{~g})$ over 3 steps. $\mathbf{R}_{\mathrm{F}}=0.4\left(\mathrm{Et}_{2} \mathrm{O} /\right.$ Acetone $\left.=10: 1\right) .{ }^{1} \mathbf{H}-\mathrm{NMR}\left(\mathrm{CDCl}_{3}, 500 \mathrm{MHz}\right): \delta(\mathrm{ppm})$ $0.01\left(\mathrm{~s}, 6 \mathrm{H},\left(\mathrm{CH}_{3}\right)_{2} \mathrm{Si}\right), 0.85\left(\mathrm{~s}, 9 \mathrm{H},\left(\mathrm{CH}_{3}\right)_{3} \mathrm{CSi}\right), 2.45$ (s, 3H, $\left.\mathrm{CH}_{3} \mathrm{Ar}\right), 2.59\left(\mathrm{t}, 2 \mathrm{H},{ }^{3} J_{H H}=6.4\right.$ $\mathrm{Hz}, \mathrm{CH}_{2} \mathrm{CH}_{2} \mathrm{OTBDMS}$ ), 3.56 (s, 3H, $\mathrm{CH}_{3} \mathrm{O}$ ), 3.77 (t, $2 \mathrm{H},{ }^{3} J_{H H}=6.1 \mathrm{~Hz}, \mathrm{CH}_{2} \mathrm{CH}_{2} \mathrm{OTBDMS}$ ), $5.61(\mathrm{~m}, 1 \mathrm{H}, \mathrm{CH}=\mathrm{C}), 7.34-7.36\left(\mathrm{~m}, 2 \mathrm{H}, \mathrm{CH}_{\mathrm{m}}-\mathrm{Ar}\right), 7.88-7.90\left(\mathrm{~m}, 2 \mathrm{H}, \mathrm{CH}_{0}-\mathrm{Ar}\right) .{ }^{13} \mathrm{C}\left\{{ }^{1} \mathrm{H}\right\}-$ NMR $\left(\mathrm{CDCl}_{3}, 126 \mathrm{MHz}\right): \delta(\mathrm{ppm})-5.4\left(\left(\mathrm{CH}_{3}\right)_{2} \mathrm{Si}\right), 18.3\left(\left(\mathrm{CH}_{3}\right)_{3} \mathrm{CSi}\right), 21.8\left(\mathrm{CH}_{3}-\mathrm{Ar}\right), 25.9$ $\left(\left(\mathrm{CH}_{3}\right)_{3} \mathrm{CSi}\right), 38.8\left(\mathrm{CH}_{2} \mathrm{CH}_{2} \mathrm{OTBDMS}\right), 51.5\left(\mathrm{CH}_{3} \mathrm{O}\right), 59.4\left(\mathrm{CH}_{2} \mathrm{CH}_{2} \mathrm{OTBDMS}\right), 111.8(\mathrm{CH}=\mathrm{C})$, $128.6\left(\mathrm{CH}_{o}-\mathrm{Ar}\right), 129.8\left(\mathrm{CH}_{m}-\mathrm{Ar}\right), 133.5\left(C_{\text {ipso }}-\mathrm{Ar}\right), 145.5\left(C_{p^{-}} \mathrm{Ar}\right), 157.2(\mathrm{CH}=C), 163.4(C=\mathrm{O})$. LRMS $(I C)=$ found $[M+H]^{+}=416$. IR (neat): $v\left(\mathrm{~cm}^{-1}\right)=2952,2930,2858,1733,1673,1598$, $1461,1435,1377,1317,1289,1255,1218,1171,1132,1091,1063,1017,970,928,873$, 834, 813, 775, 709, 687, 629, 590.

MeOOC $\overbrace{\text { OTBDMS }}^{\text {Ph }}$

(Z)-4-((tert-butyldimethylsilyl)oxy)-2-phenylbut-1-en-1-yl prepared in situ by diluting $\mathrm{PhMgBr}\left(3.6 \mathrm{mmol}, 1.2 \mathrm{~mL}\right.$ from a $3 \mathrm{M}$ solution in $\mathrm{Et}_{2} \mathrm{O}$ ) with $\mathrm{THF}$ (2.4 mL) and adding $\mathrm{ZnCl}_{2}$ ( $3.6 \mathrm{~mL}$ from a $1 \mathrm{M}$ solution in THF) while stirring for 30 minutes. Inside the glovebox, a Young valve Schlenk was charged with $\left[\mathrm{Pd}\left(\mathrm{PPh}_{3}\right)_{4}\right](0.12 \mathrm{mmol}, 139$ $\mathrm{mg})$. THF (17 mL) and (Z)-4-((tert-butyldimethylsilyl)oxy)-2-(tosyloxy)but-1-en-1-yl acetate ( $2.4 \mathrm{mmol}, 995 \mathrm{mg}$ ) were added outside the glovebox. The latter solution was transferred by cannula to the alkylzinc suspension and stirred at room temperature for $3 \mathrm{~h}$ then quenched with saturated aqueous $\mathrm{NH}_{4} \mathrm{Cl}$ solution and extracted with $\mathrm{Et}_{2} \mathrm{O}(3 \times 20 \mathrm{~mL})$. The organic phases were combined, dried over $\mathrm{Na}_{2} \mathrm{SO}_{4}$ and evaporated in vacuo. The crude mixture was purified by flash chromatography on silica gel $\left(\mathrm{Et}_{2} \mathrm{O} /\right.$ Acetone $\left.=99: 1\right)$ to afford the desired product in $59 \%$ yield $(433 \mathrm{mg})$ as a colourless oil. $\mathbf{R}_{\mathrm{F}}=0.4\left(\mathrm{Et}_{2} \mathrm{O} /\right.$ Acetone $\left.=95: 5\right) .{ }^{1} \mathrm{H}-\mathrm{NMR}$ $\left(\mathrm{CDCl}_{3}, 500 \mathrm{MHz}\right): \delta(\mathrm{ppm}) 0.00\left(\mathrm{~s}, 6 \mathrm{H},\left(\mathrm{CH}_{3}\right)_{2} \mathrm{Si}\right), 0.87$ (s, 9H, $\left.\left(\mathrm{CH}_{3}\right)_{3} \mathrm{CSi}\right), 2.65\left(\mathrm{td}, 2 \mathrm{H}_{5},{ }^{3} \mathrm{~J}_{H H}\right.$ $\left.=6.6 \mathrm{~Hz},{ }^{4} \mathrm{~J}_{\mathrm{HH}}=1.1 \mathrm{~Hz}, \mathrm{CH}_{2} \mathrm{CH}_{2} \mathrm{OTBDMS}\right), 3.55\left(\mathrm{~s}, 3 \mathrm{H}, \mathrm{CH}_{3} \mathrm{O}\right), 3.63\left(\mathrm{t}, 2 \mathrm{H},{ }^{3} J_{H H}=6.6 \mathrm{~Hz}\right.$, $\mathrm{CH}_{2} \mathrm{CH}_{2} \mathrm{OTBDMS}$ ), 5.93 (m, 1H, CH=C), $7.16-7.18$ (m, 2H, $\left.\mathrm{CH}_{0}-\mathrm{Ar}\right), 7.29-7.36(\mathrm{~m}, 2 \mathrm{H}$, $\left.\mathrm{CH}_{m}-\mathrm{Ar} ; 1 \mathrm{H}, \mathrm{CH}_{p}-\mathrm{Ar}\right) .{ }^{13} \mathrm{C}\left\{{ }^{1} \mathrm{H}\right\}-\mathrm{NMR}\left(\mathrm{CDCl}_{3}, 126 \mathrm{MHz}\right): \delta(\mathrm{ppm})-5.3\left(\left(\mathrm{CH}_{3}\right)_{2} \mathrm{Si}\right), 18.4$ $\begin{array}{lllllll}\left(\left(\mathrm{CH}_{3}\right)_{3} \mathrm{CSi}\right), \quad 26.0 & \left(\left(\mathrm{CH}_{3}\right)_{3} \mathrm{CSi}\right), \quad 43.7 \quad\left(\mathrm{CH}_{2} \mathrm{CH}_{2} \mathrm{OTBDMS}\right), \quad 51.2 & \left(\mathrm{CH}_{3} \mathrm{O}\right), \quad 60.7\end{array}$ $\left(\mathrm{CH}_{2} \mathrm{CH}_{2} \mathrm{OTBDMS}\right), 118.5(\mathrm{CH}=\mathrm{C}), 127.3\left(\mathrm{CH}_{0}-\mathrm{Ar}\right), 127.9\left(\mathrm{CH}_{p}-\mathrm{Ar}\right), 128.0\left(\mathrm{CH}_{m}-\mathrm{Ar}\right), 139.7$ (C-Ar), $156.9(\mathrm{CH}=C), 166.4(C=\mathrm{O})$. LRMS $(\mathrm{IC})=$ found $[\mathrm{M}+\mathrm{Na}]^{+}=344$. IR (neat): $v\left(\mathrm{~cm}^{-1}\right)=$ 2952, 2932, 2894, 1730, 1642, 1494, 1468, 1434, 1384, 1369, 1255, 1226, 1163, 1102 , $1052,1006,932,835,775,698,665$. 
(Z)-4-hydroxy-2-phenylbut-1-en-1-yl acetate (4n): The product was MeOOC synthesized according to GP4 starting from (Z)-4-((tertbutyldimethylsilyl)oxy)-2-phenylbut-1-en-1-yl acetate (1.25 mmol, 401 $\mathrm{mg})$. The desired product was obtained in $69 \%$ yield $(177 \mathrm{mg})$ as a pale yellow oil after flash chromatography on silica gel $\left(\mathrm{Et}_{2} \mathrm{O} /\right.$ Acetone $\left.=8: 2\right) \cdot \mathbf{R}_{\mathrm{F}}=0.2\left(\mathrm{Et}_{2} \mathrm{O} /\right.$ Acetone $\left.=8: 2\right) .{ }^{1} \mathbf{H}$-NMR $\left(\mathrm{CDCl}_{3}, 500 \mathrm{MHz}\right): \delta(\mathrm{ppm}) 1.34\left(\mathrm{bt}, 1 \mathrm{H},{ }^{3} \mathrm{~J}_{\mathrm{HH}}=5.5 \mathrm{~Hz}, \mathrm{OH}\right), 2.72\left(\mathrm{td}, 2 \mathrm{H},{ }^{3} \mathrm{~J}_{H H}=6.3 \mathrm{~Hz},{ }^{4} \mathrm{~J}_{H H}\right.$ $=1.1 \mathrm{~Hz}, \mathrm{CH}_{2} \mathrm{CH}_{2} \mathrm{OH}$ ), 3.55 (s, 3H, $\mathrm{CH}_{3} \mathrm{O}$ ), 3.68 (q, $2 \mathrm{H},{ }^{3} \mathrm{~J}_{H H}=6.1 \mathrm{~Hz}, \mathrm{CH}_{2} \mathrm{CH}_{2} \mathrm{OH}$ ), 5.99 (bt, $\left.1 \mathrm{H},{ }^{4} J_{H H}=1.1 \mathrm{~Hz}, \mathrm{CH}=\mathrm{C}\right), 7.18-7.20\left(\mathrm{~m}, 2 \mathrm{H}, \mathrm{CH}_{0}-\mathrm{Ar}\right), 7.31-7.39\left(\mathrm{~m}, 2 \mathrm{H}, \mathrm{CH}_{m}-\mathrm{Ar} ; 1 \mathrm{H}\right.$, $\mathrm{CH}_{\mathrm{p}}$-Ar). ${ }^{13} \mathrm{C}\left\{{ }^{1} \mathrm{H}\right\}-\mathrm{NMR}\left(\mathrm{CDCl}_{3}, 126 \mathrm{MHz}\right): \delta(\mathrm{ppm})=43.4\left(\mathrm{CH}_{2} \mathrm{CH}_{2} \mathrm{OH}\right), 51.3\left(\mathrm{CH}_{3} \mathrm{O}\right), 60.2$ $\left(\mathrm{CH}_{2} \mathrm{CH}_{2} \mathrm{OH}\right), 119.0(\mathrm{CH}=\mathrm{C}), 127.3\left(\mathrm{CH}_{o}-\mathrm{Ar}\right), 128.1\left(\mathrm{CH}_{p}-\mathrm{Ar}\right), 128.2\left(\mathrm{CH}_{m}-\mathrm{Ar}\right), 139.3(\mathrm{C}-\mathrm{Ar})$, 156.3 $(\mathrm{CH}=\mathrm{C}), 166.1(\mathrm{C}=\mathrm{O})$. LRMS $(\mathrm{IC})=$ found $\left[\mathrm{M}+\mathrm{NH}_{4}\right]^{+}=224$. IR (neat): $v\left(\mathrm{~cm}^{-1}\right)=3410$, $2950,2919,2853,1710,1640,1493,1435,1377,1283,1228,1163,1039,918,844,773$, 699.

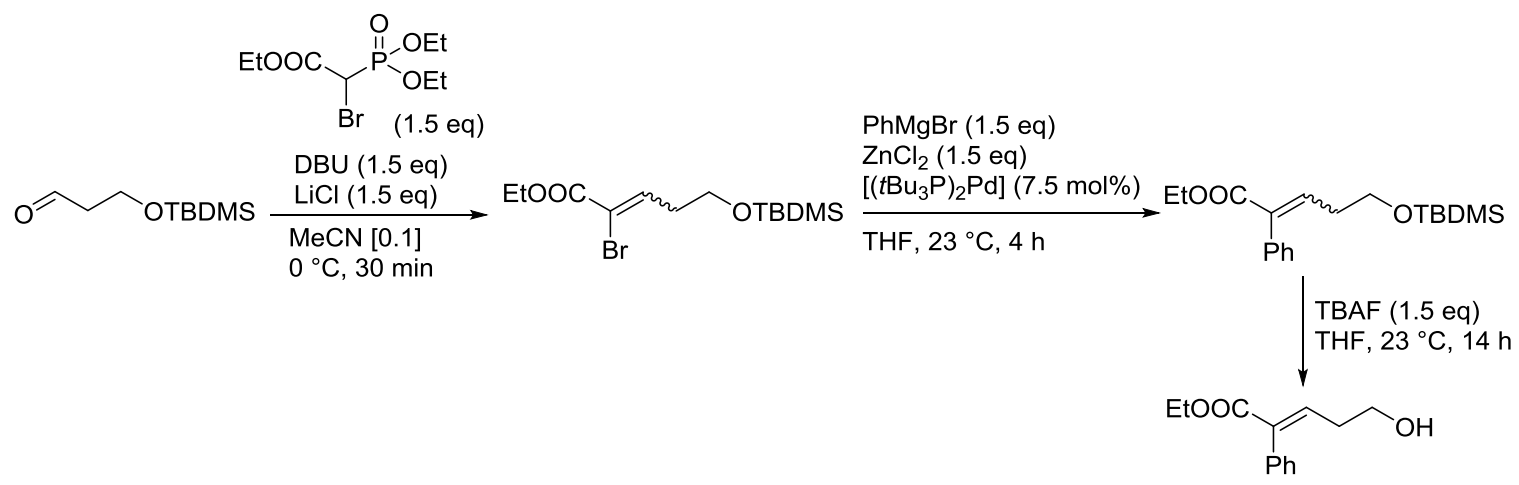

Figure S24: Synthesis of Ethyl $(E)$-5-hydroxy-2-phenylpent-2-enoate (40)<smiles>CCOC(=O)C=CCCO[Mg]C(=O)OC</smiles>

Ethyl 2-bromo-5-((tert-butyldimethylsilyl)oxy)pent-2-enoate: The compound was prepared according to GP2 starting from the 3((tert-butyldimethylsilyl)oxy)propanal $(20.89 \mathrm{mmol}, 3.93 \mathrm{~g})$. The product was obtained in $75 \%$ yield (4.93 $\mathrm{g}, E / Z=1: 4$ ) after purification by flash chromatography on silica gel (Pentane/Diethyl ether 9:1). (Z)-isomer: $\mathbf{R}_{\mathbf{f}}=0.7$ (Pentane/Diethyl ether 9:1). IR (neat): $v\left(\mathrm{~cm}^{-}\right.$ $\left.{ }^{1}\right)=2931,2858,1723,1628,1468,1367,1254,1094,1044,970,937,834,775,747,663$. ${ }^{1} \mathrm{H}-\mathrm{NMR}\left(\mathrm{CDCl}_{3}, 500 \mathrm{MHz}\right): \delta(\mathrm{ppm}) 0.06\left(\mathrm{~s}, 6 \mathrm{H},\left(\mathrm{CH}_{3}\right)_{2} \mathrm{Si}\right), 0.89\left(\mathrm{~s}, 9 \mathrm{H},\left(\mathrm{CH}_{3}\right)_{3} \mathrm{CSi}\right), 1.32(\mathrm{t}$, ${ }^{3} J_{\mathrm{HH}}=7.1 \mathrm{~Hz}, 3 \mathrm{H}, \mathrm{CH}_{3} \mathrm{CH}_{2} \mathrm{O}$ ), 2.55 (q, $\left.{ }^{3} \mathrm{~J}_{\mathrm{HH}}=6.4 \mathrm{~Hz}, 2 \mathrm{H}, \mathrm{CH}_{2} \mathrm{CH}_{2} \mathrm{OTBDMS}\right), 3.75$ (t, ${ }^{3} \mathrm{~J}_{\mathrm{HH}}=$ $6.4 \mathrm{~Hz}, 2 \mathrm{H}, \mathrm{CH}_{2} \mathrm{CH}_{2} \mathrm{OTBDMS}$ ), 4.27 (q, ${ }^{3} \mathrm{~J}_{\mathrm{HH}}=7.1 \mathrm{~Hz}, 2 \mathrm{H}, \mathrm{CH}_{3} \mathrm{CH}_{2} \mathrm{O}$ ), $7.38\left(\mathrm{t},{ }^{3} \mathrm{~J}_{\mathrm{HH}}=6.9 \mathrm{~Hz}\right.$, $1 \mathrm{H}, \mathrm{C}=\mathrm{CH}) \cdot{ }^{13} \mathrm{C}\left\{{ }^{1} \mathrm{H}\right\}-\mathrm{NMR}\left(\mathrm{CDCl}_{3}, 126 \mathrm{MHz}\right): \delta(\mathrm{ppm})-5.2\left(\left(\mathrm{CH}_{3}\right)_{2} \mathrm{Si}\right), 14.3\left(\mathrm{CH}_{3} \mathrm{CH}_{2} \mathrm{O}\right), 18.4$ $\left(\left(\mathrm{CH}_{3}\right)_{3} \mathrm{CSi}\right), 26.0$ (( $\left.\left.\mathrm{CH}_{3}\right)_{3} \mathrm{CSi}\right), 35.9\left(\mathrm{CH}_{2} \mathrm{CH}_{2} \mathrm{OTBDMS}\right), 60.8\left(\mathrm{CH}_{2} \mathrm{CH}_{2} \mathrm{OTBDMS}\right), 62.5$ $\left(\mathrm{CH}_{3} \mathrm{CH}_{2} \mathrm{O}\right), 117.6(\mathrm{C}=\mathrm{CH}), 143.4(\mathrm{C}=\mathrm{CH}), 162.6(\mathrm{C}=\mathrm{O})$. LRMS $(\mathrm{IC})$ found $[\mathrm{M}+\mathrm{H}]^{+}=338$. 
EtOOC $\mathrm{Ph}_{\mathrm{Ph}}$ OTBDMS

Ethyl 5-((tert-butyldimethylsilyl)oxy)-2-phenylpent-2-enoate:

The reaction was run according to GP6B starting from ethyl 2bromo-5-((tert-butyldimethylsilyl)oxy)pent-2-enoate (14.6 mmol, $4.93 \mathrm{~g})$. The reaction was complete after $4 \mathrm{~h}$. Purification by column chromatography on silica gel (from Pentane to Pentane/Diethyl ether 98:2) afforded the (Z)-product in $3.5 \%$ yield (165 mg), the $(E)$-product in $3.5 \%$ yield $(165 \mathrm{mg})$ and the mixture of the two isomers in $77 \%$ yield $(3.75 \mathrm{~g}, E / Z=4: 1)$. $(Z)$-isomer: $\mathbf{R}_{\mathbf{f}}=0.6$ (Pentane/Diethyl ether 9:1). IR (neat): $v\left(\mathrm{~cm}^{-1}\right)=2930,2858,1718$, 1467, 1371, 1254, 1193, 1092, 1029, 970, 938, 835, 775, 696, 661. ${ }^{1} \mathrm{H}-\mathrm{NMR}\left(\mathrm{CDCl}_{3}, 500\right.$ $\mathrm{MHz}): \delta(\mathrm{ppm}) 0.07\left(\mathrm{~s}, 6 \mathrm{H},\left(\left(\mathrm{CH}_{3}\right)_{2} \mathrm{Si}\right), 0.90\left(\mathrm{~s}, 9 \mathrm{H},\left(\left(\mathrm{CH}_{3}\right)_{3} \mathrm{CSi}\right), 1.31\left(\mathrm{t},{ }^{3} J_{\mathrm{HH}}=7.1 \mathrm{~Hz}, 3 \mathrm{H}\right.\right.\right.$, $\mathrm{CH}_{3} \mathrm{CH}_{2} \mathrm{O}$ ), 2.67 (q, ${ }^{3} \mathrm{JHH}_{\mathrm{HH}}=6.6 \mathrm{~Hz}, 2 \mathrm{H}, \mathrm{CH}_{2} \mathrm{CH}_{2} \mathrm{OTBDMS}$ ), 3.77 (t, ${ }^{3} J_{\mathrm{HH}}=6.5 \mathrm{~Hz}, 2 \mathrm{H}$, $\mathrm{CH}_{2} \mathrm{CH}_{2} \mathrm{OTBDMS}$ ), 4.29 (q, $2 \mathrm{H},{ }^{3} \mathrm{~J}_{\mathrm{HH}}=7.1 \mathrm{~Hz}, \mathrm{CH}_{3} \mathrm{CH}_{2} \mathrm{O}$ ), $6.26\left(\mathrm{t}, 1 \mathrm{H},{ }^{3} \mathrm{~J}_{\mathrm{HH}}=7.4 \mathrm{~Hz}, \mathrm{C}=\mathrm{CH}\right.$ ), $7.29\left(\mathrm{~m}, 1 \mathrm{H}, \mathrm{CH} \mathrm{H}_{p}-\mathrm{Ar}\right), 7.32\left(\mathrm{~m}, 4 \mathrm{H}, \mathrm{CH}_{0}-\mathrm{Ar} ; \mathrm{CH}_{m}-\mathrm{Ar}\right) .{ }^{13} \mathrm{C}\left\{{ }^{1} \mathrm{H}\right\}-\mathrm{NMR}\left(\mathrm{CDCl}_{3}, 126 \mathrm{MHz}\right): \delta$ $($ ppm $)=-4.7\left(\left(\mathrm{CH}_{3}\right)_{2} \mathrm{Si}\right), \quad 14.4\left(\mathrm{CH}_{3} \mathrm{CH}_{3} \mathrm{O}\right), 18.5 \quad\left(\left(\mathrm{CH}_{3}\right)_{3} \mathrm{CSi}\right), 26.1 \quad\left(\left(\mathrm{CH}_{3}\right)_{3} \mathrm{CSi}\right), \quad 33.8$ $\left(\mathrm{CH}_{2} \mathrm{CH}_{2} \mathrm{OTBDMS}\right), 60.9\left(\mathrm{CH}_{3} \mathrm{CH}_{2} \mathrm{O}\right), 62.5\left(\mathrm{CH}_{2} \mathrm{CH}_{2} \mathrm{OTBDMS}\right), 127.3\left(\mathrm{CH}_{o^{-}} \mathrm{Ar}\right), 127.7\left(\mathrm{CH}_{p^{-}}\right.$ $\mathrm{Ar}), 128.4\left(\mathrm{CH}_{m}-\mathrm{Ar}\right), 136.1(\mathrm{C}=\mathrm{CH}), 136.4(\mathrm{C}=\mathrm{CH}), 138.0(\mathrm{C}-\mathrm{Ar}), 168.3(\mathrm{C}=\mathrm{O})$. LRMS (IC) = found $[\mathrm{M}+\mathrm{H}]^{+}=335$. $(E)$-isomer: $\mathbf{R}_{\mathrm{f}}=0.6$ (Pentane/Diethyl ether 9:1). IR (neat): $v\left(\mathrm{~cm}^{-1}\right)=$ 2930, 2858, 1714, 1636, 1467, 1366, 1247, 1213, 1182, 1093, 1046, 939, 833, 774, 701, 662. ' $\mathrm{H}-\mathrm{NMR}\left(\mathrm{CDCl}_{3}, 500 \mathrm{MHz}\right): \delta(\mathrm{ppm}) 0.03\left(\mathrm{~s}, 6 \mathrm{H},\left(\mathrm{CH}_{3}\right)_{2} \mathrm{Si}\right), 0.88\left(\mathrm{~s}, 9 \mathrm{H},\left(\mathrm{CH}_{3}\right)_{3} \mathrm{CSi}\right)$, $1.26\left(\mathrm{t},{ }^{3} \mathrm{JHH}_{\mathrm{HH}}=7.1 \mathrm{~Hz}, 3 \mathrm{H}, \mathrm{CH}_{3} \mathrm{CH}_{2} \mathrm{O}\right.$ ), $2.32\left(\mathrm{q},{ }^{3} \mathrm{~J}_{\mathrm{HH}}=6.5 \mathrm{~Hz}, 2 \mathrm{H}, \mathrm{CH}_{2} \mathrm{CH}_{2} \mathrm{OTBDMS}\right.$ ), 3.68 (t, $\left.{ }^{3} J_{\mathrm{HH}}=6.5 \mathrm{~Hz}, 2 \mathrm{H}, \mathrm{CH}_{2} \mathrm{CH}_{2} \mathrm{OTBDMS}\right), 4.21\left(\mathrm{q},{ }^{3} \mathrm{~J}_{\mathrm{HH}}=7.1 \mathrm{~Hz}, 2 \mathrm{H}, \mathrm{CH}_{3} \mathrm{CH}_{2} \mathrm{O}\right), 7.10\left(\mathrm{t},{ }^{3} \mathrm{~J}_{\mathrm{HH}}=\right.$ $7.6 \mathrm{~Hz}, 1 \mathrm{H}, \mathrm{C}=\mathrm{CH}), 7.20\left(\mathrm{~m}, 1 \mathrm{H}, \mathrm{CH}_{p}-\mathrm{Ar}\right), 7.36\left(\mathrm{~m}, 2 \mathrm{H}, \mathrm{CH}_{0}-\mathrm{Ar}\right), 7.31\left(\mathrm{~m}, 2 \mathrm{H}, \mathrm{CH}_{m}-\mathrm{Ar}\right)$. ${ }^{13} \mathrm{C}\left\{{ }^{1} \mathrm{H}\right\}-N M R\left(\mathrm{CDCl}_{3}, 126 \mathrm{MHz}\right): \delta(\mathrm{ppm})=-5.2\left(\left(\mathrm{CH}_{3}\right)_{2} \mathrm{Si}\right), 14.4\left(\mathrm{CH}_{3} \mathrm{CH}_{2} \mathrm{O}\right), 18.5$

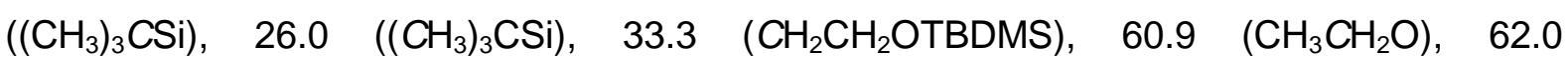
$\left(\mathrm{CH}_{2} \mathrm{CH}_{2} \mathrm{OTBDMS}\right), 127.5\left(\mathrm{CH}_{p}-\mathrm{Ar}\right), 128.1\left(\mathrm{CH}_{o}-\mathrm{Ar}\right), 129.9\left(\mathrm{CH}_{m}-\mathrm{Ar}\right), 135.4(\mathrm{C}=\mathrm{CH}), 135.5$ (C-Ar), $141.6(\mathrm{C}=\mathrm{CH}), 167.2(\mathrm{C}=\mathrm{O})$. LRMS (IC) found $[\mathrm{M}+\mathrm{H}]^{+}=335$.

Etooc $\begin{aligned} & \text { Ethyl (E)-5-hydroxy-2-phenylpent-2-enoate (4n): The reaction was run } \\ & \text { according to GP3 starting from ethyl 5-((tert-butyldimethylsilyl)oxy)-2- }\end{aligned}$ phenylpent-2-enoate $(11.21 \mathrm{mmol}, 3.75 \mathrm{~g})$. Purification by column chromatography on silica gel (Pentane/Diethyl ether from 7:3 to $1: 1$ ) afforded $4 \mathbf{n}$ in $67 \%$ yield $(1.31 \mathrm{~g})$ as an orange oil (Note: the Z-isomer cyclizes in this condition and was removed during purification). $\mathbf{R}_{\mathbf{f}}=0.2$ (Pentane/Diethyl ether 1:1). IR (neat): $v\left(\mathrm{~cm}^{-1}\right)=3426,2966,1707,1636,1495,1444,1368$, 1240, 1183, 1095, 1043, 909, 865, 774, 702. ${ }^{1} \mathrm{H}-\mathrm{NMR}\left(\mathrm{CDCl}_{3}, 500 \mathrm{MHz}\right): \delta(\mathrm{ppm}) 1.26(\mathrm{t}$, $\left.{ }^{3} J_{\mathrm{HH}}=7.1 \mathrm{~Hz}, 3 \mathrm{H}, \mathrm{CH}_{3} \mathrm{CH}_{2} \mathrm{O}\right), 1.66(\mathrm{bs}, 1 \mathrm{H}, \mathrm{OH}), 2.37\left(\mathrm{~m}, 2 \mathrm{H}, \mathrm{CH}_{2} \mathrm{CH}_{2} \mathrm{OH}\right), 3.71\left(\mathrm{t},{ }^{3} \mathrm{JHH}_{\mathrm{HH}}=6.4\right.$ $\mathrm{Hz}, 2 \mathrm{H}, \mathrm{CH}_{2} \mathrm{CH}_{2} \mathrm{OH}$ ), 4.21 (q, ${ }^{3} \mathrm{~J}_{\mathrm{HH}}=7.1 \mathrm{~Hz}, 2 \mathrm{H}, \mathrm{CH}_{3} \mathrm{CH}_{2} \mathrm{O}$ ), 7.09 (t, ${ }^{3} J_{\mathrm{HH}}=7.6 \mathrm{~Hz}, 1 \mathrm{H}$, $\mathrm{C}=\mathrm{CH}), 7.19\left(\mathrm{~m}, 2 \mathrm{H}, \mathrm{CH}_{o}-\mathrm{Ar}\right), 7.36(\mathrm{~m}, 2 \mathrm{H}, \mathrm{CH}-\mathrm{Ar}), 7.32\left(\mathrm{~m}, 1 \mathrm{H}, \mathrm{CH} \mathrm{H}^{-} \mathrm{Ar}\right) .{ }^{13} \mathrm{C}\left\{{ }^{1} \mathrm{H}\right\}-\mathrm{NMR}$ 
$\left(\mathrm{CDCl}_{3}, 126 \mathrm{MHz}\right): \delta(\mathrm{ppm}) 14.4\left(\mathrm{CH}_{3} \mathrm{CH}_{2} \mathrm{O}\right), 33.1\left(\mathrm{CH}_{2} \mathrm{CH}_{2} \mathrm{OH}\right), 61.1\left(\mathrm{CH}_{2} \mathrm{CH}_{2} \mathrm{OH}\right), 61.7$ $\left(\mathrm{CH}_{3} \mathrm{CH}_{2} \mathrm{O}\right), 127.7\left(\mathrm{CH}_{p}-\mathrm{Ar}\right), 128.2\left(\mathrm{CH}_{m}-\mathrm{Ar}\right), 129.8\left(\mathrm{CH}_{o}-\mathrm{Ar}\right), 135.3(\mathrm{C}-\mathrm{Ar}), 136.3(\mathrm{C}=\mathrm{CH})$, $140.7(\mathrm{C}=\mathrm{CH}), 167.2(\mathrm{C}=\mathrm{O})$. LRMS $(\mathrm{IC})=$ found $[\mathrm{M}+\mathrm{H}]^{+}=221$.

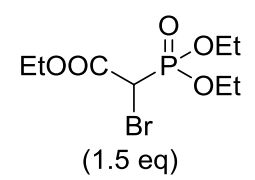

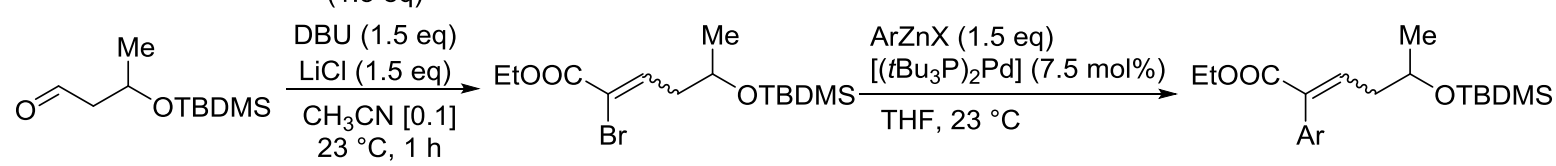

Ar:<smiles>[R]c1ccc(C)cc1</smiles>

$$
\begin{array}{ll}
\mathrm{R}=o-\mathrm{MeO} & (\mathbf{4 p}) \\
\mathrm{R}=p-\mathrm{MeO} & (\mathbf{4 q}) \\
\mathrm{R}=p-\mathrm{CF}_{3} & (4 \mathrm{r}) \\
\mathrm{R}=p-\mathrm{F} & (\mathbf{4 s})
\end{array}
$$

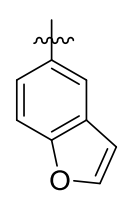

(4u)

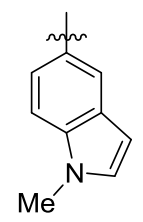

$(4 \mathrm{v})$

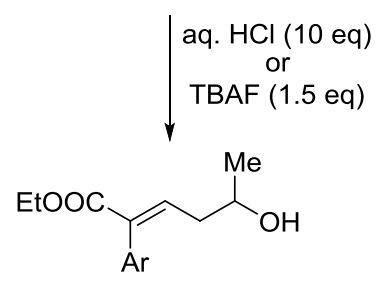

Preparation of $\underline{\operatorname{ArZnX}}$

4p, 4q, 4s: $\mathrm{ArMgBr}(1.5 \mathrm{eq})+\mathrm{ZnCl}_{2}(1.5 \mathrm{eq})$

$4 r, 4 u, 4 v: \operatorname{ArBr}(1.5$ eq $)+n B u L i(1.5$ eq $)+\mathrm{ZnCl}_{2}(1.5$ eq $)$ 4t: $\mathrm{ArBr}(1.5$ eq $)+i \mathrm{PrMgCl}(1.5$ eq $)+\mathrm{ZnCl}_{2}(1.5$ eq $)$

Figure S25: Synthesis of $\alpha, \beta$-unsaturated esters $4 p-v$

EtOOC $Y_{\mathrm{Br}}^{\mathrm{O}} \stackrel{\mathrm{N}-\mathrm{OEt}}{\mathrm{P}-\mathrm{OEt}}$

Ethyl 2-bromo-2-(diethoxyphosphoryl)acetate: To a solution of triethyl phosphonacetate $(44.6 \mathrm{mmol}, 10 \mathrm{~g})$ in THF (190 mL), NaH (53.5 mmol, 1.28

g) was added portionwise at $0{ }^{\circ} \mathrm{C}$. After 30 minutes, $\mathrm{Br}_{2}(53.5 \mathrm{mmol}, 8.55 \mathrm{~g}$ ) was carefully added and stirred for an additional 30 minutes. Conversion was monitored by ${ }^{1} \mathrm{H}-\mathrm{NMR}\left(\sim 89 \%\right.$ ) before quenching with a saturated aqueous $\mathrm{NH}_{4} \mathrm{Cl}$ solution $(100 \mathrm{~mL})$ and extracting with diethyl ether $(3 \times 100 \mathrm{~mL})$. The organic phases were combined, washed with brine, dried over $\mathrm{Na}_{2} \mathrm{SO}_{4}$ and the volatiles removed in vacuo. Purification by flash chromatography on a small pad of silica gel (Pentane/EtOAc $=4: 6$ ) afforded ethyl 2-bromo2-(diethoxyphosphoryl)acetate ${ }^{26}$ in $89 \%$ yield $(12.03 \mathrm{~g}$ ) as a colourless oil.

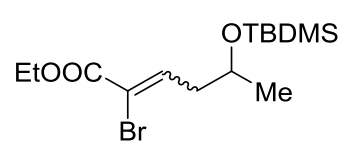

Ethyl 2-bromo-5-((tert-butyldimethylsilyl)oxy)hex-2-enoate: The reaction was performed according to GP2 using 3-((tertbutyldimethylsilyl)oxy)butanal $(1.7,8.4 \mathrm{mmol})$. Purification by flash chromatography (Pentane/Diethyl ether = 20:1) afforded ethyl 2-bromo-5-((tertbutyldimethylsilyl)oxy)hex-2-enoate in $82 \%$ yield $(2.38 \mathrm{~g}, E / Z=2.8: 1)$ as a light yellow oil. $\mathbf{R}_{\mathrm{F}}=0.6\left(\right.$ Pentane/Et $\left.{ }_{2} \mathrm{O}=9: 1\right) .{ }^{1} \mathrm{H}-\mathrm{NMR}\left(\mathrm{CDCl}_{3}, 500 \mathrm{MHz}\right): \delta(\mathrm{ppm})$ Major Isomer: $0.05(\mathrm{~m}$, 
$\left.6 \mathrm{H},\left(\mathrm{CH}_{3}\right)_{2} \mathrm{Si}\right), 0.89$, (s, 9H, $\left.\left(\mathrm{CH}_{3}\right)_{3} \mathrm{CSi}\right), 1.20$ (d, 3H, $\left.{ }^{3} \mathrm{~J}_{\mathrm{HH}}=6.1 \mathrm{~Hz}, \mathrm{CH}_{3} \mathrm{CHOTBDMS}\right), 1.32$ (t, $\left.3 \mathrm{H},{ }^{3} \mathrm{~J}_{\mathrm{HH}}=7.1 \mathrm{~Hz}, \mathrm{CH}_{3} \mathrm{CH}_{2} \mathrm{O}\right), 2.46\left(\mathrm{~m}, 2 \mathrm{H}, \mathrm{CH}_{2} \mathrm{CHOTBDMS}\right), 4.00\left(\mathrm{~h}, 1 \mathrm{H},{ }^{3} J_{H H}=6.1 \mathrm{~Hz}\right.$, $\mathrm{CH}_{3} \mathrm{CHOTBDMS}$ ), 4.28 (q, 2H, ${ }^{3} \mathrm{~J}_{\mathrm{HH}}=7.1 \mathrm{~Hz}, \mathrm{CH}_{3} \mathrm{CH}_{2} \mathrm{O}$ ), 7.39 (t, $1 \mathrm{H},{ }^{3} \mathrm{~J}_{\mathrm{HH}}=7.1 \mathrm{~Hz}, \mathrm{C}=\mathrm{CH}$ ). Minor Isomer: $0.05\left(\mathrm{~m}, 6 \mathrm{H},\left(\mathrm{CH}_{3}\right)_{2} \mathrm{Si}\right), 0.88,\left(\mathrm{~s}, 9 \mathrm{H},\left(\mathrm{CH}_{3}\right)_{3} \mathrm{CSi}\right), 1.16\left(\mathrm{~d}, 3 \mathrm{H},{ }^{3} \mathrm{~J}_{H H}=6.1 \mathrm{~Hz}\right.$, $\mathrm{CH}_{3} \mathrm{CHOTBDMS}$ ), 1.34 (t, 3H, ${ }^{3} \mathrm{~J}_{\mathrm{HH}}=7.1 \mathrm{~Hz}, \mathrm{CH}_{3} \mathrm{CH}_{2} \mathrm{O}$ ), 2.63 (m, 2H, $\mathrm{CH}_{2} \mathrm{CHOTBDMS}$ ), $3.94\left(3,1 \mathrm{H}, \mathrm{CH}_{2} \mathrm{CHOTBDMS}\right), 4.27$ (q, $2 \mathrm{H},{ }^{3} \mathrm{~J}_{H H}=7.1 \mathrm{~Hz}, \mathrm{CH}_{3} \mathrm{CH}_{2} \mathrm{O}$ ), $6.77\left(\mathrm{t}, 1 \mathrm{H},{ }^{3} J_{H H}=7.7\right.$ $\mathrm{Hz}, \mathrm{C}=\mathrm{CH}) .{ }^{13} \mathrm{C}\left\{{ }^{1} \mathrm{H}\right\}-\mathrm{NMR}\left(\mathrm{CDCl}_{3}, 126 \mathrm{MHz}\right): \delta(\mathrm{ppm})$ Major Isomer: $-4.4\left(\left(\mathrm{CH}_{3}\right)_{2} \mathrm{Si}\right), 14.3$ $\left(\mathrm{CH}_{3} \mathrm{CH}_{2} \mathrm{O}\right), \quad 18.2 \quad\left(\left(\mathrm{CH}_{3}\right)_{3} \mathrm{CSi}\right), \quad 24.2 \quad\left(\mathrm{CH}_{3} \mathrm{CHOTBDMS}\right), \quad 25.9 \quad\left(\left(\mathrm{CH}_{3}\right)_{3} \mathrm{CSi}\right), \quad 42.3$ ( $\left.\mathrm{CH}_{2} \mathrm{CHOTBDMS}\right), 62.5\left(\mathrm{CH}_{3} \mathrm{CH}_{2} \mathrm{O}\right), 67.1\left(\mathrm{CH}_{2} \mathrm{CHOTBDMS}\right), 117.5(\mathrm{C}=\mathrm{CH}), 143.5(\mathrm{C}=\mathrm{CH})$, $162.5(\mathrm{C}=\mathrm{O})$. Minor Isomer: -3.9 $\left(\left(\mathrm{CH}_{3}\right)_{2} \mathrm{Si}\right), 14.3\left(\mathrm{CH}_{3} \mathrm{CH}_{2} \mathrm{O}\right), 18.2\left(\left(\mathrm{CH}_{3}\right)_{3} \mathrm{CSi}\right), 24.0$ $\left(\mathrm{CH}_{3} \mathrm{COTBDMS}\right), 25.9\left(\left(\mathrm{CH}_{3}\right)_{3} \mathrm{CSi}\right), 41.2 \quad\left(\mathrm{CH}_{2} \mathrm{CHOTBDMS}\right), 62.2\left(\mathrm{CH}_{3} \mathrm{CH}_{2} \mathrm{O}\right), 67.7$ ( $\left.\mathrm{CH}_{2} \mathrm{CHOTBDMS}\right), 112.1(\mathrm{C}=\mathrm{CH}), 145.8(\mathrm{C}=\mathrm{CH}), 163.1(\mathrm{C}=\mathrm{O})$. LRMS $(\mathrm{IC})=$ found $[\mathrm{M}+\mathrm{H}]^{+}=$ 324. IR (neat): $v\left(\mathrm{~cm}^{-1}\right)=2956,2931,2890,2858,1726,1628,1466,1446,1370,1254$, 1213, 1129, 1090, 1040, 998, 903, 833, 806, 774, 745, 662.

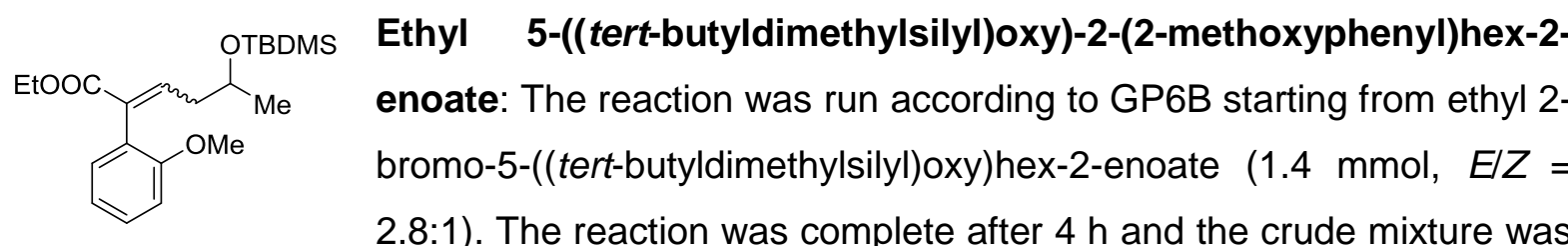

purified by flash chromatography (Pentane/Diethyl ether $=30: 1$ ) to give the title compound in $86 \%$ yield ( $450 \mathrm{mg}, E / Z=3: 1$ ) as a colourless oil. The product was engaged in the next step without further purification.

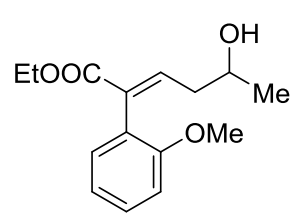

Ethyl (E)-5-hydroxy-2-(2-methoxyphenyl)hex-2-enoate (4p): To a solution of ethyl 5-((tert-butyldimethylsilyl)oxy)-2-(2-methoxyphenyl)hex-2enoate (430 mg, $1.07 \mathrm{mmol})$ in THF $(11 \mathrm{~mL})$, aqueous $\mathrm{HCl}(2 \mathrm{M}, 5 \mathrm{~mL})$ was added at $0{ }^{\circ} \mathrm{C}$ and stirred for $24 \mathrm{~h}$ at room temperature. The reaction was quenched with water $(30 \mathrm{~mL})$, extracted with diethyl ether $(3 \times 30 \mathrm{~mL})$, dried over anhydrous $\mathrm{Na}_{2} \mathrm{SO}_{4}$ and concentrated in vacuo. The residue was treated with $\mathrm{HCl}$ in diethyl ether $(1 \mathrm{M}, 11 \mathrm{~mL})$ and stirred for $4 \mathrm{~h}$. The volatiles were removed under vacuo and the crude mixture was purified by flash chromatography (Pentane/Diethyl ether= 5:1). Compound $4 \mathrm{p}$ was obtained in $56 \%$ yield $(180 \mathrm{mg})$ as a colourless oil. $\mathbf{R}_{\mathbf{f}}=0.2$ (Pentane/Diethyl ether =1:2). IR (neat): $v\left(\mathrm{~cm}^{-1}\right)=2971,1710,1248,1047,756 .{ }^{1} \mathbf{H}$ NMR $\left(400 \mathrm{MHz}, \mathrm{CDCl}_{3}\right) \delta(\mathrm{ppm}) 1.17\left(\mathrm{~d},{ }^{3} \mathrm{~J}_{\mathrm{HH}}=6.2 \mathrm{~Hz}, 3 \mathrm{H}, \mathrm{CH}_{3} \mathrm{CHOH}\right), 1.23\left(\mathrm{t},{ }^{3} \mathrm{~J}_{H H}=7.1 \mathrm{~Hz}, 3 \mathrm{H}\right.$, $\mathrm{CH}_{3} \mathrm{CH}_{2} \mathrm{O}$ ), $2.14-2.29\left(\mathrm{~m}, 2 \mathrm{H}, \mathrm{CH}_{2} \mathrm{CHOH}\right), 3.77$ (s, 3H, $\left.\mathrm{CH}_{3} \mathrm{OAr}\right), 3.88-3.98(\mathrm{~m}, 1 \mathrm{H}$, $\mathrm{CHOH}), 4.12-4.27\left(\mathrm{~m}, 2 \mathrm{H}, \mathrm{CH}_{3} \mathrm{CH}_{2} \mathrm{O}\right), 6.88-7.01(\mathrm{~m}, 2 \mathrm{H}, \mathrm{CH}-\mathrm{Ar}), 7.04-7.13(\mathrm{~m}, 1 \mathrm{H}$, 
$\mathrm{C}=\mathrm{CH} ; 1 \mathrm{H}, \mathrm{CH}-\mathrm{Ar}), 7.29-7.36(\mathrm{~m}, 1 \mathrm{H}, \mathrm{CH}-\mathrm{Ar}) .{ }^{13} \mathrm{C}\left\{{ }^{1} \mathrm{H}\right\}$ NMR $\left(101 \mathrm{MHz}, \mathrm{CDCl}_{3}\right) \delta(\mathrm{ppm})$ $14.4\left(\mathrm{CH}_{3} \mathrm{CH}\right), 23.4\left(\mathrm{CH}_{3} \mathrm{CHOH}\right), 39.4\left(\mathrm{CH}_{2} \mathrm{CHOH}\right), 55.7\left(\mathrm{CH}_{3} \mathrm{OAr}\right), 60.8\left(\mathrm{CH}_{3} \mathrm{CH}_{2} \mathrm{O}\right), 67.2$ (CHOH), 111.0 (CH-Ar), 120.6 (CH-Ar), 124.6 (C-Ar), 129.4 ( $\mathrm{CH}-\mathrm{Ar}), 131.1(\mathrm{CH}-\mathrm{Ar}), 133.5$ $(\mathrm{C}=\mathrm{CH}), 140.6(\mathrm{CH}-\mathrm{Ar}), 157.1(\mathrm{COMe}), 167.1(\mathrm{C}=\mathrm{O})$. LRMS (IC) found $\left[\mathrm{M}+\mathrm{NH}_{4}\right]^{+}=282$.

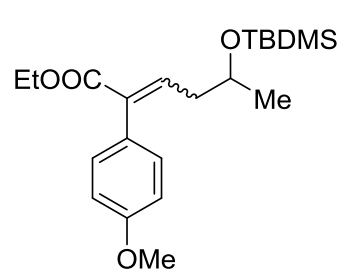

Ethyl 5-((tert-butyldimethylsilyl)oxy)-2-(4-methoxyphenyl)hex-2enoate: : The reaction was run according to GP6B starting from ethyl 2-bromo-5-((tert-butyldimethylsilyl)oxy)hex-2-enoate $(1.4 \mathrm{mmol}, E / Z=$ 2.8:1). The reaction was complete after $4 \mathrm{~h}$ and the crude mixture was filtered through a pad of silica gel and used in the next step without further purification.

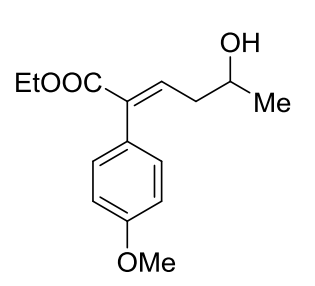

Ethyl (E)-5-hydroxy-2-(4-methoxyphenyl)hex-2-enoate (4q): To a solution of ethyl 5-((tert-butyldimethylsilyl)oxy)-2-(4-methoxyphenyl)hex-2enoate $(1.4 \mathrm{mmol})$ in THF (14 mL), aqueous $\mathrm{HCl}(2 \mathrm{M}, 7 \mathrm{~mL})$ was added at $0{ }^{\circ} \mathrm{C}$ and stirred at room temperature for $24 \mathrm{~h}$. The reaction was quenched with water $(40 \mathrm{~mL})$, extracted with diethyl ether $(3 \times 40 \mathrm{~mL})$, dried over anhydrous $\mathrm{Na}_{2} \mathrm{SO}_{4}$ and concentrated in vacuo. Purification by column chromatography on silica gel (Pentane/Diethyl ether from $5: 1$ to $1: 2$ ) gave $\mathbf{4 q}$ in $59 \%$ yield (220 mg, $E / Z=4.5: 1)$. The mixture was treated with $\mathrm{HCl}$ in diethyl ether $(1 \mathrm{M}, 11 \mathrm{~mL}$ ) for $1 \mathrm{~h}$ at room temperature. After evaporation of the volatiles, the crude mixture was purified by flash chromatography on silica gel (Pentane/Diethyl ether $=5: 1$ ) to give $4 q$ in $73 \%$ yield (160 $\mathrm{mg}, E / Z>50: 1$ ) as a colourless oil. $\mathbf{R}_{\mathbf{f}}=0.2$ (Pentane/Diethyl ether $\left.=1: 2\right)$. IR (neat): $v\left(\mathrm{~cm}^{-1}\right)$ $=3461,2972,1710,1609,1513,1247,1176,1035,835 .{ }^{1} \mathbf{H}$ NMR $\left(400 \mathrm{MHz}, \mathrm{CDCl}_{3}\right) \delta(\mathrm{ppm})$ $1.18\left(\mathrm{~d},{ }^{3} \mathrm{~J}_{\mathrm{HH}}=6.2 \mathrm{~Hz}, 3 \mathrm{H}, \mathrm{CH}_{3} \mathrm{CHOH}\right), 1.27\left(\mathrm{t},{ }^{3} \mathrm{~J}_{\mathrm{HH}}=7.1 \mathrm{~Hz}, 3 \mathrm{H}, \mathrm{CH}_{3} \mathrm{CH}_{2} \mathrm{O}\right), 1.37-1.44$ (m, $1 \mathrm{H}, \mathrm{OH}), 2.20-2.39\left(\mathrm{~m}, 2 \mathrm{H}, \mathrm{CH}_{2} \mathrm{CHOH}\right), 3.83$ (s, 3H, $\left.\mathrm{CH}_{3} \mathrm{OAr}\right), 3.90-4.03(\mathrm{~m}, 1 \mathrm{H}$, $\mathrm{CHOH}$ ), 4.21 (q, $\left.{ }^{3} \mathrm{~J}_{\mathrm{HH}}=7.1 \mathrm{~Hz}, 2 \mathrm{H}, \mathrm{CH}_{3} \mathrm{CH}_{2} \mathrm{O}\right), 6.85-6.95(\mathrm{~m}, 2 \mathrm{H}, \mathrm{CH}-\mathrm{Ar}), 7.07\left(\mathrm{t},{ }^{3} \mathrm{~J}_{\mathrm{HH}}=\right.$ $7.6 \mathrm{~Hz}, 1 \mathrm{H}, \mathrm{C}=\mathrm{CH}), 7.10-7.13(\mathrm{~m}, 2 \mathrm{H}, \mathrm{CH}-\mathrm{Ar}) .{ }^{13} \mathrm{C}\left\{{ }^{1} \mathrm{H}\right\} \mathbf{N M R}\left(101 \mathrm{MHz}, \mathrm{CDCl}_{3}\right) \delta(\mathrm{ppm})$ $14.4\left(\mathrm{CH}_{3} \mathrm{CH}_{2} \mathrm{O}\right), 23.5\left(\mathrm{CH}_{3} \mathrm{CHOH}\right), 39.2\left(\mathrm{CH}_{2} \mathrm{CHOH}\right), 55.4\left(\mathrm{CH}_{3} \mathrm{OAr}\right), 61.0\left(\mathrm{CH}_{3} \mathrm{CH}_{2} \mathrm{O}\right), 67.5$ $(\mathrm{CHOH}), 113.6(\mathrm{CH}-\mathrm{Ar}), 127.5(\mathrm{C}=\mathrm{CH}), 131.1(\mathrm{CH}-\mathrm{Ar}), 135.8(\mathrm{C}-\mathrm{Ar}), 140.1(\mathrm{C}=\mathrm{CH}), 159.0$ $(\mathrm{COCH} 3), 167.4(\mathrm{C}=\mathrm{O})$. LRMS (IC) found $\left[\mathrm{M}+\mathrm{NH}_{4}\right]^{+}=282$. 


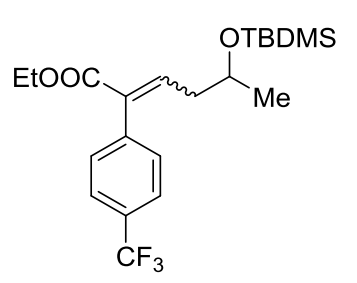

5-((tert-butyldimethylsilyl)oxy)-2-(4-

(trifluoromethyl)phenyl)hex-2-enoate: To a solution of 1-bromo-4(trifluoromethyl)benzene (337 mg, $1.5 \mathrm{mmol})$ in anhydrous THF (2 mL) at $-78{ }^{\circ} \mathrm{C}, n$-butyl lithium ( $1.5 \mathrm{mmol}, 1.6 \mathrm{M}$ in hexane) was added dropwise. The reaction mixture was stirred at $-78{ }^{\circ} \mathrm{C}$ for $1 \mathrm{~h}$ before a solution of $\mathrm{ZnCl}_{2}(1.5 \mathrm{mmol})$ in THF $(1.0 \mathrm{M})$ was added dropwise at the same temperature. The resulting homogeneous solution was stirred at room temperature for $1 \mathrm{~h}$. Ethyl 2-bromo5-((tert-butyldimethylsilyl)oxy)hex-2-enoate $(350 \mathrm{mg}, 1.0 \mathrm{mmol})$ and $\left[\left(t \mathrm{Bu}_{3} \mathrm{P}\right)_{2} \mathrm{Pd}\right](36 \mathrm{mg}$, $0.05 \mathrm{mmol}$ ) were dissolved in THF $(4 \mathrm{~mL})$ and then transferred to the alkylzinc solution by syringe. The mixture was stirred at room temperature for $17 \mathrm{~h}$, quenched with a saturated aqueous $\mathrm{NH}_{4} \mathrm{Cl}$ solution, extracted with diethyl ether $(3 \times 5 \mathrm{~mL})$, dried over anhydrous $\mathrm{Na}_{2} \mathrm{SO}_{4}$ and concentrated in vacuo. The residue was purified by flash chromatography on silica gel (Pentane/Diethyl ether $=40: 1)$ to give predominantly the $(E)$-isomer of the product in $48 \%$ yield (200 mg). $\mathbf{R}_{\mathbf{f}}=0.4$ (Pentane/Diethyl ether $\left.=10: 1\right)$. IR (neat): $v\left(\mathrm{~cm}^{-1}\right)=2957$, 1717, 1325, 1128, 837. ' $\mathbf{H}$ NMR $\left(400 \mathrm{MHz}, \mathrm{CDCl}_{3}\right) \delta(\mathrm{ppm}) 0.04\left(\mathrm{~d},{ }^{3} J_{\mathrm{HH}}=2.4 \mathrm{~Hz}, 6 \mathrm{H}\right.$, $\left.\left(\mathrm{CH}_{3}\right)_{2} \mathrm{Si}\right), 0.88\left(\mathrm{~s}, 9 \mathrm{H},\left(\mathrm{CH}_{3}\right)_{3} \mathrm{CSi}\right), 1.11\left(\mathrm{~d},{ }^{3} \mathrm{~J}_{\mathrm{HH}}=6.0 \mathrm{~Hz}, 3 \mathrm{H}, \mathrm{CH}_{3} \mathrm{CHOTBDMS}\right), 1.27\left(\mathrm{t},{ }^{3} \mathrm{~J}_{\mathrm{HH}}\right.$ $=7.1 \mathrm{~Hz}, 3 \mathrm{H}, \mathrm{CH}_{3} \mathrm{CH}_{2} \mathrm{O}$ ), $2.12-2.31\left(\mathrm{~m}, 2 \mathrm{H}, \mathrm{CH}_{2} \mathrm{CHOTBDMS}\right), 3.91$ (tdd, ${ }^{3} \mathrm{JHH}_{\mathrm{HH}}=7.2,{ }^{3} \mathrm{~J}_{\mathrm{HH}}=$ $\left.6.1,4.9 \mathrm{~Hz}, 1 \mathrm{H}, \mathrm{CH}_{2} \mathrm{CHOTBDMS}\right), 4.22\left(\mathrm{q},{ }^{3} J_{\mathrm{HH}}=7.1 \mathrm{~Hz}, 2 \mathrm{H}, \mathrm{CH}_{3} \mathrm{CH}_{2} \mathrm{O}\right.$ ), $7.19\left(\mathrm{t},{ }^{3} J_{\mathrm{HH}}=7.7\right.$ $\mathrm{Hz}, 1 \mathrm{H}, \mathrm{C}=\mathrm{CH}), 7.32\left(\mathrm{~d},{ }^{3} \mathrm{~J}_{\mathrm{HH}}=8.0 \mathrm{~Hz}, 2 \mathrm{H}, \mathrm{CH}-\mathrm{Ar}\right), 7.62\left(\mathrm{~d},{ }^{3} J_{\mathrm{HH}}=8.0 \mathrm{~Hz}, 2 \mathrm{H}, \mathrm{CH}-\mathrm{Ar}\right)$. ${ }^{13} \mathrm{C}\left\{{ }^{1} \mathrm{H}\right\}$ NMR $\left(101 \mathrm{MHz}, \mathrm{CDCl}_{3}\right) \delta(\mathrm{ppm})-4.7\left(\left(\mathrm{CH}_{3}\right)_{2} \mathrm{Si}\right),-4.4\left(\left(\mathrm{CH}_{3}\right)_{2} \mathrm{Si}\right), 14.4\left(\mathrm{CH}_{3} \mathrm{CH}_{2} \mathrm{O}\right)$, $18.2\left(\left(\mathrm{CH}_{3}\right)_{3} \mathrm{CSi}\right), 24.2\left(\mathrm{CH}_{3} \mathrm{CHOTBDMS}\right), 26.0\left(\left(\mathrm{CH}_{3}\right)_{3} \mathrm{CSi}\right), 39.6\left(\mathrm{CH}_{2} \mathrm{CHOTBDMS}\right), 61.2$ $\left(\mathrm{CH}_{3} \mathrm{CH}_{2} \mathrm{O}\right), 68.0\left(\mathrm{CH}_{2} \mathrm{CHOTBDMS}\right), 124.4$ (q, $\left.{ }^{1} \mathrm{~J}_{\mathrm{CF}}=273.71 \mathrm{~Hz}, \mathrm{CF}_{3}\right), 125.0$ (q, ${ }^{3} \mathrm{~J}_{\mathrm{CF}}=3.8$ $\mathrm{Hz}, \mathrm{CH}-\mathrm{Ar}), 129.1$ (q, $\left.{ }^{2} J_{\mathrm{CF}}=32.5, C^{C C F}\right), 130.4(\mathrm{CH}-\mathrm{Ar}), 134.0(\mathrm{C}=\mathrm{CH}), 139.3(\mathrm{C}-\mathrm{Ar}), 143.2$ $(\mathrm{C}=\mathrm{CH}), 166.5(\mathrm{C}=\mathrm{O})$. LRMS $(\mathrm{IC})$ found $\left[\mathrm{M}+\mathrm{NH}_{4}\right]^{+}=417$.

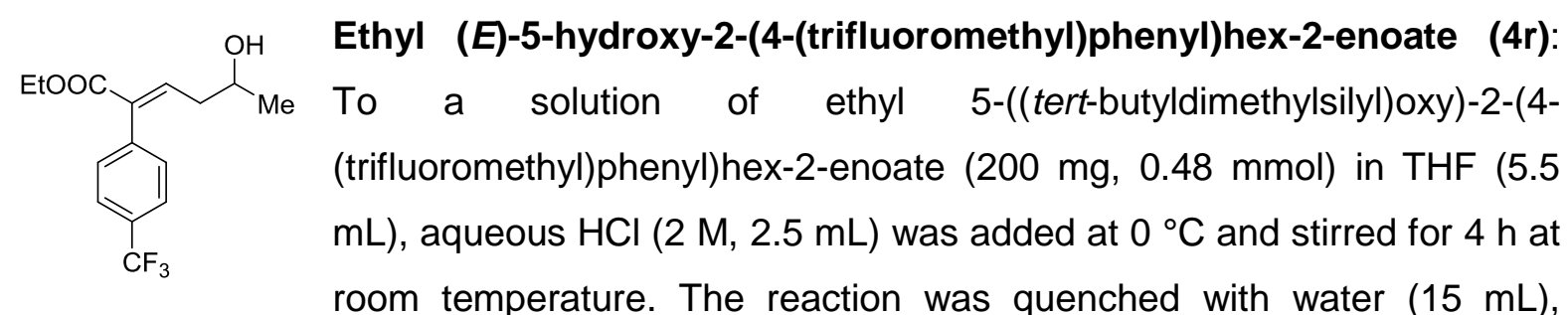
extracted with diethyl ether $(3 \times 15 \mathrm{~mL})$, dried over anhydrous $\mathrm{Na}_{2} \mathrm{SO}_{4}$ and concentrated in vacuo. The crude mixture was purified by flash chromatography on silica gel (Pentane/Diethyl ether $=5: 1$ ) to give $\mathbf{4 r}$ in $69 \%$ yield $\left(100 \mathrm{mg}\right.$ ) as a colourless oil. $\mathbf{R}_{\mathbf{f}}=0.4$ (Pentane/Diethyl ether = 1:2). IR (neat): $v\left(\mathrm{~cm}^{-1}\right)=3425,2975,1707,1323,1250,1163$, 1065, $847{ }^{1} \mathrm{H}$ NMR $\left(400 \mathrm{MHz}, \mathrm{CDCl}_{3}\right) \delta(\mathrm{ppm}) 1.19\left(\mathrm{~d},{ }^{3} \mathrm{~J}_{\mathrm{HH}}=6.2 \mathrm{~Hz}, 3 \mathrm{H}, \mathrm{CH}_{3} \mathrm{CHOH}\right), 1.27$ $\left(\mathrm{t},{ }^{3} \mathrm{~J}_{\mathrm{HH}}=7.1 \mathrm{~Hz}, 3 \mathrm{H}, \mathrm{CH}_{3} \mathrm{CH}_{2} \mathrm{O}\right), 1.39\left(\mathrm{~d},{ }^{3} \mathrm{~J}_{\mathrm{HH}}=4.5 \mathrm{~Hz}, 1 \mathrm{H}, \mathrm{OH}\right), 2.24$ (ddd, ${ }^{3} J_{\mathrm{HH}}=7.4,6.2$ 
$\mathrm{Hz},{ }^{2} J_{\mathrm{HH}}=1.3 \mathrm{~Hz}, 2 \mathrm{H}, \mathrm{CH}_{2} \mathrm{CHOH}$ ), 3.97 (qd, ${ }^{3} J_{\mathrm{HH}}=6.2,4.4 \mathrm{~Hz}, 1 \mathrm{H}, \mathrm{CH}_{2} \mathrm{CHOH}$ ), 4.22 (q, $\left.{ }^{3} J_{\mathrm{HH}}=7.1 \mathrm{~Hz}, 2 \mathrm{H}, \mathrm{CH}_{3} \mathrm{CH}_{2} \mathrm{O}\right), 7.19\left(\mathrm{t},{ }^{3} \mathrm{~J}_{\mathrm{HH}}=7.6 \mathrm{~Hz}, 1 \mathrm{H}, \mathrm{C}=\mathrm{CH}\right), 7.29-7.36(\mathrm{~m}, 2 \mathrm{H}, \mathrm{CH}-\mathrm{Ar})$, $7.63\left(\mathrm{dt},{ }^{3} \mathrm{~J}_{\mathrm{HH}}=7.6,{ }^{5} \mathrm{~J}_{\mathrm{HH}}=1.0 \mathrm{~Hz}, 2 \mathrm{H}, \mathrm{CH}-\mathrm{Ar}\right) \cdot{ }^{13} \mathrm{C}\left\{{ }^{1} \mathrm{H}\right\} \mathbf{N M R}\left(101 \mathrm{MHz}, \mathrm{CDCl}_{3}\right) \delta$ (ppm) 14.3 $\left(\mathrm{CH}_{3} \mathrm{CH}_{2} \mathrm{O}\right), 23.6\left(\mathrm{CH}_{3} \mathrm{CHOH}\right), 39.1\left(\mathrm{CH}_{2} \mathrm{CHOH}\right), 61.3\left(\mathrm{CH}_{3} \mathrm{CH}_{2} \mathrm{O}\right), 67.3\left(\mathrm{CH}_{2} \mathrm{CHOH}\right), 124.3$ (q, ${ }^{1} J_{\mathrm{CF}}=272.9 \mathrm{~Hz}, \mathrm{CF}_{3}$ ), 125.1 (q, ${ }^{3} \mathrm{~J}_{\mathrm{CF}}=3.8 \mathrm{~Hz}, \mathrm{CH}-\mathrm{Ar}$ ), 129.6 (q, ${ }^{2} J_{\mathrm{CF}}=32.5, \mathrm{CCF}_{3}$ ), $130.3(\mathrm{CH}-\mathrm{Ar}), 135.0(\mathrm{C}=\mathrm{CH}), 139.1(\mathrm{C}-\mathrm{Ar}), 141.9(\mathrm{C}=\mathrm{CH}), 166.5(\mathrm{C}=\mathrm{O})$. LRMS (IC) found $\left[\mathrm{M}+\mathrm{NH}_{4}\right]^{+}=303$.

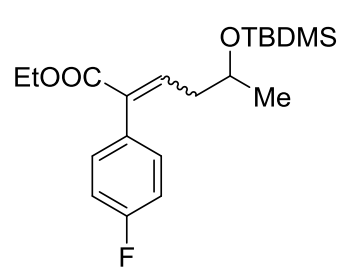

Ethyl 5-((tert-butyldimethylsilyl)oxy)-2-(4-fluorophenyl)hex-2enoate: The reaction was run according to GP6B starting from ethyl 2bromo-5-((tert-butyldimethylsilyl)oxy)hex-2-enoate $(1.4 \mathrm{mmol}, E / Z=$ 2.8:1). The reaction was complete after $4 \mathrm{~h}$ and the crude mixture was filtered through a pad of silica gel and then used for the next step without any further purification.

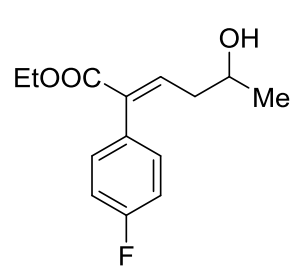

Ethyl (E)-2-(4-fluorophenyl)-5-hydroxyhex-2-enoate (4s): The product was prepared according to GP4 starting from ethyl 5-((tertbutyldimethylsilyl)oxy)-2-(4-fluorophenyl)hex-2-enoate $(1.4 \mathrm{mmol})$. The crude mixture was purified by flash chromatography on silica gel (Pentane/Diethyl ether $=1: 1)$ to give $(\boldsymbol{E})-\mathbf{4 s}$ in $48 \%$ yield $(170 \mathrm{mg}) . \mathbf{R}_{\mathbf{f}}=$ 0.3 (Pentane/Diethyl ether = 1:1). IR (neat): $v\left(\mathrm{~cm}^{-1}\right)=3441,2974,1711,1511,1251,1221$, 1043, 841. ${ }^{1} \mathrm{H}$ NMR $\left(400 \mathrm{MHz}, \mathrm{CDCl}_{3}\right) \delta(\mathrm{ppm}) 1.19\left(\mathrm{~d},{ }^{3} \mathrm{~J}_{\mathrm{HH}}=6.1 \mathrm{~Hz}, 3 \mathrm{H}, \mathrm{CH}_{3} \mathrm{CHOH}\right), 1.27$ $\left(\mathrm{t},{ }^{3} \mathrm{~J}_{\mathrm{HH}}=7.1 \mathrm{~Hz}, 3 \mathrm{H}, \mathrm{CH}_{3} \mathrm{CH}_{2} \mathrm{O}\right), 1.40\left(\mathrm{~d},{ }^{3} J_{\mathrm{HH}}=4.6 \mathrm{~Hz}, 1 \mathrm{H}, \mathrm{OH}\right), 2.17-2.34(\mathrm{~m}, 2 \mathrm{H}$, $\mathrm{CH}_{2} \mathrm{CHOH}$ ), $3.84-4.07\left(\mathrm{~m}, 1 \mathrm{H}, \mathrm{CH}_{2} \mathrm{CHOH}\right.$ ), 4.21 (q, ${ }^{3} \mathrm{JHH}_{\mathrm{HH}}=7.1 \mathrm{~Hz}, 2 \mathrm{H}, \mathrm{CH}_{3} \mathrm{CH}_{2} \mathrm{O}$ ), $6.99-$ $7.23(\mathrm{~m}, 1 \mathrm{H}, \mathrm{C}=\mathrm{CH} ; 4 \mathrm{H}, \mathrm{CH}-\mathrm{Ar}) .{ }^{13} \mathrm{C}\left\{{ }^{1} \mathrm{H}\right\} \mathrm{NMR}\left(101 \mathrm{MHz}, \mathrm{CDCl}_{3}\right) \delta$ (ppm) $14.2\left(\mathrm{CH}_{3} \mathrm{CH}_{2} \mathrm{O}\right)$, $23.4\left(\mathrm{CH}_{3} \mathrm{CHO}\right), 39.0\left(\mathrm{CH}_{2} \mathrm{CHOH}\right), 61.0\left(\mathrm{CH}_{3} \mathrm{CH}_{2} \mathrm{O}\right), 67.3\left(\mathrm{CH}_{2} \mathrm{CHOH}\right), 115.0\left(\mathrm{~d},{ }^{2} J_{\mathrm{CF}}=22.2\right.$ $\mathrm{Hz}, \mathrm{CH}-\mathrm{Ar}$ ), 131.0 (d, $\left.{ }^{4} \mathrm{~J}_{\mathrm{CF}}=4.0 \mathrm{~Hz}, C-\mathrm{Ar}\right), 131.4$ (d, $\left.{ }^{3} \mathrm{~J}_{\mathrm{CF}}=8.0 \mathrm{~Hz}, C \mathrm{H}-\mathrm{Ar}\right), 135.1(\mathrm{C}=\mathrm{CH})$, $140.8(\mathrm{C}=\mathrm{CH}), 162.2\left(\mathrm{~d},{ }^{1} J_{\mathrm{CF}}=45.4 \mathrm{~Hz}, \mathrm{CF}\right), 166.8(\mathrm{C}=\mathrm{O})$. LRMS (IC) found $\left[\mathrm{M}+\mathrm{NH}_{4}\right]^{+}=$ 253.

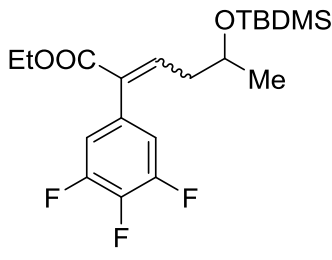

Ethyl 5-((tert-butyldimethylsilyl)oxy)-2-(3,4,5-trifluorophenyl)hex-

2-enoate: To a solution of the 5-bromo-1,2,3-trifluorobenzene (2.1 $\mathrm{mmol})$ in anhydrous THF $(2 \mathrm{~mL})$ at $0{ }^{\circ} \mathrm{C}$, isopropyl magnesium chloride (2.3 mmol, $2.0 \mathrm{M}$ in THF) was added dropwise and stirred at $0{ }^{\circ} \mathrm{C}$ for $1 \mathrm{~h}$. Subsequently, a THF solution of $\mathrm{ZnCl}_{2}(2.3 \mathrm{mmol}, 1.0 \mathrm{M})$ was added dropwise 
at $0{ }^{\circ} \mathrm{C}$ and the resulting mixture was stirred at room temperature for $1 \mathrm{~h}$. Ethyl 2-bromo-5((tert-butyldimethylsilyl)oxy)hex-2-enoate $(1.4 \mathrm{mmol}, E / Z=2.8: 1)$ and $\left[\left(t_{\mathrm{Bu}} \mathrm{P}\right){ }_{2} \mathrm{Pd}\right](53.6 \mathrm{mg})$ in THF (4 mL) were added slowly by syringe and the resulting mixture was stirred at room temperature. After $4 \mathrm{~h}$, the reaction was quenched with a saturated aqueous $\mathrm{NH}_{4} \mathrm{Cl}$ solution, extracted with diethyl ether $(3 \times 10 \mathrm{~mL})$, dried over anhydrous $\mathrm{Na}_{2} \mathrm{SO}_{4}$ and concentrated in vacuo. The crude mixture was filtered through a pad of silica gel and engaged in the next step without further purification.

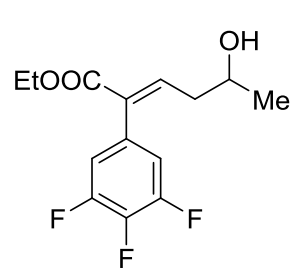

Ethyl (E)-5-hydroxy-2-(3,4,5-trifluorophenyl)hex-2-enoate (4t): To a solution of ethyl 5-((tert-butyldimethylsilyl)oxy)-2-(3,4,5trifluorophenyl)hex-2-enoate $(1.4 \mathrm{mmol})$ in THF $(14 \mathrm{~mL})$, aqueous $\mathrm{HCl}(2$ $\mathrm{M}, 7 \mathrm{~mL}$ ) was added at $0{ }^{\circ} \mathrm{C}$ and stirred at room temperature for $24 \mathrm{~h}$.

The reaction was quenched with water $(40 \mathrm{~mL})$, extracted with diethyl ether $(3 \times 40 \mathrm{~mL})$, dried over anhydrous $\mathrm{Na}_{2} \mathrm{SO}_{4}$ and concentrated in vacuo. The crude mixture was purified by flash chromatography on silica gel (Pentane/Diethyl ether $=1: 1$ ) to give $4 \mathbf{t}$ in $47 \%$ yield $(190 \mathrm{mg}) . \mathbf{R}_{\mathbf{f}}=0.2$ (Pentane/Diethyl ether $\left.=1: 1\right)$. IR (neat): $v\left(\mathrm{~cm}^{-1}\right)=$ 3399, 2980, 1712, 1531, 1254, 1042, 668. 'H NMR (400 MHz, $\left.\mathrm{CDCl}_{3}\right) \delta(\mathrm{ppm}) 1.21$ (d, ${ }^{3} \mathrm{~J}_{\mathrm{HH}}$ $\left.=6.2 \mathrm{~Hz}, 3 \mathrm{H}, \mathrm{CH}_{3} \mathrm{CHOH}\right), 1.28\left(\mathrm{t},{ }^{3} \mathrm{~J}_{\mathrm{HH}}=7.1 \mathrm{~Hz}, 3 \mathrm{H}, \mathrm{CH}_{3} \mathrm{CH}_{2} \mathrm{O}\right), 1.40\left(\mathrm{~d},{ }^{3} J_{\mathrm{HH}}=4.6 \mathrm{~Hz}, 1 \mathrm{H}\right.$, $\mathrm{OH}), 2.19-2.32\left(\mathrm{~m}, 2 \mathrm{H}, \mathrm{CH}_{2} \mathrm{CHOH}\right), 3.90-4.07\left(\mathrm{~m}, 1 \mathrm{H}, \mathrm{CH}_{2} \mathrm{CHOH}\right), 4.22$ (q, ${ }^{3} J_{H H}=7.1$ $\left.\mathrm{Hz}, 2 \mathrm{H}, \mathrm{CH}_{3} \mathrm{CH}_{2} \mathrm{O}\right), 6.78-6.92(\mathrm{~m}, 2 \mathrm{H}, \mathrm{CH}-\mathrm{Ar}), 7.17\left(\mathrm{t},{ }^{3} \mathrm{JHH}_{\mathrm{H}}=7.6 \mathrm{~Hz}, 1 \mathrm{H}, \mathrm{C}=\mathrm{CH}\right) .{ }^{13} \mathrm{C}\left\{{ }^{1} \mathrm{H}\right\}$ NMR (101 MHz, CDCl $) \delta(p p m) 14.3\left(\mathrm{CH}_{3} \mathrm{CH}_{2} \mathrm{O}\right), 23.7\left(\mathrm{CH}_{3} \mathrm{CHOH}\right), 39.0\left(\mathrm{CH}_{2} \mathrm{CHOH}\right), 61.5$ $\left(\mathrm{CH}_{3} \mathrm{CH}_{2} \mathrm{O}\right), 67.1\left(\mathrm{CH}_{2} \mathrm{CHOH}\right), 113.6-116.4$ (m, CH-Ar), $131.2\left(\mathrm{td},{ }^{3} J_{\mathrm{CF}}=8.3,{ }^{4} J_{\mathrm{CF}}=5.1 \mathrm{~Hz}\right.$, C-Ar), $133.5(C=\mathrm{CH}), 139.4\left(\mathrm{dt},{ }^{1} J_{\mathrm{CF}}=252.1,{ }^{2} J_{\mathrm{CF}}=15.3 \mathrm{~Hz}, C F_{p}-\mathrm{Ar}\right), 142.6(\mathrm{C}=\mathrm{CH}), 150.9$ (ddd, ${ }^{1} J_{\mathrm{CF}}=250.1,{ }^{2} J_{\mathrm{CF}}=10.0,{ }^{3} J_{\mathrm{CF}}=4.2 \mathrm{~Hz}, C F_{m}-\mathrm{Ar}$ ), 166.0 (C=O). LRMS (IC) found $\left[\mathrm{M}+\mathrm{NH}_{4}\right]^{+}=306$.

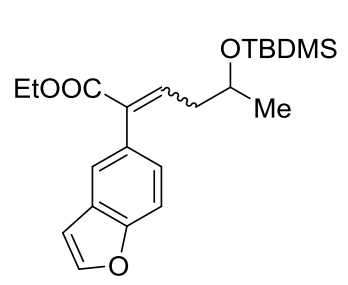

Ethyl 2-(benzofuran-5-yl)-5-((tert-butyldimethylsilyl)oxy)hex-2enoate: To a solution of the 5-bromo-benzofuran (414 $\mathrm{mg}, 2.1 \mathrm{mmol})$ in anhydrous THF $(2 \mathrm{~mL})$ at $-78^{\circ} \mathrm{C}, n$-butyl lithium $(2.2 \mathrm{mmol}, 1.0 \mathrm{M}$ in hexane) was added dropwise and stirred for $1 \mathrm{~h}$. A THF solution of $\mathrm{ZnCl}_{2}(2.1 \mathrm{mmol}, 1.0 \mathrm{M})$ was added dropwise at the same temperature and the resulting homogeneous solution was stirred at room temperature for $1 \mathrm{~h}$. Ethyl 2bromo-5-((tert-butyldimethylsilyl)oxy)hex-2-enoate $(1.4 \mathrm{mmol}, E / Z=2.8: 1)$ and $\left[\left(t \mathrm{Bu}_{3} \mathrm{P}\right)_{2} \mathrm{Pd}\right]$ (54 mg) in THF (4 mL) were added slowly by syringe. The resulting mixture was stirred at room temperature. After $4 \mathrm{~h}$, the reaction was quenched with a saturated aqueous $\mathrm{NH}_{4} \mathrm{Cl}$ 
solution, extracted with diethyl ether $(3 \times 10 \mathrm{~mL})$, dried over anhydrous $\mathrm{Na}_{2} \mathrm{SO}_{4}$, and concentrated in vacuo. The crude mixture was purified by flash chromatography to give the desired product in $64 \%$ yield $(350 \mathrm{mg}, E / Z=2.5: 1$ ) which was used in the next step directly.

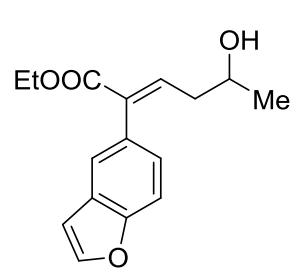

Ethyl (E)-2-(benzofuran-5-yl)-5-hydroxyhex-2-enoate (4u): Ethyl 2(benzofuran-5-yl)-5-((tert-butyldimethylsilyl)oxy)hex-2-enoate $(350 \mathrm{mg}$, $0.9 \mathrm{mmol}, E / Z=2.5: 1)$ was dissolved in THF $(4.3 \mathrm{~mL})$ and TBAF $(1.0 \mathrm{M}$ in THF, $2.7 \mathrm{mmol}$ ) was added slowly at $0{ }^{\circ} \mathrm{C}$. The mixture was warmed to room temperature. After $2 \mathrm{~h}$, the reaction was quenched with a saturated aqueous $\mathrm{NH}_{4} \mathrm{Cl}$ solution $(5 \mathrm{~mL})$, extracted with diethyl ether $(3 \times 20 \mathrm{~mL})$, dried over anhydrous $\mathrm{Na}_{2} \mathrm{SO}_{4}$ and concentrated in vacuo. The crude mixture was purified by flash chromatography on silica gel (Pentane/Diethyl ether $=1: 1$ ) to give $4 \mathbf{u}$ in $36 \%$ yield $(90 \mathrm{mg})$. $\mathbf{R}_{\mathbf{f}}=0.3$ (Pentane/Diethyl ether $=1: 2$ ). IR (neat): $v\left(\mathrm{~cm}^{-1}\right)=3435,2974,1702,1245,1109$, 1030, 911, 770, 730. ${ }^{1} \mathrm{H}$ NMR (400 MHz, $\left.\mathrm{CDCl}_{3}\right) \delta(\mathrm{ppm}) 1.13\left(\mathrm{~d},{ }^{3} \mathrm{~J}_{\mathrm{HH}}=6.2 \mathrm{~Hz}, 3 \mathrm{H}\right.$, $\mathrm{CH}_{3} \mathrm{CHOH}$ ), 1.25 (t, ${ }^{3} \mathrm{~J}_{\mathrm{HH}}=7.1 \mathrm{~Hz}, 3 \mathrm{H}, \mathrm{CH}_{3} \mathrm{CH}_{2} \mathrm{O}$ ), 2.24 (dd, ${ }^{3} \mathrm{~J}_{\mathrm{HH}}=7.5,6.2 \mathrm{~Hz}, 2 \mathrm{H}$, $\mathrm{CH}_{2} \mathrm{CHOH}$ ), $3.84-3.98\left(\mathrm{~m}, 1 \mathrm{H}, \mathrm{CH}_{2} \mathrm{CHOH}\right.$ ), 4.19 (q, ${ }^{3} \mathrm{~J}_{\mathrm{HH}}=7.1 \mathrm{~Hz}, 2 \mathrm{H}, \mathrm{CH}_{3} \mathrm{CH}_{2} \mathrm{O}$ ), 6.80 $\left(\mathrm{dd},{ }^{3} J_{\mathrm{HH}}=2.2,{ }^{5} \mathrm{~J}_{\mathrm{HH}}=1.0 \mathrm{~Hz}, 1 \mathrm{H}, \mathrm{CH}\right.$-Het), 7.10 (dd, ${ }^{3} J_{\mathrm{HH}}=8.5,{ }^{4} J_{\mathrm{HH}}=1.7 \mathrm{~Hz}, 1 \mathrm{H}, \mathrm{CH}$-Het), $7.13\left(\mathrm{t},{ }^{3} \mathrm{~J}_{\mathrm{HH}}=7.6 \mathrm{~Hz}, 1 \mathrm{H}, \mathrm{C}=\mathrm{CH}\right), 7.41\left(\mathrm{dd},{ }^{4} \mathrm{~J}_{\mathrm{HH}}=1.7 \mathrm{~Hz},{ }^{5} J_{\mathrm{HH}}=0.7 \mathrm{~Hz}, 1 \mathrm{H}, \mathrm{CH}-\mathrm{Het}\right), 7.49$ (ddd, ${ }^{3} J_{\mathrm{HH}}=8.4,{ }^{5} \mathrm{~J}_{\mathrm{HH}}=0.7,{ }^{5} \mathrm{~J}_{\mathrm{HH}}=1.0 \mathrm{~Hz}, 1 \mathrm{H}, \mathrm{CH}$-Het), 7.67 (d, ${ }^{3} \mathrm{~J}_{\mathrm{HH}}=2.2 \mathrm{~Hz}, 1 \mathrm{H}, \mathrm{CH}$-Het). ${ }^{13} \mathrm{C}\left\{{ }^{1} \mathrm{H}\right\}$ NMR $\left(101 \mathrm{MHz}, \mathrm{CDCl}_{3}\right) \delta(\mathrm{ppm}) 14.5\left(\mathrm{CH}_{3} \mathrm{CH}_{2} \mathrm{O}\right), 23.6\left(\mathrm{CH}_{3} \mathrm{CHOH}\right), 39.5$ $\left(\mathrm{CH}_{2} \mathrm{CHOH}\right), 61.2\left(\mathrm{CH}_{3} \mathrm{CH}_{2} \mathrm{O}\right), 67.6\left(\mathrm{CH}_{2} \mathrm{CHOH}\right), 107.0(\mathrm{CH}-\mathrm{Het}), 111.1(\mathrm{CH}-\mathrm{Het}), 122.8$ $(\mathrm{CH}-\mathrm{Het}), 126.6(\mathrm{CH}-\mathrm{Het}), 127.6(\mathrm{C}$-Het$), 130.5(\mathrm{C}=\mathrm{CH}), 136.3(\mathrm{C}-\mathrm{Het}), 141.1(\mathrm{C}=\mathrm{CH})$, 145.9 ( $\mathrm{CH}$-Het), 154.6 (C-Het), $167.4(\mathrm{C}=\mathrm{O})$. LRMS (IC) found $\left[\mathrm{M}+\mathrm{NH}_{4}\right]^{+}=292$.

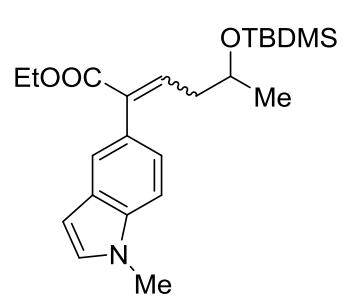

5-((tert-butyldimethylsilyl)oxy)-2-(1-methyl-1H-indol-5-

yl)hex-2-enoate: To a solution of the 5-bromo- $N$-Methyl indole (437 $\mathrm{mg}, 2.1 \mathrm{mmol})$ in anhydrous THF $(2 \mathrm{~mL})$ at $-78^{\circ} \mathrm{C}, n$-butyl lithium $(2.2$ mmol, $1.0 \mathrm{M}$ in hexane) was added dropwise. After $1 \mathrm{~h}$ at $-78{ }^{\circ} \mathrm{C}$, a THF solution of $\mathrm{ZnCl}_{2}(2.1 \mathrm{mmol}, 1.0 \mathrm{M})$ was added dropwise at the same temperature. The resulting homogeneous solution was stirred at room temperature for $1 \mathrm{~h}$ before ethyl 2-bromo-5-((tert-butyldimethylsilyl)oxy)hex-2-enoate (1.4 mmol, $E / Z=2.8: 1)$ and $\left[\left(t_{B} u_{3} P\right)_{2} \mathrm{Pd}\right](54 \mathrm{mg}, 0.105 \mathrm{mmol})$ in THF $(4 \mathrm{~mL})$ were added slowly by syringe. The resulting mixture was stirred at room temperature for $4 \mathrm{~h}$. The reaction was then quenched with a saturated aqueous $\mathrm{NH}_{4} \mathrm{Cl}$ solution, extracted with diethyl ether $(3 \times 10 \mathrm{~mL})$, dried over anhydrous $\mathrm{Na}_{2} \mathrm{SO}_{4}$, and concentrated in vacuo. The crude mixture was purified by flash 
chromatography on silica gel (Pentane/Diethyl ether $=10: 1)$ to give the desired product in $52 \%$ yield (440 mg, $E / Z=5: 1$ ). This mixture was used in the next step directly.

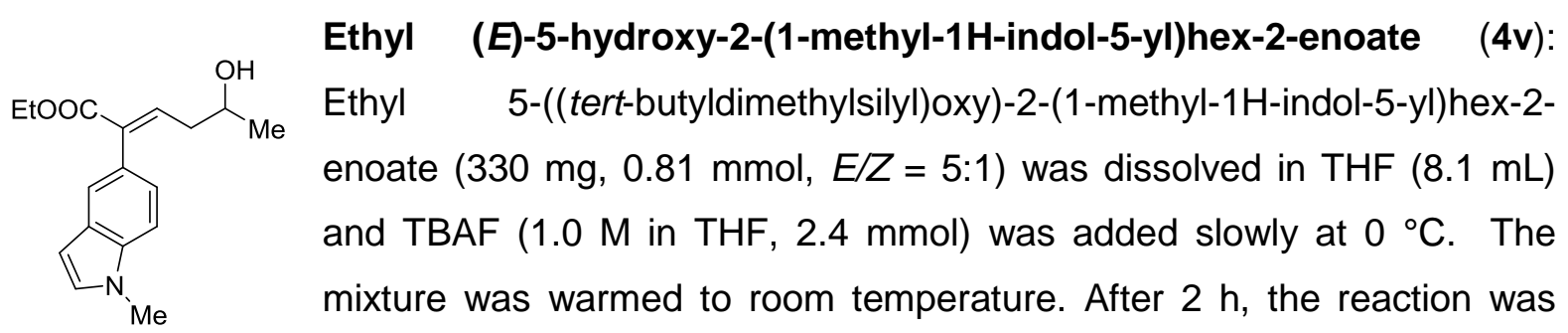
quenched with a saturated aqueous $\mathrm{NH}_{4} \mathrm{Cl}$ solution $(5 \mathrm{~mL})$, extracted with diethyl ether $(3 \times$ $20 \mathrm{~mL}$ ), dried over anhydrous $\mathrm{Na}_{2} \mathrm{SO}_{4}$ and concentrated in vacuo. The crude mixture was purified by flash chromatography on silica gel (Pentane/Diethyl ether $=1: 1$ ) to give $\mathbf{4} \mathbf{v}$ in $65 \%$ yield (152 mg) as a yellow oil. $\mathbf{R}_{\mathbf{f}}=0.2$ (Pentane/Diethyl ether $=1: 2$ ). IR (neat): $v\left(\mathrm{~cm}^{-1}\right.$ ) $=3437,2971,1705,1246,1042,728 .{ }^{1} \mathrm{H}$ NMR $\left(400 \mathrm{MHz}, \mathrm{CDCl}_{3}\right) \delta(\mathrm{ppm}) 1.13\left(\mathrm{~d},{ }^{3} \mathrm{JHH}_{\mathrm{HH}}=6.2\right.$ $\left.\mathrm{Hz}, 3 \mathrm{H}, \mathrm{CH}_{3} \mathrm{CHOH}\right), 1.25\left(\mathrm{t},{ }^{3} \mathrm{JHH}_{\mathrm{HH}}=7.1 \mathrm{~Hz}, 3 \mathrm{H}, \mathrm{CH}_{3} \mathrm{CH}_{2} \mathrm{O}\right), 1.42(\mathrm{~m}, 1 \mathrm{H}, \mathrm{OH}), 2.26\left(\mathrm{dd},{ }^{3} J_{\mathrm{HH}}\right.$ $\left.=7.5,6.2 \mathrm{~Hz}, 2 \mathrm{H}, \mathrm{CH}_{2} \mathrm{CHOH}\right), 3.80\left(\mathrm{~s}, 3 \mathrm{H}, \mathrm{CH}_{3} \mathrm{~N}\right), 3.88-3.97\left(\mathrm{~m}, 1 \mathrm{H}, \mathrm{CH}_{2} \mathrm{CHOH}\right), 4.19$ (q, ${ }^{3} J_{\mathrm{HH}}=7.1 \mathrm{~Hz}, 2 \mathrm{H}, \mathrm{CH}_{3} \mathrm{CH}_{2} \mathrm{O}$ ), 6.46 (dd, $\left.{ }^{3} J_{\mathrm{HH}}=3.1,{ }^{5} J_{\mathrm{HH}}=0.9 \mathrm{~Hz}, 1 \mathrm{H}, \mathrm{CH}-\mathrm{Het}\right), 7.01$ (dd, ${ }^{3} J_{\mathrm{HH}}$ $=8.4,{ }^{4} J_{\mathrm{HH}}=1.6 \mathrm{~Hz}, 1 \mathrm{H}, \mathrm{CH}$-Het), $7.06-7.18(\mathrm{~m}, 1 \mathrm{H}, \mathrm{CH}$-Het; $1 \mathrm{H}, \mathrm{C}=\mathrm{CH}), 7.33$ (ddd, ${ }^{3} J_{\mathrm{HH}}=$ $8.4,{ }^{5} J_{\mathrm{HH}}=0.8 \mathrm{~Hz},{ }^{5} \mathrm{~J}_{\mathrm{HH}}=0.9 \mathrm{~Hz}, 1 \mathrm{H}, \mathrm{CH}$-Het), $7.38\left(\mathrm{dd},{ }^{4} \mathrm{~J}_{\mathrm{HH}}=1.7,{ }^{5} \mathrm{~J}_{\mathrm{HH}}=0.8 \mathrm{~Hz}, 1 \mathrm{H}, \mathrm{CH}-\right.$ Het). ${ }^{13} \mathrm{C}\left\{{ }^{1} \mathrm{H}\right\}$ NMR (101 MHz, $\left.\mathrm{CDCl}_{3}\right) \delta(\mathrm{ppm}) 14.0\left(\mathrm{CH}_{3} \mathrm{CH}_{2} \mathrm{O}\right), 23.1\left(\mathrm{CH}_{3} \mathrm{CH}\right), 32.7\left(\mathrm{CH}_{3} \mathrm{~N}\right)$, $39.0\left(\mathrm{CH}_{2} \mathrm{CHOH}\right), 60.6\left(\mathrm{CH}_{3} \mathrm{CH}_{2} \mathrm{O}\right), 67.2\left(\mathrm{CH}_{2} \mathrm{CHOH}\right), 100.7(\mathrm{CH}-\mathrm{Het}), 108.6(\mathrm{CH}-\mathrm{Het})$, 121.7 ( $\mathrm{CH}-\mathrm{Het}), 123.2$ (CH-Het), 126.2 (C-Het), 128.0 (C-Het), $129.2(\mathrm{CH}-\mathrm{Het}), 135.9(\mathrm{C}-\mathrm{N})$, $136.7(\mathrm{C}=\mathrm{CH}), 139.7(\mathrm{C}=\mathrm{CH}), 167.4(\mathrm{C}=\mathrm{O})$. LRMS $(\mathrm{IC})$ found $[\mathrm{M}+\mathrm{H}]^{+}=288$.

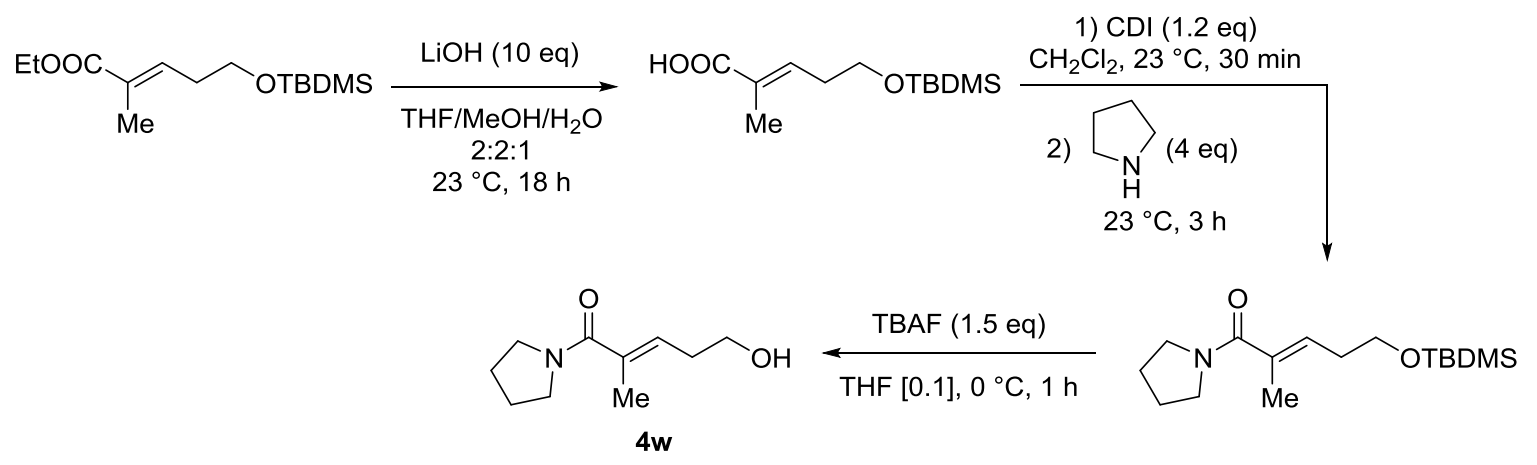

Figure S26: Synthesis of $(E)$-5-hydroxy-2-methyl-1-(pyrrolidin-1-yl)pent-2-en-1-one (4w) 


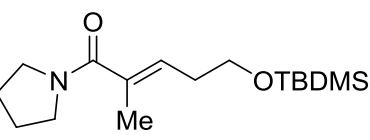

(E)-5-((tert-butyldimethylsilyl)oxy)-2-methyl-1-(pyrrolidin-1yl)pent-2-en-1-one: Ethyl (E)-5-((tert-butyldimethylsilyl)oxy)-2methylpent-2-enoate $(7.34 \mathrm{mmol})$ was dissolved in a solution of THF, MeOH, $\mathrm{H}_{2} \mathrm{O}(2: 2: 1,183 \mathrm{~mL})$ and $\mathrm{LiOH}(73.4 \mathrm{mmol}, 1.76 \mathrm{~g})$ was added and stirred at room temperature. After $16 \mathrm{~h}$, the reaction was quenched with $\mathrm{AcOH}$ ( $\mathrm{pH}$ adjusted to 5-6) and extracted with diethyl ether $(3 \times 100 \mathrm{~mL})$ then the organic phases were combined, dried over anhydrous $\mathrm{Na}_{2} \mathrm{SO}_{4}$ and evaporated in vacuo. The residue was redissolved in $\mathrm{CH}_{2} \mathrm{Cl}_{2}$ (32 $\mathrm{mL})$ and 1,1'-carbonyldiimidazole (CDI) $(8.4 \mathrm{mmol}, 1.36 \mathrm{~g})$ was added and stirred at room temperature for 30 minutes. Pyrrolidine $(28 \mathrm{mmol}, 2.3 \mathrm{~mL})$ was added in one portion and stirred for $3 \mathrm{~h}$ at room temperature. The reaction was quenched with a saturated aqueous $\mathrm{NH}_{4} \mathrm{Cl}$ solution (30 mL), extracted with EtOAc $(3 \times 30 \mathrm{~mL})$ then the organic phases combined, dried over anhydrous $\mathrm{Na}_{2} \mathrm{SO}_{4}$ and evaporated. The crude oil was purified by flash chromatography on silica gel (Diethyl ether) to afford (E)-5-((tert-butyldimethylsilyl)oxy)-2methyl-1-(pyrrolidin-1-yl)pent-2-en-1-one as yellow oil in $51 \%$ yield $(1.11 \mathrm{~g})$ over two steps. $\mathbf{R}_{\mathbf{f}}=0.4$ (Diethyl ether). IR (neat): $v\left(\mathrm{~cm}^{-1}\right)=2953,2930,2859,1618,1462,1436,1378$, 1253, 1224, 1198, 1160, 1093, 1006, 936, 834, 775, 740, 664, 599, 568. ${ }^{1}$ H NMR $(500 \mathrm{MHz}$, $\left.\mathrm{CDCl}_{3}\right) \delta(\mathrm{ppm}) 0.04\left(\mathrm{~s}, 6 \mathrm{H},\left(\mathrm{CH}_{3}\right)_{2} \mathrm{Si}\right), 0.87\left(\mathrm{~s}, 9 \mathrm{H},\left(\mathrm{CH}_{3}\right)_{3} \mathrm{CSi}\right), 1.84\left(\mathrm{~s}, 3 \mathrm{H}, \mathrm{CH}_{3} \mathrm{C}=\mathrm{CH}\right), 1.84$ - 1.86 (bm, 4H, $\left(\mathrm{CH}_{2} \mathrm{CH}_{2}\right)_{2} \mathrm{~N}$ ), 2.32 (q, $2 \mathrm{H},{ }^{3} \mathrm{~J}_{\mathrm{HH}}=7.0 \mathrm{~Hz}, \mathrm{CH}_{2} \mathrm{CH}_{2} \mathrm{OTBDMS}$ ), $3.42-3.44$ (bm, 2H, $\left.\left(\mathrm{CH}_{2} \mathrm{CH}_{2}\right)_{2} \mathrm{~N}\right), 3.46-3.48\left(\mathrm{bm}, 2 \mathrm{H},\left(\mathrm{CH}_{2} \mathrm{CH}_{2}\right)_{2} \mathrm{~N}\right), 3.65$ (t, $2 \mathrm{H},{ }^{3} J_{H H}=6.7 \mathrm{~Hz}$, $\left.\mathrm{CH}_{2} \mathrm{CH}_{2} \mathrm{OTBDMS}\right), 5.66(\mathrm{~m}, 1 \mathrm{H}, \mathrm{C}=\mathrm{CH}) .{ }^{13} \mathrm{C}\left\{{ }^{1} \mathrm{H}\right\} \mathrm{NMR}\left(126 \mathrm{MHz}, \mathrm{CDCl}_{3}\right) \delta$ (ppm) -5.2 $\left(\left(\mathrm{CH}_{3}\right)_{2} \mathrm{Si}\right), 14.1\left(\mathrm{CH}_{3} \mathrm{C}=\mathrm{CH}\right), 18.4\left(\left(\mathrm{CH}_{3}\right)_{3} \mathrm{CSi}\right), 24.6\left(\left(\mathrm{CH}_{2} \mathrm{CH}_{2}\right)_{2} \mathrm{~N}\right), 26.0\left(\left(\mathrm{CH}_{3}\right)_{3} \mathrm{CSi}\right), 26.3$ $\left.\left(\mathrm{CH}_{2} \mathrm{CH}_{2}\right)_{2} \mathrm{~N}\right), 31.6\left(\mathrm{CH}_{2} \mathrm{CH}_{2} \mathrm{OTBDMS}\right), 45.5 \quad\left(\left(\mathrm{CH}_{2} \mathrm{CH}_{2}\right)_{2} \mathrm{~N}\right), 49.0 \quad\left(\left(\mathrm{CH}_{2} \mathrm{CH}_{2}\right)_{2} \mathrm{~N}\right), \quad 62.2$ $\left(\mathrm{CH}_{2} \mathrm{CH}_{2} \mathrm{OTBDMS}\right), 128.4(\mathrm{C}=\mathrm{CH}), 134.4(\mathrm{C}=\mathrm{CH}), 172.0(\mathrm{C}=\mathrm{O})$. LRMS (IC) found $[\mathrm{M}+\mathrm{H}]^{+}=$ 299.

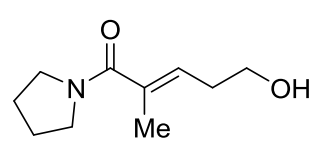

(E)-5-hydroxy-2-methyl-1-(pyrrolidin-1-yl)pent-2-en-1-one (4w): The product was prepared according to GP3 starting from (E)-5-((tertbutyldimethylsilyl)oxy)-2-methyl-1-(pyrrolidin-1-yl)pent-2-en-1-one (3.74 mmol, $1.11 \mathrm{~g}$ ). After column chromatography on silica gel (Diethyl ether then Acetone) the product $\mathbf{4} \mathbf{w}$ was obtained in $32 \%$ yield $(213 \mathrm{mg})$ as a pale yellow oil. $\mathbf{R}_{\mathbf{f}}=0.2$ $\left(\mathrm{CH}_{2} \mathrm{Cl}_{2} /\right.$ Acetone $\left.=1: 1\right)$. IR (neat): $v\left(\mathrm{~cm}^{-1}\right)=3376,2952,2928,2876,1657,1590,1436$, 1378, 1341, 1252, 1227, 1186, 1159, 1051, 895, 870, 744, 653, 602, 576. ${ }^{1}$ H NMR (500 $\left.\mathrm{MHz}, \mathrm{CDCl}_{3}\right) \delta(\mathrm{ppm}) 1.68$ (bs, $\left.1 \mathrm{H}, \mathrm{OH}\right), 1.86-1.92\left(\mathrm{bm}, 4 \mathrm{H},\left(\mathrm{CH}_{2} \mathrm{CH}_{2}\right)_{2} \mathrm{~N}, 3 \mathrm{H}, \mathrm{CH}_{3} \mathrm{C}=\mathrm{CH}\right)$, $2.39\left(\mathrm{q}, 2 \mathrm{H},{ }^{3} \mathrm{~J}_{\mathrm{HH}}=6.7 \mathrm{~Hz}, \mathrm{CH}_{2} \mathrm{CH}_{2} \mathrm{OH}\right), 3.42-3.45\left(\mathrm{bm}, 4 \mathrm{H},\left(\mathrm{CH}_{2} \mathrm{CH}_{2}\right)_{2} \mathrm{~N}\right), 3.71\left(\mathrm{t}, 2 \mathrm{H},{ }^{3} \mathrm{~J}_{H H}\right.$ $\left.=6.4 \mathrm{~Hz}, \mathrm{CH}_{2} \mathrm{CH}_{2} \mathrm{OH}\right), 5.69(\mathrm{~m}, 1 \mathrm{H}, \mathrm{C}=\mathrm{CH}) .{ }^{13} \mathbf{C}\left\{{ }^{1} \mathrm{H}\right\} \mathbf{N M R}\left(126 \mathrm{MHz}, \mathrm{CDCl}_{3}\right) \delta(\mathrm{ppm}) 14.2$ $\left.\left.\left(\mathrm{CH}_{3} \mathrm{C}=\mathrm{CH}\right), 24.6\left(\left(\mathrm{CH}_{2} \mathrm{CH}_{2}\right)_{2} \mathrm{~N}\right), 26.3\left(\mathrm{CH}_{2} \mathrm{CH}_{2}\right)_{2} \mathrm{~N}\right), 31.4\left(\mathrm{CH}_{2} \mathrm{CH}_{2} \mathrm{OH}\right), 45.8\left(\mathrm{CH}_{2} \mathrm{CH}_{2}\right)_{2} \mathrm{~N}\right)$, 
$\left.48.9\left(\mathrm{CH}_{2} \mathrm{CH}_{2}\right)_{2} \mathrm{~N}\right), 61.9\left(\mathrm{CH}_{2} \mathrm{CH}_{2} \mathrm{OH}\right), 127.8(\mathrm{C}=\mathrm{CH}), 135.5(\mathrm{C}=\mathrm{CH}), 171.9(\mathrm{C}=\mathrm{O})$. LRMS (IC) found $[\mathrm{M}+\mathrm{H}]^{+}=184,[\mathrm{M}+\mathrm{Na}]^{+}=206$.

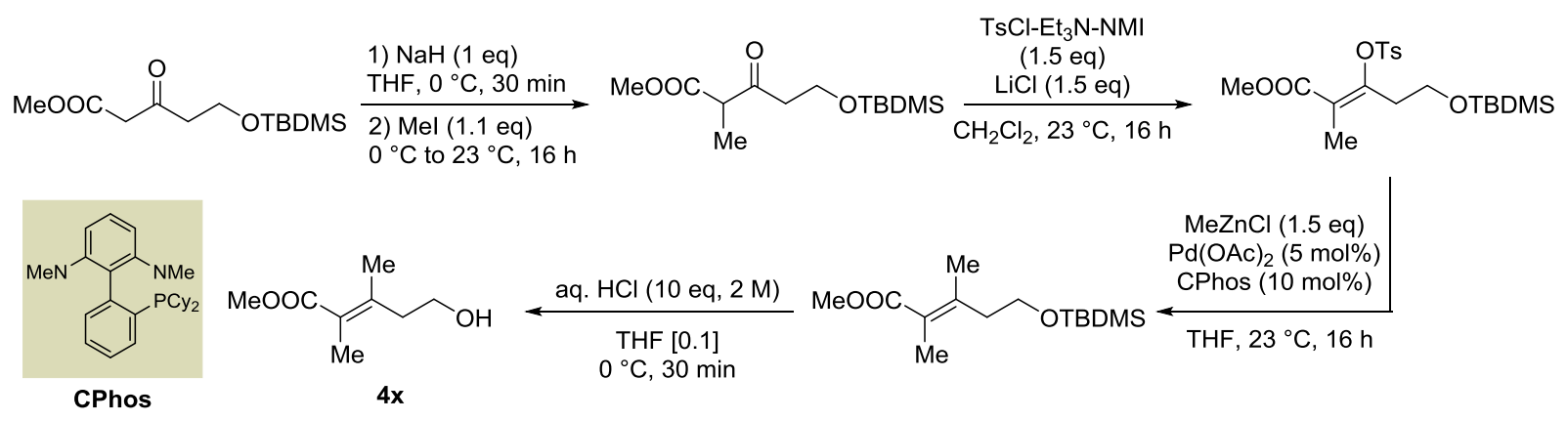

Figure S27: Synthesis of Methyl $(E)$-5-hydroxy-2,3-dimethylpent-2-enoate (4x)<smiles>CCOCCC(=O)C([N])C(C)=O</smiles>

\section{Methyl}

5-((tert-butyldimethylsilyl)oxy)-2-methyl-3-

oxopentanoate: In a round bottom flask, the crude $\beta$-ketoester (15.76 mmol) was dissolved in THF $(79 \mathrm{~mL})$ and cooled to $0{ }^{\circ} \mathrm{C}$. After the addition of $\mathrm{NaH}(15.76 \mathrm{mmol}, 378 \mathrm{mg})$, the mixture was stirred 30 minutes at $0{ }^{\circ} \mathrm{C}$. Next, methyl iodide $(17.34 \mathrm{mmol}, 1.1 \mathrm{~mL})$ was added at $0{ }^{\circ} \mathrm{C}$ and the reaction stirred for $16 \mathrm{~h}$ at room temperature. The reaction was quenched with a saturated aqueous $\mathrm{NH}_{4} \mathrm{Cl}$ solution $(20 \mathrm{~mL})$, extracted with diethyl ether $(3 \times 80 \mathrm{~mL})$, dried over $\mathrm{Na}_{2} \mathrm{SO}_{4}$ and concentrated in vacuo. The residual oil was purified by flash chromatography on silica gel (Pentane/Diethyl ether $=95: 5)$ to afford methyl 5-((tert-butyldimethylsilyl)oxy)-2-methyl-3-oxopentanoate in $50 \%$ yield $\left(2.16 \mathrm{~g}\right.$ ) over 2 steps as a colourless oil. $\mathbf{R}_{\mathbf{f}}=0.4$ (Pentane/Diethyl ether). IR (neat): $v\left(\mathrm{~cm}^{-1}\right)=2954,2932,2858,1748,1718,1653,1618,1461,1436,1388,1331,1253$, 1203, 1090, 1005, 921, 831, 776, 732, 663. ${ }^{1} \mathrm{H}-\mathrm{NMR}\left(\mathrm{CDCl}_{3}, 500 \mathrm{MHz}\right): \delta(\mathrm{ppm}) 0.05$ (d, ${ }^{3} J_{\mathrm{HH}}=6.2 \mathrm{~Hz}, 6 \mathrm{H},\left(\mathrm{CH}_{3}\right)_{2} \mathrm{Si}$, ), $0.87\left(\mathrm{~s}, 9 \mathrm{H},\left(\mathrm{CH}_{3}\right)_{3} \mathrm{CSi}\right), 1.34\left(\mathrm{~d},{ }^{3} \mathrm{~J}_{\mathrm{HH}}=7.2 \mathrm{~Hz}, 2 \mathrm{H}, \mathrm{CH}_{3} \mathrm{CH}\right)$, $2.74\left(\mathrm{td},{ }^{3} \mathrm{~J}_{\mathrm{HH}}=6.2,{ }^{2} \mathrm{~J}_{\mathrm{HH}}=2.0 \mathrm{~Hz}, 2 \mathrm{H}, \mathrm{CH}_{2} \mathrm{CH}_{2} \mathrm{OTBDMS}\right.$ ), 3.59 (q, ${ }^{3} \mathrm{~J}_{\mathrm{HH}}=7.2 \mathrm{~Hz}, 1 \mathrm{H}$, $\mathrm{CH}_{3} \mathrm{CH}$ ), 3.73 (s, 3H, CH $\mathrm{H}_{3} \mathrm{O}$ ), 3.89 (t, $\left.{ }^{3} \mathrm{JHH}_{\mathrm{HH}}=6.3 \mathrm{~Hz}, 1 \mathrm{H}, \mathrm{CH}_{2} \mathrm{CH}_{2} \mathrm{OTBDMS}\right) .{ }^{13} \mathrm{C}\left\{{ }^{1} \mathrm{H}\right\}-\mathrm{NMR}$ $\left(\mathrm{CDCl}_{3}, 126 \mathrm{MHz}\right): \delta(\mathrm{ppm}) \quad-5.4\left(\left(\mathrm{CH}_{3}\right)_{2} \mathrm{Si}\right), 12.6\left(\mathrm{CH}_{3} \mathrm{CH}\right), 18.4 \quad\left(\left(\mathrm{CH}_{3}\right)_{3} \mathrm{CSi}\right), 26.0$ (( $\left.\left.\mathrm{CH}_{3}\right)_{3} \mathrm{CSi}\right), 44.4\left(\mathrm{CH}_{2} \mathrm{CH}_{2} \mathrm{OTBDMS}\right), 52.5\left(\mathrm{CH}_{3} \mathrm{O}\right), 53.4\left(\mathrm{CH}_{3} \mathrm{CH}\right), 58.8\left(\mathrm{CH}_{2} \mathrm{CH}_{2} \mathrm{OTBDMS}\right)$, $171.0\left(\mathrm{CH}_{3} \mathrm{O}_{2} \mathrm{C}\right), 204.9(\mathrm{CHC}(\mathrm{O}))$. LRMS (IC) found $[\mathrm{M}+\mathrm{HCOOH}]^{+}=320$.

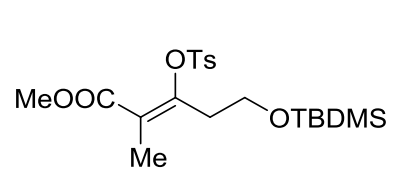

\section{Methyl}

(Z)-5-((tert-butyldimethylsilyl)oxy)-2-methyl-3-

(tosyloxy)pent-2-enoate: In a round-bottom flask, methyl 5-((tertbutyldimethylsilyl)oxy)-2-methyl-3-oxopentanoate $(6.15 \mathrm{mmol}, 1.69$ g) was dissolved in $\mathrm{CH}_{2} \mathrm{Cl}_{2}(31 \mathrm{~mL})$. Next, $\mathrm{Et}_{3} \mathrm{~N}$ (9.23 mmol, $\left.1.3 \mathrm{~mL}\right)$, NMI (9.23 mmol, 0.7 $\mathrm{mL})$, $\mathrm{LiCl}(30.75 \mathrm{mmol}, 1.3 \mathrm{~g})$ and $\mathrm{TsCl}(9.23 \mathrm{mmol}, 1.76 \mathrm{~g})$ were added sequentially at $0^{\circ} \mathrm{C}$ 
and the reaction was stirred at room temperature for $16 \mathrm{~h}$. The reaction was quenched with a saturated aqueous $\mathrm{NH}_{4} \mathrm{Cl}$ solution $(20 \mathrm{~mL})$, extracted with diethyl ether $(3 \times 30 \mathrm{~mL})$, dried over $\mathrm{Na}_{2} \mathrm{SO}_{4}$ and concentrated in vacuo. ${ }^{25}$ The residual oil was purified by flash chromatography on silica gel (Pentane/Diethyl ether 9:1) to afford (Z)-tosylate in 59\% yield $(1.47 \mathrm{~g})$ as a pale yellow oil. $(Z)$-isomer: $\mathbf{R}_{\mathbf{f}}=0.5$ (Pentane/Diethyl ether 8:2). IR (neat): $v\left(\mathrm{~cm}^{-1}\right)=2930,2861,1725,1375,1258,1193,1086,1019,776,733,666,548 .{ }^{1} \mathbf{H}-\mathbf{N M R}$ $\left(\mathrm{CDCl}_{3}, 500 \mathrm{MHz}\right): \delta(\mathrm{ppm})-0.01\left(\mathrm{~s}, 6 \mathrm{H},\left(\mathrm{CH}_{3}\right)_{2} \mathrm{Si}\right), 0.83\left(\mathrm{~s}, 9 \mathrm{H},\left(\mathrm{CH}_{3}\right)_{3} \mathrm{CSi}\right) .1 .91(\mathrm{~m}, 3 \mathrm{H}$, $\mathrm{CH}_{3} \mathrm{C}=\mathrm{C}$ ), 2.45 (s, 3H, CH $\mathrm{Ar}$ ), 2.59 (t, ${ }^{3} \mathrm{JHH}_{\mathrm{HH}}=6.5 \mathrm{~Hz}, 2 \mathrm{H}, \mathrm{CH}_{2} \mathrm{CH}_{2} \mathrm{OTBDMS}$ ), 3.57 (s, 3H, $\mathrm{CH}_{3} \mathrm{O}$ ), $3.72\left(\mathrm{t},{ }^{3} \mathrm{JHH}_{\mathrm{HH}}=6.5 \mathrm{~Hz}, 2 \mathrm{H}, \mathrm{CH}_{2} \mathrm{CH}_{2} \mathrm{OTBDMS}\right), 7.33-7.35\left(\mathrm{~d},{ }^{3} \mathrm{~J}_{\mathrm{HH}}=8.2 \mathrm{~Hz}, 2 \mathrm{H}, \mathrm{CH}_{m^{-}}\right.$ Ar), $7.80-7.82\left(\mathrm{~d},{ }^{3} \mathrm{~J}_{\mathrm{HH}}=8.2 \mathrm{~Hz}, 2 \mathrm{H}, \mathrm{CH}-\mathrm{Ar}\right) .{ }^{13} \mathrm{C}\left\{{ }^{1} \mathrm{H}\right\}-\mathrm{NMR}\left(\mathrm{CDCl}_{3}, 126 \mathrm{MHz}\right): \delta(\mathrm{ppm})-5.4$ $\left(\left(\mathrm{CH}_{3}\right)_{2} \mathrm{Si}\right), \quad 15.4 \quad\left(\mathrm{CH}_{3} \mathrm{C}=\mathrm{C}\right), \quad 18.3 \quad\left(\left(\mathrm{CH}_{3}\right)_{3} \mathrm{CSi}\right), \quad 21.8 \quad\left(\mathrm{CH}_{3} \mathrm{Ar}\right), \quad 25.9 \quad\left(\left(\mathrm{CH}_{3}\right)_{3} \mathrm{CSi}\right), \quad 35.5$ $\left(\mathrm{CH}_{2} \mathrm{CH}_{2} \mathrm{OTBDMS}\right), 52.0\left(\mathrm{CH}_{3} \mathrm{O}\right), 59.7\left(\mathrm{CH}_{2} \mathrm{CH}_{2} \mathrm{OTBDMS}\right), 122.0\left(\mathrm{CH}_{3} \mathrm{C}=\mathrm{C}\right), 128.2\left(\mathrm{CH}_{0^{-}}\right.$ $\mathrm{Ar}), 129.9\left(\mathrm{CH}_{m}-\mathrm{Ar}\right), 134.1$ ( $\left.C_{\text {ipso }}-\mathrm{Ar}\right), 148.7\left(\mathrm{CH}_{3} \mathrm{C}=C\right), 145.3\left(C_{p}-\mathrm{Ar}\right), 167.1(C=\mathrm{O})$. LRMS (IC) found $[\mathrm{M}+\mathrm{H}]^{+}=430$.

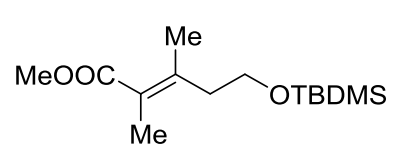

Methyl (E)-5-((tert-butyldimethylsilyl)oxy)-2,3-dimethylpent-2enoate: In a Schlenk, the electrophilic zinc reagent was prepared in situ by diluting $\mathrm{MeMgBr}(4.91 \mathrm{mmol}, 1.6 \mathrm{~mL}, 3 \mathrm{M}$ in diethyl ether) in THF (3.3 mL) and adding $4.9 \mathrm{~mL}$ of a solution of $\mathrm{ZnCl}_{2}(1 \mathrm{M}$ in THF). The resulting suspension was stirred 30 minutes before the addition of CPhos $(0.327 \mathrm{mmol}, 143 \mathrm{mg})$ along with a solution of $\mathrm{Pd}(\mathrm{OAc})_{2}(0.164 \mathrm{mmol}, 37 \mathrm{mg})$ in THF $(2 \mathrm{~mL})$. In another Schlenk tube, methyl (Z)-5-((tert-butyldimethylsilyl)oxy)-2-methyl-3-(tosyloxy)pent-2-enoate (3.27 $\mathrm{mmol}$ ) was dissolved in THF ( $25 \mathrm{~mL})$ and added to the alkylzinc suspension by cannula then stirred at room temperature. After $16 \mathrm{~h}$, the reaction was quenched with a saturated aqueous $\mathrm{NH}_{4} \mathrm{Cl}$ solution $(10 \mathrm{~mL})$, extracted with diethyl ether $(3 \times 30 \mathrm{~mL})$ and dried over $\mathrm{Na}_{2} \mathrm{SO}_{4}{ }^{27}$ The crude oil was purified by flash chromatography (Pentane/Diethyl ether 98:2) to afford the $(E)$-product in $85 \%$ yield $(758 \mathrm{mg})$ as a colourless oil. $(E)$-isomer: $\mathbf{R}_{\mathbf{f}}=0.8$ (Pentane/Diethyl ether = 8:2). IR (neat): $v\left(\mathrm{~cm}^{-1}\right)=2953,2931,2895,2859,1718,1636$, 1465, 1434, 1382, 1283, 1254, 1209, 1089, 1006, 911, 833, 774, 663. 'H-NMR $\left(\mathrm{CDCl}_{3}, 500\right.$ $\mathrm{MHz}): \delta(\mathrm{ppm})=0.04\left(\mathrm{~s}, 6 \mathrm{H},\left(\mathrm{CH}_{3}\right)_{2} \mathrm{Si}\right), 0.88\left(\mathrm{~s}, 9 \mathrm{H},\left(\mathrm{CH}_{3}\right)_{3} \mathrm{CSi}\right), 1.87\left(\mathrm{~s}, 3 \mathrm{H}, \mathrm{CH}_{3} \mathrm{C}=\mathrm{C}\right), 1.98$ $\left(\mathrm{m}, 3 \mathrm{H}, \mathrm{C}=\mathrm{CCH}_{3}\right), 2.39\left(\mathrm{t},{ }^{3} J_{H H}=7.1 \mathrm{~Hz}, 2 \mathrm{H}, \mathrm{CH}_{2} \mathrm{CH}_{2} \mathrm{OTBDMS}\right), 3.68\left(\mathrm{t},{ }^{3} J_{H H}=7.1 \mathrm{~Hz}, 2 \mathrm{H}\right.$, $\left.\mathrm{CH}_{2} \mathrm{CH}_{2} \mathrm{OTBDMS}\right), 3.71$ (s, 3H, $\left.\mathrm{CH}_{3} \mathrm{O}\right) \cdot{ }^{13} \mathrm{C}\left\{{ }^{1} \mathrm{H}\right\}-\mathrm{NMR}\left(\mathrm{CDCl}_{3}, 126 \mathrm{MHz}\right): \delta(\mathrm{ppm})=-5.3$ $\left(\left(\mathrm{CH}_{3}\right)_{2} \mathrm{Si}\right), 15.7 \quad\left(\mathrm{CH}_{3} \mathrm{C}=\mathrm{C}\right), 18.4 \quad\left(\left(\mathrm{CH}_{3}\right)_{3} \mathrm{CSi}\right), 21.7 \quad\left(\mathrm{C}=\mathrm{CCH}_{3}\right), 26.0 \quad\left(\left(\mathrm{CH}_{3}\right)_{3} \mathrm{CSi}\right), \quad 39.6$ $\left(\mathrm{CH}_{2} \mathrm{CH}_{2} \mathrm{OTBDMS}\right), \quad 51.4\left(\mathrm{CH}_{3} \mathrm{O}\right), 61.1 \quad\left(\mathrm{CH}_{2} \mathrm{CH}_{2} \mathrm{OTBDMS}\right), 124.3 \quad\left(\mathrm{CH}_{3} \mathrm{C}=\mathrm{C}\right), 143.4$ $\left(\mathrm{C}=\mathrm{CCH}_{3}\right), 170.3(\mathrm{C}=\mathrm{O})$. LRMS (IC) $(\mathrm{IS})=$ found $[\mathrm{M}+\mathrm{H}]^{+}=273$. 
$\mathrm{MeOOC}$

Methyl (E)-5-hydroxy-2,3-dimethylpent-2-enoate $(4 x)$ : The reaction was run according to GP4 starting from methyl (E)-5-((tertbutyldimethylsilyl)oxy)-2,3-dimethylpent-2-enoate (2.78 mmol). After purification by flash chromatography on silica gel (Pentane/Diethyl ether 1:1), 4x was obtained in $95 \%$ yield $\left(420 \mathrm{mg}\right.$ ) as a colourless oil. $(E)$-isomer: $\mathbf{R}_{\mathbf{f}}=0.3$ (Pentane/Diethyl ether 1:1). IR (neat): $v\left(\mathrm{~cm}^{-1}\right)=3413,2941,1706,1633,1435,1377,1283,1210,1099$, 1037, 991, 873, 773, 532. ${ }^{1} \mathrm{H}-\mathrm{NMR}\left(\mathrm{C}_{6} \mathrm{D}_{6}, 500 \mathrm{MHz}\right): \delta(\mathrm{ppm}) 1.41$ (bs, $\left.1 \mathrm{H}, \mathrm{OH}\right), 1.81(\mathrm{~m}$, $2 \mathrm{H}, \mathrm{CH}_{3} \mathrm{C}=\mathrm{C}$ ), 1.90 (q, $\left.{ }^{3} \mathrm{~J}_{\mathrm{HH}}=1.5 \mathrm{~Hz}, 2 \mathrm{H}, \mathrm{C}=\mathrm{CCH}_{3}\right), 2.36\left(\mathrm{t},{ }^{3} \mathrm{~J}_{\mathrm{HH}}=6.8 \mathrm{~Hz}, 2 \mathrm{H}, \mathrm{CH}_{2} \mathrm{CH}_{2} \mathrm{OH}\right)$, $3.63\left(\mathrm{~s}, 3 \mathrm{H}, \mathrm{CH}_{3} \mathrm{O}\right), 3.65$ (bt, $\left.{ }^{3} \mathrm{~J}_{\mathrm{HH}}=5.9 \mathrm{~Hz}, 2 \mathrm{H}, \mathrm{CH}_{2} \mathrm{CH}_{2} \mathrm{OH}\right) \cdot{ }^{13} \mathrm{C}\left\{{ }^{1} \mathrm{H}\right\}-\mathrm{NMR}\left(\mathrm{C}_{6} \mathrm{D}_{6}, 126 \mathrm{MHz}\right)$ :

$\delta(\mathrm{ppm})=15.7\left(\mathrm{CH}_{3} \mathrm{C}=\mathrm{C}\right), 21.4\left(\mathrm{C}=\mathrm{CCH}_{3}\right), 39.4\left(\mathrm{CH}_{2} \mathrm{CH}_{2} \mathrm{OH}\right), 50.9\left(\mathrm{CH}_{3} \mathrm{O}\right), 60.3$ $\left(\mathrm{CH}_{2} \mathrm{CH}_{2} \mathrm{OH}\right), 124.9\left(\mathrm{CH}_{3} \mathrm{C}=\mathrm{C}\right), 143.5\left(\mathrm{C}=\mathrm{CCH}_{3}\right), 169.5(\mathrm{C}=\mathrm{O})$. GC-MS found $[\mathrm{M}]=158$ (1\%); [M] - $\mathrm{H}_{2} \mathrm{O}=140$ (38\%), [M] - $\mathrm{CH}_{3} \mathrm{OH}=128$ (59\%); [M] $-\mathrm{CH}_{2} \mathrm{CH}_{2} \mathrm{OH}=110$ (15\%); [M] $-3 \mathrm{CH}_{3},-\mathrm{OH}=96$ (87\%), [M] $-\mathrm{CH}_{3} \mathrm{O},-2 \mathrm{CH}_{3},-\mathrm{OH}=79$ (52\%); [M] $-\mathrm{CH}_{3} \mathrm{OOC},-\mathrm{CH}_{3},-$ $\mathrm{OH}=67(100 \%) ;[\mathrm{M}]-\mathrm{CH}_{3} \mathrm{OOC},-2 \mathrm{CH}_{3},-\mathrm{OH}=53(62 \%)$.

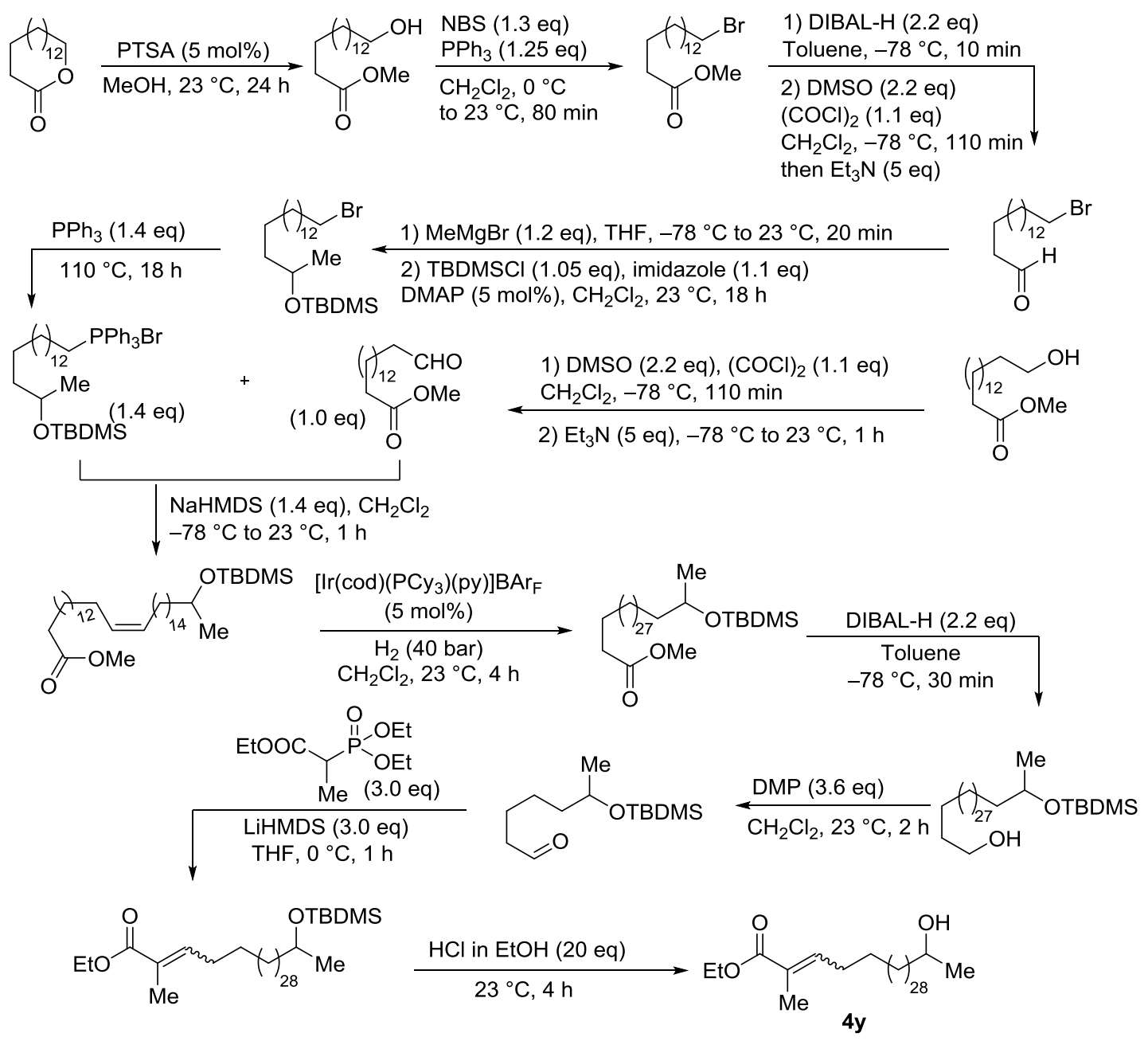

Figure S28: Synthesis of Ethyl 34-hydroxy-2-methylpentatriacont-2-enoate (4y) 


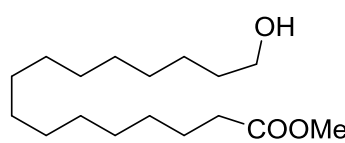

Methyl 16-Hydroxyhexadecanoate: This compound was prepared from the corresponding lactone according to the literature ${ }^{28}$ and used without further purification.

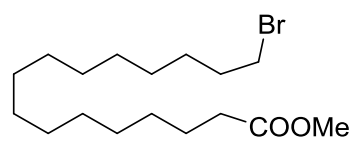

Methyl 16-bromohexadecanoate: Triphenylphosphine $(6.5 \mathrm{~g}, 25$ mmol) was added to a stirred solution of methyl 16hydroxyhexadecanoate $(5.72 \mathrm{~g}, 20 \mathrm{mmol})$ in $\mathrm{CH}_{2} \mathrm{Cl}_{2}(100 \mathrm{~mL})$ before $\mathrm{NaHCO}_{3}(0.14 \mathrm{~g})$ was added. The mixture was cooled to $0^{\circ} \mathrm{C}$ and $\mathrm{N}$-bromosuccinimide (NBS) (4.34 g, $26 \mathrm{mmol}$ ) was added in three portions over 20 minutes. After $1 \mathrm{~h}$ at room temeperature Tlc analysis revealed complete disappearance of the starting material. A saturated aqueous $\mathrm{Na}_{2} \mathrm{~S}_{2} \mathrm{O}_{3}$ solution $(120 \mathrm{~mL}$ ) was added and the organic layer was separated. The aqueous phase was extracted with $\mathrm{CH}_{2} \mathrm{Cl}_{2}(2 \times 30 \mathrm{~mL})$ and the combined organic layers were washed with water $(100 \mathrm{~mL})$, dried over $\mathrm{Na}_{2} \mathrm{SO}_{4}$, and evaporated. The residue was purified by flash chromatography on silica gel (Pentane/Diethyl ether $=10: 1$ ) to give the title compound as a white solid in $93 \%$ yield $(6.5 \mathrm{~g})$. All spectroscopic analyses were in agreement with those reported in the literature. ${ }^{29}$

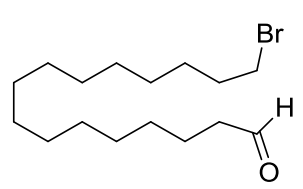

16-bromohexadecanal: To a solution of methyl 16bromohexadecanoate $(3.2 \mathrm{~g}, 10 \mathrm{mmol})$ in toluene $(50 \mathrm{~mL})$ at $-78^{\circ} \mathrm{C}$, diisobutylaluminium hydride (DIBAL-H) $(21 \mathrm{~mL}, 1 \mathrm{M}$ in THF) was added dropwise and stirred for 10 minutes at this temperature before warming up to $0{ }^{\circ} \mathrm{C}$ and stirring for a addtional 30 minutes. The reaction was quenched with a saturated aqueous $\mathrm{NH}_{4} \mathrm{Cl}$ solution $(2 \mathrm{~mL})$ at $0^{\circ} \mathrm{C}$ and diluted with diethyl ether $(100 \mathrm{~mL})$. Stirring was continued for an additional $3 \mathrm{~h}$. The mixture was extracted with diethyl ether $(2 \times 30 \mathrm{~mL})$, dried over anhydrous $\mathrm{Na}_{2} \mathrm{SO}_{4}$ and concentrated in vacuo. The crude mixture was used for the following Swern oxidation (according to GP5). After purification by flash chromatography on silica gel (Pentane/Diethyl ether $=60: 1)$ the product was obtained in $77 \%$ yield $(2.4 \mathrm{~g})$ over two steps. $\mathbf{R}_{\mathbf{f}}=0.7$ (Pentane/Diethyl ether $\left.=10: 1\right)$. IR (neat): $v\left(\mathrm{~cm}^{-1}\right)=2917,2849,1701,1470,1209$, 719. ${ }^{1} \mathbf{H}$ NMR $\left(400 \mathrm{MHz}, \mathrm{CDCl}_{3}\right) \delta(\mathrm{ppm}) 1.17-1.38\left(\mathrm{~m}, 2 \mathrm{H},\left(\mathrm{CH}_{2}\right)_{10}\right), 1.39-1.51(\mathrm{~m}, 2 \mathrm{H}$, $\left.\mathrm{CH}_{2} \mathrm{CH}_{2} \mathrm{CH}_{2} \mathrm{Br}\right), 1.58$ - $1.72\left(\mathrm{~m}, 2 \mathrm{H}, \mathrm{CH}_{2} \mathrm{CH}_{2} \mathrm{CHO}\right), 1.79-1.96\left(\mathrm{~m}, 2 \mathrm{H}, \mathrm{CH}_{2} \mathrm{CH}_{2} \mathrm{CH}_{2} \mathrm{Br}\right), 2.42$ (td, ${ }^{3} J_{\mathrm{HH}}=7.4,1.9 \mathrm{~Hz}, 2 \mathrm{H}, \mathrm{CH}_{2} \mathrm{CH}_{2} \mathrm{CHO}$ ), 3.41 (t, ${ }^{3} \mathrm{~J}_{\mathrm{HH}}=6.9 \mathrm{~Hz}, 2 \mathrm{H}, \mathrm{CH}_{2} \mathrm{CH}_{2} \mathrm{CH}_{2} \mathrm{Br}$ ), 9.76 (t, $\left.{ }^{3} J_{\mathrm{HH}}=1.9 \mathrm{~Hz}, 1 \mathrm{H}, \mathrm{CHO}\right) .{ }^{13} \mathrm{C}\left\{{ }^{1} \mathrm{H}\right\} \mathbf{N M R}\left(101 \mathrm{MHz}, \mathrm{CDCl}_{3}\right) \delta(\mathrm{ppm}) 22.2\left(\mathrm{CH}_{2} \mathrm{CH}_{2} \mathrm{CHO}\right), 28.3$ $\left(\mathrm{CH}_{2} \mathrm{CH}_{2} \mathrm{CH}_{2} \mathrm{Br}\right), 28.9\left(\mathrm{CH}_{2}\right), 29.3\left(\mathrm{CH}_{2}\right), 29.5\left(\mathrm{CH}_{2}\right), 29.6\left(\mathrm{CH}_{2}\right), 29.6\left(\mathrm{CH}_{2}\right), 29.7\left(\mathrm{CH}_{2}\right), 29.7$ $\left(\mathrm{CH}_{2}\right), 29.7\left(\mathrm{CH}_{2}\right), 29.8\left(\mathrm{CH}_{2}\right)$, $33.0\left(\mathrm{CH}_{2} \mathrm{CH}_{2} \mathrm{CH}_{2} \mathrm{Br}\right), 34.2\left(\mathrm{CH}_{2} \mathrm{CH}_{2} \mathrm{CH}_{2} \mathrm{Br}\right), 44.1\left(\mathrm{CH}_{2} \mathrm{CHO}\right)$, $203.1(\mathrm{CHO})$. LRMS (IC) found $\left[\mathrm{M}+\mathrm{NH}_{4}\right]^{+}=336$. 


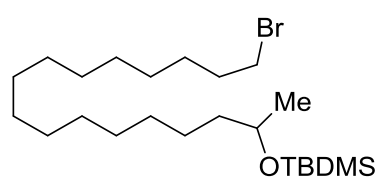

((17-bromoheptadecan-2-yl)oxy)(tert-butyl)dimethylsilane: To a solution of 16-bromohexadecanal $(1.7 \mathrm{~g}, 5.3 \mathrm{mmol})$ in THF $(75 \mathrm{~mL})$,

$\operatorname{MeMgBr}(2.65 \mathrm{~mL}, 7.95 \mathrm{mmol}, 3 \mathrm{M}$ in THF) was added dropwise at $-78{ }^{\circ} \mathrm{C}$. The mixture was warmed up to room temperature and stirred for 20 minutes then it was quenched with aqueous $\mathrm{HCl}(1 \mathrm{~N}, 8 \mathrm{~mL})$ at $0{ }^{\circ} \mathrm{C}$ and stirred for 30 minutes. The mixture was dried over anhydrous $\mathrm{Na}_{2} \mathrm{SO}_{4}$ then filtrated through a pad of silica gel and flushed with diethyl ether $(50 \mathrm{~mL})$. The volatiles were evaporated in vacuo to give $1.7 \mathrm{~g}$ of crude product, which was redissolved in $\mathrm{CH}_{2} \mathrm{Cl}_{2}(40 \mathrm{~mL})$ at $0{ }^{\circ} \mathrm{C}$. Imidazole $(0.42 \mathrm{~g}, 5.5 \mathrm{mmol})$ and DMAP (70 mg, 4 mol\%) were added and stirred for 10 minutes. TBDMSCl (791 mg, $4.72 \mathrm{mmol}$ ) was added and stirred overnight at room temperature then quenched with saturated aqueous $\mathrm{NH}_{4} \mathrm{Cl}$ solution and extracted with $\mathrm{CH}_{2} \mathrm{Cl}_{2}(3 \times 40 \mathrm{~mL})$. The combined organic phases were dried over $\mathrm{Na}_{2} \mathrm{SO}_{4}$ and concentrated in vacuo. The crude mixture was purified by flash chromatography (Pentane/Diethyl ether $=40: 1$ ) to give the product in $88 \%$ yield $(2.1 \mathrm{~g})$ over two steps. $\mathbf{R}_{\mathbf{f}}=0.9$ (Pentane/Diethyl ether $=10: 1$ ). IR (neat): $v\left(\mathrm{~cm}^{-1}\right)=2926,2855,2349$, 1464, 1373, 1253, 1061, 835, 774, 526. ${ }^{1} \mathrm{H}$ NMR (400 MHz, CDCl $)_{3} \delta$ (ppm) 0.04 (s, 6H, $\left.\left(\mathrm{CH}_{3}\right)_{2} \mathrm{Si}\right), 0.88\left(\mathrm{~s}, 9 \mathrm{H},\left(\mathrm{CH}_{3}\right)_{3} \mathrm{CSi}\right), 1.11\left(\mathrm{~d},{ }^{3} \mathrm{~J}_{\mathrm{HH}}=6.0 \mathrm{~Hz}, 3 \mathrm{H}, \mathrm{CH}_{3} \mathrm{CHOTBDMS}\right), 1.23-1.46$ $\left(\mathrm{m}, 26 \mathrm{H},\left(\mathrm{CH}_{2}\right)_{13}\right), 1.94-1.78\left(\mathrm{~m}, 2 \mathrm{H}, \mathrm{CH}_{2} \mathrm{CH}_{2} \mathrm{Br}\right), 3.41\left(\mathrm{t},{ }^{3} \mathrm{~J}_{\mathrm{HH}}=6.9 \mathrm{~Hz}, 2 \mathrm{H}, \mathrm{CH}_{2} \mathrm{CH}_{2} \mathrm{Br}\right)$, $3.66-3.85$ (m, 1H, CH $\left.{ }_{3} \mathrm{CHOTBDMS}\right) .{ }^{13} \mathrm{C}\left\{{ }^{1} \mathrm{H}\right\}$ NMR $\left(101 \mathrm{MHz}, \mathrm{CDCl}_{3}\right) \delta(\mathrm{ppm})-4.5$ $\left(\left(\mathrm{CH}_{3}\right)_{2} \mathrm{Si}\right), \quad-4.2\left(\left(\mathrm{CH}_{3}\right)_{2} \mathrm{Si}\right), 18.3\left(\left(\mathrm{CH}_{3}\right)_{3} \mathrm{CSi}\right), 24.0\left(\mathrm{CH}_{3} \mathrm{CHOTBDMS}\right), 26.0\left(\mathrm{CH}_{2}\right), 26.1$ ((CH$\left.)_{3} \mathrm{CSi}\right), 28.3\left(\mathrm{CH}_{2}\right), 28.9\left(\mathrm{CH}_{2}\right), 29.6\left(\mathrm{CH}_{2}\right), 29.7\left(\mathrm{CH}_{2}\right), 29.8\left(\mathrm{CH}_{2}\right), 29.8\left(\mathrm{CH}_{2}\right), 29.8$ $\left(\mathrm{CH}_{2}\right), 29.8\left(\mathrm{CH}_{2}\right), 33.0\left(\mathrm{CH}_{2} \mathrm{CH}_{2} \mathrm{Br}\right), 34.2\left(\mathrm{CH}_{2} \mathrm{CH}_{2} \mathrm{Br}\right), 39.9\left(\mathrm{CH}_{2} \mathrm{CHOTBDMS}\right), 68.8$ $\left(\mathrm{CH}_{2} \mathrm{CHOTBDMS}\right)$. LRMS (IC) found $\left[\mathrm{M}+\mathrm{NH}_{4}\right]^{+}=468$.

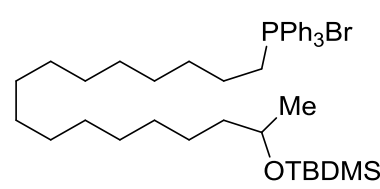

Bromo(16-((tert-butyldimethylsilyl)oxy)heptadecyl)triphenyl-I5phosphane: In a round-bottom flask, triphenylphosphine $(1.68 \mathrm{~g}$, $6.44 \mathrm{mmol})$ and ((17-bromoheptadecan-2-yl)oxy)(tertbutyl)dimethylsilane $(2.1 \mathrm{~g}, 4.6 \mathrm{mmol})$ were added. The reaction mixture was heated at $110{ }^{\circ} \mathrm{C}$ and monitored by ${ }^{1} \mathrm{H}$ NMR until the starting material was consumed completely (ca. $24 \mathrm{~h}$ ). The resulting oil was engaged in the next step without further purification.

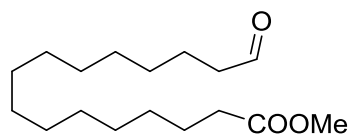

Methyl 16-oxohexadecanoate: This compound was prepared according to a literature procedure, ${ }^{29}$ starting from methyl 16 hydroxyhexadecanoate $(1.43 \mathrm{~g}, 5 \mathrm{mmol})$. The crude mixture was filtered through silica gel affording the product in $91 \%$ yield $(1.3 \mathrm{~g})$. All spectroscopic analyses were in agreement with those reported in the literature. ${ }^{30}$ 


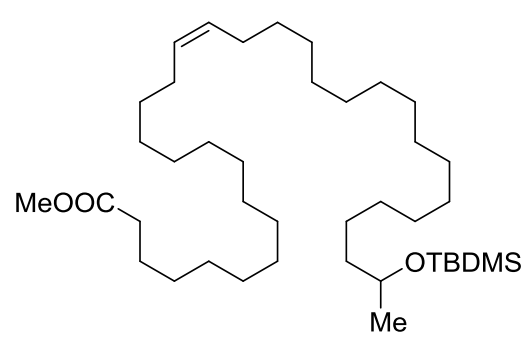

Methyl (Z)-32-((tert-butyldimethylsilyl)oxy)tritriacont-16enoate:

Bromo-(16-((tert-

butyldimethylsilyl)oxy)heptadecyl)triphenyl-I5-phosphane ( $3.5 \mathrm{mmol}$ ) was dissolved in $\mathrm{CH}_{2} \mathrm{Cl}_{2}(20 \mathrm{~mL})$ and sodium hexamethydisilazide (NaHMDS, $1.75 \mathrm{~mL}$ from a $2 \mathrm{M}$ sol. in THF) was added at $0{ }^{\circ} \mathrm{C}$ and stirred for 15 minutes. Methyl 16-oxohexadecanoate in $\mathrm{CH}_{2} \mathrm{Cl}_{2}(10 \mathrm{~mL})$ was added dropwise at $-78{ }^{\circ} \mathrm{C}$ and stirred at room temperature for $1 \mathrm{~h}$. The reaction was quenched with a saturated aqueous $\mathrm{NH}_{4} \mathrm{Cl}$ solution $(10 \mathrm{~mL})$, extracted with $\mathrm{CH}_{2} \mathrm{Cl}_{2}(2 \times 20 \mathrm{~mL})$, dried over anhydrous $\mathrm{Na}_{2} \mathrm{SO}_{4}$ and concentrated in vacuo. The crude mixture was purified by flash chromatography (Pentane/Diethyl ether = $30: 1)$ to give the title product as a colourless oil in $36 \%$ yield $\left(570 \mathrm{mg}\right.$ ). $\mathbf{R}_{\mathbf{f}}=0.6$ (Pentane/Diethyl ether = 10:1). IR (neat): $v\left(\mathrm{~cm}^{-1}\right)=2925,2854,1744,1463,1372,835,774$. ${ }^{1} \mathrm{H}$ NMR $\left(400 \mathrm{MHz}, \mathrm{CDCl}_{3}\right) \delta(\mathrm{ppm}) 0.04\left(\mathrm{~s}, 6 \mathrm{H},\left(\mathrm{CH}_{3}\right)_{2} \mathrm{Si}\right), 0.88\left(\mathrm{~s}, 9 \mathrm{H},\left(\mathrm{CH}_{3}\right)_{3} \mathrm{CSi}\right), 1.11$ (d, $\left.{ }^{3} J_{H H}=6.0 \mathrm{~Hz}, 3 \mathrm{H}, \mathrm{CH}_{3} \mathrm{CHOTBDMS}\right), 1.16-1.49\left(\mathrm{~m}, 50 \mathrm{H}, 25 \times \mathrm{CH}_{2}\right), 1.56-1.68(\mathrm{~m}, 2 \mathrm{H}$, $\left.\mathrm{CH}_{2} \mathrm{CH}_{2} \mathrm{COOMe}\right), 1.90-2.08\left(\mathrm{~m}, 4 \mathrm{H}, \mathrm{CH}_{2} \mathrm{CH}=\mathrm{CHCH}_{2}\right), 2.30\left(\mathrm{td},{ }^{3} J_{\mathrm{HH}}=7.6,{ }^{2} J_{\mathrm{HH}}=1.1 \mathrm{~Hz}\right.$, $2 \mathrm{H}, \mathrm{CH}_{2} \mathrm{CH}_{2} \mathrm{COOMe}$ ), 3.66 (s, 3H, $\mathrm{CH}_{3} \mathrm{O}$ ), 3.72 - 3.83 (m, 1H, $\mathrm{CH}_{2} \mathrm{CHOTBDMS}$ ), 5.34 (td, $\left.{ }^{3} J_{\mathrm{HH}}=4.5,2.4 \mathrm{~Hz}, 2 \mathrm{H}, \mathrm{CH}=\mathrm{CH}\right) \cdot{ }^{13} \mathrm{C}\left\{{ }^{1} \mathrm{H}\right\} \operatorname{NMR}\left(101 \mathrm{MHz}, \mathrm{CDCl}_{3}\right) \delta(\mathrm{ppm})-4.5\left(\left(\mathrm{CH}_{3}\right)_{2} \mathrm{Si}\right),-$ $4.2\left(\left(\mathrm{CH}_{3}\right)_{2} \mathrm{Si}\right), 18.3\left(\left(\mathrm{CH}_{3}\right)_{3} \mathrm{CSi}\right), 24.0\left(\mathrm{CH}_{3} \mathrm{CHOTBDMS}\right), 25.1\left(\mathrm{CH}_{2} \mathrm{CH}_{2} \mathrm{COOMe}\right), 26.0$ $\left(\mathrm{CH}_{2}\right), \quad 26.1\left(\left(\mathrm{CH}_{3}\right)_{3} \mathrm{Csi}\right), \quad 27.4\left(\mathrm{CH}_{2} \mathrm{CH}=\mathrm{CHCH}_{2}\right), \quad 29.3-29.9\left(23 \times \mathrm{CH}_{2}\right), \quad 34.3$ $\left(\mathrm{CH}_{2} \mathrm{CH}_{2} \mathrm{COOMe}\right), 39.9\left(\mathrm{CH}_{2} \mathrm{CHOTBDMS}\right), 51.6\left(\mathrm{CH}_{3} \mathrm{O}\right), 68.8\left(\mathrm{CH}_{2} \mathrm{CHOTBDMS}\right), 130.0$ $(\mathrm{CH}=\mathrm{CH}), 174.5(\mathrm{C}=\mathrm{O})$. LRMS (IC) found $\left[\mathrm{M}+\mathrm{NH}_{4}\right]^{+}=655$.

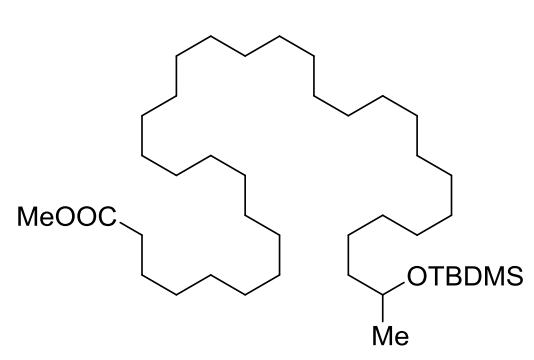

After $4 \mathrm{~h}$ stirring, the autoclave was carefully opened to release $\mathrm{H}_{2}$ and the mixture was diluted with $\mathrm{CH}_{2} \mathrm{Cl}_{2}(10 \mathrm{~mL})$, quenched with water $(15 \mathrm{~mL})$, extracted with $\mathrm{CH}_{2} \mathrm{Cl}_{2}(3 \times 10$ $\mathrm{mL}$ ), dried over anhydrous $\mathrm{Na}_{2} \mathrm{SO}_{4}$ and concentrated in vacuo to give the desired product in $92 \%$ yield $\left(458 \mathrm{mg}\right.$ ) as a white wax. $\mathbf{R}_{\mathbf{f}}=0.6$ (Pentane/Diethyl ether $\left.=10: 1\right)$. IR (neat): $v\left(\mathrm{~cm}^{-}\right.$ $\left.{ }^{1}\right)=2918,2850,1743,1462,1372,732 .{ }^{1} \mathbf{H}$ NMR (400 MHz, CDCl $\left.{ }_{3}\right) \delta$ (ppm) 0.04 (s, 6H, $\left.\left(\mathrm{CH}_{3}\right)_{2} \mathrm{Si}\right), 0.88\left(\mathrm{~s}, 9 \mathrm{H},\left(\mathrm{CH}_{3}\right)_{3} \mathrm{CSi}\right), 1.11\left(\mathrm{~d},{ }^{3} \mathrm{~J}_{\mathrm{HH}}=6.1 \mathrm{~Hz}, 3 \mathrm{H}, \mathrm{CH}_{3} \mathrm{CHOTBDMS}\right), 1.23-1.41$ $\left(\mathrm{m}, 56 \mathrm{H},\left(\mathrm{CH}_{2}\right)_{28}\right), 1.62\left(\mathrm{t},{ }^{3} \mathrm{~J}_{\mathrm{HH}}=7.3 \mathrm{~Hz}, 2 \mathrm{H}, \mathrm{CH}_{2} \mathrm{CH}_{2} \mathrm{COOMe}\right), 2.30\left(\mathrm{t},{ }^{3} \mathrm{~J}_{\mathrm{HH}}=7.6 \mathrm{~Hz}, 2 \mathrm{H}\right.$, $\mathrm{CH}_{2} \mathrm{CH}_{2} \mathrm{COOMe}$ ), 3.66 (s, 3H, $\mathrm{CH}_{3} \mathrm{O}$ ), $3.70-3.81$ (m, $\left.1 \mathrm{H}, \mathrm{CH}_{2} \mathrm{CHOTBDMS}\right) .{ }^{13} \mathrm{C}\left\{{ }^{1} \mathrm{H}\right\}$ NMR 


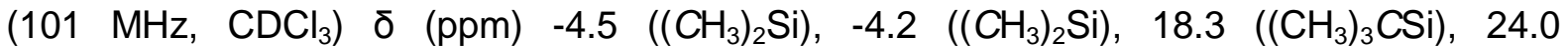
( $\left.\mathrm{CH}_{3} \mathrm{CHOTBDMS}\right), 25.1\left(\mathrm{CH}_{2} \mathrm{CH}_{2} \mathrm{COOMe}\right), 26.0\left(\mathrm{CH}_{2}\right), 26.1\left(\left(\mathrm{CH}_{3}\right)_{3} \mathrm{Csi}\right), 29.3-29.8(27 \times$ $\begin{array}{lllllll}\left.\mathrm{CH}_{2}\right), & 34.3 & \left(\mathrm{CH}_{2} \mathrm{CH}_{2} \mathrm{COOMe}\right), \quad 40.0 \quad\left(\mathrm{CH}_{2} \mathrm{CHOTBDMS}\right), \quad 51.6 \quad\left(\mathrm{CH}_{3} \mathrm{O}\right), \quad 68.8\end{array}$ $\left(\mathrm{CH}_{2} \mathrm{CHOTBDMS}\right), 174.5(\mathrm{C}=\mathrm{O})$. LRMS (IC) found $[\mathrm{M}+\mathrm{H}]^{+}=640$.

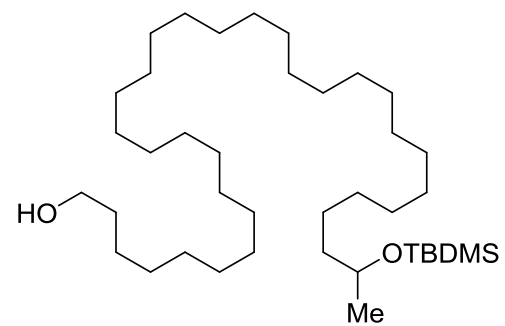

32-((tert-butyldimethylsilyl)oxy)tritriacontan-1-ol: To a solution of methyl 32-((tertbutyldimethylsilyl)oxy)tritriacontanoate (458 mg, $0.72 \mathrm{mmol}$ ) in toluene $(2.5 \mathrm{~mL})$ at $-78^{\circ} \mathrm{C}$, DIBAL-H $(1.6 \mathrm{~mL}, 1 \mathrm{M}$ in THF) was added dropwise and stirred for 10 minutes. The mixture was warmed to $0{ }^{\circ} \mathrm{C}$ and stirred for 30 minutes, then quenched with saturated aqueous $\mathrm{NH}_{4} \mathrm{Cl}$ solution $(0.5 \mathrm{~mL})$ at the same temperature. The mixture was diluted with diethyl ether $(10 \mathrm{~mL})$, stirred for $3 \mathrm{~h}$ and then dried by adding anhydrous $\mathrm{Na}_{2} \mathrm{SO}_{4}$. After $1 \mathrm{~h}, \mathrm{Na}_{2} \mathrm{SO}_{4}$ was removed by filtration, washed with diethyl ether (3 $\times 10 \mathrm{~mL}$ ) and the organic phase concentrated in vacuo to give the title compound in $93 \%$ yield $(410 \mathrm{mg})$. It was used for next step without further purification.

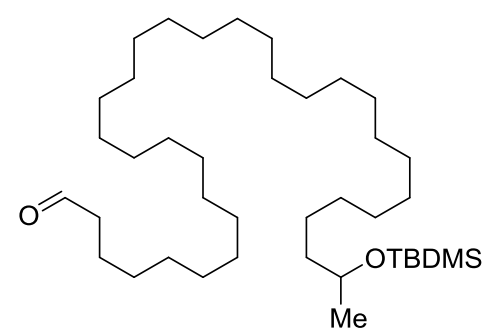

32-((tert-butyldimethylsilyl)oxy)tritriacontanal: To a solution of Dess-Martin periodinane (DMP, $0.57 \mathrm{mmol}, 240$ $\mathrm{mg})$ in $\quad \mathrm{CH}_{2} \mathrm{Cl}_{2} \quad(5 \quad \mathrm{~mL}), \quad 32-(($ tertbutyldimethylsilyl)oxy)tritriacontan-1-ol $(0.47 \mathrm{mmol}, 288 \mathrm{mg})$ in $\mathrm{CH}_{2} \mathrm{Cl}_{2}(3 \mathrm{~mL})$ was added dropwise at $0{ }^{\circ} \mathrm{C}$. After 40 minutes at room temperature, another portion of DMP $(0.57$ $\mathrm{mmol}, 240 \mathrm{mg}$ ) was added and stirred 40 minutes. This sequence was repeated one more time. The mixture was diluted with $\mathrm{CH}_{2} \mathrm{Cl}_{2}(15 \mathrm{~mL})$ and a solution of $\mathrm{K}_{2} \mathrm{CO}_{3}(1.5 \mathrm{~g})$ in water $(10 \mathrm{~mL})$ was added. The biphasic system was stirred for 20 minutes. The aqueous phase was extracted with $\mathrm{CH}_{2} \mathrm{Cl}_{2}(2 \times 3 \mathrm{~mL})$ and the combined organic phases were dried over anhydrous $\mathrm{Na}_{2} \mathrm{SO}_{4}$ and evaporated in vacuo. The residue was purified by flash chromatography on silica gel (Pentane/Diethyl ether $=10: 1$ ) to afford the title compound in $70 \%$ yield $\left(200 \mathrm{mg}\right.$ ) as a colourless oil. $\mathbf{R}_{\mathbf{f}}=0.8$ (Pentane/Diethyl ether $\left.=10: 1\right)$. IR (neat): $v\left(\mathrm{~cm}^{-1}\right)=2917,2850,1725,1463,1353,1135,835 .{ }^{1} \mathbf{H}$ NMR $\left(400 \mathrm{MHz}, \mathrm{CDCl}_{3}\right) \delta(\mathrm{ppm})$ 0.04 (s, 6H, $\left.\left(\mathrm{CH}_{3}\right)_{2} \mathrm{Si}\right), 0.88$ (s, 9H, $\left.\left(\mathrm{CH}_{3}\right)_{3} \mathrm{Csi}\right), 1.11$ (d, $\left.{ }^{3} \mathrm{~J}_{\mathrm{HH}}=6.0 \mathrm{~Hz}, 3 \mathrm{H}, \mathrm{CH}_{3} \mathrm{CHOTBDMS}\right)$, $1.18-1.43\left(\mathrm{~m}, 56 \mathrm{H},\left(\mathrm{CH}_{2}\right)_{28}\right), 1.58-1.70\left(\mathrm{~m}, 2 \mathrm{H}, \mathrm{CH}_{2} \mathrm{CH}_{2} \mathrm{CHO}\right), 2.42\left(\mathrm{td},{ }^{3} \mathrm{~J}_{\mathrm{HH}}=7.4,1.9 \mathrm{~Hz}\right.$, $2 \mathrm{H}, \mathrm{CH}_{2} \mathrm{CH}_{2} \mathrm{CHO}$ ), $3.69-3.83\left(\mathrm{~m}, 1 \mathrm{H}, \mathrm{CH}_{3} \mathrm{CHOTBDMS}\right), 9.76\left(\mathrm{t},{ }^{3} J_{\mathrm{HH}}=1.9 \mathrm{~Hz}, 1 \mathrm{H}, \mathrm{CHO}\right)$. ${ }^{13} \mathrm{C}\left\{{ }^{1} \mathrm{H}\right\}$ NMR $\left(101 \mathrm{MHz}, \mathrm{CDCl}_{3}\right) \delta(\mathrm{ppm})-4.5\left(\left(\mathrm{CH}_{3}\right)_{2} \mathrm{Si}\right),-4.2\left(\left(\mathrm{CH}_{3}\right)_{2} \mathrm{Si}\right), 18.3\left(\left(\mathrm{CH}_{3}\right)_{3} \mathrm{CSi}\right)$, $22.2\left(\mathrm{CH}_{2} \mathrm{CH}_{2} \mathrm{CHO}\right), 24.0\left(\mathrm{CH}_{3} \mathrm{CHOTBDMS}\right), 26.0\left(\mathrm{CH}_{2}\right), 26.1\left(\left(\mathrm{CH}_{3}\right)_{3} \mathrm{CSi}\right), 29.3\left(\mathrm{CH}_{2}\right), 29.5$ 
$\left(\mathrm{CH}_{2}\right), 29.6\left(\mathrm{CH}_{2}\right), 29.7\left(\mathrm{CH}_{2}\right), 29.8-29.9\left(\mathrm{CH}_{2} \times 22\right), 39.9\left(\mathrm{CH}_{2} \mathrm{CHOTBDMS}\right), 44.1$ $\left(\mathrm{CH}_{2} \mathrm{CH}_{2} \mathrm{CHO}\right), 68.8\left(\mathrm{CH}_{2} \mathrm{CHOTBDMS}\right), 203.1$ (CHO). LRMS (IC) found $[\mathrm{M}+\mathrm{H}]^{+}=610$.

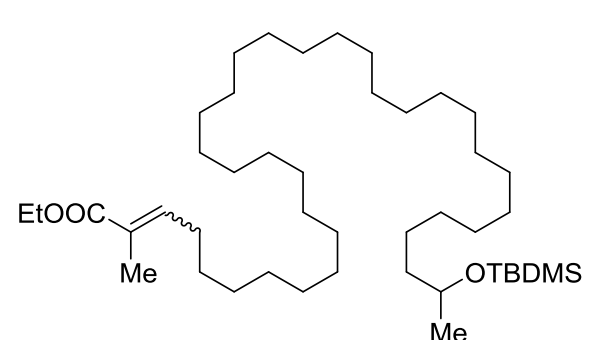

Ethyl methylpentatriacont-2-enoate:

hexamethyldisilazide (LiHMDS, $1 \mathrm{mmol}, 1 \mathrm{~mL}, 1.0 \mathrm{M}$ in THF) was added dropwise to a solution of triethylphosphopropionate $(236 \mathrm{mg}, 0.99 \mathrm{mmol})$ in THF $(3 \mathrm{~mL})$ at $0{ }^{\circ} \mathrm{C}$. After 15 minutes, 32-((tertbutyldimethylsilyl)oxy)tritriacontanal (200 mg, $0.33 \mathrm{mmol}$ ) in THF (2 mL) was added dropwise. After $1 \mathrm{~h}$, the reaction was quenched with a saturated aqueous $\mathrm{NH}_{4} \mathrm{Cl}$ solution $(0.5 \mathrm{~mL})$, washed with water $(15 \mathrm{~mL})$, extracted with diethyl ether $(3 \times 10 \mathrm{~mL})$, dried over $\mathrm{Na}_{2} \mathrm{SO}_{4}$ and then concentrated in vacuo. The residue was purified by flash chromatography (Hexane/Diethyl ether $=10: 1$ ) to afford the title compound in $87 \%$ yield $(200 \mathrm{mg}, E / Z=2.3: 1$ ) as a colourless wax. $\mathbf{R}_{\mathbf{f}}=0.6$ (Pentane/Diethyl ether $=10: 1$ ). IR (neat): $v\left(\mathrm{~cm}^{-1}\right)=2919$, 2850, 1717, 1650, 1469, 1371, 1254, 835. (E)-isomer: ${ }^{1} \mathbf{H}$ NMR $\left(400 \mathrm{MHz}, \mathrm{CDCl}_{3}\right) \delta$ (ppm) 0.04 (s, 6H, $\left.\left(\mathrm{CH}_{3}\right)_{2} \mathrm{Si}\right), 0.88$ (s, 9H, $\left.\left(\mathrm{CH}_{3}\right)_{3} \mathrm{CSi}\right), 1.11$ (d, $\left.{ }^{3} \mathrm{~J}_{\mathrm{HH}}=6.1 \mathrm{~Hz}, 3 \mathrm{H}, \mathrm{CH}_{3} \mathrm{CHOTBDMS}\right)$, $1.24-1.41\left(\mathrm{~m}, 3 \mathrm{H}, \mathrm{CH}_{3} \mathrm{CH}_{2} \mathrm{O} ; 56 \mathrm{H},\left(\mathrm{CH}_{2}\right)_{28}\right), 1.41-1.47\left(\mathrm{~m}, 2 \mathrm{H}, \mathrm{CH}_{2}\right), 1.80-1.84(\mathrm{~m}, 3 \mathrm{H}$, $\mathrm{CH}_{3} \mathrm{C}=\mathrm{CH}$ ), $2.12-2.20\left(\mathrm{~m}, 2 \mathrm{H}, \mathrm{C}=\mathrm{CHCH}_{2}\right.$ ), $3.69-3.84$ (m, 1H, $\left.\mathrm{CH}_{3} \mathrm{CHOTBDMS}\right), 4.19$ (q, $\left.{ }^{3} J_{\mathrm{HH}}=7.1 \mathrm{~Hz}, 2 \mathrm{H}, \mathrm{CH}_{3} \mathrm{CH} \mathrm{O}\right), 6.76\left(\mathrm{tq},{ }^{3} \mathrm{~J}_{\mathrm{HH}}=7.5,{ }^{4} \mathrm{~J}_{\mathrm{HH}}=1.4 \mathrm{~Hz}, 1 \mathrm{H}, \mathrm{C}=\mathrm{CH}\right) .{ }^{13} \mathrm{C}\left\{{ }^{1} \mathrm{H}\right\} \mathbf{N M R}$ $\left(101 \mathrm{MHz}, \mathrm{CDCl}_{3}\right) \quad \delta(\mathrm{ppm})-4.5 \quad\left(\left(\mathrm{CH}_{3}\right)_{2} \mathrm{Si}\right),-4.2 \quad\left(\left(\mathrm{CH}_{3}\right)_{2} \mathrm{Si}\right), 12.5 \quad\left(\mathrm{CH}_{3} \mathrm{C}=\mathrm{CH}\right), 14.5$ $\left(\mathrm{CH}_{3} \mathrm{CH}_{2} \mathrm{O}\right), 18.3\left(\left(\mathrm{CH}_{3}\right)_{3} \mathrm{C}=\mathrm{CH}\right), 24.0\left(\mathrm{CH}_{3} \mathrm{CHOTBDMS}\right), 26.0\left(\mathrm{CH}_{2}\right), 26.1\left(\left(\mathrm{CH}_{3}\right)_{3} \mathrm{CSi}\right), 28.7$ - $29.9\left(28 \times \mathrm{CH}_{2}\right), 39.9\left(\mathrm{CH}_{2} \mathrm{CHOTBDMS}\right), 60.5\left(\mathrm{CH}_{3} \mathrm{CH}_{2} \mathrm{O}\right), 68.8\left(\mathrm{CH}_{3} \mathrm{CHOTBDMS}\right), 127.8$ $(C=\mathrm{CH}), 142.6(\mathrm{C}=\mathrm{CH}), 168.51(\mathrm{C}=\mathrm{O}) .(Z)$-isomer: ${ }^{1} \mathrm{H}$ NMR $\left(400 \mathrm{MHz}, \mathrm{CDCl}_{3}\right) \delta(\mathrm{ppm}) 0.04$ (s, 6H, $\left.\left(\mathrm{CH}_{3}\right)_{2} \mathrm{Si}\right), 0.88\left(\mathrm{~s}, 9 \mathrm{H},\left(\mathrm{CH}_{3}\right)_{3} \mathrm{CSi}\right), 1.11\left(\mathrm{~d},{ }^{3} \mathrm{~J}_{\mathrm{HH}}=6.1 \mathrm{~Hz}, 3 \mathrm{H}, \mathrm{CH}_{3} \mathrm{CHOTBDMS}\right), 1.24$ - $1.41\left(\mathrm{~m}, 3 \mathrm{H}, \mathrm{CH}_{3} \mathrm{CH}_{2} \mathrm{O} ; 56 \mathrm{H},\left(\mathrm{CH}_{2}\right)_{28}\right), 1.41-1.47\left(\mathrm{~m}, 2 \mathrm{H}, \mathrm{CH}_{2}\right), 1.88-1.91(\mathrm{~m}, 3 \mathrm{H}$, $\mathrm{CH}_{3} \mathrm{C}=\mathrm{CH}$ ), $2.39-2.50$ (m, 2H, C=CHCH${ }_{2}$ ), $3.69-3.84$ (m, 1H, $\left.\mathrm{CH}_{3} \mathrm{CHOTBDMS}\right), 4.19$ (q, $\left.{ }^{3} J_{\mathrm{HH}}=7.1 \mathrm{~Hz}, 2 \mathrm{H}, \mathrm{CH}_{3} \mathrm{CH}_{2} \mathrm{O}\right), 5.92\left(\mathrm{tq},{ }^{3} \mathrm{~J}_{\mathrm{HH}}=7.6,{ }^{4} \mathrm{~J}_{\mathrm{HH}}=1.6 \mathrm{~Hz}, 1 \mathrm{H}, \mathrm{C}=\mathrm{CH}\right) .{ }^{13} \mathrm{C}\left\{{ }^{1} \mathrm{H}\right\} \mathbf{N M R}$ $\left(101 \mathrm{MHz}, \mathrm{CDCl}_{3}\right) \quad \delta(\mathrm{ppm})-4.5 \quad\left(\left(\mathrm{CH}_{3}\right)_{2} \mathrm{Si}\right),-4.2 \quad\left(\left(\mathrm{CH}_{3}\right)_{2} \mathrm{Si}\right), 14.5 \quad\left(\mathrm{CH}_{3} \mathrm{CH}_{2} \mathrm{O}\right), 18.3$ $\left(\left(\mathrm{CH}_{3}\right)_{3} \mathrm{CSi}\right), 20.8\left(\mathrm{CH}_{3} \mathrm{C}=\mathrm{CH}\right), 24.0\left(\mathrm{CH}_{3} \mathrm{CHOTBDMS}\right), 26.0\left(\mathrm{CH}_{2}\right), 26.1\left(\left(\mathrm{CH}_{3}\right)_{3} \mathrm{CSi}\right), 28.7-$ $29.8\left(28 \times \mathrm{CH}_{2}\right), 39.9\left(\mathrm{CH}_{2} \mathrm{CHOTBDMS}\right), 60.1\left(\mathrm{CH}_{3} \mathrm{CH}_{2} \mathrm{O}\right), 68.8\left(\mathrm{CH}_{3} \mathrm{CHOTBDMS}\right), 128.6$ $(C=\mathrm{CH}), 143.3(\mathrm{C}=\mathrm{CH}), 168.51(\mathrm{C}=\mathrm{O})$. LRMS $(\mathrm{IC})$ found $[\mathrm{M}+\mathrm{H}]^{+}=694$. 


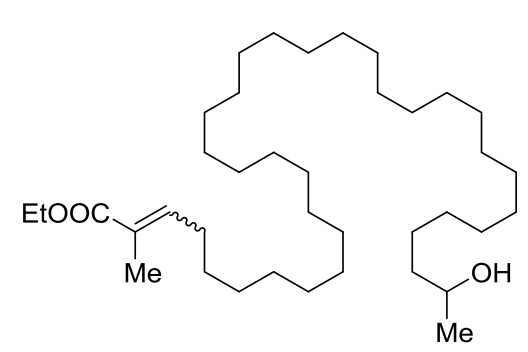

Ethyl 34-hydroxy-2-methylpentatriacont-2-enoate (4y): Ethyl 34-((tert-butyldimethylsilyl)oxy)-2-methylpentatriacont2-enoate $(270 \mathrm{mg}, 0.39 \mathrm{mmol})$ was added to a solution of $\mathrm{HCl}$ in ethanol $(1.25 \mathrm{M}, 6.24 \mathrm{~mL}, 7.8 \mathrm{mmol})$ at room temperature. After stirring for $3 \mathrm{~h}$, the reaction was diluted with water $(15 \mathrm{~mL})$, extracted with diethyl ether $(3 \times 30 \mathrm{~mL})$, dried over anhydrous $\mathrm{Na}_{2} \mathrm{SO}_{4}$ and concentrated in vacuo. The residue was purified by flash chromatography on silica gel (Pentane/Diethyl ether $=2: 1$ ) to afford $\mathbf{4 y}$ in $75 \%$ yield (170 $\mathrm{mg}, E / Z=2.9: 1$ ) as white wax. $\mathbf{R}_{\mathbf{f}}=0.3$ (Pentane/Diethyl ether $\left.=2: 1\right)$. IR (neat): $v\left(\mathrm{~cm}^{-1}\right)=$ 2916, 2849, 1710, 1648, 1462, 1370, 728. (E)-isomer: ${ }^{1} \mathbf{H}$ NMR $\left(400 \mathrm{MHz}, \mathrm{CDCl}_{3}\right) \delta$ (ppm) $1.19\left(\mathrm{~d},{ }^{3} \mathrm{~J}_{\mathrm{HH}}=6.1 \mathrm{~Hz}, 3 \mathrm{H}, \mathrm{CH}_{3} \mathrm{CHOH}\right), 1.24-1.49\left(\mathrm{~m}, 3 \mathrm{H}, \mathrm{CH}_{3} \mathrm{CH}_{2} \mathrm{O} ; 58 \mathrm{H},\left(\mathrm{CH}_{2}\right)_{29} ; 1 \mathrm{H}\right.$, $\mathrm{OH}), 1.79-1.85\left(\mathrm{~m}, 3 \mathrm{H}, \mathrm{CH}_{3} \mathrm{C}=\mathrm{CH}\right), 2.10-2.22\left(\mathrm{~m}, 2 \mathrm{H}, \mathrm{C}=\mathrm{CHCH}_{2}\right), 3.74-3.85(\mathrm{~m}, 1 \mathrm{H}$, $\mathrm{CH}_{3} \mathrm{CHOH}$ ), 4.19 (q, ${ }^{3} \mathrm{~J}_{\mathrm{HH}}=7.1 \mathrm{~Hz}, 2 \mathrm{H}, \mathrm{CH}_{3} \mathrm{CH}_{2} \mathrm{O}$ ), 6.76 (tq, ${ }^{3} J_{\mathrm{HH}}=7.5,{ }^{4} \mathrm{~J}_{\mathrm{HH}}=1.4 \mathrm{~Hz}, 1 \mathrm{H}$, $\mathrm{C}=\mathrm{CH}) \cdot{ }^{13} \mathrm{C}\left\{{ }^{1} \mathrm{H}\right\}$ NMR $\left(101 \mathrm{MHz}, \mathrm{CDCl}_{3}\right) \delta(\mathrm{ppm}) 12.5\left(\mathrm{CH}_{3} \mathrm{C}=\mathrm{CH}\right), 14.5\left(\mathrm{CH}_{3} \mathrm{CH}_{2} \mathrm{O}\right), 23.6$ $\left(\mathrm{CH}_{3} \mathrm{CHOH}\right), 25.9\left(\mathrm{CH}_{2}\right), 28.7-29.8\left(28 \times \mathrm{CH}_{2}\right), 39.5\left(\mathrm{CH}_{2} \mathrm{CHOH}\right), 60.5\left(\mathrm{CH}_{3} \mathrm{CH}_{2} \mathrm{O}\right), 68.4$ $\left(\mathrm{CH}_{2} \mathrm{CHOH}\right), 127.8(\mathrm{C}=\mathrm{CH}), 142.6(\mathrm{C}=\mathrm{CH}), 168.5(\mathrm{C}=\mathrm{O})$. (Z)-isomer: ${ }^{1} \mathrm{H}$ NMR $(400 \mathrm{MHz}$, $\left.\mathrm{CDCl}_{3}\right) \delta(\mathrm{ppm}) 1.19\left(\mathrm{~d},{ }^{3} \mathrm{~J}_{\mathrm{HH}}=6.1 \mathrm{~Hz}, 3 \mathrm{H}, \mathrm{CH}_{3} \mathrm{CHOH}\right), 1.24-1.49\left(\mathrm{~m}, 3 \mathrm{H}, \mathrm{CH}_{3} \mathrm{CH}_{2} \mathrm{O} ; 58 \mathrm{H}\right.$, $\left.\left(\mathrm{CH}_{2}\right)_{29} ; 1 \mathrm{H}, \mathrm{OH}\right), 1.79-1.85(\mathrm{~m}, 2 \mathrm{H}), 1.87-1.92\left(\mathrm{~m}, 3 \mathrm{H}, \mathrm{CH}_{3} \mathrm{C}=\mathrm{CH}\right), 2.37-2.50(\mathrm{~m}, 2 \mathrm{H}$, $\left.\mathrm{C}=\mathrm{CHCH}_{2}\right), 3.74-3.85\left(\mathrm{~m}, 1 \mathrm{H}, \mathrm{CH}_{3} \mathrm{CHOH}\right), 4.19\left(\mathrm{q},{ }^{3} J_{\mathrm{HH}}=7.1 \mathrm{~Hz}, 2 \mathrm{H}, \mathrm{CH}_{3} \mathrm{CH}_{2} \mathrm{O}\right.$ ), 5.92 (tq, $\left.{ }^{3} J_{\mathrm{HH}}=7.6,{ }^{4} \mathrm{~J}_{\mathrm{HH}}=1.6 \mathrm{~Hz}, 1 \mathrm{H}, \mathrm{C}=\mathrm{CH}=\right) .{ }^{13} \mathrm{C}\left\{{ }^{1} \mathrm{H}\right\} \mathbf{N M R}\left(101 \mathrm{MHz}, \mathrm{CDCl}_{3}\right) \delta(\mathrm{ppm}) 14.5$ $\left(\mathrm{CH}_{3} \mathrm{CH}_{2} \mathrm{O}\right), 20.8\left(\mathrm{CH}_{3} \mathrm{C}=\mathrm{CH}\right), 23.6\left(\mathrm{CH}_{3} \mathrm{CHOH}\right), 25.9\left(\mathrm{CH}_{2}\right), 28.7-29.9\left(28 \times \mathrm{CH}_{2}\right), 39.5$ $\left(\mathrm{CH}_{2} \mathrm{CHOH}\right), 60.2\left(\mathrm{CH}_{3} \mathrm{CH}_{2} \mathrm{O}\right), 68.4\left(\mathrm{CH}_{3} \mathrm{CHOH}\right), 127.1(\mathrm{C}=\mathrm{CH}), 143.3(\mathrm{C}=\mathrm{CH}), 168.50$ $(C=\mathrm{O})$. LRMS (IC) found $[\mathrm{M}+\mathrm{H}]^{+}=580$.

\subsection{Characterization of isomerization products}

Ethyl 5-oxopentanoate (3a): $19 \mathrm{mg}, 53 \%$ yield. Colourless oil, ${ }^{1} \mathbf{H}$ NMR
$\left(400 \mathrm{MHz}, \mathrm{CDCl}_{3}\right) \delta(\mathrm{ppm}) 1.25\left(\mathrm{t},{ }^{3} \mathrm{~J}_{\mathrm{HH}}=7.1 \mathrm{~Hz}, 3 \mathrm{H}\right), 1.86-2.03(\mathrm{~m}, 2 \mathrm{H})$, $2.36\left(\mathrm{t},{ }^{3} J_{H H}=7.2 \mathrm{~Hz}, 2 \mathrm{H}\right), 2.53\left(\mathrm{td},{ }^{3} J_{H H}=7.2,1.3 \mathrm{~Hz}, 2 \mathrm{H}\right), 4.13\left(\mathrm{q},{ }^{3} J_{H H}=7.1 \mathrm{~Hz}, 2 \mathrm{H}\right), 9.77$ $\left(\mathrm{t},{ }^{3} J_{H H}=1.4 \mathrm{~Hz}, 1 \mathrm{H}\right)$. The other spectroscopic analyses were in agreement with those reported in the literature. ${ }^{31}$

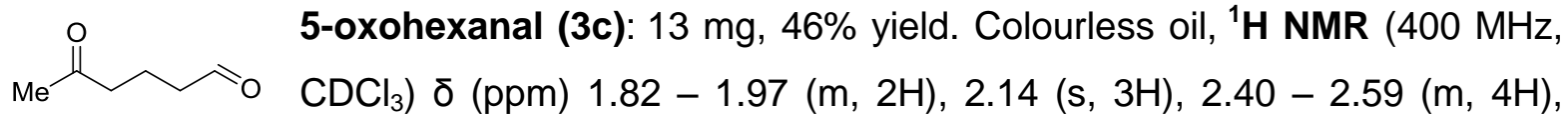
$9.76\left(\mathrm{t},{ }^{3} \mathrm{~J}_{\mathrm{HH}}=1.4 \mathrm{~Hz}, 1 \mathrm{H}\right)$. All other spectroscopic analyses were in agreement with those reported in the literature. ${ }^{32}$ 


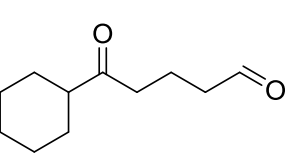

5-cyclohexyl-5-oxopentanal (3d): $28 \mathrm{mg}, 61 \%$ yield. Colourless oil. ${ }^{1} \mathbf{H}$

NMR $\left(400 \mathrm{MHz}, \mathrm{CDCl}_{3}\right) \delta(\mathrm{ppm}) 1.17-1.36(\mathrm{~m}, 5 \mathrm{H}), 1.63-1.71(\mathrm{~m}$, $1 \mathrm{H}), 1.74-1.83(\mathrm{~m}, 4 \mathrm{H}), 1.90\left(\mathrm{q},{ }^{3} J_{H H}=7.1 \mathrm{~Hz}, 2 \mathrm{H}\right), 2.22-2.38(\mathrm{~m}$, $1 \mathrm{H}), 2.43-2.57(\mathrm{~m}, 4 \mathrm{H}), 9.75\left(\mathrm{t},{ }^{3} J_{H H}=1.5 \mathrm{~Hz}, 1 \mathrm{H}\right)$. All other spectroscopic analyses were in agreement with those reported in the literature. ${ }^{32}$

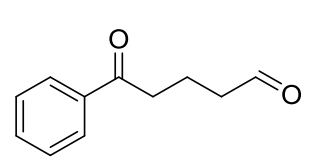

5-oxo-5-phenylpentanal (3e): $33 \mathrm{mg}, 75 \%$ yield. Colourless oil. ${ }^{1} \mathbf{H}$ NMR $\left(400 \mathrm{MHz}, \mathrm{CDCl}_{3}\right) \delta(\mathrm{ppm}) 2.01-2.13(\mathrm{~m}, 2 \mathrm{H}), 2.60\left(\mathrm{td},{ }^{3} J_{H H}=7.1,1.3\right.$ $\mathrm{Hz}, 2 \mathrm{H}), 3.05\left(\mathrm{t},{ }^{3} \mathrm{~J}_{\mathrm{HH}}=7.0 \mathrm{~Hz}, 2 \mathrm{H}\right), 7.40-7.51(\mathrm{~m}, 2 \mathrm{H}), 7.51-7.64(\mathrm{~m}$, $1 \mathrm{H}), 7.83-8.10(\mathrm{~m}, 2 \mathrm{H}), 9.82\left(\mathrm{t},{ }^{3} J_{\mathrm{HH}}=1.3 \mathrm{~Hz}, 1 \mathrm{H}\right)$. All other spectroscopic analyses were in agreement with those reported in the literature. ${ }^{32}$

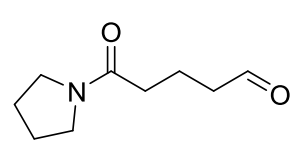

5-oxo-5-(pyrrolidin-1-yl)pentanal (3f): $21 \mathrm{mg}, 50 \%$ yield. Colourless oil. $\mathbf{R}_{\mathbf{f}}=0.1$ (Diethyl ether/EtOAc = 1:2). IR (neat): $v\left(\mathrm{~cm}^{-1}\right)=2953,2875$, 1719, 1631, 1433. ${ }^{1} \mathrm{H}$ NMR $\left(500 \mathrm{MHz}, \mathrm{CD}_{2} \mathrm{Cl}_{2}\right) \delta(\mathrm{ppm}) 1.79-1.88(\mathrm{~m}$, $\left.2 \mathrm{H},\left(\mathrm{CH}_{2} \mathrm{CH}_{2}\right)_{2} \mathrm{~N}\right), 1.88-1.98\left(\mathrm{~m}, 2 \mathrm{H},\left(\mathrm{CH}_{2} \mathrm{CH}_{2}\right)_{2} \mathrm{~N} ; 2 \mathrm{H}, \mathrm{CH}_{2} \mathrm{CH}_{2} \mathrm{CH}_{2} \mathrm{CHO}\right), 2.27\left(\mathrm{t},{ }^{3} J_{\mathrm{HH}}=7.2\right.$ $\mathrm{Hz}, 2 \mathrm{H}, \mathrm{CH}_{2} \mathrm{CH}_{2} \mathrm{CH}_{2} \mathrm{CHO}$ ), 2.50 (td, ${ }^{3} J_{\mathrm{HH}}=7.1,{ }^{3} \mathrm{~J}_{\mathrm{HH}}=1.5 \mathrm{~Hz}, 2 \mathrm{H}, \mathrm{CH}_{2} \mathrm{CH}_{2} \mathrm{CH}_{2} \mathrm{CHO}$ ), $3.28-$ $3.50\left(\mathrm{~m}, 4 \mathrm{H},\left(\mathrm{CH}_{2} \mathrm{CH}_{2}\right)_{2} \mathrm{~N}\right), 9.74\left(\mathrm{t},{ }^{3} \mathrm{~J}_{\mathrm{HH}}=1.5 \mathrm{~Hz}, 1 \mathrm{H}, \mathrm{CHO}\right) .{ }^{13} \mathrm{C}\left\{{ }^{1} \mathrm{H}\right\} \mathrm{NMR}(126 \mathrm{MHz}$,

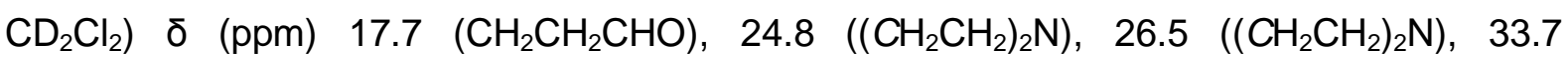
$\left(\mathrm{CH}_{2} \mathrm{CH}_{2} \mathrm{CH}_{2} \mathrm{CHO}\right), 43.7\left(\mathrm{CH}_{2} \mathrm{CH}_{2} \mathrm{CH}_{2} \mathrm{CHO}\right), 45.9\left(\left(\mathrm{CH}_{2} \mathrm{CH}_{2}\right)_{2} \mathrm{~N}\right), 46.8\left(\left(\mathrm{CH}_{2} \mathrm{CH}_{2}\right)_{2} \mathrm{~N}\right), 170.6$ $(\mathrm{C}=\mathrm{O}), 202.6(\mathrm{CHO})$. LRMS (IC) found $[\mathrm{M}+\mathrm{H}]^{+}=170$

O Me Ethyl 5-oxohexanoate (3g): $30 \mathrm{mg}, 94 \%$ yield. Colourless oil. ${ }^{\mathbf{1}} \mathbf{H}$ NMR Eto $\left(400 \mathrm{MHz}, \mathrm{CDCl}_{3}\right) \delta(\mathrm{ppm}) 1.25\left(\mathrm{t},{ }^{3} \mathrm{~J}_{\mathrm{HH}}=7.1 \mathrm{~Hz}, 3 \mathrm{H}\right), 1.84-1.96(\mathrm{~m}$, $2 \mathrm{H}), 2.14(\mathrm{~s}, 3 \mathrm{H}), 2.32\left(\mathrm{t},{ }^{3} \mathrm{~J}_{\mathrm{HH}}=7.2 \mathrm{~Hz}, 2 \mathrm{H}\right), 2.50\left(\mathrm{t},{ }^{3} \mathrm{~J}_{\mathrm{HH}}=7.2 \mathrm{~Hz}, 2 \mathrm{H}\right), 4.12\left(\mathrm{q},{ }^{3} \mathrm{~J}_{\mathrm{HH}}=7.1\right.$ $\mathrm{Hz}, 2 \mathrm{H})$. All other spectroscopic analyses were in agreement with those reported in the literature. $^{33}$

$\begin{array}{ll}M e & \text { Heptane-2,6-dione (3h): } 19 \mathrm{mg}, 59 \% \text { yield. Colourless oil. }{ }^{1} \mathbf{H} \text { NMR (400 }\end{array}$ $=7.1 \mathrm{~Hz}, 4 \mathrm{H})$. All other spectroscopic analyses were in agreement with those reported in the literature. ${ }^{34}$

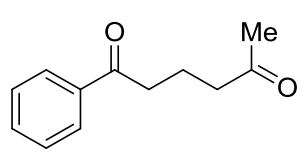

1-phenylhexane-1,5-dione (3i): $44 \mathrm{mg}, 94 \%$ yield. Colourless oil. ${ }^{1} \mathbf{H}$ NMR $\left(400 \mathrm{MHz}, \mathrm{CDCl}_{3}\right) \delta(\mathrm{ppm}) 1.94-2.08(\mathrm{~m}, 2 \mathrm{H}), 2.16(\mathrm{~s}, 3 \mathrm{H}), 2.58$ $\left(\mathrm{t},{ }^{3} \mathrm{~J}_{\mathrm{HH}}=7.0 \mathrm{~Hz}, 2 \mathrm{H}\right), 3.02\left(\mathrm{t},{ }^{3} \mathrm{~J}_{\mathrm{HH}}=7.0 \mathrm{~Hz}, 2 \mathrm{H}\right), 7.46\left(\mathrm{dd},{ }^{3} \mathrm{~J}_{\mathrm{HH}}=8.4,6.9\right.$ 
$\mathrm{Hz}, 2 \mathrm{H}), 7.53-7.65(\mathrm{~m}, 1 \mathrm{H}), 7.91-8.03(\mathrm{~m}, 2 \mathrm{H})$. All other spectroscopic analyses were in agreement with those reported in the literature. ${ }^{35}$

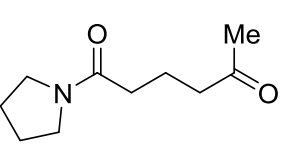

1-(pyrrolidin-1-yl)hexane-1,5-dione (3j): $44 \mathrm{mg}, 96 \%$ yield. Colorless oil. $\mathbf{R}_{\mathbf{f}}=0.5(\mathrm{MeOH} / \mathrm{EtOAc}=1: 10)$. IR (neat): $v\left(\mathrm{~cm}^{-1}\right)=2962,2950$, 2874, 1710, 1630, 1433, 1354, 1278, 1125. ' $\mathbf{H}$ NMR (500 MHz, $\left.\mathrm{CD}_{2} \mathrm{Cl}_{2}\right)$ $\delta(\mathrm{ppm}) 1.73-1.85\left(\mathrm{~m}, 4 \mathrm{H},\left(\mathrm{CH}_{2} \mathrm{CH}_{2}\right)_{2} \mathrm{~N}\right), 1.86-1.96\left(\mathrm{~m}, 2 \mathrm{H}, \mathrm{CH}_{2} \mathrm{CH}_{2} \mathrm{CH}_{2} \mathrm{CO}\right), 2.08$ (s, 3H, $\mathrm{CH}_{3} \mathrm{CO}$ ), $2.22\left(\mathrm{t},{ }^{3} \mathrm{~J}_{\mathrm{HH}}=7.3 \mathrm{~Hz}, 2 \mathrm{H}, \mathrm{CH}_{2} \mathrm{CH}_{2} \mathrm{CH}_{2} \mathrm{CO}\right), 2.49$ (t, ${ }^{3} J_{\mathrm{HH}}=7.1 \mathrm{~Hz}, 2 \mathrm{H}$, $\left.\mathrm{CH}_{2} \mathrm{CH}_{2} \mathrm{CH}_{2} \mathrm{CO}\right), 3.29-3.42\left(\mathrm{~m}, 4 \mathrm{H},\left(\mathrm{CH}_{2} \mathrm{CH}_{2}\right)_{2} \mathrm{~N}\right) .{ }^{13} \mathrm{C}\left\{{ }^{1} \mathrm{H}\right\} \mathrm{NMR}\left(126 \mathrm{MHz}, \mathrm{CD}_{2} \mathrm{Cl}_{2}\right) \delta(\mathrm{ppm})$ $19.3\left(\left(\mathrm{CH}_{2} \mathrm{CH}_{2}\right)_{2} \mathrm{~N}\right), 24.8\left(\left(\mathrm{CH}_{2} \mathrm{CH}_{2}\right)_{2} \mathrm{~N}\right), 26.5\left(\mathrm{CH}_{2} \mathrm{CH}_{2} \mathrm{CH}_{2} \mathrm{CO}\right), \quad 30.0\left(\mathrm{CH}_{3} \mathrm{CO}\right), \quad 33.9$ $\left(\mathrm{CH}_{2} \mathrm{CH}_{2} \mathrm{CH}_{2} \mathrm{CO}\right), 43.2\left(\mathrm{CH}_{2} \mathrm{CH}_{2} \mathrm{CH}_{2} \mathrm{CO}\right), 45.9\left(\left(\mathrm{CH}_{2} \mathrm{CH}_{2}\right)_{2} \mathrm{~N}\right), 46.8\left(\left(\mathrm{CH}_{2} \mathrm{CH}_{2}\right)_{2} \mathrm{~N}\right), 171.0$ $(C=\mathrm{O}), 208.8\left(\mathrm{COCH}_{3}\right)$. LRMS (IC) found $[\mathrm{M}+\mathrm{H}]^{+}=184$.

Ethyl 6-oxohexanoate (3k): $44 \mathrm{mg}, 56 \%$ yield. Colourless oil. ${ }^{1} \mathrm{H}$ NMR Eto $\left.1400 \mathrm{MHz}, \mathrm{CDCl}_{3}\right) \delta(\mathrm{ppm}) 1.25\left(\mathrm{t},{ }^{3} J_{\mathrm{HH}}=7.1 \mathrm{~Hz}, 3 \mathrm{H}\right), 1.60-1.73(\mathrm{~m}$, $4 \mathrm{H}), 2.24-2.55(\mathrm{~m}, 4 \mathrm{H}), 4.13\left(\mathrm{q},{ }^{3} \mathrm{~J}_{\mathrm{HH}}=7.1 \mathrm{~Hz}, 2 \mathrm{H}\right), 9.77\left(\mathrm{q},{ }^{3} \mathrm{~J}_{\mathrm{HH}}=1.9 \mathrm{~Hz}, 1 \mathrm{H}\right)$. All other spectroscopic analyses were in agreement with those reported in the literature. ${ }^{36}$<smiles>CCOC(=O)CCCCCCC(N)=O</smiles>

Ethyl 8-oxononanoate (3I): $42 \mathrm{mg}, 84 \%$ yield. Colourless oil. $\mathbf{R}_{\mathbf{f}}=$ 0.3 (Pentane/Diethyl ether $=2: 1)$. IR (neat): $v\left(\mathrm{~cm}^{-1}\right)=2936,2860$, 1732, 1176, 1034. ${ }^{1} \mathrm{H}$ NMR $\left(400 \mathrm{MHz}, \mathrm{CDCl}_{3}\right) \delta(\mathrm{ppm}) 1.25\left(\mathrm{t},{ }^{3} \mathrm{~J}_{\mathrm{HH}}=\right.$ $\left.7.1 \mathrm{~Hz}, 3 \mathrm{H}, \mathrm{CH}_{3} \mathrm{CH}_{2} \mathrm{O}\right), 1.28-1.36\left(\mathrm{~m}, 4 \mathrm{H},\left(\mathrm{CH}_{2}\right)_{2} \mathrm{CH}_{2} \mathrm{CH}_{2}\left(\mathrm{CH}_{2}\right)_{2} \mathrm{COCH}_{3}\right), 1.52-1.69$ (m, $\left.4 \mathrm{H}, \mathrm{CH}_{2} \mathrm{CH}_{2}\left(\mathrm{CH}_{2}\right)_{2} \mathrm{CH}_{2} \mathrm{CH}_{2} \mathrm{COCH}_{3}\right), 2.13\left(\mathrm{~s}, 3 \mathrm{H}, \mathrm{CH}_{3} \mathrm{CO}\right), 2.28$ (t, ${ }^{3} J_{\mathrm{HH}}=7.5 \mathrm{~Hz}, 2 \mathrm{H}$, $\left.\mathrm{CH}_{2}\left(\mathrm{CH}_{2}\right)_{5} \mathrm{CO}\right), 2.42$ (t, $\left.{ }^{3} J_{\mathrm{HH}}=7.4 \mathrm{~Hz}, 2 \mathrm{H},\left(\mathrm{CH}_{2}\right)_{5} \mathrm{CH}_{2} \mathrm{CO}\right), 4.12\left(\mathrm{q},{ }^{3} J_{\mathrm{HH}}=7.1 \mathrm{~Hz}, 2 \mathrm{H}\right.$,

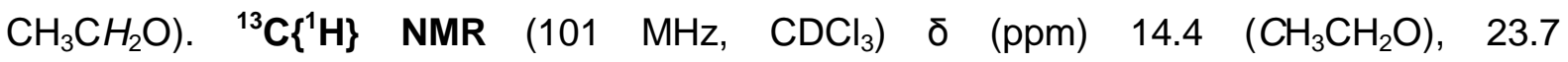
$\left(\left(\mathrm{CH}_{2}\right)_{4} \mathrm{CH}_{2} \mathrm{CH}_{2} \mathrm{CO}\right), 24.9\left(\mathrm{CH}_{2}\left(\mathrm{CH}_{2}\right)_{5}\right), 28.9\left(\left(\mathrm{CH}_{2}\right)_{5} \mathrm{CH}_{2} \mathrm{CO}\right), 29.0\left(\left(\mathrm{CH}_{2}\right)_{2} \mathrm{CH}_{2}\left(\mathrm{CH}_{2}\right)_{3} \mathrm{CO}\right)$, $30.0\left(\mathrm{CH}_{3} \mathrm{CO}\right)$, $34.4\left(\left(\mathrm{CH}_{2}\right)_{5} \mathrm{CH}_{2} \mathrm{CO}\right), 43.8\left(\mathrm{CH}_{2}\left(\mathrm{CH}_{2}\right)_{5} \mathrm{CO}\right), 60.4\left(\mathrm{CH}_{3} \mathrm{CH}_{2} \mathrm{O}\right), 173.9$ (COOEt), $209.3(C=\mathrm{O})$. LRMS (IC) found $[\mathrm{M}-\mathrm{H}]^{+}=199$.

Ethyl 6-methyl-8-oxooctanoate $(3 \mathrm{~m}): 36 \mathrm{mg}, 72 \%$ yield.<smiles>COC(=O)CCCCC(C)CC=O</smiles>
Colourless oil. $\mathbf{R}_{\mathbf{f}}=0.6$ (Pentane/Diethyl ether $=3: 2$ ). IR (neat): $v\left(\mathrm{~cm}^{-1}\right)=2933,2718,1727,1462,1374,1239,1168,1104,1033$, 858, 738. ${ }^{1} \mathbf{H}$ NMR $\left(500 \mathrm{MHz}, \mathrm{CDCl}_{3}\right) \delta(\mathrm{ppm}) 0.96\left(\mathrm{~d}, 3 \mathrm{H},{ }^{3} \mathrm{~J}_{\mathrm{HH}}=6.7 \mathrm{~Hz}, \mathrm{CHCH}_{3}\right), 1.22-$ $1.41\left(\mathrm{~m}, 4 \mathrm{H}, \mathrm{CH}_{2} \mathrm{CH}_{2}\left(\mathrm{CH}_{2}\right)_{2}\right), 1.25\left(\mathrm{t}, 3 \mathrm{H},{ }^{3} \mathrm{~J}_{\mathrm{HH}}=7.1 \mathrm{~Hz}, \mathrm{CH}_{3} \mathrm{CH}_{2} \mathrm{O}\right), 1.58-1.64(\mathrm{~m}, 2 \mathrm{H}$, $\left.\mathrm{CH}_{2} \mathrm{CH}_{2}\left(\mathrm{CH}_{2}\right)_{2}\right), 2.05\left(\mathrm{dh}, 1 \mathrm{H},{ }^{3} J_{\mathrm{HH}}=13.9,6.9 \mathrm{~Hz}, \mathrm{CHCH}_{3}\right), 2.20-2.26\left(\mathrm{ddd}, 1 \mathrm{H},{ }^{3} J_{\mathrm{HH}}=\right.$ 16.1, 7.8, $\left.{ }^{2} \mathrm{~J}_{\mathrm{HH}}=2.5 \mathrm{~Hz}, \mathrm{CH}_{2} \mathrm{CHO}\right), 2.29\left(\mathrm{t}, 2 \mathrm{H},{ }^{3} \mathrm{~J}_{\mathrm{HH}}=7.5 \mathrm{~Hz}, \mathrm{CH}_{2} \mathrm{CH}_{2}\left(\mathrm{CH}_{2}\right)_{2}\right), 2.36-2.40$ 
(ddd, $1 \mathrm{H},{ }^{3} \mathrm{~J}_{\mathrm{HH}}=16.1,5.7,{ }^{2} \mathrm{~J}_{\mathrm{HH}}=1.9 \mathrm{~Hz}, \mathrm{CH}_{2} \mathrm{CHO}$ ), 4.12 (q, $2 \mathrm{H},{ }^{3} \mathrm{~J}_{\mathrm{HH}}=7.1 \mathrm{~Hz}, \mathrm{CH}_{3} \mathrm{CH}_{2} \mathrm{O}$ ), $9.75\left(\mathrm{bd}, 1 \mathrm{H},{ }^{3} \mathrm{~J}_{\mathrm{HH}}=2.2 \mathrm{~Hz}, \mathrm{CHO}\right) .{ }^{13} \mathrm{C}\left\{{ }^{1} \mathrm{H}\right\} \quad \mathrm{NMR}\left(101 \mathrm{MHz}, \mathrm{CDCl}_{3}\right) \delta(\mathrm{ppm}) 14.4$ $\left(\mathrm{CH}_{3} \mathrm{CH}_{2} \mathrm{O}\right), 20.0\left(\mathrm{CHCH}_{3}\right), 25.1\left(\mathrm{CH}_{2} \mathrm{CH}_{2} \mathrm{CH}_{2} \mathrm{CH}_{2}\right), 26.6\left(\mathrm{CH}_{2} \mathrm{CH}_{2} \mathrm{CH}_{2} \mathrm{CH}_{2}\right), 28.1\left(\mathrm{CHCH}_{3}\right)$, $34.4\left(\mathrm{CH}_{2} \mathrm{CH}_{2} \mathrm{CH}_{2} \mathrm{CH}_{2}\right), 36.6\left(\mathrm{CH}_{2} \mathrm{CH}_{2} \mathrm{CH}_{2} \mathrm{CH}_{2}\right), 51.1\left(\mathrm{CH}_{2} \mathrm{CHO}\right), 60.4\left(\mathrm{CH}_{3} \mathrm{CH}_{2} \mathrm{O}\right), 173.8$ $(\mathrm{C}=\mathrm{O}), 203.0(\mathrm{CHO})$. LRMS (IC) found $[\mathrm{M}+\mathrm{H}]^{+}=201$.

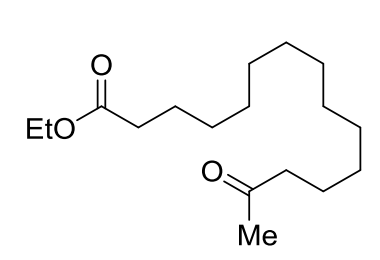

Ethyl 14-oxopentadecanoate (3n): $60 \mathrm{mg}$, 85\% yield. Colourless solid. $\mathbf{M p}$ : $37^{\circ} \mathrm{C} . \mathbf{R}_{\mathbf{f}}=0.5$ (Pentane/Diethyl ether $=2: 1$ ). IR (neat): $v\left(\mathrm{~cm}^{-1}\right)=2987,2920,1731,1465,1172,1030 .{ }^{1} \mathbf{H}$ NMR $(400 \mathrm{MHz}$, $\left.\mathrm{CDCl}_{3}\right) \delta(\mathrm{ppm}) 1.24-1.30\left(\mathrm{~m}, 3 \mathrm{H}, \mathrm{CH}_{3} \mathrm{CH}_{2} \mathrm{O} ; 16 \mathrm{H}, \mathrm{COCH}_{2}\left(\mathrm{CH}_{2}\right)_{8}\right)$, $1.55-1.67\left(\mathrm{~m}, 2 \mathrm{H}, \mathrm{COCH}_{2} \mathrm{CH}_{2} ; 2 \mathrm{H}, \mathrm{EtOCOCH}_{2} \mathrm{CH}_{2}\right), 2.13\left(\mathrm{~s}, 3 \mathrm{H}, \mathrm{CH}_{3} \mathrm{CO}\right), 2.28\left(\mathrm{t},{ }^{3} \mathrm{JHH}_{\mathrm{HH}}=\right.$ $7.5 \mathrm{~Hz}, 2 \mathrm{H}, \mathrm{EtOCOCH}_{2}$ ), $2.41\left(\mathrm{t},{ }^{3} \mathrm{~J}_{\mathrm{HH}}=7.5 \mathrm{~Hz}, 2 \mathrm{H}, \mathrm{CH}_{3} \mathrm{COCH}_{2}\right), 4.12\left(\mathrm{q},{ }^{3} \mathrm{~J}_{\mathrm{HH}}=7.1 \mathrm{~Hz}, 2 \mathrm{H}\right.$,

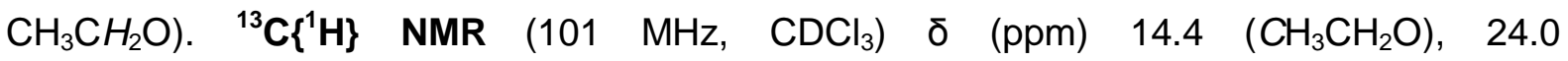
$\left(\mathrm{CH}_{3} \mathrm{COCH}_{2} \mathrm{CH}_{2}\right), 25.1\left(\mathrm{EtOCOCH}_{2} \mathrm{CH}_{2}\right), 29.3\left(\mathrm{CH}_{2}\right), 29.3\left(\mathrm{CH}_{2}\right), 29.4\left(\mathrm{CH}_{2}\right), 29.5\left(\mathrm{CH}_{2}\right)$, $29.6\left(\mathrm{CH}_{2}\right), \quad 29.7\left(\mathrm{CH}_{2}\right), \quad 30.0\left(\mathrm{CH}_{3} \mathrm{CO}\right), \quad 34.6\left(\mathrm{CH}_{3} \mathrm{COCH}_{2}\right), \quad 44.0(\mathrm{EtOOCCH}), 60.3$ $\left(\mathrm{CH}_{3} \mathrm{CH}_{2} \mathrm{O}\right), 174.1$ (EtOCO), $209.6\left(\mathrm{COCH}_{3}\right)$. LRMS (IC) found $[\mathrm{M}+\mathrm{H}]^{+}=285 ;\left[\mathrm{M}+\mathrm{NH}_{4}\right]^{+}=$ 302.<smiles>CCOC(=O)C(CCCC(=O)[N+](=O)[O-])C[N+](=O)[O-]</smiles>

Ethyl 2-ethyl-6-oxoheptanoate (3p): $18 \mathrm{mg}, 73 \%$ yield. Colourless oil. $\mathbf{R}_{\boldsymbol{f}}=0.6$ (Pentane/Diethyl ether 1:1). IR (neat): $v\left(\mathrm{~cm}^{-1}\right)=2962,2942$, 2878, 1730, 1721, 1459, 1411, 1367, 1264, 1226, 1180, 1153, 1094, 1028, 958, 859, 776, 752, 726, 595. ${ }^{1} \mathrm{H}-\mathrm{NMR}\left(\mathrm{CDCl}_{3}, 500 \mathrm{MHz}\right): \delta(\mathrm{ppm}) 0.88\left(\mathrm{t},{ }^{3} J_{\mathrm{HH}}=7.4\right.$ $\left.\mathrm{Hz}, 3 \mathrm{H}, \mathrm{CHCH}_{2} \mathrm{CH}_{3}\right), 1.25\left(\mathrm{t},{ }^{3} \mathrm{~J}_{\mathrm{HH}}=7.1 \mathrm{~Hz}, 3 \mathrm{H}, \mathrm{CH}_{3} \mathrm{CH}_{2} \mathrm{O}\right), 1.42-1.64\left(\mathrm{~m}, 62 \mathrm{H}, \mathrm{CHCH}_{2} \mathrm{CH}_{3}\right.$; $2 \mathrm{H}, \mathrm{CH}_{2} \mathrm{CH}_{2} \mathrm{CH}_{2} \mathrm{CO} ; 2 \mathrm{H}, \mathrm{CH}_{2} \mathrm{CH}_{2} \mathrm{CH}_{2} \mathrm{CO}$ ), 2.12 (s, 3H, COCH$), 2.25\left(\mathrm{~m}, 1 \mathrm{H}, \mathrm{CHCH}_{2} \mathrm{CH}_{3}\right.$ ), 2.42 (bt, $2 \mathrm{H},{ }^{3} \mathrm{~J}_{\mathrm{HH}}=6.9 \mathrm{~Hz}, \mathrm{CH}_{2} \mathrm{CH}_{2} \mathrm{CH}_{2} \mathrm{CO}$ ), 4.13 (qd, $2 \mathrm{H},{ }^{3} J_{\mathrm{HH}}=7.1 \mathrm{~Hz}, 1.3 \mathrm{~Hz}, \mathrm{CH}_{3} \mathrm{CH}_{2} \mathrm{O}$ ). ${ }^{13} \mathrm{C}\left\{{ }^{1} \mathrm{H}\right\}-N M R\left(\mathrm{CDCl}_{3}, 126 \mathrm{MHz}\right): \delta(\mathrm{ppm}) 11.9\left(\mathrm{CHCH}_{2} \mathrm{CH}_{3}\right), 14.5\left(\mathrm{CH}_{3} \mathrm{CH}_{2} \mathrm{O}\right), 21.8$ $\left(\mathrm{CH}_{2} \mathrm{CH}_{2} \mathrm{CH}_{2} \mathrm{CO}\right), \quad 25.6\left(\mathrm{CHCH}_{2} \mathrm{CH}_{3}\right), \quad 30.0 \quad\left(\mathrm{COCH}_{3}\right), \quad 31.5 \quad\left(\mathrm{CH}_{2} \mathrm{CH}_{2} \mathrm{CH}_{2} \mathrm{CO}\right), \quad 43.6$ $\left(\mathrm{CH}_{2} \mathrm{CH}_{2} \mathrm{CH}_{2} \mathrm{CO}\right), 47.2\left(\mathrm{CHCH}_{2} \mathrm{CH}_{3}\right), 60.3\left(\mathrm{CH}_{3} \mathrm{CH}_{2} \mathrm{O}\right), 176.2\left(\mathrm{EtO}_{2} \mathrm{C}\right), 208.8\left(\mathrm{COCH}_{3}\right)$. LRMS (IC) found $[\mathrm{M}+\mathrm{H}]^{+}=201$.

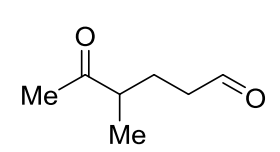

4-methyl-5-oxohexanal (5a): $19 \mathrm{mg}, 60 \%$ yield. Colourless oil. $\mathbf{R}_{\mathrm{f}}=0.1$ (Pentane/Diethyl ether $=2: 1)$. IR (neat): $v\left(\mathrm{~cm}^{-1}\right)=2930,1709,1459$, 1359, 1169. ${ }^{1} \mathrm{H}$ NMR $\left(400 \mathrm{MHz}, \mathrm{CDCl}_{3}\right) \delta(\mathrm{ppm}) 1.13\left(\mathrm{~d},{ }^{3} \mathrm{~J}_{\mathrm{HH}}=7.1 \mathrm{~Hz}, 3 \mathrm{H}\right.$, $\left.\mathrm{CH}_{3} \mathrm{CH}\right), 1.63-1.76\left(\mathrm{~m}, 1 \mathrm{H}, \mathrm{CH}_{2} \mathrm{CH}_{2} \mathrm{CHO}\right) 1.92-2.07\left(\mathrm{~m}, 1 \mathrm{H}, \mathrm{CH}_{2} \mathrm{CH}_{2} \mathrm{CHO}\right), 2.16(\mathrm{~s}, 3 \mathrm{H}$, $\left.\mathrm{CH}_{3} \mathrm{CO}\right), 2.39-2.51\left(\mathrm{~m}, 2 \mathrm{H}, \mathrm{CH}_{2} \mathrm{CH}_{2} \mathrm{CHO}\right), 2.51-2.66\left(\mathrm{~m}, 1 \mathrm{H}, \mathrm{CH}_{3} \mathrm{CH}\right), 9.76\left(\mathrm{t},{ }^{3} J_{\mathrm{HH}}=1.4\right.$ $\mathrm{Hz}, 1 \mathrm{H}, \mathrm{CHO}) .{ }^{13} \mathrm{C}\left\{{ }^{1} \mathrm{H}\right\} \mathrm{NMR}\left(101 \mathrm{MHz}, \mathrm{CDCl}_{3}\right) \delta(\mathrm{ppm}) 16.6\left(\mathrm{CH}_{3} \mathrm{CH}\right), 24.6\left(\mathrm{CH}_{2} \mathrm{CH}_{2} \mathrm{CHO}\right)$, 
$28.4\left(\mathrm{CH}_{3} \mathrm{CO}\right), 41.5\left(\mathrm{CH}_{2} \mathrm{CHO}\right), 46.2\left(\mathrm{CH}_{3} \mathrm{CH}\right), 201.8(\mathrm{CHO}), 211.8\left(\mathrm{CH}_{3} \mathrm{CO}\right)$. LRMS (IC) found $[\mathrm{M}-\mathrm{OH}]^{+}=111$.

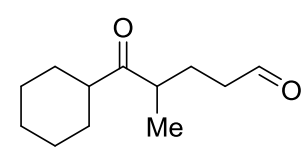

5-cyclohexyl-4-methyl-5-oxopentanal (5b): $32 \mathrm{mg}, \quad 65 \%$ yield. Colourless oil. $\mathbf{R}_{\mathbf{f}}=0.3$ (Pentane/Diethyl ether $\left.=2: 1\right)$. IR (neat): $v\left(\mathrm{~cm}^{-1}\right)=$ 3409, 2928, 2854, 1722, 1703, 1449, 992. ${ }^{1} \mathrm{H}$ NMR (400 MHz, $\left.\mathrm{CDCl}_{3}\right) \delta$ (ppm) $1.08\left(\mathrm{~d},{ }^{3} \mathrm{~J}_{\mathrm{HH}}=7.0 \mathrm{~Hz}, 3 \mathrm{H}, \mathrm{CH}_{3} \mathrm{CH}\right), 1.14-1.44$ (m, 5H, CH $\left.\mathrm{C}_{2} \mathrm{Cy}\right), 1.59-1.84(\mathrm{~m}, 4 \mathrm{H}$, $\mathrm{CH}_{2}$-Cy), $1.59-1.66\left(\mathrm{~m}, 1 \mathrm{H}, \mathrm{CH}_{2} \mathrm{CH}_{2} \mathrm{CHO}\right), 1.92-2.01\left(\mathrm{~m}, 1 \mathrm{H}, \mathrm{CH}_{2} \mathrm{CH}_{2} \mathrm{CHO}\right.$ ), $2.30-2.51$ $\left(\mathrm{m}, 2 \mathrm{H}, \mathrm{CH}_{2} \mathrm{CH}_{2} \mathrm{CHO} ; 1 \mathrm{H}, \mathrm{CH}-\mathrm{Cy}\right), 2.66-2.86\left(\mathrm{~m}, 1 \mathrm{H}, \mathrm{CH}_{3} \mathrm{CH}\right), 9.74\left(\mathrm{t},{ }^{3} J_{\mathrm{HH}}=1.5 \mathrm{~Hz}, 1 \mathrm{H}\right.$, $\mathrm{CHO}) .{ }^{13} \mathrm{C}\left\{{ }^{1} \mathrm{H}\right\}$ NMR (101 MHz, $\left.\mathrm{CDCl}_{3}\right) \delta(\mathrm{ppm}) 17.1\left(\mathrm{CH}_{3} \mathrm{CH}\right), 24.9\left(\mathrm{CH}_{2} \mathrm{CH}_{2} \mathrm{CHO}\right), 25.7$ $\left(\mathrm{CH}_{2}-\mathrm{Cy}\right), 25.9\left(\mathrm{CH}_{2}-\mathrm{Cy}\right), 28.4\left(\mathrm{CH}_{2}-\mathrm{Cy}\right), 28.8\left(\mathrm{CH}_{2}-\mathrm{Cy}\right), 41.7\left(\mathrm{CH}_{2} \mathrm{CH}_{2} \mathrm{CHO}\right), 43.50$ $\left(\mathrm{CH}_{3} \mathrm{CH}\right), 49.9$ ( $\left.\mathrm{CH}-\mathrm{Cy}\right), 202.0(\mathrm{CHO}), 216.9$ (CyCO). LRMS (IC) found $[\mathrm{M}+\mathrm{H}]^{+}=197$.

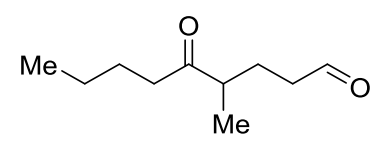

4-methyl-5-oxononanal (5c): $24 \mathrm{mg}, 57 \%$ yield. Colourless oil. $\mathbf{R}_{\mathrm{f}}=$ 0.1 (Pentane/Diethyl ether $=10: 1)$. IR (neat): $v\left(\mathrm{~cm}^{-1}\right)=3488,2958$, 2930, 1709, 1459, 1127, 731. ${ }^{1} \mathbf{H}$ NMR (400 MHz, $\left.\mathrm{CDCl}_{3}\right) \delta(\mathrm{ppm})$ $0.90\left(\mathrm{t},{ }^{3} \mathrm{~J}_{\mathrm{HH}}=7.3 \mathrm{~Hz}, 3 \mathrm{H}, \mathrm{CH}_{3} \mathrm{CH}_{2} \mathrm{CH}_{2} \mathrm{CH}_{2}\right.$ ), $1.10\left(\mathrm{~d},{ }^{3} J_{\mathrm{HH}}=7.0 \mathrm{~Hz}, 3 \mathrm{H}, \mathrm{CH}_{3} \mathrm{CH}\right.$ ), $1.25-$ $1.34\left(\mathrm{~m}, 2 \mathrm{H}, \mathrm{CH}_{3} \mathrm{CH}_{2} \mathrm{CH}_{2} \mathrm{CH}_{2}\right), 1.46-1.59\left(\mathrm{~m}, 2 \mathrm{H}, \mathrm{CH}_{3} \mathrm{CH}_{2} \mathrm{CH}_{2} \mathrm{CH}_{2}\right), 1.60-1.76(\mathrm{~m}, 1 \mathrm{H}$, $\mathrm{CH}_{2} \mathrm{CH}_{2} \mathrm{CHO}$ ), $1.87-2.07$ (m, $\left.1 \mathrm{H}, \mathrm{CH}_{2} \mathrm{CH}_{2} \mathrm{CHO}\right), 2.31-2.53\left(\mathrm{~m}, 2 \mathrm{H}, \mathrm{CH}_{3} \mathrm{CH}_{2} \mathrm{CH}_{2} \mathrm{CH}_{2} \mathrm{CO}\right.$ ), $2.57\left(\mathrm{td},{ }^{3} \mathrm{JHH}_{\mathrm{HH}}=7.3,6.1 \mathrm{~Hz}, 1 \mathrm{H}, \mathrm{CH}_{3} \mathrm{CH}\right), 9.75\left(\mathrm{t},{ }^{3} \mathrm{~J}_{\mathrm{HH}}=1.4 \mathrm{~Hz}, 1 \mathrm{H}, \mathrm{CHO}\right) .{ }^{13} \mathrm{C}\left\{{ }^{1} \mathrm{H}\right\}$ NMR $(101$ $\left.\mathrm{MHz}, \mathrm{CDCl}_{3}\right) \delta(\mathrm{ppm}) 14.0\left(\mathrm{CH}_{3} \mathrm{CH}_{2} \mathrm{CH}_{2} \mathrm{CH}_{2}\right), 16.8\left(\mathrm{CH}_{3} \mathrm{CH}\right), 22.5\left(\mathrm{CH}_{3} \mathrm{CH}_{2} \mathrm{CH}_{2} \mathrm{CH}_{2}\right), 24.8$ $\left(\mathrm{CH}_{3} \mathrm{CH}_{2} \mathrm{CH}_{2} \mathrm{CH}_{2}\right), 25.9\left(\mathrm{CH}_{2} \mathrm{CH}_{2} \mathrm{CHO}\right), 41.1\left(\mathrm{CH}_{3} \mathrm{CH}_{2} \mathrm{CH}_{2} \mathrm{CH}_{2}\right), 41.6\left(\mathrm{CH}_{2} \mathrm{CH}_{2} \mathrm{CHO}\right), 45.3$ $\left(\mathrm{CH}_{3} \mathrm{CH}\right), 201.9(\mathrm{CHO}), 214.1\left(\mathrm{COCH}_{3}\right)$. LRMS (IC) found $[\mathrm{M}+\mathrm{H}]^{+}=171 ;\left[\mathrm{M}+\mathrm{NH}_{4}\right]^{+}=188$.

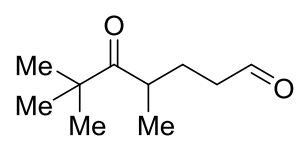

(4,6,6-trimethyl-5-oxoheptanal (5d): $27 \mathrm{mg}, 64 \%$ yield. Colourless oil. $\mathbf{R}_{\mathbf{f}}=0.2$ (Pentane/Diethyl ether $=4: 1$ ). IR (neat): $v\left(\mathrm{~cm}^{-1}\right)=2969,2874$, 2338, 1724, 1702, 1478, 1368, 1055. ${ }^{1} \mathrm{H}$ NMR $\left(400 \mathrm{MHz}, \mathrm{CDCl}_{3}\right) \delta(\mathrm{ppm})$

$1.06\left(\mathrm{~d},{ }^{3} \mathrm{~J}_{\mathrm{HH}}=6.8 \mathrm{~Hz}, 3 \mathrm{H}, \mathrm{CH}_{3} \mathrm{CH}\right), 1.15\left(\mathrm{~s}, 9 \mathrm{H},\left(\mathrm{CH}_{3}\right)_{3} \mathrm{CCO}\right), 1.61-1.78(\mathrm{~m}, 1 \mathrm{H}$, $\mathrm{CH}_{2} \mathrm{CH}_{2} \mathrm{CHO}$ ), $1.86-2.01\left(\mathrm{~m}, 1 \mathrm{H}, \mathrm{CH}_{2} \mathrm{CH}_{2} \mathrm{CHO}\right.$ ), 2.39 (td, ${ }^{3} J_{\mathrm{HH}}=7.4 \mathrm{~Hz}, 1.5 \mathrm{~Hz}, 2 \mathrm{H}$, $\left.\mathrm{CH}_{2} \mathrm{CH}_{2} \mathrm{CHO}\right), 2.94-3.24\left(\mathrm{~m}, 1 \mathrm{H}, \mathrm{CH}_{3} \mathrm{CH}\right), 9.74\left(\mathrm{t},{ }^{3} \mathrm{JHH}_{\mathrm{HH}}=1.5 \mathrm{~Hz}, 1 \mathrm{H}, \mathrm{CHO}\right) .{ }^{13} \mathrm{C}\left\{{ }^{1} \mathrm{H}\right\}$ NMR $\left(101 \mathrm{MHz}, \mathrm{CDCl}_{3}\right) \delta$ (ppm) 18.41( $\left.\mathrm{CH}_{3} \mathrm{CH}\right), 26.10\left(\left(\mathrm{CH}_{3}\right)_{3} \mathrm{C}\right), 26.15\left(\mathrm{CH}_{2} \mathrm{CH}_{2} \mathrm{CHO}\right), 38.48$ $\left(\mathrm{CH}_{3} \mathrm{CH}\right), 41.54\left(\mathrm{CH}_{2} \mathrm{CH}_{2} \mathrm{CHO}\right), 44.67\left(\left(\mathrm{CH}_{3}\right)_{3} \mathrm{C}\right), 201.79(\mathrm{CHO}), 219.02\left(\left(\mathrm{CH}_{3}\right)_{3} \mathrm{CC}=\mathrm{O}\right)$. LRMS (IC) found $[\mathrm{M}+\mathrm{H}]^{+}=171$. 


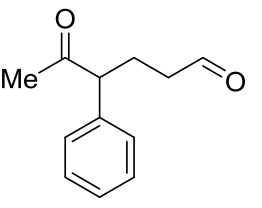

5-oxo-4-phenylhexanal (5e): $20 \mathrm{mg}, 63 \%$ yield. Colourless oil. $\mathbf{R}_{\mathbf{f}}=0.3$ (Pentane/Diethyl ether $=1: 1)$. IR (neat): $v\left(\mathrm{~cm}^{-1}\right)=2926,1709,1356,1158$, 700. ${ }^{1} \mathbf{H}$ NMR (400 MHz, $\left.\mathrm{CDCl}_{3}\right) \delta(\mathrm{ppm}) 1.97-2.42(\mathrm{~m}, 4 \mathrm{H}$, $\mathrm{CH}_{2} \mathrm{CH}_{2} \mathrm{CHO}$ ), 2.05 (s, 3H, $\mathrm{CH}_{3} \mathrm{CO}$ ), $3.61-3.77(\mathrm{~m}, 1 \mathrm{H}, \mathrm{CH}), 7.14-7.20$ (m, 2H, CH-Ar), $7.27-7.39$ (m, 3H, CH-Ar), 9.69 (t, $\left.{ }^{3} \mathrm{~J}_{\mathrm{HH}}=1.2 \mathrm{~Hz}, 1 \mathrm{H}, \mathrm{CHO}\right) .{ }^{13} \mathrm{C}\left\{{ }^{1} \mathrm{H}\right\} \mathbf{N M R}$ (101 MHz, $\left.\mathrm{CDCl}_{3}\right) \delta(\mathrm{ppm}) 24.3\left(\mathrm{CH}_{2} \mathrm{CH}_{2} \mathrm{CHO}\right), 29.2\left(\mathrm{CH}_{3} \mathrm{CO}\right), 41.5\left(\mathrm{CH}_{2} \mathrm{CH}_{2} \mathrm{CHO}\right), 58.4$ $(\mathrm{CH}), 127.8$ (CH-Ar), 128.4 (CH-Ar), 129.3 (CH-Ar), $138.2(\mathrm{C}-\mathrm{Ar}), 201.9(\mathrm{CHO}), 207.75$ $\left(\mathrm{CH}_{3} \mathrm{CO}\right)$.LRMS (IC) found $[\mathrm{M}+\mathrm{H}]^{+}=208$.

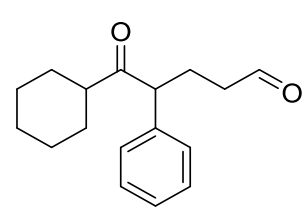

5-cyclohexyl-5-oxo-4-phenylpentanal (5f): $18 \mathrm{mg}, \quad 28 \%$ yield. Colourless oil. $\mathbf{R}_{\mathbf{f}}=0.5$ (Pentane/diethyl ether $=2: 1$ ). IR (neat): $v\left(\mathrm{~cm}^{-1}\right)=$ 2969, 2874, 2338, 1724, 1702, 1478, 1368, 1055. 'H NMR (400 MHz, $\left.\mathrm{CDCl}_{3}\right) \delta(\mathrm{ppm}) 1.03-1.86\left(\mathrm{~m}, 10 \mathrm{H}, \mathrm{CH}_{2}-\mathrm{Cy}\right), 1.91-2.41(\mathrm{~m}, 5 \mathrm{H}$, $\mathrm{CH}_{2} \mathrm{CHO} ; \mathrm{CH}_{2} \mathrm{CH}_{2} \mathrm{CHO}$; $\left.\mathrm{CH}-\mathrm{Cy}\right), 3.74-3.92(\mathrm{~m}, 1 \mathrm{H}, \mathrm{CH}), 7.13-7.22$ (m, 2H, CH-Ar), 7.26 - $7.36(\mathrm{~m}, 3 \mathrm{H}, \mathrm{CH}-\mathrm{Ar}), 9.69\left(\mathrm{t},{ }^{3} \mathrm{~J}_{\mathrm{HH}}=1.3 \mathrm{~Hz}, 1 \mathrm{H}, \mathrm{CHO}\right) .{ }^{13} \mathrm{C}\left\{{ }^{1} \mathrm{H}\right\} \mathbf{N M R}\left(101 \mathrm{MHz}, \mathrm{CDCl}_{3}\right) \delta$ (ppm) $25.2\left(\mathrm{CH}_{2} \mathrm{CH}_{2} \mathrm{CHO}\right), 25.3\left(\mathrm{CH}_{2}-\mathrm{Cy}\right), 25.9\left(\mathrm{CH}_{2}-\mathrm{Cy}\right), 26.0\left(\mathrm{CH}_{2}-\mathrm{Cy}\right), 28.2\left(\mathrm{CH}_{2}-\mathrm{Cy}\right)$, 29.4 ( $\left.\mathrm{CH}_{2}-\mathrm{Cy}\right), 41.7\left(\mathrm{CH}_{2} \mathrm{CH}_{2} \mathrm{CHO}\right), 50.1$ ( $\left.\mathrm{CH}-\mathrm{Cy}\right), 55.9$ ( $\left.\mathrm{CH}-\mathrm{Ar}\right), 127.6(\mathrm{CH}-\mathrm{Ar}), 128.6(\mathrm{CH}-$ $\mathrm{Ar}), 129.1(\mathrm{CH}-\mathrm{Ar}), 138.2(\mathrm{C}-\mathrm{Ar}), 202.0(\mathrm{CHO}), 212.9\left(\mathrm{COCH}_{3}\right)$. LRMS (IC) found $[\mathrm{M}+\mathrm{H}]^{+}=$ 259.

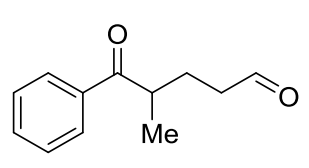

4-methyl-5-oxo-5-phenylpentanal (5g): $35 \mathrm{mg}, 73 \%$ yield. Orange oil.

$\mathbf{R}_{\mathbf{f}}=0.6$ Pentane/Diethyl ether $\left.=1: 1\right)$. IR (neat): $v\left(\mathrm{~cm}^{-1}\right)$ 2971, 2934, 2827, 2725, 1721, 1678, 1569, 1579, 1448, 1411, 1374, 1375, 1279, 1227, 1180, 1100, 1078, 1001, 971, 933, 905, 793, 702, 655. ${ }^{1} \mathrm{H}-\mathrm{NMR}\left(\mathrm{CDCl}_{3}, 500 \mathrm{MHz}\right): \delta$ (ppm) $1.22\left(\mathrm{~d},{ }^{3} \mathrm{~J}_{\mathrm{HH}}=7.0 \mathrm{~Hz}, 3 \mathrm{H}, \mathrm{CH}_{3} \mathrm{CH}\right), 1.77-1.84\left(\mathrm{~m}, 1 \mathrm{H}, \mathrm{CH}_{2} \mathrm{CH}_{2} \mathrm{CHO}\right), 2.11-2.18$ (m, $1 \mathrm{H}, \mathrm{CH}_{2} \mathrm{CH}_{2} \mathrm{CHO}$ ), $2.41-2.57\left(\mathrm{~m}, 2 \mathrm{H}, \mathrm{CH}_{2} \mathrm{CH}_{2} \mathrm{CHO}\right), 3.55\left(\mathrm{~h},{ }^{3} \mathrm{~J}_{\mathrm{HH}}=7.0 \mathrm{~Hz}, 1 \mathrm{H}\right.$, $\mathrm{CH}_{3} \mathrm{CH}$ ), $7.46-7.49\left(\mathrm{~m}, 2 \mathrm{H}, \mathrm{CH}_{m}-\mathrm{Ar}\right), 7.55-7.58\left(\mathrm{~m}, 1 \mathrm{H}, \mathrm{CH}_{p}-\mathrm{Ar}\right), 7.95-7.96\left(\mathrm{~m}, 2 \mathrm{H}, \mathrm{CH}_{0^{-}}\right.$ Ar), $9.76\left(\mathrm{~m}, 1 \mathrm{H}, \mathrm{CH}_{2} \mathrm{CH}_{2} \mathrm{CHO}\right) .{ }^{13} \mathrm{C}\left\{{ }^{1} \mathrm{H}\right\}-\mathrm{NMR}\left(\mathrm{CDCl}_{3}, 126 \mathrm{MHz}\right): \delta(\mathrm{ppm}) 17.8\left(\mathrm{CH}_{3} \mathrm{CH}\right)$, $25.5\left(\mathrm{CH}_{2} \mathrm{CH}_{2} \mathrm{CHO}\right), 39.7\left(\mathrm{CH}_{3} \mathrm{CH}\right), 41.6\left(\mathrm{CH}_{2} \mathrm{CH}_{2} \mathrm{CHO}\right), 128.4\left(\mathrm{CH}_{o}-\mathrm{Ar}\right), 128.9\left(\mathrm{CH}_{m}-\mathrm{Ar}\right)$, $133.3\left(\mathrm{CH}_{p}-\mathrm{Ar}\right), 136.4(\mathrm{C}-\mathrm{Ar}), 202.0(\mathrm{CHO}), 203.1\left(\mathrm{COCH}_{3}\right)$. LRMS (IC) found $[\mathrm{M}+\mathrm{H}]^{+}=191$.

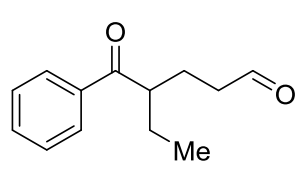

4-benzoylhexanal (5h): $21 \mathrm{mg}, 41 \%$ yield. Yellow oil. $\mathbf{R}_{\boldsymbol{f}}=0.6$ (Pentane/Diethyl ether $=1: 1)$. IR (neat): $v\left(\mathrm{~cm}^{-1}\right)=2964,2723,1751$, 1676, 1596, 1577, 1447, 1382, 1219, 1001, 886, 785, 699, 535. 'H-NMR $\left(\mathrm{CDCl}_{3}, 500 \mathrm{MHz}\right): \delta(\mathrm{ppm}) \quad 0.90\left(\mathrm{t},{ }^{3} J_{\mathrm{HH}}=7.5 \mathrm{~Hz}, 3 \mathrm{H}, \mathrm{CH}_{2} \mathrm{CH}_{3}\right), 1.52-1.61(\mathrm{~m}, 1 \mathrm{H}$, $\left.\mathrm{CH}_{2} \mathrm{CH}_{3}\right), 1.76-1.83\left(\mathrm{~m}, 1 \mathrm{H}, \mathrm{CH}_{2} \mathrm{CH}_{3}\right), 1.84-1.92\left(\mathrm{~m}, 1 \mathrm{H}, \mathrm{CH}_{2} \mathrm{CH}_{2} \mathrm{CHO}\right)$, 2.06-2.14 (m, 
$\left.1 \mathrm{H}, \mathrm{CH}_{2} \mathrm{CH}_{2} \mathrm{CHO}\right), 2.35-2.41\left(\mathrm{~m}, 1 \mathrm{H}, \mathrm{CH}_{2} \mathrm{CH}_{2} \mathrm{CHO}\right.$ ), $2.47-2.53\left(\mathrm{~m}, 1 \mathrm{H}, \mathrm{CH}_{2} \mathrm{CH}_{2} \mathrm{CHO}\right.$ ), 3.43 - $3.48(\mathrm{~m}, 1 \mathrm{H}, \mathrm{ArCOCH}), 7.46-7.49\left(\mathrm{~m}, 2 \mathrm{H}, \mathrm{CH}_{m}-\mathrm{Ar}\right), 7.56-7.59\left(\mathrm{~m}, 1 \mathrm{H}, \mathrm{CH}_{p}-\mathrm{Ar}\right)$, $7.96-7.94\left(\mathrm{~m}, 2 \mathrm{H}, \mathrm{CH}_{\circ}-\mathrm{Ar}\right), 9.74(\mathrm{bm}, 1 \mathrm{H}, \mathrm{CHO}) .{ }^{13} \mathrm{C}\left\{{ }^{1} \mathrm{H}\right\}-\mathrm{NMR}\left(\mathrm{CDCl}_{3}, 126 \mathrm{MHz}\right): \delta(\mathrm{ppm})$ $11.7\left(\mathrm{CH}_{2} \mathrm{CH}_{3}\right), 23.5\left(\mathrm{CH}_{2} \mathrm{CH}_{2} \mathrm{CHO}\right), 25.7\left(\mathrm{CH}_{2} \mathrm{CH}_{3}\right), 41.6\left(\mathrm{CH}_{2} \mathrm{CH}_{2} \mathrm{CHO}\right), 46.4(\mathrm{ArCOCH})$, $128.4\left(\mathrm{CH}_{o}-\mathrm{Ar}\right), 128.9\left(\mathrm{CH}_{m}-\mathrm{Ar}\right), 133.3\left(\mathrm{CH}_{p}-\mathrm{Ar}\right), 137.3(\mathrm{C}-\mathrm{Ar}), 202.0(\mathrm{CHO}), 203.7(\mathrm{ArCO})$. LRMS (IC) found $[\mathrm{M}+\mathrm{Na}]^{+}=225$.

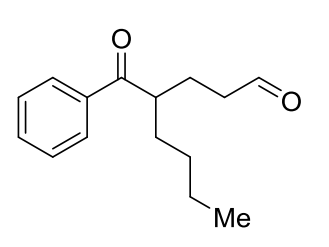

4-benzoyloctanal (5i): $36 \mathrm{mg}, 62 \%$ yield. Yellow oil. $\mathbf{R}_{\mathbf{f}}=0.5$ (Pentane/Diethyl ether $=7: 3$ ). IR (neat): $v\left(\mathrm{~cm}^{-1}\right)=2928,2857,2718$, 1723, 1677, 1594, 1577, 1447, 1376, 1229, 1003, 934, 703. ' $\mathrm{H}-\mathrm{NMR}$ $\left(\mathrm{CDCl}_{3}, 500 \mathrm{MHz}\right): \delta(\mathrm{ppm}) \quad 0.84\left(\mathrm{~m}, 3 \mathrm{H}, \mathrm{CH}_{2} \mathrm{CH}_{2} \mathrm{CH}_{2} \mathrm{CH}_{3}\right), 1.25-1.28(\mathrm{~m}$, $\left.4 \mathrm{H}, \mathrm{CH}_{2} \mathrm{CH}_{2} \mathrm{CH}_{2} \mathrm{CH}_{3}\right), 1.49-1.76\left(\mathrm{~m}, 2 \mathrm{H}, \mathrm{CH}_{2} \mathrm{CH}_{2} \mathrm{CH}_{2} \mathrm{CH}_{3}\right), 1.89-2.08(\mathrm{~m}, 2 \mathrm{H}$, $\mathrm{CH}_{2} \mathrm{CH}_{2} \mathrm{CHO}$ ), $2.34-2.41$ (m, 1H, $\mathrm{CH}_{2} \mathrm{CH}_{2} \mathrm{CHO}$ ), $2.46-2.53\left(\mathrm{~m}, 1 \mathrm{H}, \mathrm{CH}_{2} \mathrm{CH}_{2} \mathrm{CHO}\right), 3.47-$ $3.53(\mathrm{~m}, 1 \mathrm{H}, \mathrm{ArCOCH}), 7.46-7.49\left(\mathrm{~m}, 2 \mathrm{H}, \mathrm{CH}_{m}-\mathrm{Ar}\right), 7.56-7.59\left(\mathrm{~m}, 1 \mathrm{H}, \mathrm{CH}_{p}-\mathrm{Ar}\right), 7.93-$ $7.95\left(\mathrm{~m}, 2 \mathrm{H}, \mathrm{CH} \mathrm{o}^{-} \mathrm{Ar}\right), 9.73(\mathrm{~m}, 1 \mathrm{H}, \mathrm{CHO}) .{ }^{13} \mathrm{C}\left\{{ }^{1} \mathrm{H}\right\}-\mathrm{NMR}\left(\mathrm{CDCl}_{3}, 126 \mathrm{MHz}\right): \delta(\mathrm{ppm}) 14.0$ $\left(\mathrm{CH}_{2} \mathrm{CH}_{2} \mathrm{CH}_{2} \mathrm{CH}_{3}\right), 22.6\left(\mathrm{CH}_{2} \mathrm{CH}_{2} \mathrm{CH}_{2} \mathrm{CH}_{3}\right), 23.7\left(\mathrm{CH}_{2} \mathrm{CH}_{2} \mathrm{CHO}\right), 29.5\left(\mathrm{CH}_{2} \mathrm{CH}_{2} \mathrm{CH}_{2} \mathrm{CH}_{3}\right), 32.4$ $\left(\mathrm{CH}_{2} \mathrm{CH}_{2} \mathrm{CH}_{2} \mathrm{CH}_{3}\right), 41.7\left(\mathrm{CH}_{2} \mathrm{CH}_{2} \mathrm{CHO}\right), 45.0(\mathrm{ArCOCH}), 128.0\left(\mathrm{CH}_{p}-\mathrm{Ar}\right), 128.9\left(\mathrm{CH}_{m}-\mathrm{Ar}\right)$, $133.3\left(\mathrm{CH}_{p}-\mathrm{Ar}\right), 137.3(\mathrm{C}-\mathrm{Ar}), 202.0(\mathrm{CHO}), 203.8(\mathrm{ArCO})$. LRMS (IC) found $[\mathrm{M}+\mathrm{H}]^{+}=233$.

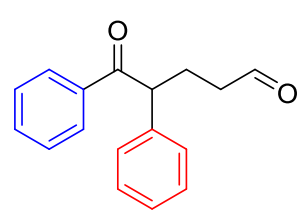

5-oxo-4,5-diphenylpentanal (5j): $19 \mathrm{mg}, 36 \%$ yield. Orange oil. $\mathbf{R}_{\mathbf{f}}=0.5$ (Pentane/Diethyl ether $=1: 1)$. IR (neat): $v\left(\mathrm{~cm}^{-1}\right)=3061,3028,2929$, $2827,2725,1721,1678,1597,1580,1492,1448,1390,1353,1257$, $1231,1205,1176,1100,1072,1002,964,934,847,754,696,617 .{ }^{1} \mathrm{H}-$ NMR $\left(\mathrm{CDCl}_{3}, 500 \mathrm{MHz}\right): \delta$ (ppm) 2.12-2.19 (m, 1H, $\left.\mathrm{CHCH}_{2} \mathrm{CH}_{2} \mathrm{CHO}\right), 2.38-2.46(\mathrm{~m}, 1 \mathrm{H}$, $\left.\mathrm{CHCH}_{2} \mathrm{CH}_{2} \mathrm{CHO}\right), 2.43\left(\mathrm{~m}, 2 \mathrm{H}, \mathrm{CHCH}_{2} \mathrm{CH}_{2} \mathrm{CHO}\right), 4.60-4.63\left(\mathrm{~m}, 1 \mathrm{H}, \mathrm{CHCH}_{2} \mathrm{CH}_{2} \mathrm{CHO}\right), 7.19$

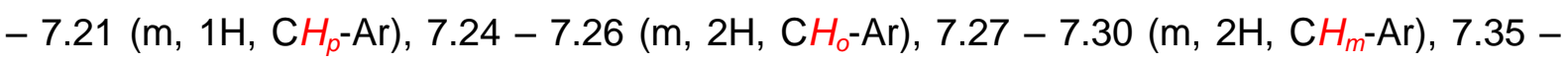
$7.38\left(\mathrm{~m}, 2 \mathrm{H}, \mathrm{CH}_{m}-\mathrm{Ar}\right), 7.45-7.48$ (m, 1H, CHo $\left.-\mathrm{Ar}\right), 7.92-7.94$ (m, 2H, CHo $\left.-\mathrm{Ar}\right), 9.71(\mathrm{~s}, 1 \mathrm{H}$ $\left.\mathrm{CHCH}_{2} \mathrm{CH}_{2} \mathrm{CHO}\right) .{ }^{13} \mathrm{C}\left\{{ }^{1} \mathrm{H}\right\}-\mathrm{NMR}\left(\mathrm{CDCl}_{3}, 126 \mathrm{MHz}\right): \delta$ (ppm) $26.1\left(\mathrm{CHCH}_{2} \mathrm{CH}_{2} \mathrm{CHO}\right), 41.6$ $\left(\mathrm{CHCH}_{2} \mathrm{CH}_{2} \mathrm{CHO}\right), 52.4\left(\mathrm{CHCH}_{2} \mathrm{CH}_{2} \mathrm{CHO}\right), 127.5\left(\mathrm{CH}_{p}-\mathrm{Ar}\right), 128.3\left(\mathrm{CH}_{o}-\mathrm{Ar}\right), 128.7\left(\mathrm{CH}_{m}-\mathrm{Ar}\right)$, $128.9\left(\mathrm{CH}_{o}-\mathrm{Ar}\right), 129.2\left(\mathrm{CH}_{m}-\mathrm{Ar}\right), 133.2\left(\mathrm{CH}_{p}-\mathrm{Ar}\right), 136.2(\mathrm{C}-\mathrm{Ar}), 138.7(\mathrm{C}-\mathrm{Ar}), 199.3(\mathrm{ArCO})$, $202.3(\mathrm{CHO})$. LRMS (IC) found $[\mathrm{M}+\mathrm{H}]^{+}=253$.

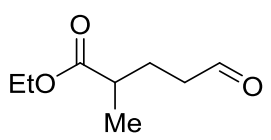

Ethyl 2-methyl-5-oxopentanoate (5k): $21 \mathrm{mg}, 53 \%$ yield. Colourless oil. $\mathbf{R}_{\mathbf{f}}=0.2$ (Pentane/Diethyl ether $\left.=2: 1\right)$. IR (neat): $v\left(\mathrm{~cm}^{-1}\right)=2978,2936$, 1732, 1460, 1376, 1171, 1027. ${ }^{1} \mathbf{H}$ NMR $\left(400 \mathrm{MHz}, \mathrm{CDCl}_{3}\right) \delta$ (ppm) 1.18 $\left(\mathrm{d},{ }^{3} \mathrm{~J}_{\mathrm{HH}}=7.0 \mathrm{~Hz}, 3 \mathrm{H}, \mathrm{CH}_{3} \mathrm{CH}\right), 1.26\left(\mathrm{t},{ }^{3} \mathrm{~J}_{\mathrm{HH}}=7.1 \mathrm{~Hz}, 3 \mathrm{H}, \mathrm{CH}_{3} \mathrm{CH}_{2} \mathrm{O}\right), 1.72-1.88(\mathrm{~m}, 1 \mathrm{H}$, 
$\left.\mathrm{CH}_{2} \mathrm{CH}_{2} \mathrm{CHO}\right), 1.89-2.03\left(\mathrm{~m}, 1 \mathrm{H}, \mathrm{CH}_{2} \mathrm{CH}_{2} \mathrm{CHO}\right), 2.38-2.57\left(\mathrm{~m}, 1 \mathrm{H}, \mathrm{CH}_{3} \mathrm{CH} ; 2 \mathrm{H}\right.$, $\mathrm{CH}_{2} \mathrm{CH}_{2} \mathrm{CHO}$ ), 4.13 (q, ${ }^{3} J_{\mathrm{HH}}=7.1 \mathrm{~Hz}, 2 \mathrm{H}, \mathrm{CH}_{3} \mathrm{CH}_{2} \mathrm{O}$ ), 9.77 (s, $\left.1 \mathrm{H}, \mathrm{CHO}\right) .{ }^{13} \mathrm{C}\left\{{ }^{1} \mathrm{H}\right\}$ NMR (101 $\left.\mathrm{MHz}, \mathrm{CDCl}_{3}\right) \delta$ (ppm) $14.4\left(\mathrm{CH}_{3} \mathrm{CH}_{2} \mathrm{O}\right), 17.3\left(\mathrm{CH}_{3} \mathrm{CH}\right), 25.9\left(\mathrm{CH}_{2} \mathrm{CH}_{2} \mathrm{CHO}\right), 38.9\left(\mathrm{CH}_{3} \mathrm{CH}\right)$, $41.7\left(\mathrm{CH}_{2} \mathrm{CH}_{2} \mathrm{CHO}\right), 60.6\left(\mathrm{CH}_{3} \mathrm{CH}_{2} \mathrm{O}\right), 176.0$ (COOEt), 201.7 (CHO). LRMS (IC) found [M$\mathrm{H}]^{+}=141$.

Me Ethyl 2-methyl-5-oxohexanoate (5I): $39 \mathrm{mg}, 91 \%$ yield. Colourless oil. $\mathbf{R}_{\mathbf{f}}$
$\mathrm{Me}$ 1719, 1462, 1369, 1161, 1026. ' ${ }^{\mathbf{H}}$ NMR $\left(400 \mathrm{MHz}, \mathrm{CDCl}_{3}\right) \delta(\mathrm{ppm}) 1.16\left(\mathrm{~d},{ }^{3} \mathrm{~J}_{\mathrm{HH}}=7.0 \mathrm{~Hz}\right.$, $3 \mathrm{H}, \mathrm{CH}_{3} \mathrm{CH}$ ), $1.25\left(\mathrm{t},{ }^{3} \mathrm{~J}_{\mathrm{HH}}=7.1 \mathrm{~Hz}, 3 \mathrm{H}, \mathrm{CH}_{3} \mathrm{CH}_{2} \mathrm{O}\right), 1.70-1.96\left(\mathrm{~m}, 2 \mathrm{H}, \mathrm{CH}_{2} \mathrm{CH}_{2} \mathrm{CO}\right), 2.13(\mathrm{~s}$, $3 \mathrm{H}, \mathrm{CH}_{3} \mathrm{CO}$ ), $2.32-2.55\left(\mathrm{~m}, 2 \mathrm{H}, \mathrm{CH}_{2} \mathrm{CH}_{2} \mathrm{CO} ; 1 \mathrm{H}, \mathrm{CH}_{3} \mathrm{CH}\right.$ ), 4.12 (q, ${ }^{3} J_{\mathrm{HH}}=7.1 \mathrm{~Hz}, 2 \mathrm{H}$, $\left.\mathrm{CH}_{3} \mathrm{CH}_{2} \mathrm{O}\right) .{ }^{13} \mathrm{C}\left\{{ }^{1} \mathrm{H}\right\}$ NMR (101 MHz, $\left.\mathrm{CDCl}_{3}\right) \delta(\mathrm{ppm}) 14.4\left(\mathrm{CH}_{3} \mathrm{CH}_{2} \mathrm{O}\right), 17.3\left(\mathrm{CH}_{3} \mathrm{CH}\right), 27.5$ $\left(\mathrm{CH}_{2} \mathrm{CH}_{2} \mathrm{CO}\right), 30.1\left(\mathrm{CH}_{3} \mathrm{CO}\right), 38.9\left(\mathrm{CH}_{3} \mathrm{CH}\right), 41.2\left(\mathrm{CH}_{2} \mathrm{CH}_{2} \mathrm{CO}\right), 60.5\left(\mathrm{CH}_{3} \mathrm{CH}_{2} \mathrm{O}\right), 176.3$ (EtOOC), $208.2\left(\mathrm{COCH}_{3}\right)$. LRMS (IC) found $[\mathrm{M}+\mathrm{H}]^{+}=173$.

O Me Ethyl 3-methyl-5-oxopentanoate (5m): $22 \mathrm{mg}, 56 \%$ yield. Colourless oil. Eto $10 \mathbf{R}_{\mathbf{f}}=0.2$ ( $n$-Pentane/Diethyl ether $\left.=10: 1\right)$. IR (neat): $v\left(\mathrm{~cm}^{-1}\right)=2952,1874$, 1719, 1631, 1433. ${ }^{1} \mathrm{H}$ NMR $\left(400 \mathrm{MHz}, \mathrm{CDCl}_{3}\right) \delta(\mathrm{ppm}) 1.03\left(\mathrm{~d},{ }^{3} \mathrm{~J}_{\mathrm{HH}}=6.6 \mathrm{~Hz}, 3 \mathrm{H}, \mathrm{CH}_{3} \mathrm{CH}\right)$, $1.26\left(\mathrm{t},{ }^{3} \mathrm{~J}_{\mathrm{HH}}=7.1 \mathrm{~Hz}, 3 \mathrm{H}, \mathrm{CH}_{3} \mathrm{CH}_{2} \mathrm{O}\right), 2.20-2.65\left(\mathrm{~m}, 5 \mathrm{H}, \mathrm{CH}_{2} \mathrm{CHCH}_{2}\right), 4.13\left(\mathrm{q},{ }^{3} \mathrm{~J}_{\mathrm{HH}}=7.1\right.$ $\left.\mathrm{Hz}, 2 \mathrm{H}, \mathrm{CH}_{3} \mathrm{CH}_{2} \mathrm{O}\right), 9.75\left(\mathrm{t},{ }^{3} \mathrm{JHH}_{\mathrm{HH}}=1.8 \mathrm{~Hz}, 1 \mathrm{H}, \mathrm{CHO}\right) .{ }^{13} \mathrm{C}\left\{{ }^{1} \mathrm{H}\right\}$ NMR $\left(101 \mathrm{MHz}, \mathrm{CDCl}_{3}\right) \delta$ (ppm) $14.4\left(\mathrm{CH}_{3} \mathrm{CH}_{2} \mathrm{O}\right), 20.3\left(\mathrm{CH}_{3} \mathrm{CH}\right), 25.4\left(\mathrm{CH}_{3} \mathrm{CH}\right), 41.1(\mathrm{EtOOCCH}), 50.3\left(\mathrm{CH}_{2} \mathrm{CHO}\right)$, $60.6\left(\mathrm{CH}_{3} \mathrm{CH}_{2} \mathrm{O}\right), 172.4$ (EtOOC), $201.7(\mathrm{CHO})$. LRMS (IC) found $[\mathrm{M}+\mathrm{H}]^{+}=159$.

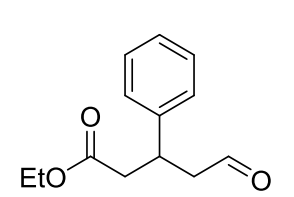

Ethyl 5-oxo-3-phenylpentanoate (5n): $21 \mathrm{mg}, 40 \%$ yield. Colourless oil. $\mathbf{R}_{\mathbf{f}}=0.3$ (Diethyl ether/Acetone $=8: 2$ ). IR (neat): $v\left(\mathrm{~cm}^{-1}\right)=2957,2924$, 2857, 1726, 1672, 1605, 1495, 1453, 1437, 1367, 1318, 1258, 1195, 1160, 1083, 1012, 848, 764, 700, 569. ${ }^{1} \mathrm{H}$ NMR (400 MHz, $\left.\mathrm{CD}_{2} \mathrm{Cl}_{2}\right) \delta$ (ppm) $2.61-2.71$ (m, $\left.2 \mathrm{H}, \mathrm{CH}_{2} \mathrm{CHAr}\right), 2.75-2.85\left(\mathrm{~m}, 2 \mathrm{H}, \mathrm{CH}_{2} \mathrm{CHO}\right), 3.58\left(\mathrm{~s}, 3 \mathrm{H}, \mathrm{CH}_{3} \mathrm{O}\right), 3.71\left(\mathrm{p}, 1 \mathrm{H},{ }^{3} \mathrm{~J}_{H H}=7.4\right.$ $\mathrm{Hz}, \mathrm{CH}_{2} \mathrm{CHPh}$ ), $7.21-7.24$ (m, 2H, CHo $-\mathrm{Ar} ; 1 \mathrm{H}, \mathrm{CH}_{p}-\mathrm{Ar}$ ), $7.29-7.33$ (m, 2H, CH $\left.\mathrm{H}_{m}-\mathrm{Ar}\right), 9.64$ $\left(\mathrm{t}, 1 \mathrm{H},{ }^{3} \mathrm{~J}_{H H}=1.7 \mathrm{~Hz}, \mathrm{CHO}\right) .{ }^{13} \mathrm{C}\left\{{ }^{1} \mathrm{H}\right\}$ NMR $\left(101 \mathrm{MHz}, \mathrm{CD}_{2} \mathrm{Cl} \mathrm{l}_{2} \delta(\mathrm{ppm})=36.5\left(\mathrm{CH}_{2} \mathrm{CHAr}\right)\right.$, $41.0\left(\mathrm{CH}_{2} \mathrm{CHAr}\right), 49.8\left(\mathrm{CH}_{2} \mathrm{CHO}\right), 51.9\left(\mathrm{CH}_{3} \mathrm{O}\right), 127.3\left(\mathrm{CH}_{\mathrm{p}}-\mathrm{Ar}\right), 127.7\left(\mathrm{CH}_{o}-\mathrm{Ar}\right), 129.1\left(\mathrm{CH}_{m^{-}}\right.$ $\mathrm{Ar}), 143.2(C-A r), 172.2(\mathrm{EtOOC}), 201.0(\mathrm{CHO})$. LRMS (IC) found $[\mathrm{M}+\mathrm{H}]^{+}=207 ;[\mathrm{M}+\mathrm{Na}]^{+}=$ 229. 


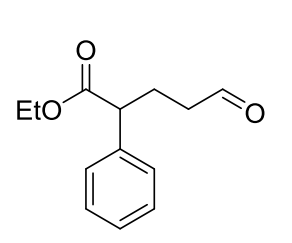

Ethyl 5-oxo-2-phenylpentanoate (5o): $12 \mathrm{mg}, 45 \%$ yield. Clear oil. $\mathbf{R}_{\mathrm{f}}=$ 0.5 (Pentane/Diethyl ether $=6: 4)$. IR (neat): $v\left(\mathrm{~cm}^{-1}\right)=2981,2726,1724$, 1602, 1494, 1453, 1370, 1257, 1216, 1159, 1096, 1070, 1025, 853, 768, 734, 699. ${ }^{1} \mathrm{H}-\mathrm{NMR}\left(\mathrm{CDCl}_{3}, 500 \mathrm{MHz}\right): \delta(\mathrm{ppm})=1.20\left(\mathrm{t},{ }^{3} \mathrm{~J}_{\mathrm{HH}}=7.1 \mathrm{~Hz}, 3 \mathrm{H}\right.$, $\mathrm{CH}_{3} \mathrm{CH}_{2} \mathrm{O}$ ), $2.12-2.36\left(\mathrm{~m}, 2 \mathrm{H}, \mathrm{CH}_{2} \mathrm{CH}_{2} \mathrm{CHO}\right.$ ), $2.40-2.43\left(\mathrm{~m}, 2 \mathrm{H}, \mathrm{CH}_{2} \mathrm{CH}_{2} \mathrm{CHO}\right.$ ), 3.58 (t, $\left.{ }^{3} J_{\mathrm{HH}}=7.5 \mathrm{~Hz}, 1 \mathrm{H}, \mathrm{ArCH}\right), 4.06-4.18\left(\mathrm{~m}, 2 \mathrm{H}, \mathrm{CH}_{3} \mathrm{CH}_{2} \mathrm{O}\right), 7.20-7.28\left(\mathrm{~m}, 2 \mathrm{H}, \mathrm{CH}_{0}-\mathrm{Ar}\right), 7.26$ (m, 1H, CH $\left.H_{p}-\mathrm{Ar}\right), 7.31-734\left(\mathrm{~m}, 2 \mathrm{H}, \mathrm{CH}_{m}-\mathrm{Ar}\right), 9.71(\mathrm{~m}, 1 \mathrm{H}, \mathrm{CHO}) \cdot{ }^{13} \mathrm{C}\left\{{ }^{1} \mathrm{H}\right\}-\mathrm{NMR}\left(\mathrm{CDCl}_{3}, 126\right.$ $\mathrm{MHz}): \delta$ (ppm) $14.2\left(\mathrm{CH}_{3} \mathrm{CH}_{2} \mathrm{O}\right), 25.8\left(\mathrm{CH}_{2} \mathrm{CH}_{2} \mathrm{CHO}\right), 41.6\left(\mathrm{CH}_{2} \mathrm{CH}_{2} \mathrm{CHO}\right), 50.6(\mathrm{ArCH}), 61.0$ $\left(\mathrm{CH}_{3} \mathrm{CH}_{2} \mathrm{O}\right), 127.6\left(\mathrm{CH}_{p}-\mathrm{Ar}\right), 128.0\left(\mathrm{CH}_{\mathrm{o}}-\mathrm{Ar}\right), 128.9\left(\mathrm{CH}_{m}-\mathrm{Ar}\right), 138.4$ (C-Ar), 173.5 (EtOOC), $201.4(\mathrm{CHO})$. LRMS (IC) $=$ found $[\mathrm{M}+\mathrm{H}]^{+}=221$.

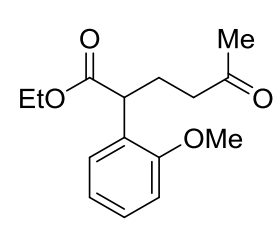

Ethyl 2-(2-methoxyphenyl)-5-oxohexanoate (5p): $55 \mathrm{mg}, 83 \%$ yield. Colourless oil. $\mathbf{R}_{\mathbf{f}}=0.3$ (Pentane/Diethyl ether $\left.=2: 1\right)$. IR (neat): $v\left(\mathrm{~cm}^{-1}\right)=$ 2959, 1722, 1494, 1245, 1156, 1245, 1156, 1028, 756, 535. 'H NMR (400 $\left.\mathrm{MHz}, \mathrm{CDCl}_{3}\right) \delta(\mathrm{ppm}) 1.19\left(\mathrm{t},{ }^{3} \mathrm{JHH}_{\mathrm{HH}}=7.1 \mathrm{~Hz}, 3 \mathrm{H}, \mathrm{CH}_{3} \mathrm{CH}_{2} \mathrm{O}\right), 2.08(\mathrm{~s}, 3 \mathrm{H}$, $\mathrm{CH}_{3} \mathrm{CO}$ ), $1.92-2.06\left(\mathrm{~m}, 2 \mathrm{H}, \mathrm{CH}_{2} \mathrm{CH}_{2} \mathrm{CO} ; 2 \mathrm{H}, \mathrm{CH}_{2} \mathrm{CO}\right), 2.23-2.49\left(\mathrm{~m}, 2 \mathrm{H}, \mathrm{CH}_{2} \mathrm{CH}_{2} \mathrm{CO} ; 2 \mathrm{H}\right.$, $\mathrm{CH}_{2} \mathrm{CO}$ ), 3.81 (s, 3H, CH $\mathrm{CHAr}_{3}$, 3.97 (t, $\left.{ }^{3} \mathrm{~J}_{\mathrm{HH}}=7.3 \mathrm{~Hz}, 1 \mathrm{H}, \mathrm{ArCH}\right), 4.12$ (q, ${ }^{3} \mathrm{~J}_{\mathrm{HH}}=7.1 \mathrm{~Hz}, 2 \mathrm{H}$, $\mathrm{CH}_{3} \mathrm{CH}_{2} \mathrm{O}$ ), $6.83-6.89(\mathrm{~m}, 1 \mathrm{H}, \mathrm{CH}-\mathrm{Ar}), 6.90-6.97(\mathrm{~m}, 1 \mathrm{H}, \mathrm{CH}-\mathrm{Ar}), 7.17-7.26(\mathrm{~m}, 2 \mathrm{H}, \mathrm{CH}-$ $\mathrm{Ar}) .{ }^{13} \mathrm{C}\left\{{ }^{1} \mathrm{H}\right\}$ NMR $\left(101 \mathrm{MHz}, \mathrm{CDCl}_{3}\right) \delta(\mathrm{ppm}) 14.3\left(\mathrm{CH}_{3} \mathrm{CH}_{2} \mathrm{O}\right), 26.2\left(\mathrm{CH}_{2} \mathrm{CH}_{2} \mathrm{CO}\right), 30.0$ $\left(\mathrm{CH}_{3} \mathrm{CO}\right), 41.4\left(\mathrm{CH}_{2} \mathrm{CH}_{2} \mathrm{CO}\right), 43.7(\mathrm{ArCH}), 55.6\left(\mathrm{CH}_{3} \mathrm{OAr}\right), 60.7\left(\mathrm{CH}_{3} \mathrm{CH}_{2} \mathrm{O}\right), 110.8(\mathrm{CH}-\mathrm{Ar})$, 120.9 (CH-Ar), 127.6 (C-Ar), 128.4 (CH-Ar), 128.6 (CH-Ar), 157.0 (COMe), 174.1 (EtOOC), $208.4(\mathrm{CHO})$. LRMS (IC) found $[\mathrm{M}+\mathrm{H}]^{+}=256$.

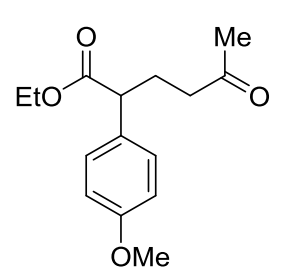

Ethyl 2-(4-methoxyphenyl)-5-oxohexanoate: (5q): $64 \mathrm{mg}, 97 \%$ yield. Colourless oil. $\mathbf{R}_{\mathbf{f}}=0.3$ (Pentane/Diethyl ether $=2: 1$ ). IR (neat): $v\left(\mathrm{~cm}^{-1}\right)=$ 2954, 1722, 1512, 1248, 1176, 1156, 1032. ${ }^{1} \mathbf{H}$ NMR $\left(400 \mathrm{MHz}, \mathrm{CDCl}_{3}\right) \delta$ (ppm) $1.19\left(\mathrm{t},{ }^{3} \mathrm{~J}_{\mathrm{HH}}=7.1 \mathrm{~Hz}, 3 \mathrm{H}, \mathrm{CH}_{3} \mathrm{CH}_{2} \mathrm{O}\right), 2.08\left(\mathrm{~s}, 3 \mathrm{H}, \mathrm{CH}_{3} \mathrm{CO}\right), 1.98-$ $2.06\left(\mathrm{~m}, 1 \mathrm{H}, \mathrm{CH}_{2} \mathrm{CH}_{2} \mathrm{CO}\right), 2.21-2.33\left(\mathrm{~m}, 1 \mathrm{H}, \mathrm{CH}_{2} \mathrm{CH}_{2} \mathrm{CO}\right), 2.34-2.41$ (m, 2H, $\mathrm{CH}_{2} \mathrm{CH}_{2} \mathrm{CO}$ ), 3.50 (t, ${ }^{3} \mathrm{~J}_{\mathrm{HH}}=7.7 \mathrm{~Hz}, 1 \mathrm{H}, \mathrm{ArCH}$ ), 3.79 (s, 3H, $\mathrm{CH}_{3} \mathrm{OAr}$ ), $3.99-4.26$ (m, 2H, $\left.\mathrm{CH}_{3} \mathrm{CH}_{2} \mathrm{O}\right), 6.80-6.88$ (m, 2H, CH-Ar), $7.15-7.22$ (m, 2H, CH-Ar). ${ }^{13}{ }^{1}\left\{{ }^{1} \mathrm{H}\right\}$ NMR (101 MHz, CDCl $\left.{ }_{3}\right) \delta(\mathrm{ppm}) 14.3\left(\mathrm{CH}_{3} \mathrm{CH}_{2} \mathrm{O}\right), 27.3\left(\mathrm{CH}_{2} \mathrm{CH}_{2} \mathrm{CO}\right), 30.1\left(\mathrm{CH}_{3} \mathrm{CO}\right), 41.2$ $\left(\mathrm{CH}_{2} \mathrm{CH}_{2} \mathrm{CO}\right), 49.8$ ( $\left.\mathrm{ArCH}\right), 55.4\left(\mathrm{CH}_{3} \mathrm{OAr}\right), 60.9\left(\mathrm{CH}_{3} \mathrm{CH}_{2} \mathrm{O}\right), 114.2(\mathrm{CH}-\mathrm{Ar}), 129.1(\mathrm{CH}-\mathrm{Ar})$, 130.7 (C-Ar), 159.0 (COMe), 173.9 (EtOOC), 208.0 (CHO). LRMS (IC) found $\left[\mathrm{M}_{+} \mathrm{NH}_{4}\right]^{+}=$ 282. 


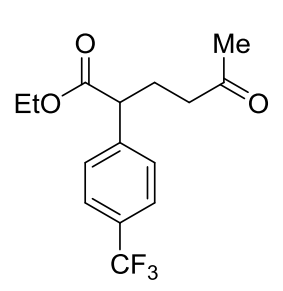

Ethyl 5-oxo-2-(4-(trifluoromethyl)phenyl)hexanoate (5r): $56 \mathrm{mg}, 74 \%$ yield. Colourless oil. $\mathbf{R}_{\mathbf{f}}=0.4$ (Pentane/Diethyl ether $=2: 1$ ). IR (neat): $v\left(\mathrm{~cm}^{-1}\right)=2940,1728,1326,1164,1069 .{ }^{1} \mathbf{H}$ NMR $\left(400 \mathrm{MHz}, \mathrm{CDCl}_{3}\right) \delta$ (ppm) $1.21\left(\mathrm{t},{ }^{3} \mathrm{~J}_{\mathrm{HH}}=7.1 \mathrm{~Hz}, 3 \mathrm{H}, \mathrm{CH}_{3} \mathrm{CH}_{2} \mathrm{O}\right), 2.10\left(\mathrm{~s}, 3 \mathrm{H}, \mathrm{CH}_{3} \mathrm{CO}\right), 2.00-$ $2.10\left(\mathrm{~m}, 2 \mathrm{H}, \quad \mathrm{CH}_{2} \mathrm{CH}_{2} \mathrm{CO} ; 2 \mathrm{H}, \mathrm{CH}_{2} \mathrm{CH}_{2} \mathrm{CO}\right) 2.26-2.44(\mathrm{~m}, 2 \mathrm{H}$, $\mathrm{CH}_{2} \mathrm{CH}_{2} \mathrm{CO} ; \mathrm{CH}_{2} \mathrm{CH}_{2} \mathrm{CO}$ ), 3.65 (t, $\left.{ }^{3} \mathrm{~J}_{\mathrm{HH}}=7.7 \mathrm{~Hz}, 1 \mathrm{H}, \mathrm{ArCH}\right), 4.05-4.22\left(\mathrm{~m}, 2 \mathrm{H}, \mathrm{CH}_{3} \mathrm{CH} \mathrm{H}_{2}\right.$ ), $7.41\left(\mathrm{~d},{ }^{3} \mathrm{~J}_{\mathrm{HH}}=8.1 \mathrm{~Hz}, 2 \mathrm{H}, \mathrm{CH}-\mathrm{Ar}\right), 7.58\left(\mathrm{~d},{ }^{3} \mathrm{~J}_{\mathrm{HH}}=8.1 \mathrm{~Hz}, 2 \mathrm{H}, \mathrm{CH}-\mathrm{Ar}\right) .{ }^{13} \mathrm{C}\left\{{ }^{1} \mathrm{H}\right\}$ NMR $(101$ $\left.\mathrm{MHz}, \quad \mathrm{CDCl}_{3}\right) \quad \delta \quad(\mathrm{ppm}) \quad 14.2\left(\mathrm{CH}_{3} \mathrm{CH}_{2} \mathrm{O}\right), 27.2\left(\mathrm{CH}_{2} \mathrm{CH}_{2} \mathrm{CO}\right), \quad 30.1 \quad\left(\mathrm{CH}_{3} \mathrm{CO}\right), 40.9$ $\left(\mathrm{CH}_{2} \mathrm{CH}_{2} \mathrm{CO}\right.$ ), $50.4(\mathrm{ArCH}), 61.3\left(\mathrm{CH}_{3} \mathrm{CH}_{2} \mathrm{O}\right), 124.2$ (q, $\left.{ }^{1} \mathrm{~J}_{\mathrm{CF}}=273.0 \mathrm{~Hz}, \mathrm{CF}_{3}\right), 125.8$ (q, ${ }^{3} \mathrm{~J}_{\mathrm{CF}}$ $=3.8 \mathrm{~Hz}, \mathrm{CH}-\mathrm{Ar}$ ), 128.5 (CH-Ar), 129.9 (q, $\left.{ }^{2} J_{\mathrm{CF}}=32.5, \mathrm{CCF}_{3}\right), 142.7$ (C-Ar), 173.0 (EtOOC), $207.6\left(\mathrm{CH}_{3} \mathrm{CO}\right)$. LRMS (IC) found $\left[\mathrm{M}+\mathrm{NH}_{4}\right]^{+}=320$.

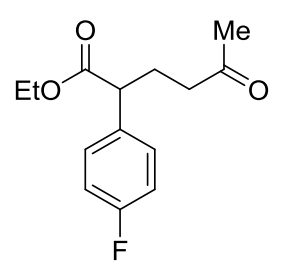

Ethyl 2-(4-fluorophenyl)-5-oxohexanoate (5s): $58 \mathrm{mg}, 92 \%$ yield. Colourless oil. $\mathbf{R}_{\mathbf{f}}=0.4$ (Pentane/Diethyl ether $=2: 1$ ). IR (neat): $v\left(\mathrm{~cm}^{-1}\right)=$ 2980, 1726, 1604, 1510, 1224, 1157, 840. ${ }^{1} \mathrm{H}$ NMR $\left(400 \mathrm{MHz}, \mathrm{CDCl}_{3}\right) \delta$ (ppm) $1.20\left(\mathrm{t},{ }^{3} \mathrm{~J}_{\mathrm{HH}}=7.1 \mathrm{~Hz}, 3 \mathrm{H}, \mathrm{CH}_{3} \mathrm{CH}_{2} \mathrm{O}\right), 2.09$ (s, 3H, $\left.\mathrm{CH}_{3} \mathrm{CO}\right), 1.98-$ $2.08\left(\mathrm{~m}, 1 \mathrm{H}, \mathrm{CH}_{2} \mathrm{CH}_{2} \mathrm{CO}\right), 2.23-2.34\left(\mathrm{~m}, 1 \mathrm{H}, \mathrm{CH}_{2} \mathrm{CH}_{2} \mathrm{CO}\right), 2.34-2.43(\mathrm{~m}, 2 \mathrm{H}$, $\mathrm{CH}_{2} \mathrm{CH}_{2} \mathrm{CO}$ ), $3.56\left(\mathrm{t},{ }^{3} \mathrm{~J}_{\mathrm{HH}}=7.6 \mathrm{~Hz}, 1 \mathrm{H}, \mathrm{ArCH}\right), 4.04-4.20\left(\mathrm{~m}, 2 \mathrm{H}, \mathrm{CH}_{3} \mathrm{CH}_{2} \mathrm{O}\right), 6.97-7.05$ (m, 2H, CH-Ar), $7.22-7.28$ (m, 2H, CH-Ar). ${ }^{13} \mathbf{C}\left\{{ }^{1} \mathrm{H}\right\}$ NMR $\left(101 \mathrm{MHz}, \mathrm{CDCl}_{3}\right) \delta$ (ppm) 14.3 $\left(\mathrm{CH}_{3} \mathrm{CH}_{2} \mathrm{O}\right), 27.4\left(\mathrm{CH}_{2} \mathrm{CH}_{2} \mathrm{CO}\right), 30.1\left(\mathrm{CH}_{3} \mathrm{CO}\right), 41.1\left(\mathrm{CH}_{2} \mathrm{CH}_{2} \mathrm{CO}\right), 49.9(\mathrm{ArCH}), 61.1$ $\left(\mathrm{CH}_{3} \mathrm{CH}_{2} \mathrm{O}\right), 115.7$ (d, $\left.{ }^{2} J_{\mathrm{CF}}=21.2 \mathrm{~Hz}, \mathrm{CH}-\mathrm{Ar}\right), 129.6$ (d, $\left.{ }^{3} J_{\mathrm{CF}}=8.1 \mathrm{~Hz}, \mathrm{CH}-\mathrm{Ar}\right), 134.4\left(\mathrm{~d},{ }^{4} \mathrm{~J}_{\mathrm{CF}}\right.$ $=3.2 \mathrm{~Hz}, \mathrm{C}-\mathrm{Ar}), 161.3\left(\mathrm{~d},{ }^{1} \mathrm{~J}_{\mathrm{CF}}=45.4 \mathrm{~Hz}, \mathrm{C}-\mathrm{F}\right), 173.6$ (EtOOC), $207.8\left(\mathrm{CH}_{3} \mathrm{CO}\right)$. LRMS (IC) found $[\mathrm{M}+\mathrm{H}]^{+}=253$.

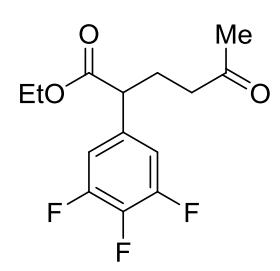

Ethyl 5-oxo-2-(3,4,5-trifluorophenyl)hexanoate (5t): $43 \mathrm{mg}, 60 \%$ yield. Colourless oil. $\mathbf{R}_{\mathbf{f}}=0.2$ (Pentane/Diethyl ether $=2: 1$ ). IR (neat): $v\left(\mathrm{~cm}^{-1}\right)=$ 2981, 1725, 1620, 1530, 1451, 1361, 1160, 1041, 866. '1H NMR (400 $\left.\mathrm{MHz}, \mathrm{CDCl}_{3}\right) \delta(\mathrm{ppm}) 1.22\left(\mathrm{t},{ }^{3} \mathrm{~J}_{\mathrm{HH}}=7.1 \mathrm{~Hz}, 3 \mathrm{H}, \mathrm{CH}_{3} \mathrm{CH}_{2} \mathrm{O}\right), 2.12(\mathrm{~s}, 3 \mathrm{H}$, $\left.\mathrm{CH}_{3} \mathrm{CO}\right), 1.93-2.05\left(\mathrm{~m}, 1 \mathrm{H}, \mathrm{CH}_{2} \mathrm{CH}_{2} \mathrm{CO}\right), 2.19-2.33(\mathrm{~m}, 1 \mathrm{H}$, $\mathrm{CH}_{2} \mathrm{CH}_{2} \mathrm{CO}$ ), $2.40\left(\mathrm{t},{ }^{3} \mathrm{~J}_{\mathrm{HH}}=7.2 \mathrm{~Hz}, 2 \mathrm{H}, \mathrm{CH}_{2} \mathrm{CH}_{2} \mathrm{CO}\right.$ ), $3.52\left(\mathrm{t},{ }^{3} \mathrm{~J}_{\mathrm{HH}}=7.8 \mathrm{~Hz}, 1 \mathrm{H}, \mathrm{ArCH}\right), 4.03$ - $4.24\left(\mathrm{~m}, 2 \mathrm{H}, \mathrm{CH}_{3} \mathrm{CH}_{2} \mathrm{O}\right), 6.85-7.06$ (m, 2H, CH-Ar). ${ }^{13} \mathrm{C}\left\{{ }^{1} \mathrm{H}\right\}$ NMR $\left(101 \mathrm{MHz}, \mathrm{CDCl}_{3}\right) \delta$ (ppm) $14.23\left(\mathrm{CH}_{3} \mathrm{CH}_{2} \mathrm{O}\right), 27.16\left(\mathrm{CH}_{2} \mathrm{CH}_{2} \mathrm{CO}\right), 30.17\left(\mathrm{CH}_{3} \mathrm{CO}\right), 40.71\left(\mathrm{CH}_{2} \mathrm{CH}_{2} \mathrm{CO}\right), 49.76$ $(\mathrm{ArCH}), 61.49\left(\mathrm{CH}_{3} \mathrm{CH}_{2} \mathrm{O}\right), 112.20-112.41$ (m, CH-Ar ), 134.92 (td, ${ }^{3} \mathrm{~J}_{\mathrm{HH}}=7.5,4.9 \mathrm{~Hz}, \mathrm{C}$ $\mathrm{Ar}$ ), $139.24\left(\mathrm{dt},{ }^{1} J_{\mathrm{CF}}=251.6,{ }^{2} J_{\mathrm{CF}}=15.1 \mathrm{~Hz}, C_{p}-\mathrm{Ar}\right.$ ), 151.34 (ddd, ${ }^{1} J_{\mathrm{CF}}=250.4,{ }^{2} J_{\mathrm{CF}}=9.8$, $\left.{ }^{3} J_{\mathrm{CF}}=4.0 \mathrm{~Hz}, \mathrm{CF}_{m^{-}} \mathrm{Ar}\right), 172.57(\mathrm{EtOOC}), 207.32\left(\mathrm{CH}_{3} \mathrm{CO}\right)$. LRMS (IC) found $[\mathrm{M}+\mathrm{H}]^{+}=289$. 


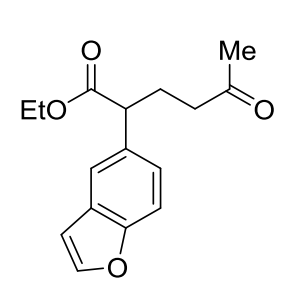

Ethyl 2-(benzofuran-5-yl)-5-oxohexanoate (5u): $49 \mathrm{mg}, 72 \%$ yield. Colourless oil. $\mathbf{R}_{\mathbf{f}}=0.3$ (Pentane/Diethyl ether $=2: 1$ ). IR (neat): $v\left(\mathrm{~cm}^{-1}\right)=$ 2980, 1723, 1468, 1158, 1030, 745. '1 $\mathbf{H}$ NMR (400 MHz, $\left.\mathrm{CDCl}_{3}\right) \delta(\mathrm{ppm})$ $1.18\left(\mathrm{t},{ }^{3} \mathrm{~J}_{\mathrm{HH}}=7.1 \mathrm{~Hz}, 3 \mathrm{H}, \mathrm{CH}_{3} \mathrm{CH}_{2} \mathrm{O}\right.$ ), 2.04 (s, 3H, $\mathrm{CH}_{3} \mathrm{CO}$ ), $1.98-2.15$ (m, $2 \mathrm{H}, \mathrm{CH}_{2} \mathrm{CH}_{2} \mathrm{CO} ; 2 \mathrm{H}, \mathrm{CH}_{2} \mathrm{CH}_{2} \mathrm{CO}$ ), 2.22 - 2.47 (m, 2H, $\mathrm{CH}_{2} \mathrm{CH}_{2} \mathrm{CO}$; $2 \mathrm{H}, \mathrm{CH}_{2} \mathrm{CH}_{2} \mathrm{CO}$ ), 3.64 (dd, $\left.{ }^{3} \mathrm{JHH}_{\mathrm{HH}}=8.2,7.1 \mathrm{~Hz}, 1 \mathrm{H}, \mathrm{ArCH}\right), 3.96-4.20\left(\mathrm{~m}, 2 \mathrm{H}, \mathrm{CH}_{3} \mathrm{CH} \mathrm{H}_{2}\right.$ ), $6.77\left(\mathrm{dd},{ }^{3} J_{\mathrm{HH}}=2.2,{ }^{5} \mathrm{~J}_{\mathrm{HH}}=1.0 \mathrm{~Hz}, 1 \mathrm{H}, \mathrm{CH}-\mathrm{Het}\right), 7.21\left(\mathrm{dd},{ }^{3} \mathrm{~J}_{\mathrm{HH}}=8.6,{ }^{4} \mathrm{~J}_{\mathrm{HH}}=1.9 \mathrm{~Hz}, 1 \mathrm{H}, \mathrm{CH}-\right.$ Het), 7.45 (dt, ${ }^{3} J_{H H}=8.6,{ }^{5} J_{H H}=0.8 \mathrm{~Hz}, 1 \mathrm{H}, \mathrm{CH}$ - Het), $7.52\left(\mathrm{~d},{ }^{4} J_{\mathrm{HH}}=1.8 \mathrm{~Hz}, 1 \mathrm{H}, \mathrm{CH}\right.$ - Het), $7.65\left(\mathrm{~d},{ }^{3} \mathrm{~J}_{\mathrm{HH}}=2.2 \mathrm{~Hz}, 1 \mathrm{H}, \mathrm{CH}\right.$-Het). ${ }^{13} \mathrm{C}\left\{{ }^{1} \mathrm{H}\right\}$ NMR $\left(101 \mathrm{MHz}, \mathrm{CDCl}_{3}\right) \delta(\mathrm{ppm}) 14.3$ $\left(\mathrm{CH}_{3} \mathrm{CH}_{2} \mathrm{O}\right), 27.9\left(\mathrm{CH}_{2} \mathrm{CH}_{2} \mathrm{CO}\right), 30.1\left(\mathrm{CH}_{3} \mathrm{CO}\right), 41.3\left(\mathrm{CH}_{2} \mathrm{CH}_{2} \mathrm{CO}\right), 50.7(\mathrm{ArCH}), 61.2$ $\left(\mathrm{CH}_{3} \mathrm{CH}_{2} \mathrm{O}\right), 106.9$ (CH-Het), 111.7 (CH-Het), 120.9 (CH-Het), 124.7 (CH-Het), 128.2 (CHet), 133.8 (C-Het), 146.0 ( $\mathrm{CH}$-Het), 154.7 (C-Het), 174.1 (EtOOC), 207.9 ( $\left.\mathrm{CH}_{3} \mathrm{CO}\right)$. LRMS (IC) found $\left[\mathrm{M}+\mathrm{NH}_{4}\right]^{+}=292$.

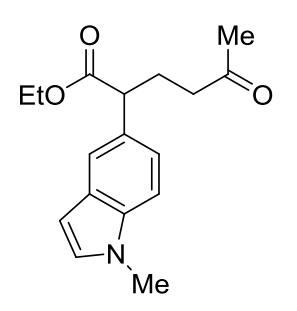

Ethyl 2-(1-methyl-1H-indol-5-yl)-5-oxohexanoate (5v): $44 \mathrm{mg}, 61 \%$ yield. Colourless oil. $\mathbf{R}_{\mathbf{f}}=0.45$ (Pentane/Diethyl ether $=2: 1$ ). IR (neat): $v\left(\mathrm{~cm}^{-1}\right)=2945,1722,1492,1156,725 .{ }^{1} \mathbf{H}$ NMR $\left(400 \mathrm{MHz}, \mathrm{CDCl}_{3}\right) \delta$ (ppm) $1.18\left(\mathrm{t},{ }^{3} \mathrm{~J}_{\mathrm{HH}}=7.1 \mathrm{~Hz}, 3 \mathrm{H}, \mathrm{CH}_{3} \mathrm{CH}_{2} \mathrm{O}\right), 2.03\left(\mathrm{~s}, 3 \mathrm{H}, \mathrm{CH}_{3} \mathrm{CO}\right), 2.00-$ $2.13\left(\mathrm{~m}, 2 \mathrm{H}, \mathrm{CH}_{2} \mathrm{CH}_{2} \mathrm{CO} ; 2 \mathrm{H}, \mathrm{CH}_{2} \mathrm{CH}_{2} \mathrm{CO}\right), 2.21-2.47(\mathrm{~m}, 2 \mathrm{H}$, $\mathrm{CH}_{2} \mathrm{CH}_{2} \mathrm{CO} ; 2 \mathrm{H}, \mathrm{CH}_{2} \mathrm{CH}_{2} \mathrm{CO}$ ), 3.60 (dd, ${ }^{3} \mathrm{JHH}_{\mathrm{HH}}=8.4,6.6 \mathrm{~Hz}, 1 \mathrm{H}, \mathrm{ArCH}$ ), 3.77 (s, 3H, $\mathrm{NCH}_{3}$ ), $3.94-4.23\left(\mathrm{~m}, 2 \mathrm{H}, \mathrm{CH}_{3} \mathrm{CH}_{2} \mathrm{O}\right), 6.42\left(\mathrm{dd},{ }^{3} \mathrm{~J}_{\mathrm{HH}}=3.2,{ }^{5} \mathrm{~J}_{\mathrm{HH}}=0.9 \mathrm{~Hz}, 1 \mathrm{H}, \mathrm{CH}-\mathrm{Het}\right), 7.07$ (d, ${ }^{3} J_{\mathrm{HH}}=3.1 \mathrm{~Hz}, 1 \mathrm{H}, \mathrm{CH}$ - Het), $7.12\left(\mathrm{dd},{ }^{3} \mathrm{~J}_{\mathrm{HH}}=8.5,{ }^{4} \mathrm{~J}_{\mathrm{HH}}=1.8 \mathrm{~Hz}, 1 \mathrm{H}, \mathrm{CH}\right.$ - Het), 7.29 (dt, ${ }^{3} \mathrm{~J}_{\mathrm{HH}}$ $=8.5,{ }^{5} J_{\mathrm{HH}}=0.8 \mathrm{~Hz}, 1 \mathrm{H}, \mathrm{CH}$ - Het $), 7.47\left(\mathrm{~d},{ }^{4} \mathrm{~J}_{\mathrm{HH}}=1.7 \mathrm{~Hz}, 1 \mathrm{H}, \mathrm{CH}-\mathrm{Het}\right) .{ }^{13} \mathrm{C}\left\{{ }^{1} \mathrm{H}\right\} \mathbf{N M R}(101$ $\left.\mathrm{MHz}, \mathrm{CDCl}_{3}\right) \delta(\mathrm{ppm}) 14.3\left(\mathrm{CH}_{3} \mathrm{CH}_{2} \mathrm{O}\right), 27.9\left(\mathrm{CH}_{2} \mathrm{CH}_{2} \mathrm{CO}\right), 30.1\left(\mathrm{CH}_{3} \mathrm{CO}\right), 33.2\left(\mathrm{CH}_{3} \mathrm{~N}\right), 41.4$ $\left(\mathrm{CH}_{2} \mathrm{CH}_{2} \mathrm{CO}\right), 50.9(\mathrm{ArCH}), 61.0\left(\mathrm{CH}_{3} \mathrm{CH}_{2} \mathrm{O}\right), 101.1$ (CH-Het), $109.8(\mathrm{CH}-\mathrm{Het}), 120.3(\mathrm{CH}-$ Het), 121.7 (CH-Het), 129.1 (C-Het), 129.8 (CH-Het), 129.9 (C-Het), 136.5 (C-Het), 174.5 (EtOOC), $208.1\left(\mathrm{CH}_{3} \mathrm{CO}\right)$. LRMS (IC) found $[\mathrm{M}+\mathrm{H}]^{+}=288$.

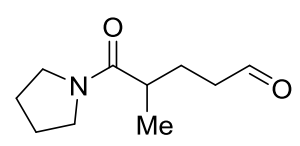

4-methyl-5-oxo-5-(pyrrolidin-1-yl)pentanal (5w): $20 \mathrm{mg}, 44 \%$ yield.

Yellow oil. $\mathbf{R}_{\mathbf{f}}=0.1$ (Diethyl ether $\left.=6: 4\right)$. IR (neat): $v\left(\mathrm{~cm}^{-1}\right)=2952,2924$, 2856, 1724, 1633, 1459, 1440, 1374, 1341, 1254, 1227, 1190, 1170, 1116, 860. ${ }^{1} \mathrm{H}-\mathrm{NMR}\left(\mathrm{CDCl}_{3}, 500 \mathrm{MHz}\right): \delta(\mathrm{ppm})=1.11\left(\mathrm{~d},{ }^{3} \mathrm{~J}_{\mathrm{HH}}=6.8 \mathrm{~Hz}, 3 \mathrm{H}, \mathrm{CH} \mathrm{H}_{3} \mathrm{CH}\right), 1.65-$ $\left.1.74\left(\mathrm{~m}, 1 \mathrm{H}, \mathrm{CH}_{2} \mathrm{CH}_{2} \mathrm{CHO}\right), 1.80-186\left(\mathrm{~m}, 2 \mathrm{H},\left(\mathrm{CH}_{2} \mathrm{CH}_{2}\right)_{2} \mathrm{~N}\right), 1.90-1.97\left(\mathrm{~m}, 2 \mathrm{H}, \mathrm{CH}_{2} \mathrm{CH}_{2}\right)_{2} \mathrm{~N}\right)$, 1.97-2.04 (m, $\left.1 \mathrm{H}, \mathrm{CH}_{2} \mathrm{CH}_{2} \mathrm{CHO}\right)$, 2.35-2.52 (m, 2H, $\left.\mathrm{CH}_{2} \mathrm{CH}_{2} \mathrm{CHO}\right), 2.54-2.63(\mathrm{~m}, 1 \mathrm{H}$, $\left.\mathrm{CH}_{3} \mathrm{CH}\right)$, 3.38-3.49 (m, 4H, $\left.\left(\mathrm{CH}_{2} \mathrm{CH}_{2}\right)_{2} \mathrm{~N}\right), 9.72(\mathrm{~m}, 1 \mathrm{H}, \mathrm{CHO}) .{ }^{13} \mathbf{C}\left\{{ }^{1} \mathrm{H}\right\}-\mathrm{NMR}\left(\mathrm{CDCl}_{3}, 126\right.$ $\mathrm{MHz}): \delta(\mathrm{ppm}) 17.5\left(\mathrm{CH}_{3} \mathrm{CH}\right), 24.4\left(\left(\mathrm{CH}_{2} \mathrm{CH}_{2}\right)_{2} \mathrm{~N}\right), 26.1\left(\mathrm{CH}_{2} \mathrm{CH}_{2} \mathrm{CHO}\right), 26.3\left(\left(\mathrm{CH}_{2} \mathrm{CH}_{2}\right)_{2} \mathrm{~N}\right)$, 
$37.1\left(\mathrm{CHCH}_{3}\right), 41.8\left(\mathrm{CH}_{2} \mathrm{CH}_{2} \mathrm{CHO}\right), 45.9\left(\left(\mathrm{CH}_{2} \mathrm{CH}_{2}\right)_{2} \mathrm{~N}\right), 46.6\left(\left(\mathrm{CH}_{2} \mathrm{CH}_{2}\right)_{2} \mathrm{~N}\right), 174.2(\mathrm{NC}=\mathrm{O})$, $202.5(\mathrm{HC}=\mathrm{O})$. LRMS $(\mathrm{IC})=$ found $[\mathrm{M}+\mathrm{H}]^{+}=184,[\mathrm{M}+\mathrm{Na}]^{+}=206$.

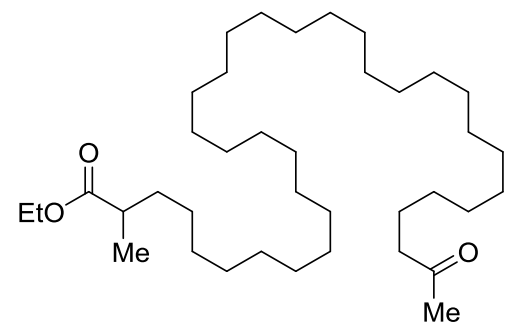

Ethyl 2-methyl-34-oxopentatriacontanoate (5y): $42 \mathrm{mg}$, $72 \%$ yield. Colourless wax. $\mathbf{R}_{\mathrm{f}}=0.3($ Pentane/Diethyl ether $=$ 6:1). IR (neat): $v\left(\mathrm{~cm}^{-1}\right)=2916,2849,1710,1648,1462$, 1370, 728. ${ }^{1} \mathrm{H}$ NMR $\left(400 \mathrm{MHz}, \mathrm{CDCl}_{3}\right) \delta(\mathrm{ppm}) 1.13\left(\mathrm{~d},{ }^{3} \mathrm{JHH}_{\mathrm{H}}\right.$ $\left.=7.0 \mathrm{~Hz}, 3 \mathrm{H}, \mathrm{CH}_{3} \mathrm{CH}\right), 1.22-1.29\left(\mathrm{~m}, 3 \mathrm{H}, \mathrm{CH}_{3} \mathrm{CH}_{2} \mathrm{O} ; 56 \mathrm{H}\right.$,

$\left.\left(\mathrm{CH}_{2}\right)_{28}\right), 1.35-1.42\left(\mathrm{~m}, 2 \mathrm{H}, \mathrm{CH}_{2} \mathrm{CHCOOEt} ; 2 \mathrm{H}, \mathrm{CH}_{2} \mathrm{CH}_{2} \mathrm{CO}\right), 1.54-1.70(\mathrm{~m}, 2 \mathrm{H}$, $\mathrm{CH}_{2} \mathrm{CHCOOEt} ; 2 \mathrm{H}, \mathrm{CH}_{2} \mathrm{CH}_{2} \mathrm{CO}$ ), 2.13 (s, 3H, $\mathrm{CH}_{3} \mathrm{CO}$ ), 2.32 - 2.47 (m, 1H, CHCOOEt; $2 \mathrm{H}$, $\left.\mathrm{CH}_{2} \mathrm{COCH} 3\right), 4.12\left(\mathrm{q},{ }^{3} \mathrm{JHH}_{\mathrm{HH}}=7.1 \mathrm{~Hz}, 2 \mathrm{H}, \mathrm{CH}_{3} \mathrm{CH}_{2} \mathrm{O}\right) .{ }^{13} \mathrm{C}\left\{{ }^{1} \mathrm{H}\right\} \mathrm{NMR}\left(101 \mathrm{MHz}, \mathrm{CDCl}_{3}\right) \delta(\mathrm{ppm})$ $14.4\left(\mathrm{CH}_{3} \mathrm{CH}_{2} \mathrm{O}\right)$, $17.2\left(\mathrm{CH}_{3} \mathrm{CH}\right), 24.0\left(\mathrm{CH}_{2} \mathrm{CH}_{2} \mathrm{CO}\right), 27.4-29.9\left(\left(\mathrm{CH}_{2}\right)_{28}\right), 30.0\left(\mathrm{CH}_{3} \mathrm{CO}\right)$, $34.0\left(\mathrm{CH}_{2} \mathrm{CH}_{2} \mathrm{COOEt}\right), 39.7\left(\mathrm{CH}_{3} \mathrm{CH}\right), 44.0\left(\mathrm{CH}_{2} \mathrm{CO}\right), 60.2\left(\mathrm{CH}_{3} \mathrm{CH}_{2} \mathrm{O}\right), 177.1$ (EtOOC), $209.5\left(\mathrm{CH}_{3} \mathrm{CO}\right)$. LRMS (IC) found $[\mathrm{M}+\mathrm{H}]^{+}=580$.

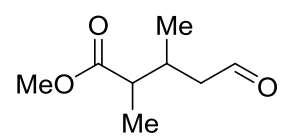

Methyl 2,3-dimethyl-5-oxopentanoate (5x) $(\mathrm{dr}=50: 50): 16 \mathrm{mg}, 40 \%$ yield. Colourless oil. $\mathbf{R}_{\mathbf{f}}=0.6$ (Pentane/Diethyl ether $=6: 4$ ). IR (neat): $v\left(\mathrm{~cm}^{-1}\right)=2949,2886,2724,1725,1637,1457,1436,1381,1257,1197$, 1167, 1094, 1035, 972, 839, 776. ${ }^{1} \mathrm{H}-\mathrm{NMR}\left(\mathrm{C}_{6} \mathrm{D}_{6}, 500 \mathrm{MHz}\right): \delta(\mathrm{ppm})=0.68\left(\mathrm{~d},{ }^{3} \mathrm{JHH}_{\mathrm{HH}}=6.8\right.$ $\left.\mathrm{Hz}, 3 \mathrm{H}, \mathrm{CH}\left(\mathrm{CH}_{3}\right) \mathrm{CH}_{2}\right), 0.86\left(\mathrm{~d},{ }^{3} \mathrm{JHH}_{\mathrm{HH}}=7.1 \mathrm{~Hz}, 3 \mathrm{H}, \mathrm{C}(\mathrm{O}) \mathrm{CH}_{3}\right), 1.71-1.78(\mathrm{~m}, 1 \mathrm{H}$, $\left.\mathrm{CH}\left(\mathrm{CH}_{3}\right) \mathrm{CH}_{2}\right), 1.91-1.96\left(\mathrm{~m}, 1 \mathrm{H}, \mathrm{CH}\left(\mathrm{CH}_{3}\right) \mathrm{CH}_{2}\right), 2.12-2.20(\mathrm{~m}, 1 \mathrm{H}, \mathrm{C}(\mathrm{O}) \mathrm{CH}), 2.22-2.31$ (m, $1 \mathrm{H}, \mathrm{CH}\left(\mathrm{CH}_{3}\right) \mathrm{CH}_{2}$ ), $3.28-3.29$ (s, 3H, $\mathrm{CH}_{3} \mathrm{O}$ ), $9.25-9.27$ (m, 1H, CHO). ${ }^{13} \mathrm{C}\left\{{ }^{1} \mathrm{H}\right\}-\mathrm{NMR}$ $\left(\mathrm{C}_{6} \mathrm{D}_{6}, 126 \mathrm{MHz}\right): \delta$ (ppm) 13.1, $13.8\left(\mathrm{C}(\mathrm{O}) \mathrm{CHCH}_{3}\right), 16.7,17.4\left(\mathrm{CH}\left(\mathrm{CH}_{3}\right) \mathrm{CH}_{2}\right) .36 .7$ $\left(\mathrm{CH}\left(\mathrm{CH}_{3}\right) \mathrm{CH}_{2}\right), 43.9\left(\mathrm{C}(\mathrm{O}) \mathrm{CHCH}_{3}\right), 48.5,47.9\left(\mathrm{CH}\left(\mathrm{CH}_{3}\right) \mathrm{CH}_{2}\right), 50.9,51.0\left(\mathrm{CH}_{3} \mathrm{O}\right)$, 175.0, $175.2\left(\mathrm{CH}_{3} \mathrm{OOC}\right), 199.9,200.0(\mathrm{CHO})$. LRMS $(\mathrm{IC})=$ found $[\mathrm{M}+\mathrm{Na}]^{+}=181,[\mathrm{M}+\mathrm{MeOH}+\mathrm{H}]^{+}=$ 191. 


\subsection{Mechanistic studies}

\subsubsection{Evidence for de-coordinative process}

\subsubsection{Synthesis of $2 m$}

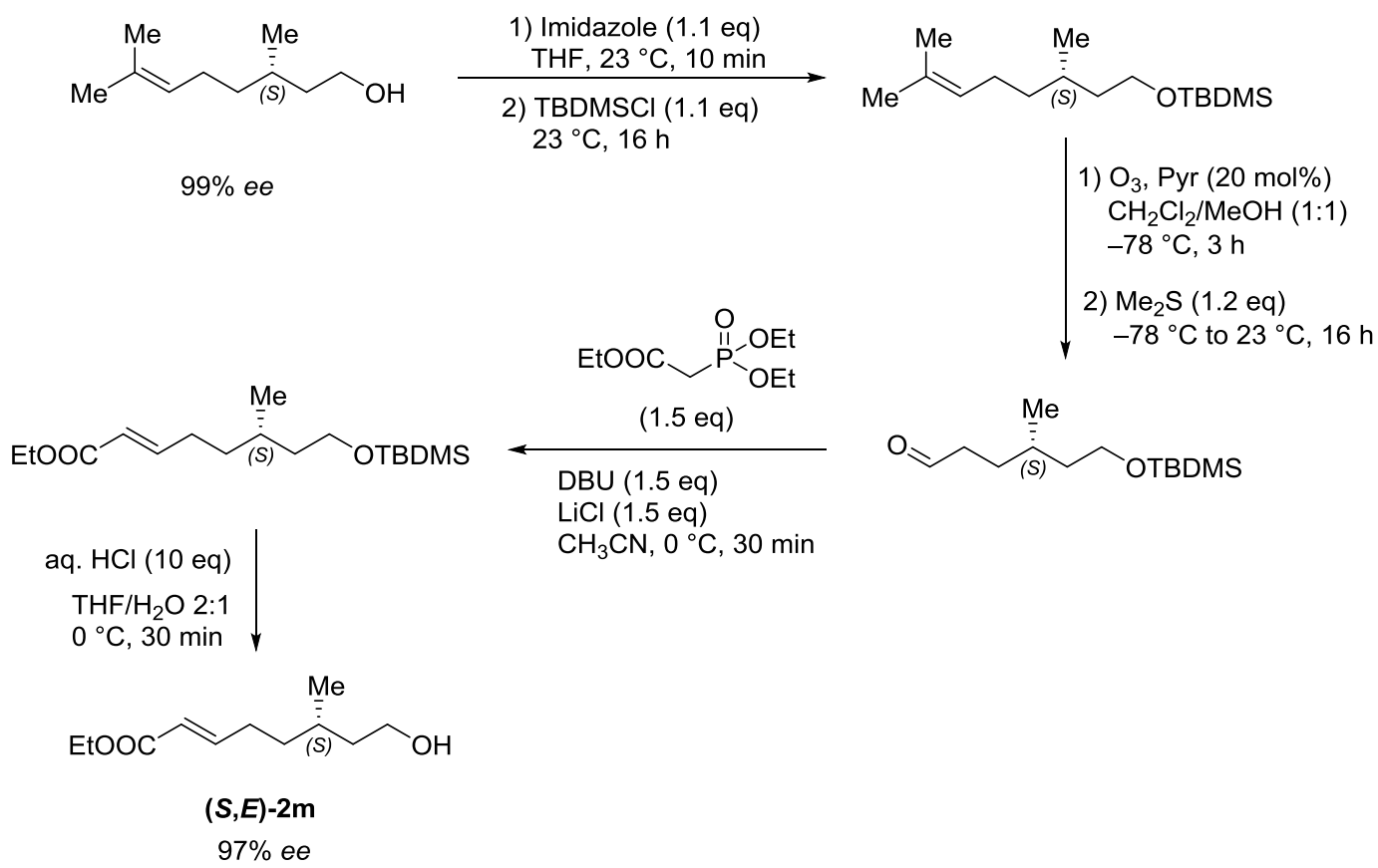

Figure S29: Synthesis of Ethyl $(S, E)$-8-hydroxy-6-methyloct-2-enoate (2m)

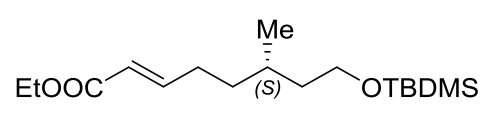

Ethyl (S,E)-8-((tert-butyldimethylsilyl)oxy)-6-methyloct-2quantitative yield according to a procedure reported in the literature. ${ }^{37}$ Subsequent ozonolysis was performed following a known protocol ${ }^{38}$ and the resulting aldehyde was used in the next step without further purification. . The olefination step was run according to GP2 starting from 6-((tert-butyldimethylsilyl)oxy)-4-methylhexanal $(2.17 \mathrm{mmol}, 530 \mathrm{mg})$. The residual oil was purified by flash chromatography on silica gel (Pentane/Diethyl ether = 95:5) to afford ethyl $(S, E)-8$-((tert-butyldimethylsilyl)oxy)-6-methyloct-2-enoate in $86 \%$ yield (588 $\mathrm{mg}$ ) as a colourless oil. $\mathbf{R}_{\mathbf{f}}=0.75$ (Pentane/Diethyl ether $\left.=9: 1\right)$. IR (neat): $v\left(\mathrm{~cm}^{-1}\right)=2955$, 2930, 2858, 1722, 1655, 1464, 1389, 1366, 1306, 1255, 1197, 1169, 1093, 1047, 983, 939,

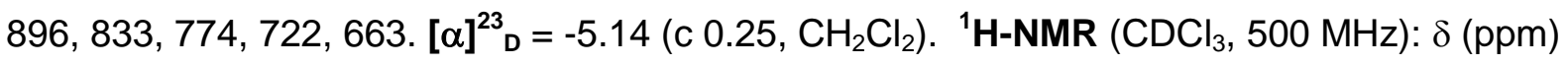
$0.04\left(\mathrm{~s}, 6 \mathrm{H},\left(\mathrm{CH}_{3}\right)_{2} \mathrm{Si}\right), 0.87-0.90\left(\mathrm{~m}, 3 \mathrm{H}, \mathrm{CH}_{3} \mathrm{CH} ; 9 \mathrm{H},\left(\mathrm{CH}_{3}\right)_{3} \mathrm{CSi}\right), 1.28$ (s, 3H, $\mathrm{CH}_{3} \mathrm{CH} \mathrm{O}_{2}$ ), 1.27-1.36 (m, $\left.1 \mathrm{H}, \mathrm{CH}_{2} \mathrm{CH}_{2} \mathrm{CH} ; 1 \mathrm{H}, \mathrm{CHCH}_{2} \mathrm{CH}_{2}\right), 1.44-1.50\left(\mathrm{~m}, 1 \mathrm{H}, \mathrm{CH}_{2} \mathrm{CH}_{2} \mathrm{CH}\right), 1.51$ $-1.61\left(\mathrm{~m}, 1 \mathrm{H}, \mathrm{CH}_{2} \mathrm{CH}_{2} \mathrm{CH} ; 1 \mathrm{H}, \mathrm{CHCH}_{2} \mathrm{CH}\right), 2.17-2.26\left(\mathrm{~m},{ }^{3} \mathrm{~J}_{\mathrm{HH}}=7.1 \mathrm{~Hz}, 2 \mathrm{H}, \mathrm{CH}_{2} \mathrm{CH}_{2} \mathrm{CH}\right)$, $4.18\left(\mathrm{t},{ }^{3} \mathrm{~J}_{\mathrm{HH}}=6.8 \mathrm{~Hz}, 2 \mathrm{H}, \mathrm{CH}_{3} \mathrm{CH}_{2} \mathrm{O}\right), 5.79-5.83(\mathrm{~m}, 1 \mathrm{H}, \mathrm{CH}=\mathrm{CH}), 6.96\left(\mathrm{dt},{ }^{3} \mathrm{~J}_{\mathrm{HH}}=15.5,6.9\right.$ $\left.\mathrm{Hz}, \quad 1 \mathrm{H}, \quad \mathrm{CH}_{2} \mathrm{CH}=\mathrm{CH}\right) .{ }^{13} \mathrm{C}\left\{{ }^{1} \mathrm{H}\right\}-\mathrm{NMR}\left(\mathrm{CDCl}_{3}, 126 \mathrm{MHz}\right): \delta(\mathrm{ppm})-5.1\left(\left(\mathrm{CH}_{3}\right)_{2} \mathrm{Si}\right), 14.4$ $\left(\mathrm{CH}_{3} \mathrm{CH}_{2} \mathrm{O}\right), 18.5\left(\left(\mathrm{CH}_{3}\right)_{3} \mathrm{CSi}\right), 19.6\left(\mathrm{CH}_{3} \mathrm{CH}\right), 26.1\left(\left(\mathrm{CH}_{3}\right)_{3} \mathrm{CSi}\right), 29.2\left(\mathrm{CH}_{2} \mathrm{CH}_{2} \mathrm{CH}\right), 29.9$ 
$\left(\mathrm{CH}_{2} \mathrm{CH}_{2} \mathrm{CH}\right)$, $35.4\left(\mathrm{CH}_{2} \mathrm{CH}_{2} \mathrm{CH}\right), 39.8\left(\mathrm{CHCH}_{2} \mathrm{CH}_{2}\right), 60.3\left(\mathrm{CH}_{3} \mathrm{CH}_{2} \mathrm{O}\right), 61.3\left(\mathrm{CHCH}_{2} \mathrm{CH}_{2}\right)$, $121.3(\mathrm{CH}=\mathrm{CH}), 149.6(\mathrm{CH}=\mathrm{CH}), 166.9(\mathrm{C}=\mathrm{O})$. LRMS $(\mathrm{IC})$ found $[\mathrm{M}+\mathrm{Na}]^{+}=337$.

Ethyl $(S, E)$-8-hydroxy-6-methyloct-2-enoate $(2 \mathrm{~m})$ : The reaction chromatography on silica gel (Pentane/Diethyl ether $=6: 4)(S, E)-2 m$ was obtained in $99 \%$ yield (352 mg) as a colourless oil. $\mathbf{R}_{\mathbf{f}}=0.3$ (Pentane/Diethyl ether $\left.=6: 4\right)$. IR (neat): $v\left(\mathrm{~cm}^{-1}\right)=$ 3409, 2925, 1713, 1653, 1457, 1369, 1268, 1175, 1121, 1046, 984, 918, 849, 732, 647. $[\alpha]^{23}{ }_{\mathrm{D}}=-7.91\left(\mathrm{c} 0.25, \mathrm{CH}_{2} \mathrm{Cl}_{2}\right) .{ }^{1} \mathrm{H}-\mathrm{NMR}\left(\mathrm{CDCl}_{3}, 500 \mathrm{MHz}\right): \delta(\mathrm{ppm}) 0.90\left(\mathrm{~d}, 3 \mathrm{H},{ }^{3} J_{H H}=6.6\right.$ $\mathrm{Hz}, \mathrm{CH}_{3} \mathrm{CH}$ ), $1.22-1.34\left(\mathrm{~m}, 1 \mathrm{H}, \mathrm{CH}_{2} \mathrm{CH}_{2} \mathrm{CH}\right), 1.27$ (s, $\left.{ }^{3} \mathrm{~J}_{\mathrm{HH}}=7.1 \mathrm{~Hz}, 3 \mathrm{H}, \mathrm{CH}_{3} \mathrm{CH}_{2} \mathrm{O}\right), 1.37$ - $1.43\left(\mathrm{~m}, 1 \mathrm{H}, \mathrm{CHCH}_{2} \mathrm{CH}_{2} \mathrm{OH}\right), 1.45-1.52\left(\mathrm{~m}, 1 \mathrm{H}, \mathrm{CH}_{2} \mathrm{CH}_{2} \mathrm{CH} ; 1 \mathrm{H}, \mathrm{OH}\right), 1.57-1.64(\mathrm{~m}$, $\left.1 \mathrm{H}, \mathrm{CHCH}_{2} \mathrm{CH} ; 1 \mathrm{H}, \mathrm{CHCH}_{2} \mathrm{CH}_{2}\right), 2.14-2.29\left(\mathrm{~m}, 1 \mathrm{H}, \mathrm{CH}_{2} \mathrm{CH}_{2} \mathrm{CH}\right), 3.63-3.72(\mathrm{~m}, 2 \mathrm{H}$, $\mathrm{CHCH}_{2} \mathrm{CH}_{2} \mathrm{OH}$ ), 4.17 (q, ${ }^{3} \mathrm{~J}_{\mathrm{HH}}=7.1 \mathrm{~Hz}, 2 \mathrm{H}, \mathrm{CH}_{3} \mathrm{CH}_{2} \mathrm{O}$ ), 5.81 (dt, ${ }^{3} \mathrm{~J}_{\mathrm{HH}}=15.6 \mathrm{~Hz},{ }^{4} \mathrm{~J}_{\mathrm{HH}}=1.6$ $\mathrm{Hz}, 1 \mathrm{H}, \mathrm{CH}=\mathrm{CH}), 6.95\left(\mathrm{~m},{ }^{3} \mathrm{~J}_{\mathrm{HH}}=15.6 \mathrm{~Hz}, 6.9 \mathrm{~Hz}, 1 \mathrm{H}, \mathrm{CH}=\mathrm{CH}\right) \cdot{ }^{13} \mathrm{C}\left\{{ }^{1} \mathrm{H}\right\}-\mathrm{NMR}\left(\mathrm{CDCl}_{3}, 126\right.$ $\mathrm{MHz}): \delta$ (ppm) $14.4\left(\mathrm{CH}_{3} \mathrm{CH}_{2} \mathrm{O}\right), 19.4\left(\mathrm{CH}_{3} \mathrm{CH}\right), 29.8\left(\mathrm{CH}_{2} \mathrm{CH}_{2} \mathrm{CH}\right), 29.2\left(\mathrm{CH}_{2} \mathrm{CH}_{2} \mathrm{CH}\right), 35.4$ $\left(\mathrm{CH}_{2} \mathrm{CH}_{2} \mathrm{CH}\right), 39.8\left(\mathrm{CHCH}_{2} \mathrm{CH}_{2}\right), 60.3\left(\mathrm{CH}_{3} \mathrm{CH}_{2} \mathrm{O}\right), 61.0\left(\mathrm{CHCH}_{2} \mathrm{CH}_{2}\right), 121.4\left(\mathrm{CH}=\mathrm{CHCH}_{2}\right)$, $149.4\left(\mathrm{CH}=\mathrm{CHCH}_{2}\right), 166.9(\mathrm{C}=\mathrm{O})$. LRMS $(\mathrm{IC})$ found $[\mathrm{M}+\mathrm{H}]^{+}=201$.

\subsubsection{Isomerization of $2 \mathrm{~m}$}<smiles>CCOC(=O)C=CCC[C@@H](C)CCO</smiles>

$(S, E)-2 m$

$0.25 \mathrm{mmol}$

$97 \%$ ee
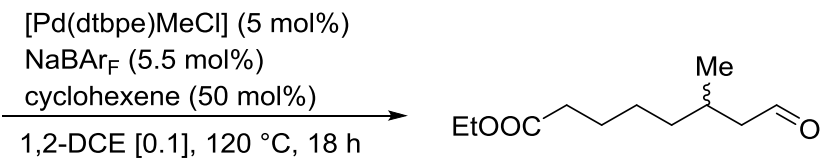

$3 \mathrm{~m}$

Conv. $95 \%$

Yield $75 \%$

$0 \%$ ee

The reaction was performed according to GP1. The enantiomeric excess (ee) of $\mathbf{2 m}$ (Figure S30) was measured by HPLC (column OJ-H, Hexane/iPrOH 98:2, $1 \mathrm{~mL} / \mathrm{min}, \lambda 205 \mathrm{~nm}$ ) and those of $3 \mathrm{~m}$ (Figure S31) by GC (column HYDRODEX $\gamma$-DiMOM, method $100{ }^{\circ} \mathrm{C}$ for 120 minutes then $100-170{ }^{\circ} \mathrm{C}$ in 70 minutes). 
mAU

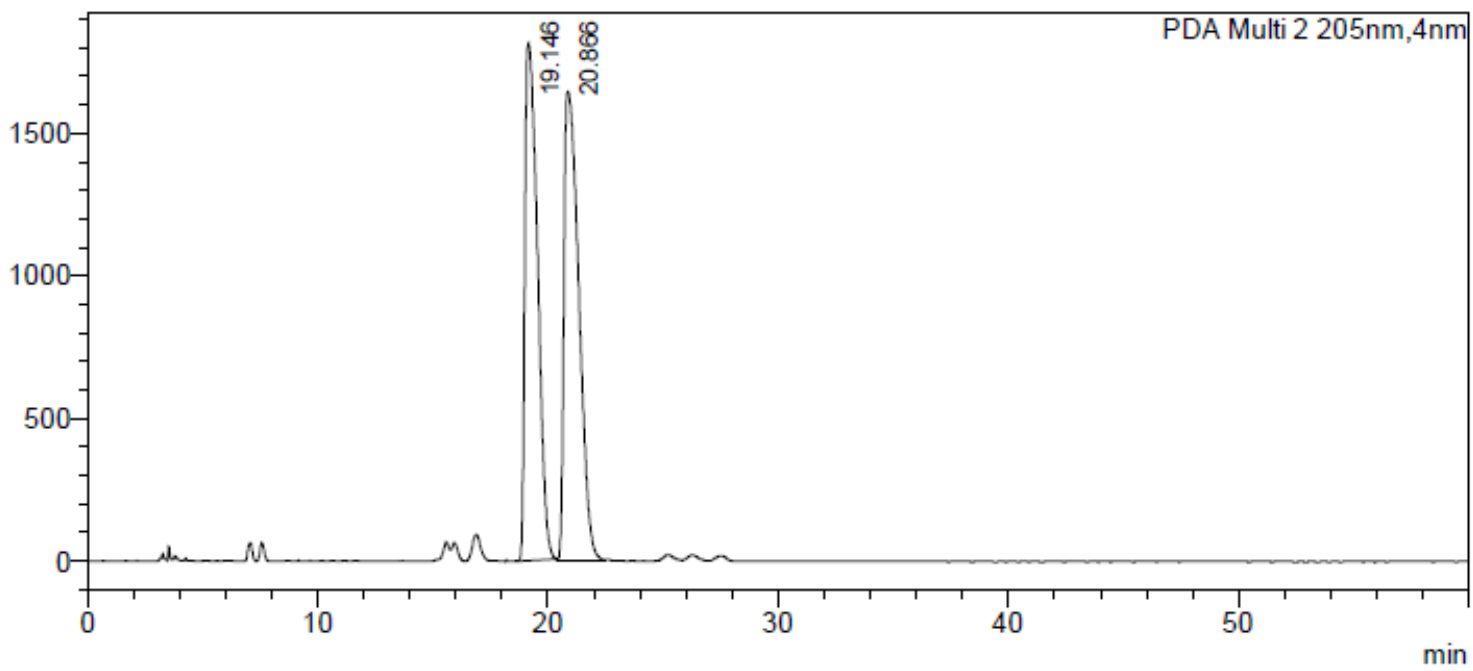

<Peak Table>

PDA Ch2 205nm

\begin{tabular}{|r|r|r}
\hline Peak\# & Ret. Time & Area $\%$ \\
\hline
\end{tabular}

\begin{tabular}{|c|c|c|}
\hline & 490 & \\
\hline 1 & 19.146 & 48.467 \\
\hline 2 & 20.866 & 51.533 \\
\hline Total & & 100.000 \\
\hline
\end{tabular}

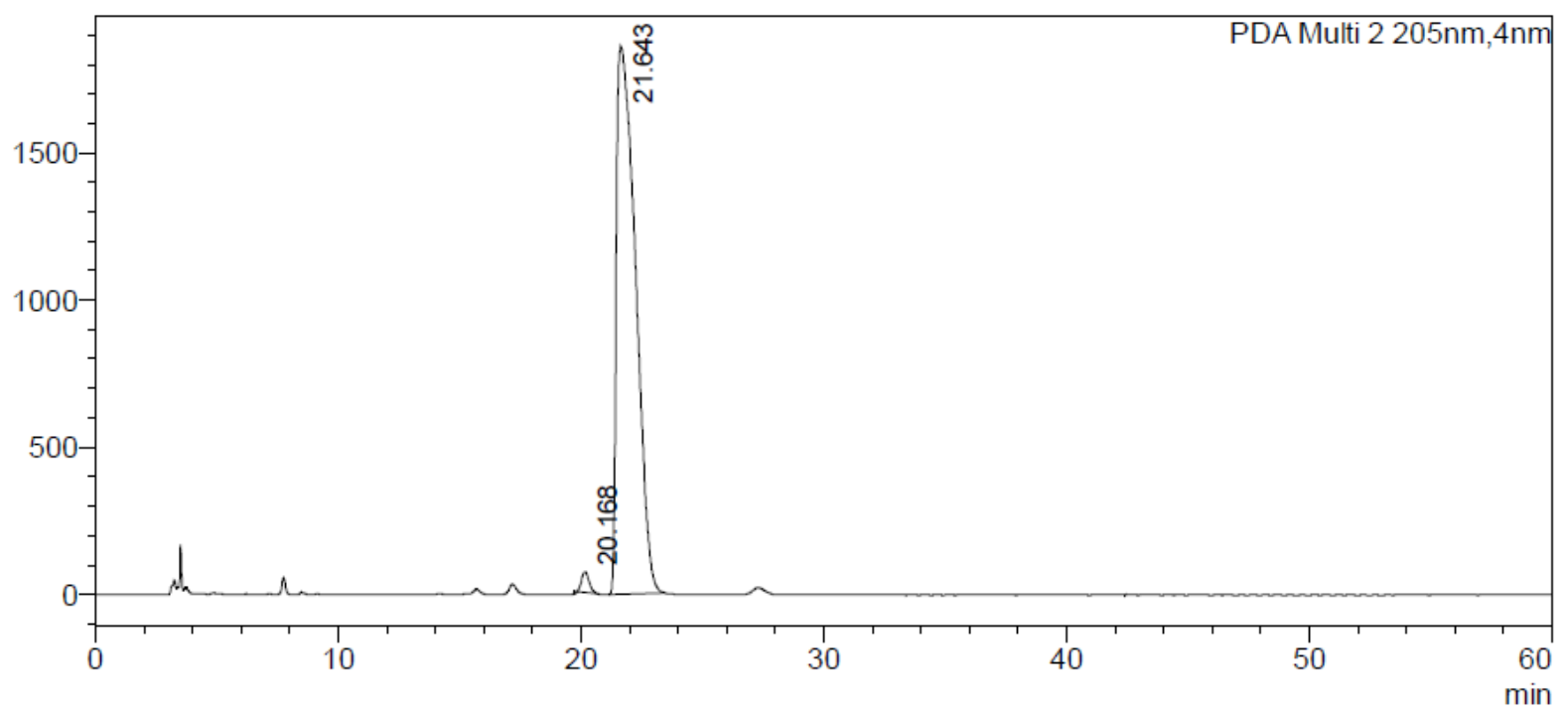

PDA Ch2 205nm

\begin{tabular}{|r|r|r|}
\hline Peak\# & Ret. Time & \multicolumn{1}{|c|}{ Area\% } \\
\hline 1 & 20.168 & 1.488 \\
\hline 2 & 21.643 & 98.512 \\
\hline Total & & 100.000 \\
\hline
\end{tabular}

Figure S30: HPLC traces of racemic $\mathbf{2 m}$ (top) and enantio-enriched $\mathbf{2 m}$ (bottom) 

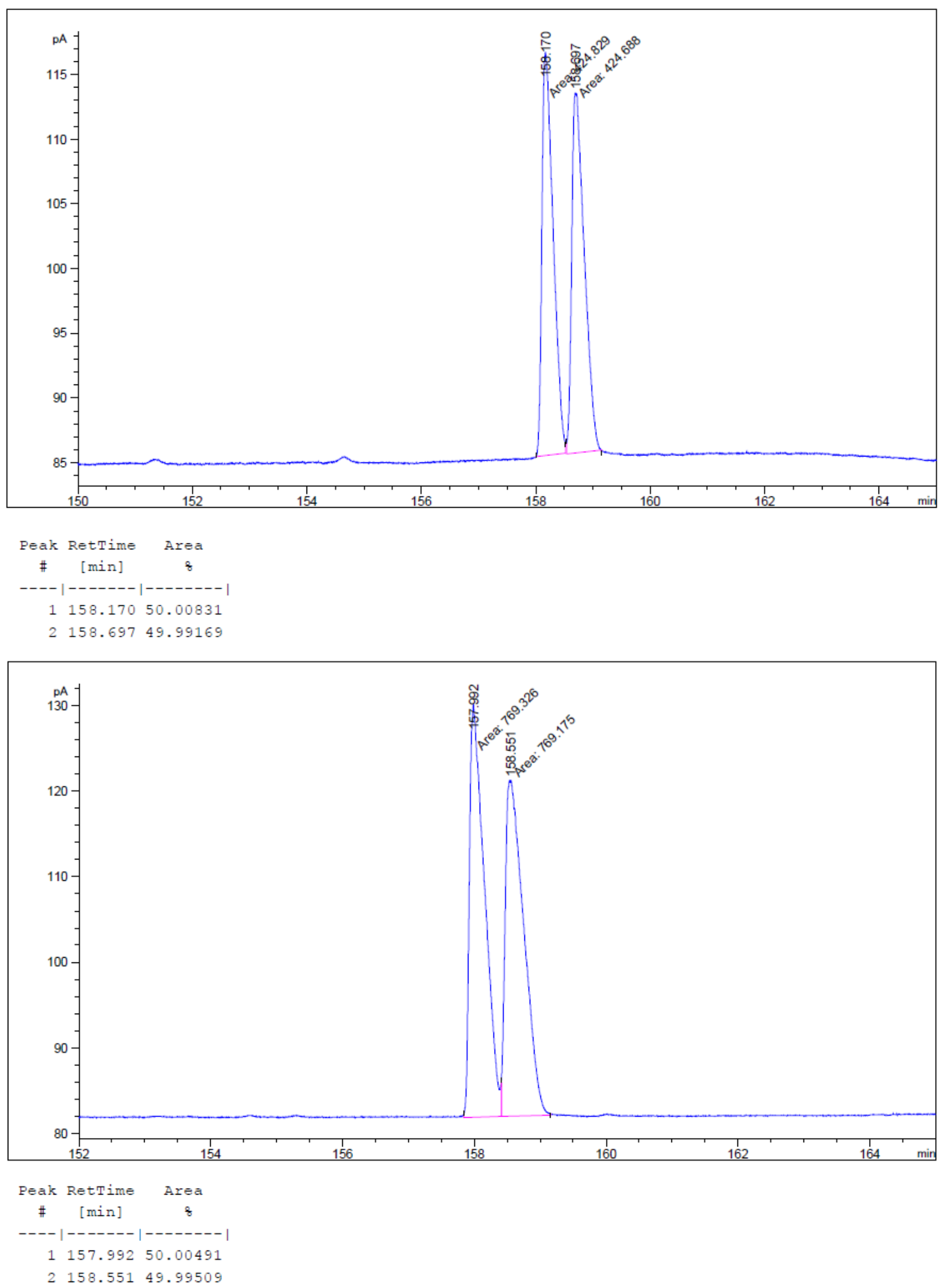

Figure S31: GC traces of $\mathbf{3 m}$, starting from racemic $\mathbf{2 m}$ (top) and enantio-enriched $\mathbf{2 m}$ (bottom) 


\subsubsection{Assessment of the bidirectional nature of the process}

\subsubsection{Synthesis of 20}

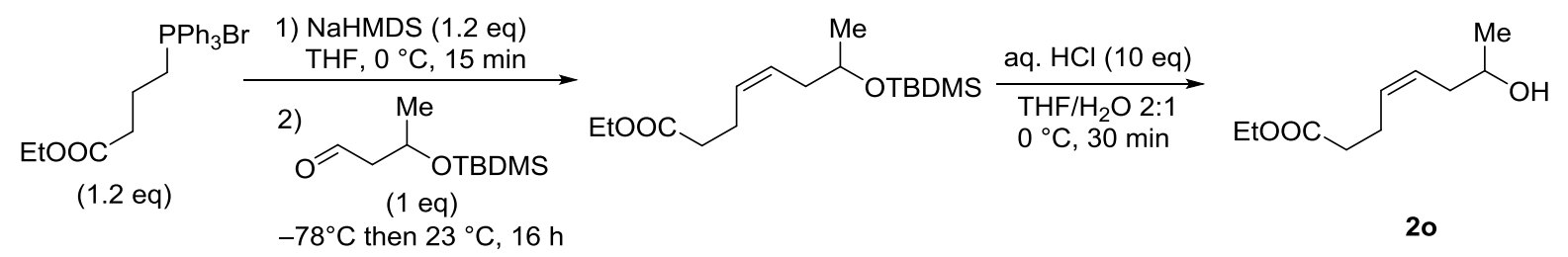

Figure S32: Synthesis of Ethyl (Z)-7-hydroxyoct-4-enoate (20)

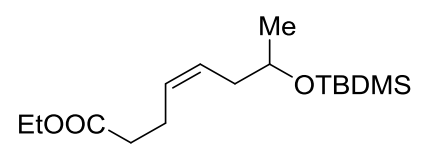

Ethyl (Z)-7-((tert-butyldimethylsilyl)oxy)oct-4-enoate: Ethyl 4(bromotriphenyl-15-phosphanyl)butanoate was prepared from ethyl 4-bromobutanoate according to a known procedure reported in the literature..$^{39}$ In a 3-neck flask, equipped with a dropping funnel, ethyl 4-(bromotriphenyl15-phosphanyl)butanoate $(4.56 \mathrm{mmol}, 2 \mathrm{~g})$ was dissolved in THF $(18 \mathrm{~mL})$ and cooled to $0{ }^{\circ} \mathrm{C}$ before the dropwise addition of NaHMDS (4.56 mmol, $2.3 \mathrm{~mL}$ from a $2 \mathrm{M}$ solution in THF). After 15 minutes, this solution was cooled to $-78{ }^{\circ} \mathrm{C}$ and $3-($ (tertbutyldimethylsilyl)oxy)butanal (3.8 mmol, $769 \mathrm{mg}$ ) in THF (15 mL) added dropwise. After 16 $\mathrm{h}$ at room temperature, the reaction was quenched with water, extracted with diethyl ether (3 $\times 30 \mathrm{~mL}$ ), the organic phases combined, dried over $\mathrm{Na}_{2} \mathrm{SO}_{4}$ and the solvent evaporated. The residue was purified by flash chromatography on silica gel (Pentane/Diethyl ether $=98: 2$ ) to afford ethyl (Z)-7-((tert-butyldimethylsilyl)oxy)oct-4-enoate in $58 \%$ yield $(661 \mathrm{mg})$ as a pale yellow oil. $\mathbf{R}_{\mathbf{f}}=0.6$ (Pentane/Diethyl ether $=9: 1$ ). IR (neat) $v\left(\mathrm{~cm}^{-1}\right)=2957,2931,2858$, 1736, 1463, 1373, 1252, 1161, 1131, 1087, 1049, 1004, 832, 809, 774, 725, 662. 'H NMR $\left(500 \mathrm{MHz}, \mathrm{CDCl}_{3}\right) \delta$ (ppm) $0.04\left(\mathrm{~s}, 3 \mathrm{H},\left(\mathrm{CH}_{3}\right)_{2} \mathrm{Si}\right), 0.05\left(\mathrm{~s}, 3 \mathrm{H},\left(\mathrm{CH}_{3}\right)_{2} \mathrm{Si}\right), 0.88(\mathrm{~s}, 9 \mathrm{H}$, $\left.\left(\mathrm{CH}_{3}\right)_{3} \mathrm{CSi}\right), 1.12\left(\mathrm{~d},{ }^{3} \mathrm{JHH}_{\mathrm{HH}}=6.1 \mathrm{~Hz}, 3 \mathrm{H}, \mathrm{CH}_{3} \mathrm{CH}\right), 1.25\left(\mathrm{t},{ }^{3} J_{\mathrm{HH}}=7.1 \mathrm{~Hz}, 3 \mathrm{H}, \mathrm{CH}_{3} \mathrm{CH}_{2} \mathrm{O}\right), 2.13-$ $2.25\left(\mathrm{~m}, 2 \mathrm{H}, \mathrm{CH}_{2} \mathrm{CH}\right), 2.32-2.39\left(\mathrm{~m}, 2 \mathrm{H}, \mathrm{CH}_{2} \mathrm{CH}_{2} \mathrm{CH}=\mathrm{CH} ; 2 \mathrm{H}, \mathrm{CH}_{2} \mathrm{CH}_{2} \mathrm{CH}=\mathrm{CH}\right)$, $3.82(\mathrm{~h}$, $\left.{ }^{3} \mathrm{JHH}_{\mathrm{HH}}=6.1 \mathrm{~Hz}, 1 \mathrm{H}, \mathrm{CH}_{2} \mathrm{CH}\right), 4.11\left(\mathrm{q},{ }^{3} \mathrm{JHH}_{\mathrm{HH}}=7.1 \mathrm{~Hz}, 2 \mathrm{H}, \mathrm{CH}_{3} \mathrm{CH}_{2} \mathrm{O}\right), 5.39-5.48(\mathrm{~m}, 1 \mathrm{H}$, $\left.\left.\mathrm{CH}_{2} \mathrm{CH}_{2} \mathrm{CH}=\mathrm{CH} ; 1 \mathrm{H}, \mathrm{CH}_{2} \mathrm{CH}_{2} \mathrm{CH}=\mathrm{CH}\right) .{ }^{13} \mathrm{C}^{1}{ }^{1} \mathrm{H}\right\} \quad \mathrm{NMR}\left(126 \mathrm{MHz}, \mathrm{CDCl}_{3}\right) \delta(\mathrm{ppm})-4.4$ $\left(\left(\mathrm{CH}_{3}\right)_{2} \mathrm{Si}\right)$, - $4.5\left(\left(\mathrm{CH}_{3}\right)_{2} \mathrm{Si}\right), 14.2\left(\mathrm{CH}_{3} \mathrm{CH}_{2} \mathrm{O}\right), 18.3\left(\left(\mathrm{CH}_{3}\right)_{3} \mathrm{CSi}\right), 23.1\left(\mathrm{CH}_{2} \mathrm{CH}_{2} \mathrm{CH}=\mathrm{CH}\right), 23.7$ $\left(\mathrm{CH}_{3} \mathrm{CH}\right), 26.0\left(\left(\mathrm{CH}_{3}\right)_{3} \mathrm{CSi}\right), 34.5\left(\mathrm{CH}_{2} \mathrm{CH}_{2} \mathrm{CH}=\mathrm{CH}\right), 37.6\left(\mathrm{CH}_{2} \mathrm{CH}\right), 60.5\left(\mathrm{CH}_{3} \mathrm{CH}_{2} \mathrm{O}\right), 68.7$ $\left(\mathrm{CH}_{2} \mathrm{CH}\right), 127.9\left(\mathrm{CH}_{2} \mathrm{CH}_{2} \mathrm{CH}=\mathrm{CH}\right), 129.1\left(\mathrm{CH}_{2} \mathrm{CH}_{2} \mathrm{CH}=\mathrm{CH}\right), 173.3(\mathrm{C}=\mathrm{O})$. LRMS (IC) found $[\mathrm{M}+\mathrm{Na}]^{+}=319$.<smiles>CCOC(=O)CC/C=C\CC(O)O</smiles>

Ethyl (Z)-7-hydroxyoct-4-enoate (20): The reaction was performed according to GP4 starting from ethyl (Z)-7-((tertbutyldimethylsilyl)oxy)oct-4-enoate $(2.20 \mathrm{mmol}, 661 \mathrm{mg})$. After 
purification by flash chromatography on silica gel (Pentane/Diethyl ether $=7: 3) 20$ was obtained in $78 \%$ yield $\left(314 \mathrm{mg}\right.$ ) as a pale yellow oil. $\mathbf{R}_{\mathbf{f}}=0.2$ ( $n$-Pentane/Diethyl ether $\left.=6: 4\right)$. ${ }^{1} \mathrm{H}$ NMR $\left(500 \mathrm{MHz}, \mathrm{CDCl}_{3}\right) \delta(\mathrm{ppm}) 1.22\left(\mathrm{~d},{ }^{3} \mathrm{~J}_{\mathrm{HH}}=6.2 \mathrm{~Hz}, 3 \mathrm{H}, \mathrm{CH}_{3} \mathrm{CH}\right), 1.25\left(\mathrm{t},{ }^{3} \mathrm{~J}_{\mathrm{HH}}=7.1\right.$, $3 \mathrm{H}, \mathrm{CH}_{3} \mathrm{CH}_{2} \mathrm{O}$ ), 1.59 (bs, $\left.1 \mathrm{H}, \mathrm{OH}\right), 2.18-2.23\left(\mathrm{~m}, 1 \mathrm{H}, \mathrm{CH}_{2} \mathrm{CH}\right), 2.26-2.31(\mathrm{~m}, 1 \mathrm{H}$, $\left.\mathrm{CH}_{2} \mathrm{CH}\right), 2.34-2.45\left(\mathrm{~m}, 2 \mathrm{H}, \mathrm{CH}_{2} \mathrm{CH}_{2} \mathrm{CH}=\mathrm{CH} ; 2 \mathrm{H}, \mathrm{CH}_{2} \mathrm{CH}_{2} \mathrm{CH}=\mathrm{CH}\right), 3.81-3.87(\mathrm{~m}, 1 \mathrm{H}$, $\mathrm{CH}_{2} \mathrm{CH}$ ), $4.13\left(\mathrm{t}, 2 \mathrm{H},{ }^{3} \mathrm{~J}_{\mathrm{HH}}=7.1 \mathrm{~Hz}, \mathrm{CH}_{3} \mathrm{CH}_{2} \mathrm{O}\right), 5.45-5.54\left(\mathrm{~m}, 1 \mathrm{H}, \mathrm{CH}_{2} \mathrm{CH}_{2} \mathrm{CH}=\mathrm{CH} ; 1 \mathrm{H}\right.$, $\left.\mathrm{CH}_{2} \mathrm{CH}_{2} \mathrm{CH}=\mathrm{CH}\right) \cdot{ }^{13} \mathrm{C}\left\{{ }^{1} \mathrm{H}\right\} \quad$ NMR (126 $\left.\mathrm{MHz}, \mathrm{CDCl}_{3}\right) \delta(\mathrm{ppm}) 14.4\left(\mathrm{CH}_{3} \mathrm{CH}_{2} \mathrm{O}\right), 23.0$ $\left(\mathrm{CH}_{2} \mathrm{CH}_{2} \mathrm{CH}=\mathrm{CH}\right), 23.1\left(\mathrm{CH}_{3} \mathrm{CH}\right), 34.2\left(\mathrm{CH}_{2} \mathrm{CH}_{2} \mathrm{CH}=\mathrm{CH}\right), 37.2\left(\mathrm{CH}_{2} \mathrm{CH}\right), 60.6\left(\mathrm{CH}_{3} \mathrm{CH}_{2} \mathrm{O}\right)$, $67.7\left(\mathrm{CH}_{2} \mathrm{CH}\right), 127.1\left(\mathrm{CH}_{2} \mathrm{CH}_{2} \mathrm{CH}=\mathrm{CH}\right), 131.0\left(\mathrm{CH}_{2} \mathrm{CH}_{2} \mathrm{CH}=\mathrm{CH}\right), 173.4(\mathrm{C}=\mathrm{O})$. LRMS (IC) found $[\mathrm{M}+\mathrm{H}]^{+}=187$. IR (neat): $v\left(\mathrm{~cm}^{-1}\right)=3417,2971,2932,1732,1451,1422,1373,1378$, 1258, 1163, 1120, 1163, 1120, 1077, 1041, 941, 885, 720.

\subsubsection{Isotopic labelling experiments}

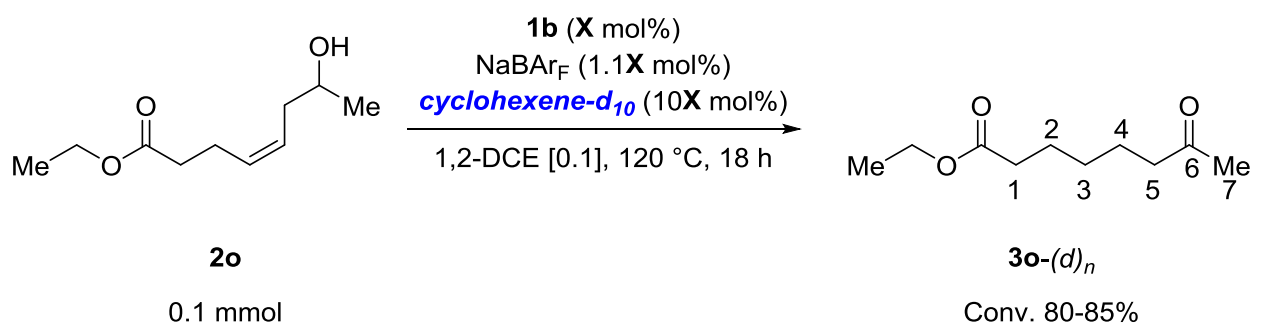

Inside the glovebox, a $5 \mathrm{~mL}$ Young valve Schlenk was charged with precatalyst $\mathbf{1 b}(\mathbf{X ~ m m o l})$, $\operatorname{NaBAr}_{F}(1.1 \mathrm{X} \mathrm{mmol})$ and 1,2-dichloroethane $(1 \mathrm{~mL})$. After 5 minutes at room temperature, cyclohexene- $d_{10}(10 \mathrm{X} \mathrm{mmol}, 98 \%$ in deuterium) was added and the mixture stirred for 5 minutes at room temperature. Substrate $20(0.1 \mathrm{mmol})$ was added, the Schlenk sealed and immersed in an oil bath pre-heated at $120^{\circ} \mathrm{C}$. After $18 \mathrm{~h}$, the reaction mixture was cooled to room temperature, the solvent was removed in vacuo and 1,1,2,2-tetrachloroethane $(0.05$ mmol) was added as internal standard. The conversion was assessed by ${ }^{1} \mathrm{H}-\mathrm{NMR}$ while the extent of deuterium incorporation was established by comparison of the ${ }^{1} \mathrm{H}-\mathrm{NMR}$ spectra of 30 and 30- $(d)_{n}$ at different catalyst loadings (Figure S34-S36).

The ${ }^{2} \mathrm{H}-\mathrm{NMR}$ spectra of the crude reaction mixtures at different catalyst loadings are shown below (Figure S37-S39) to qualitatively support the effective deuterium incorporation in 30$(d)_{n}$. 


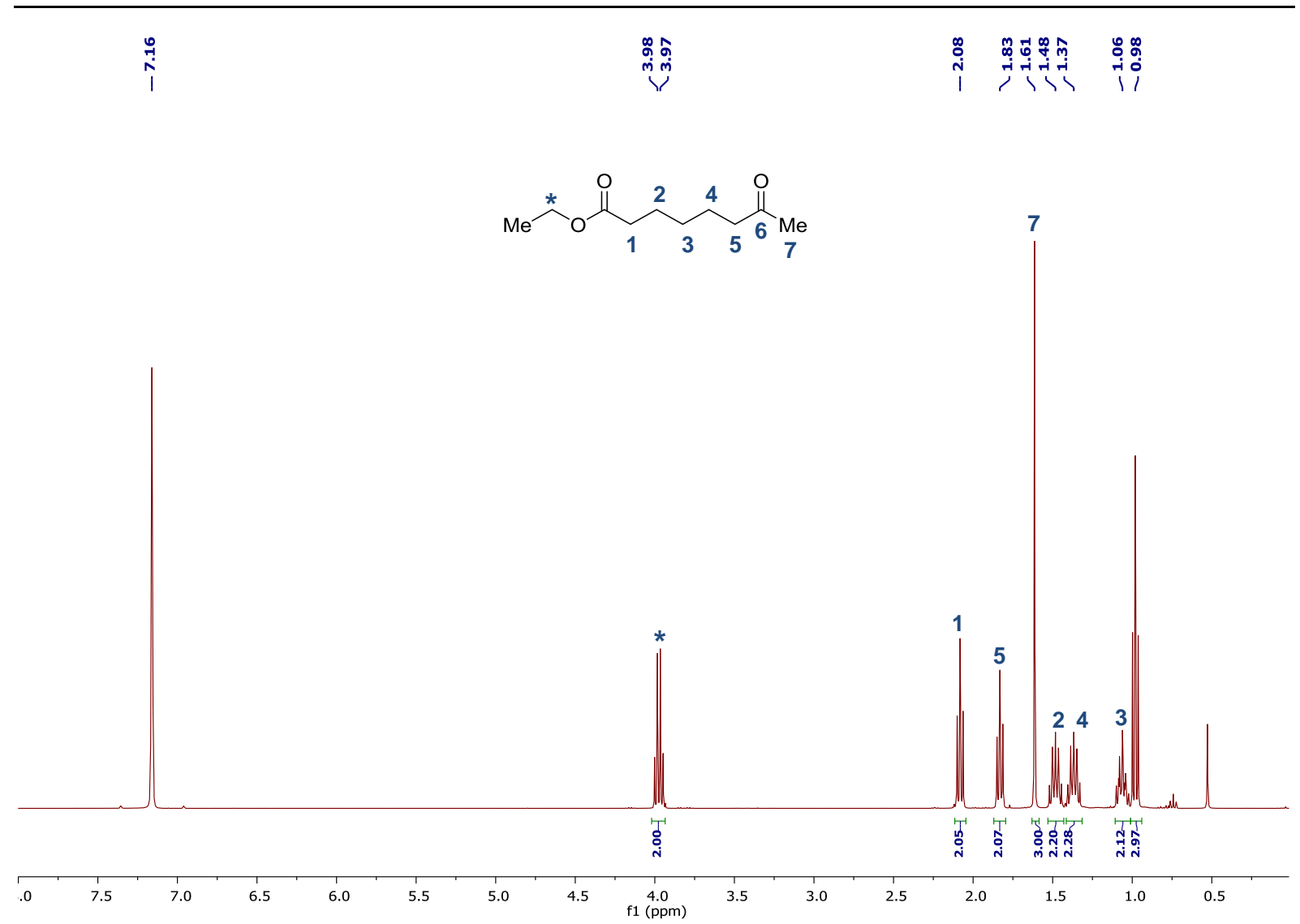

Figure S33: ${ }^{1} \mathrm{H}-\mathrm{NMR}$ in $\mathrm{C}_{6} \mathrm{D}_{6}$ of $\mathbf{3 0}$

\begin{tabular}{lllllll}
\hline $\mathbf{1 b}$ (mol\%) & pos. 1 & pos. 2 & pos. 3 & pos. 4 & pos. 5 & pos. 7 \\
\hline 10 & $2.0 \%$ & $3.0 \%$ & $3.3 \%$ & $0.9 \%$ & $3.6 \%$ & $0.7 \%$ \\
20 & $2.5 \%$ & $1.6 \%$ & $2.9 \%$ & $2.2 \%$ & $3.6 \%$ & $0.7 \%$ \\
$\mathbf{4 0}$ & $2.0 \%$ & $2.1 \%$ & $5.0 \%$ & n.d. $^{2}$ & $4.4 \%$ & $0.6 \%$ \\
\hline
\end{tabular}

${ }^{a}$ Integration higher than in 30 because of an overlap with signals from the catalyst

Table 1: Extent of deuterium incorporation at various catalyst loadings 


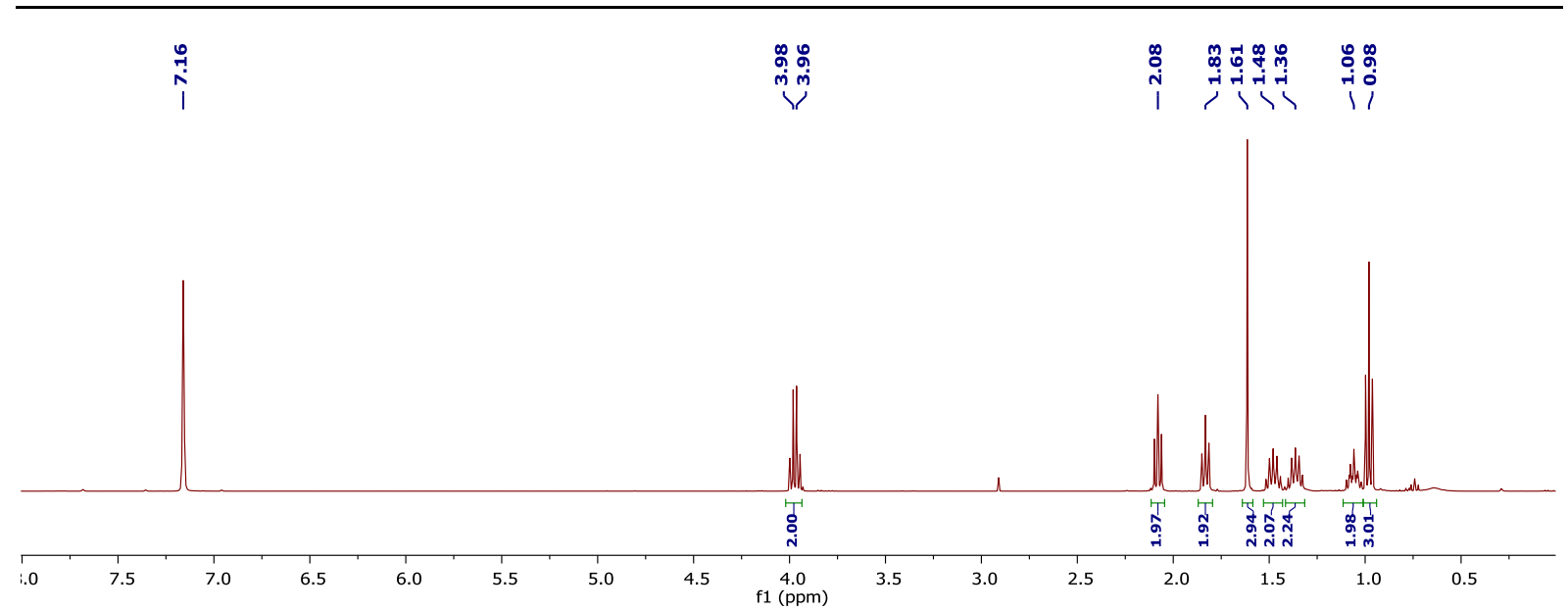

Figure S34: ${ }^{1} \mathrm{H}-\mathrm{NMR}$ in $\mathrm{C}_{6} \mathrm{D}_{6}$ of the crude reaction mixture using $10 \mathrm{~mol} \%$ of $\mathbf{1 b}$

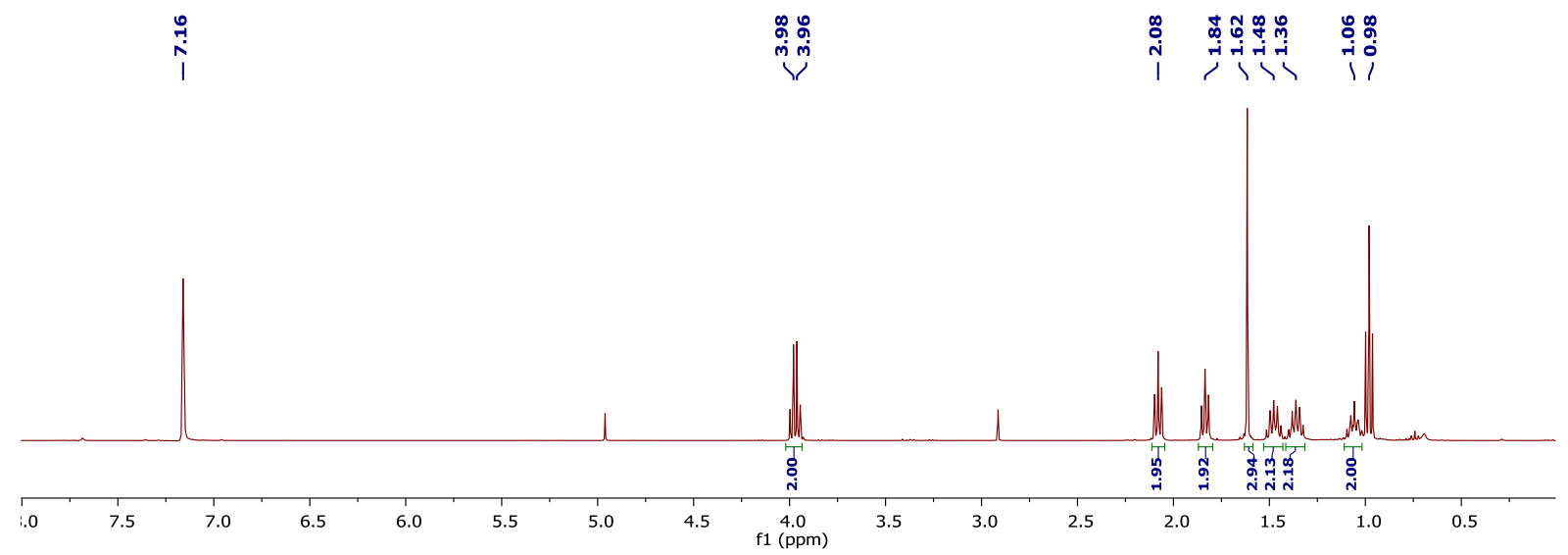

Figure S35: ${ }^{1} \mathrm{H}-\mathrm{NMR}$ in $\mathrm{C}_{6} \mathrm{D}_{6}$ of the crude reaction mixture using $20 \mathrm{~mol} \%$ of $\mathbf{1 b}$

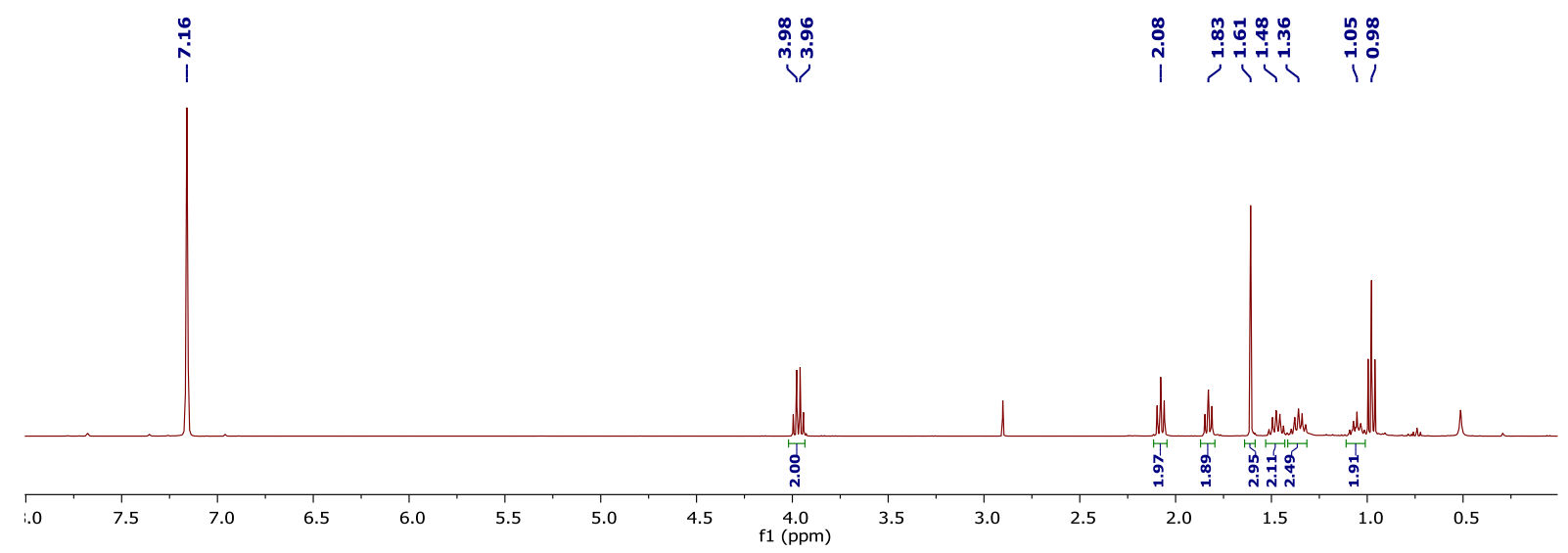

Figure S36: ${ }^{1} \mathrm{H}-\mathrm{NMR}$ in $\mathrm{C}_{6} \mathrm{D}_{6}$ of the crude reaction mixture using $40 \mathrm{~mol} \%$ of $\mathbf{1 b}$ 


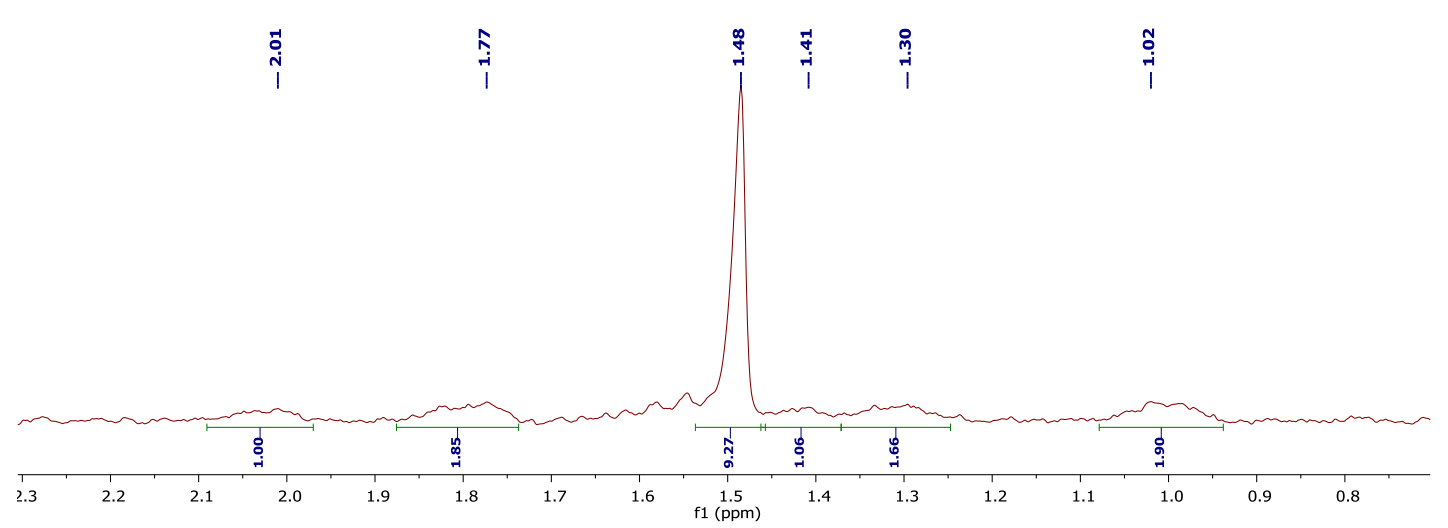

Figure S37: Expansion of ${ }^{2} \mathrm{H}-\mathrm{NMR}$ in $\mathrm{C}_{6} \mathrm{D}_{6}$ of the crude reaction mixture using $10 \mathrm{~mol} \%$ of $1 b$

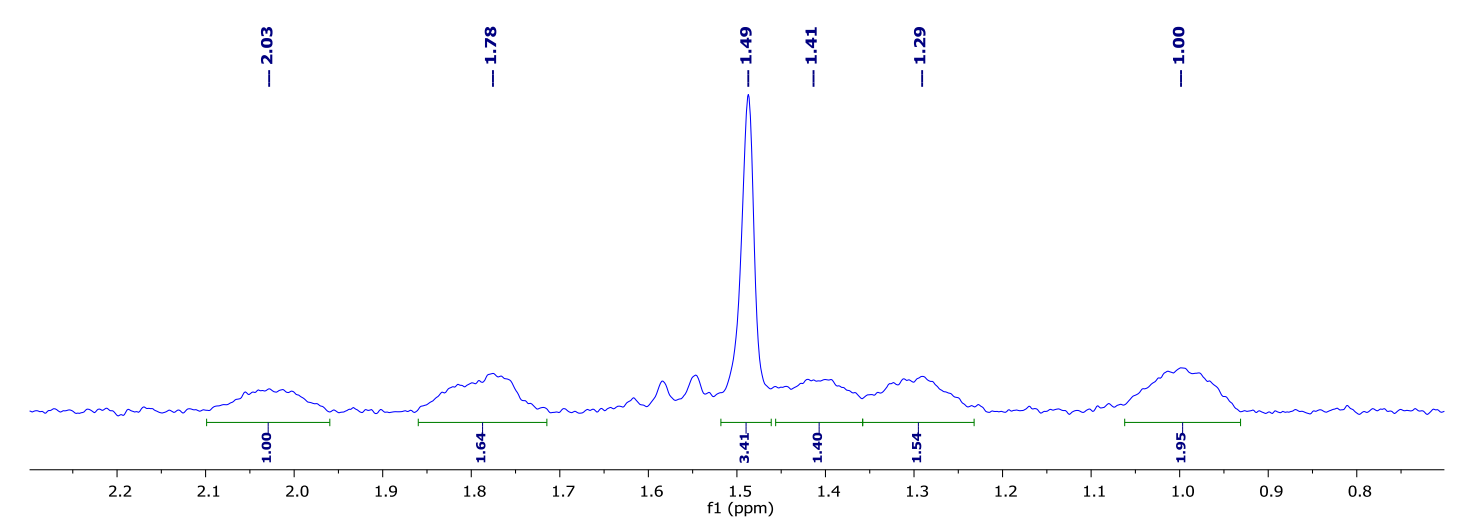

Figure S38: Expansion of ${ }^{2} \mathrm{H}-\mathrm{NMR}$ in $\mathrm{C}_{6} \mathrm{D}_{6}$ of the crude reaction mixture using $20 \mathrm{~mol} \%$ of 1b

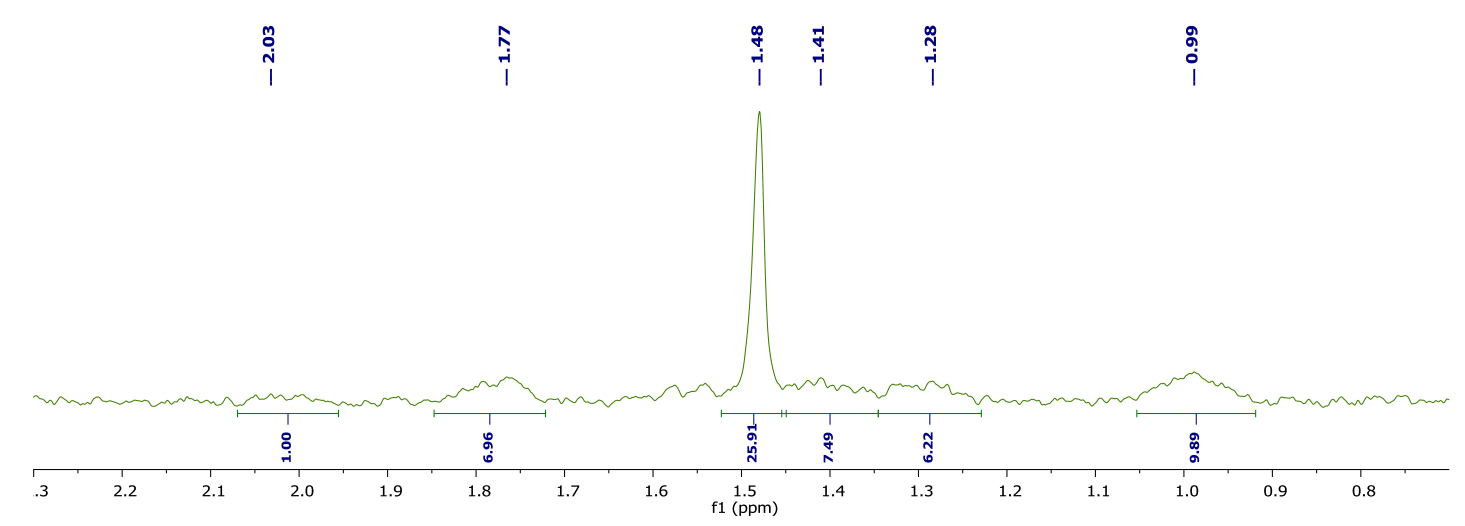

Figure S39: Expansion of ${ }^{2} \mathrm{H}-\mathrm{NMR}$ in $\mathrm{C}_{6} \mathrm{D}_{6}$ of the crude reaction mixture using $40 \mathrm{~mol} \%$ of 


\subsubsection{Assessment of site-selectivity of $[\mathrm{Pd}-\mathrm{H}]$ insertion}

\subsubsection{Synthesis of $2 p$}

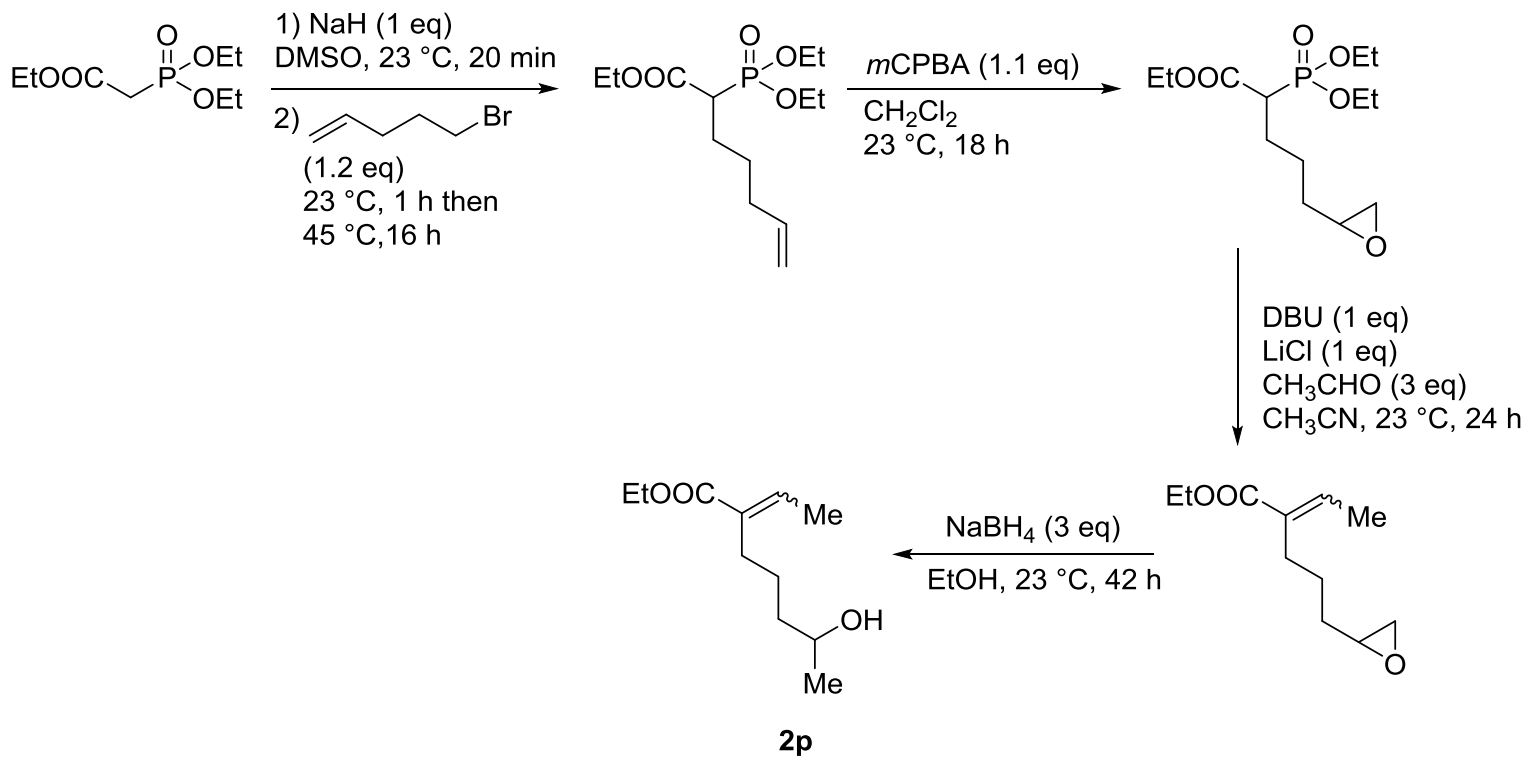

Figure S40: Synthesis of Ethyl 2-ethylidene-6-hydroxyheptanoate (2p)

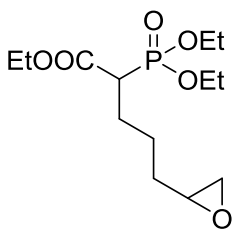

1-(diethoxyphosphoryl)-4-(oxiran-2-yl)butyl propionate: The $\alpha$ alkylation of triethyl phosphonacetate was performed according to a know procedure $^{40}$ affording ethyl 2-(diethoxyphosphoryl)hept-6-enoate in $71 \%$ yield. In a round-bottom flask the $\alpha$-alkylated phosphonacetate $(5 \mathrm{mmol}$, $1.46 \mathrm{~g}$ ) was dissolved in $\mathrm{CH}_{2} \mathrm{Cl}_{2}(12.5 \mathrm{~mL})$ and cooled to $0{ }^{\circ} \mathrm{C}$. Next, $m \mathrm{CPBA}$ (70\% wt purity, $5.5 \mathrm{mmol}, 1.36 \mathrm{~g}$ ) was added in two portions. After $18 \mathrm{~h}$ at room temperature, all starting material had been consumed (TLC monitoring) and water $(10 \mathrm{~mL})$ was added. The mixture was extracted with $\mathrm{CH}_{2} \mathrm{Cl}_{2}(3 \times 15 \mathrm{~mL})$, washed with $5 \%$ aqueous $\mathrm{NaOH}$, a saturated aqueous $\mathrm{NaHCO}_{3}$ solution, water and brine. The organic phases were combined, dried over $\mathrm{Na}_{2} \mathrm{SO}_{4}$ and the solvent removed in vacuo. The residue was purified by flash chromatography on silica gel (Diethyl ether) to afford the epoxide as a pale yellow oil in $72 \%$ $(1.1 \mathrm{~g}) . \mathbf{R}_{\mathrm{f}}=0.2$ (Diethyl ether = 6:4). ${ }^{1} \mathbf{H}$ NMR $\left(500 \mathrm{MHz}, \mathrm{CDCl}_{3}\right) \delta(\mathrm{ppm}) 1.29\left(\mathrm{td},{ }^{3} \mathrm{~J}_{\mathrm{HH}}=7.1\right.$, $\left.1.0 \mathrm{~Hz}, 3 \mathrm{H}, \mathrm{CH}_{3} \mathrm{CH}_{2} \mathrm{O}\right), 1.33\left(\mathrm{td},{ }^{3} \mathrm{~J}_{\mathrm{HH}}=7.1 \mathrm{~Hz},{ }^{4} \mathrm{~J}_{\mathrm{PH}}=3.9 \mathrm{~Hz}, 6 \mathrm{H},\left(\mathrm{CH}_{3} \mathrm{CH}_{2} \mathrm{O}\right)_{2} \mathrm{P}\right), 1.43-1.62$ (m, 2H, $\mathrm{CH}_{2} \mathrm{CH}_{2} \mathrm{CH}_{2} ; 2 \mathrm{H}, \mathrm{CH}_{2} \mathrm{CH}_{2} \mathrm{CH}_{2}$ ), $1.84-1.94$ (m, $1 \mathrm{H}, \mathrm{CH}_{2} \mathrm{CH}_{2} \mathrm{CH}_{2}$ ), $1.99-2.09$ (m, $\left.1 \mathrm{H}, \mathrm{CH}_{2} \mathrm{CH}_{2} \mathrm{CH}_{2}\right), 2.45-2.47\left(\mathrm{~m}, 1 \mathrm{H}, \mathrm{CH}(\mathrm{O}) \mathrm{CH}_{2}\right), 2.73-2.75\left(\mathrm{~m}, 1 \mathrm{H}, \mathrm{CH}(\mathrm{O}) \mathrm{CH}_{2}\right), 2.87-$ $\left.2.90\left(\mathrm{~m}, 1 \mathrm{H}, \mathrm{CH}(\mathrm{O}) \mathrm{CH}_{2}\right), 2.91-2.98(\mathrm{~m}, 1 \mathrm{H}, \mathrm{CHP}), 4.11-4.18\left(\mathrm{~m}, 4 \mathrm{H},\left(\mathrm{CH}_{3} \mathrm{CH}_{2} \mathrm{O}\right)_{2} \mathrm{P}\right)\right)$, 4.19 - $4.27\left(\mathrm{~m}, 2 \mathrm{H}, \mathrm{CH}_{3} \mathrm{CH}_{2} \mathrm{O}\right) .{ }^{13} \mathrm{C}\left\{{ }^{1} \mathrm{H}\right\}$ NMR (126 MHz, $\left.\mathrm{CDCl}_{3}\right) \delta(\mathrm{ppm}) 14.3\left(\mathrm{CH}_{3} \mathrm{CH}_{2} \mathrm{O}\right)$, $16.5\left(\mathrm{dd},{ }^{3} J_{\mathrm{CP}}=6.0,3.9 \mathrm{~Hz},\left(\mathrm{CH}_{3} \mathrm{CH}_{2} \mathrm{O}\right)_{2} \mathrm{P}\right)$ ), $25.0\left(\mathrm{dd},{ }^{3} J_{\mathrm{CP}}=15.3,5.9 \mathrm{~Hz}, \mathrm{CH}_{2} \mathrm{CH}_{2} \mathrm{CH}_{2}\right.$ ), $26.9\left(\mathrm{~m}, \mathrm{CH}_{2} \mathrm{CH}_{2} \mathrm{CH}_{2}\right.$ ), 32.2 (d, ${ }^{4} J_{\mathrm{CP}}=10.3 \mathrm{~Hz}, \mathrm{CH}_{2} \mathrm{CH}_{2} \mathrm{CH}_{2}$ ), 45.9 (d, ${ }^{1} J_{\mathrm{CP}}=131.3 \mathrm{~Hz}, \mathrm{CHP}$ ), $47.2\left(\mathrm{~d},{ }^{6} J_{\mathrm{CP}}=14.4 \mathrm{~Hz}, \mathrm{CH}(\mathrm{O}) \mathrm{CH}_{2}\right), 52.0\left(\mathrm{~d},{ }^{5} \mathrm{~J}_{\mathrm{CP}}=3.2 \mathrm{~Hz}, \mathrm{CH}(\mathrm{O}) \mathrm{CH}_{2},\right), 61.6\left(\mathrm{CH}_{3} \mathrm{CH}_{2} \mathrm{O}\right)$, 
$\left.62.9\left(\mathrm{dd},{ }^{2} J_{\mathrm{CP}}=10.7,6.6 \mathrm{~Hz},\left(\mathrm{CH}_{3} \mathrm{CH}_{2} \mathrm{O}\right){ }_{2} \mathrm{P}\right)\right), 169.3\left(\mathrm{dd},{ }^{2} J_{\mathrm{CP}}=4.9,1.0 \mathrm{~Hz}, \mathrm{C}=\mathrm{O}\right)$. LRMS (IC) found $[\mathrm{M}+\mathrm{H}]^{+}=309,[\mathrm{M}+\mathrm{Na}]^{+}=331$. IR (neat): $v\left(\mathrm{~cm}^{-1}\right)=2983,2935,2870,1731,1446$, 1392, 1369, 1332, 1250, 1209, 1158, 1097, 1019, 958, 845, 789, 760, 645.

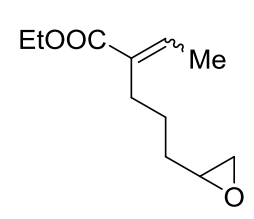

Ethyl 2-ethylidene-5-(oxiran-2-yl)pentanoate: In a 2-neck round bottom flask, 1-(diethoxyphosphoryl)-4-(oxiran-2-yl)butyl propionate $(3.4 \mathrm{mmol}$, $1.05 \mathrm{~g}$ ) was dissolved in $\mathrm{CH}_{3} \mathrm{CN}(23 \mathrm{~mL})$ and $\mathrm{LiCl}(3.4 \mathrm{mmol}, 144 \mathrm{mg})$ was added at room temperature. After 5 minutes, DBU $(3.4 \mathrm{mmol}, 0.5 \mathrm{~mL})$ was added and the mixture stirred for 5 more minutes. The reaction was cooled to $0{ }^{\circ} \mathrm{C}$ and acetaldehyde (10.2 mmol, $0.57 \mathrm{~mL})$ in $\mathrm{CH}_{3} \mathrm{CN}(10 \mathrm{~mL})$ was slowly added stirred at room temperature. After $24 \mathrm{~h}$, the reaction was quenched with a saturated aqueous $\mathrm{NH}_{4} \mathrm{Cl}$ solution $(20 \mathrm{~mL})$ and extracted with diethyl ether $(3 \times 20 \mathrm{~mL})$. The organic phases were combined and dried over $\mathrm{Na}_{2} \mathrm{SO}_{4}$ and the solvent removed in vacuo. The residual oil was purified by flash chromatography on silica gel (Pentane/Diethyl ether 7:3) to afford ethyl 2-ethylidene-5(oxiran-2-yl)pentanoate as a yellow oil in $31 \%$ yield $\left(213 \mathrm{mg}, E / Z 3.3: 1\right.$ ). $\mathbf{R}_{\mathbf{f}}=0.3$ (Diethyl ether). IR (neat): $v\left(\mathrm{~cm}^{-1}\right)=2977,2934,2861,1706,1648,1451,1384,1370,1285,1255$, 1184, 1134, 1106, 1044, 951, 918, 832, 786, 750, 581. ${ }^{1} \mathbf{H}$ NMR $\left(500 \mathrm{MHz}, \mathrm{CDCl}_{3}\right) \delta(\mathrm{ppm})$ Major Isomer: $1.28\left(\mathrm{t},{ }^{3} \mathrm{JHH}_{\mathrm{HH}} 7.1 \mathrm{~Hz}, 3 \mathrm{H}, \mathrm{CH}_{3} \mathrm{CH}_{2} \mathrm{O}\right), 1.50-1.59\left(\mathrm{~m}, 2 \mathrm{H}, \mathrm{CH}_{2} \mathrm{CH}_{2} \mathrm{CH}_{2} ; 2 \mathrm{H}\right.$, $\mathrm{CH}_{2} \mathrm{CH}_{2} \mathrm{CH}_{2}$ ), 1.79 (d, $\left.{ }^{3} \mathrm{~J}_{\mathrm{HH}}=7.1 \mathrm{~Hz}, 3 \mathrm{H}, \mathrm{CH}_{3}\right), 2.34-2.37\left(\mathrm{~m}, 2 \mathrm{H}, \mathrm{CH}_{2} \mathrm{CH}_{2} \mathrm{CH}_{2}\right), 2.46-2.47$ $\left(\mathrm{dd},{ }^{3} \mathrm{~J}_{\mathrm{HH}}=5.0,2.7 \mathrm{~Hz}, 1 \mathrm{H}, \mathrm{CH}(\mathrm{O}) \mathrm{CH}_{2}\right), 2.73-2.74\left(\mathrm{~m}, 1 \mathrm{H} \mathrm{CH}(\mathrm{O}) \mathrm{CH}_{2}\right), 2.88-2.93(\mathrm{~m}, 1 \mathrm{H}$, $\mathrm{CH}(\mathrm{O}) \mathrm{CH}_{2}$ ), 4.17 (q, ${ }^{3} \mathrm{~J}_{\mathrm{HH}}=7.1 \mathrm{~Hz}, 2 \mathrm{H}, \mathrm{CH}_{3} \mathrm{CH}_{2} \mathrm{O}$ ), 6.86 (q, ${ }^{3} \mathrm{~J}_{\mathrm{HH}}=7.1 \mathrm{~Hz}, 1 \mathrm{H}, \mathrm{C}=\mathrm{CH}$ ). Minor Isomer: $1.30\left(\mathrm{t},{ }^{3} \mathrm{~J}_{\mathrm{HH}}=7.1 \mathrm{~Hz}, 3 \mathrm{H}, \mathrm{CH}_{3} \mathrm{CH}_{2} \mathrm{O}\right), 1.50-1.59\left(\mathrm{~m}, 2 \mathrm{H}, \mathrm{CH}_{2} \mathrm{CH}_{2} \mathrm{CH}_{2} ; 2 \mathrm{H}\right.$, $\left.\mathrm{CH}_{2} \mathrm{CH}_{2} \mathrm{CH}_{2}\right), 1.95\left(\mathrm{~m}, 3 \mathrm{H}, \mathrm{CH}_{3}\right), 2.27-2.30\left(\mathrm{~m}, 2 \mathrm{H}, \mathrm{CH}_{2} \mathrm{CH}_{2} \mathrm{CH}_{2}\right), 2.44-2.46(\mathrm{~m}, 1 \mathrm{H}$, $\left.\mathrm{CH}(\mathrm{O}) \mathrm{CH}_{2}\right), 2.73-2.74\left(\mathrm{~m}, 1 \mathrm{H}, \mathrm{CH}(\mathrm{O}) \mathrm{CH}_{2}\right), 2.88-2.93\left(\mathrm{~m}, 1 \mathrm{H}, \mathrm{CH}(\mathrm{O}) \mathrm{CH}_{2}\right), 4.21\left(\mathrm{q},{ }^{3} \mathrm{JHH}_{\mathrm{HH}}=7.1\right.$ $\left.\mathrm{Hz}, 2 \mathrm{H}, \mathrm{CH}_{3} \mathrm{CH}_{2} \mathrm{O}\right), 5.98(\mathrm{~m}, 1 \mathrm{H}, \mathrm{C}=\mathrm{CH}) \cdot{ }^{13} \mathrm{C}\left\{{ }^{1} \mathrm{H}\right\}$ NMR $\left(126 \mathrm{MHz}, \mathrm{CDCl}_{3}\right) \delta$ (ppm) Major Isomer: $14.4\left(\mathrm{CH}_{3} \mathrm{CH}_{2} \mathrm{O}\right), 14.4\left(\mathrm{CH}_{3} \mathrm{CH}=\mathrm{C}\right), 25.5\left(\mathrm{CH}_{2} \mathrm{CH}_{2} \mathrm{CH}_{2}\right), 26.2\left(\mathrm{CH}_{2} \mathrm{CH}_{2} \mathrm{CH}_{2}\right), 32.3$ $\left(\mathrm{CH}_{2} \mathrm{CH}_{2} \mathrm{CH}_{2}\right), 47.2\left(\mathrm{CH}(\mathrm{O}) \mathrm{CH}_{2}\right), 52.3\left(\mathrm{CH}(\mathrm{O}) \mathrm{CH}_{2}\right), 60.5\left(\mathrm{CH}_{3} \mathrm{CH}_{2} \mathrm{O}\right), 133.1(\mathrm{C}=\mathrm{CH}), 137.6$ $(\mathrm{C}=\mathrm{CH}), 167.9(\mathrm{C}=\mathrm{O})$. Minor Isomer: $14.4\left(\mathrm{CH}_{3} \mathrm{CH}_{2} \mathrm{O}\right), 14.4\left(\mathrm{CH}_{3} \mathrm{CH}=\mathrm{C}\right), 25.6\left(\mathrm{CH}_{2} \mathrm{CH}_{2} \mathrm{CH}_{2}\right)$, $32.0\left(\mathrm{CH}_{2} \mathrm{CH}_{2} \mathrm{CH}_{2}\right), \quad 34.4\left(\mathrm{CH}_{2} \mathrm{CH}_{2} \mathrm{CH}_{2}\right), \quad 47.2\left(\mathrm{CH}(\mathrm{O}) \mathrm{CH}_{2}\right), \quad 52.3\left(\mathrm{CH}(\mathrm{O}) \mathrm{CH}_{2}\right), \quad 60.2$ $\left(\mathrm{CH}_{3} \mathrm{CH}_{2} \mathrm{O}\right), 132.7(\mathrm{C}=\mathrm{CH}), 136.6(\mathrm{C}=\mathrm{CH}), 168.2(\mathrm{C}=\mathrm{O})$. LRMS $(\mathrm{IC})$ found $[\mathrm{M}+\mathrm{K}]^{+}=237$.

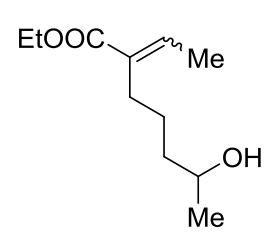

Ethyl 2-ethylidene-6-hydroxyheptanoate (2p): In a round-bottom flask, ethyl 2-ethylidene-5-(oxiran-2-yl)pentanoate $(0.77 \mathrm{mmol}, 153 \mathrm{mg})$ was dissolved in abs. EtOH (15 mL) and $\mathrm{NaBH}_{4}(2.31 \mathrm{mmol}, 88 \mathrm{mg})$ was added in a single portion. After stirring for $42 \mathrm{~h}$ at room temperature, the solvent was removed in vacuo and the crude mixture was purified by flash chromatography on silica 
gel (Pentane/Diethyl ether 7:3) to afford $\mathbf{2 p}$ in $79 \%$ yield (122 $\mathrm{mg}, E / Z 3.2: 1$ ) as a pale yellow oil. $\mathbf{R}_{\mathbf{f}}=0.5$ (Pentane/Diethyl ether $=1: 1$ ). IR (neat): $v\left(\mathrm{~cm}^{-1}\right)=3442,2965,2932,2864$, 1707, 1648, 1449, 1391, 1381, 1329, 1283, 1258, 1180, 1135, 1095, 1041, 941, 856, 749, 666. ${ }^{1} \mathbf{H}$ NMR $\left(500 \mathrm{MHz}, \mathrm{CDCl}_{3}\right) \delta(\mathrm{ppm})$ Major Isomer: $1.19\left(\mathrm{~d},{ }^{3} \mathrm{~J}_{\mathrm{HH}}=6.2 \mathrm{~Hz}, 3 \mathrm{H}\right.$, $\mathrm{CH}_{3} \mathrm{CHOH}$ ), 1.29 (t, $\left.{ }^{3} \mathrm{~J}_{\mathrm{HH}}=7.1 \mathrm{~Hz}, 3 \mathrm{H}, \mathrm{CH}_{3} \mathrm{CH}_{2} \mathrm{O}\right), 1.38-1.67\left(\mathrm{~m}, 2 \mathrm{H}, \mathrm{CH}_{2} \mathrm{CH}_{2} \mathrm{CH}_{2} ; 2 \mathrm{H}\right.$, $\left.\mathrm{CH}_{2} \mathrm{CH}_{2} \mathrm{CH}_{2} ; 1 \mathrm{H}, \mathrm{OH}\right), 1.80\left(\mathrm{~d},{ }^{3} \mathrm{~J}_{\mathrm{HH}}=7.1 \mathrm{~Hz}, 3 \mathrm{H}, \mathrm{C}=\mathrm{CHCH}_{3}\right), 2.23-2.35(\mathrm{~m}, 2 \mathrm{H}$, $\mathrm{CH}_{2} \mathrm{CH}_{2} \mathrm{CH}_{2}$ ), $3.77-3.85$ (m, $1 \mathrm{H}, \mathrm{CHOH}$ ), 4.19 (q, ${ }^{3} \mathrm{~J}_{\mathrm{HH}}=7.1 \mathrm{~Hz}, 2 \mathrm{H}, \mathrm{CH}_{3} \mathrm{CH}_{2} \mathrm{O}$ ), 6.86 (q, ${ }^{3} J_{\mathrm{HH}}=7.1 \mathrm{~Hz}, 1 \mathrm{H}, \mathrm{C}=\mathrm{CH}$ ). Minor Isomer: $1.31\left(\mathrm{t},{ }^{3} J_{\mathrm{HH}}=7.1 \mathrm{~Hz}, 3 \mathrm{H}, \mathrm{CH}_{3} \mathrm{CH}_{2} \mathrm{O}\right), 4.22\left(\mathrm{q},{ }^{3} J_{\mathrm{HH}}\right.$ $\left.=7.1 \mathrm{~Hz}, 2 \mathrm{H}, \mathrm{CH}_{3} \mathrm{CH}_{2} \mathrm{O}\right), 5.98(\mathrm{~m}, 1 \mathrm{H}, \mathrm{C}=\mathrm{CH}), 1.95\left(\mathrm{~m}, 3 \mathrm{H}, \mathrm{C}=\mathrm{CHCH}_{3}\right), 2.23-2.35(\mathrm{~m}, 2 \mathrm{H}$, $\left.\mathrm{CH}_{2} \mathrm{CH}_{2} \mathrm{CH}_{2}\right), 1.38-1.67\left(\mathrm{~m}, 2 \mathrm{H}, \mathrm{CH}_{2} \mathrm{CH}_{2} \mathrm{CH}_{2} ; 2 \mathrm{H}, \mathrm{CH}_{2} \mathrm{CH}_{2} \mathrm{CH}_{2} ; 1 \mathrm{H}, \mathrm{OH}\right), 3.77-3.85(\mathrm{~m}$, $1 \mathrm{H}, \mathrm{CHOH}), 1.16\left(\mathrm{~d}, J=6.2 \mathrm{~Hz}, 3 \mathrm{H}, \mathrm{CH}_{3} \mathrm{CHOH}\right) .{ }^{13} \mathrm{C}\left\{{ }^{1} \mathrm{H}\right\} \mathrm{NMR}\left(126 \mathrm{MHz}, \mathrm{CDCl}_{3}\right) \delta(\mathrm{ppm})$ Major Isomer: $14.4\left(\mathrm{CH}_{3} \mathrm{CH}_{2} \mathrm{O}\right), 14.4(\mathrm{C}=\mathrm{CHCH})_{3}, 23.6\left(\mathrm{CH}_{3} \mathrm{CHOH}\right), 25.3\left(\mathrm{CH}_{2} \mathrm{CH}_{2} \mathrm{CH}_{2}\right)$, $26.2\left(\mathrm{CH}_{2} \mathrm{CH}_{2} \mathrm{CH}_{2}\right)$, $38.9\left(\mathrm{CH}_{2} \mathrm{CH}_{2} \mathrm{CH}_{2}\right), 60.4\left(\mathrm{CH}_{3} \mathrm{CH}_{2} \mathrm{O}\right), 68.0(\mathrm{CHOH}), 133.4(\mathrm{C}=\mathrm{CH})$, $137.4(\mathrm{C}=\mathrm{CH}), 168.0(\mathrm{C}=\mathrm{O})$. Minor Isomer: $14.4\left(\mathrm{CH}_{3} \mathrm{CH}_{2} \mathrm{O}\right), 15.8\left(\mathrm{C}=\mathrm{CHCH}_{3}\right), 23.6$ $\left(\mathrm{CH}_{3} \mathrm{CHOH}\right), 25.4\left(\mathrm{CH}_{2} \mathrm{CH}_{2} \mathrm{CH}_{2}\right)$, $34.6\left(\mathrm{CH}_{2} \mathrm{CH}_{2} \mathrm{CH}_{2}\right), 38.8\left(\mathrm{CH}_{2} \mathrm{CH}_{2} \mathrm{CH}_{2}\right), 60.2\left(\mathrm{CH}_{3} \mathrm{CH} \mathrm{O}\right)$, $68.0(\mathrm{CHOH}), 133.0(\mathrm{C}=\mathrm{CH}), 136.3(\mathrm{C}=\mathrm{CH}) .168 .4(\mathrm{C}=\mathrm{O})$. LRMS $(\mathrm{IC})$ found $[\mathrm{M}+\mathrm{H}]^{+}=201$, $[\mathrm{M}+\mathrm{Na}]^{+}=223$

\subsubsection{Isomerization of $2 p$}

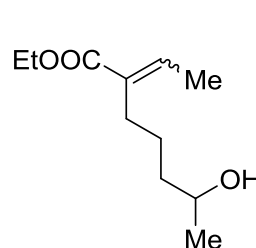

$0.25 \mathrm{mmol}$

$2 p$

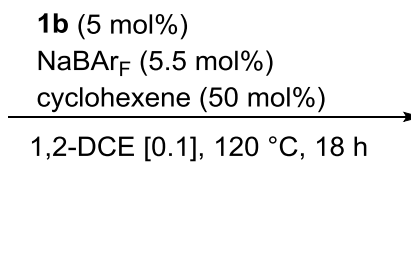

$\operatorname{NaBAr}_{F}(5.5 \mathrm{~mol} \%)$ cyclohexene $(50 \mathrm{~mol} \%)$

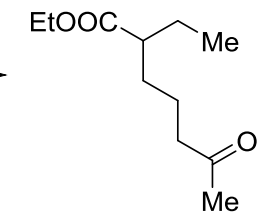

$3 p$

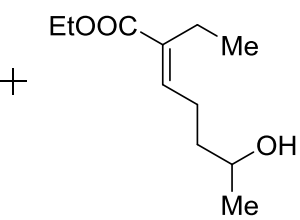

$2 \mathbf{q}$

The reaction was performed according to GP1. After $18 \mathrm{~h}$, conversion into $3 p$ was $39 \%$ (34\% yield) and consumption was $69 \%$.

Note: No formation of $\mathbf{2 q}$ was detected when the starting material was subjected to the same reaction conditions in absence of the palladium precatalyst. 


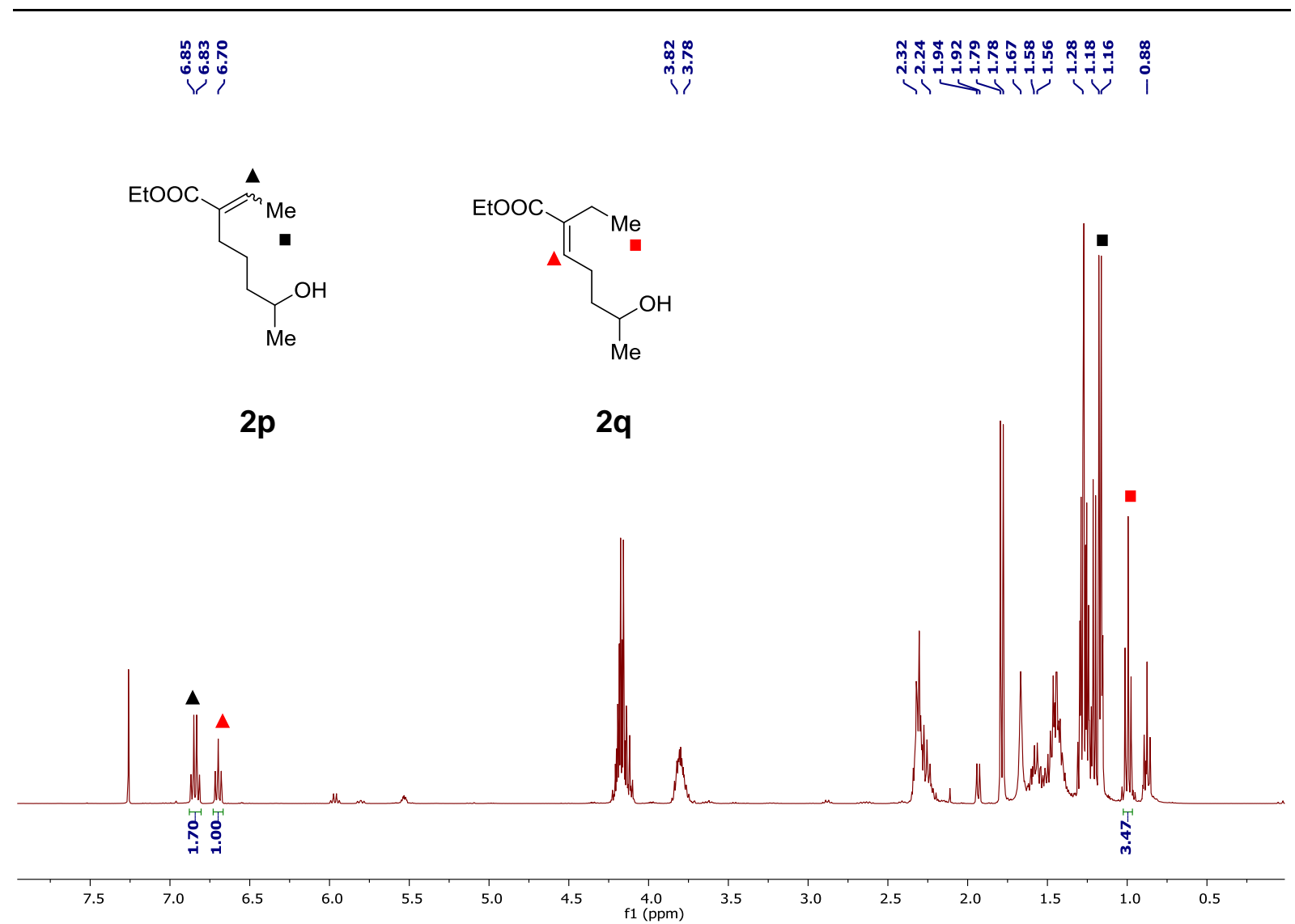

Figure S41: ${ }^{1} \mathrm{H}-\mathrm{NMR}$ spectrum of the fraction containing $\mathbf{2 p} / \mathbf{2 q}$

To confirm the identity of the second product present in this reaction mixture, spiking experiments were performed using $\mathbf{2 q}$ prepared by an independent synthesis (see below). 


\subsubsection{Independent synthesis of $2 q$}

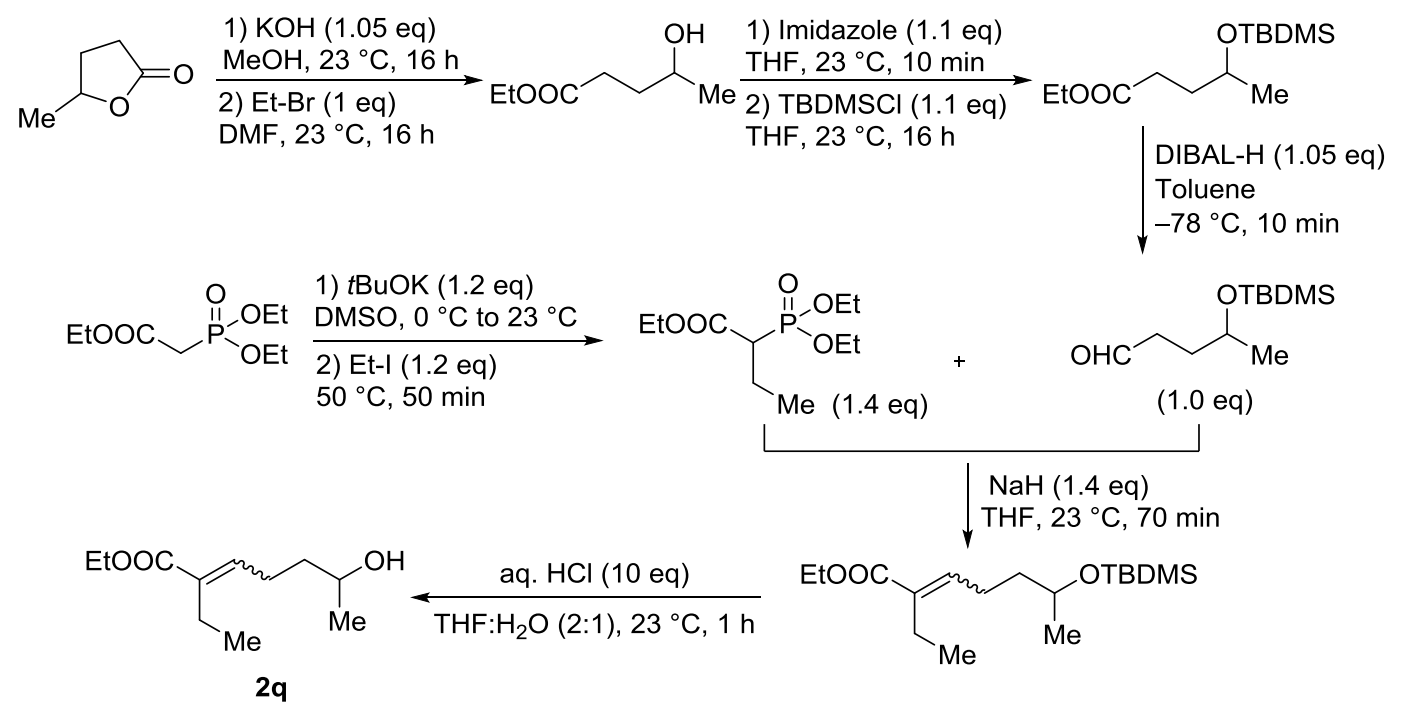

Figure S42: Synthesis of Ethyl 2-ethyl-6-hydroxyhept-2-enoate (2q)

$\overbrace{\mathrm{Me}}^{\text {OTBDMs }} \begin{aligned} & \text { 4-((tert-butyldimethylsilyl)oxy)pentanal: In a 2-neck round bottom } \\ & \text { flask, } \gamma \text {-valerolactone }(10 \mathrm{mmol}, 1 \mathrm{~g}) \text { was dissolved in } \mathrm{MeOH}(50 \mathrm{~mL}) \text { and }\end{aligned}$ $\mathrm{KOH}(10.5 \mathrm{mmol}, 589 \mathrm{mg}$ ) was added at room temperature. After $16 \mathrm{~h}$, the solvent was evaporated and the residual material redissolved in DMF $(50 \mathrm{~mL})$. To this solution was added dropwise ethylbromide $(10 \mathrm{mmol}, 1.09 \mathrm{~g})$ at room temperature. After $16 \mathrm{~h}$, the reaction was quenched with water and extracted with diethyl ether $(3 \times 50 \mathrm{~mL})$. The organic phases were combined, dried over $\mathrm{Na}_{2} \mathrm{SO}_{4}$ and the solvent evaporated to afford a clear oil that was used in the next step without further purification. ${ }^{41}$ The crude oil was dissolved in THF (26 mL), imidazole $(8.6 \mathrm{mmol}, 584 \mathrm{mg}$ ) was added and the solution stirred at room temperature for 10 minutes before the addition of TBDMSCl $(8.6 \mathrm{mmol}, 1.30 \mathrm{~g})$ in a single portion. The reaction was stirred for $16 \mathrm{~h}$ at room temperature and then quenched with a saturated aqueous $\mathrm{NaHCO}_{3}$ solution $(20 \mathrm{~mL})$, extracted with diethyl ether $(3 \times 30 \mathrm{~mL})$ and washed with brine. The organic phases were combined, dried over $\mathrm{Na}_{2} \mathrm{SO}_{4}$ and evaporated. The clear oil obtained was dissolved in toluene $(30 \mathrm{~mL})$ and cooled to $-78{ }^{\circ} \mathrm{C}$ before the dropwise addition of DIBAL-H (1.9 mL from a $1 \mathrm{M}$ solution in hexane). After 10 minutes, the reaction was quenched at $0{ }^{\circ} \mathrm{C}$ with a saturated aqueous $\mathrm{NH}_{4} \mathrm{Cl}$ solution and extracted with diethyl ether $(3 \times 30 \mathrm{~mL})$. The organic phases were combined, washed with brine, dried over $\mathrm{Na}_{2} \mathrm{SO}_{4}$ and evaporated. The residual material was purified by flash chromatography on silica gel (Pentane/Diethyl ether $=10: 1)$ affording 4-((tert-butyldimethylsilyl)oxy)pentanal as a colourless oil in $36 \%$ yield $(140 \mathrm{mg}$ ) over 4 steps. 


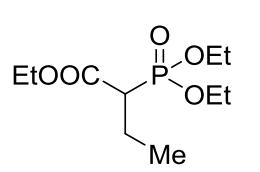

Ethyl 2-(diethoxyphosphoryl)butanoate: In a 2-neck round bottom flask, triethyl phosphonacetate $(10 \mathrm{mmol}, 2.24 \mathrm{~g})$ was dissolved in DMSO $(6.7 \mathrm{~mL})$ and cooled to $0{ }^{\circ} \mathrm{C}$. Next, tBuOK (12 mmol, $1.34 \mathrm{~g}$ ) was added and the resulting suspension was stirred at room temperature until complete consumption of the base. Ethyliodide (12 mmol, $1.87 \mathrm{~g}$ ) was added dropwise and stirred for 50 minutes at 50 ${ }^{\circ} \mathrm{C} .{ }^{42}$ The reaction was quenched with a saturated aqueous $\mathrm{NH}_{4} \mathrm{Cl}$ solution and extracted with diethyl ether $(3 \times 10 \mathrm{~mL})$. The organic phases were combined, dried over $\mathrm{Na}_{2} \mathrm{SO}_{4}$ and evaporated. After flash chromatography on silica gel (Diethyl ether) the product was obtained in $73 \%$ yield $(1.85 \mathrm{~g})$ as a colourless oil.

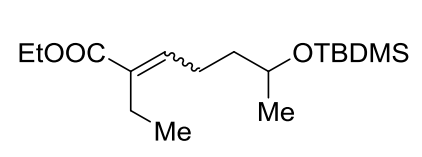

Ethyl 6-((tert-butyldimethylsilyl)oxy)-2-ethylhept-2-enoate: In a 2-necked flask, ethyl 2-(diethoxyphosphoryl)butanoate $(0.975$ mmol, $246 \mathrm{mg}$ ) was dissolved in THF (5 mL) before the addition of $\mathrm{NaH}(0.975 \mathrm{mmol}, 23 \mathrm{mg})$ in one portion. After 10 minutes at room temperature, 4-((tertbutyldimethylsilyl)oxy)pentanal $(0.65 \mathrm{mmol})$ in THF $(1 \mathrm{~mL})$ was added dropwise and stirred for $1 \mathrm{~h}$. The reaction was quenched with water $(5 \mathrm{~mL})$, extracted with diethyl ether $(3 \times 10$ $\mathrm{mL}$ ), the organic phases combined, dried over $\mathrm{Na}_{2} \mathrm{SO}_{4}$ and evaporated in vacuo. After flash chromatography on silica gel (Pentane/Diethyl ether $=10: 1$ ) the product was obtained in $67 \%$ yield (137 mg, $E / Z=1.7: 1$ ) as a colourless oil. $\mathbf{R}_{\mathbf{f}}=0.7$ (Pentane/Diethyl ether $=10: 1$ ). IR (neat): $v\left(\mathrm{~cm}^{-1}\right)=2963,2936,2886,2857,1713,1461,1446,1374,1285,1252,1201$, 1140, 1087, 1037, 996, 836, 804, 774, 613. ${ }^{1} \mathbf{H}$ NMR (500 MHz, $\left.\mathrm{CDCl}_{3}\right) \delta$ (ppm) Major Isomer: $0.05\left(\mathrm{~s}, 3 \mathrm{H},\left(\mathrm{CH}_{3}\right)_{2} \mathrm{Si}\right), 0.06\left(\mathrm{~s}, 3 \mathrm{H},\left(\mathrm{CH}_{3}\right)_{2} \mathrm{Si}\right), 0.89\left(\mathrm{~s}, 9 \mathrm{H},\left(\mathrm{CH}_{3}\right)_{3} \mathrm{CSi}\right), 1.00\left(\mathrm{t},{ }^{3} \mathrm{JHH}_{\mathrm{HH}}=\right.$ $\left.7.5 \mathrm{~Hz}, 3 \mathrm{H}, \mathrm{CCH}_{2} \mathrm{CH}_{3}\right), 1.15\left(\mathrm{~d},{ }^{3} \mathrm{~J}_{\mathrm{HH}}=6.1 \mathrm{~Hz}, 3 \mathrm{H}, \mathrm{CH}_{3} \mathrm{CHOH}\right), 1.29\left(\mathrm{t},{ }^{3} \mathrm{JHH}_{\mathrm{HH}}=7.1 \mathrm{~Hz}, 3 \mathrm{H}\right.$, $\mathrm{CH}_{3} \mathrm{CH}_{2} \mathrm{O}$ ), $1.51-1.57$ (m, $\left.2 \mathrm{H}, \mathrm{CH}_{2} \mathrm{CH}_{2} \mathrm{CHOH}\right), 2.10-2.20\left(\mathrm{~m}, 1 \mathrm{H}, \mathrm{CH}_{2} \mathrm{CH}_{2} \mathrm{CHOH}\right), 2.24-$ $2.29\left(\mathrm{~m}, 1 \mathrm{H}, \mathrm{CH}_{2} \mathrm{CH}_{2} \mathrm{CHOH}\right), 2.31$ (q, $\left.{ }^{3} J_{\mathrm{HH}}=7.5 \mathrm{~Hz}, 2 \mathrm{H}, \mathrm{CCH}_{2} \mathrm{CH}_{3}\right), 3.84\left(\mathrm{~h},{ }^{3} J_{\mathrm{HH}}=5.5 \mathrm{~Hz}\right.$, $1 \mathrm{H}, \mathrm{CH}_{2} \mathrm{CH}_{2} \mathrm{CH}$ ), 4.19 (q, ${ }^{3} \mathrm{~J}_{\mathrm{HH}}=7.1 \mathrm{~Hz}, 2 \mathrm{H}, \mathrm{CH}_{3} \mathrm{CH}_{2} \mathrm{O}$ ), 6.72 (t, ${ }^{3} \mathrm{JHH}_{\mathrm{HH}}=7.6 \mathrm{~Hz}, 1 \mathrm{H}, \mathrm{C}=\mathrm{CH}$ ), 26.3 (s, 9H, $\left.\left(\mathrm{CH}_{3}\right)_{3} \mathrm{CSi}\right)$, Minor Isomer: 0.04 (s, 3H, $\left.\left(\mathrm{CH}_{3}\right)_{2} \mathrm{Si}\right), 0.05\left(\mathrm{~s}, 3 \mathrm{H},\left(\mathrm{CH}_{3}\right)_{2} \mathrm{Si}\right), 0.88$ (s, $\left.9 \mathrm{H},\left(\mathrm{CH}_{3}\right)_{3} \mathrm{CSi}\right), 1.02\left(\mathrm{t}, 3 \mathrm{H}, \mathrm{CCH}_{2} \mathrm{CH}_{3},{ }^{3} \mathrm{~J}_{\mathrm{HH}}=7.4 \mathrm{~Hz}\right), 1.13\left(\mathrm{~d},{ }^{3} \mathrm{~J}_{\mathrm{HH}}=6.6 \mathrm{~Hz}, 3 \mathrm{H}\right.$, $\mathrm{CH}_{3} \mathrm{CHOH}$,), $1.30\left(\mathrm{t},{ }^{3} \mathrm{~J}_{\mathrm{HH}}=7.1 \mathrm{~Hz}, 3 \mathrm{H}, \mathrm{CH}_{3} \mathrm{CH}_{2} \mathrm{O}\right), 1.51-1.57\left(\mathrm{~m}, 2 \mathrm{H}, \mathrm{CH}_{2} \mathrm{CH}_{2} \mathrm{CHOH}\right), 2.24$ - $2.29\left(\mathrm{~m}, 2 \mathrm{H}, \mathrm{CCH}_{2} \mathrm{CH}_{3}\right), 2.35-2.48\left(\mathrm{~m}, 2 \mathrm{H}, \mathrm{CH}_{2} \mathrm{CH}_{2} \mathrm{CHOH}\right), 3.80\left(\mathrm{~h},{ }^{3} J_{\mathrm{HH}}=6.1 \mathrm{~Hz}, 1 \mathrm{H}\right.$, $\left.\mathrm{CH}_{2} \mathrm{CH}_{2} \mathrm{CH}\right), 4.20\left(\mathrm{q}, 2 \mathrm{H}, \mathrm{CH}_{3} \mathrm{CH}_{2} \mathrm{O},{ }^{3} \mathrm{~J}_{\mathrm{HH}}=7.1 \mathrm{~Hz}\right), 5.81-5.85(\mathrm{~m}, 1 \mathrm{H}, \mathrm{C}=\mathrm{CH}) .{ }^{13} \mathrm{C}\left\{{ }^{1} \mathrm{H}\right\}$ NMR (126 MHz, $\left.\mathrm{CDCl}_{3}\right) \delta$ (ppm) Major Isomer: -4.2, -4.6 $\left(\left(\mathrm{CH}_{3}\right)_{2} \mathrm{Si}\right), 14.1\left(\mathrm{CCH}_{2} \mathrm{CH}_{3}\right), 14.5$ $\left(\mathrm{CH}_{3} \mathrm{CH}_{2} \mathrm{O}\right), 18.3\left(\left(\mathrm{CH}_{3}\right)_{3} \mathrm{CSi}\right), 20.2\left(\mathrm{CCH}_{2} \mathrm{CH}_{3}\right), 23.8\left(\mathrm{CH}_{3} \mathrm{CHOH}\right), 24.8\left(\mathrm{CH}_{2} \mathrm{CH}_{2} \mathrm{CHO}\right), 26.0$ $\left(\left(\mathrm{CH}_{3}\right)_{3} \mathrm{CSi}\right), 38.9\left(\mathrm{CH}_{2} \mathrm{CH}_{2} \mathrm{CHOH}\right), 60.4\left(\mathrm{CH}_{3} \mathrm{CH}_{2} \mathrm{O}\right), 68.2\left(\mathrm{CH}_{2} \mathrm{CH}_{2} \mathrm{CHOH}\right), 134.2(\mathrm{C}=\mathrm{CH})$, $141.9(\mathrm{C}=\mathrm{CH}), 168.1(\mathrm{C}=\mathrm{O})$. Minor Isomer: -4.3, -4.6 $\left(\left(\mathrm{CH}_{3}\right)_{2} \mathrm{Si}\right), 13.8\left(\mathrm{CCH}_{2} \mathrm{CH}_{3}\right), 14.5$ $\left(\mathrm{CH}_{3} \mathrm{CH}_{2} \mathrm{O}\right), 18.3\left(\left(\mathrm{CH}_{3}\right)_{3} \mathrm{CSi}\right), 23.9\left(\mathrm{CH}_{3} \mathrm{CHOH}\right), 26.1\left(\left(\mathrm{CH}_{3}\right)_{3} \mathrm{CSi}\right), 26.1\left(\mathrm{CH}_{2} \mathrm{CH}_{2} \mathrm{CHOH}\right)$, 
$27.7\left(\mathrm{CCH}_{2} \mathrm{CH}_{3}\right), 39.6\left(\mathrm{CH}_{2} \mathrm{CH}_{2} \mathrm{CHOH}\right), 60.2\left(\mathrm{CH}_{3} \mathrm{CH}_{2} \mathrm{O}\right), 68.4\left(\mathrm{CH}_{2} \mathrm{CH}_{2} \mathrm{CHOH}\right), 133.9$ $(C=\mathrm{CH}), 140.1(\mathrm{C}=\mathrm{CH}), 168.5(\mathrm{C}=\mathrm{O})$. LRMS $(\mathrm{IC})$ found $[\mathrm{M}+\mathrm{Na}]^{+}=338$.

$\mathrm{CtOOC}_{\mathrm{Me}}^{\mathrm{Me}}$ Ethyl 2-ethyl-6-hydroxyhept-2-enoate (2q): In a round-bottomed flask ethyl 6-((tert-butyldimethylsilyl)oxy)-2-ethylhept-2-enoate $(0.41 \mathrm{mmol})$ was dissolved in $\mathrm{THF} / \mathrm{H}_{2} \mathrm{O} 2: 1(4 \mathrm{~mL})$ and cooled to $0{ }^{\circ} \mathrm{C}$ before the dropwise addition of aqueous $\mathrm{HCl}(0.7 \mathrm{~mL}$ from a $6 \mathrm{M}$ solution). After $1 \mathrm{~h}$ at room temperature, the reaction was diluted with water and extracted with diethyl ether $(3 \times 5 \mathrm{~mL})$ then the organic phases were combined, dried over $\mathrm{Na}_{2} \mathrm{SO}_{4}$ and evaporated in vacuo. Flash chromatography on silica gel (Pentane/Diethyl ether $=1: 1$ ) afforded the desired product in $80 \%$ yield (66 mg, $E / Z=1.7: 1$ ) as a colourless oil. $\mathbf{R}_{\mathbf{f}}=0.2$ (Pentane/Diethyl ether $=1: 1$ ). IR (neat): $v\left(\mathrm{~cm}^{-1}\right)=3418,2969,2933,1704,1464,1458,1374,1291,1240,1193,1140,1098$, 1036, 943, 847, 765. ${ }^{1} \mathbf{H}$ NMR $\left(500 \mathrm{MHz}, \mathrm{CDCl}_{3}\right) \delta(\mathrm{ppm})$ Major Isomer: $1.01\left(\mathrm{t},{ }^{3} \mathrm{JHH}_{\mathrm{HH}}=7.5\right.$ $\mathrm{Hz}, 3 \mathrm{H}, \mathrm{CCH}_{2} \mathrm{CH}_{3}$ ), $1.22\left(\mathrm{~d},{ }^{3} \mathrm{~J}_{\mathrm{HH}}=6.2 \mathrm{~Hz}, 3 \mathrm{H}, \mathrm{CH}_{3} \mathrm{CHOH}\right.$ ), 1.29 (t, ${ }^{3} J_{\mathrm{HH}}=7.1 \mathrm{~Hz} 3 \mathrm{H}$, $\mathrm{CH}_{3} \mathrm{CH}_{2} \mathrm{O}$ ), $1.54-1.61$ (m, 2H, $\mathrm{CH}_{2} \mathrm{CH}_{2} \mathrm{CHOH}$ ), 1.72, (bs, $\left.1 \mathrm{H}, \mathrm{OH}\right), 2.24-2.35(\mathrm{~m}, 2 \mathrm{H}$, $\left.\mathrm{CH}_{2} \mathrm{CH}_{2} \mathrm{CHOH} ; 2 \mathrm{H}, \mathrm{CCH}_{2} \mathrm{CH}_{3}\right), 3.83\left(\mathrm{~h},{ }^{3} \mathrm{~J}_{\mathrm{HH}}=6.3 \mathrm{~Hz}, 1 \mathrm{H}, \mathrm{CH}_{2} \mathrm{CH}_{2} \mathrm{CHOH}\right), 4.19$ (q, ${ }^{3} \mathrm{~J}_{\mathrm{HH}}=$ $7.1 \mathrm{~Hz}, 2 \mathrm{H}, \mathrm{CH}_{3} \mathrm{CH}_{2} \mathrm{O}$ ), $6.71\left(\mathrm{t},{ }^{3} \mathrm{~J}_{\mathrm{HH}}=7.6 \mathrm{~Hz}, 1 \mathrm{H}, \mathrm{C}=\mathrm{CH}\right.$ ), Minor Isomer: $1.03\left(\mathrm{t},{ }^{3} \mathrm{~J}_{\mathrm{HH}}=7.4\right.$ $\mathrm{Hz}, 3 \mathrm{H}, \mathrm{CCH}_{2} \mathrm{CH}_{3}$ ), 1.19 (d, $\left.{ }^{3} \mathrm{~J}_{\mathrm{HH}}=6.2 \mathrm{~Hz}, 3 \mathrm{H}, \mathrm{CH}_{3} \mathrm{CHOH}\right), 1.31$ (t, ${ }^{3} J_{\mathrm{HH}}=7.1 \mathrm{~Hz}, 3 \mathrm{H}$, $\mathrm{CH}_{3} \mathrm{CH}_{2} \mathrm{OH}$ ), $1.54-1.61$ (m, 2H, $\left.\mathrm{CH}_{2} \mathrm{CH}_{2} \mathrm{CHOH}\right), 1.72$, (bs, 1H, OH), $2.24-2.35(\mathrm{~m}, 2 \mathrm{H}$, $\mathrm{CH}_{2} \mathrm{CH}_{2} \mathrm{CHOH} ; 2 \mathrm{H}, \mathrm{CCH}_{2} \mathrm{CH}_{3}$ ), $3.75-3.80\left(\mathrm{~m}, 1 \mathrm{H}, \mathrm{CH}_{2} \mathrm{CH}_{2} \mathrm{CHOH}\right.$ ), 4.22 (q, 2H, $\mathrm{CH}_{3} \mathrm{CH}_{2} \mathrm{O}$, $\left.{ }^{3} J_{\mathrm{HH}}=7.1 \mathrm{~Hz}\right), 5.80-5.83(\mathrm{~m}, 1 \mathrm{H}, \mathrm{C}=\mathrm{CH}) \cdot{ }^{13} \mathrm{C}\left\{{ }^{1} \mathrm{H}\right\} \mathbf{N M R}\left(126 \mathrm{MHz}, \mathrm{CDCl}_{3}\right) \delta(\mathrm{ppm})$ Major Isomer: $13.8\left(\mathrm{CCH}_{2} \mathrm{CH}_{3}\right), 14.1\left(\mathrm{CCH}_{2} \mathrm{CH}_{3}\right), 14.4\left(\mathrm{CH}_{3} \mathrm{CH}_{2} \mathrm{O}\right), 20.2\left(\mathrm{CCH}_{2} \mathrm{CH}_{3}\right), 23.2$ $\left(\mathrm{CH}_{3} \mathrm{CHOH}\right), \quad 23.8\left(\mathrm{CH}_{3} \mathrm{CHOH}\right), 24.8\left(\mathrm{CH}_{2} \mathrm{CH}_{2} \mathrm{CHOH}\right), \quad 38.4 \quad\left(\mathrm{CH}_{2} \mathrm{CH}_{2} \mathrm{CHOH}\right), \quad 60.5$ $\left(\mathrm{CH}_{3} \mathrm{CH}_{2} \mathrm{O}\right), 67.7\left(\mathrm{CH}_{2} \mathrm{CH}_{2} \mathrm{CHOH}\right), 134.6(\mathrm{C}=\mathrm{CH}), 141.2(\mathrm{C}=\mathrm{CH}), 168.0(\mathrm{C}=\mathrm{O})$. Minor Isomer: $14.4\left(\mathrm{CH}_{3} \mathrm{CH}_{2} \mathrm{O}\right), 26.6\left(\mathrm{CCH}_{2} \mathrm{CH}_{3}\right), 27.7\left(\mathrm{CH}_{2} \mathrm{CH}_{2} \mathrm{CHOH}\right), 38.4\left(\mathrm{CH}_{2} \mathrm{CH}_{2} \mathrm{CHOH}\right)$, $60.6\left(\mathrm{CH}_{3} \mathrm{CH}_{2} \mathrm{O}\right), 66.5\left(\mathrm{CH}_{2} \mathrm{CH}_{2} \mathrm{CHOH}\right), 134.9(\mathrm{C}=\mathrm{CH}), 139.5(\mathrm{C}=\mathrm{CH}), 168.9(\mathrm{C}=\mathrm{O})$. LRMS (IC) found $[\mathrm{M}+\mathrm{Na}]^{+}=224$. 


\subsubsection{Control experiment using $3 g$}

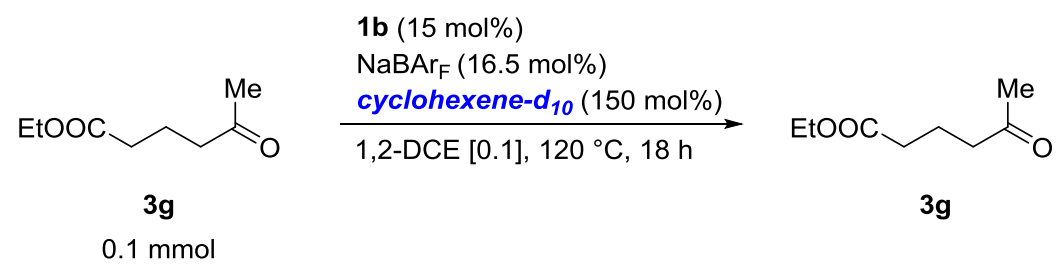

Inside the glovebox, a $5 \mathrm{~mL}$ Young valve Schlenk was charged with the precatalyst $\mathbf{1 b}$ (0.015 mmol, $7.1 \mathrm{mg}), \mathrm{NaBAr}_{\mathrm{F}}(0.0165 \mathrm{mmol}, 14.6 \mathrm{mg})$ and 1,2-dichloroethane $(1 \mathrm{~mL})$. After stirring at room temperature for 5 minutes, cyclohexene- $d_{10}(0.15 \mathrm{mmol}, 15 \mu \mathrm{L})$ was added and the mixture stirred for 5 minutes. Next, substrate $\mathbf{3 g}(0.1 \mathrm{mmol}, 15.8 \mathrm{mg})$ was added to the mixture and the sealed Schlenk was placed in an oil bath pre-heated at $120^{\circ} \mathrm{C}$. After 18 h, deuterium incorporation was assessed by ${ }^{2} \mathrm{H}$-NMR using 1,1,2,2-tetrachloroethane $(0.05$ $\mathrm{mmol}$ ) as internal standard. No deuterium incorporation was detectable. 
2.5 Optimization of the deconjugative isomerization under microwave (MW) irradiation

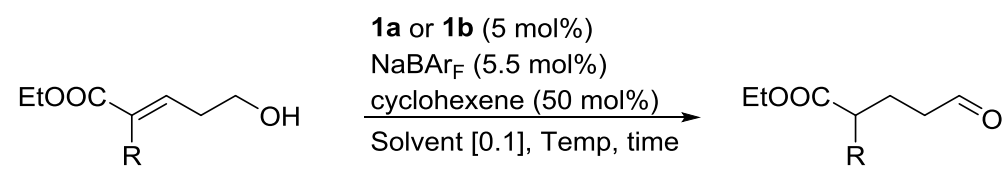

Inside the glovebox, a $5 \mathrm{~mL}$ microwave reaction vial was charged with 1a or $\mathbf{1 b}(0.0125$ mmol), $\operatorname{NaBAr}_{\mathrm{F}}(12.2 \mathrm{mg}, 0.0138 \mathrm{mmol})$ and the solvent $(2.5 \mathrm{~mL})$. After 5 minutes, cyclohexene $(0.125 \mathrm{mmol}, 13 \mu \mathrm{L})$ was added and the reaction mixture stirred for 5 minutes. Next, the appropriate substrate $(0.25 \mathrm{mmol})$ was added. The sealed reaction vial was placed in a microwave oven and stirred at the desired temperature for the appropriate time. The mixture was then cooled to room temperature and the solvent removed in vacuo.

\begin{tabular}{lccccccc}
\hline Entry & Cat. & $\mathbf{R}$ & Solvent & MW & Temp $\left({ }^{\circ} \mathbf{C}\right)$ & Time (h) & Yield (\%) \\
\hline $\mathbf{1}$ & $\mathbf{1 a}$ & Me & $1,2-\mathrm{DCE}$ & Yes & 160 & 2 & 28 \\
$\mathbf{2}$ & $\mathbf{1 a}$ & $\mathrm{Me}$ & Toluene & Yes & 160 & 2 & 50 \\
$\mathbf{3}$ & $\mathbf{1 a}$ & $\mathrm{Me}$ & $\mathrm{DMF}$ & Yes & 160 & 2 & 0 \\
$\mathbf{4}$ & $\mathbf{1 a}$ & $\mathrm{Ph}$ & $1,2-\mathrm{DCE}$ & No & 120 & 18 & 45 \\
$\mathbf{5}$ & $\mathbf{1 a}$ & $\mathrm{Ph}$ & $1,2-\mathrm{DCE}$ & Yes & 120 & 4 & 45 \\
$\mathbf{6}$ & $\mathbf{1 a}$ & $\mathrm{Ph}$ & $1,2-\mathrm{DCE}$ & Yes & 140 & 2 & 49 \\
$\mathbf{7}$ & $\mathbf{1 a}$ & $\mathrm{Ph}$ & $1,2-\mathrm{DCE}$ & Yes & 140 & 4 & 55 \\
$\mathbf{8}$ & $\mathbf{1 a}$ & $\mathrm{Ph}$ & $1,2-\mathrm{DCE}$ & Yes & 160 & 2 & 56 \\
$\mathbf{9}$ & $\mathbf{1 a}$ & $\mathrm{Ph}$ & $1,2-\mathrm{DCE}$ & Yes & 160 & 4 & 47 \\
\hline $\mathbf{1 0}$ & $\mathbf{1 b}$ & $\mathrm{Ph}$ & $1,2-\mathrm{DCE}$ & Yes & 140 & 2 & 50 \\
\hline
\end{tabular}

Table 2: Reaction optimization under microwave irradiation 


\subsubsection{Chiral ligand screening}

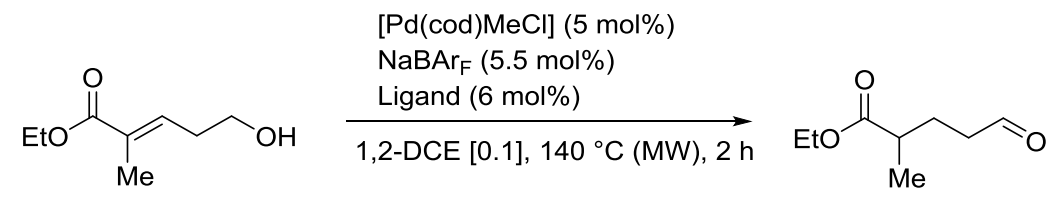<smiles>CC1CC[C@@H](C)P1c1ccccc1P1C(C)CC[C@H]1C</smiles>

$<10 \%$ conv.<smiles>CC1(C)OC(CPc2ccccc2)[C@H](CPc2ccccc2)O1</smiles>

$10 \%, 0 \%$ ee<smiles>CCC1CC[C@@H](CC)P1c1ccccc1P1C(CC)CC[C@H]1CC</smiles>

$40 \%, 10 \%$ ee<smiles>CC(c1ccccc1)C(C)[PH](=O)c1ccccc1</smiles>

$5 \%,<10 \%$ ee<smiles>c1ccc(C2CC[C@H](c3ccccc3)P2CCP2[C@H](c3ccccc3)CC[C@@H]2c2ccccc2)cc1</smiles>

$0 \%$<smiles>CC(C)C1[C@@H]2CC[C@H](C(C)C)P2c2ccccc2P2[C@@H](C(C)C)CC[C@H]12</smiles>

$25 \%, 16 \%$ ee<smiles>CC(CC(C)Pc1ccccc1)Pc1ccccc1</smiles>

$10 \%, 0 \%$ ee<smiles>CC(C)CP(C)c1ccccc1P(C)C(C)(C)C</smiles>

$25 \%,-35 \%$ ee<smiles>COc1cccc(OC)c1-c1c(OC)cccc1P(C(C)C)C(C)(C)C(C)C</smiles>

$0 \%$<smiles>CC1CC[C@H](C)P1CCP1C(C)CC[C@@H]1C</smiles>

$35 \%,<10 \%$ ee

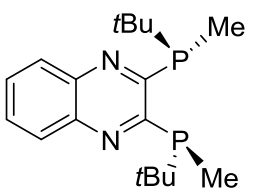

$35 \%, 33 \%$ ee

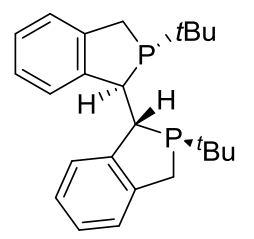

$30 \%, 27 \%$ ee

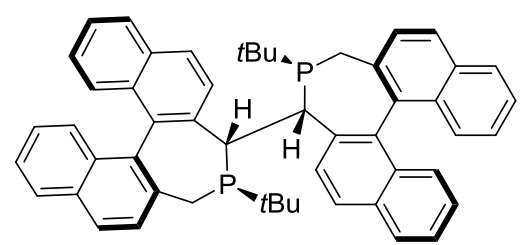

$10 \%, 53 \%$ ee

Figure S43: Ligand screening 


\subsection{General procedure for enantioselective deconjugative isomerization}

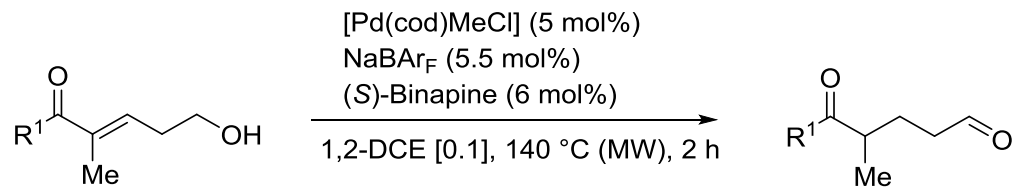

Inside the glovebox, a $5 \mathrm{~mL}$ microwave reaction vial was charged with $[\mathrm{Pd}(\mathrm{cod}) \mathrm{MeCl}]$ (3.4 $\mathrm{mg}, 0.0125 \mathrm{mmol}),(S)$-Binapine (11 $\mathrm{mg}, 0.015 \mathrm{mmol}$ ) and 1,2-dichloroethane $(2.5 \mathrm{~mL})$. The mixture was stirred for 5 minutes at room temperature then $\operatorname{NaBAr}_{F}(12.2 \mathrm{mg}, 0.0138 \mathrm{mmol})$ was added and the reaction mixture stirred 5 minutes. The appropriate substrate $(0.25 \mathrm{mmol})$ was added and the sealed reaction vial was placed in a microwave oven. After $2 \mathrm{~h}$ at $140{ }^{\circ} \mathrm{C}$, the mixture was cooled to room temperature and the solvent removed in vacuo. The crude mixture was purified by flash chromatography on silica gel to afford the analytically pure dicarbonyl product.<smiles>CC(=O)C(CCC=O)C(CCC=O)[N+]([O])=O</smiles>

4-methyl-5-oxohexanal (5a): The residue was purified by flash chromatography on silica gel (Pentane/EtOAc $=40: 1$ ) affording $\mathbf{5 a}$ in $50 \%$ yield (16 mg, 73\% ee) as a colourless oil. The enantiomeric excess was determined by GC analysis: column HYDRODEX TBDM, method: $60^{\circ} \mathrm{C}$ to $170{ }^{\circ} \mathrm{C}$ in 110 minutes then $170{ }^{\circ} \mathrm{C}$ for 30 minutes, $t_{R}=24.4$ and 26.5 min (Figure S44). Carrier gas: $\mathrm{H}_{2}$ $(60 \mathrm{~cm} / \mathrm{s}) \cdot[\alpha]^{23} \mathrm{D}=+0.71(\mathrm{c}=0.2, \mathrm{EtOH})$.

5-cyclohexyl-4-methyl-5-oxopentanal (5b): The residue was purified<smiles>O=CCCC(C(=O)C1CCCCC1)[N+](=O)[O-]</smiles>

by flash chromatography on silica gel (Pentane/EtOAc $=40: 1$ ) affording

$\mathbf{5 b}$ in $57 \%$ yield ( $28 \mathrm{mg}, 75 \%$ ee) as colourless oil. The enantiomeric excess was determined by GC analysis: column HYDRODEX Y-DiMOM, method: $60{ }^{\circ} \mathrm{C}$ to $170{ }^{\circ} \mathrm{C}$ in 110 minutes then $170^{\circ} \mathrm{C}$ for 20 minutes, $t_{R}=96.1$ and $96.5 \mathrm{~min}$ (Figure S45). Carrier gas: $\mathrm{H}_{2}(60 \mathrm{~cm} / \mathrm{s}) \cdot[\alpha]^{23}=-7.5(\mathrm{c}=0.5, \mathrm{EtOH})$.

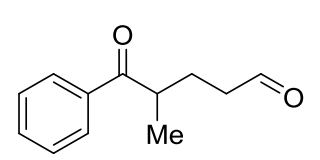

4-methyl-5-oxo-5-phenylpentanal $(5 \mathrm{~g})$ : The residue was purified by flash chromatography on silica gel (Pentane/Diethyl ether $=7: 3$ ) affording $\mathbf{5 g}$ in $99 \%$ yield (47 $\mathrm{mg}, 88 \%$ ee) as yellow oil. The enantiomeric excess was determined by HPLC analysis: AD-H column, Hexane/ $\mathrm{PrOH}$ 98:2, flow $1 \mathrm{~mL} / \mathrm{min}, \lambda=288 \mathrm{~nm}, t_{\mathrm{R}}=11.6$ and $12.7 \mathrm{~min}$ (Figure S46). $[\alpha]^{23}{ }_{\mathrm{D}}=-30.9(\mathrm{c}=0.25$, $\mathrm{CH}_{2} \mathrm{Cl}_{2}$ ). 

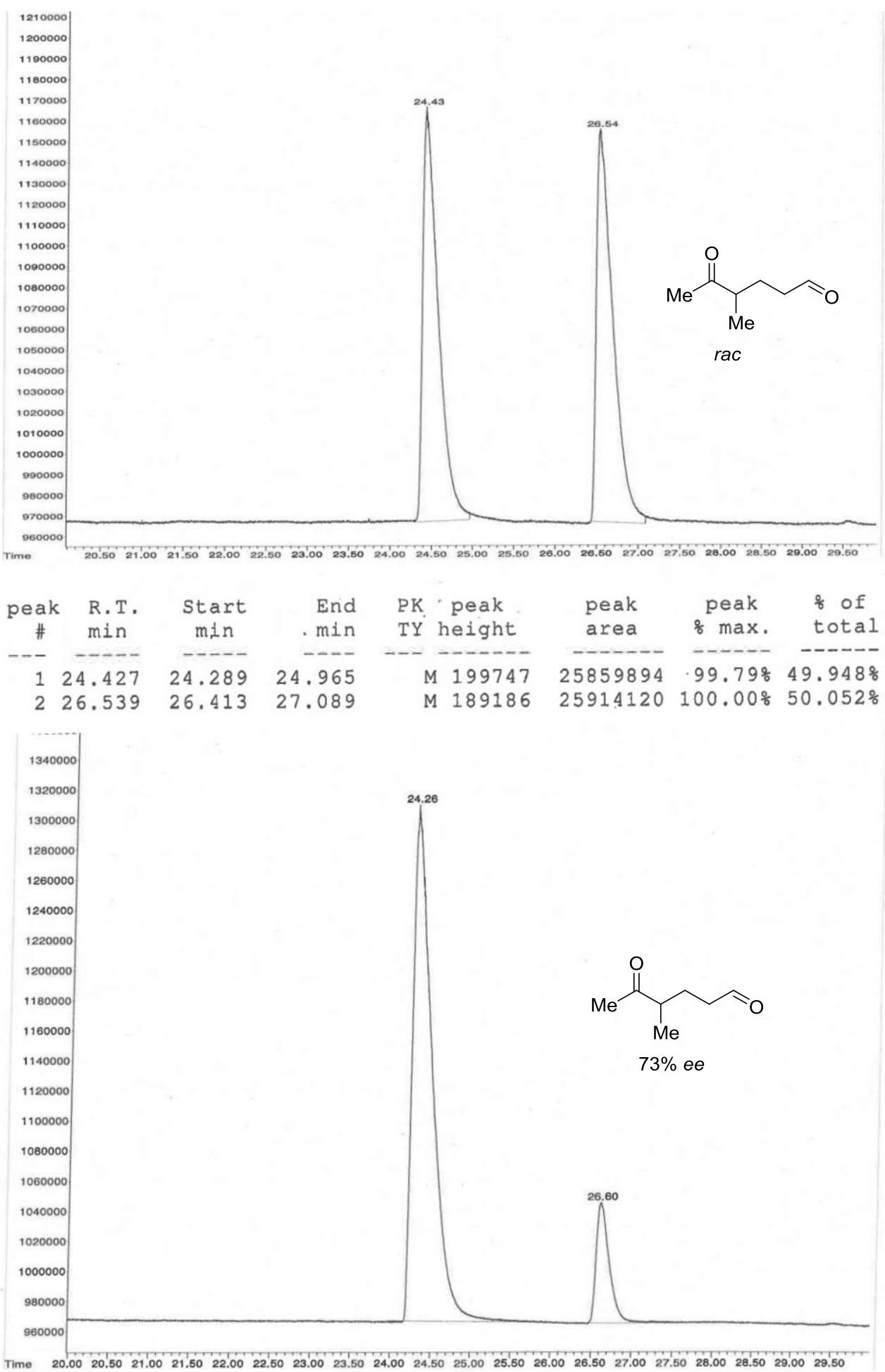

\begin{tabular}{|c|c|c|c|c|c|c|c|}
\hline $\begin{array}{c}\text { peak } \\
\#\end{array}$ & $\begin{array}{l}\text { R.T. } \\
\text { min }\end{array}$ & $\begin{array}{l}\text { Start } \\
\text { min }\end{array}$ & $\begin{array}{l}\text { End } \\
\text { min }\end{array}$ & $\begin{array}{l}\text { PK peak } \\
\text { TY height }\end{array}$ & $\begin{array}{l}\text { peak } \\
\text { area }\end{array}$ & $\begin{array}{c}\text { peak } \\
\text { o max. }\end{array}$ & $\begin{array}{l}\text { o of } \\
\text { total }\end{array}$ \\
\hline-- & $-\cdots$ & $-\cdots$ & $-\cdots$ & - - - - - & $-\cdots-$ & $\ldots-n$ & $\ldots . .$. \\
\hline 1 & 24.259 & 23.976 & 25.703 & M 343483 & 57826235 & $100.00 \%$ & $86.330 \%$ \\
\hline 2 & 26.602 & 26.294 & 27.540 & 80433 & 9156302 & $15.83 \%$ & $13.670 \%$ \\
\hline
\end{tabular}

Figure S44: GC traces of racemic 5a (top) and enantio-enriched $\mathbf{5 a}$ (bottom) 


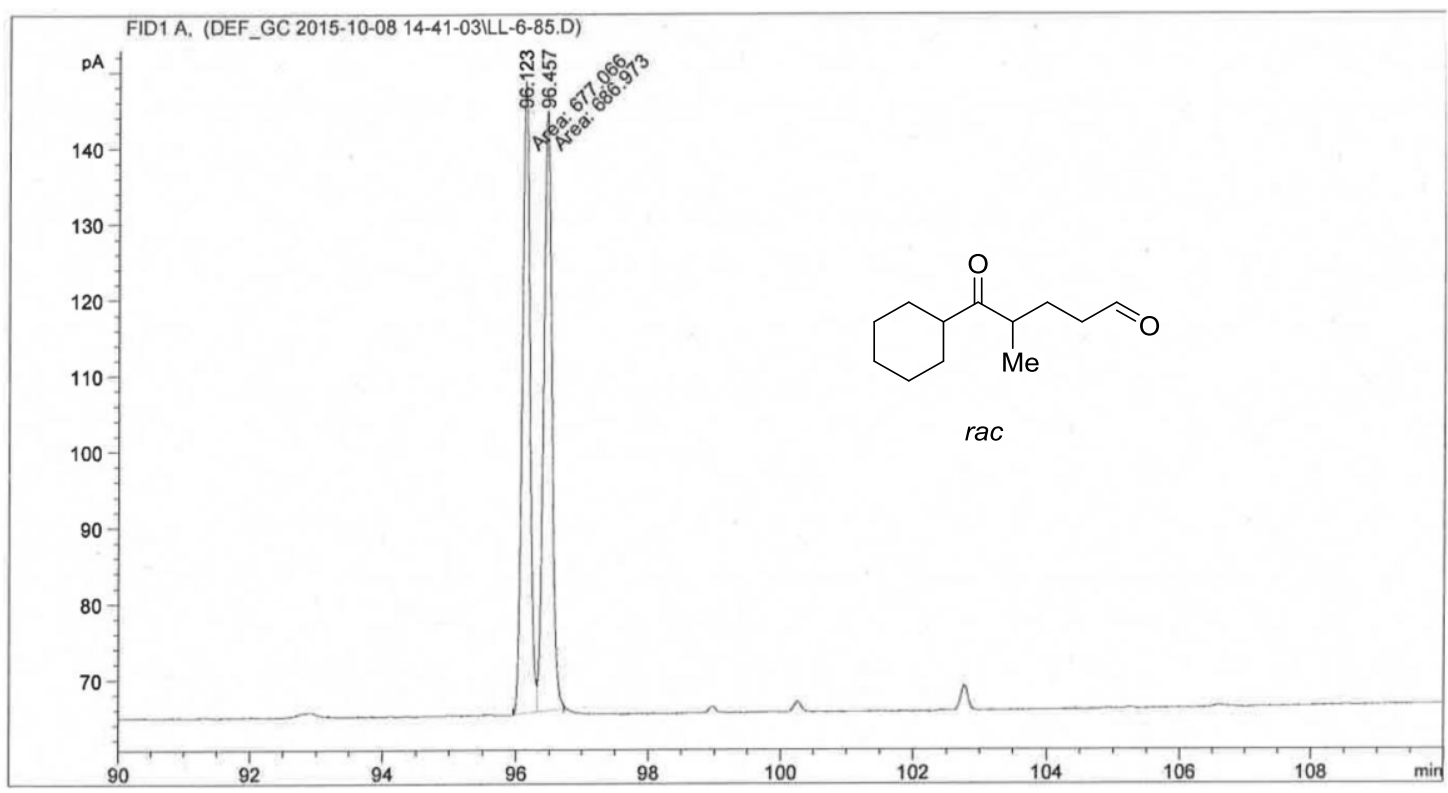

\begin{tabular}{|c|c|c|c|c|c|c|}
\hline $\begin{array}{c}\text { eak } \\
\#\end{array}$ & $\begin{array}{c}\text { RetTime } \\
\text { [min] }\end{array}$ & Type & $\begin{array}{l}\text { Width } \\
\text { [min] }\end{array}$ & $\begin{array}{r}\text { Area } \\
{\left[\mathrm{pA}^{\star} \mathrm{s}\right]}\end{array}$ & $\begin{array}{l}\text { Height } \\
{[\mathrm{pA}]}\end{array}$ & $\begin{array}{c}\text { Area } \\
\%\end{array}$ \\
\hline & & & & ------ & & -- \\
\hline 1 & & & & & & \\
\hline 2 & 6.457 & FM & & 686. & 02203 & 36316 \\
\hline
\end{tabular}
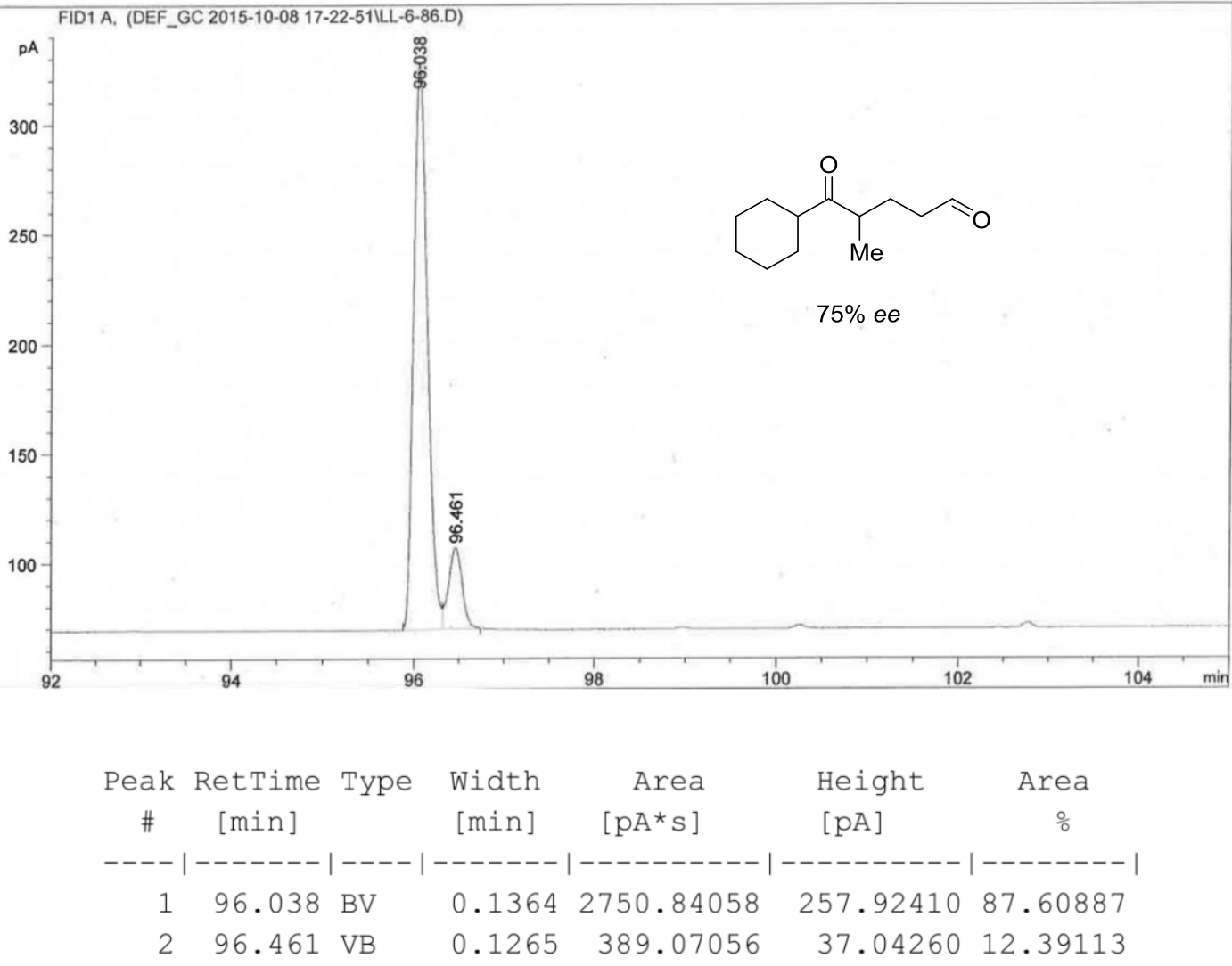

Figure S45: GC traces of racemic 5b (top) and enantio-enriched $\mathbf{5 b}$ (bottom) 
mAU

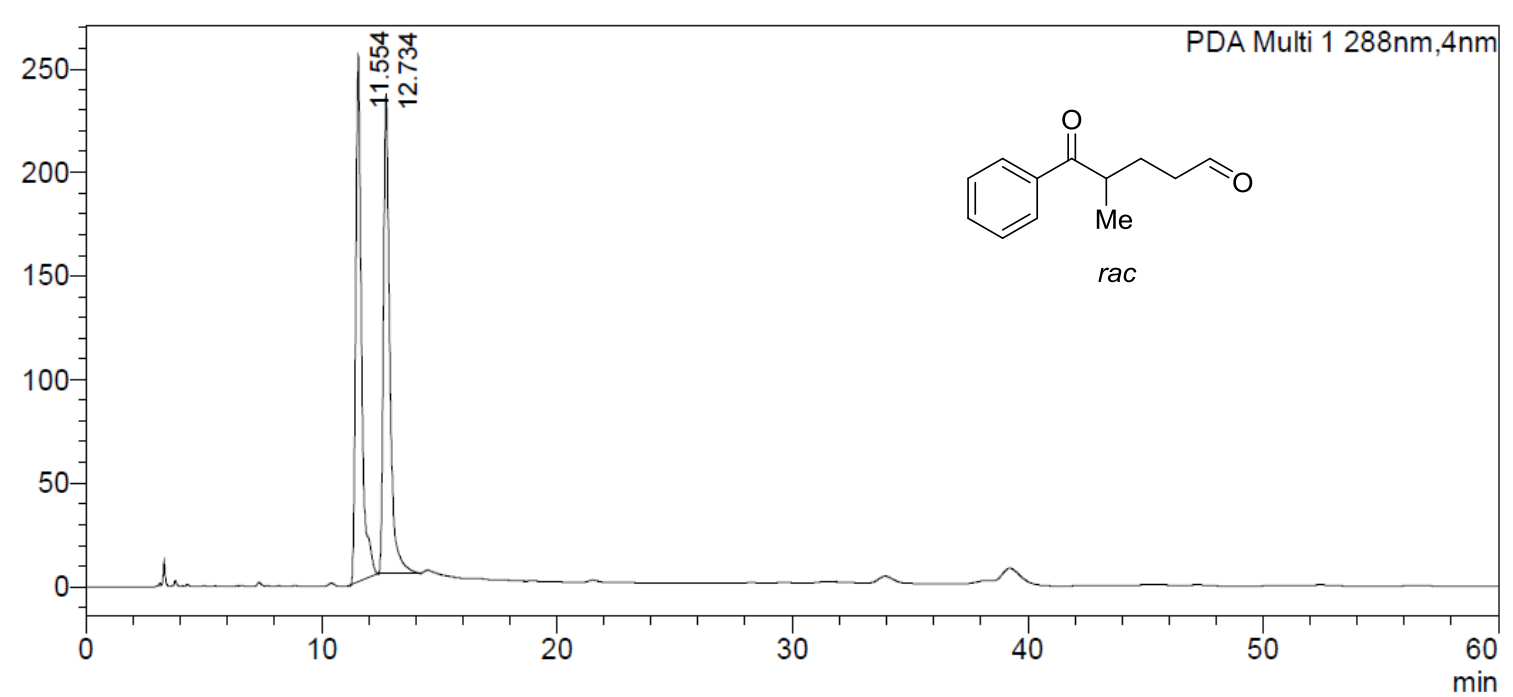

\begin{tabular}{|c|c|c|}
\hline & \multirow{3}{*}{$\frac{\text { Area } \%}{50.059}$} \\
\hline & & \\
\hline \multicolumn{2}{|c|}{\begin{tabular}{r|r} 
DA Ch1 288r \\
Deak\# Ret. Ti \\
11.5
\end{tabular}} & \\
\hline \multicolumn{2}{|c|}{\begin{tabular}{r|r}
2 & \\
\end{tabular}} & \\
\hline \multicolumn{2}{|c|}{ Total } & \\
\hline
\end{tabular}

mAU

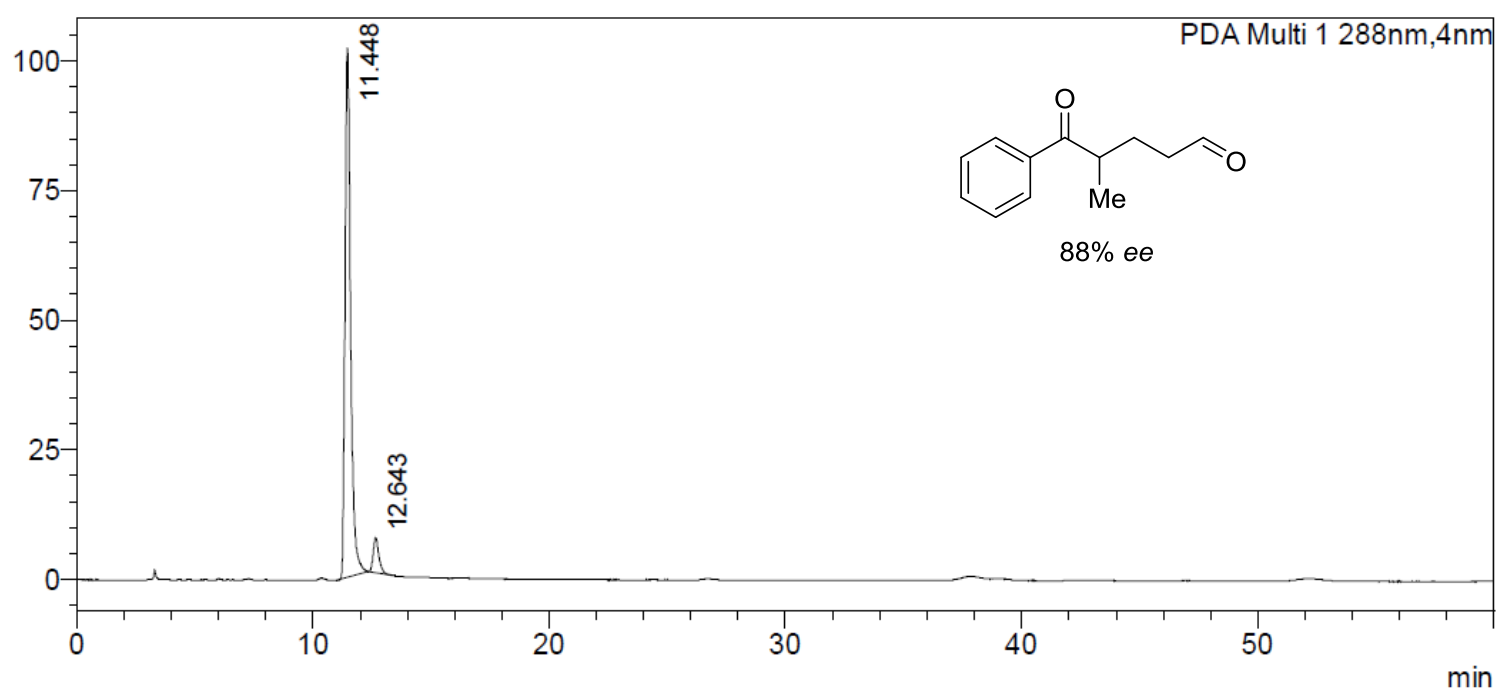

\begin{tabular}{|c|c|c|}
\hline \multirow{2}{*}{\multicolumn{3}{|c|}{$\begin{array}{l}\text { PDA Ch1 288nm } \\
\text { Peak\# Ret. Time }\end{array}$}} \\
\hline & & Area\% \\
\hline 1 & 11.448 & 93.655 \\
\hline 2 & 12.643 & \\
\hline Tota & & 1000 \\
\hline
\end{tabular}

Figure S46: HPLC traces of racemic $\mathbf{5 g}$ (top) and enantioenriched $\mathbf{5 g}$ (bottom) 


\section{References}

1. Reger, D. L.; Wright, T. D.; Little, C. A.; Lamba, J. J. S.; Smith, M. D. Inorg. Chem. 2001, 40, 3810-3814.

2. Dias, L. C.; de Lucca, E. C. Jr. Org. Lett. 2015, 17, 6278-628.

3. Fernandes, R. A.; Chavan, V. P. Tetrahedron: Asymmetry 2011, 22, 1312-1319.

4. Phillips, D. J.; Graham A. E. Synlett. 2008, 649-652.

5. Lifchits, O.; Mahlau, M.; Reisinger, C. M.; Lee, A.; Farès, C.; Polyak, I.; Gopakumar, G.; Thiel, W.; List, B. J. Am. Chem. Soc. 2013, 135, 6677-6693.

6. Li, D. R.; Murugan, A.; Falck, J. R. J. Am. Chem. Soc. 2008, 130, 46-48.

7. Kasatkin, A.; Whitby R. J. Tetrahedron 2003, 59, 9857-9864.

8. Chen, J.; Lu G.-P.; Cai, C. RSC Adv. 2015, 5, 13208-13211.

9. Matsumoto, A.; Asano, K.; Matsubara, S. Chem. Commun. 2015, 51, 11693-11696.

10. Blum, A.; Hess, W.; Studer, A. Synthesis 2004, 13, 2226-2235.

11. Yadav, J. S.; Reddy, N. M.; Reddy, P. A. N.; Ather, H.; Prasad, A. R. Synthesis 2010, 9, 1473-1478.

12. Tan, K.-T.; Chng, S.-S.; Cheng, H.-S.; Loh, T.-P. J. Am. Chem. Soc. 2003, 125, 2958-2963.

13. Evans, P. A.; Grisin, A. Lawler, M. J. J. Am. Chem. Soc. 2012, 134, 2856-2859.

14. Phillips, D. J.; Pillinger, K. S.; Li, W.; Taylor, A. E.; Graham, A. E. Chem. Commun. 2006, 2280-2282.

15. Uehling, M. R.; Suess, A. M.; Lalic, G. J. Am. Chem. Soc. 2015, 137, 1424-1427.

16. Wang, Y.; Ma, J.; Cheon, H.-S.; Kishi, Y. Angew. Chem. Int. Ed. 2007, 46, 13331336.

17. Phillips, D. J.; Pillinger, K. S.; Taylor, W. Li; Graham, A. A. Tetrahedron 2007, 63, 10528-10533.

18. Molander, G. A.; Harris, C. R. J. Org. Chem. 1997, 62, 7418-7429.

19. He, W.; Soll C. E.; Chavadi, S. S.; Zhang, G.; Warren, J. D.; Quadri L. E. N. J. Am. Chem. Soc. 2009, 131, 16744-16750.

20. Cons, B. D.; Bunt, A. J.; Bailey, C. D.; Willis, C. L. Org. Lett. 2013, 15, 2046-2049.

21. Phillips, D. J.; Graham, A. E. Synlett 2008, 5, 649-652.

22. Dodd, D. S.; Oehlschlager A. C. J. Org. Chem. 1992, 57, 2794-2803.

23. Mohapatra, D. K.; Umamaheshwar, G.; Rao, M. M.; Umadevi, D.; Yadev, J. S. RSC Adv. 2014, 4, 8335-8340.

24. Allan, K. M.; Hong, B. D.; Stoltz, B. M. Org. Biomol. Chem. 2009, 7, 4960-4964.

25. Nakatsuji, H.; Ueno, K.; Misaki, T.; Tanabe, Y. Org. Lett. 2008, 10, 2131-2134. 
26. Zhao, S.; He, Y.-H.; Wu, D.; Guan, Z. J. Fluorine Chem. 2010, 131, 597-605.

27. Han, C.; Buchwald, S. L. J. Am. Chem. Soc. 2009, 131, 7532-7533.

28. Drescher, S.; Meister, A.; Blume, A.; Karlsson, G.; Almgren, M.; Dobner, B. Chem. Eur. J. 2007, 13, 5300-5307.

29. Caplar, V.; Frkanec, L.; Vujicic, N. S.; Zinic, M. Chem. Eur. J. 2010, 16, 3066-3082.

30. Sui, B.; Yeh, E. A.-H.; Curran, D. P. J. Org. Chem. 2010, 75, 2942-2954.

31. Xu, G.; Micklatcher, M.; Silvestri, M. A.; Hartman,T. L.; Burrier, J.; Osterling, M. C.; Wargo, H.; Turpin, J. A.; Buckheit R. W.; Cushman M. Jr. J. Med. Chem. 2001, 144, 4092-4113.

32. Hsu, J.-L.; Fang, J.-M. J. Org. Chem. 2001, 66, 8573-8584.

33. Kulkarni, M. G.; Sebastian M. T. Synth. Commun. 1991, 21, 581-586.

34. Lory, P.M. J.; Jones, R. C. F.; lley, J. N.; Coles, S. J.; Hursthouse, M. B Org. Biomol. Chem. 2006, 4, 3155-3165.

35. Yu, Y.; Liebeskind, L. S. J. Org. Chem. 2004, 69, 3554-3557.

36. Cloarec, J.-M.; Charette, A. B. Org. Lett. 2004, 6, 4731-4734.

37. Basar, N.; Damodaran, K.; Liu, H.; Morris, G. A.; Sirat, H. M.; Thomas, E. J.; Curran, D. P. J. Org. Chem. 2014, 79, 7477-7490

38. G. N. Varseev, M. E. Maier, Org. Lett. 2007, 9, 1461-1464.

39. Egger, J.; Fischer, S.; Bretscher, P.; Freigang, S.; Kopf, M.; Carreira, E. M. Org. Lett. 2015, 17, 4340-4343.

40. Gastl, C.; Laschat, S. Synthesis, 2010, 15, 2643-2651.

41. Juncosa, J. I. Jr.; Groves, A. P.; Xia, G.; Silverman, R. B. Bioorg. Med. Chem. 2013, 21, 903-911.

42. Stritzke, K.; Schulz, S.; Nishida, R. Eur. J. Org. Chem. 2002, 3884-3892. 


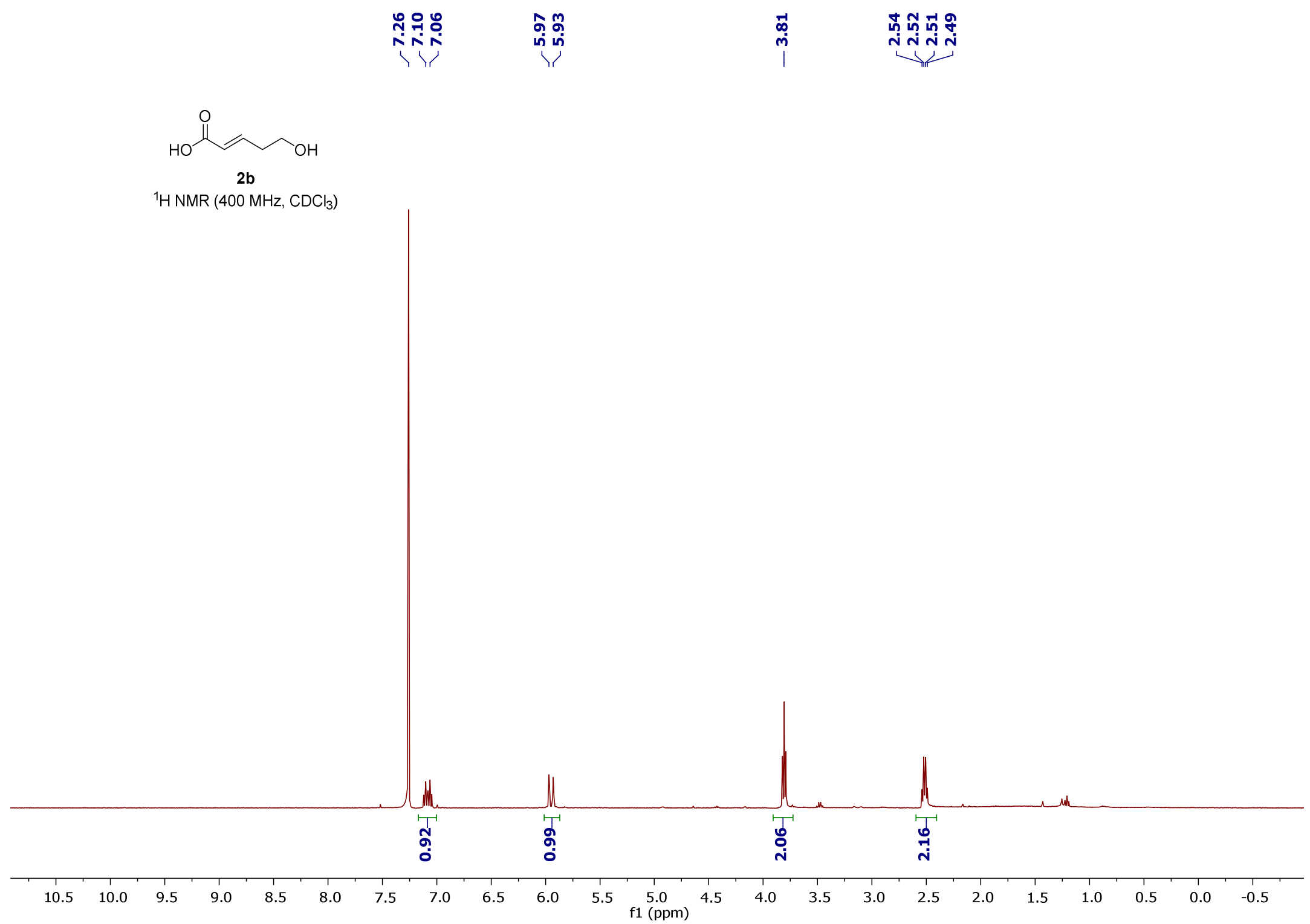




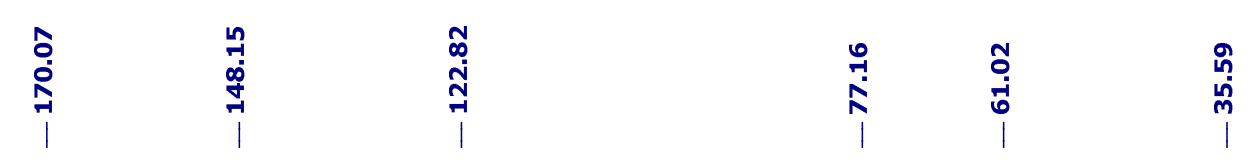

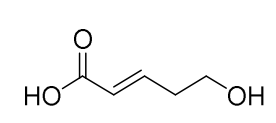

2b

${ }^{13} \mathrm{C}\left\{{ }^{1} \mathrm{H}\right\} \mathrm{NMR}\left(101 \mathrm{MHz}, \mathrm{CDCl}_{3}\right)$

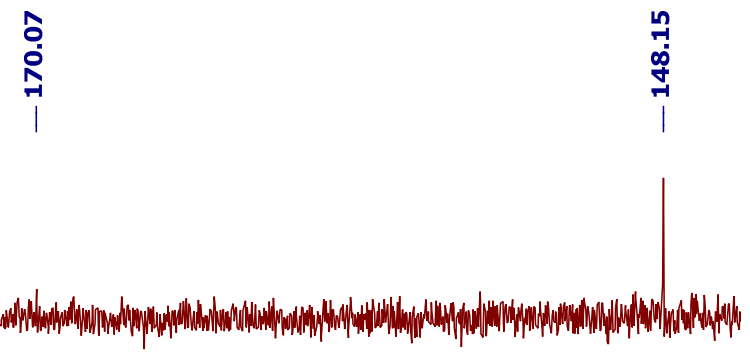

$\begin{array}{rrrr}170 & 165 & 160 & 150 \\ \text { f1 (ppm) } & 150\end{array}$

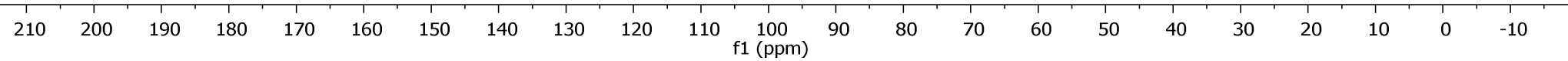




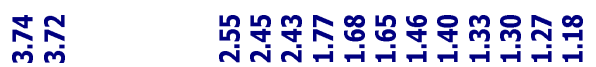

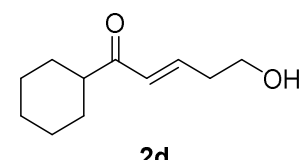

${ }^{1} \mathrm{HNMR}\left(400 \mathrm{MHz}, \mathrm{CD}_{2} \mathrm{Cl}_{2}\right)$

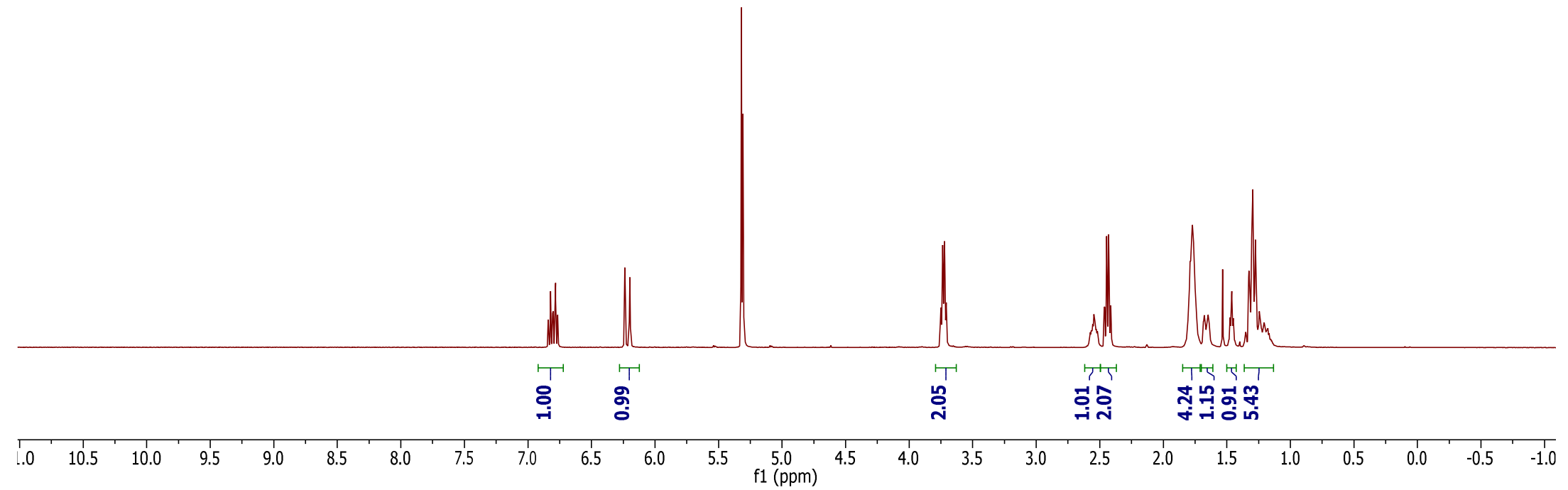




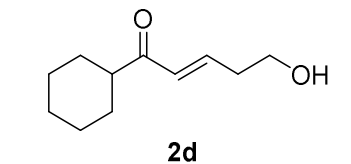

$\left.{ }^{13} \mathrm{C}^{1} \mathrm{H}\right\} \mathrm{NMR}\left(101 \mathrm{MHz}, \mathrm{CD}_{2} \mathrm{Cl}_{2}\right)$

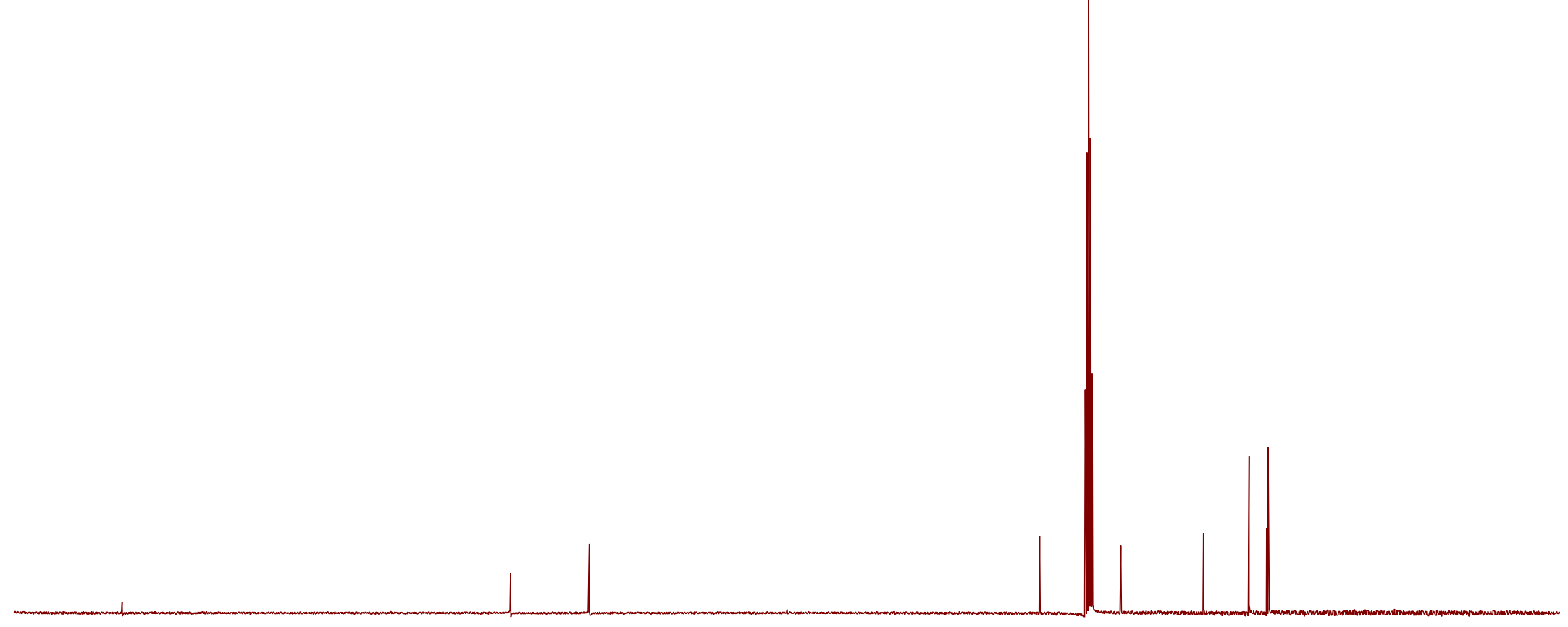

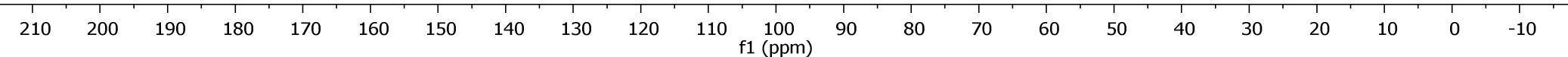




\section{ำ.}

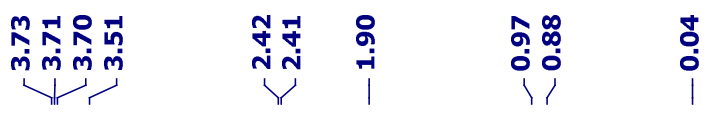

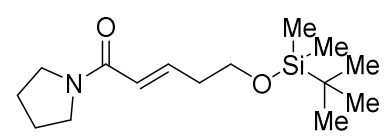

${ }^{1} \mathrm{H} \mathrm{NMR}\left(400 \mathrm{MHz}, \mathrm{CDCl}_{3}\right.$ )

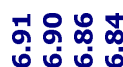

414

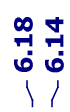

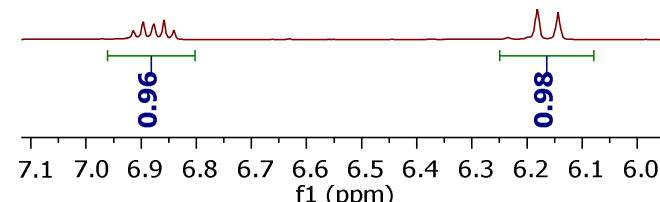

6.66 .5
$\mathrm{f} 1(\mathrm{ppm})$

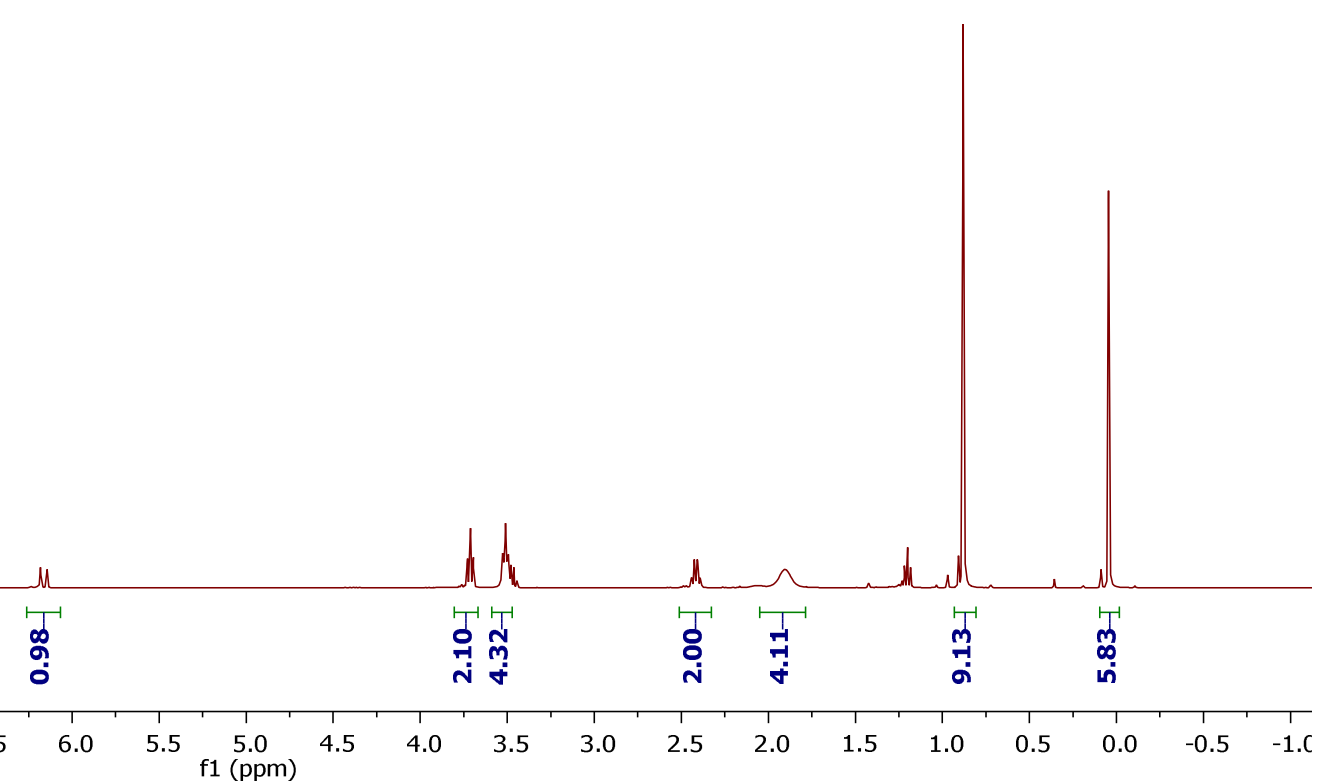





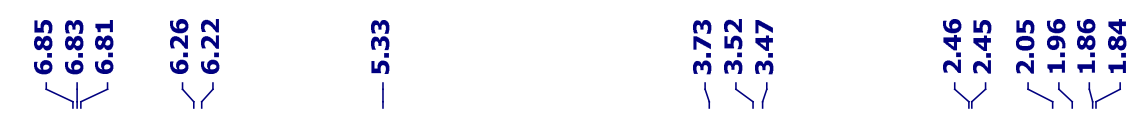

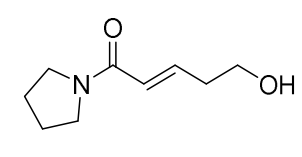

${ }^{1} \mathrm{H}$ NMR $\left(400 \mathrm{MHz}, \mathrm{CD}_{2} \mathrm{Cl}_{2}\right)$

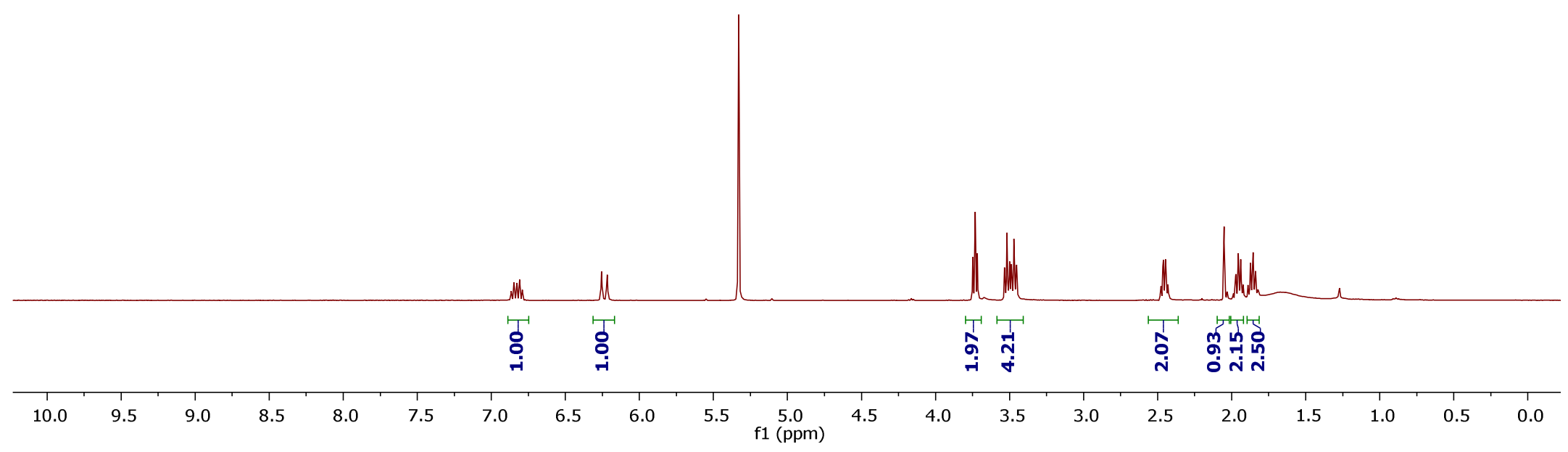




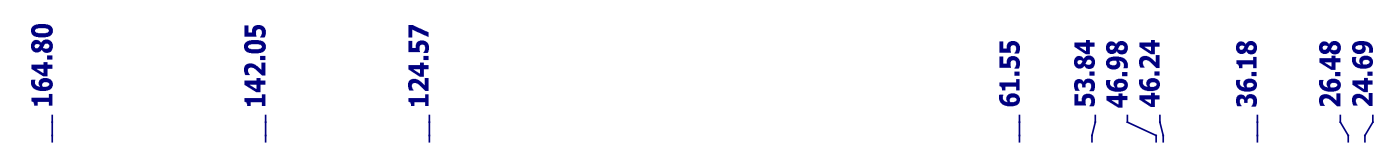

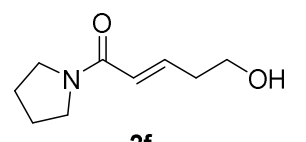

${ }^{13} \mathrm{C}\left\{{ }^{1} \mathrm{H}\right\} \mathrm{NMR}\left(101 \mathrm{MHz}, \mathrm{CD}_{2} \mathrm{Cl}_{2}\right)$

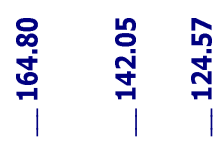

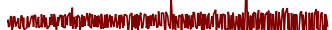

$$
160 \underset{f 1(\mathrm{ppm})}{140} 120
$$

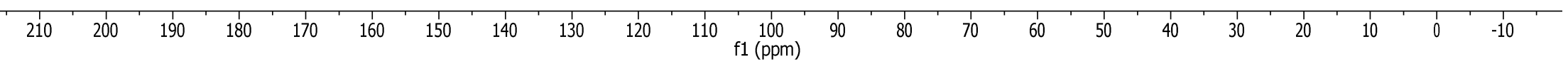




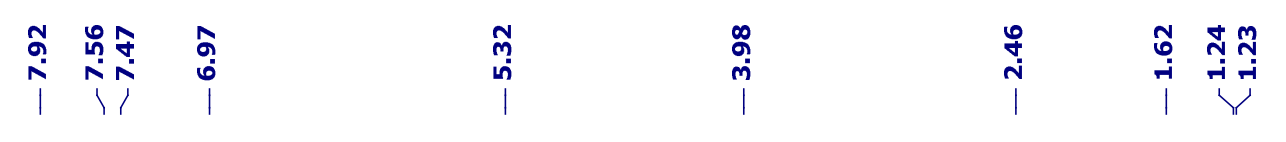
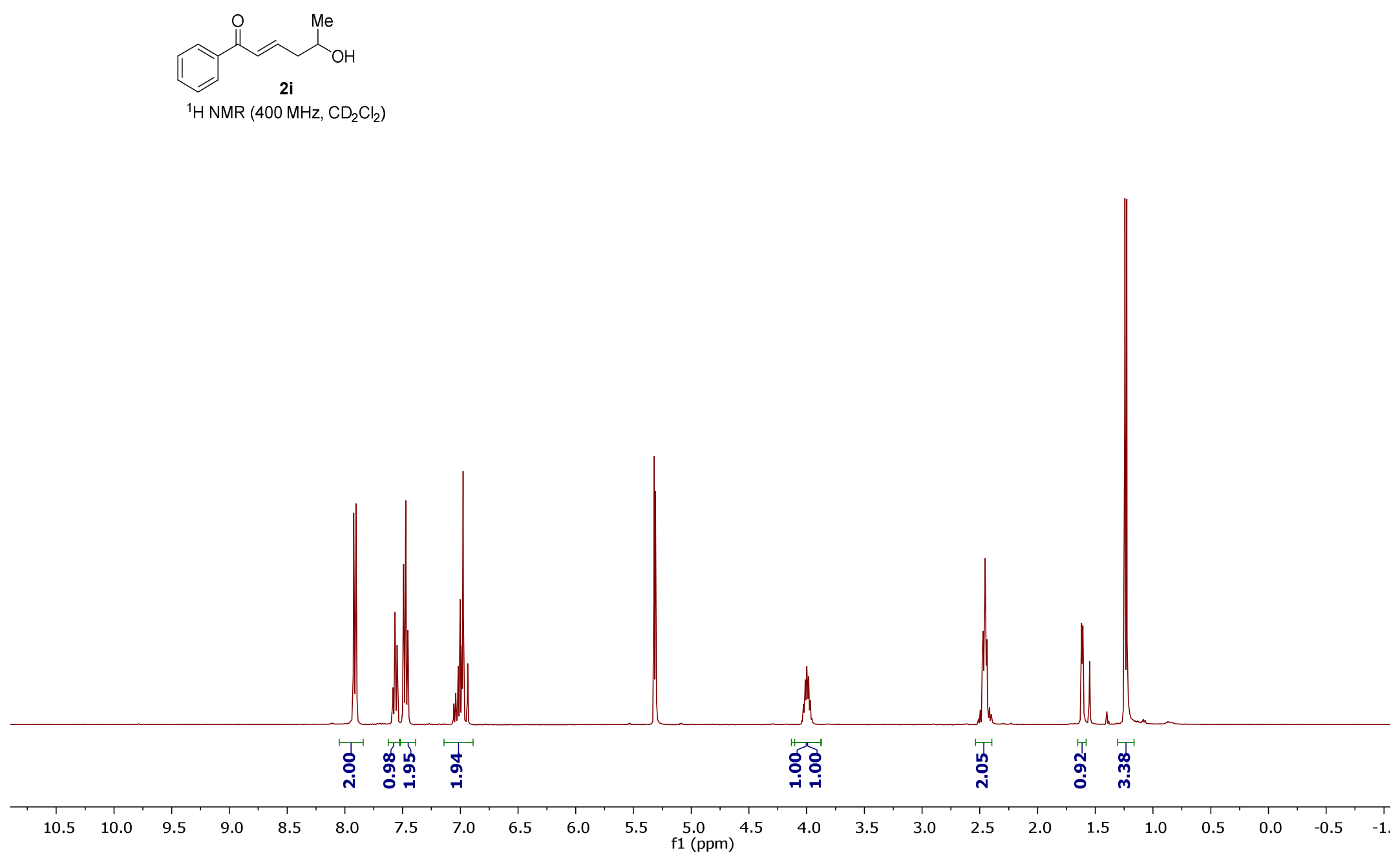


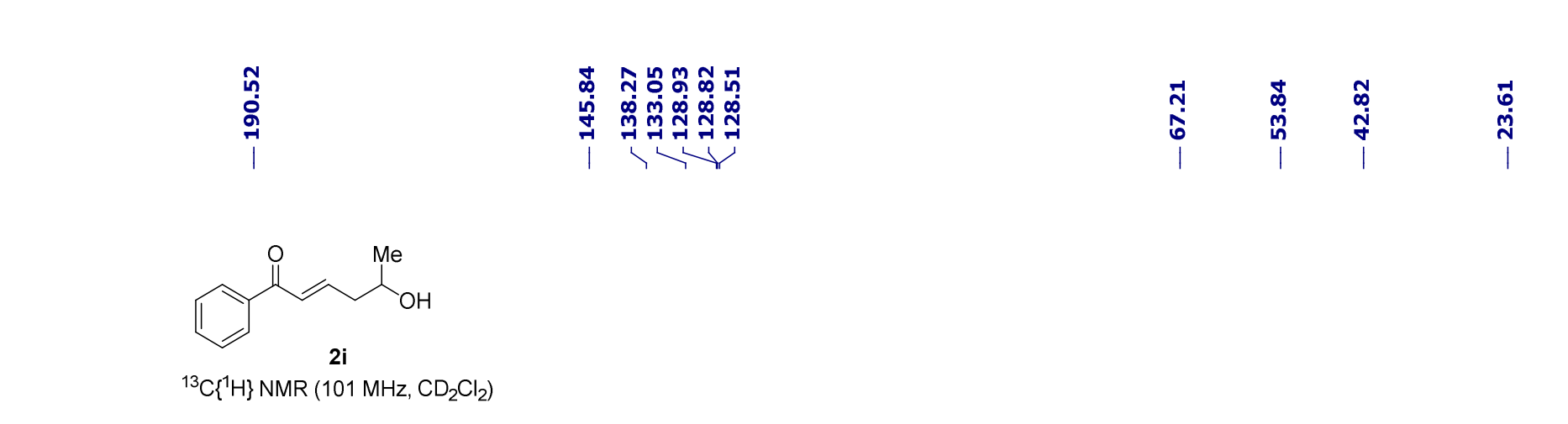




\begin{tabular}{|c|c|c|c|c|}
\hline 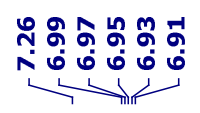 & 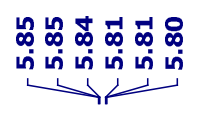 & 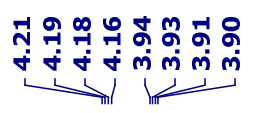 & 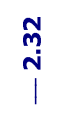 & 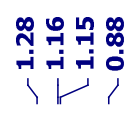 \\
\hline
\end{tabular}

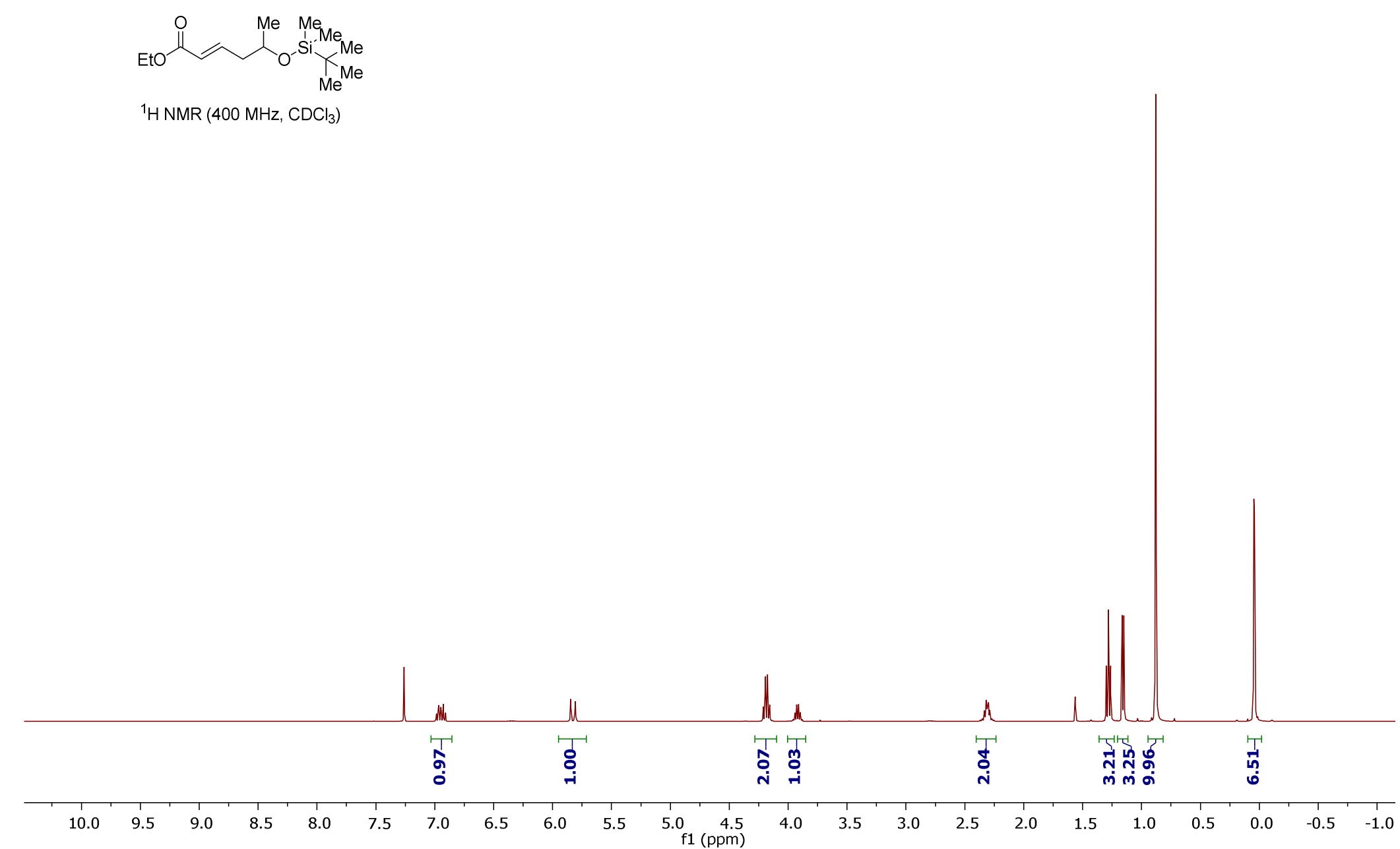




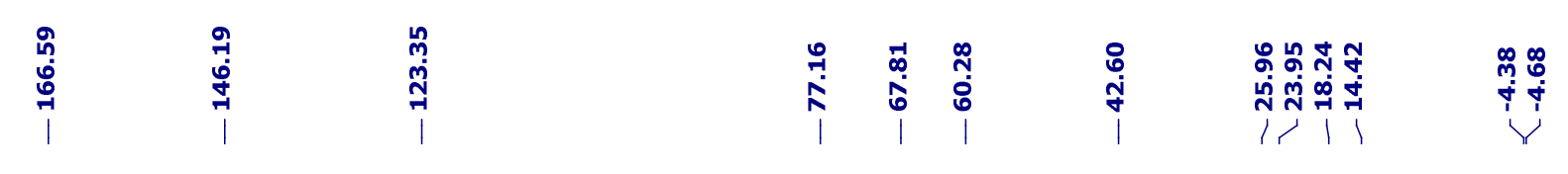

$$
\left.{ }^{13} \mathrm{C}^{1} \mathrm{H}\right\} \mathrm{NMR}\left(101 \mathrm{MHz}, \mathrm{CDCl}_{3}\right)
$$
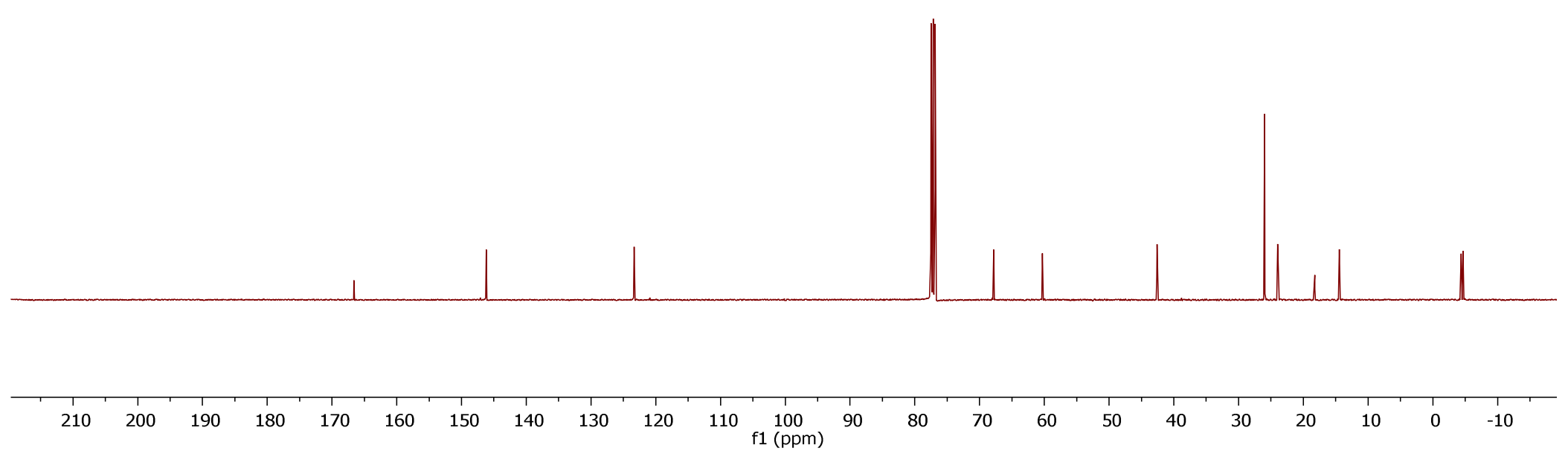


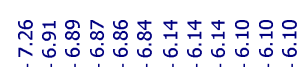

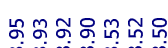

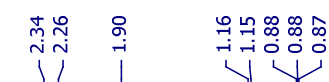

槰

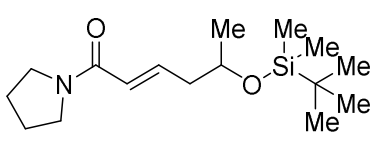

${ }^{1} \mathrm{H} \mathrm{NMR}\left(400 \mathrm{MHz}, \mathrm{CDCl}_{3}\right)$

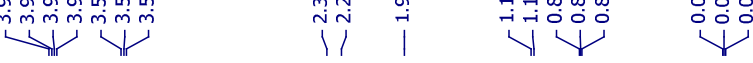

HNMR (400 MHz, $\mathrm{CDCl}_{3}$ )

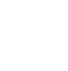




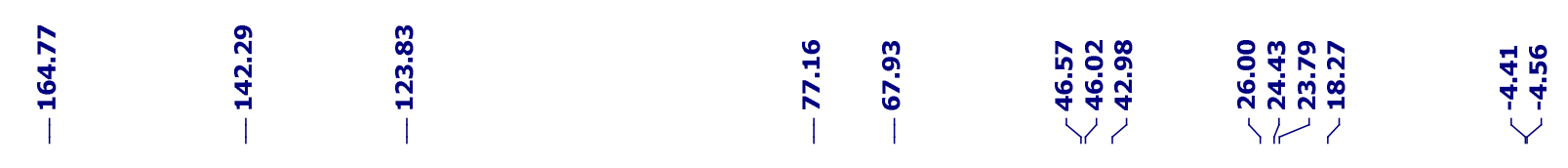

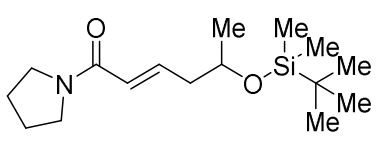

${ }^{13} \mathrm{C}\left\{{ }^{1} \mathrm{H}\right\} \mathrm{NMR}\left(101 \mathrm{MHz}, \mathrm{CDCl}_{3}\right)$

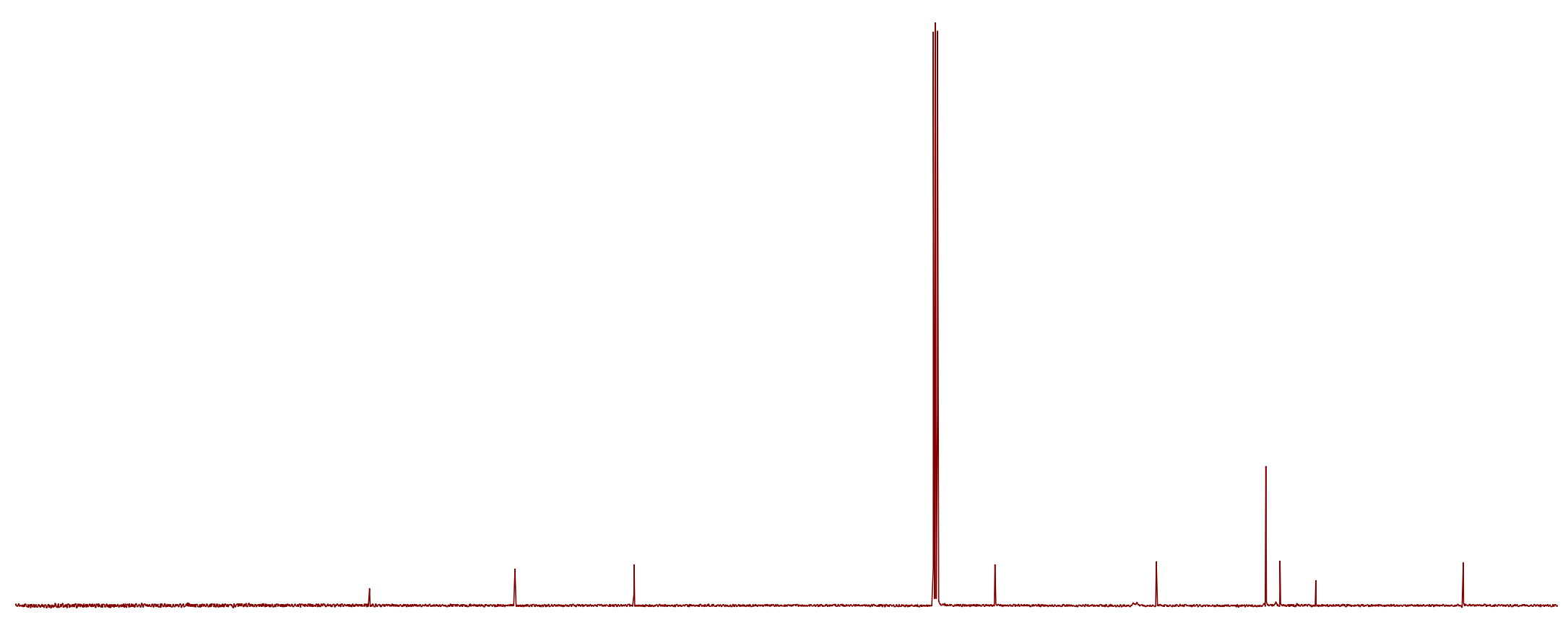

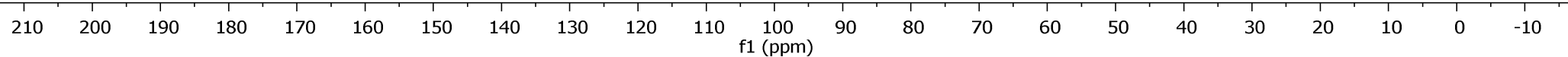



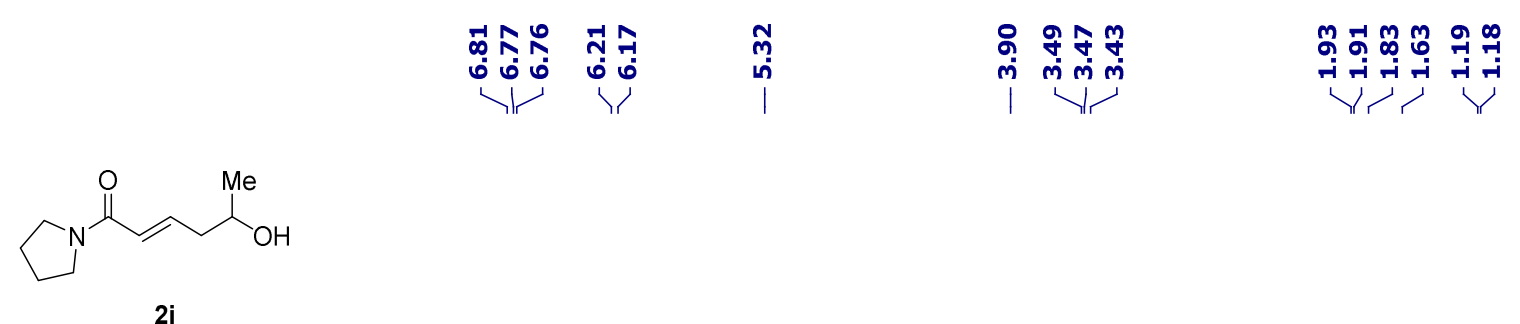

${ }^{1} \mathrm{H} \mathrm{NMR}\left(400 \mathrm{MHz}, \mathrm{CDCl}_{3}\right)$

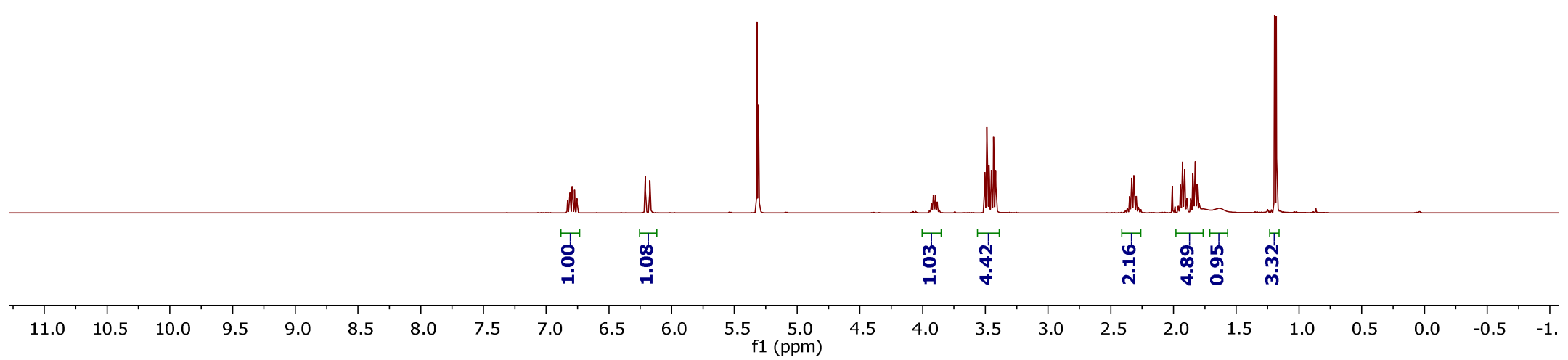




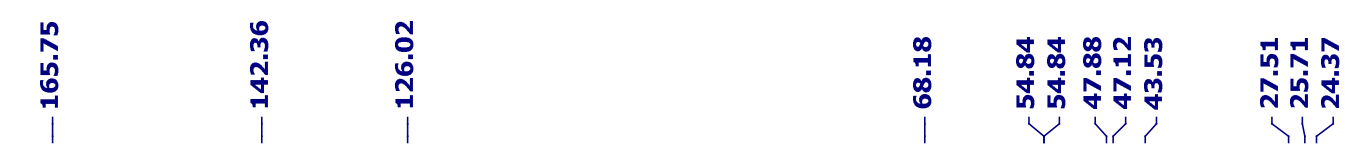

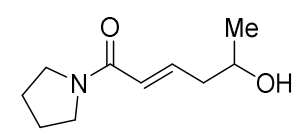

${ }^{13} \mathrm{C}\left\{{ }^{1} \mathrm{H}\right\} \mathrm{NMR}\left(101 \mathrm{MHz}, \mathrm{CDCl}_{3}\right)$

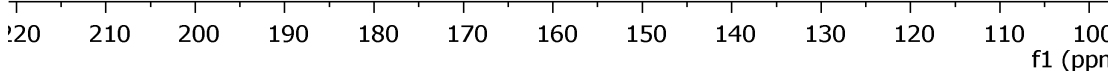




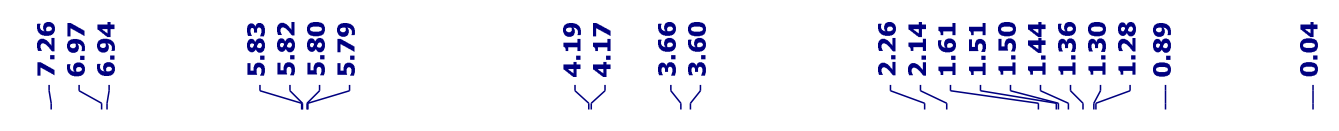

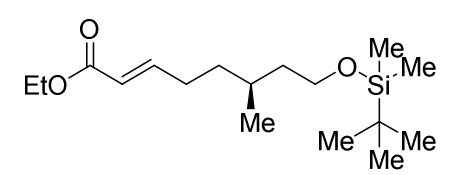

${ }^{1} \mathrm{H} \mathrm{NMR}\left(400 \mathrm{MHz}, \mathrm{CDCl}_{3}\right)$

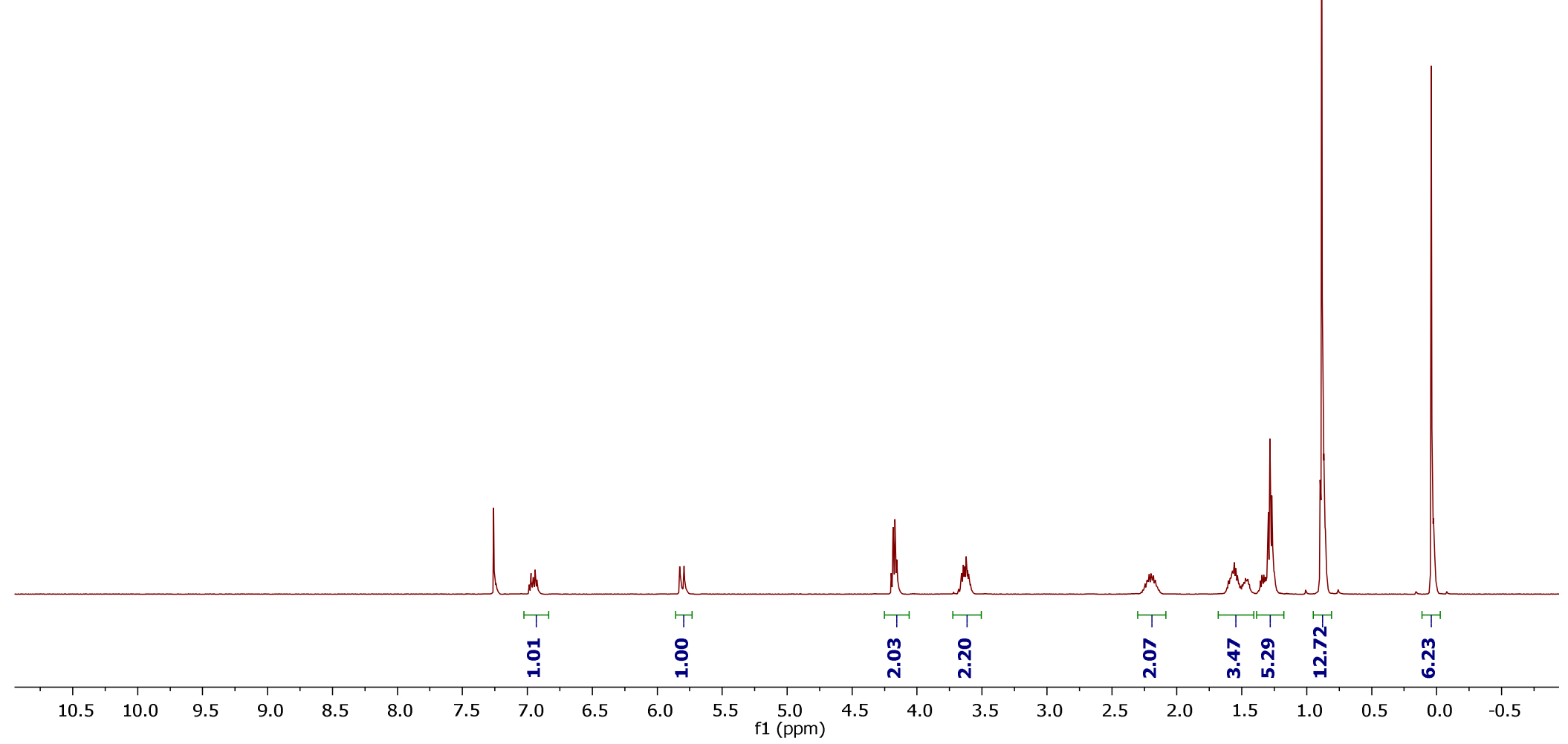




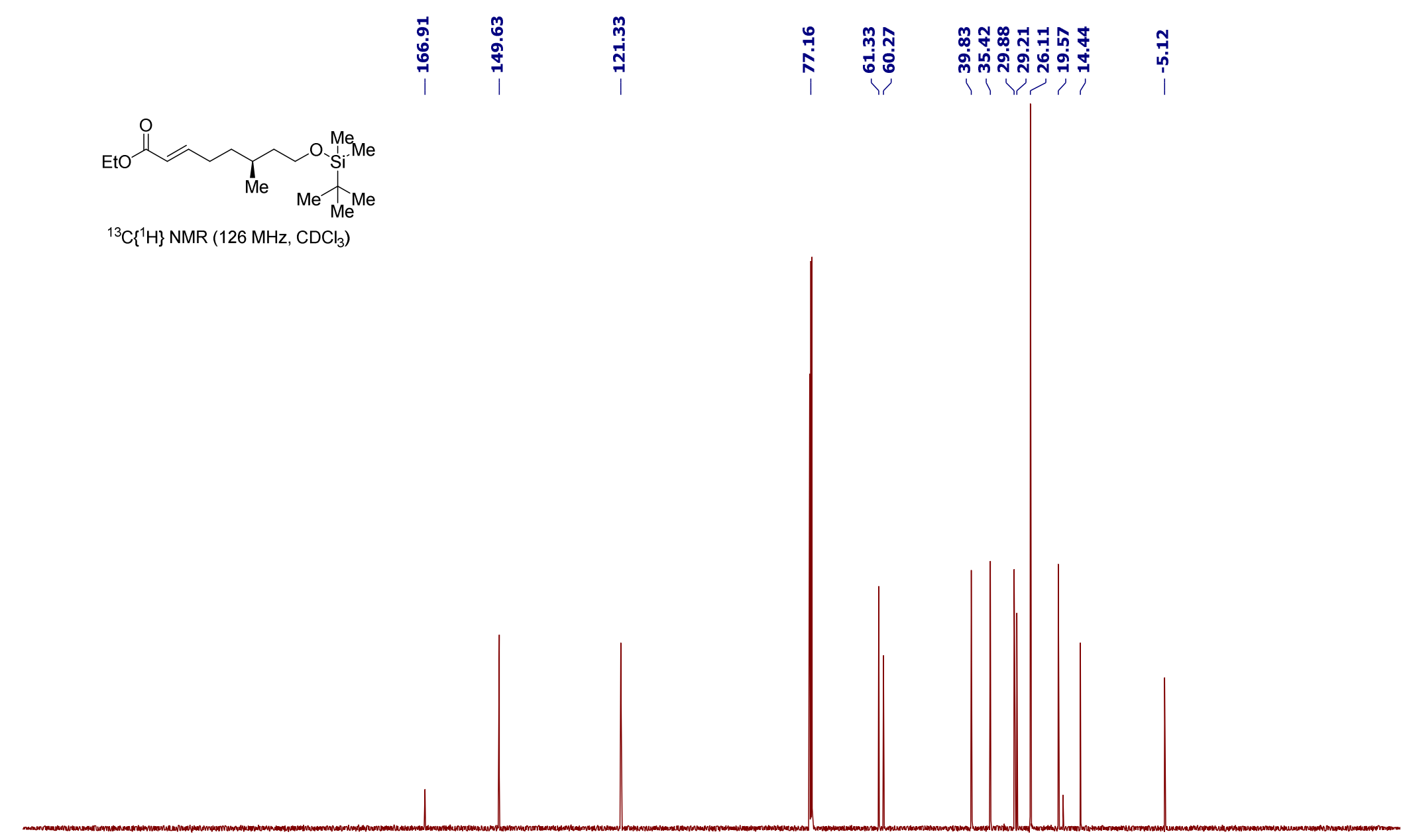
$\begin{array}{lllllllllllllllllllllllllllllllllllllll}60 & 250 & 240 & 230 & 220 & 210 & 200 & 190 & 180 & 170 & 160 & 150 & 140 & 130 & 120 & 110 & 100 & 90 & 80 & 70 & 60 & 50 & 40 & 30 & 20 & 10 & 0 & -10 & -20 & -30 & -40 & -50 & -t\end{array}$ 


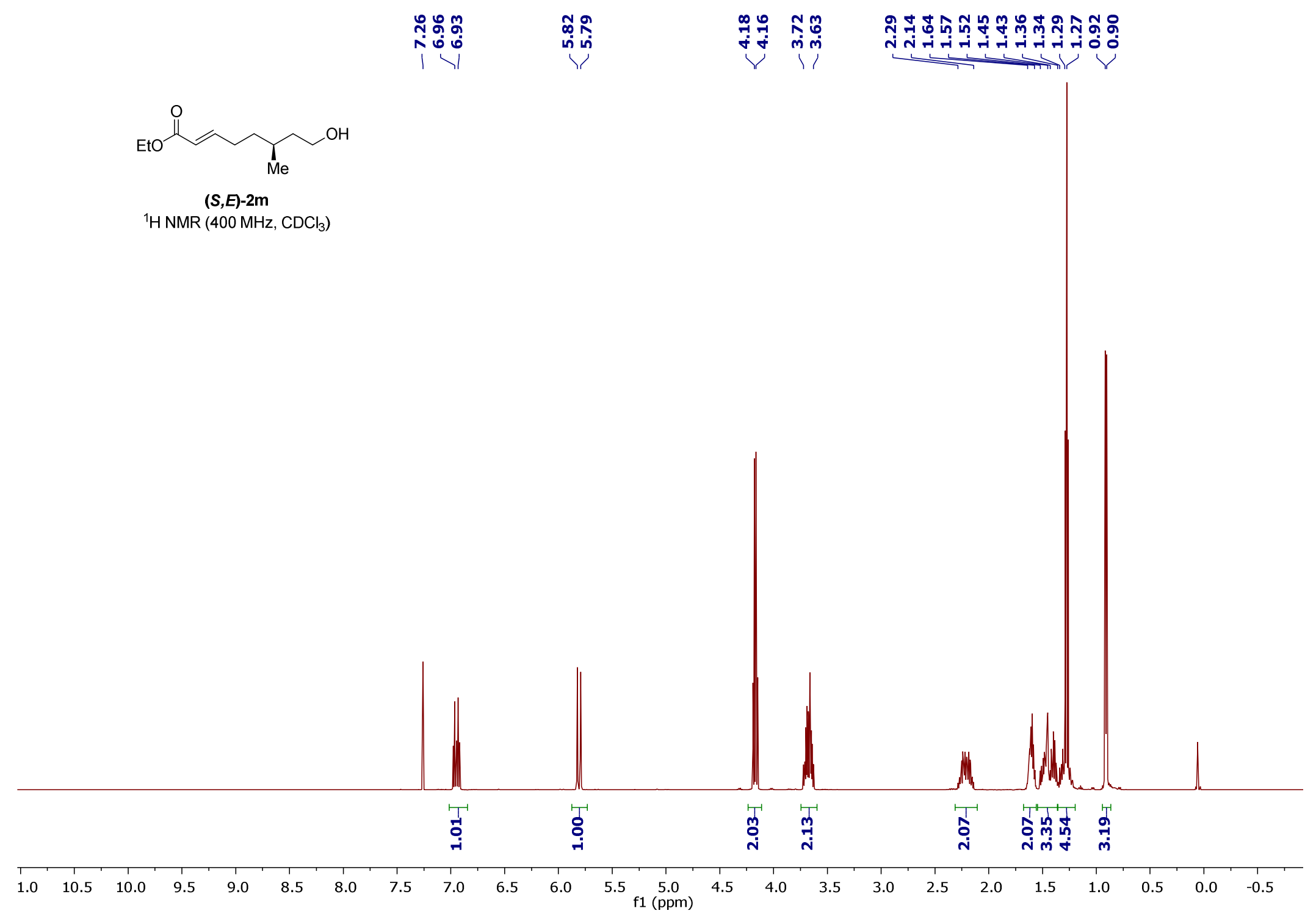




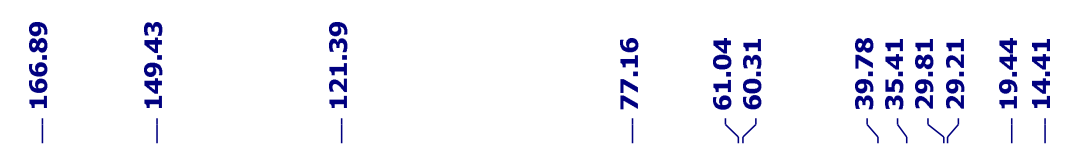

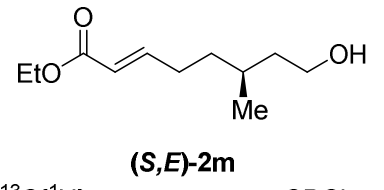

${ }^{13} \mathrm{C}\left\{{ }^{1} \mathrm{H}\right\}$ NMR $\left(126 \mathrm{MHz}, \mathrm{CDCl}_{3}\right)$

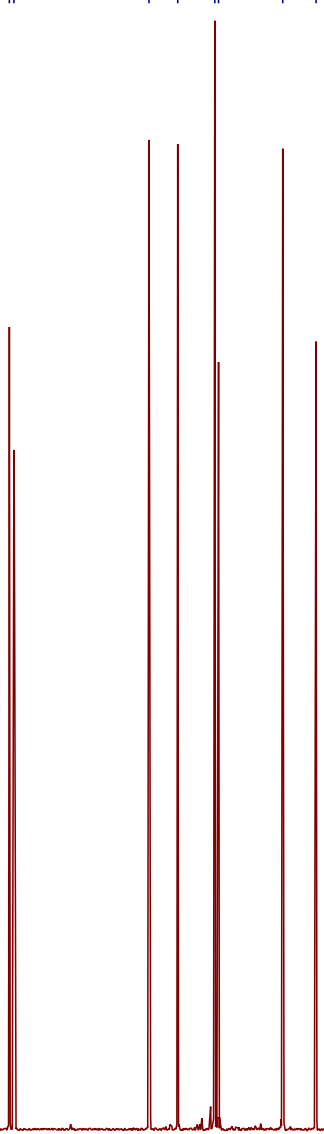

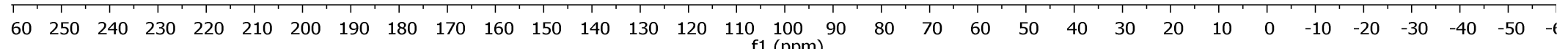
1 (ppm) 
กำ

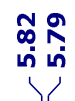

궁

品

¿̊.
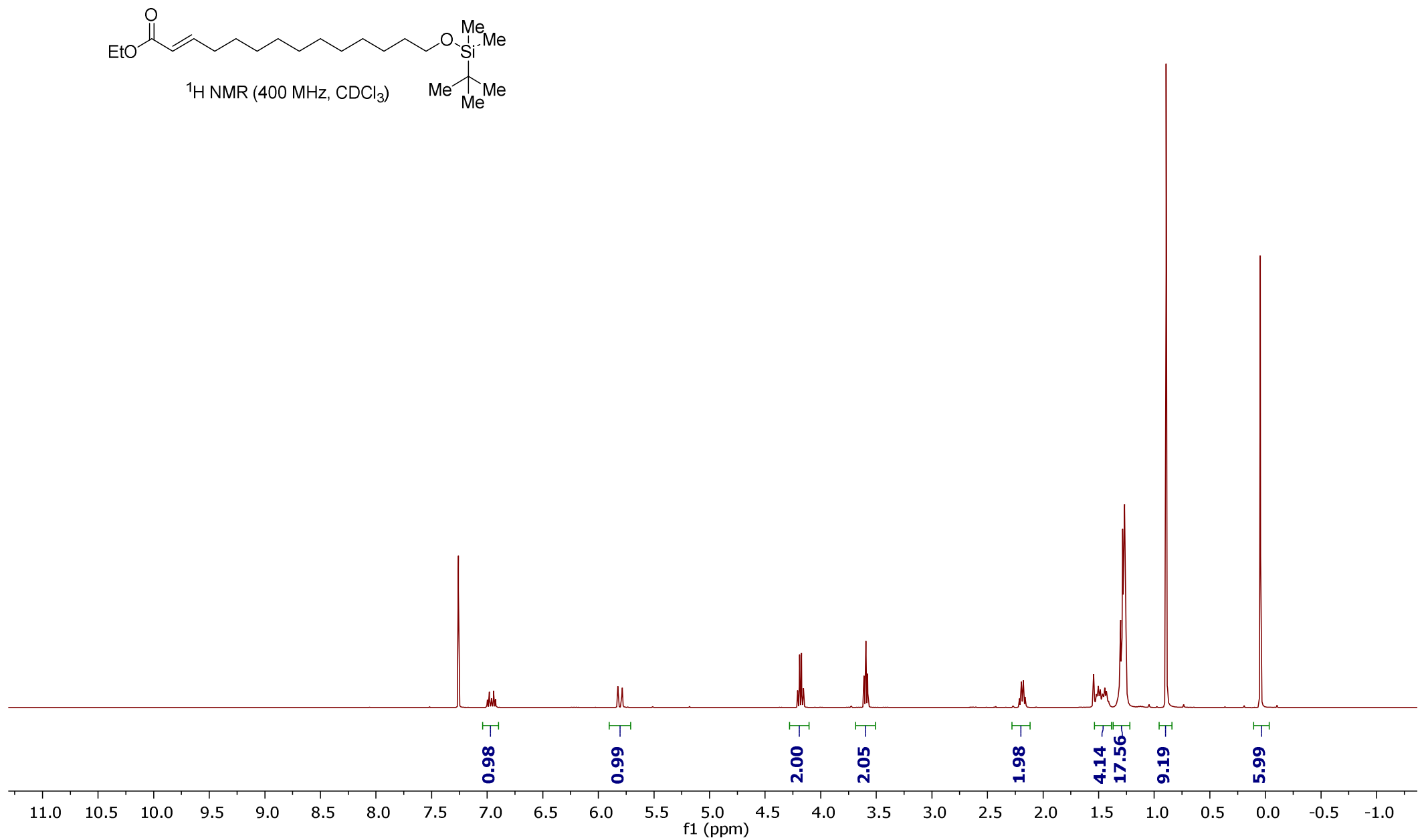


\begin{tabular}{|c|c|c|c|}
\hline $\begin{array}{l}\stackrel{0}{0} \\
\stackrel{9}{+} \\
1\end{array}$ & 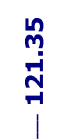 & 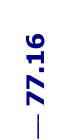 & 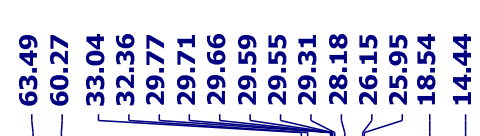 \\
\hline
\end{tabular}
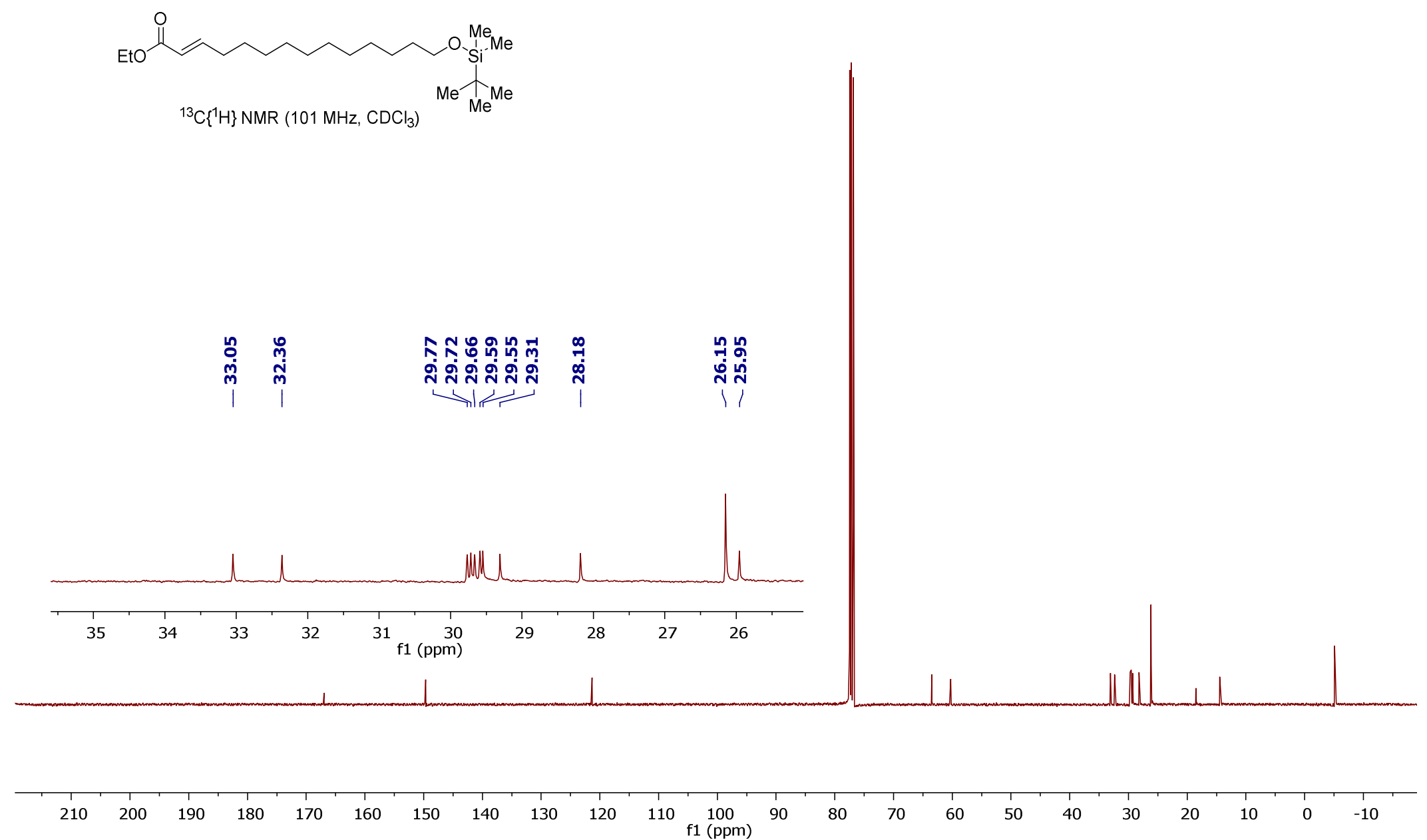

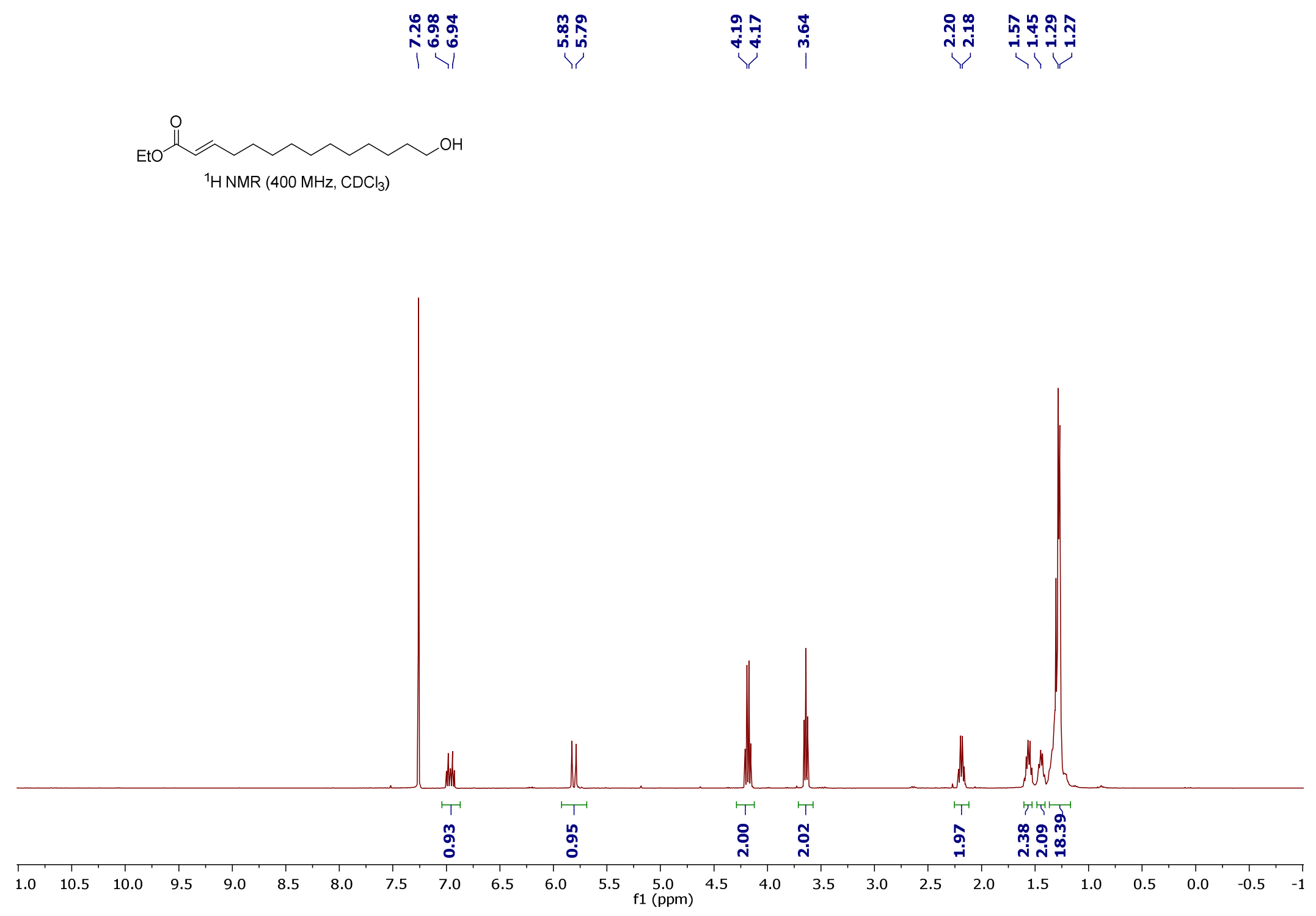


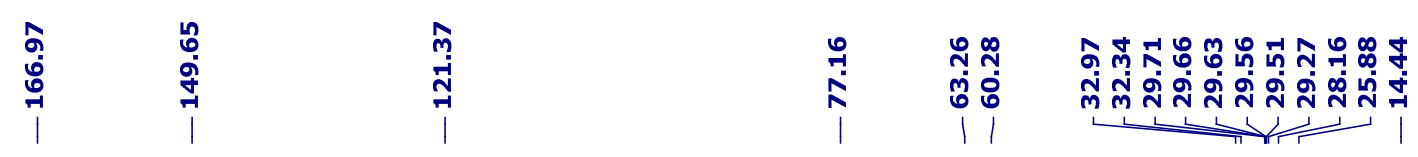

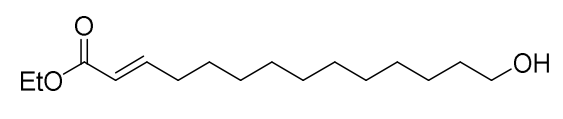

${ }^{13} \mathrm{C}\left\{{ }^{1} \mathrm{H}\right\} \mathrm{NMR}\left(101 \mathrm{MHz}, \mathrm{CDCl}_{3}\right)$
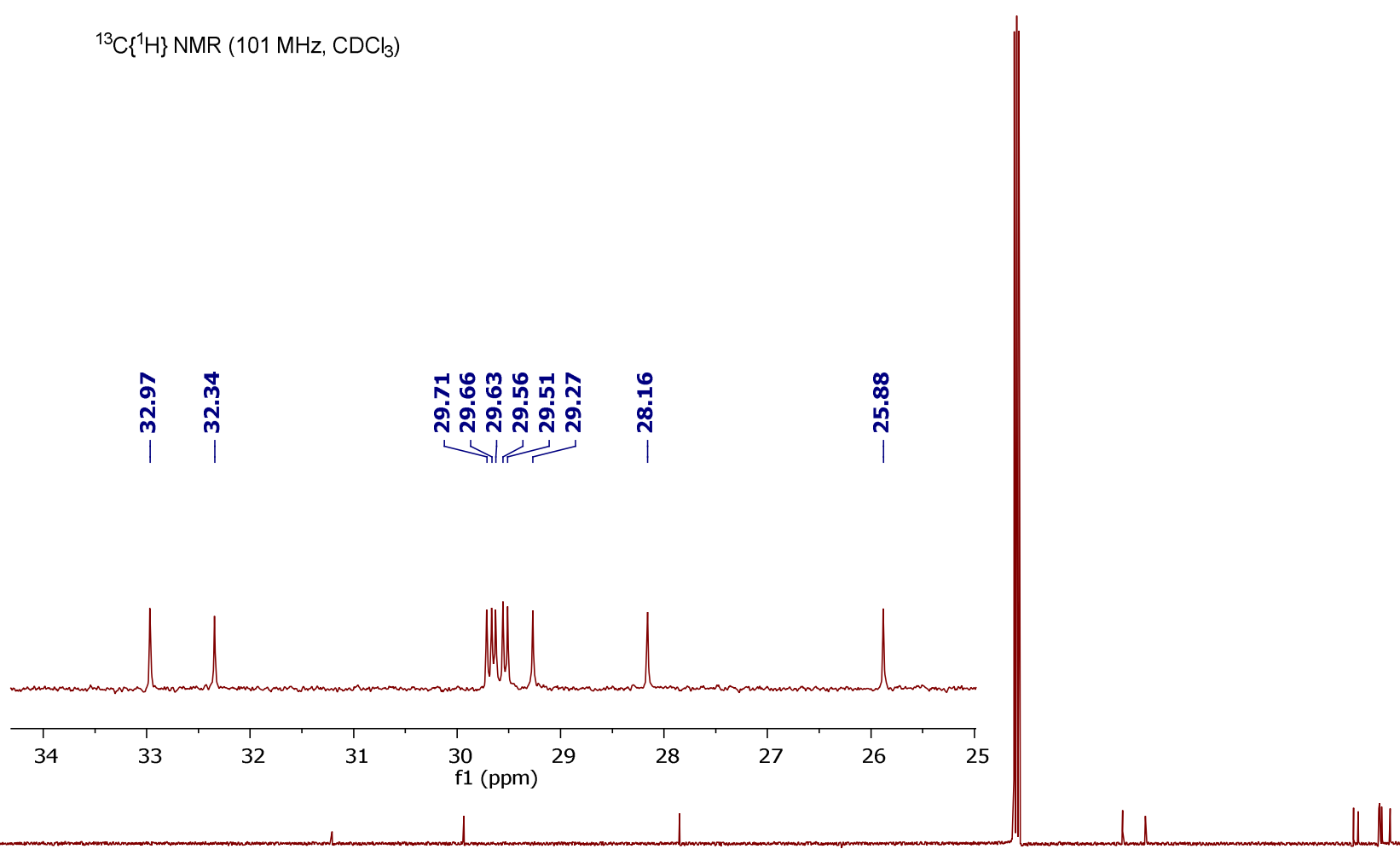

$\begin{array}{llllllllllll}210 & 200 & 190 & 180 & 170 & 160 & 150 & 140 & 130 & 120 & 110 & 100\end{array}$ 100
$\mathrm{f} 1(\mathrm{ppm})$ 

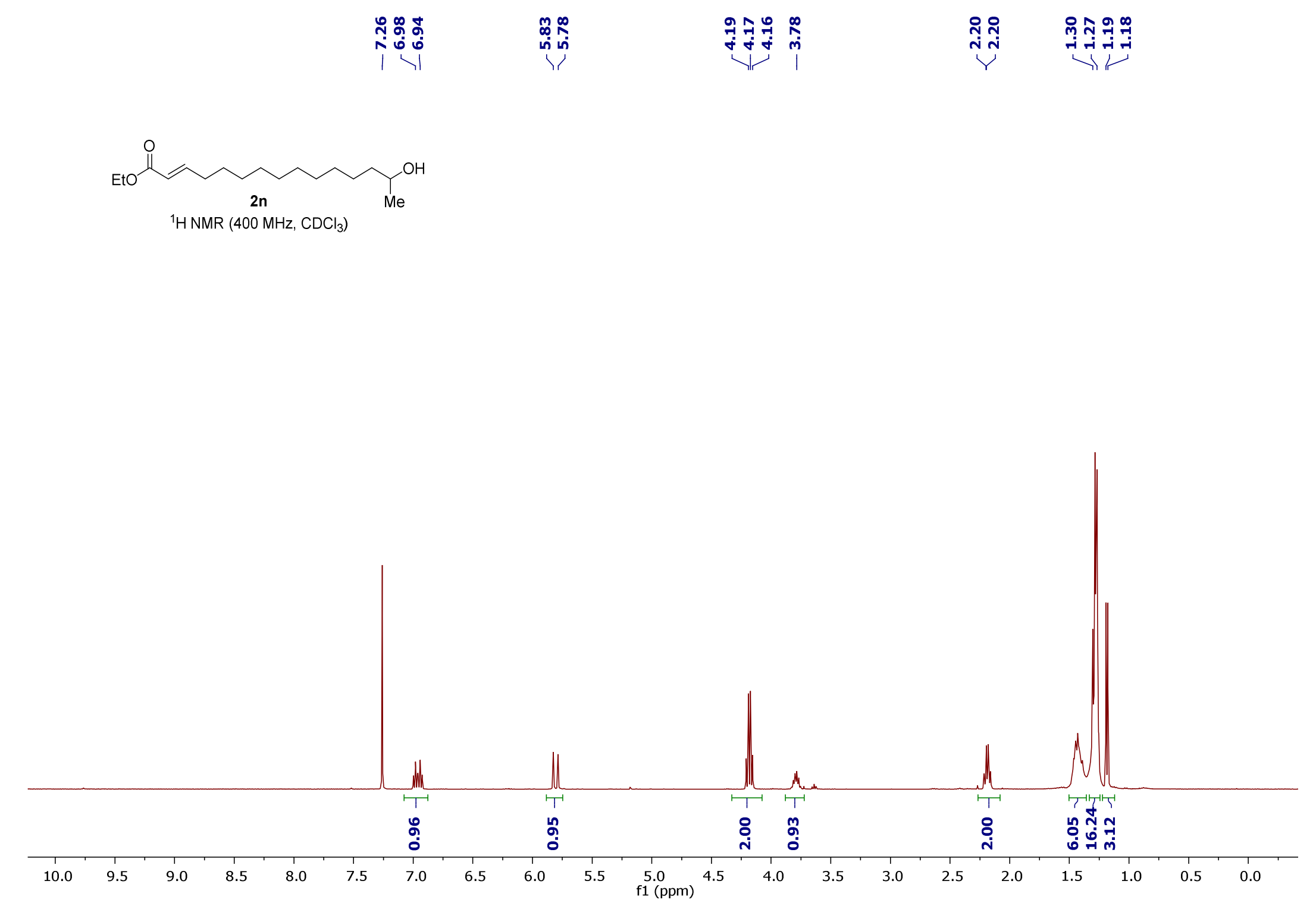

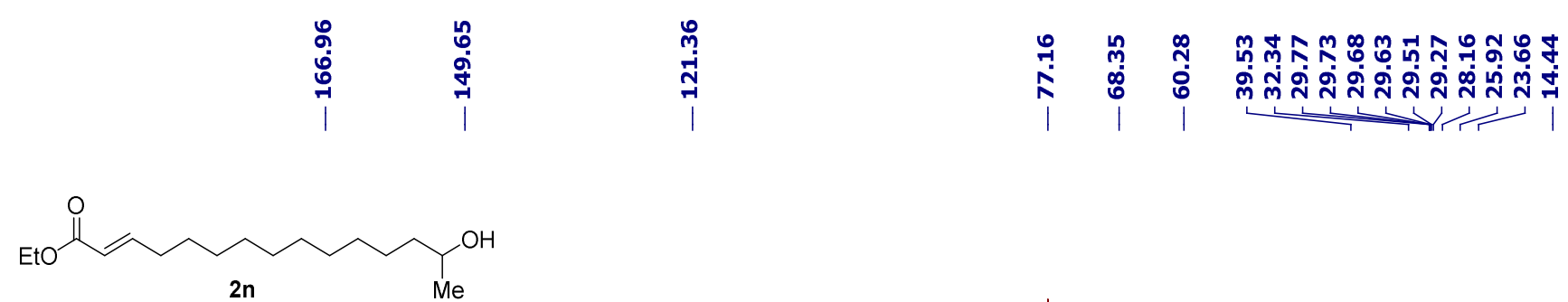

${ }^{13} \mathrm{C}\left\{{ }^{1} \mathrm{H}\right\} \mathrm{NMR}\left(101 \mathrm{MHz}, \mathrm{CDCl}_{3}\right)$

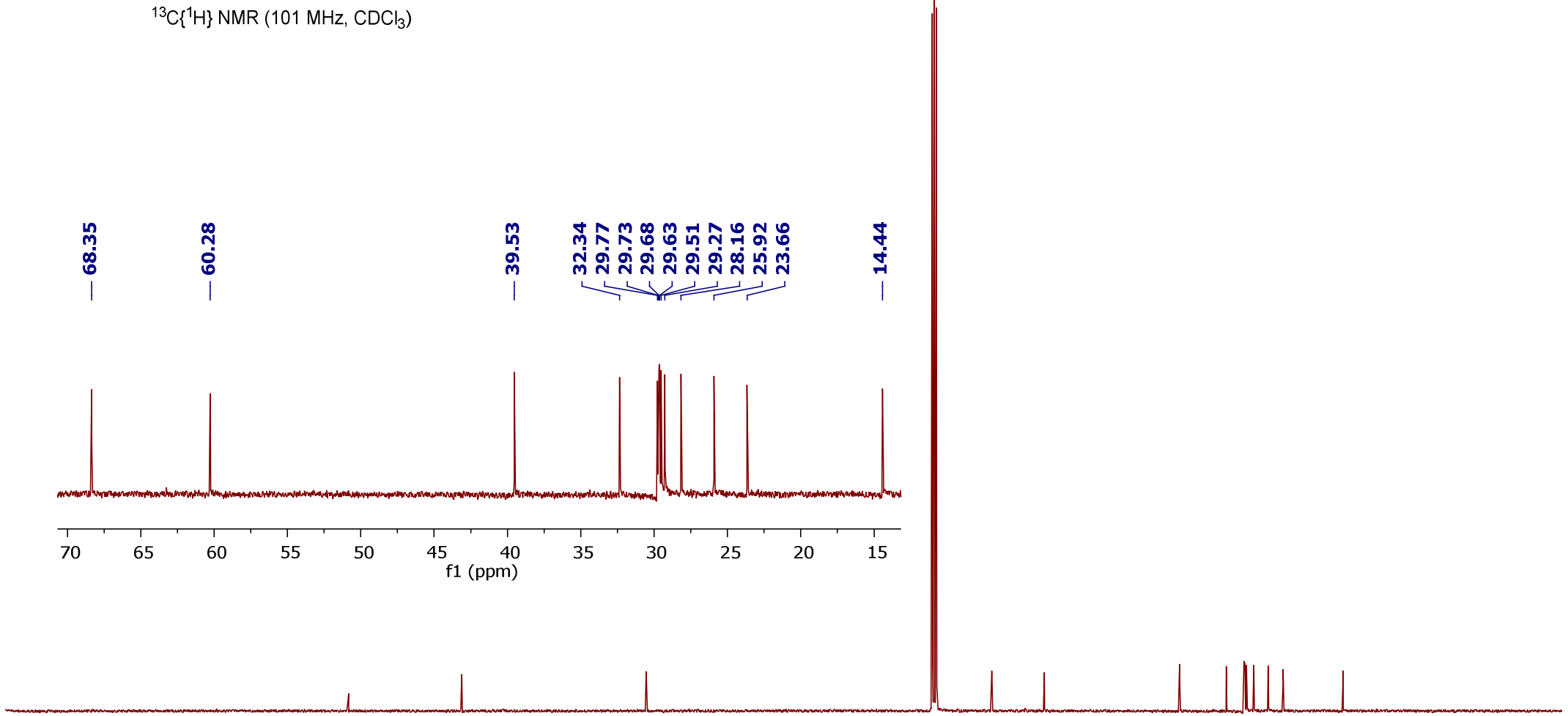

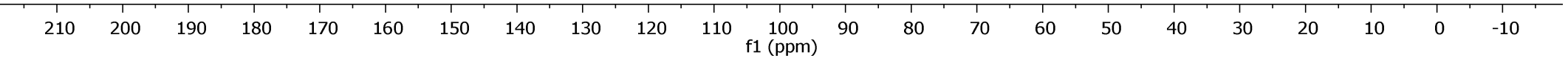




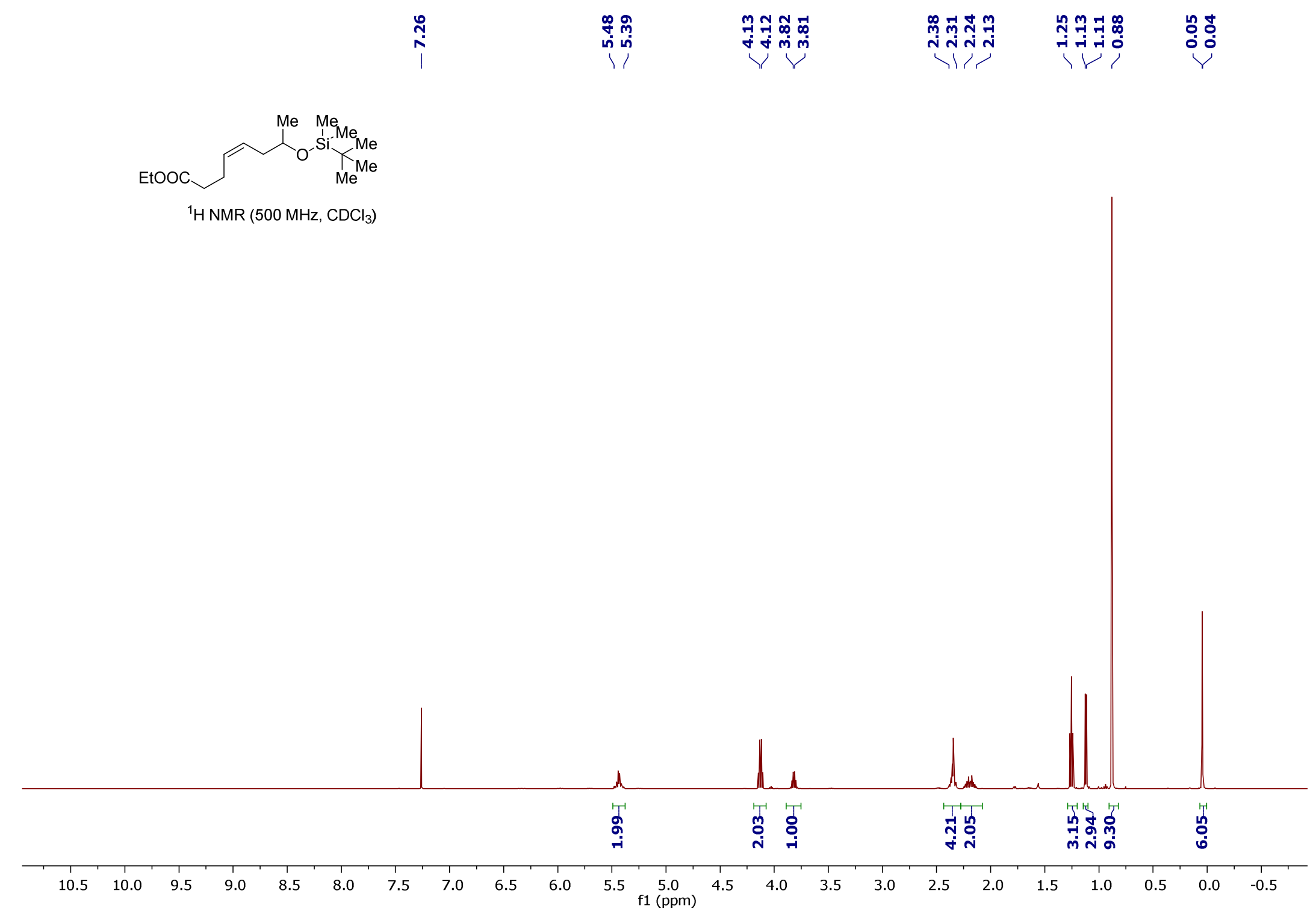




ज̂

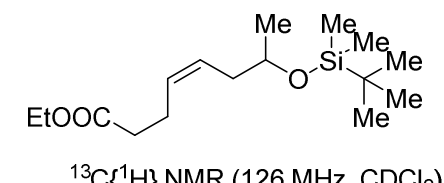

$\left.{ }^{13} \mathrm{C}^{1}{ }^{1} \mathrm{H}\right\} \mathrm{NMR}\left(126 \mathrm{MHz}, \mathrm{CDCl}_{3}\right)$

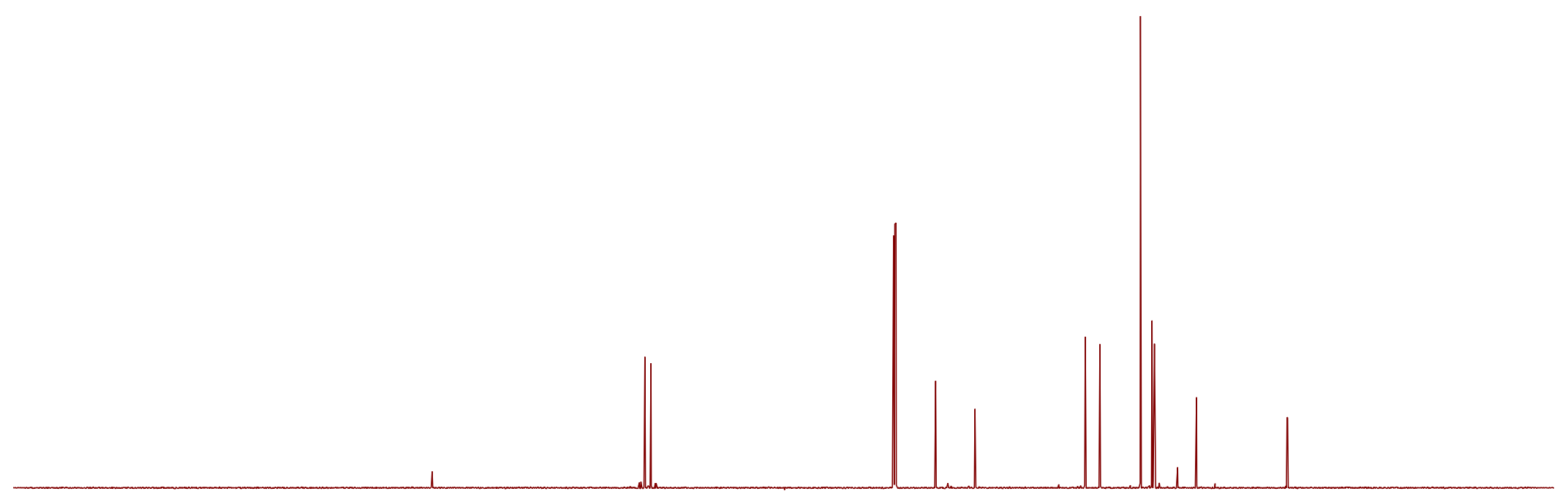

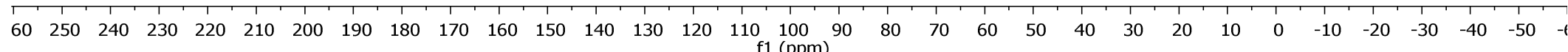



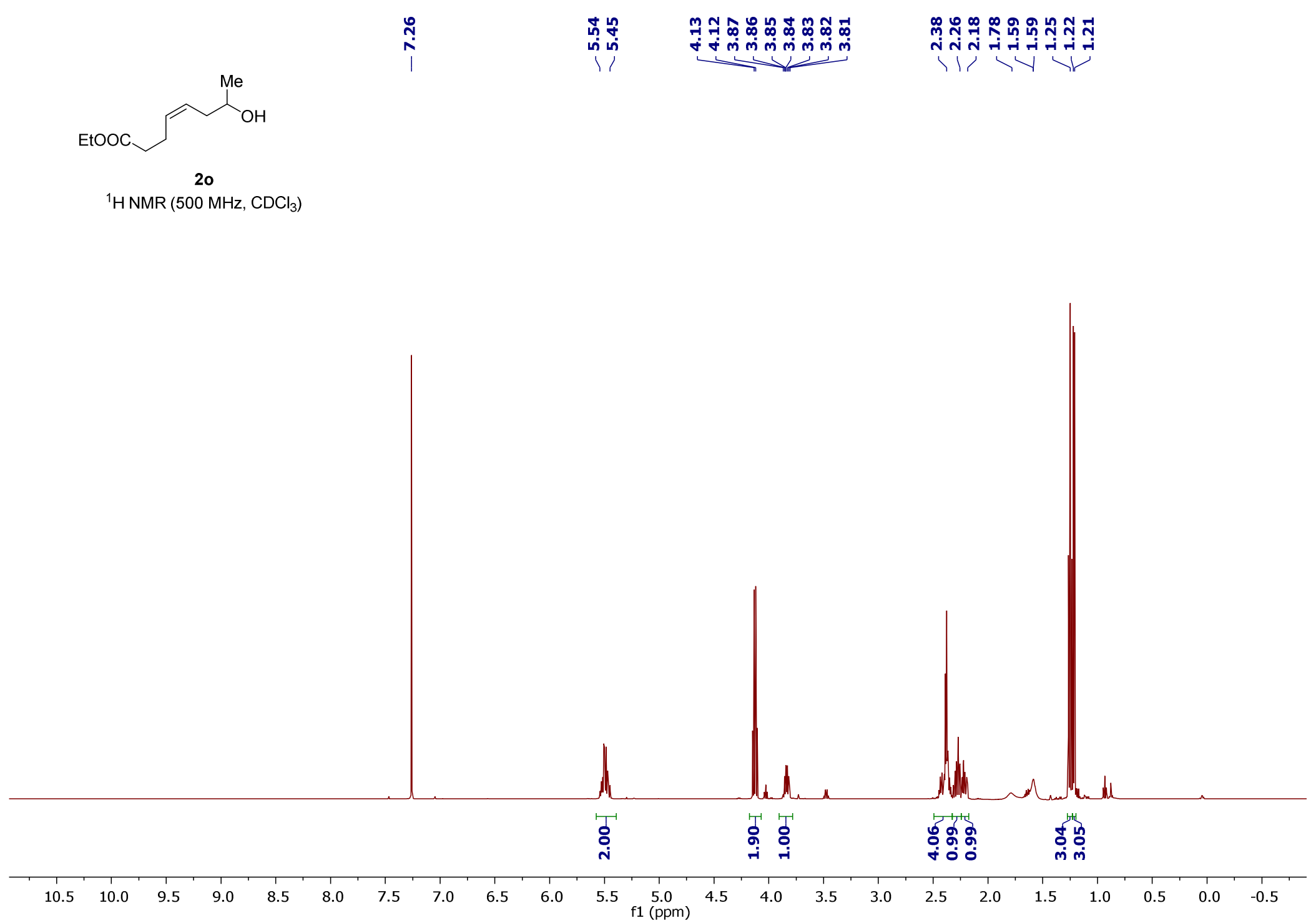


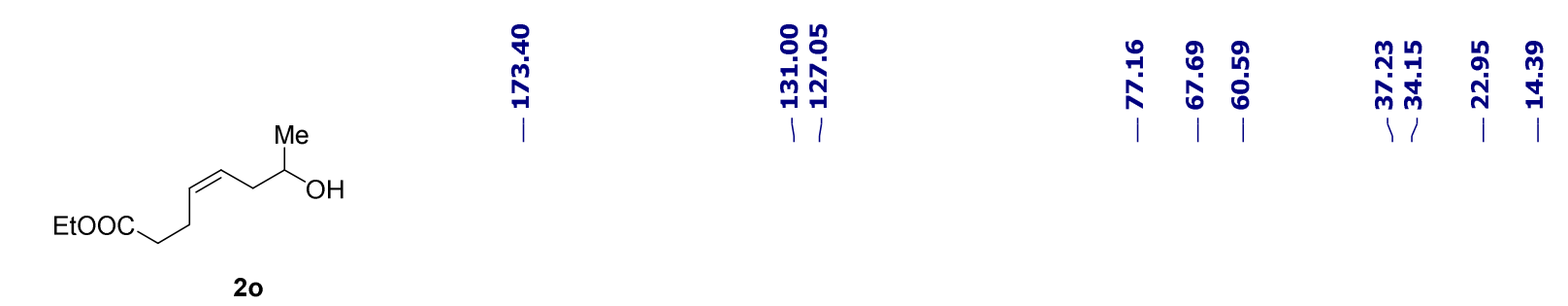

${ }^{13} \mathrm{C}\left\{{ }^{1} \mathrm{H}\right\} \mathrm{NMR}\left(126 \mathrm{MHz}, \mathrm{CDCl}_{3}\right)$

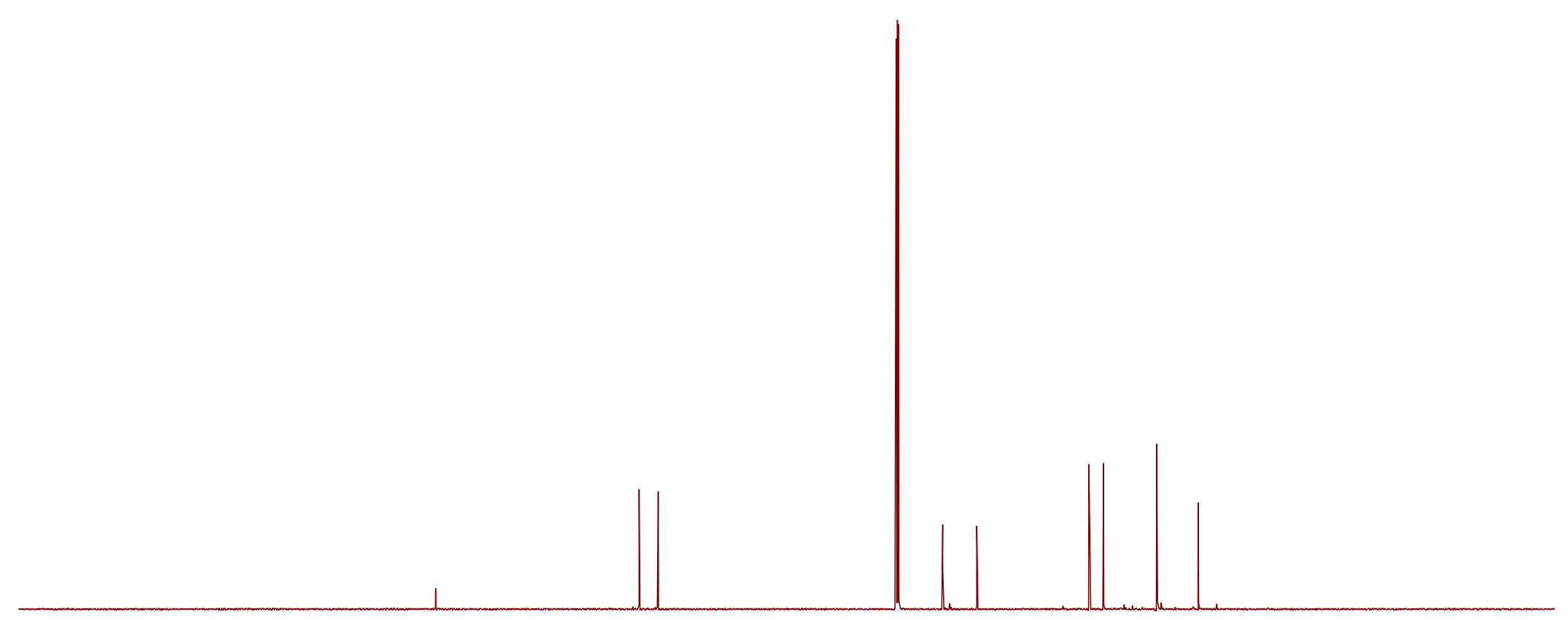

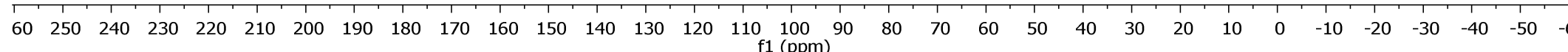




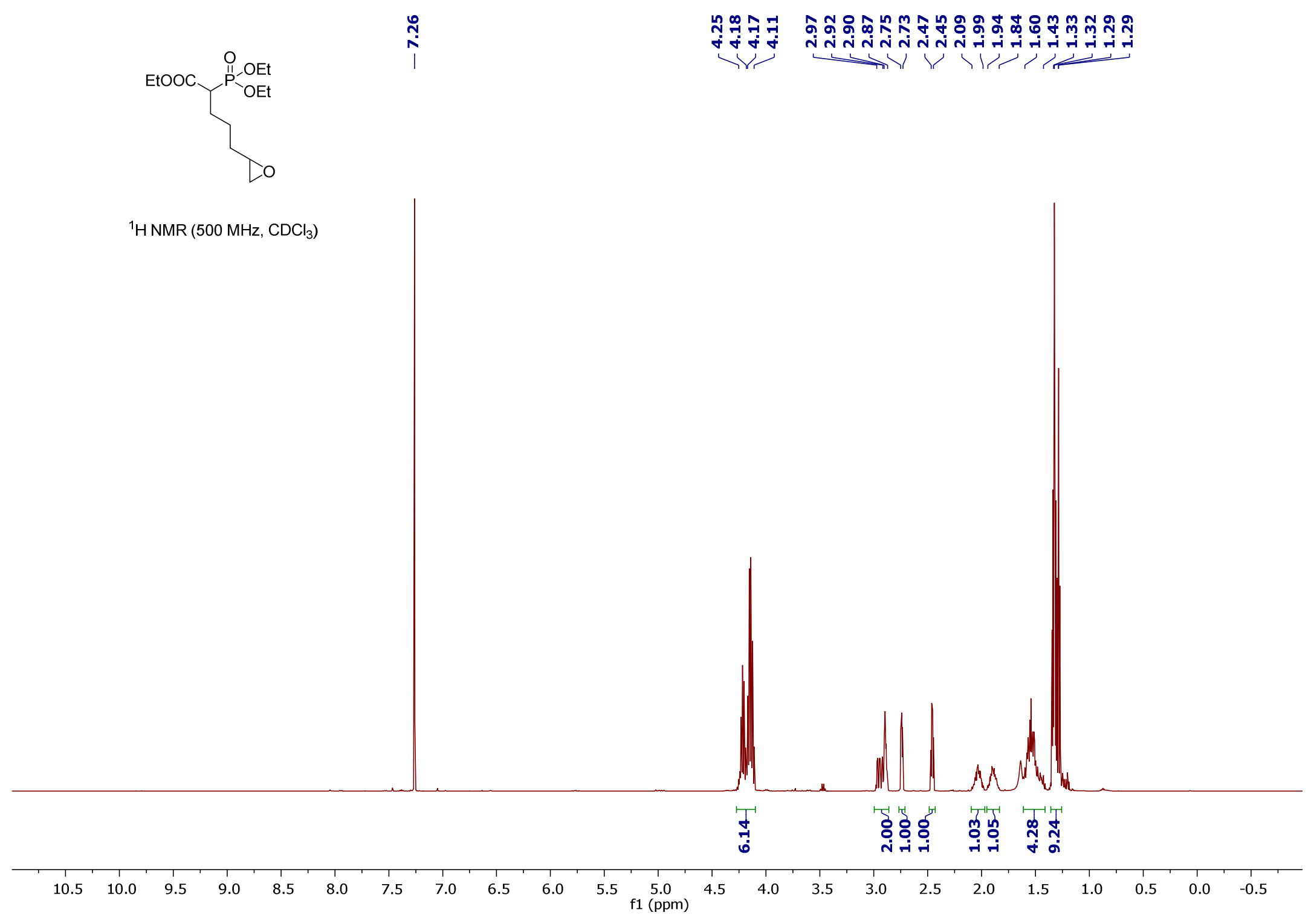




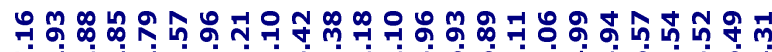

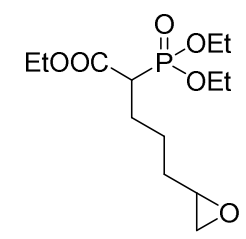

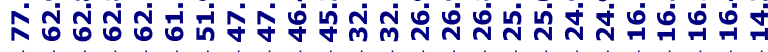

$\left.{ }^{13} \mathrm{C}^{1} \mathrm{H}\right\} \mathrm{NMR}\left(126 \mathrm{MHz}, \mathrm{CDCl}_{3}\right)$

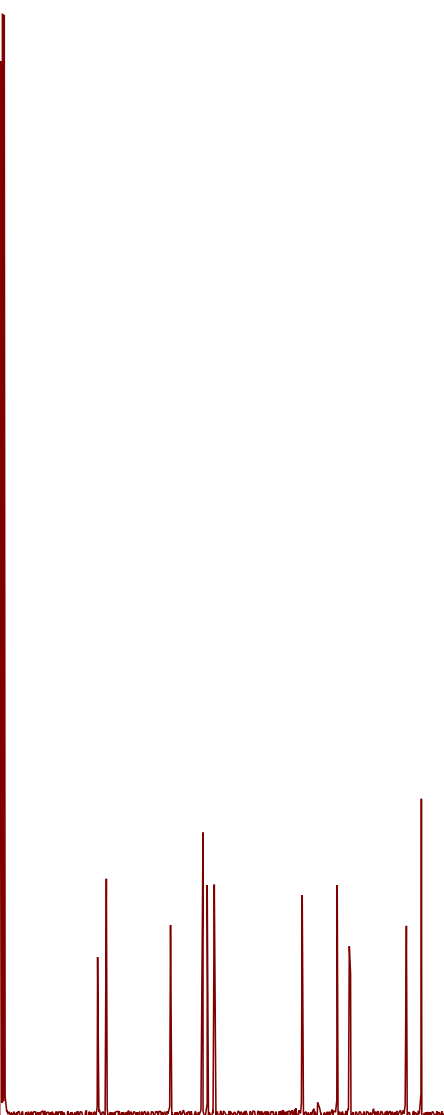

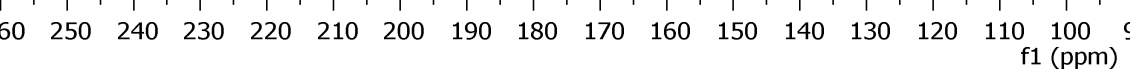
$\begin{array}{lllllllllllllll}60 & 50 & 40 & 30 & 20 & 10 & 0 & -10 & -20 & -30 & -40 & -50 & -t\end{array}$ 


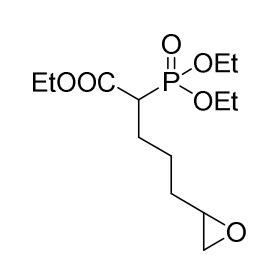

$\left.{ }^{31} \mathrm{P}^{1}{ }^{1} \mathrm{H},{ }^{13} \mathrm{C}\right\} \mathrm{NMR}\left(162 \mathrm{MHz}, \mathrm{CDCl}_{3}\right)$

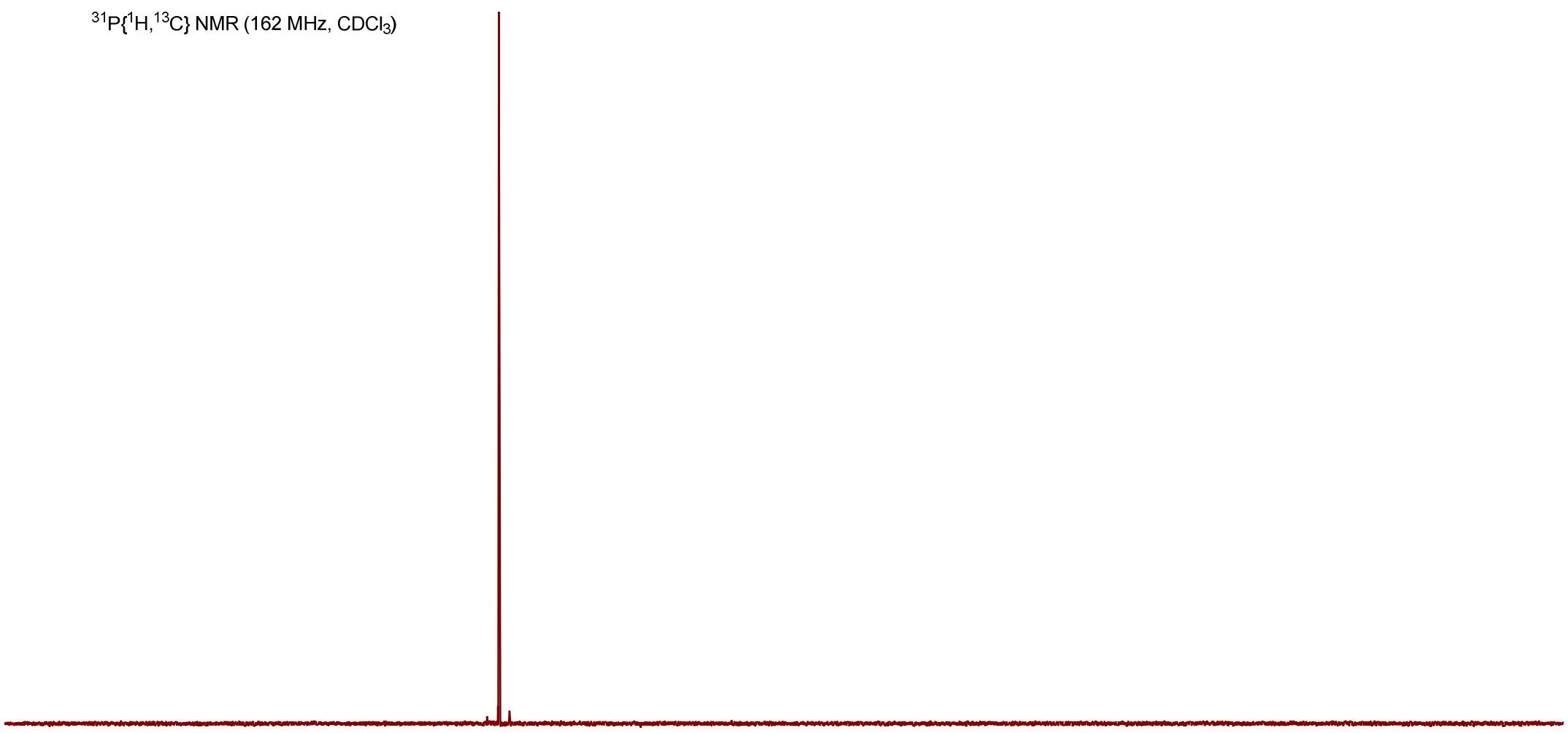

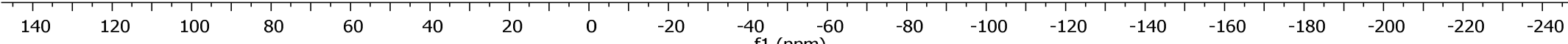




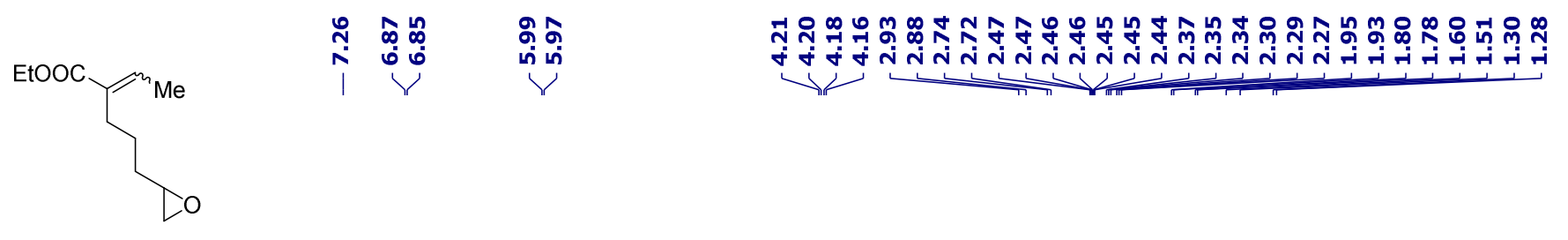

${ }^{1} \mathrm{H}$ NMR $\left(500 \mathrm{MHz}, \mathrm{CDCl}_{3}\right)$

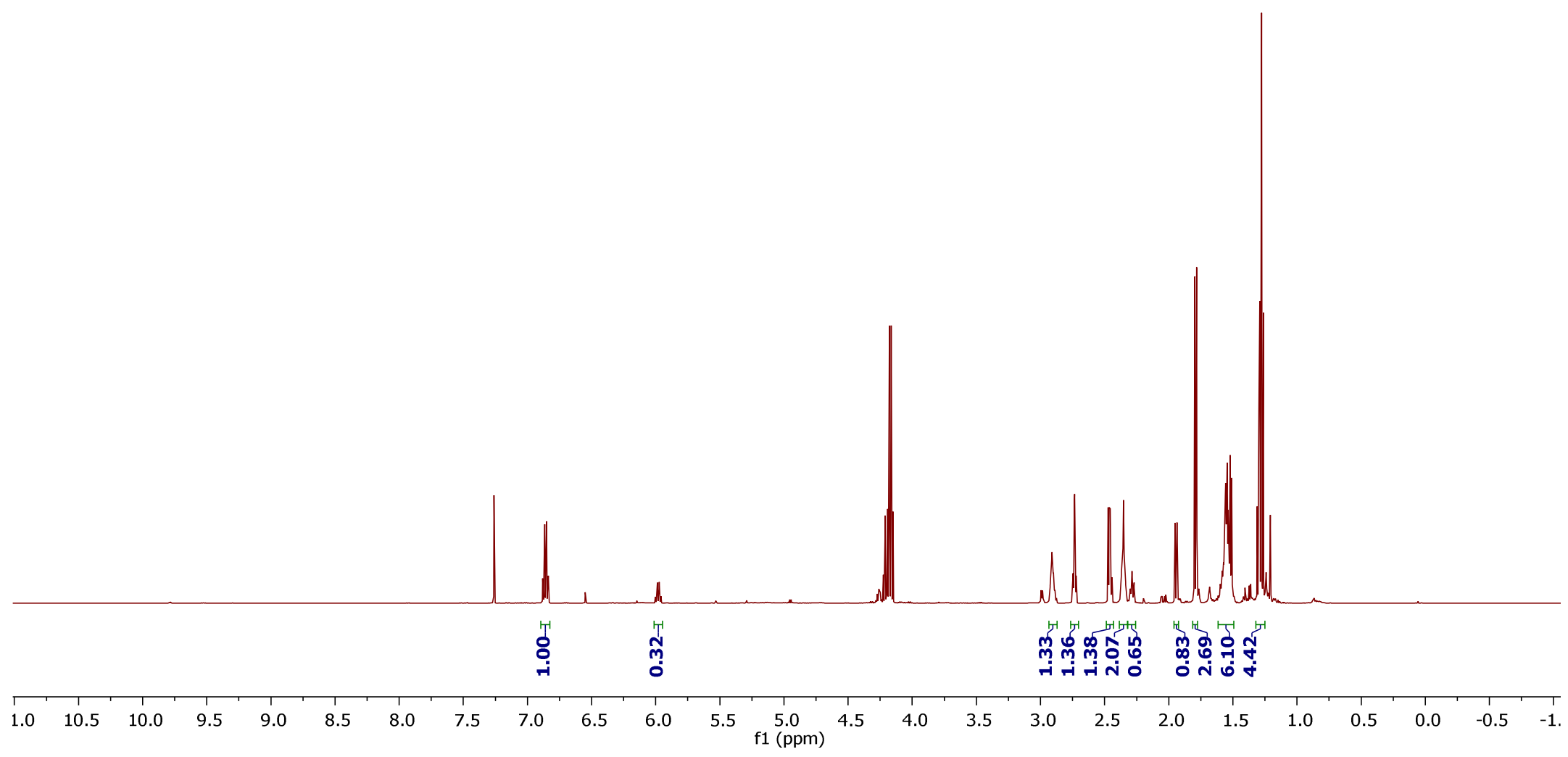




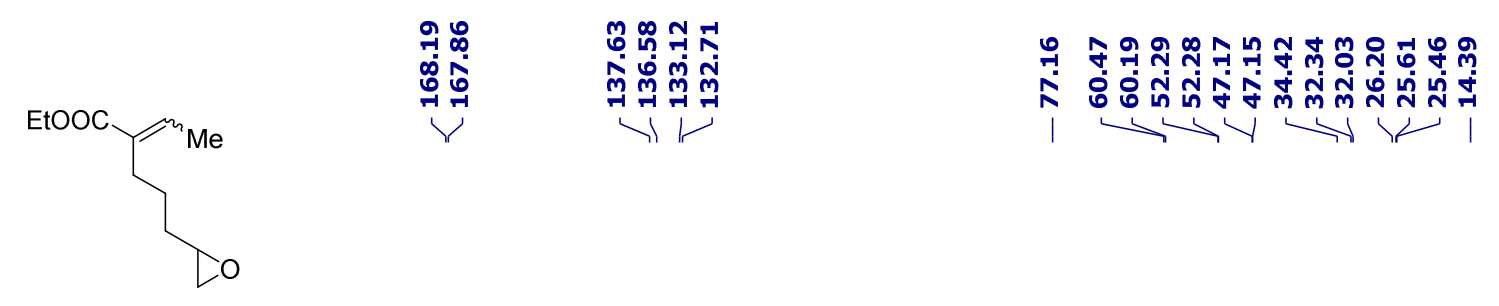

${ }^{13} \mathrm{C}\left\{{ }^{1} \mathrm{H}\right\}$ NMR $\left(126 \mathrm{MHz}, \mathrm{CDCl}_{3}\right)$

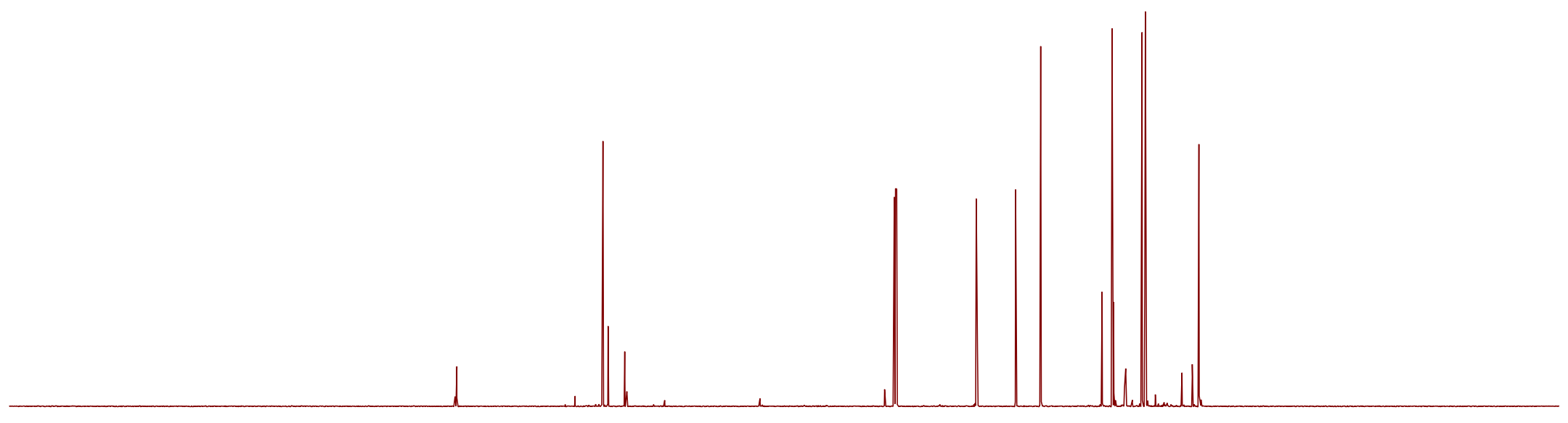

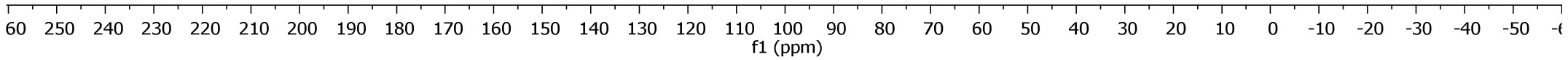




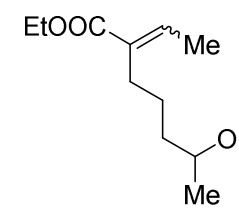

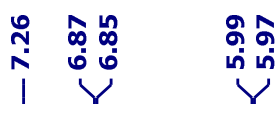

รีง

1) $M$ M 1 .

${ }^{1} \mathrm{H}$ NMR $\left(500 \mathrm{MHz}, \mathrm{CDCl}_{3}\right)$

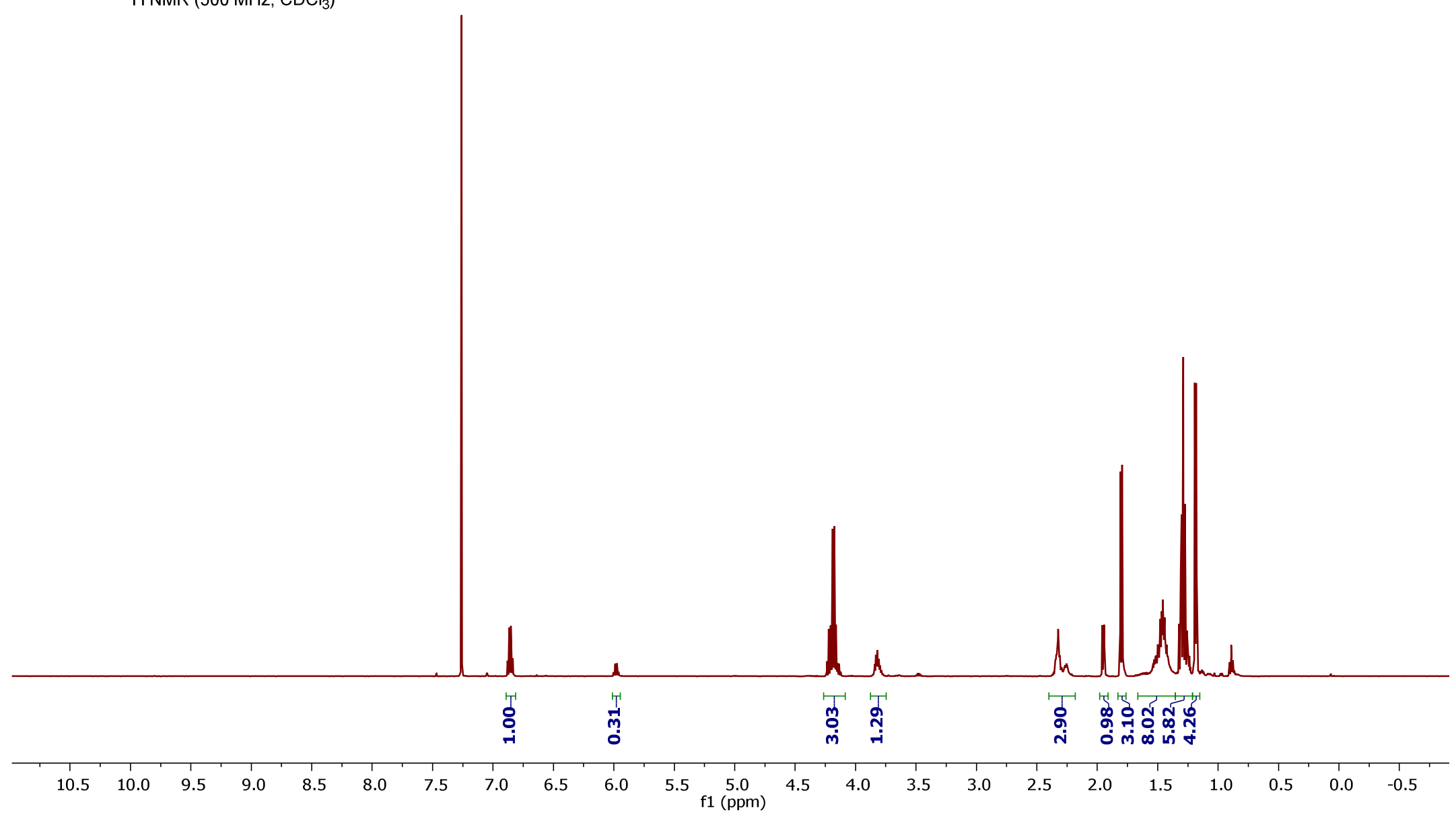




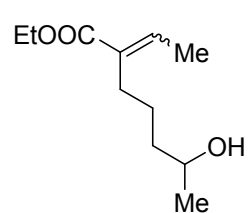

$2 p$

${ }^{13} \mathrm{C}\left\{{ }^{1} \mathrm{H}\right\}$ NMR $\left(126 \mathrm{MHz}, \mathrm{CDCl}_{3}\right)$

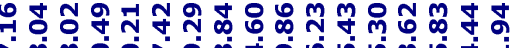

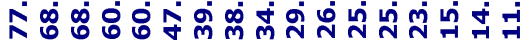

$\mathrm{Y} \times{ }^{2}$

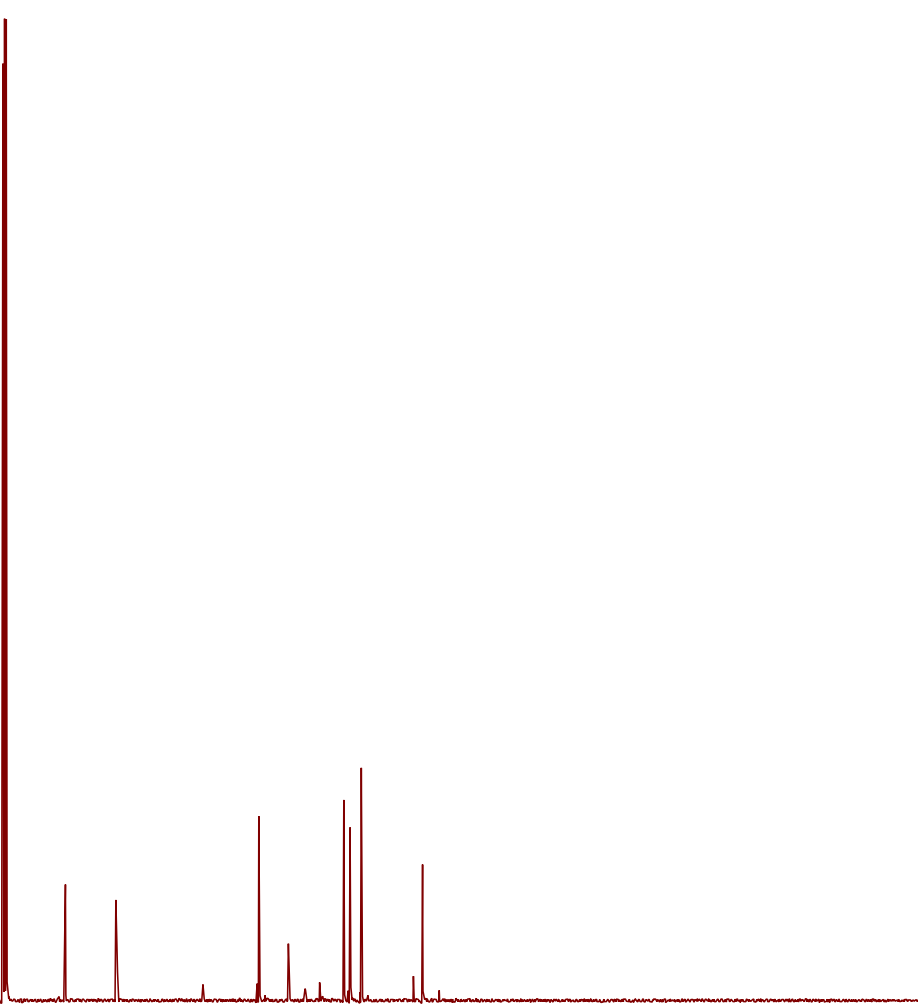

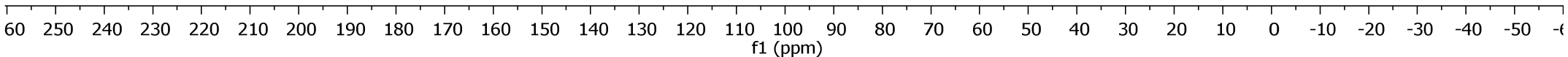



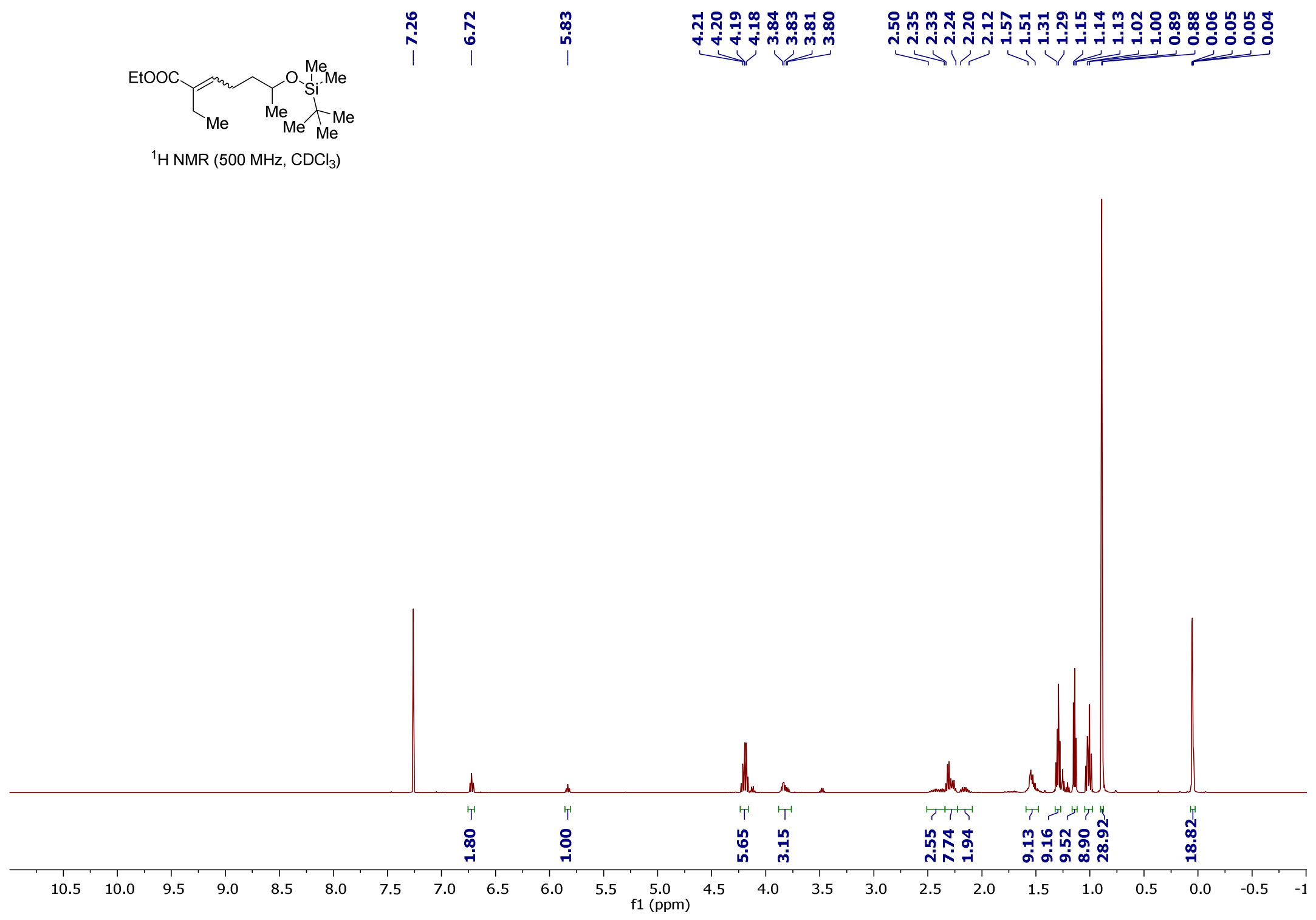


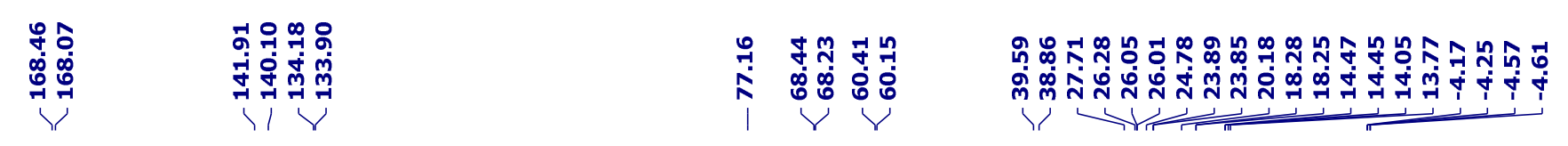

$\underbrace{\mathrm{EtOOC}}_{\mathrm{Me}} \mathrm{Me}_{\mathrm{Me}}^{\mathrm{O}} \lambda_{\mathrm{Me}}^{\mathrm{Me}}$

${ }^{13} \mathrm{C}\left\{{ }^{1} \mathrm{H}\right\} \mathrm{NMR}\left(126 \mathrm{MHz}, \mathrm{CDCl}_{3}\right)$

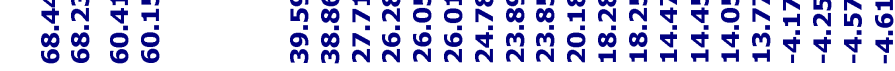

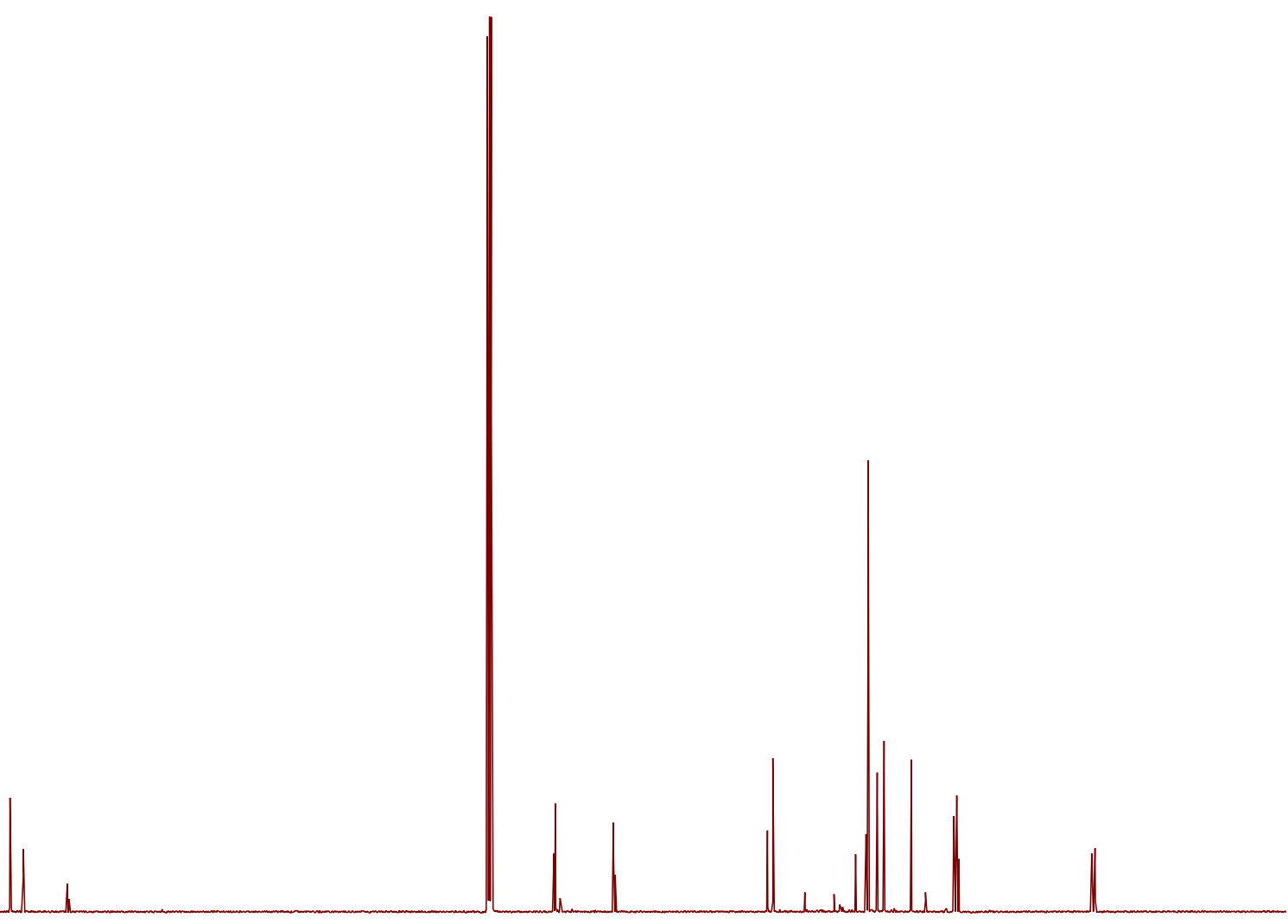

$\begin{array}{lllllllllllll}210 & 200 & 190 & 180 & 170 & 160 & 150 & 140 & 130 & 120 & 110 & 100 & 90\end{array}$ $\begin{array}{rr}100 & 90 \\ \mathrm{f} 1(\mathrm{ppm})\end{array}$ 

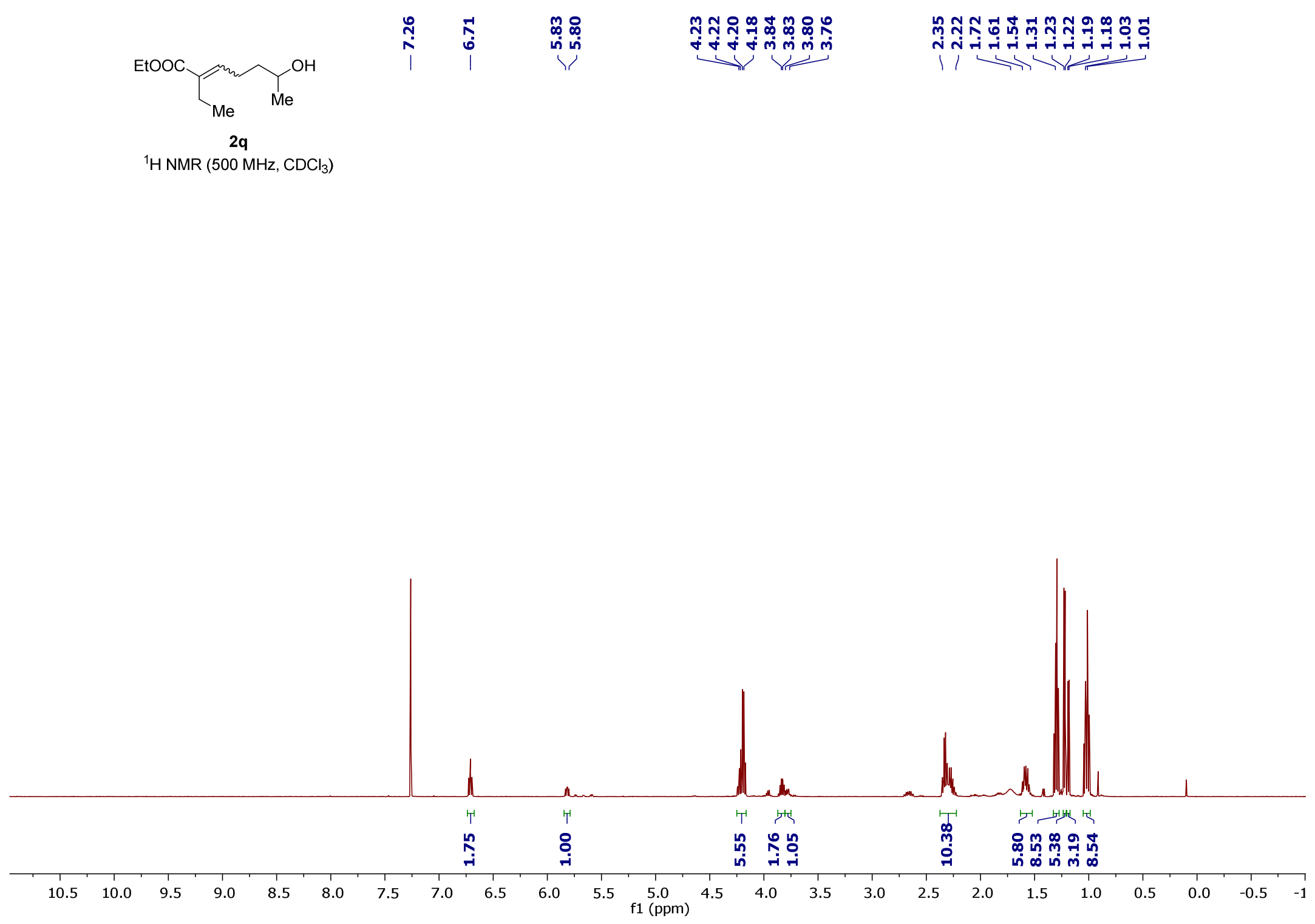


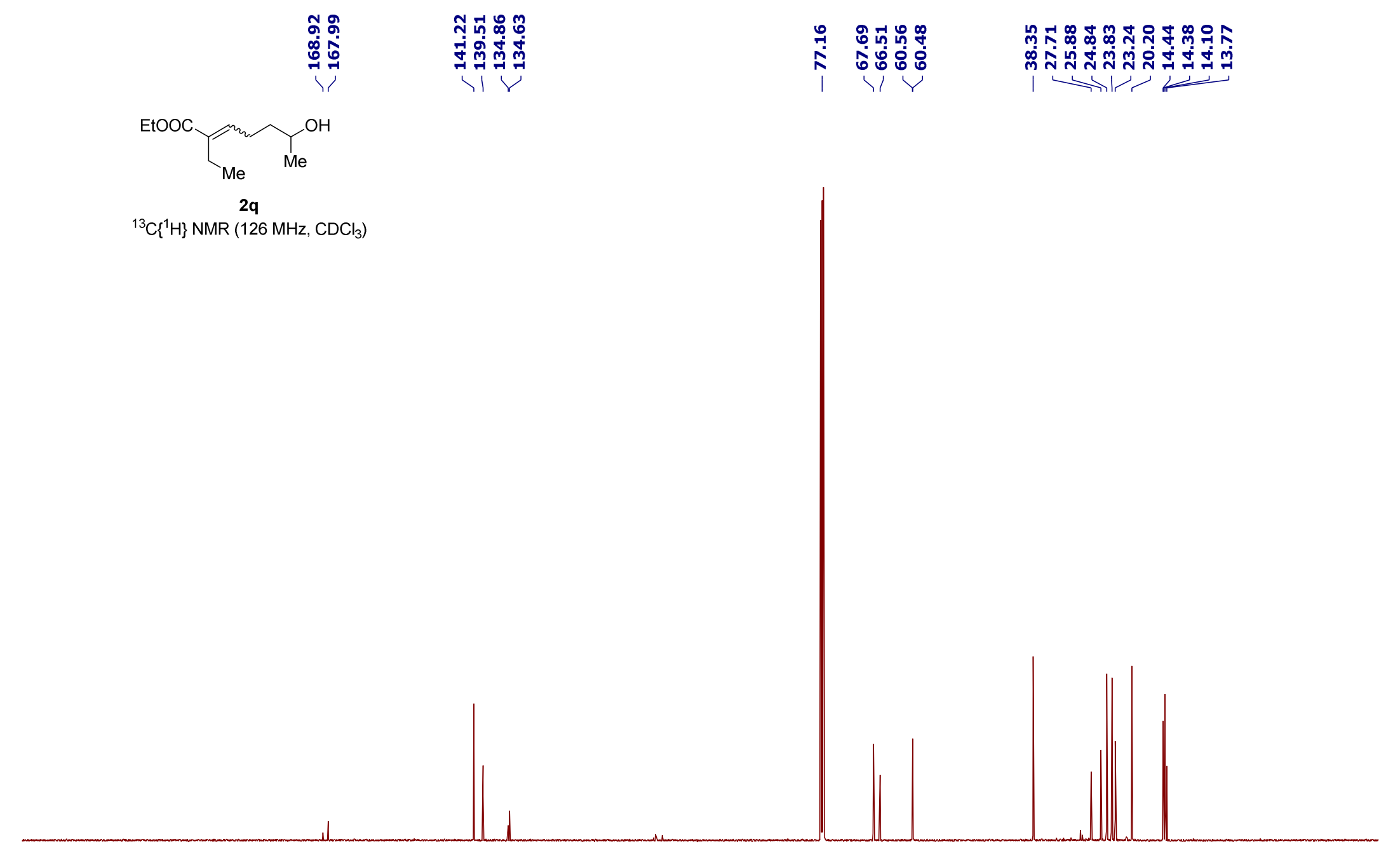

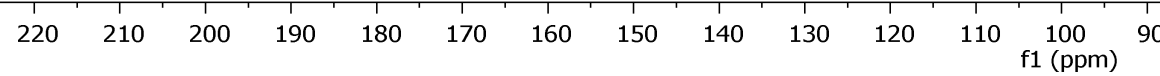



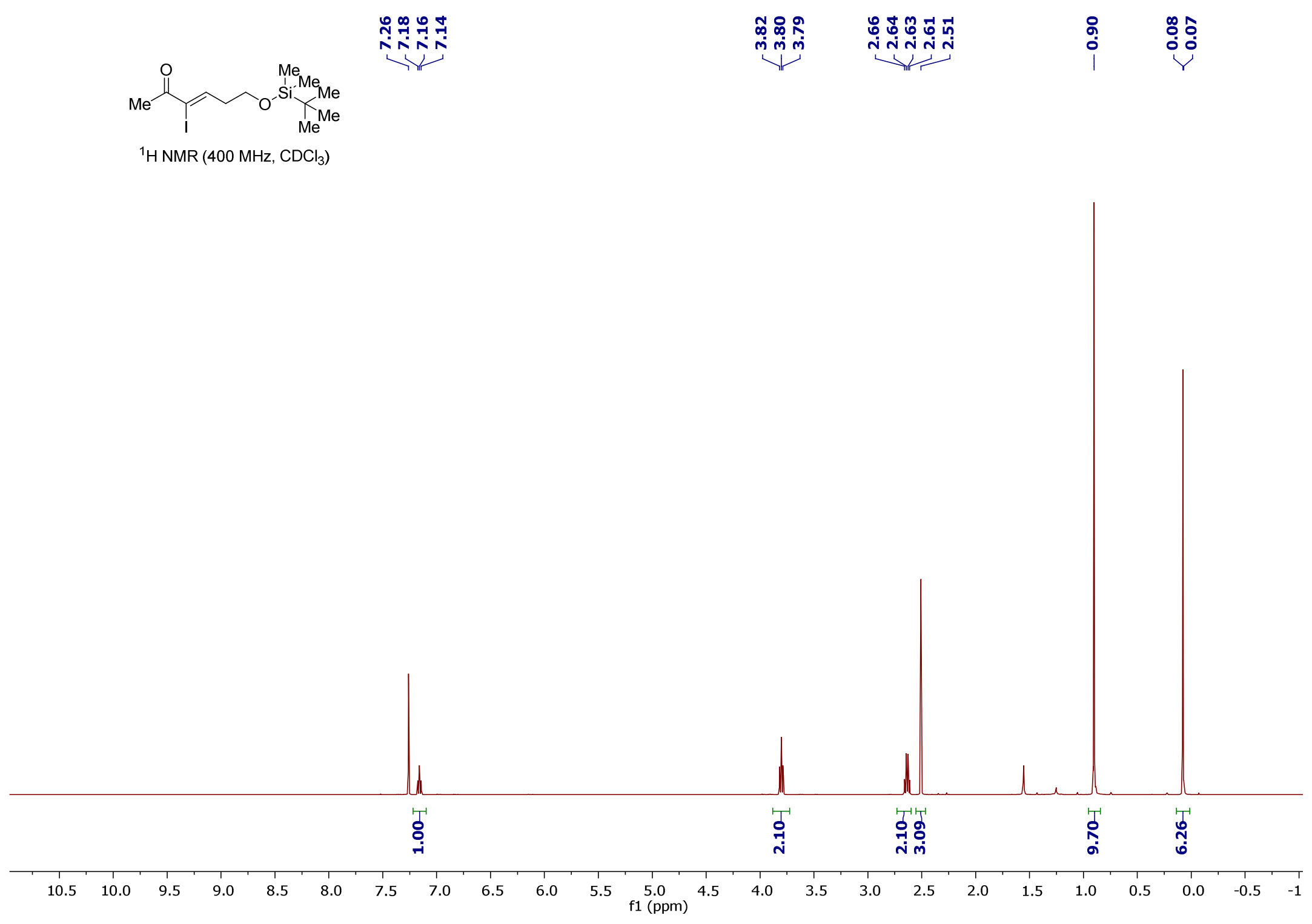


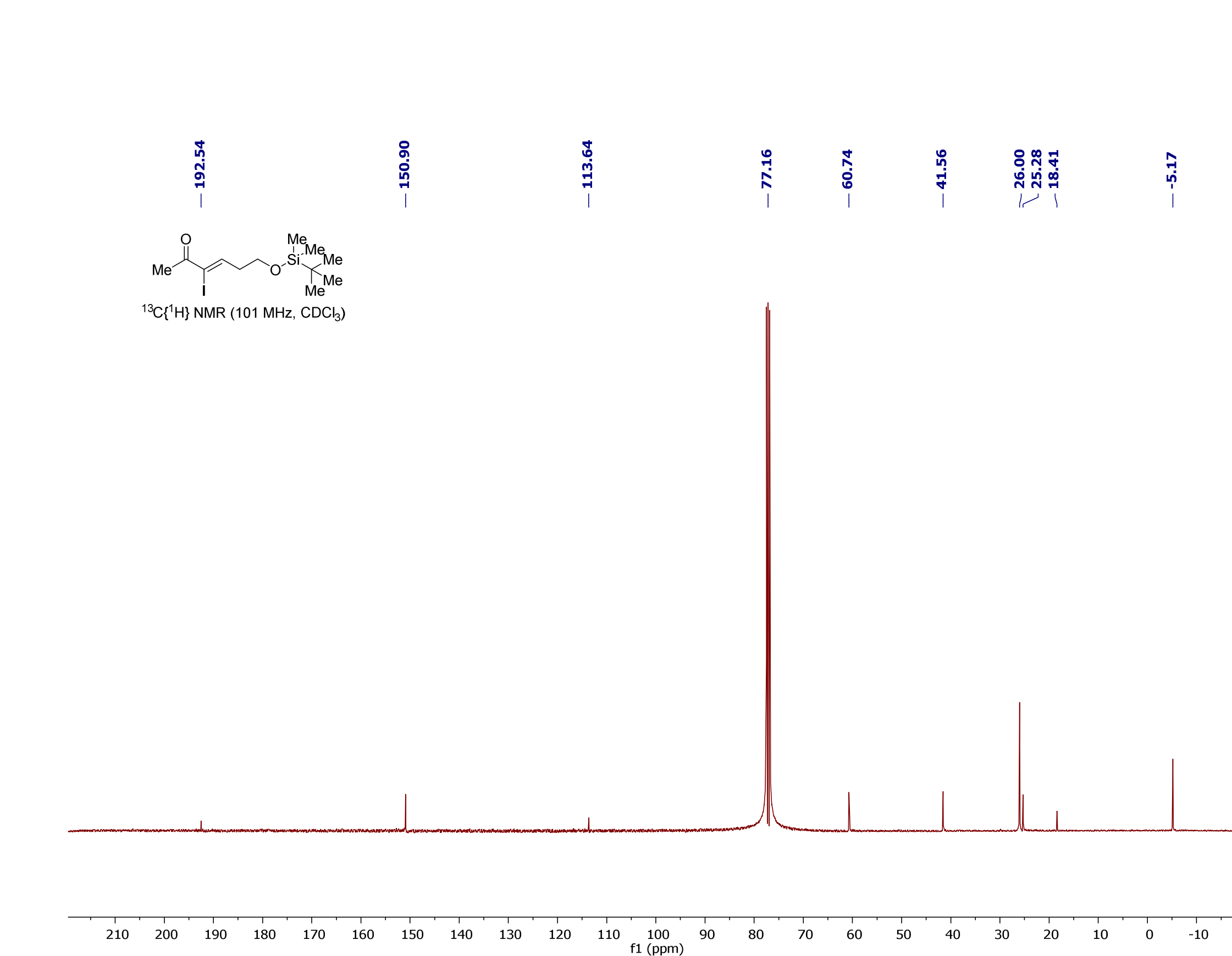




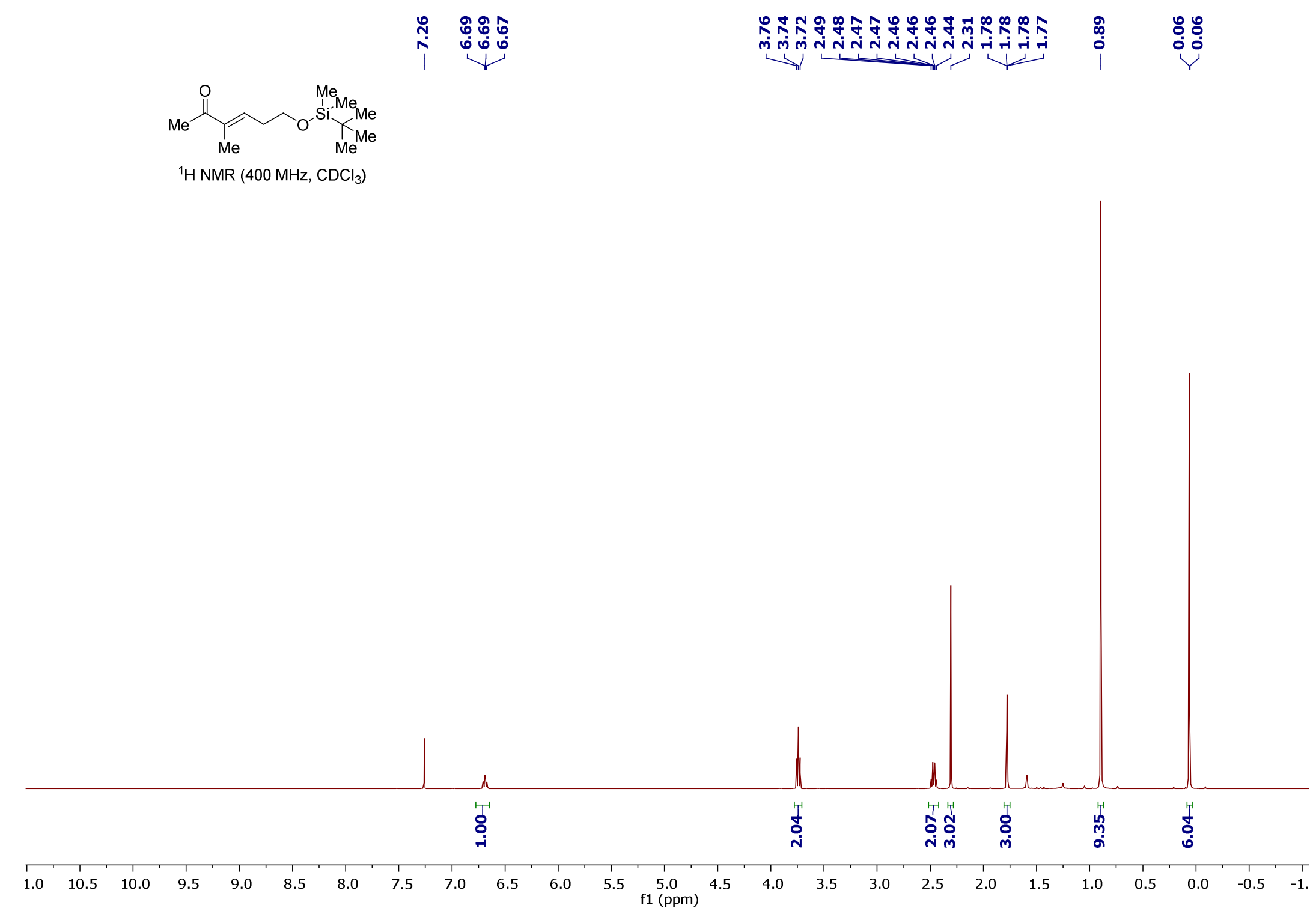




$$
\begin{array}{lll}
\omega^{\prime} \\
\hline
\end{array}
$$




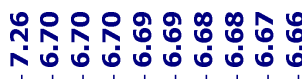

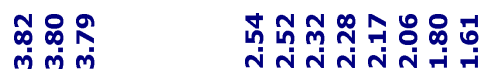

$\mathrm{Me}_{\mathrm{OH}}^{\mathrm{O}}$

${ }^{1} \mathrm{H} \mathrm{NMR}\left(400 \mathrm{MHz}, \mathrm{CDCl}_{3}\right)$

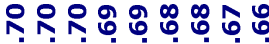

ن

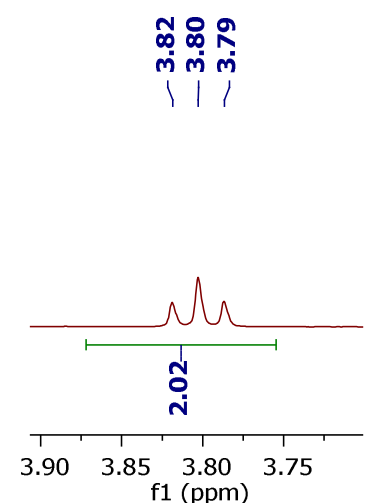

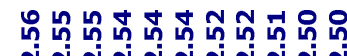

तind

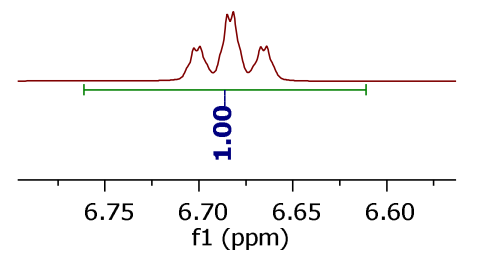

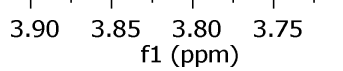
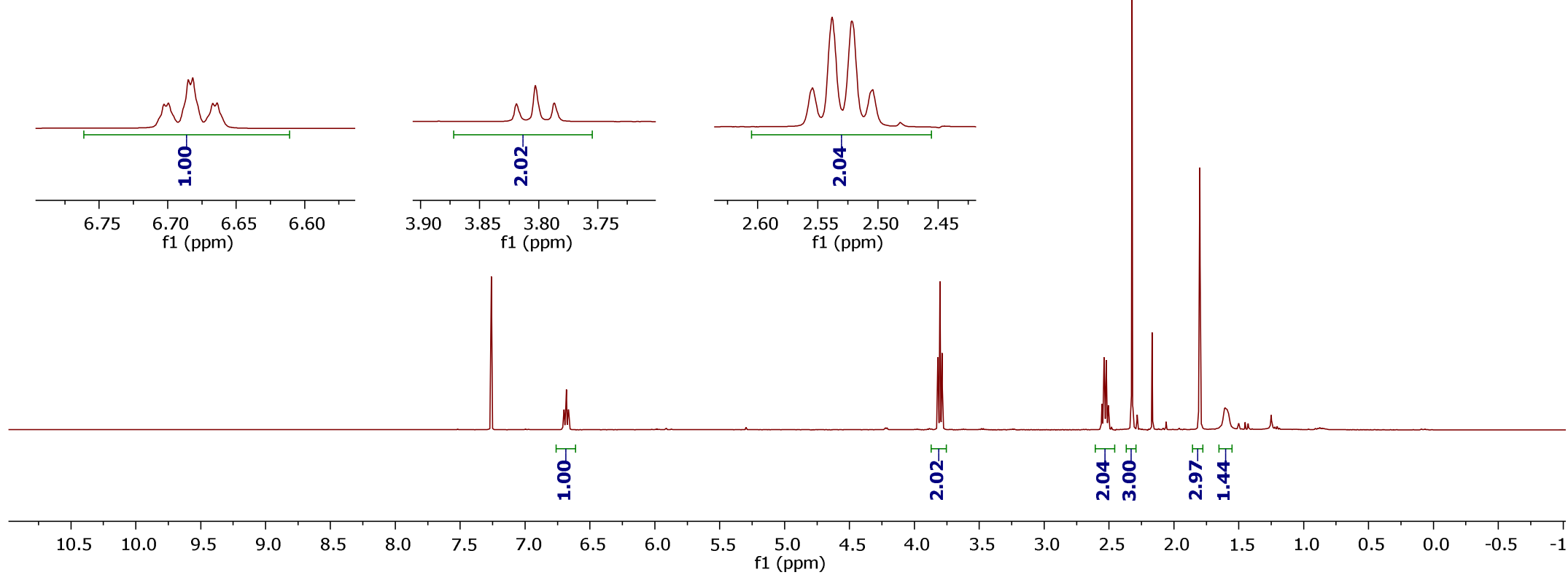


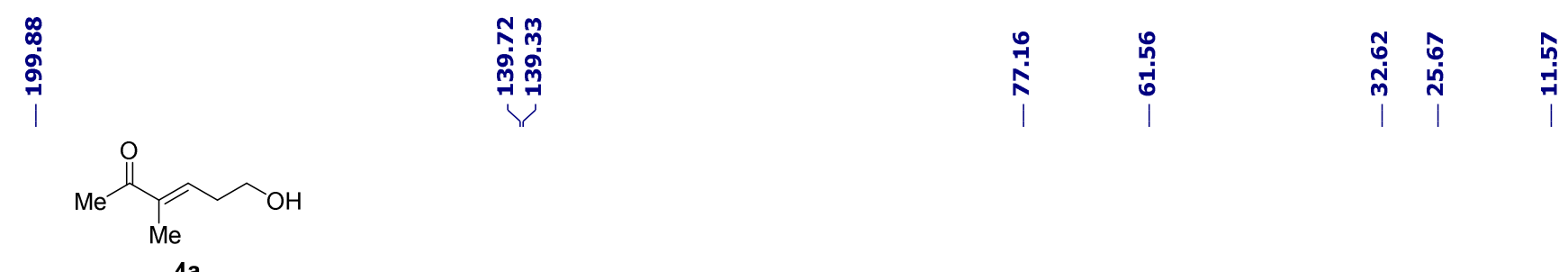

${ }^{13} \mathrm{C}\left\{{ }^{1} \mathrm{H}\right\}$ NMR $\left(101 \mathrm{MHz}, \mathrm{CDCl}_{3}\right)$

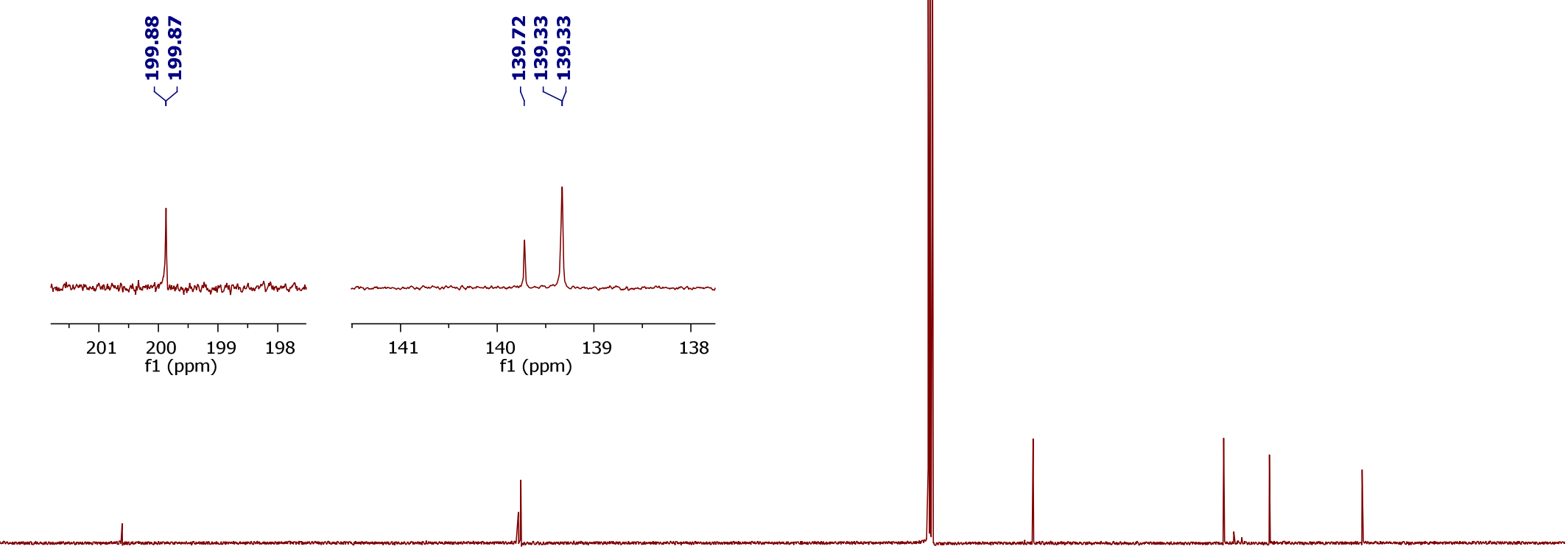

$\begin{array}{llllllllllllllllllllllllllll}210 & 200 & 190 & 180 & 170 & 160 & 150 & 140 & 130 & 120 & 110 & 100 & 90 & 80 & 70 & 60 & 50 & 40 & 30 & 20 & 10 & 0 & -10\end{array}$ 


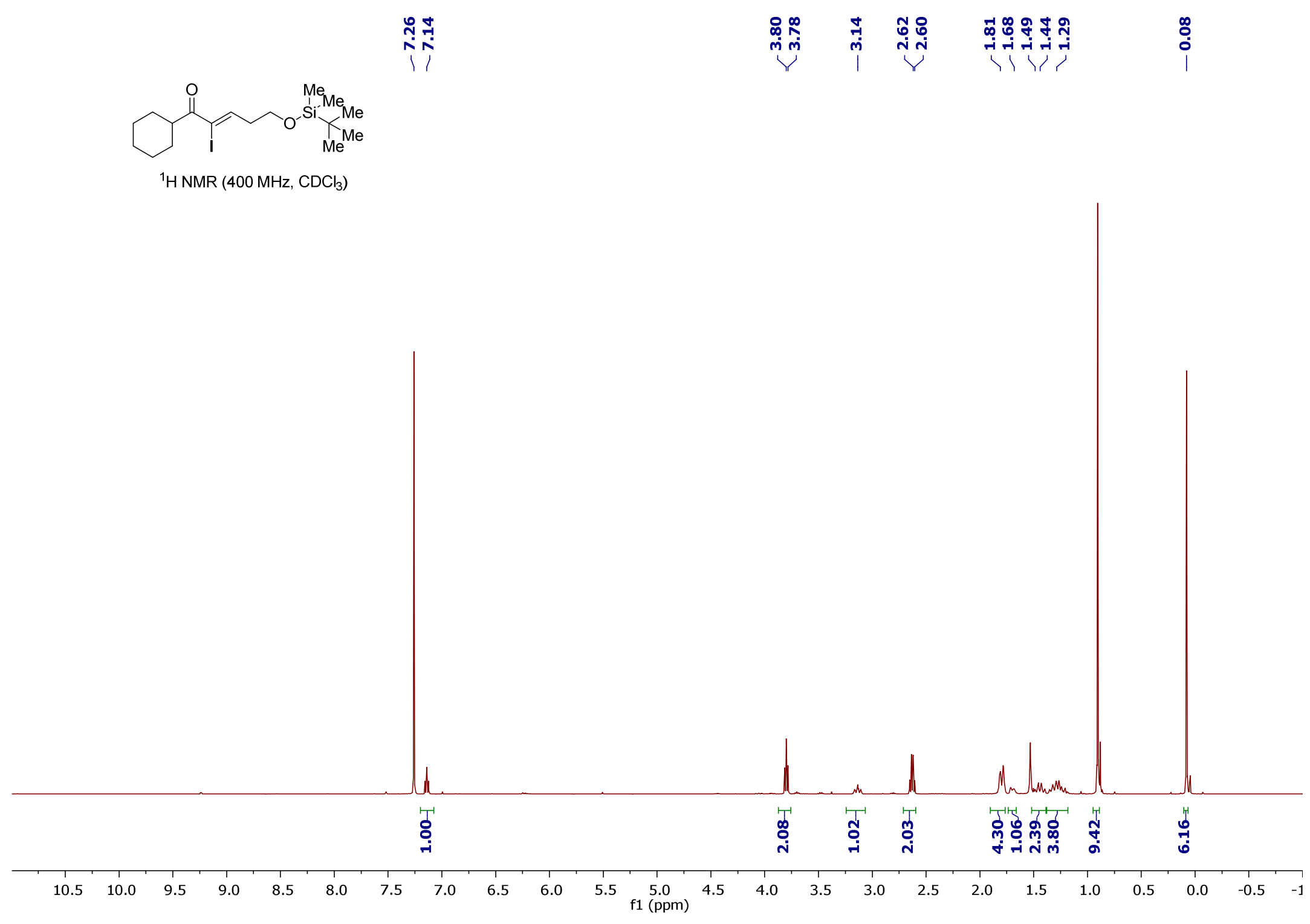




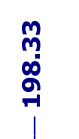

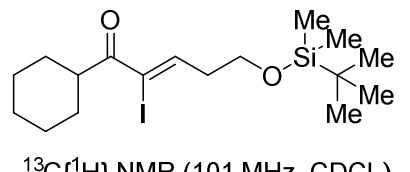

${ }^{13} \mathrm{C}\left\{{ }^{1} \mathrm{H}\right\} \mathrm{NMR}\left(101 \mathrm{MHz}, \mathrm{CDCl}_{3}\right)$

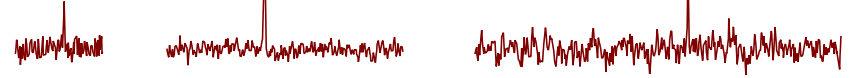

$\begin{array}{ccccc}197 & 150 & 149 & 148 & 147 \\ \mathrm{f} 1(\mathrm{ppm}) & & & \\ \mathrm{f} 1(\mathrm{ppm}) & \end{array}$

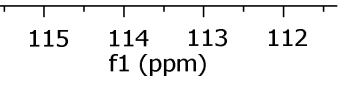

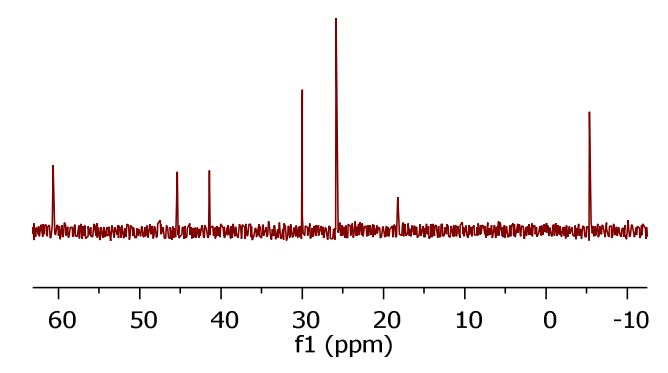



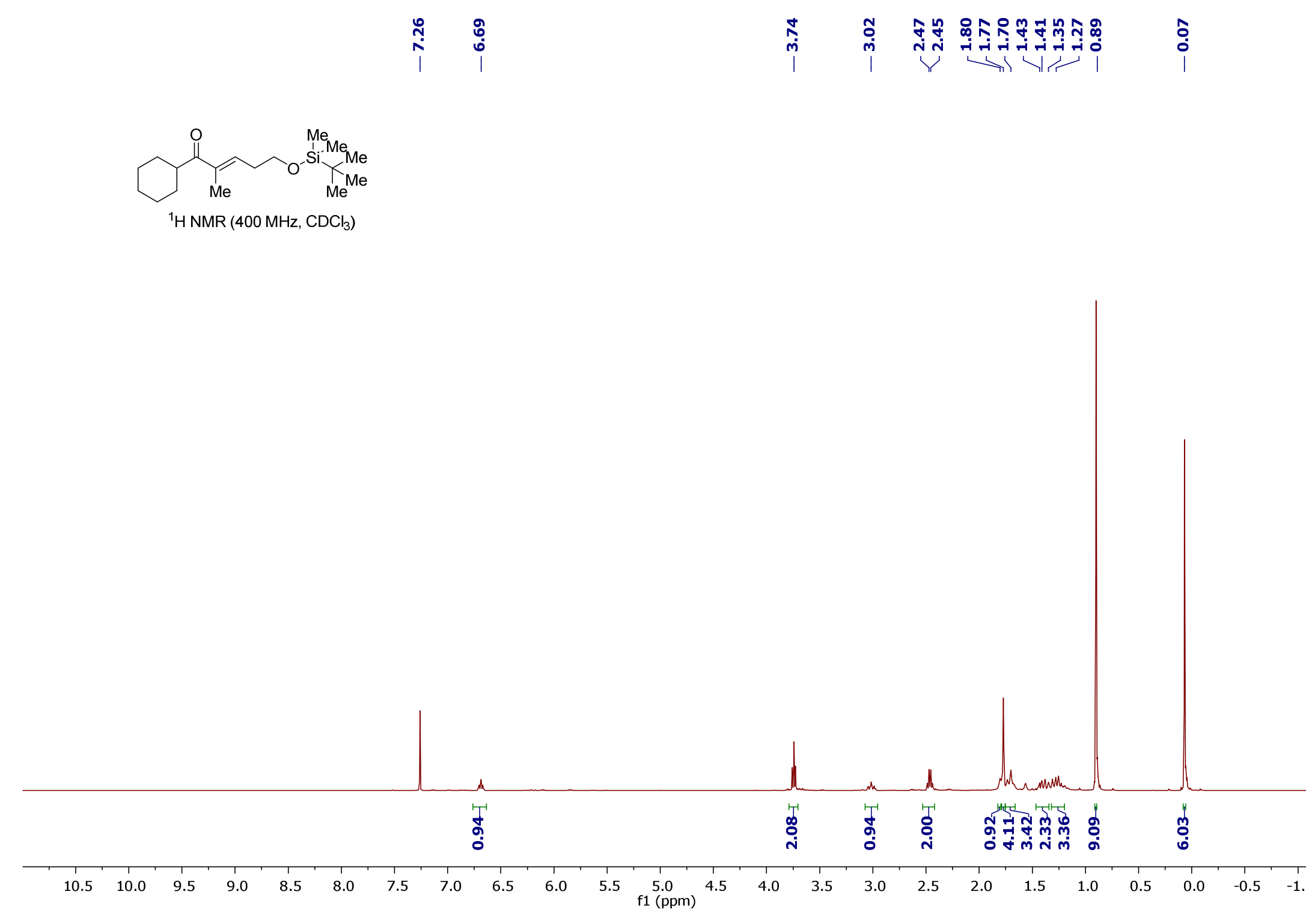


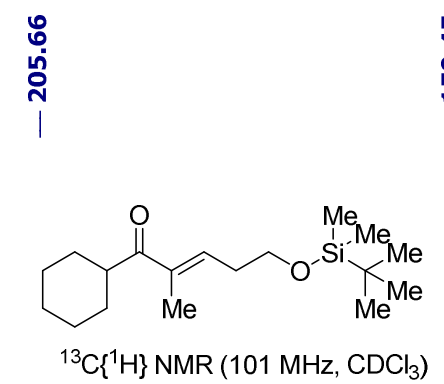

คั

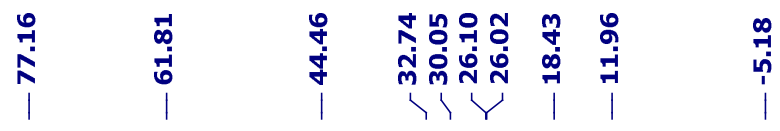

${ }^{2} \mathrm{C}\{\mathrm{H}\} \mathrm{NMR}\left(101 \mathrm{MHz}, \mathrm{CDCl}_{3}\right)$

กั่

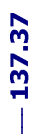

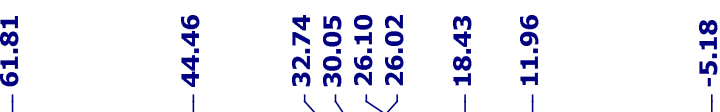

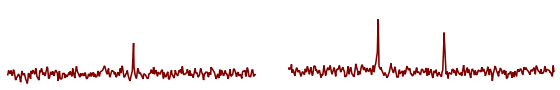

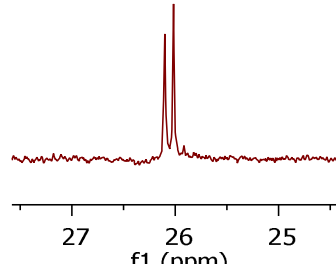

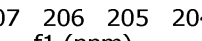

$\begin{array}{llll}139 & 138 & 137 & 136\end{array}$ 

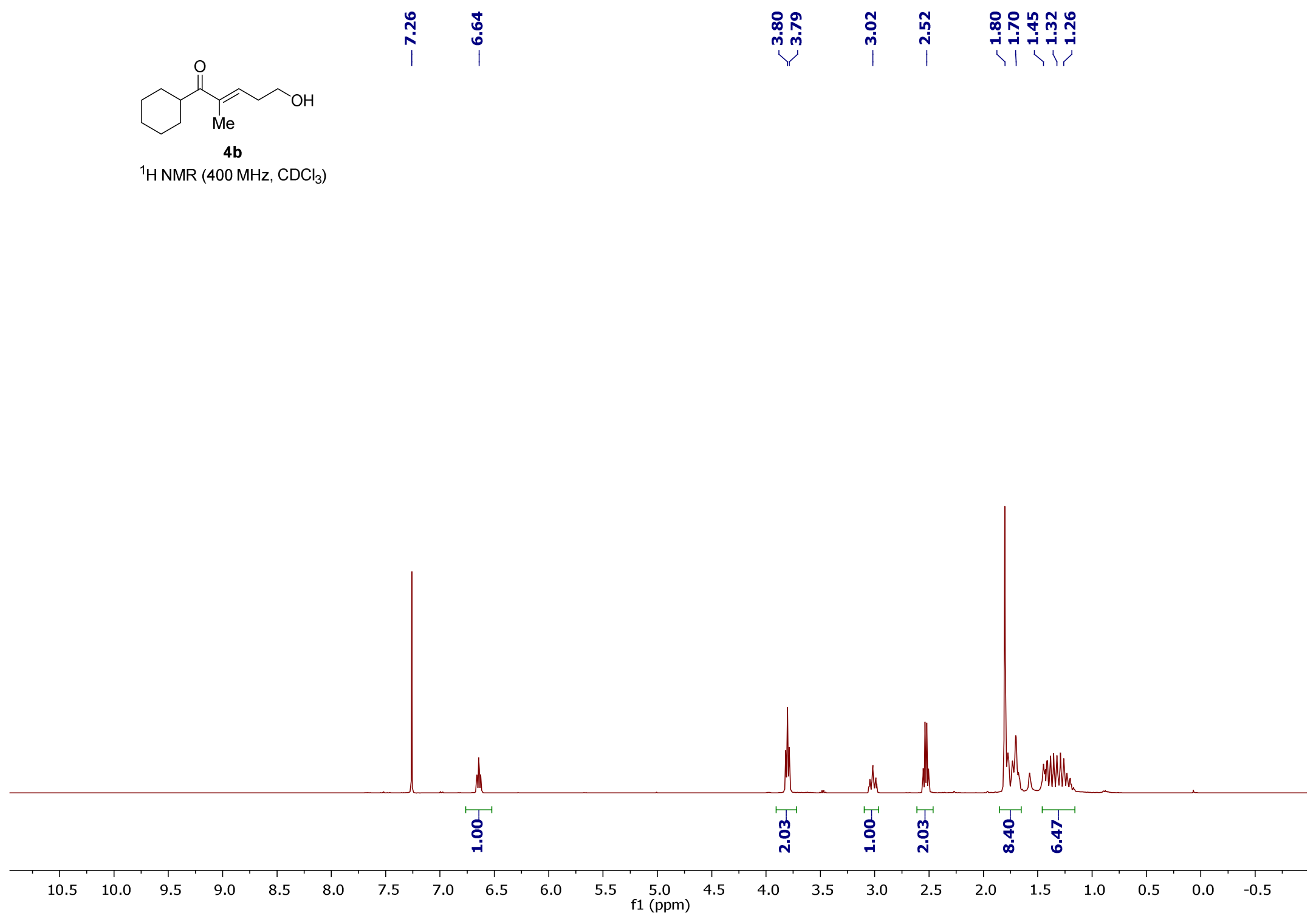
오ำ

离芦

$\begin{array}{ll}0 & 0 \\ 1 & 0 \\ 1 & 0 \\ 1 & 1\end{array}$
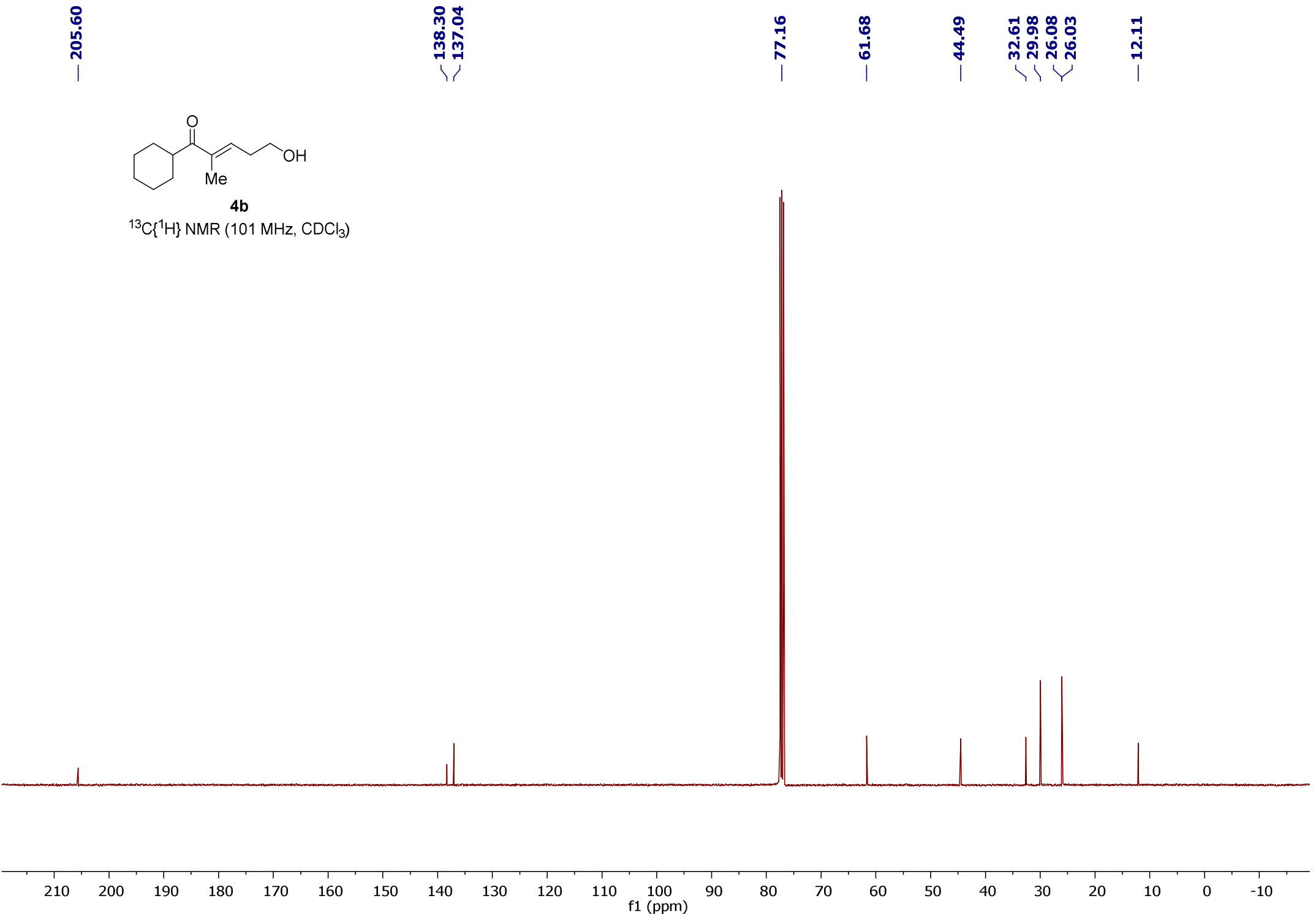
$\underbrace{\mathrm{Me}}_{\mathrm{Me}}$

${ }^{1} \mathrm{HNMR}\left(400 \mathrm{MHz}, \mathrm{CDCl}_{3}\right)$

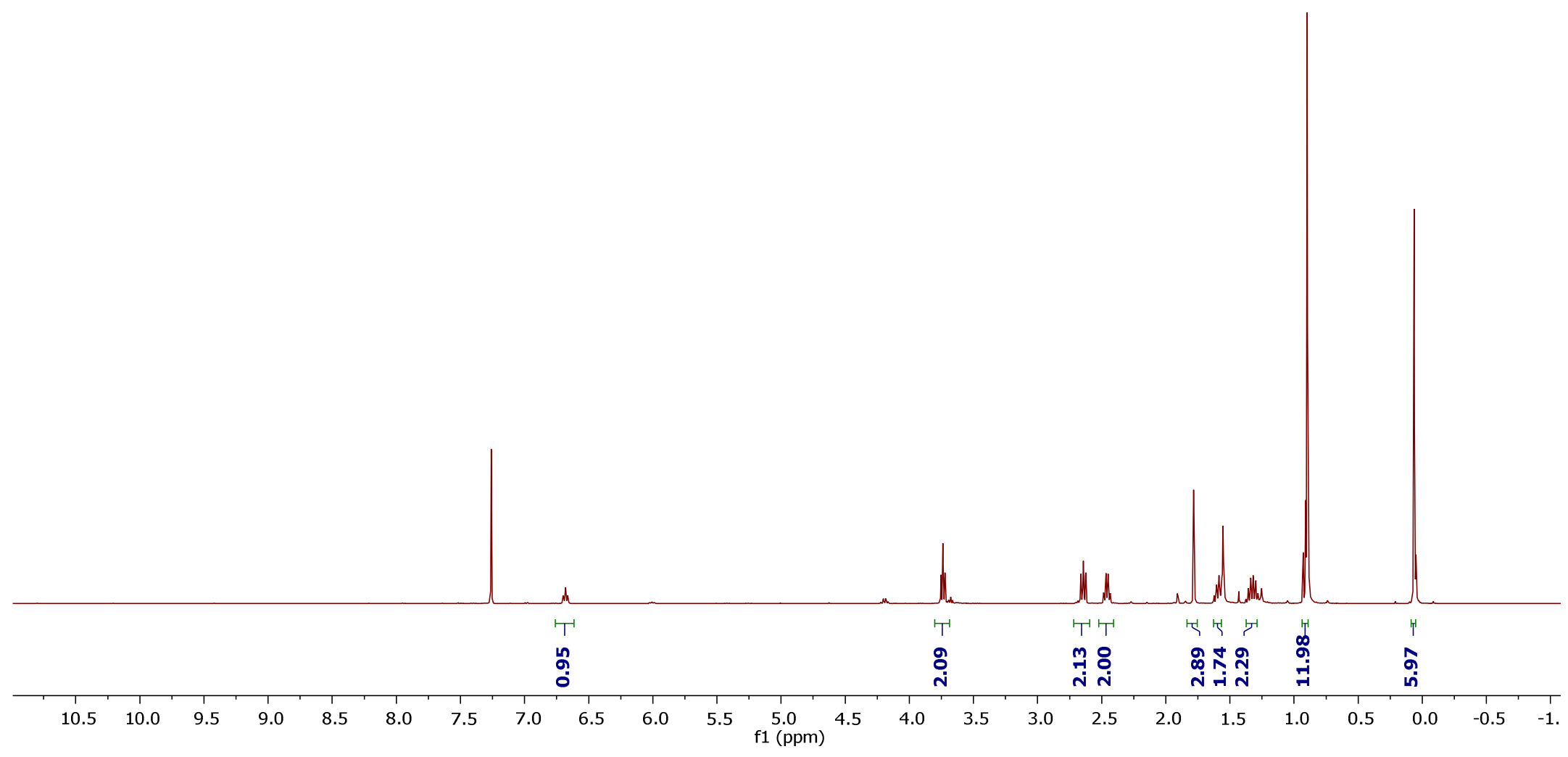



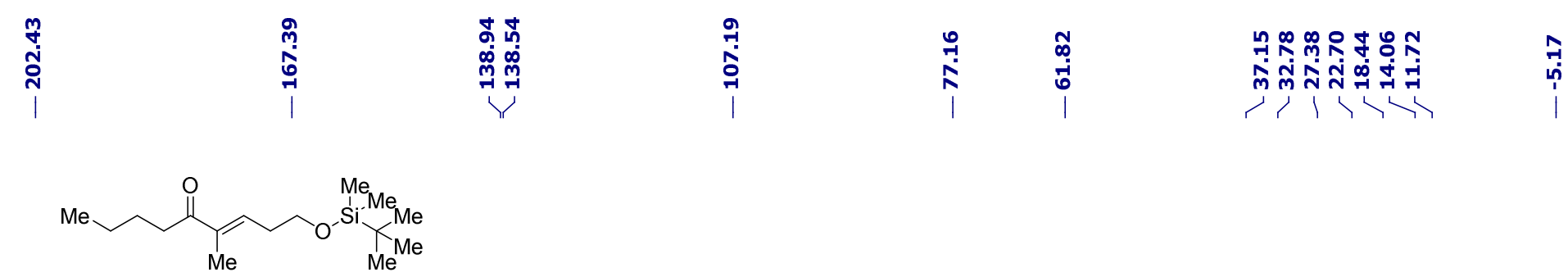

${ }^{13} \mathrm{C}\left\{{ }^{1} \mathrm{H}\right\} \mathrm{NMR}\left(101 \mathrm{MHz}, \mathrm{CDCl}_{3}\right)$

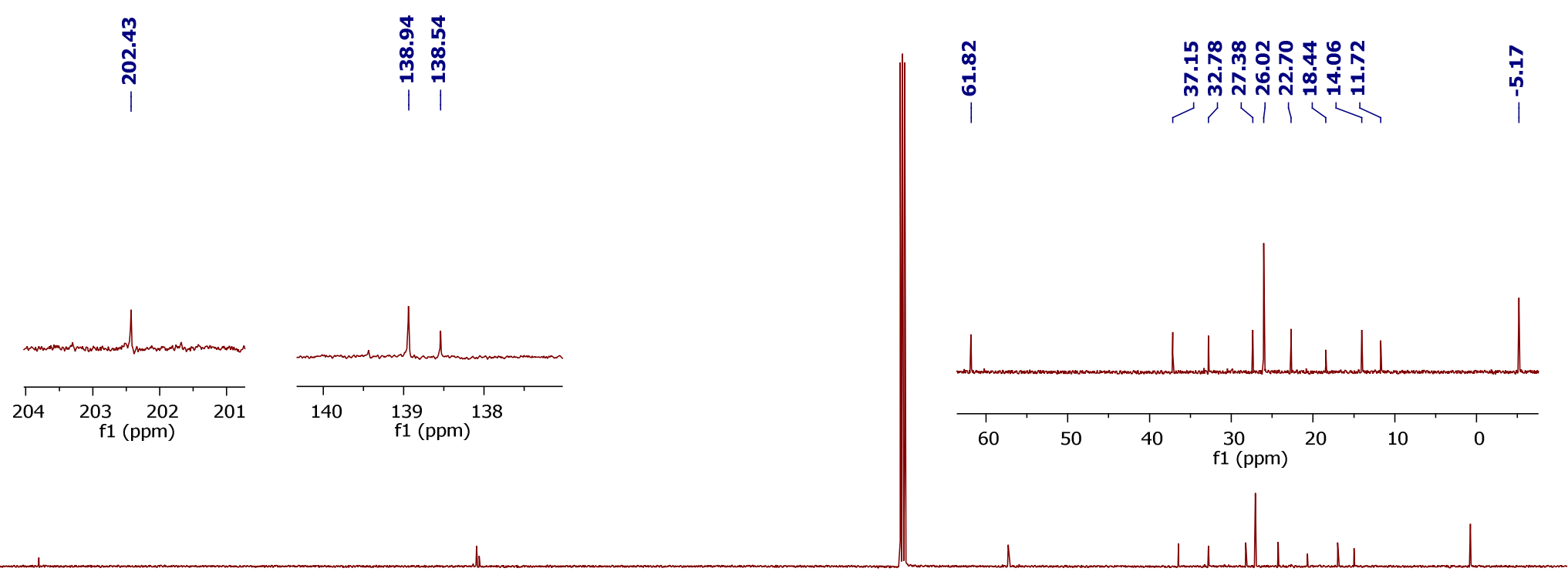

$\begin{array}{lllllllllllllllllllllllllll}210 & 200 & 190 & 180 & 170 & 160 & 150 & 140 & 130 & 120 & 110 & 100 & 100 & 80 & 70 & 60 & 50 & 40 & 30 & 20 & 10 & 0 & -10\end{array}$ 


\section{ก̊ำ}

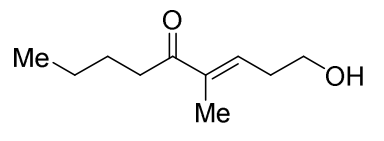

${ }^{1} \mathrm{H}$ NMR $\left(400 \mathrm{MHz}, \mathrm{CDCl}_{3}\right)$

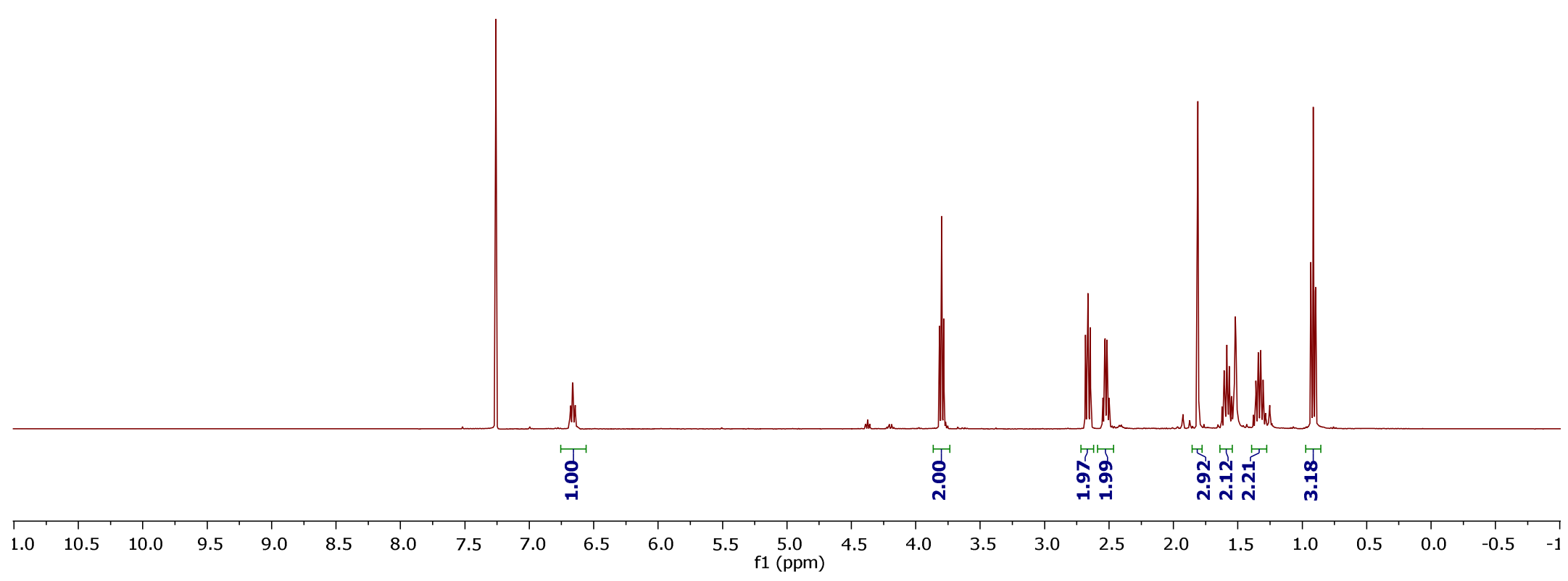




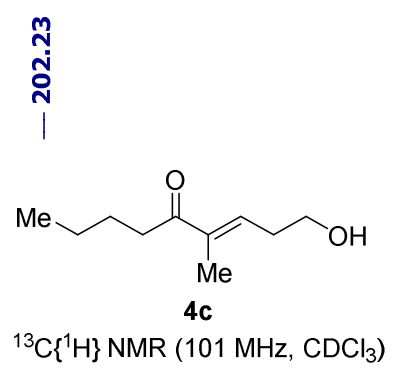

Mํㅇำ

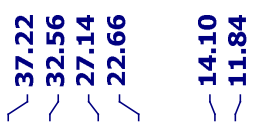

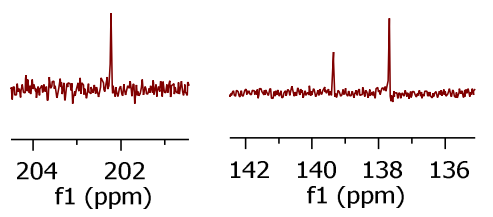

$\begin{array}{ll}0 \\ \stackrel{1}{1} & 0 \\ i & 0 \\ 1 & 1\end{array}$

ָָ

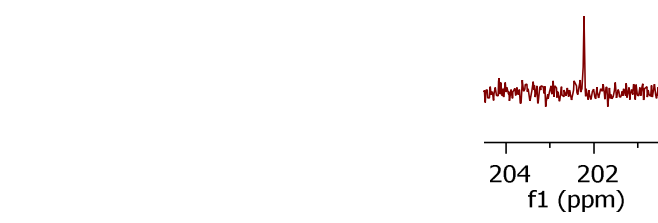

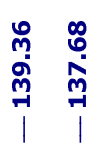

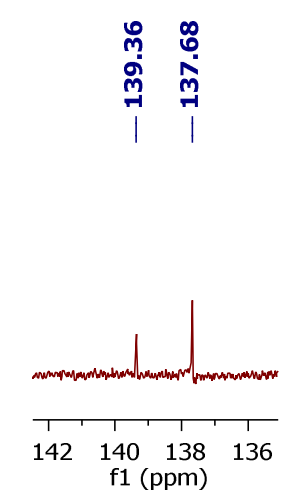




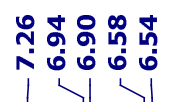

140

${ }_{\mathrm{Me}}^{\mathrm{H} M R}\left(400 \mathrm{MHz}, \mathrm{CDCl}_{3}\right)$

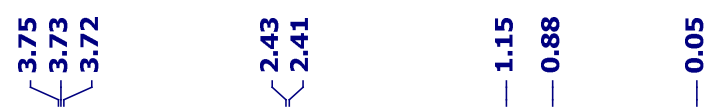

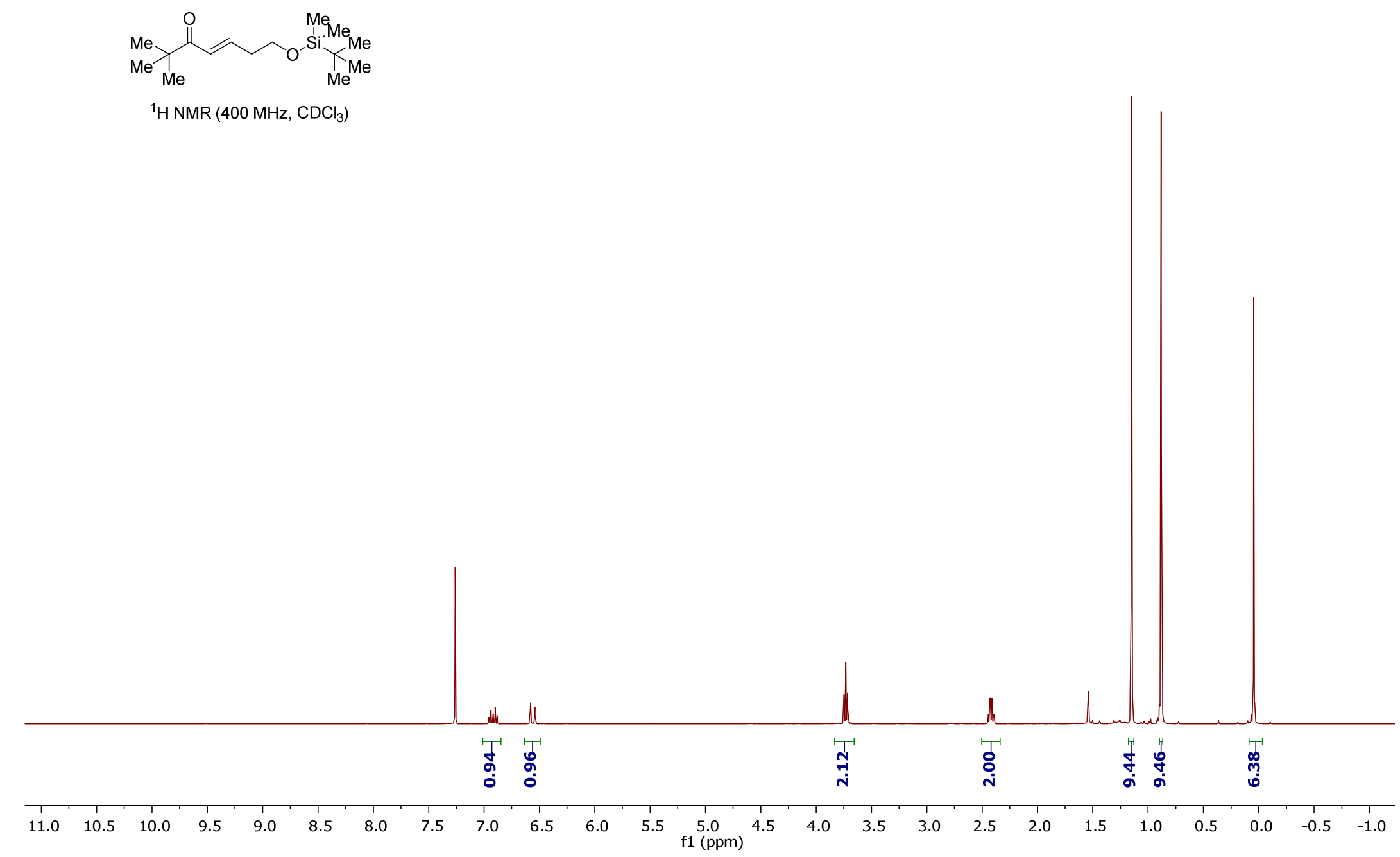




$$
\Delta^{\prime \prime}
$$




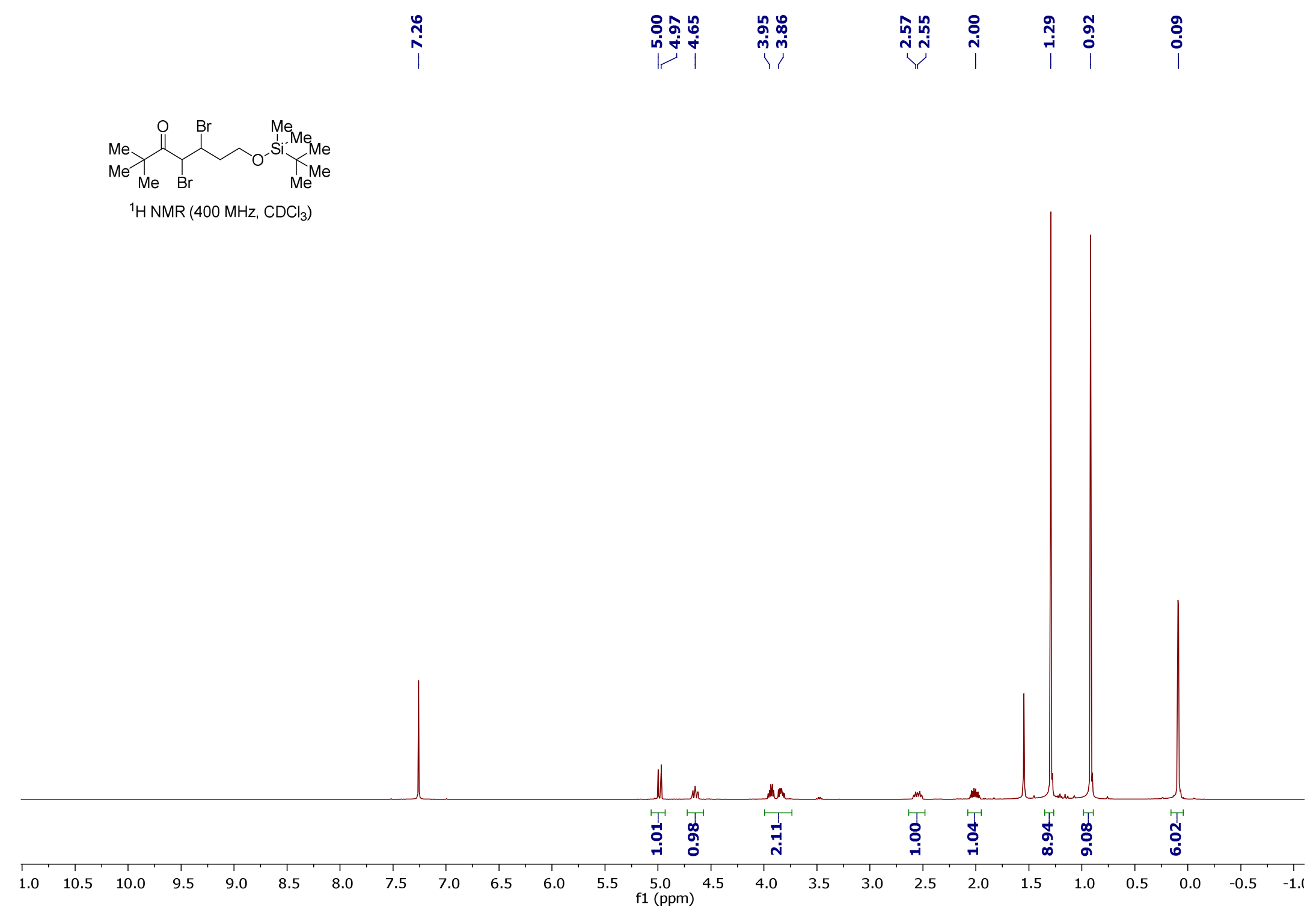




$$
=1 .
$$




苞

$$
\text { }{ }_{\mathrm{Me}}^{\mathrm{H} \mathrm{NMR}}\left(400 \mathrm{MHz}, \mathrm{CDCl}_{3}\right)
$$

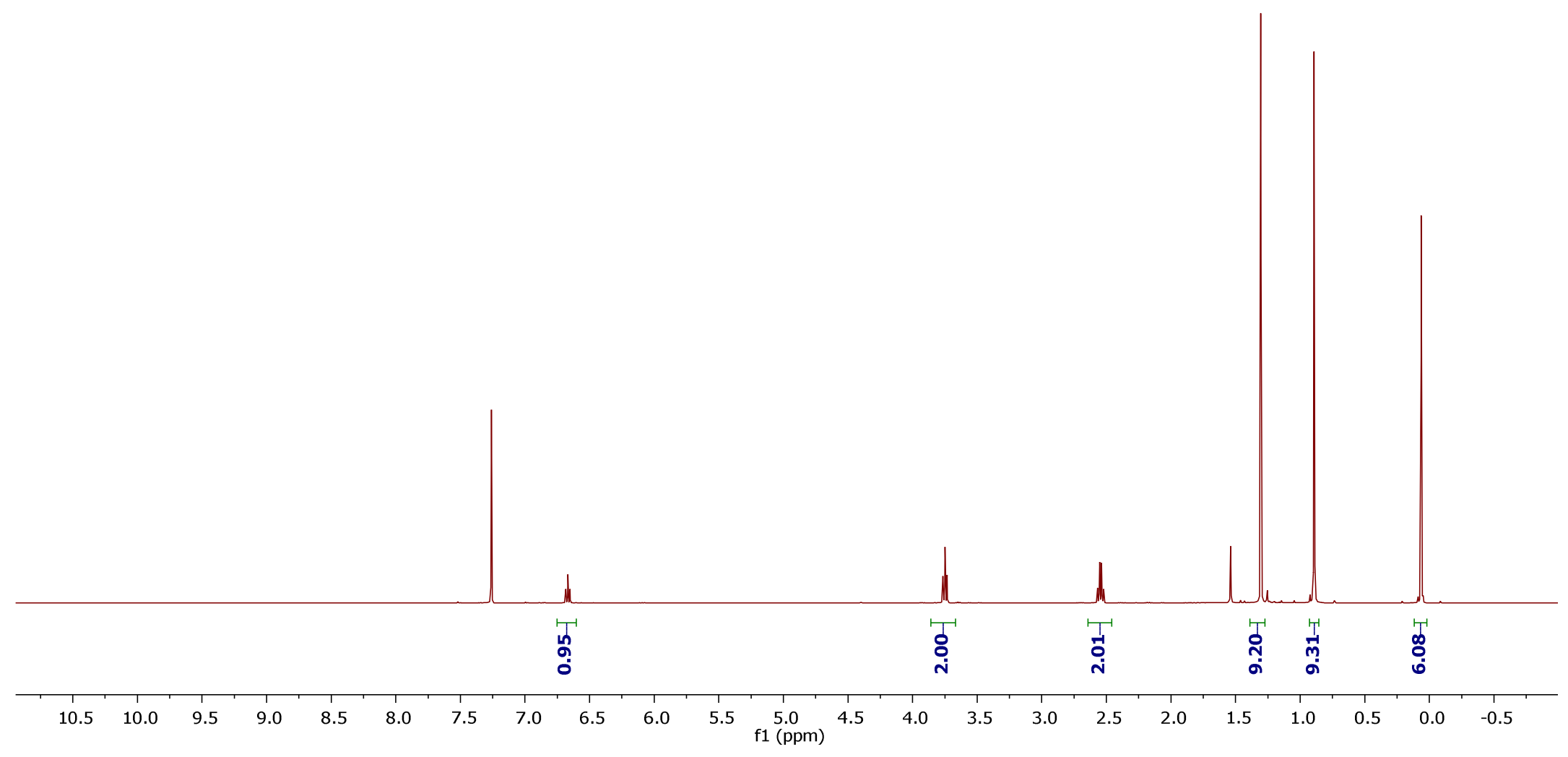




$$
\begin{array}{ll}
L^{\prime} \\
\hline
\end{array}
$$




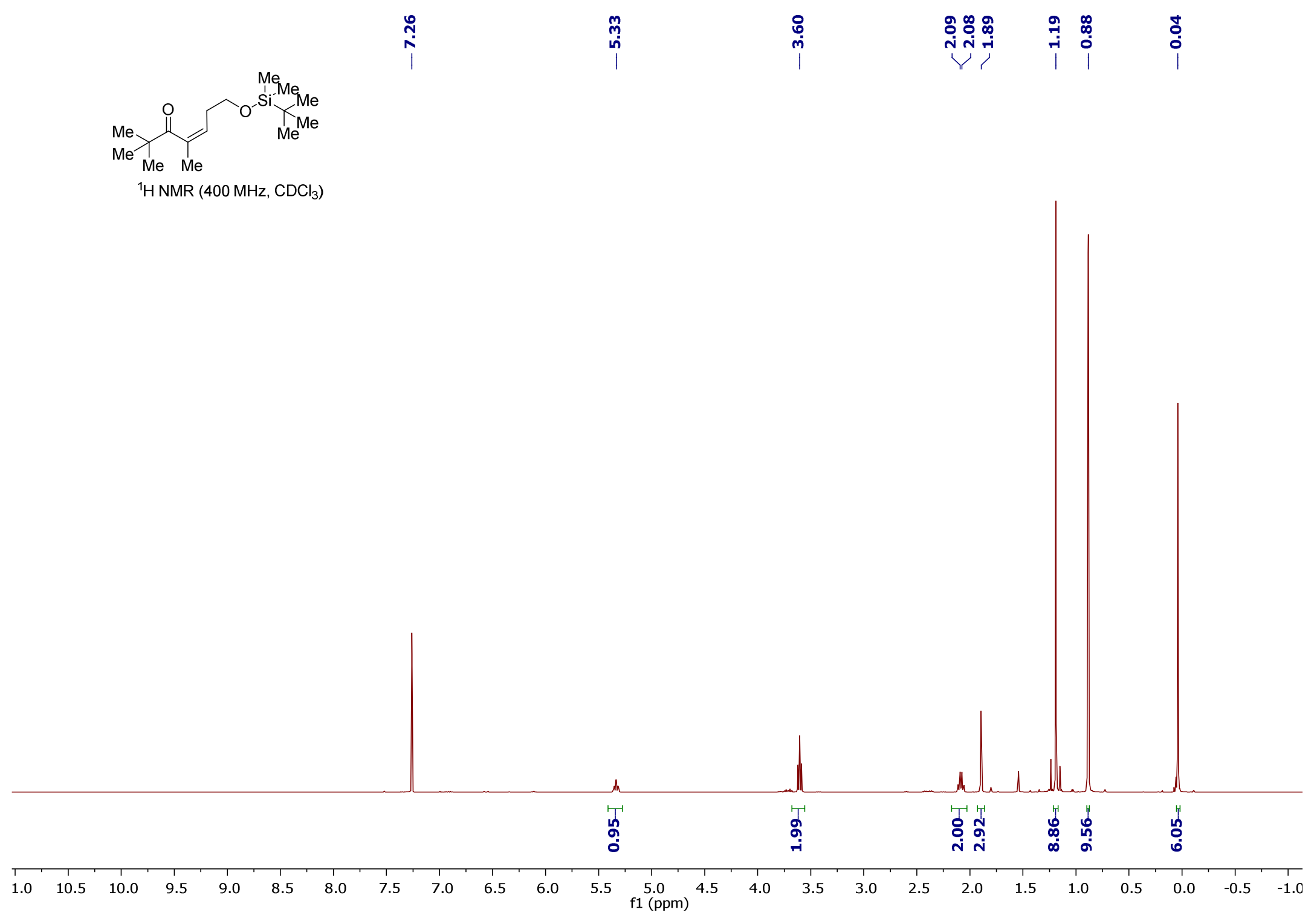




$$
\begin{array}{ll}
10 \\
\hline
\end{array}
$$




$$
{ }_{{ }_{1} \mathrm{HNMR}\left(400 \mathrm{MHz}, \mathrm{CDCl}_{3}\right)}^{\mathrm{Me}}
$$

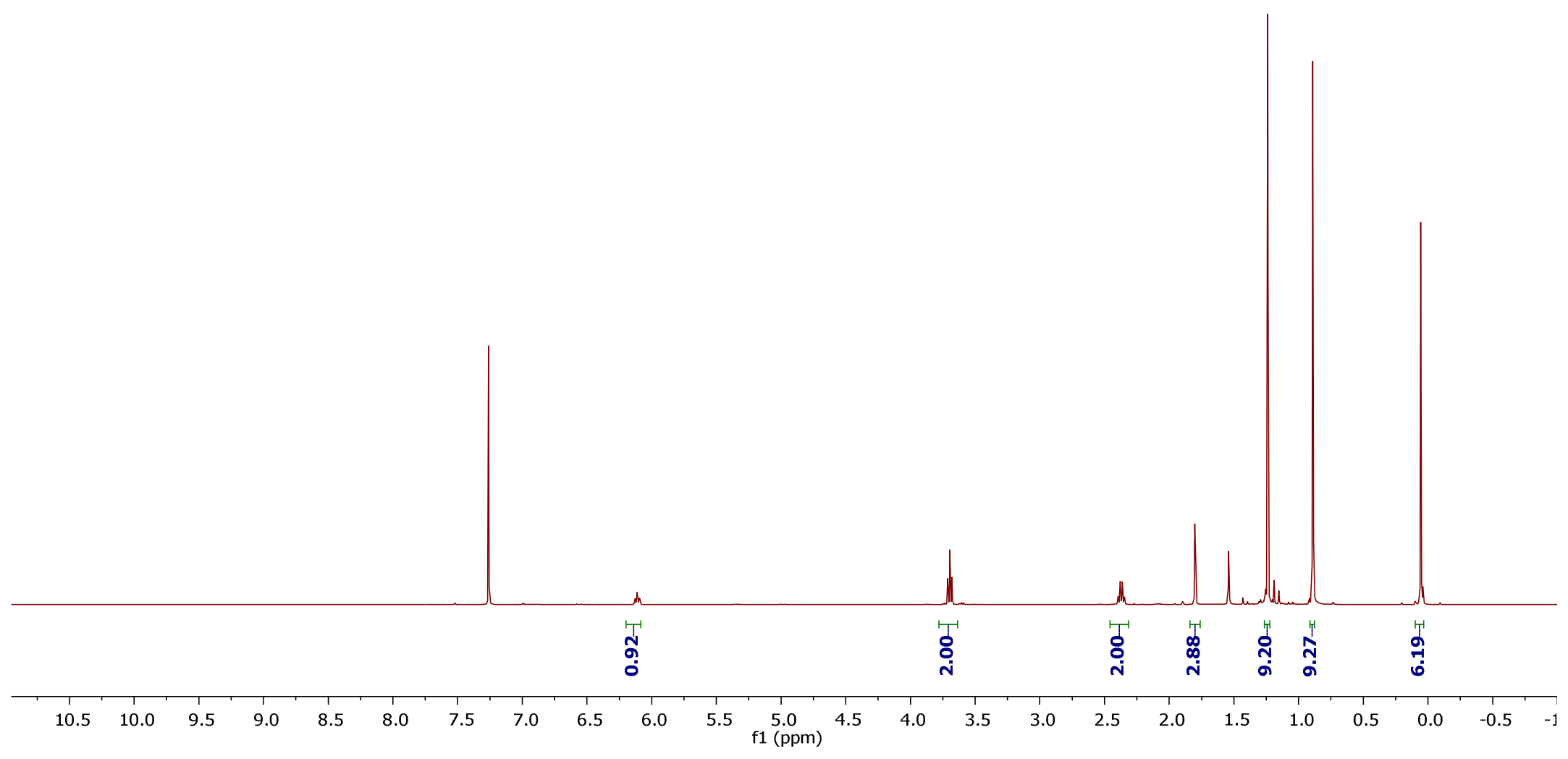




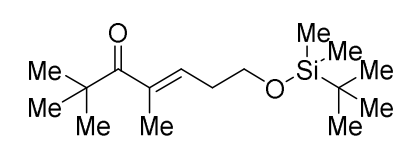

${ }^{13} \mathrm{C}\left\{{ }^{1} \mathrm{H}\right\} \mathrm{NMR}\left(101 \mathrm{MHz}, \mathrm{CDCl}_{3}\right)$

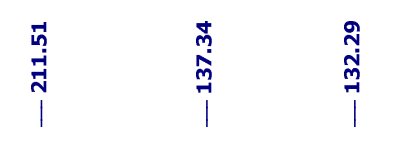
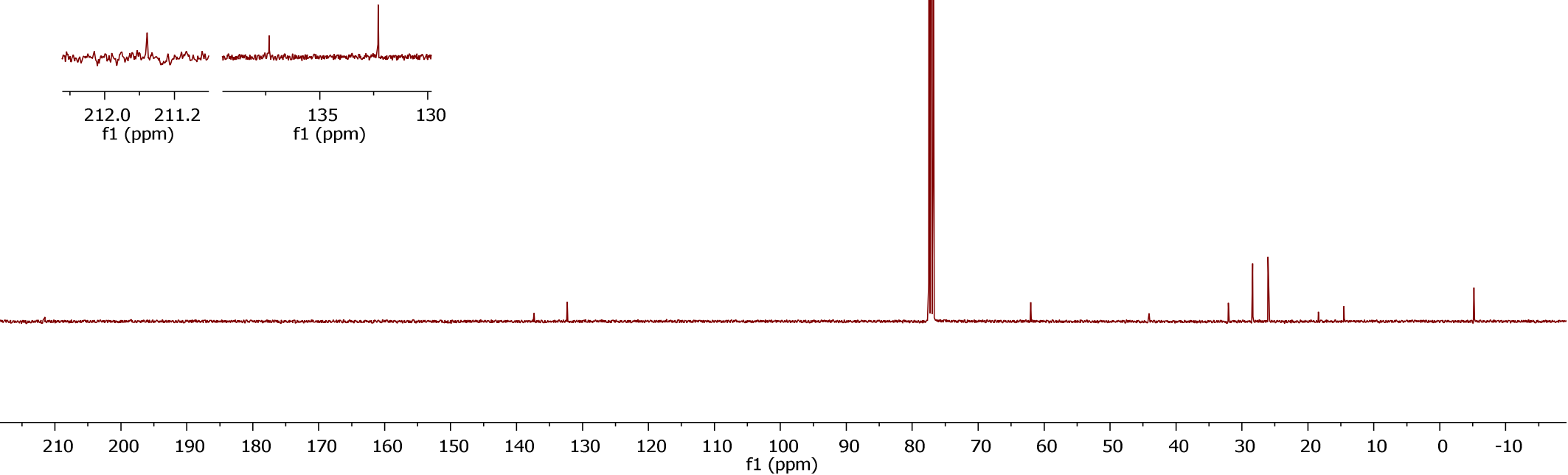

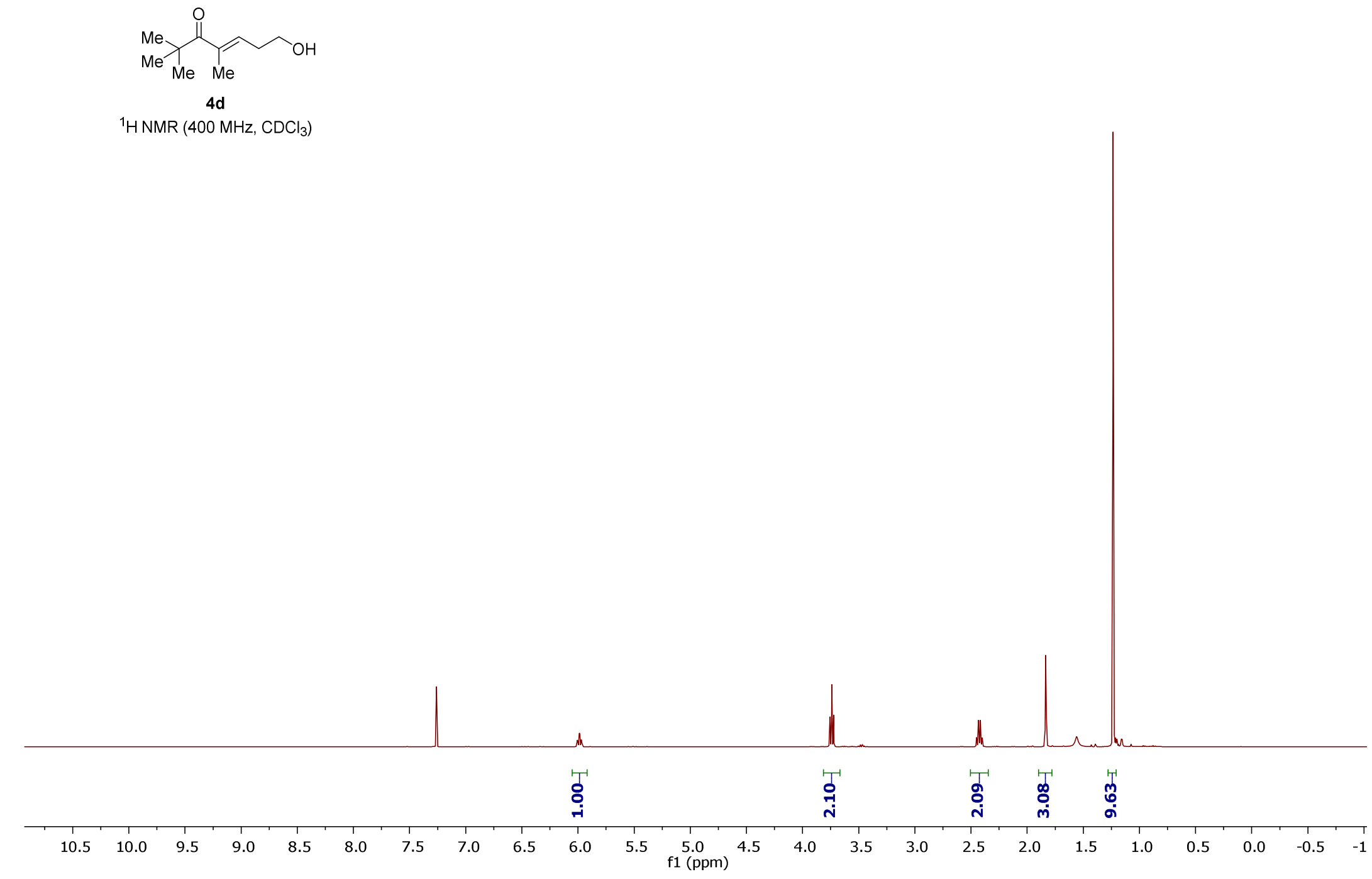


$$
="
$$




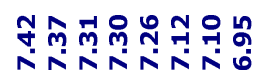

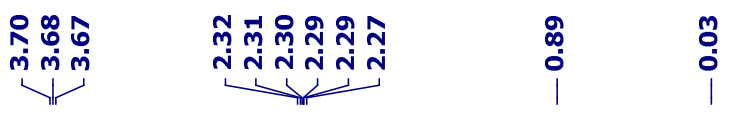

Me

${ }^{1} \mathrm{H} \mathrm{NMR}\left(400 \mathrm{MHz}, \mathrm{CDCl}_{3}\right)$

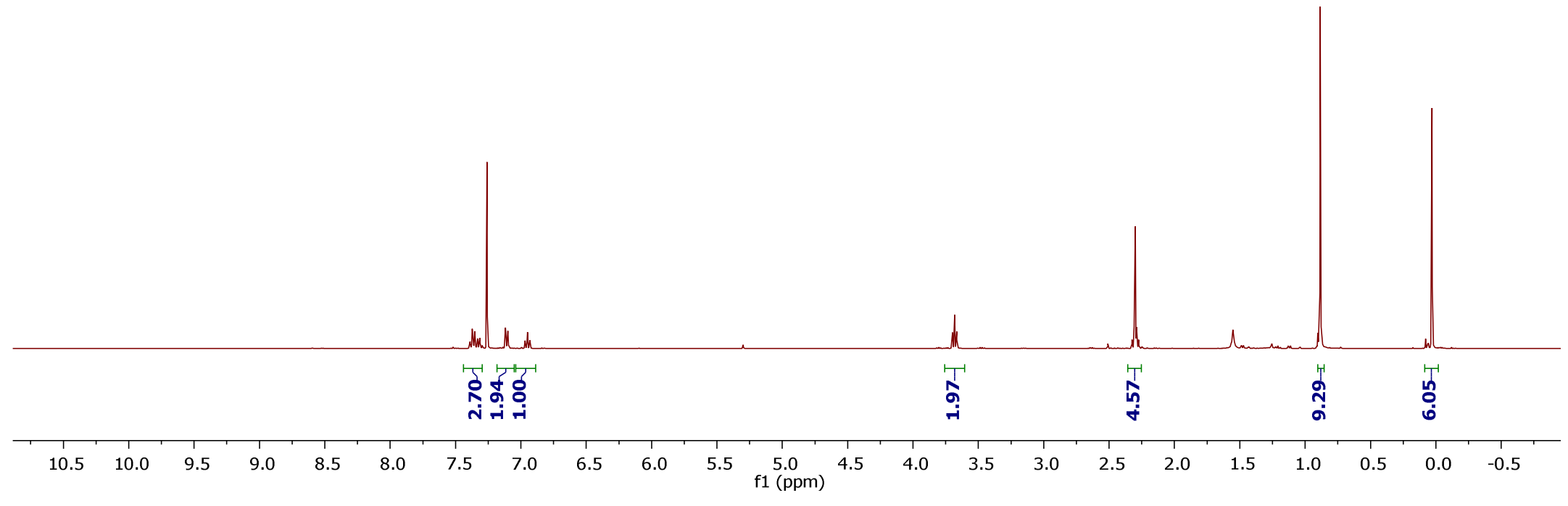




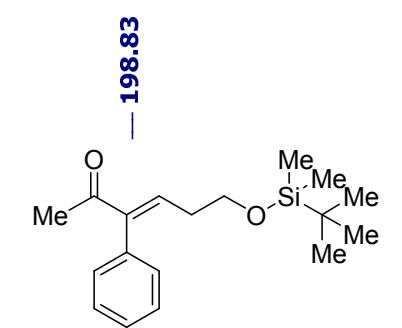

${ }^{13} \mathrm{C}\left\{{ }^{1} \mathrm{H}\right\}$ NMR $\left(101 \mathrm{MHz}, \mathrm{CDCl}_{3}\right)$

\section{웅유ำ

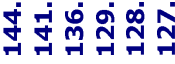 \\ |। ये।}

$\begin{array}{ll}0 \\ \stackrel{1}{i} & \stackrel{0}{0} \\ i & 0\end{array}$

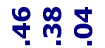

लำ

ind

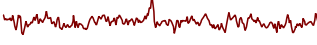

$\begin{array}{llll}199.5 & 199.0 & 198.5 & 198.0\end{array}$ f1 (ppm)

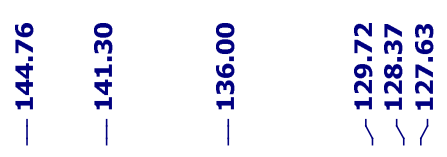

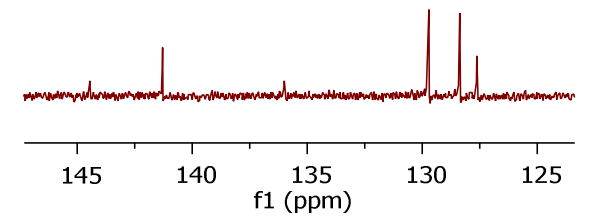




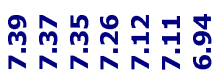

고정

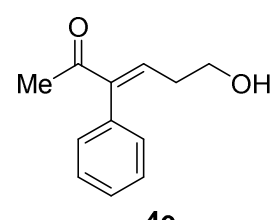

${ }^{1} \mathrm{HNMR}\left(400 \mathrm{MHz}, \mathrm{CDCl}_{3}\right)$

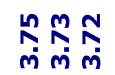

लู่

पा

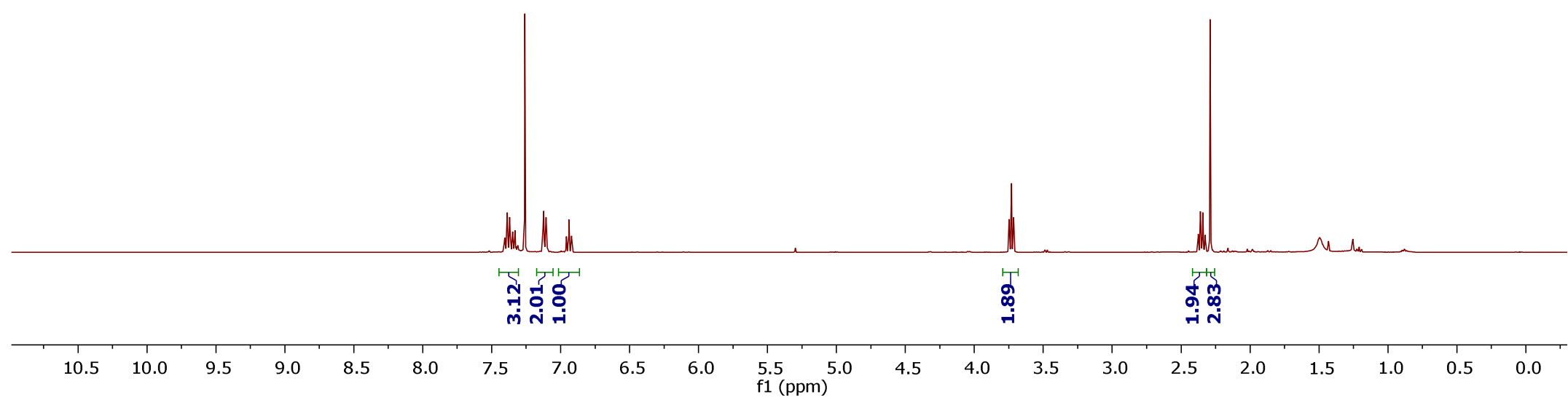




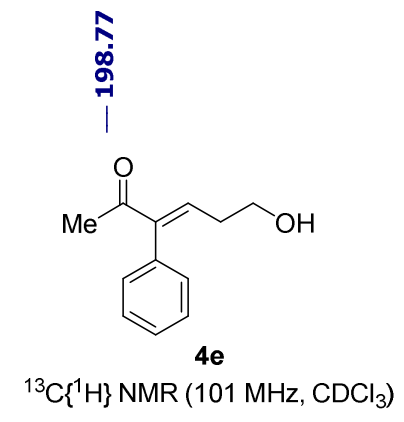

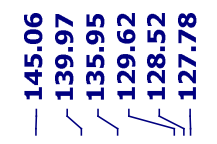
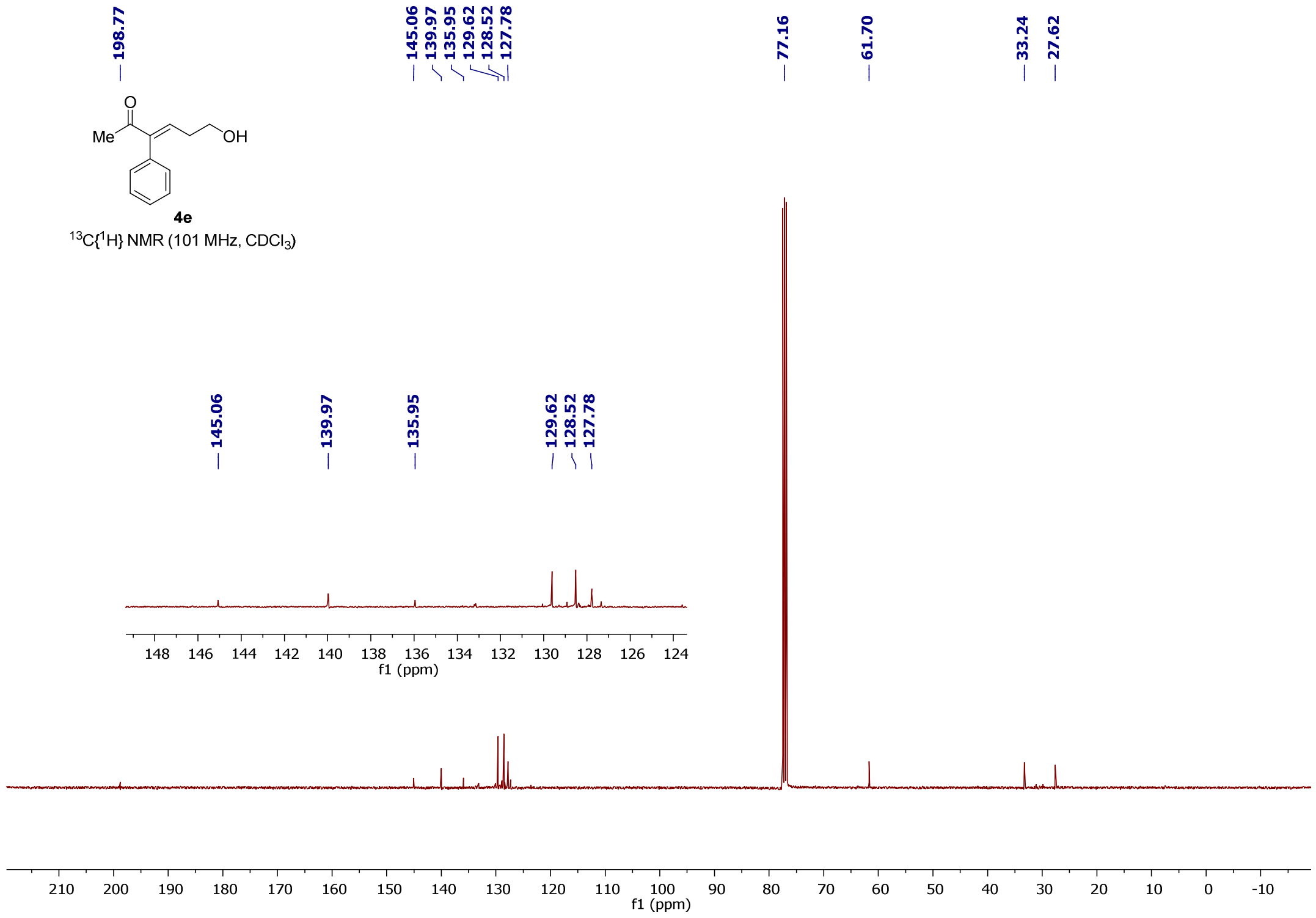
mำ

hisi

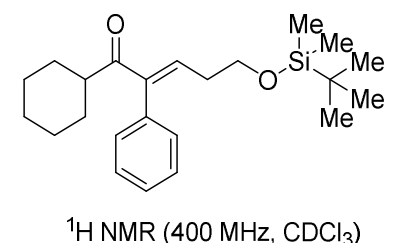

NMR $\left(400 \mathrm{MHz}, \mathrm{CDCl}_{3}\right)$
๓

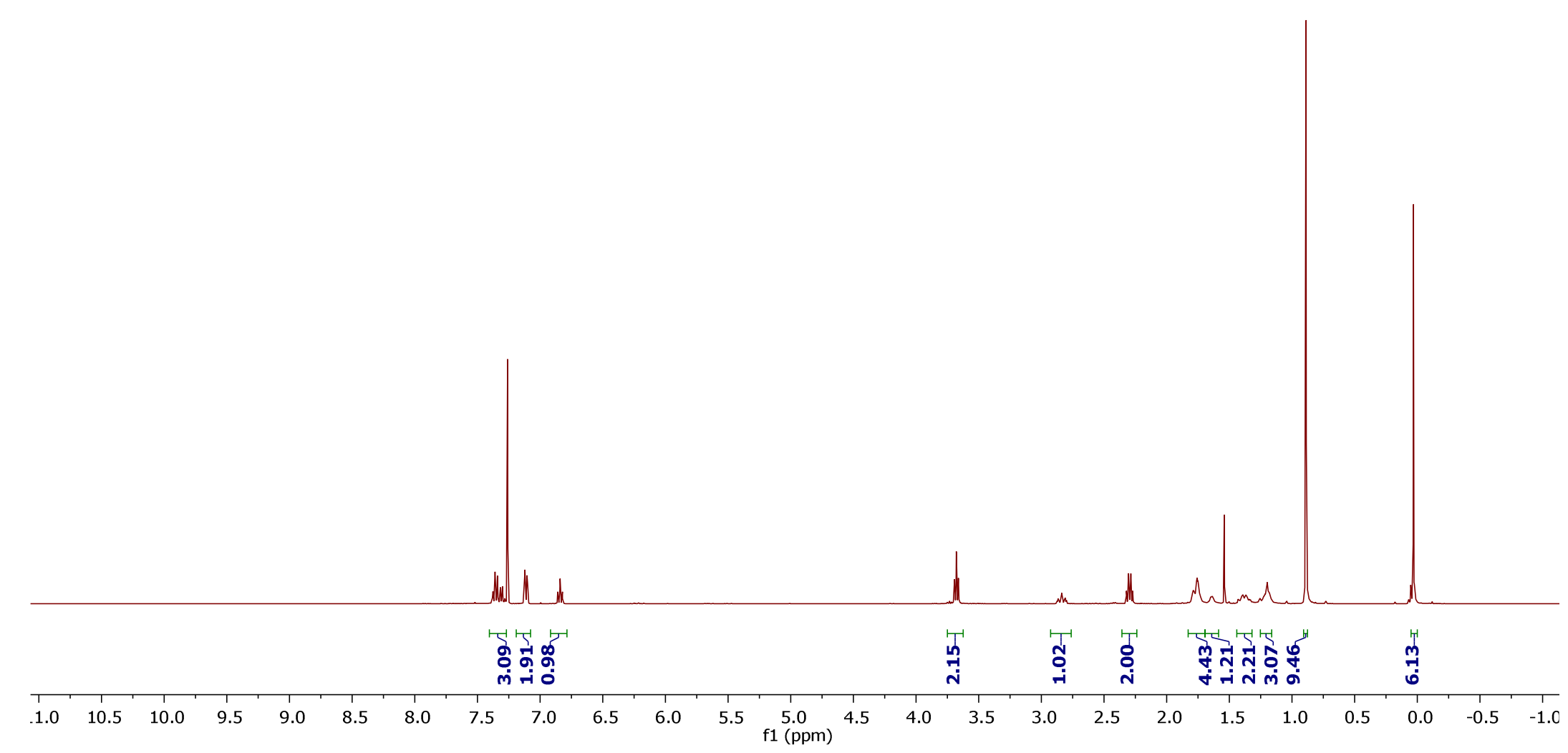




$$
=
$$




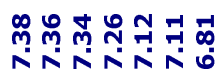

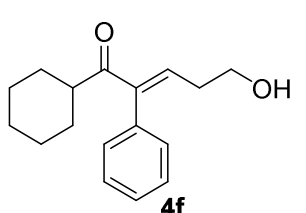

${ }^{1} \mathrm{H} \mathrm{NMR}\left(400 \mathrm{MHz}, \mathrm{CDCl}_{3}\right)$
กิ

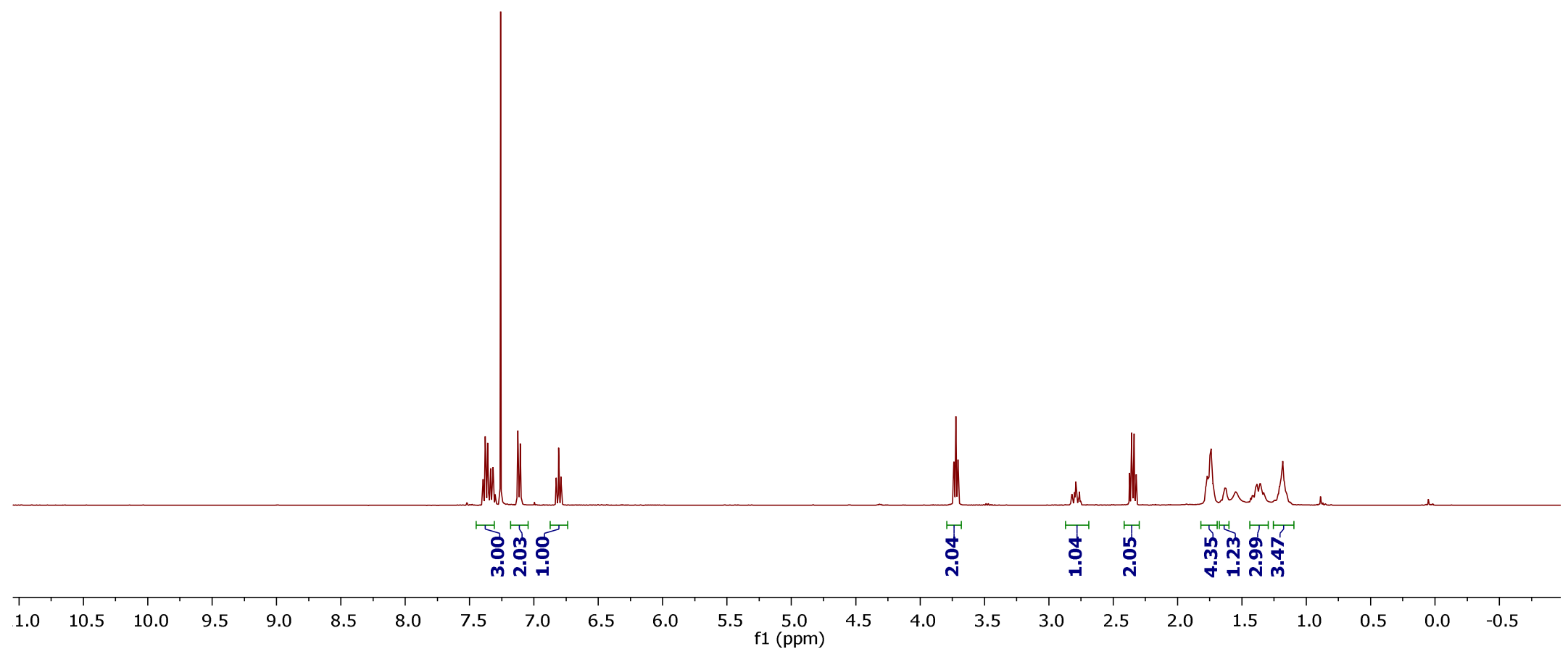




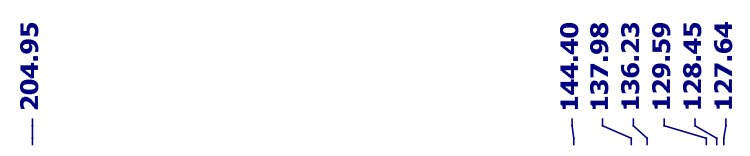
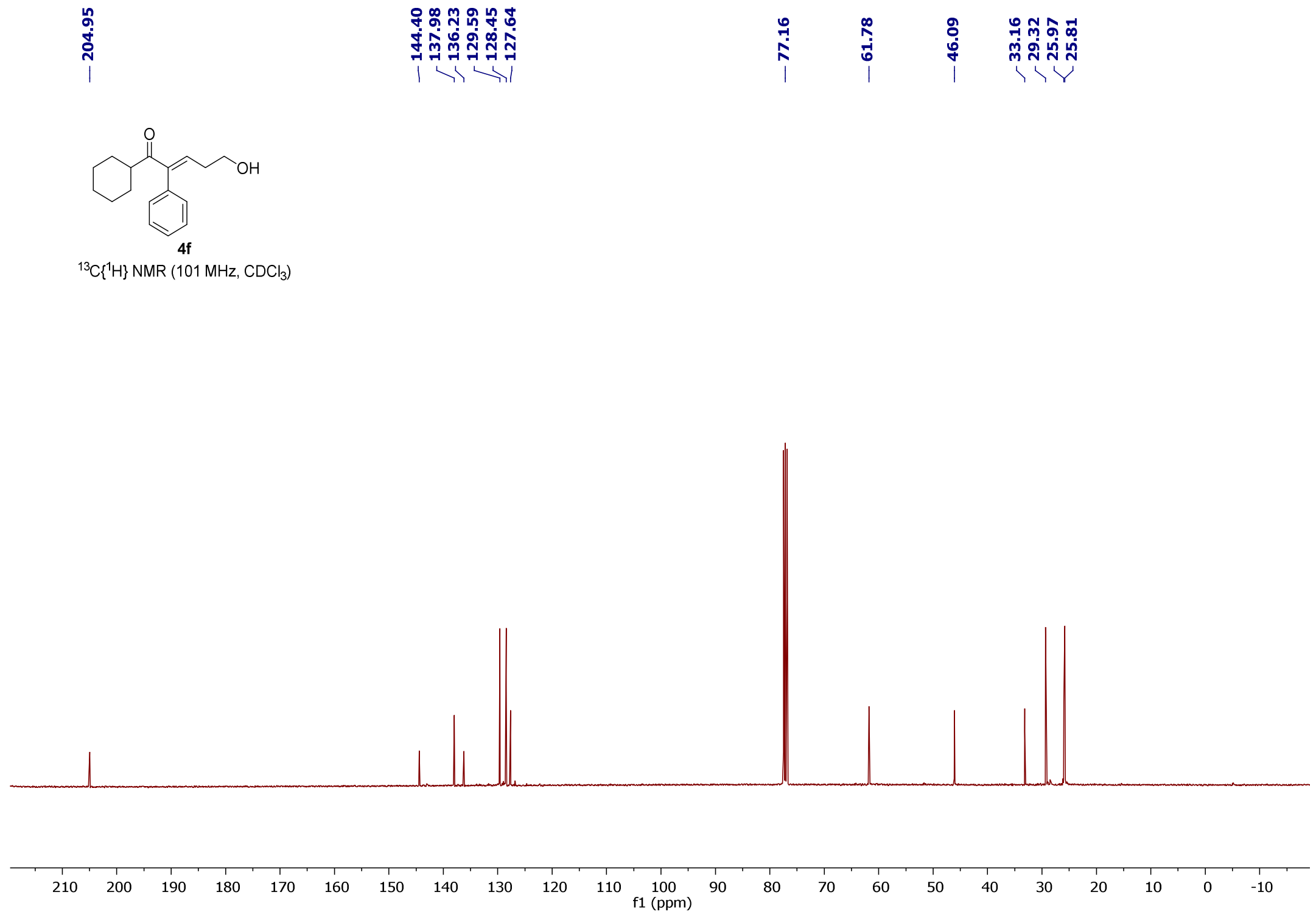


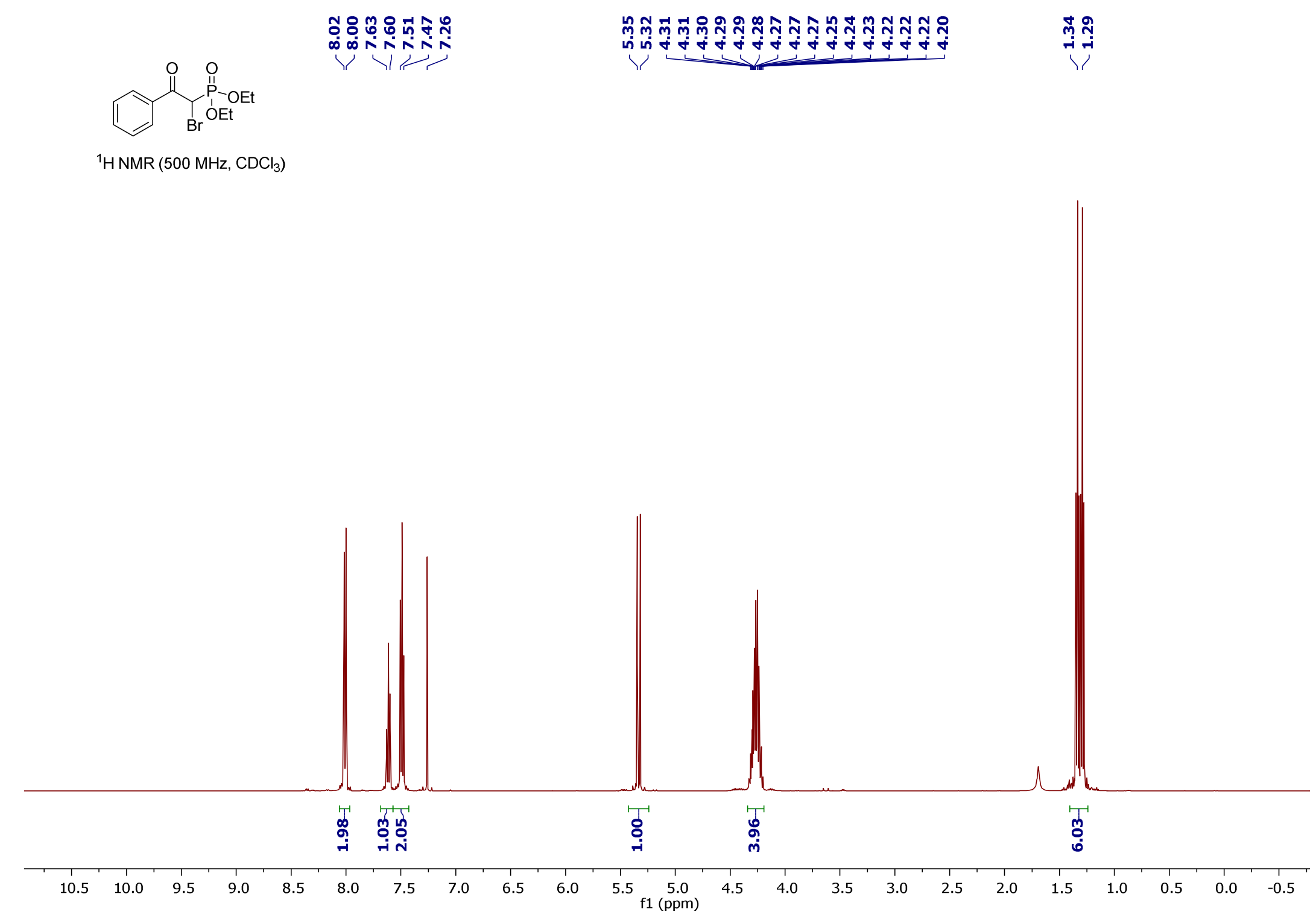



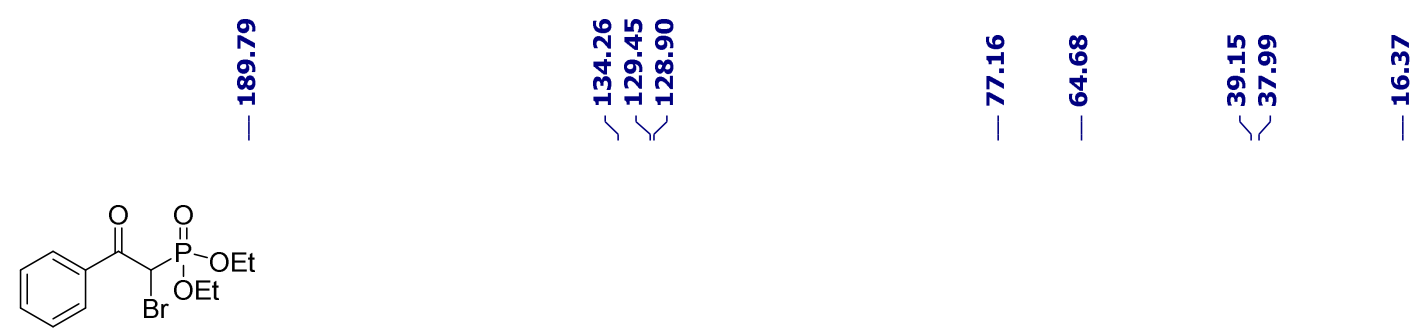

${ }^{13} \mathrm{C}\left\{{ }^{1} \mathrm{H}\right\} \mathrm{NMR}\left(126 \mathrm{MHz}, \mathrm{CDCl}_{3}\right)$

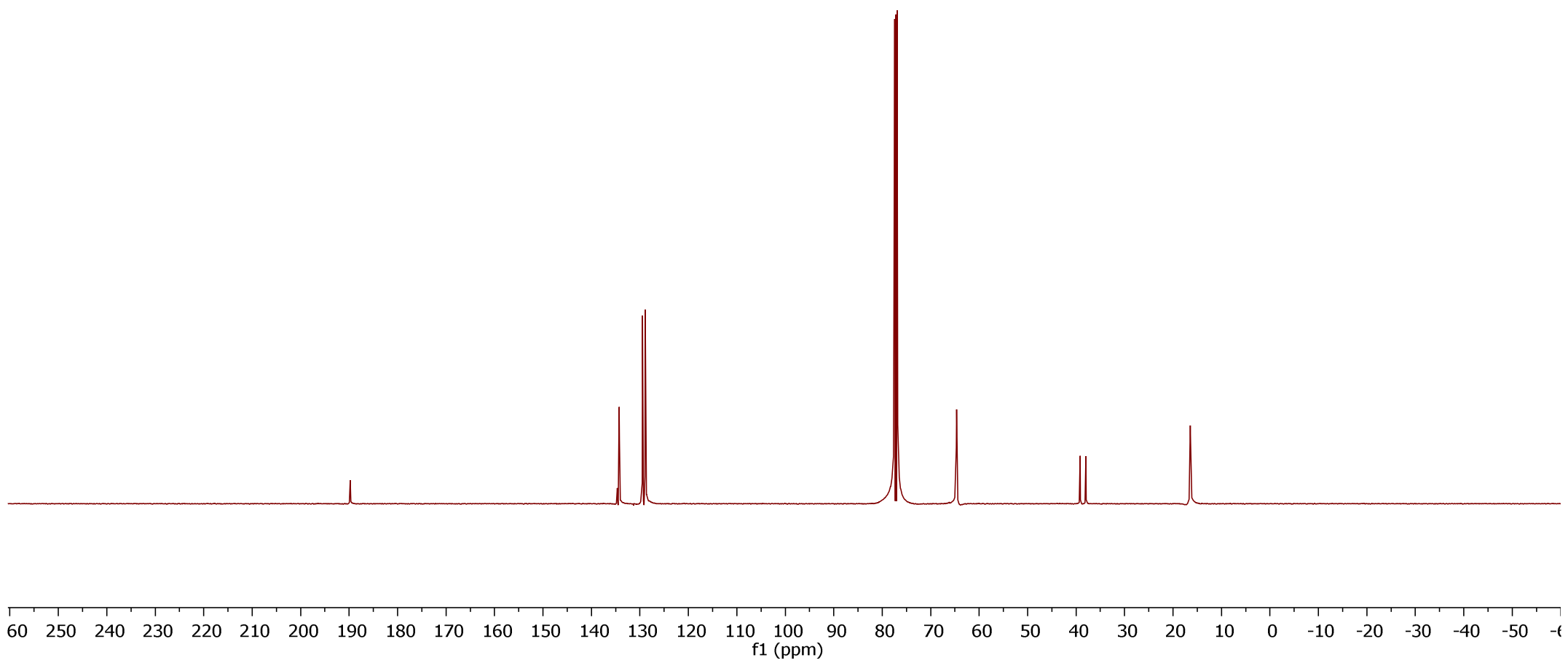




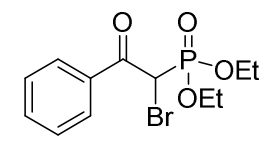

${ }^{31} \mathrm{P}\left\{{ }^{1} \mathrm{H},{ }^{13} \mathrm{C}\right\} \mathrm{NMR}\left(162 \mathrm{MHz}, \mathrm{CDCl}_{3}\right)$

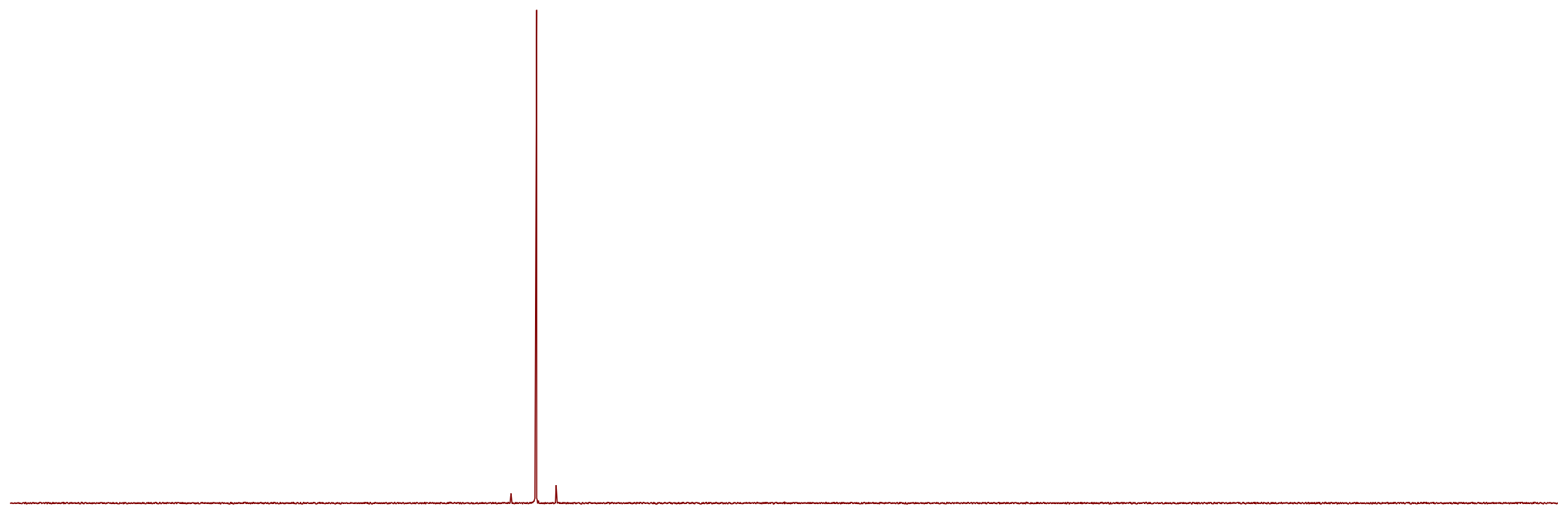

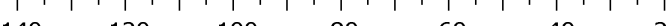

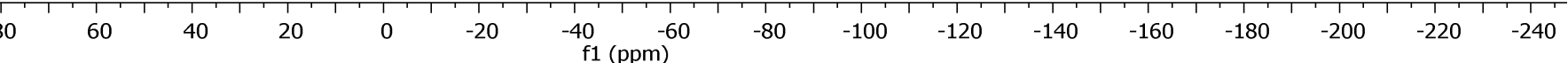




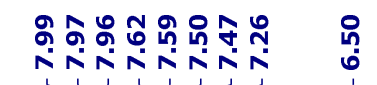

NiñNiñ

${ }^{1} \mathrm{H}$ NMR $\left(500 \mathrm{MHz}, \mathrm{CDCl}_{3}\right)$

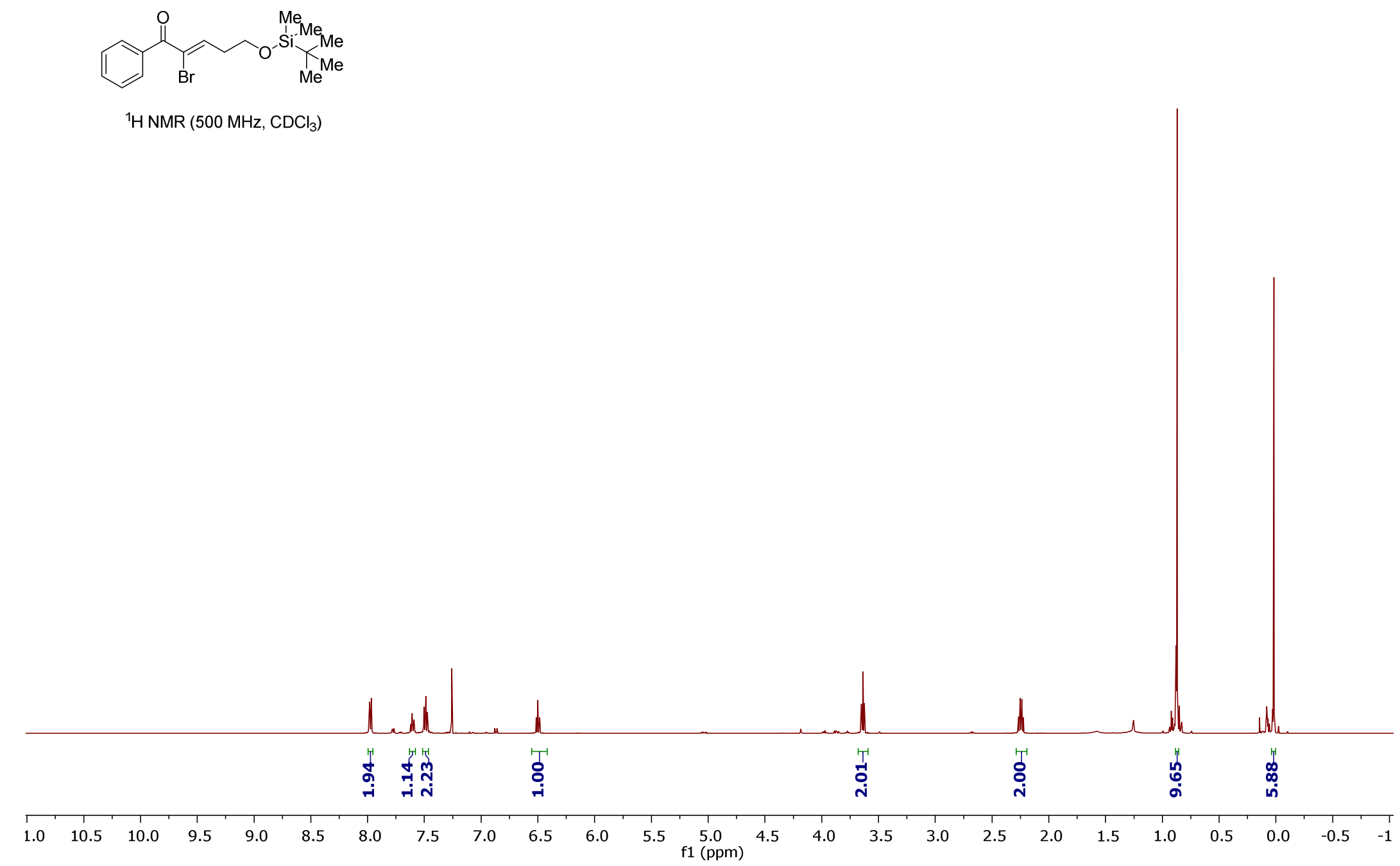

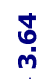

ฟูป ำ

ڤั̊ 


至

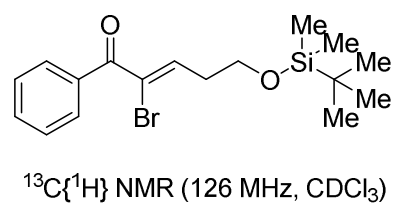

${ }^{13} \mathrm{C}\left\{{ }^{1} \mathrm{H}\right\} \mathrm{NMR}\left(126 \mathrm{MHz}, \mathrm{CDCl}_{3}\right)$

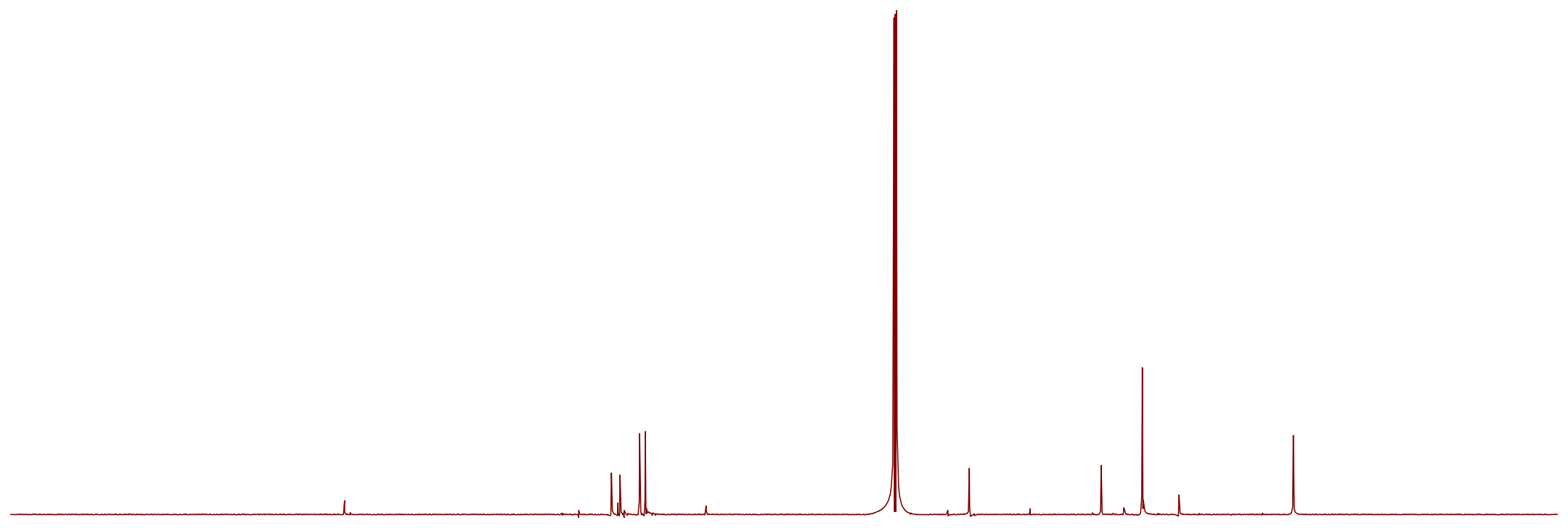

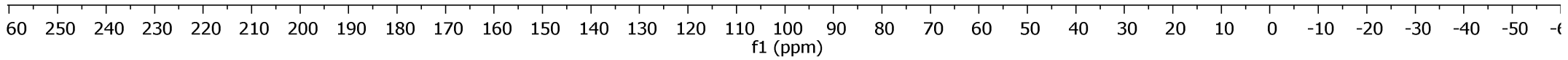




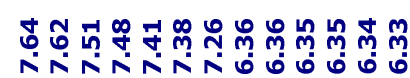

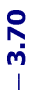

ฟñ

$\underbrace{\mathrm{Me}}_{\mathrm{Me}}$

${ }^{1} \mathrm{H} \mathrm{NMR}\left(400 \mathrm{MHz}, \mathrm{CDCl}_{3}\right)$

तो।

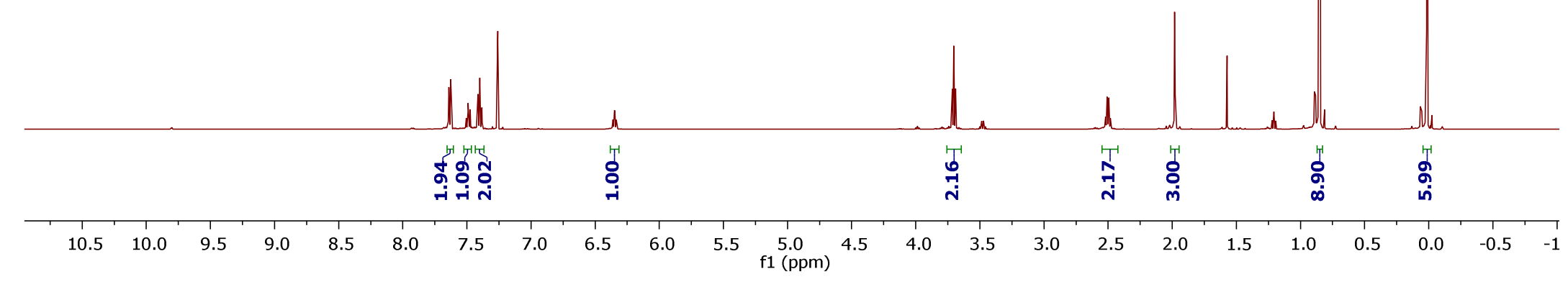



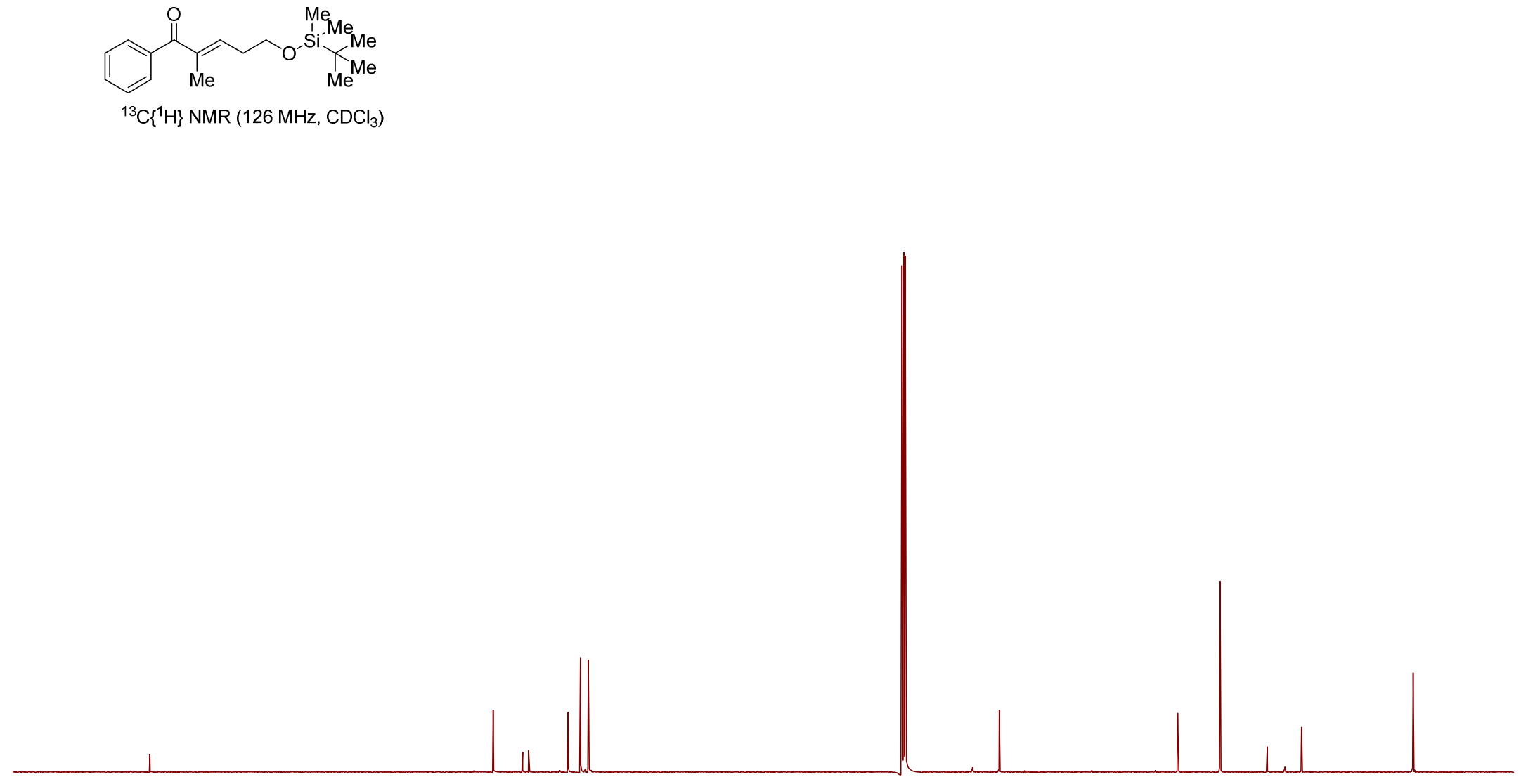

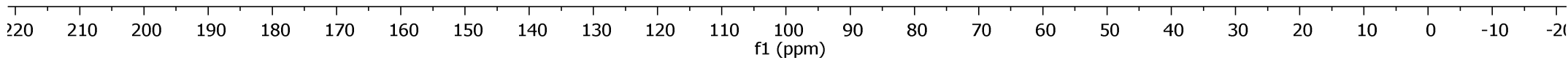




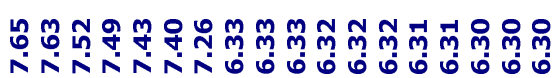

내선

inj

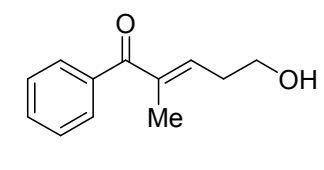

$4 \mathrm{~g}$

${ }^{1} \mathrm{H}$ NMR $\left(500 \mathrm{MHz}, \mathrm{CDCl}_{3}\right)$

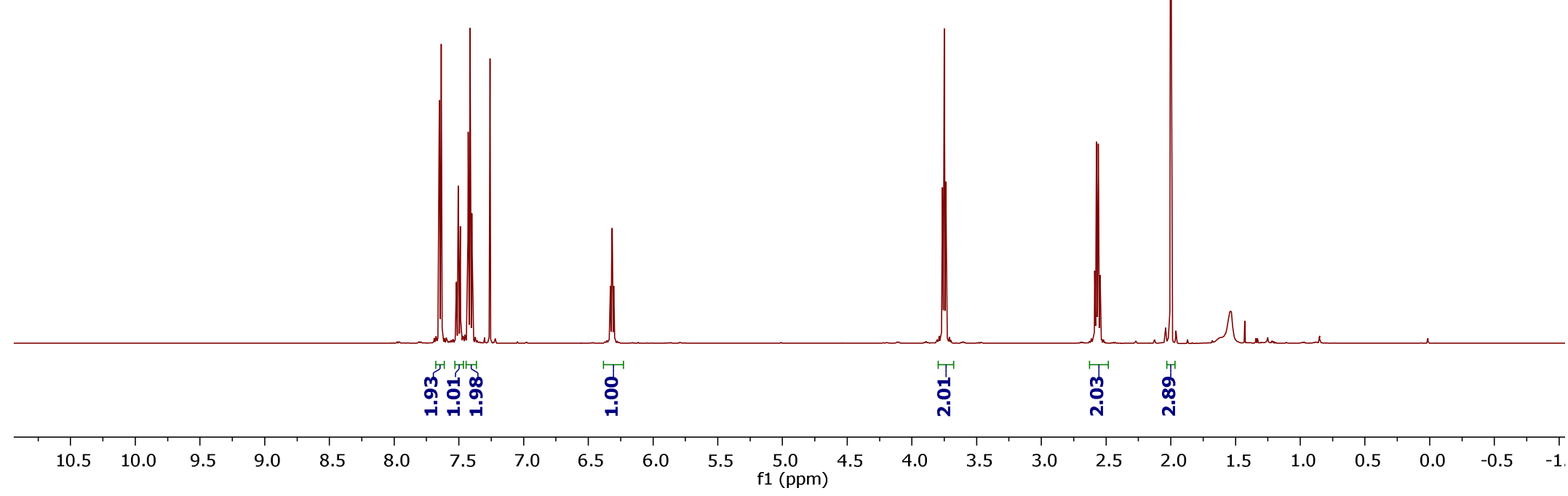




\section{ำำ웜

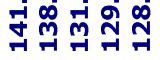

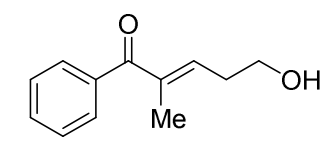

$4 \mathrm{~g}$

${ }^{13} \mathrm{C}\left\{{ }^{1} \mathrm{H}\right\} \operatorname{NMR}\left(126 \mathrm{MHz}, \mathrm{CDCl}_{3}\right)$

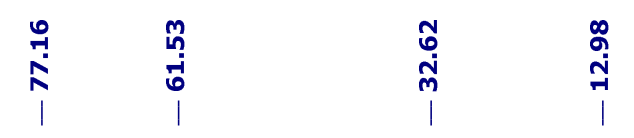

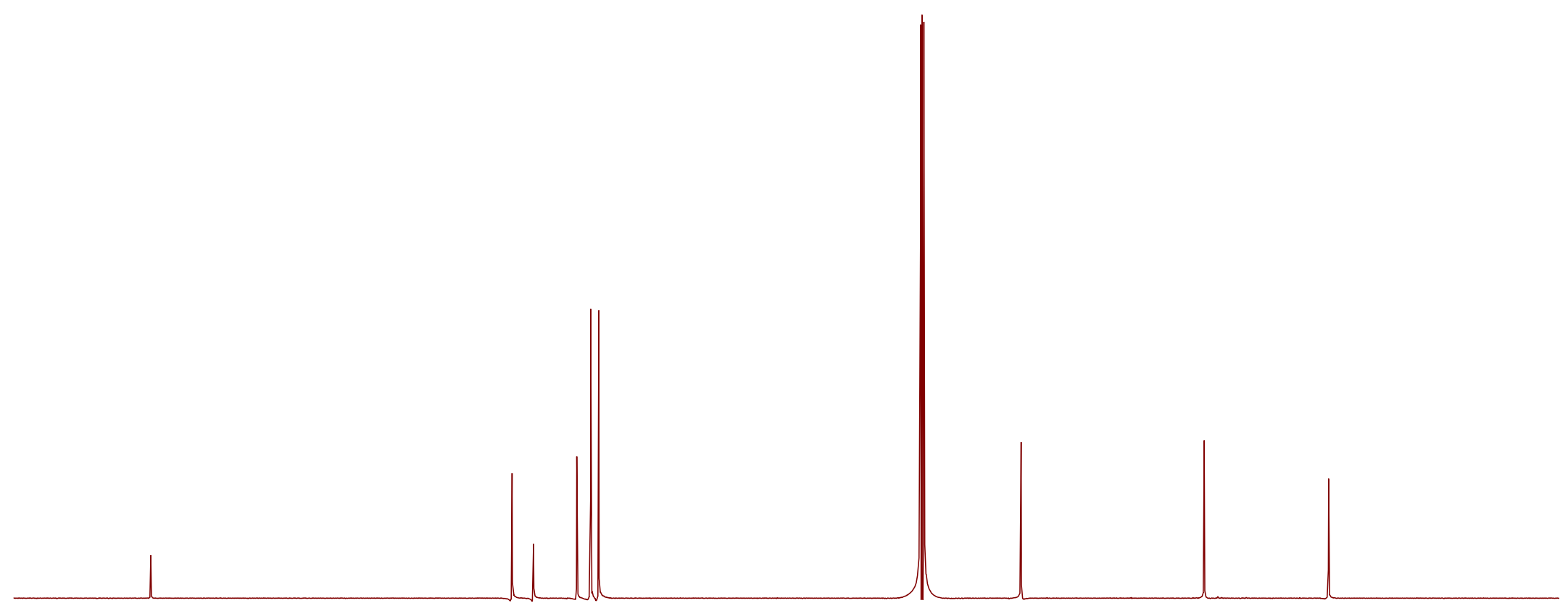

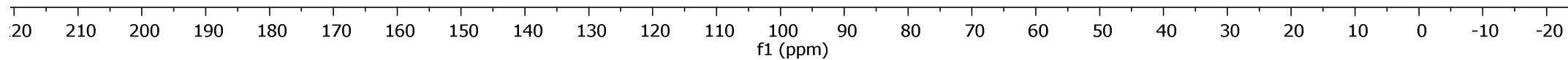




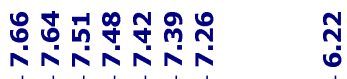

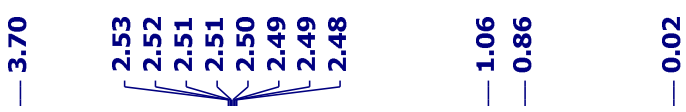

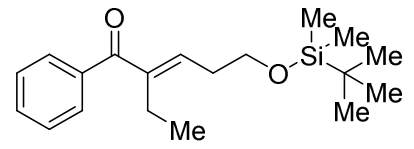

${ }^{1} \mathrm{H} \mathrm{NMR}\left(500 \mathrm{MHz}, \mathrm{CDCl}_{3}\right)$

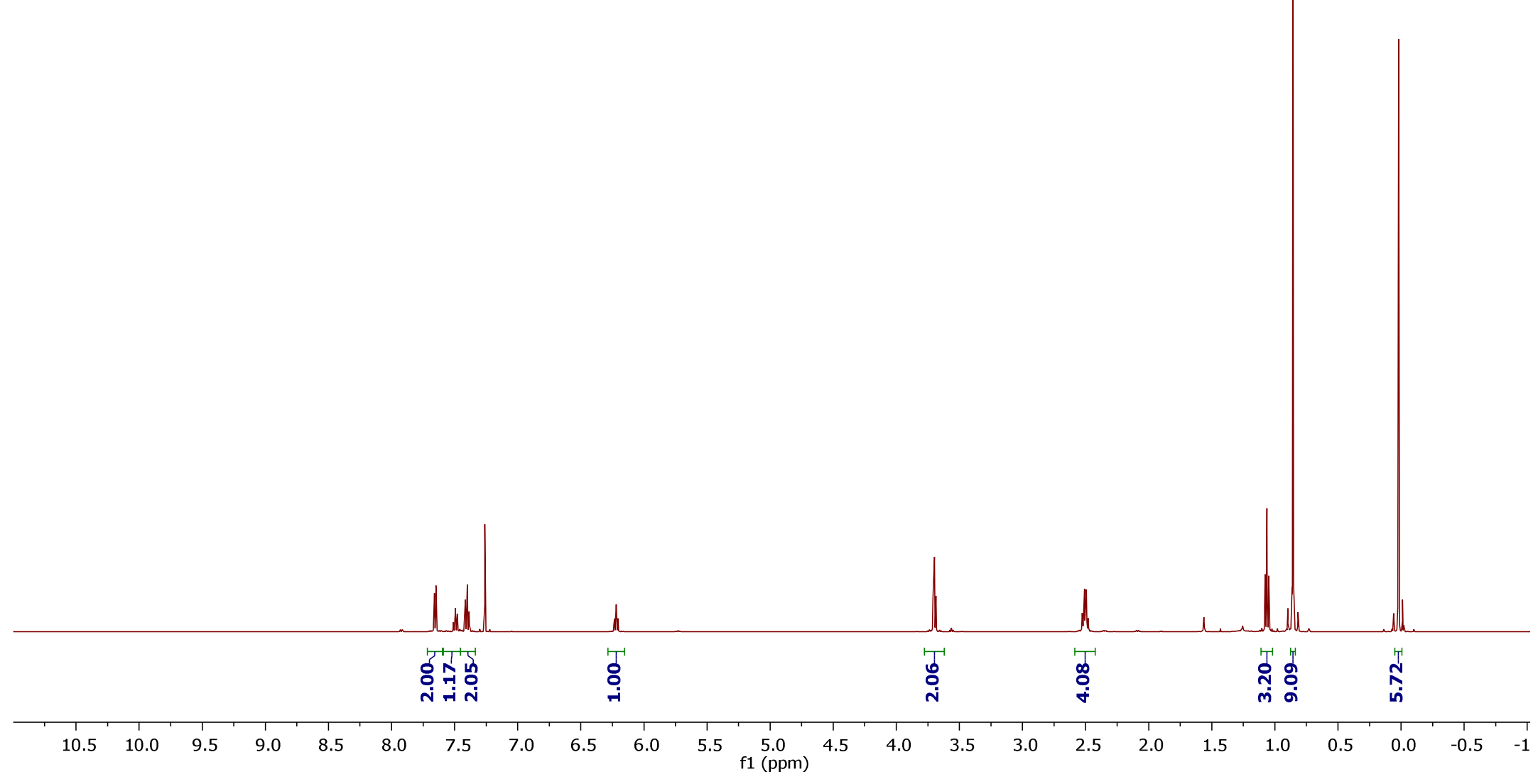




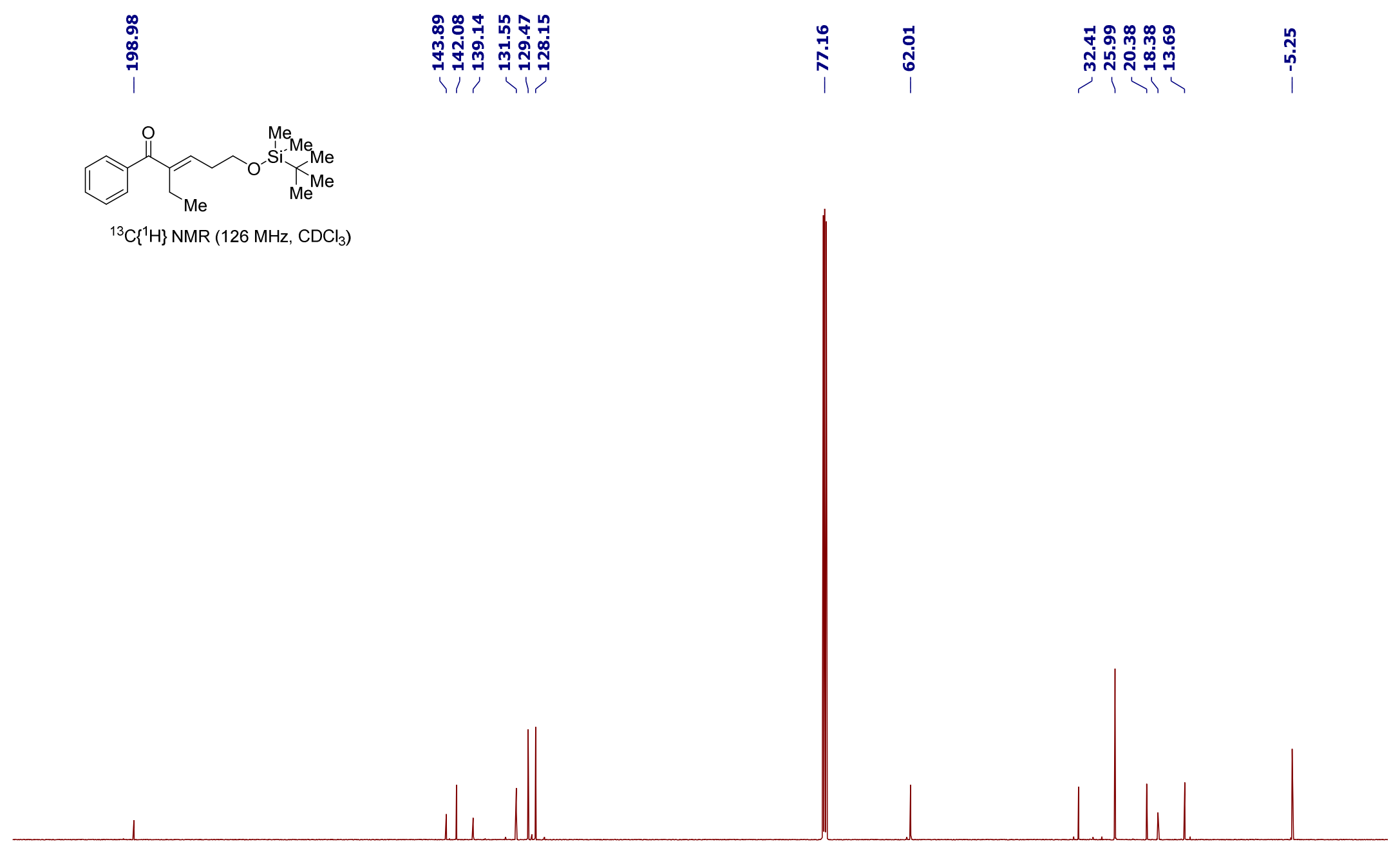

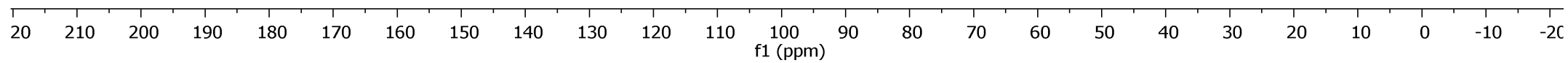




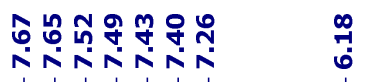

空

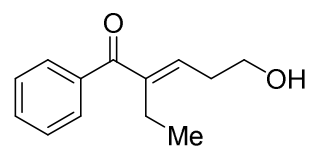

4h

${ }^{1} \mathrm{H} \mathrm{NMR} \mathrm{(500} \mathrm{MHz,} \mathrm{CDCl}_{3}$ )

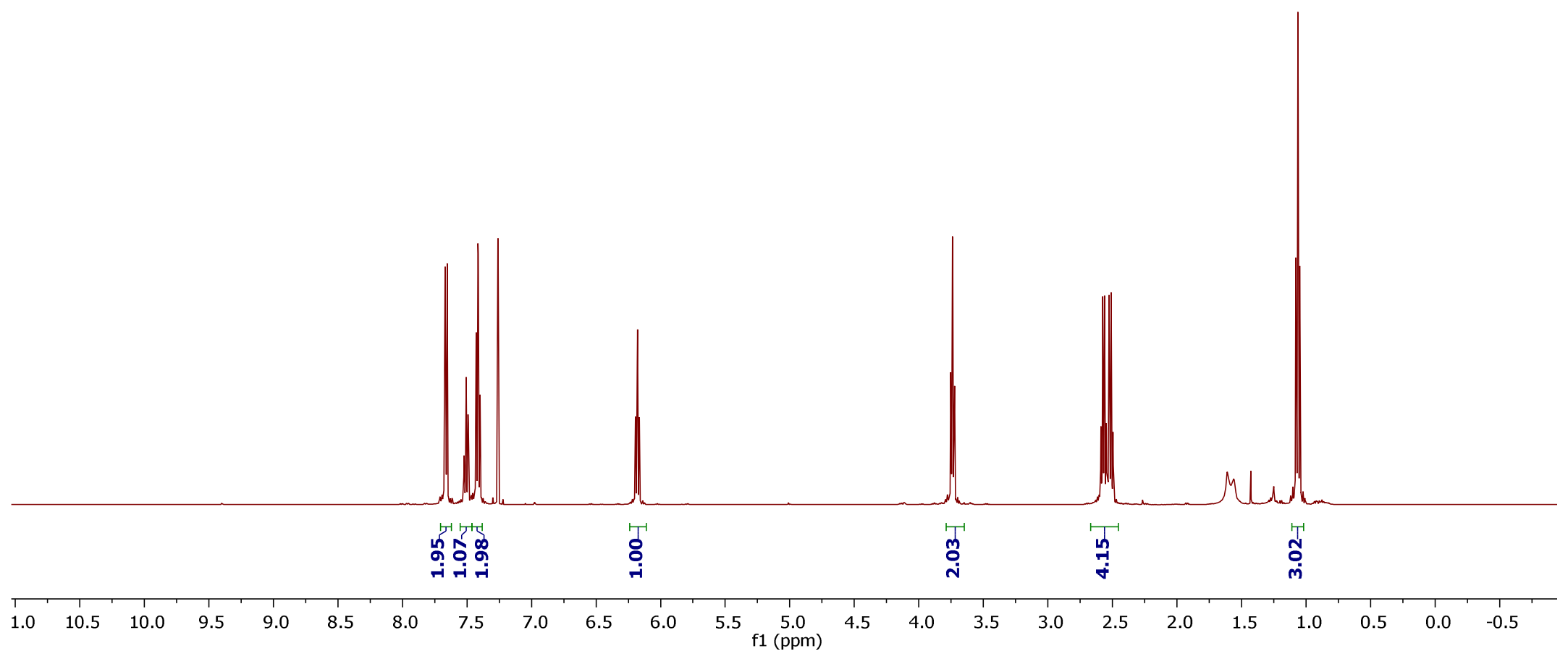



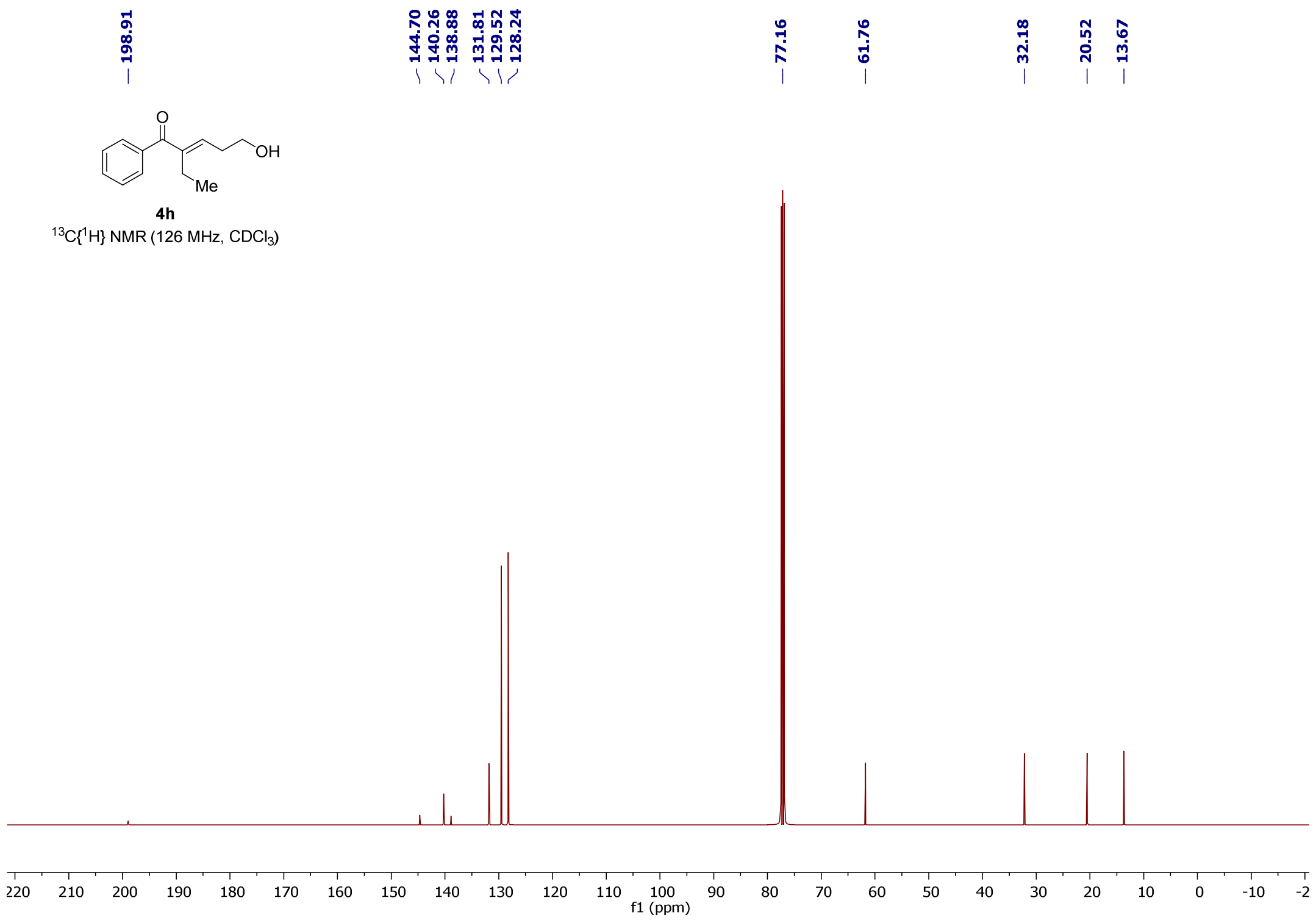
|

总

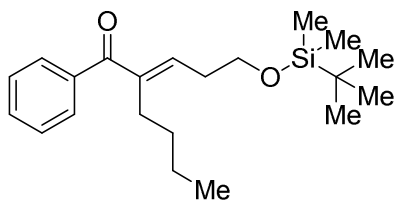

${ }^{1} \mathrm{H} \mathrm{NMR}\left(500 \mathrm{MHz}, \mathrm{CDCl}_{3}\right)$

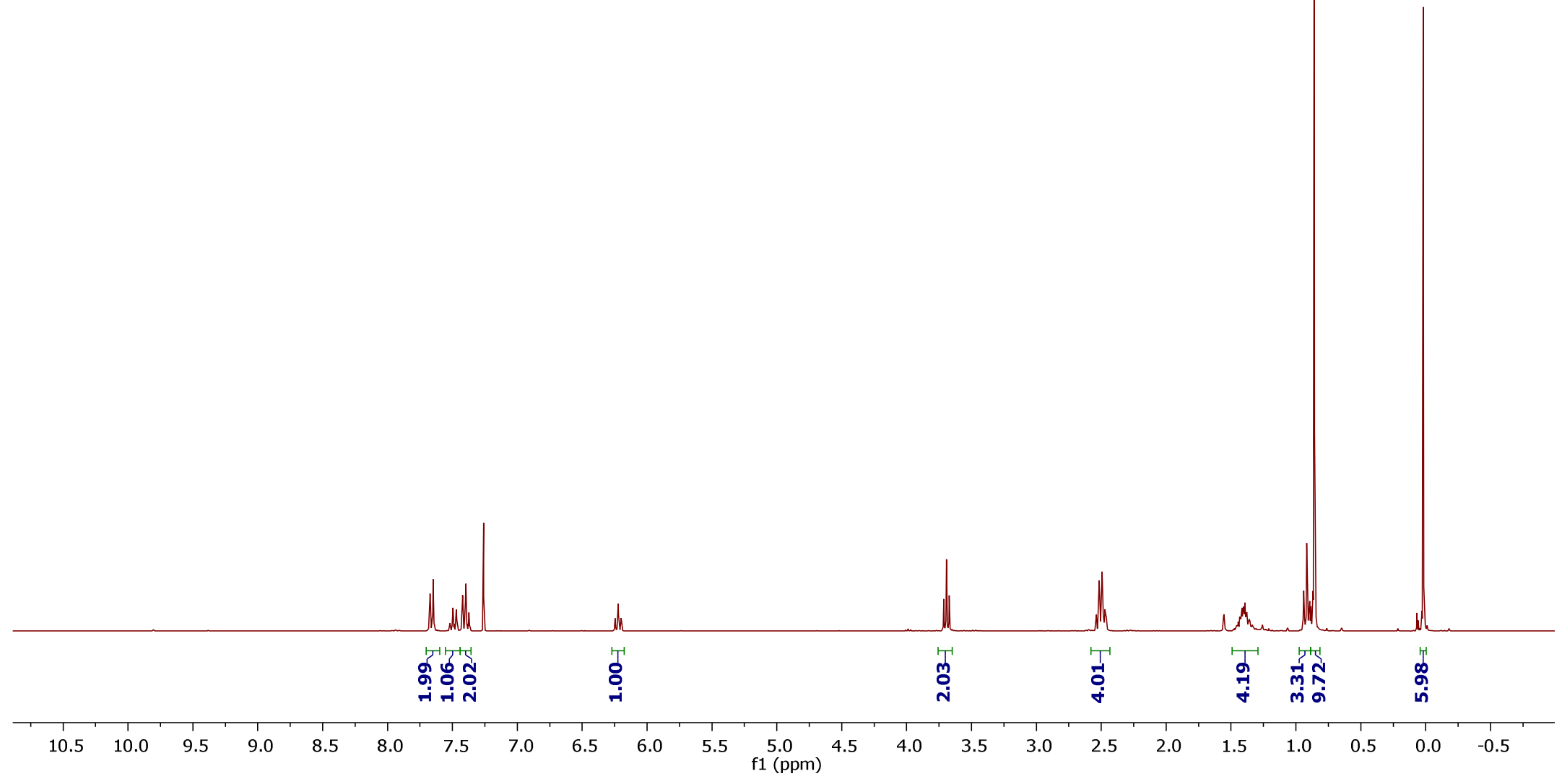




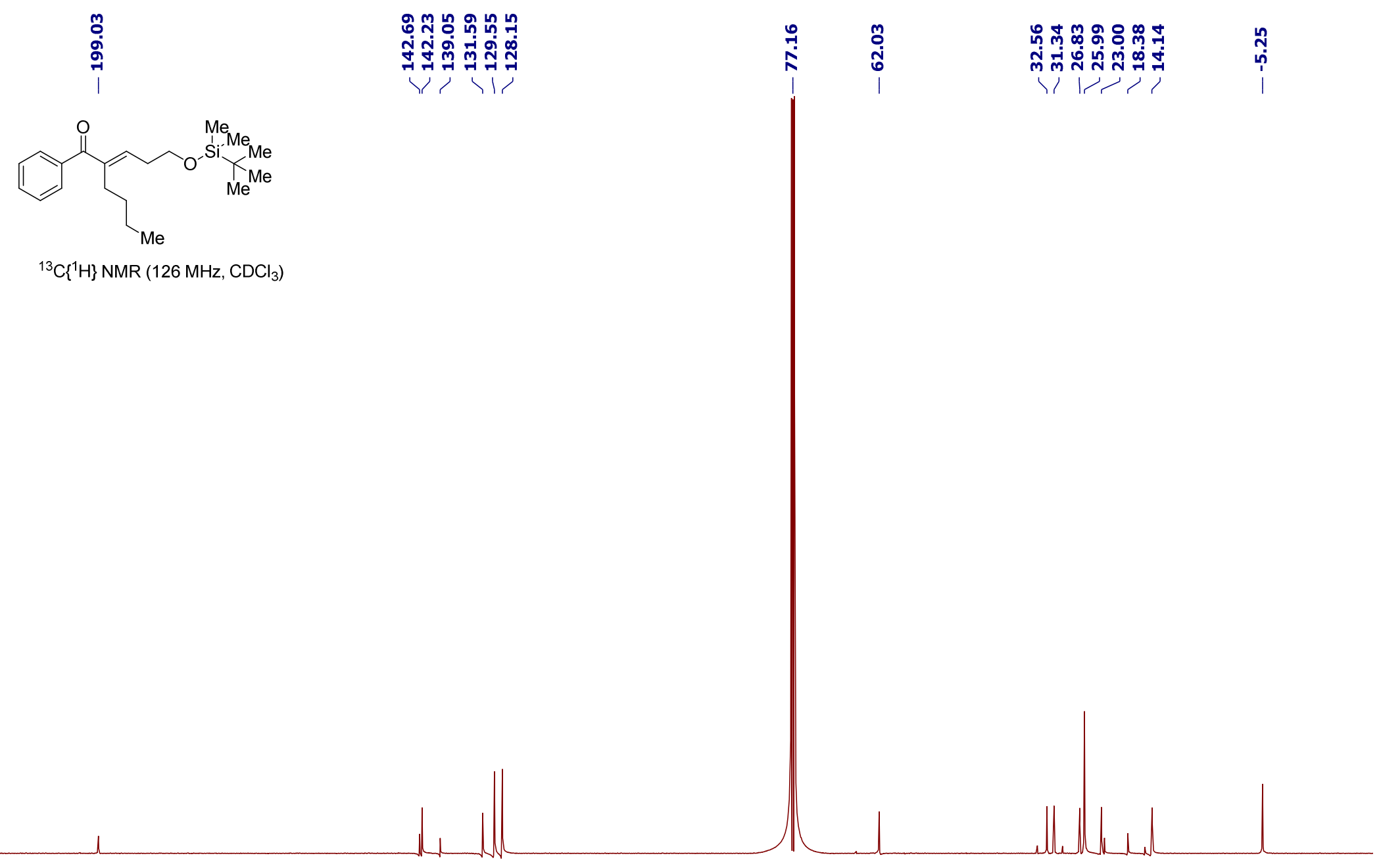

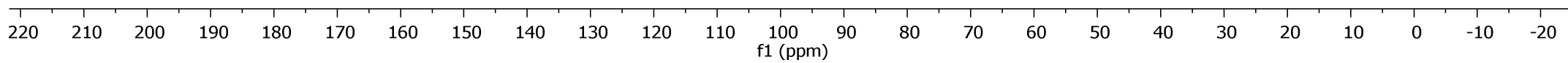




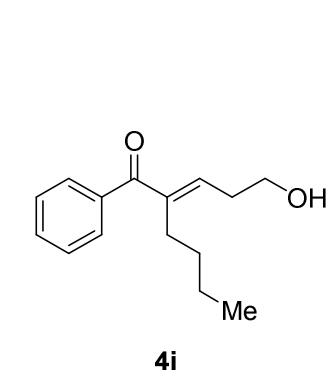

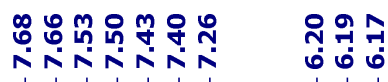

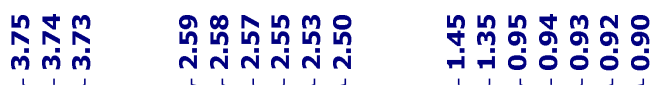

${ }^{1} \mathrm{H} \mathrm{NMR}\left(500 \mathrm{MHz}, \mathrm{CDCl}_{3}\right)$

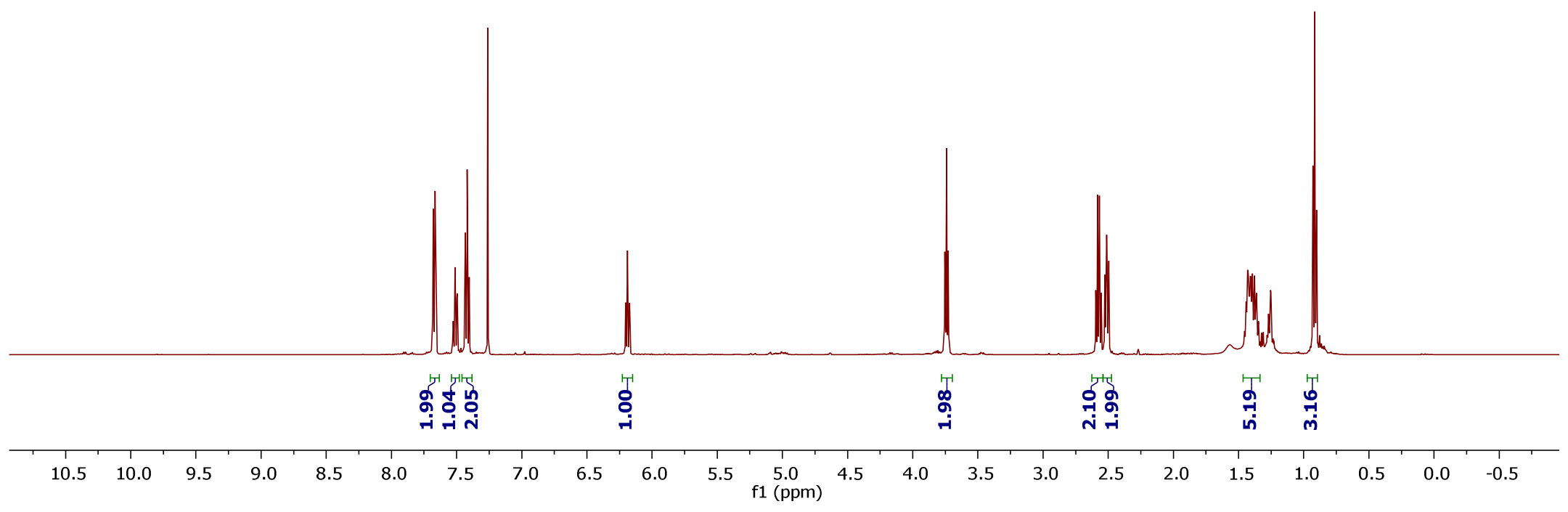




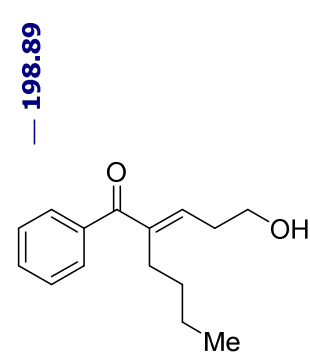

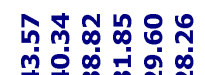

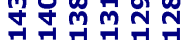

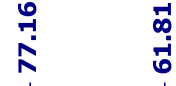

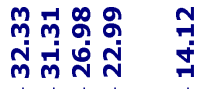

$4 \mathrm{i}$

${ }^{13} \mathrm{C}\left\{{ }^{1} \mathrm{H}\right\} \mathrm{NMR}\left(126 \mathrm{MHz}, \mathrm{CDCl}_{3}\right)$

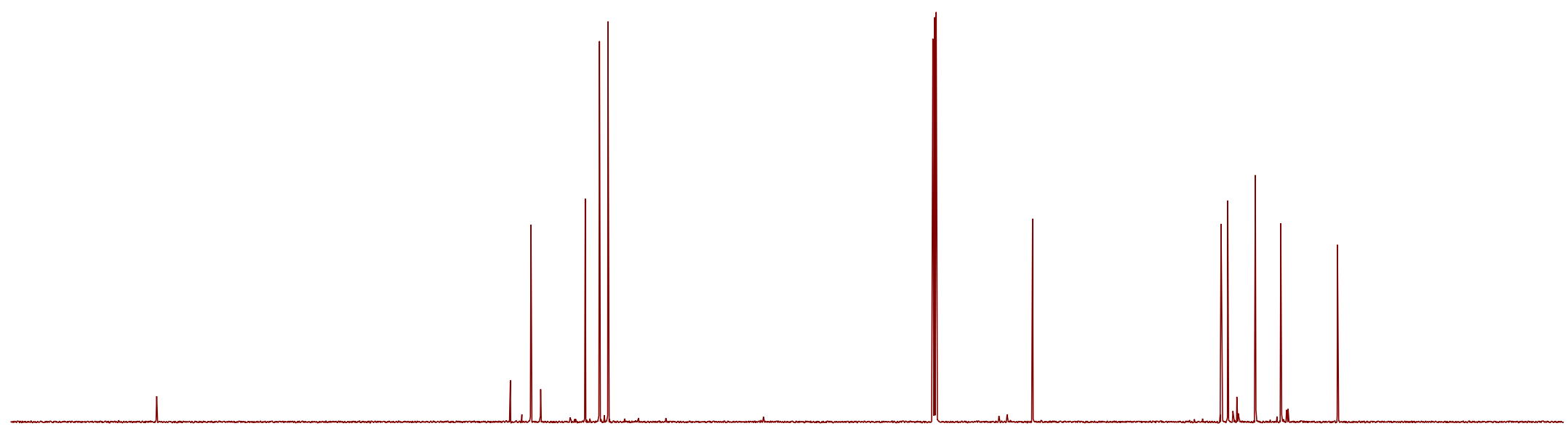

$\begin{array}{rllllllllllllllllllllllllllllll}220 & 210 & 200 & 190 & 180 & 170 & 160 & 150 & 140 & 130 & 120 & 110 & 100 & 90 & 80 & 70 & 60 & 50 & 40 & 30 & 20 & 10 & 0 & -10 & -2\end{array}$ 


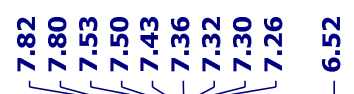

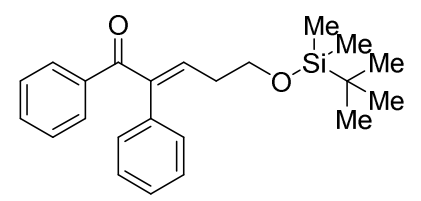

${ }^{1} \mathrm{H} \mathrm{NMR}\left(400 \mathrm{MHz}, \mathrm{CDCl}_{3}\right)$

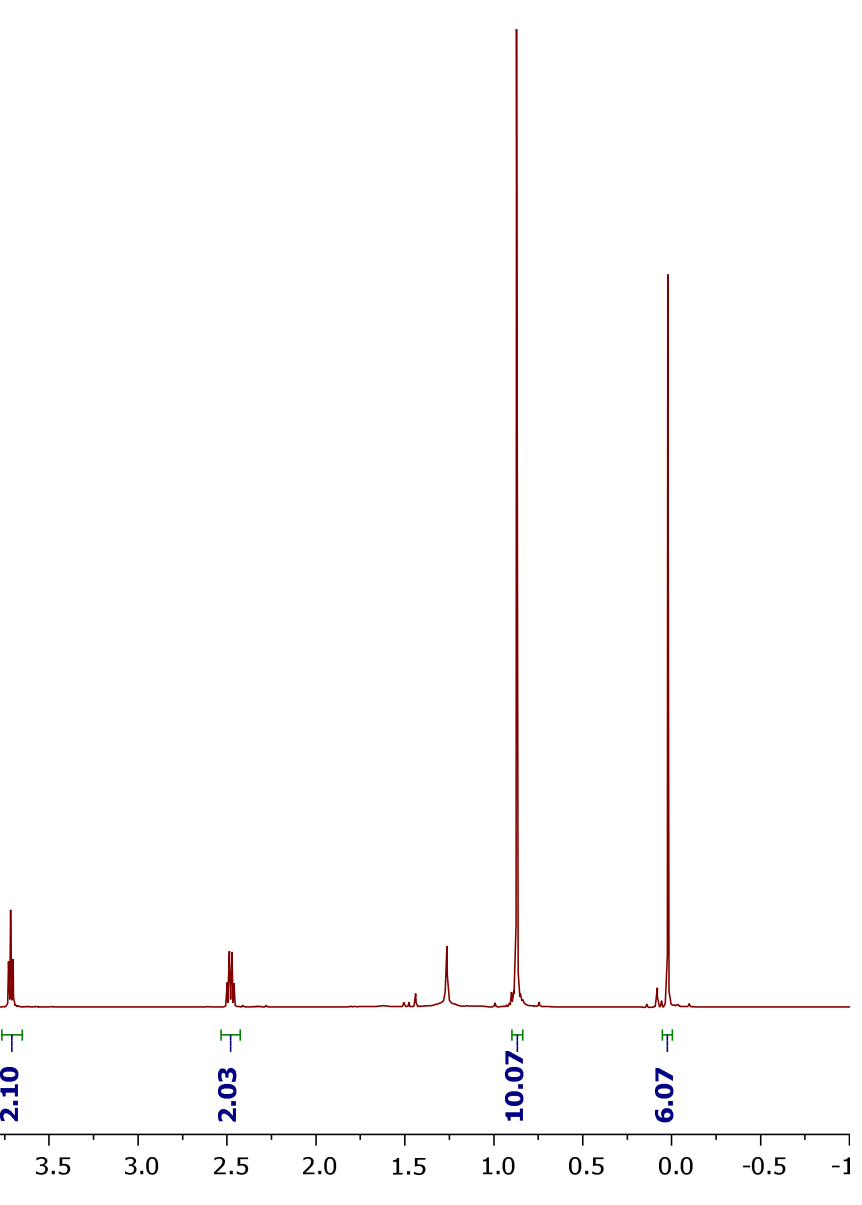




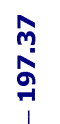

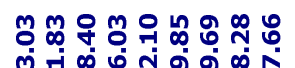

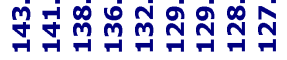

$\begin{array}{ll}\stackrel{0}{i} & \hat{d} \\ i & 0\end{array}$

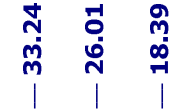

$\underset{\substack{\text { ஸे } \\ i}}{i}$

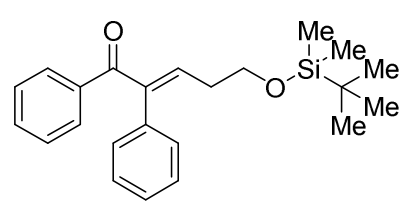

${ }^{13} \mathrm{C}\left\{{ }^{1} \mathrm{H}\right\}$ NMR $\left(126 \mathrm{MHz}, \mathrm{CDCl}_{3}\right)$

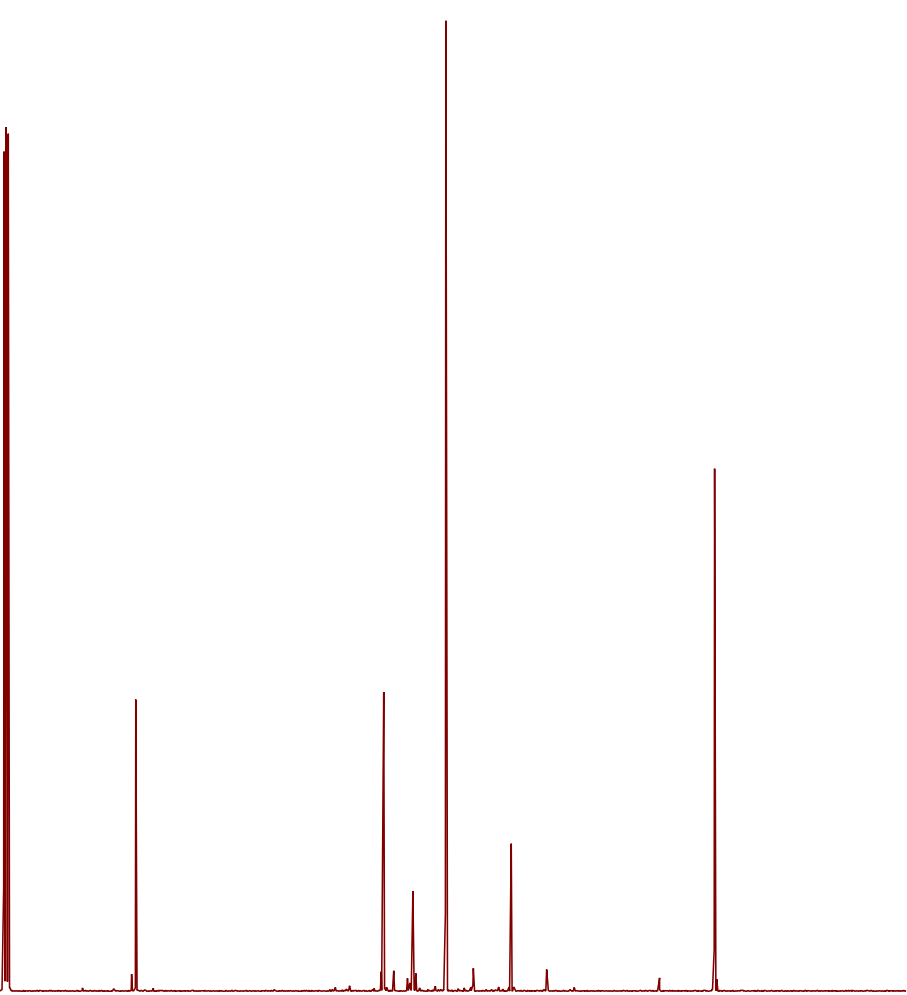

$20 \quad 210 \quad 200$

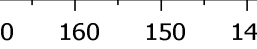




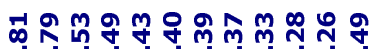

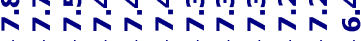

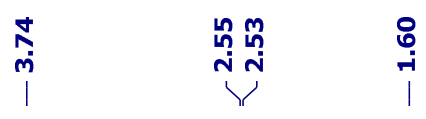

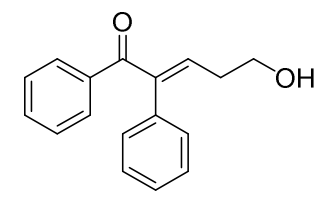

${ }^{1} \mathrm{HNMR}\left(500 \mathrm{MHz}, \mathrm{CDCl}_{3}\right)$

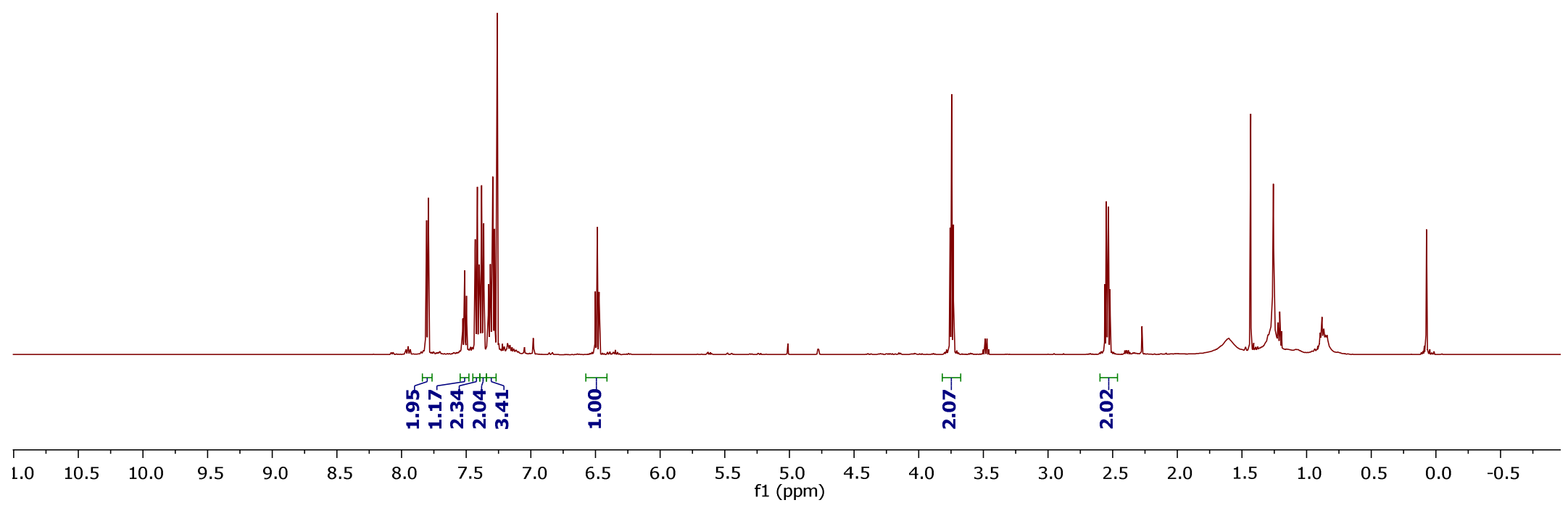



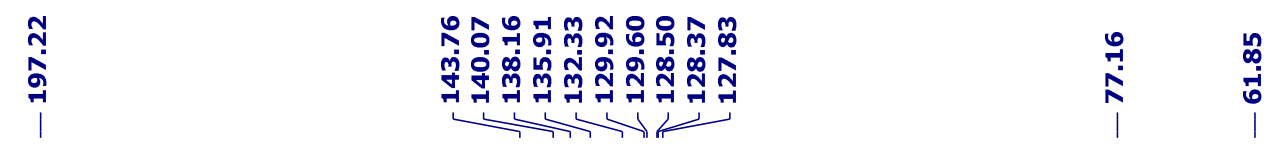

$\stackrel{\stackrel{\circ}{\dot{j}}}{m}$
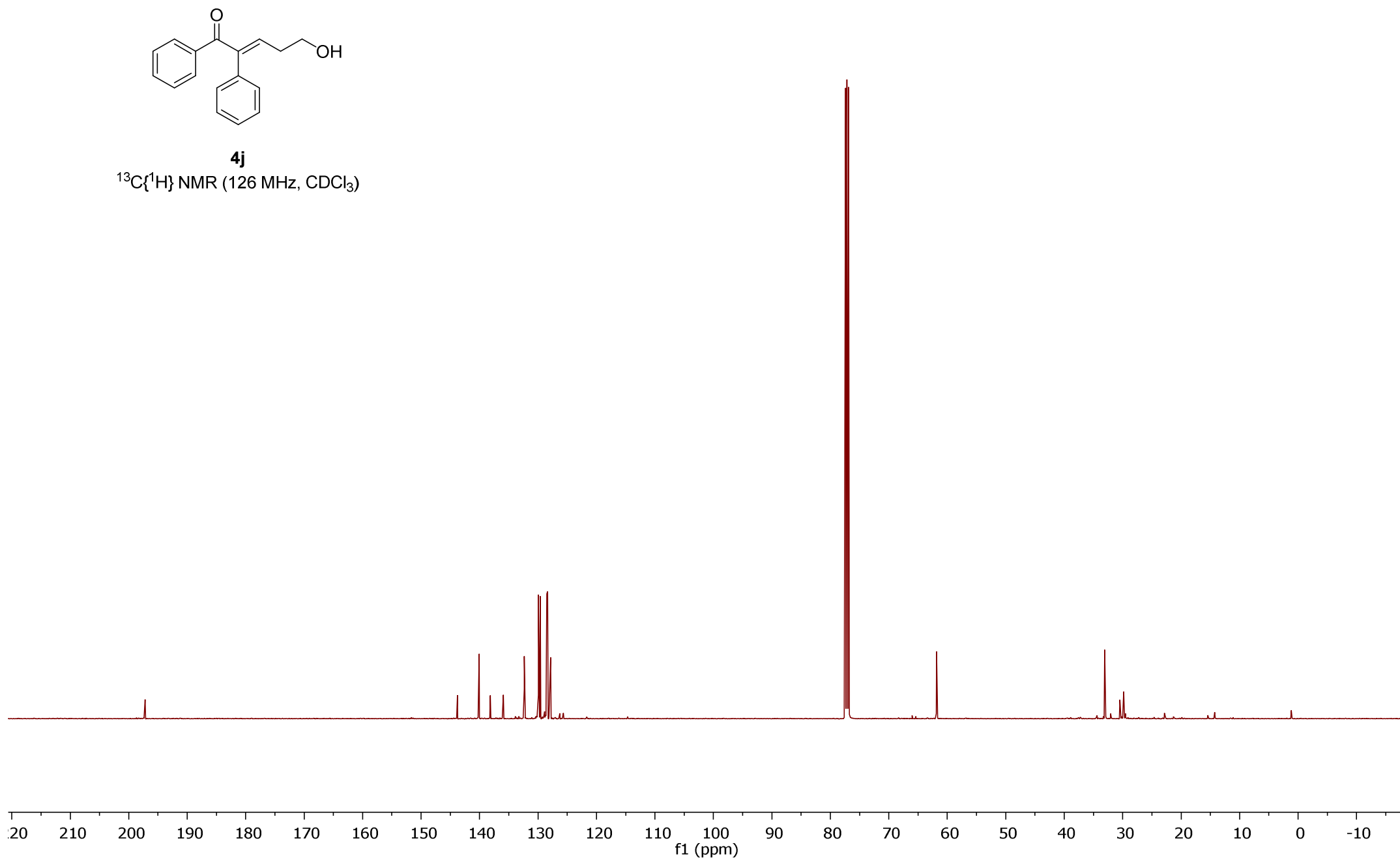


旁

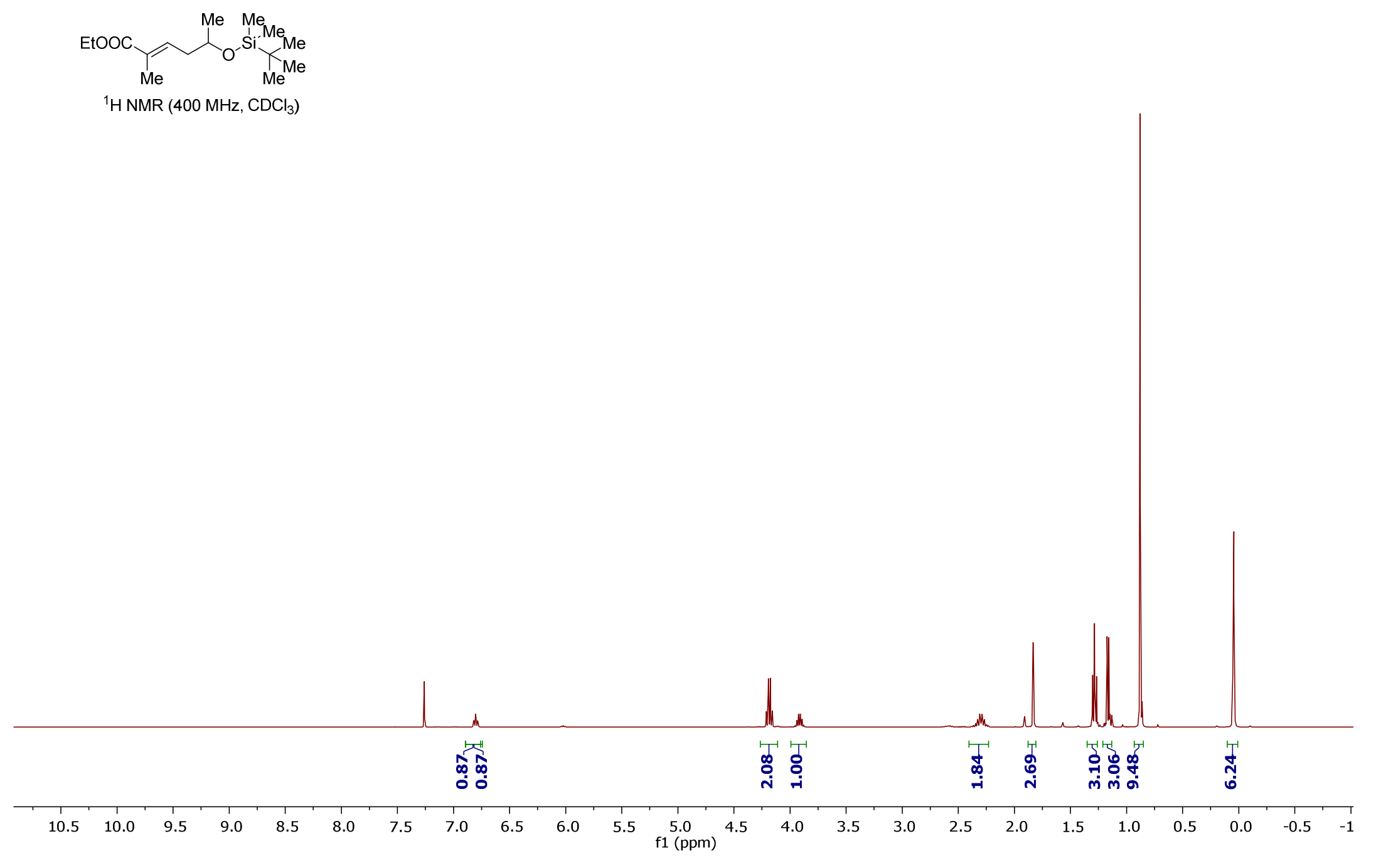




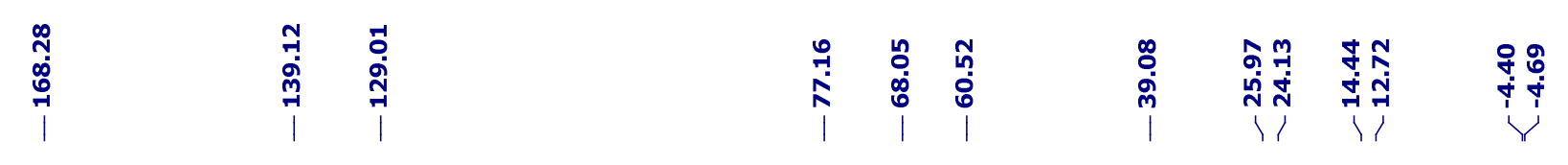

$$
{ }^{13} \mathrm{C}\{\mathrm{H}\} \mathrm{NMR}(101 \mathrm{MHz}, \mathrm{CDCl})
$$

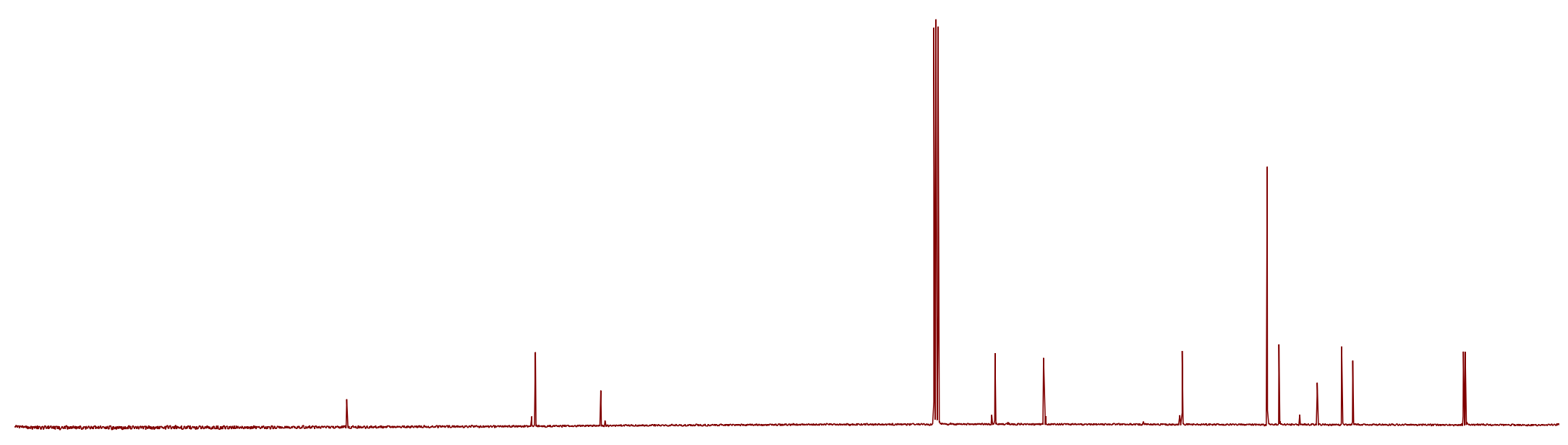

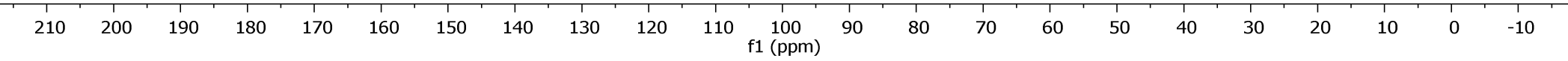




$$
\text { EtoOC }
$$

${ }^{1} \mathrm{HNMR}\left(400 \mathrm{MHz}, \mathrm{CDCl}_{3}\right)$

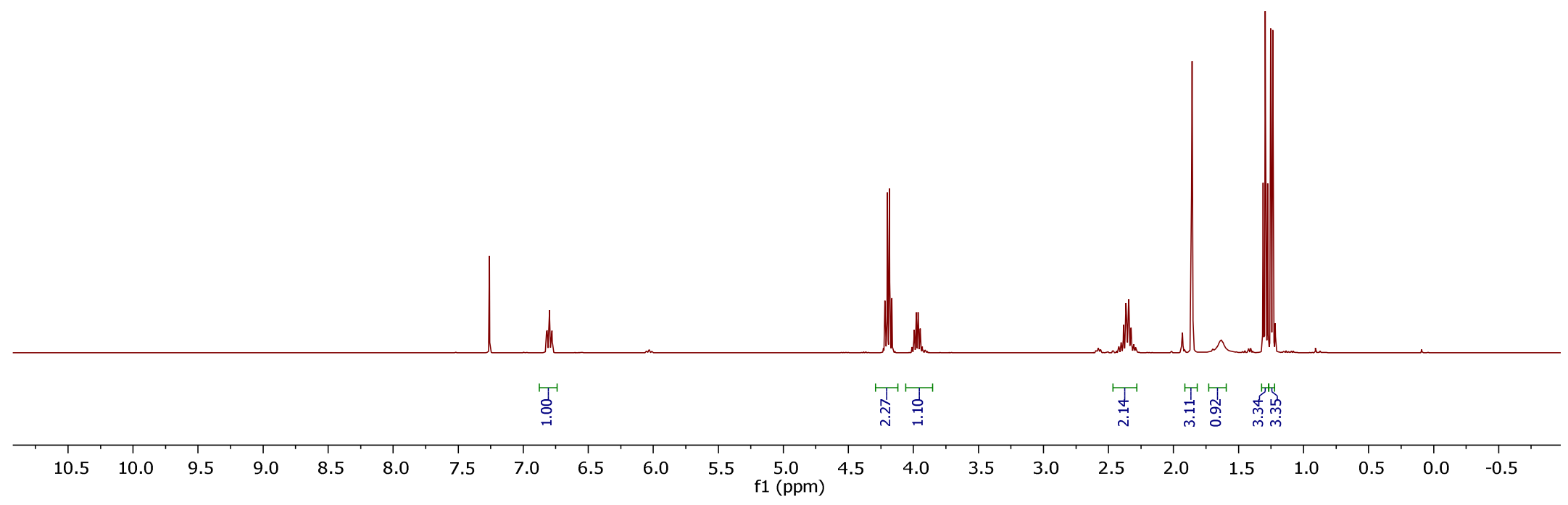




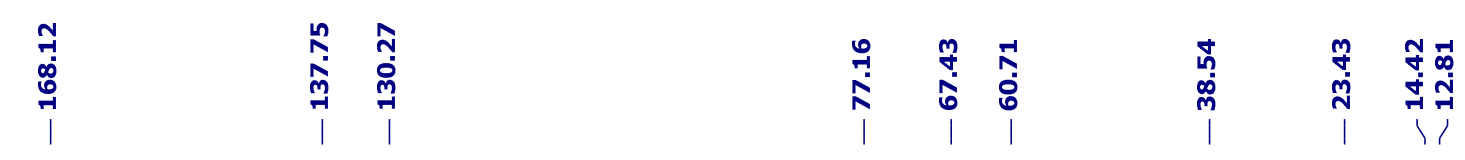

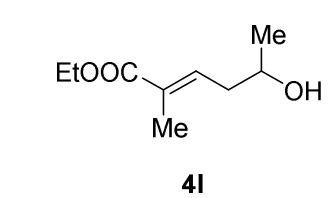

${ }^{13} \mathrm{C}\left\{{ }^{1} \mathrm{H}\right\} \mathrm{NMR}\left(101 \mathrm{MHz}, \mathrm{CDCl}_{3}\right)$

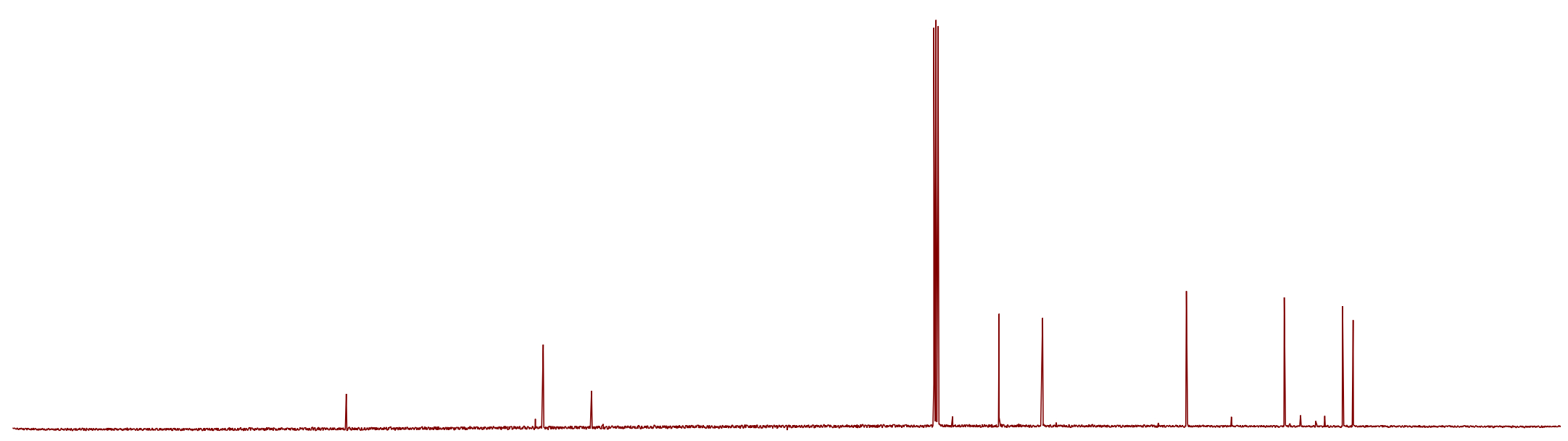

$\begin{array}{llllllllllllllllllllllllll}210 & 200 & 190 & 180 & 170 & 160 & 150 & 140 & 130 & 120 & 110 & 100 & 90 & 80 & 70 & 60 & 50 & 40 & 30 & 20 & 10 & 0 & -10\end{array}$ 

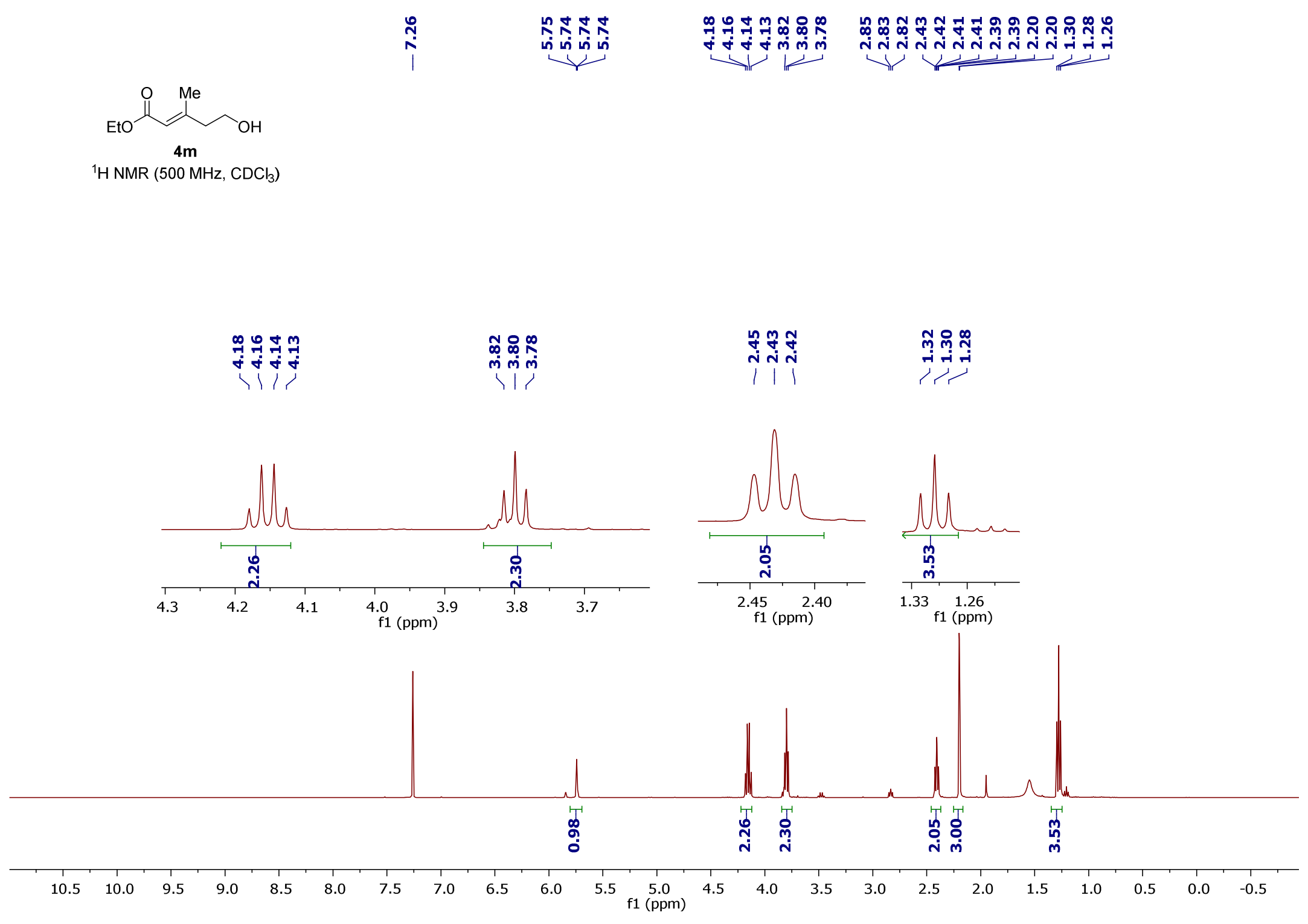


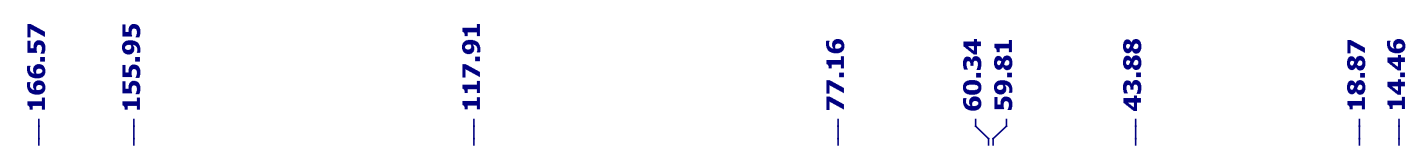

$$
\text { Eto } \stackrel{\mathrm{O}}{\mathrm{Me}} \mathrm{N}_{\mathrm{OH}}
$$

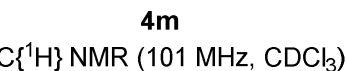
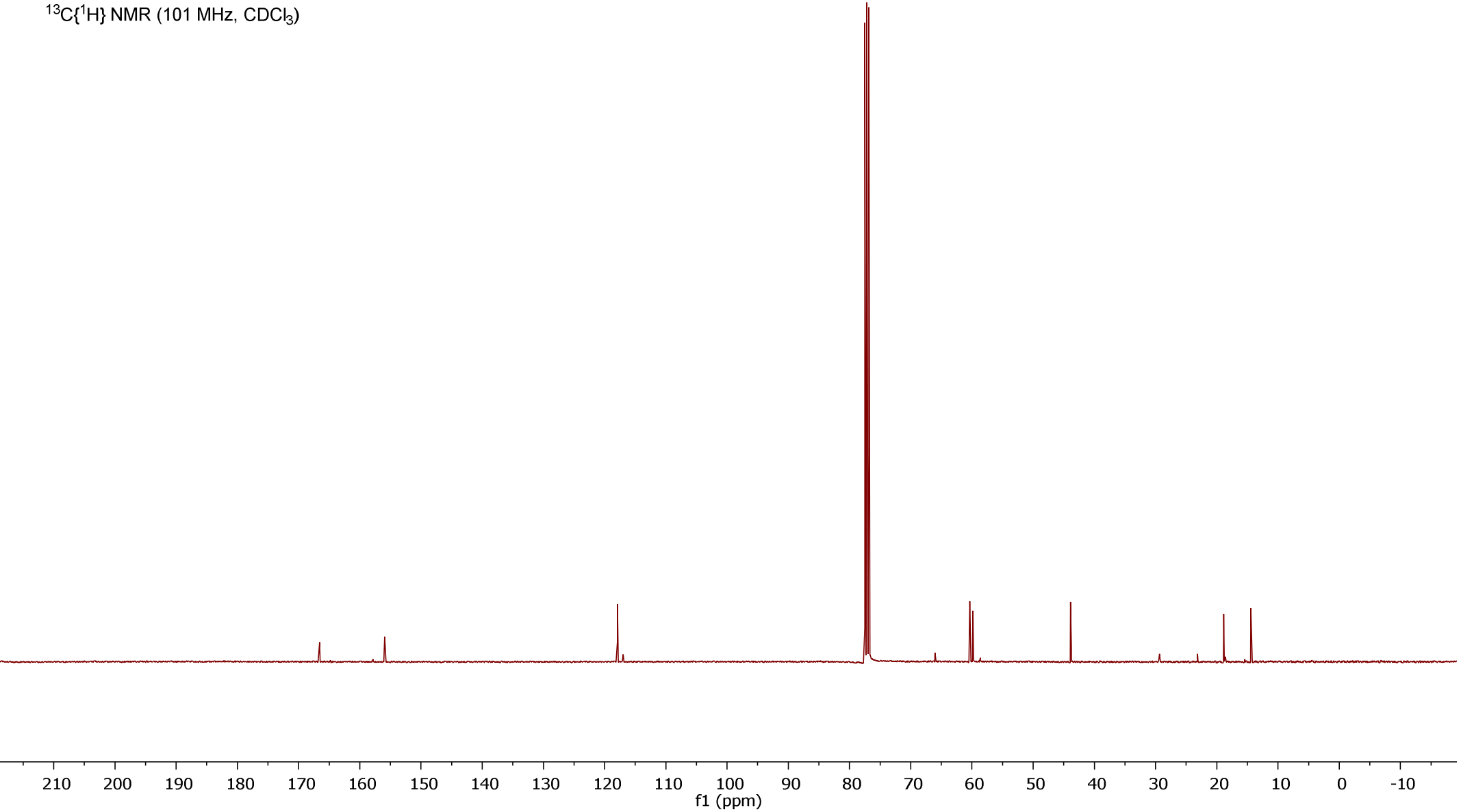


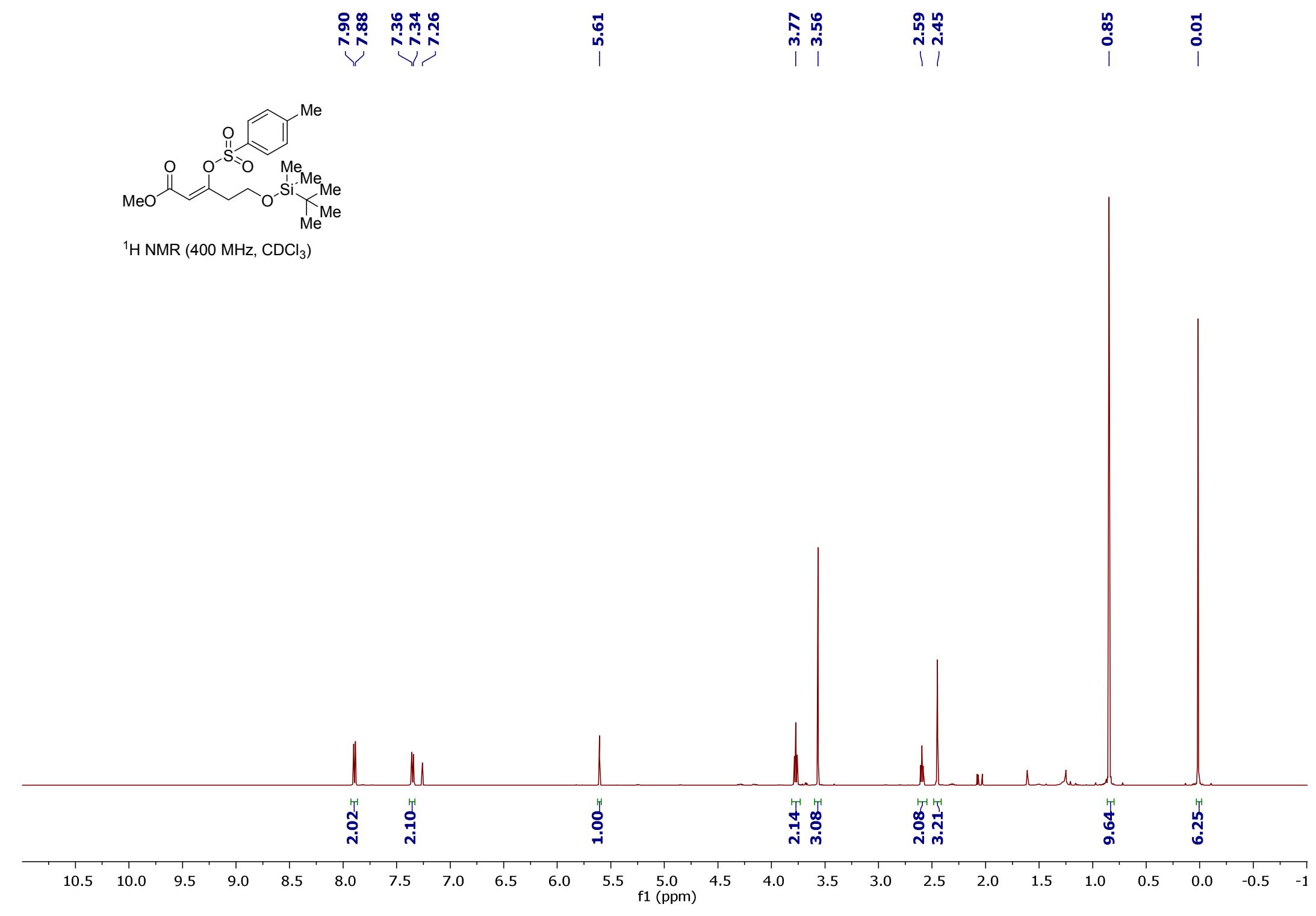




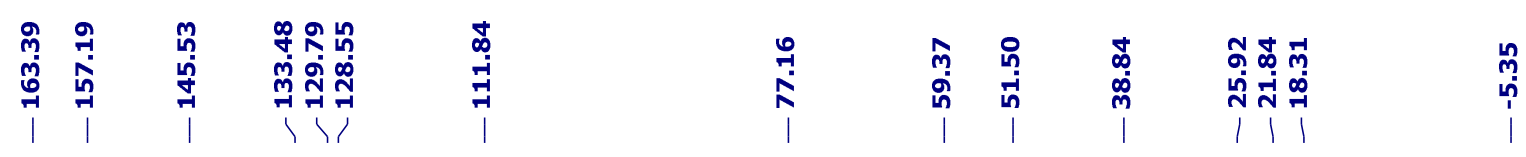

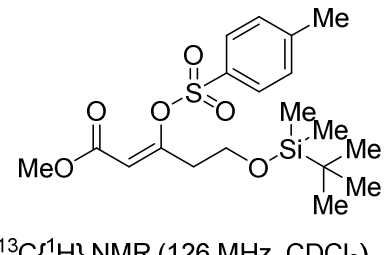

$\left.{ }^{13} \mathrm{C}^{1} \mathrm{H}\right\} \mathrm{NMR}\left(126 \mathrm{MHz}, \mathrm{CDCl}_{3}\right)$

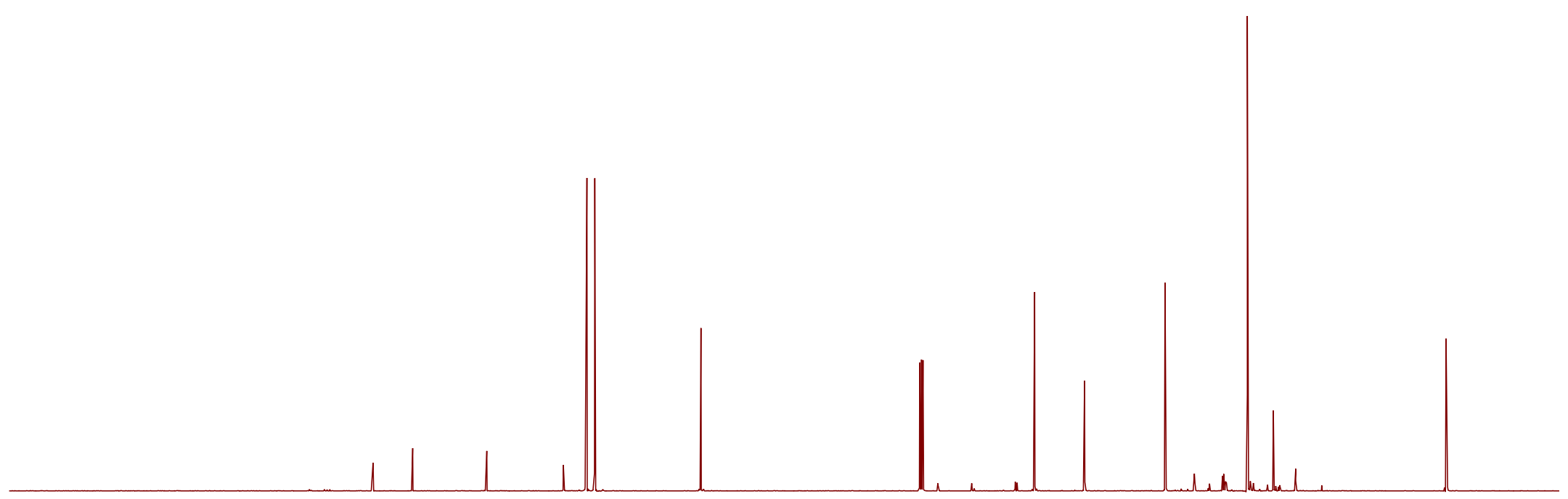

$\begin{array}{lllllllllllllllllllllllllllllll}1 & 20 & 210 & 200 & 190 & 180 & 170 & 160 & 150 & 140 & 130 & 120 & 110 & \begin{array}{c}100 \\ \mathrm{f} 1(\mathrm{ppm})\end{array} & 90 & 80 & 70 & 60 & 50 & 40 & 30 & 20 & 10 & 0 & -10 & -20\end{array}$ 


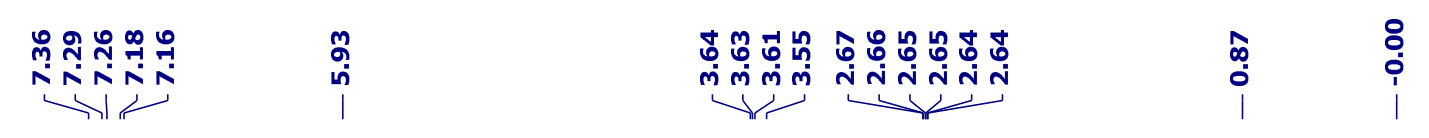
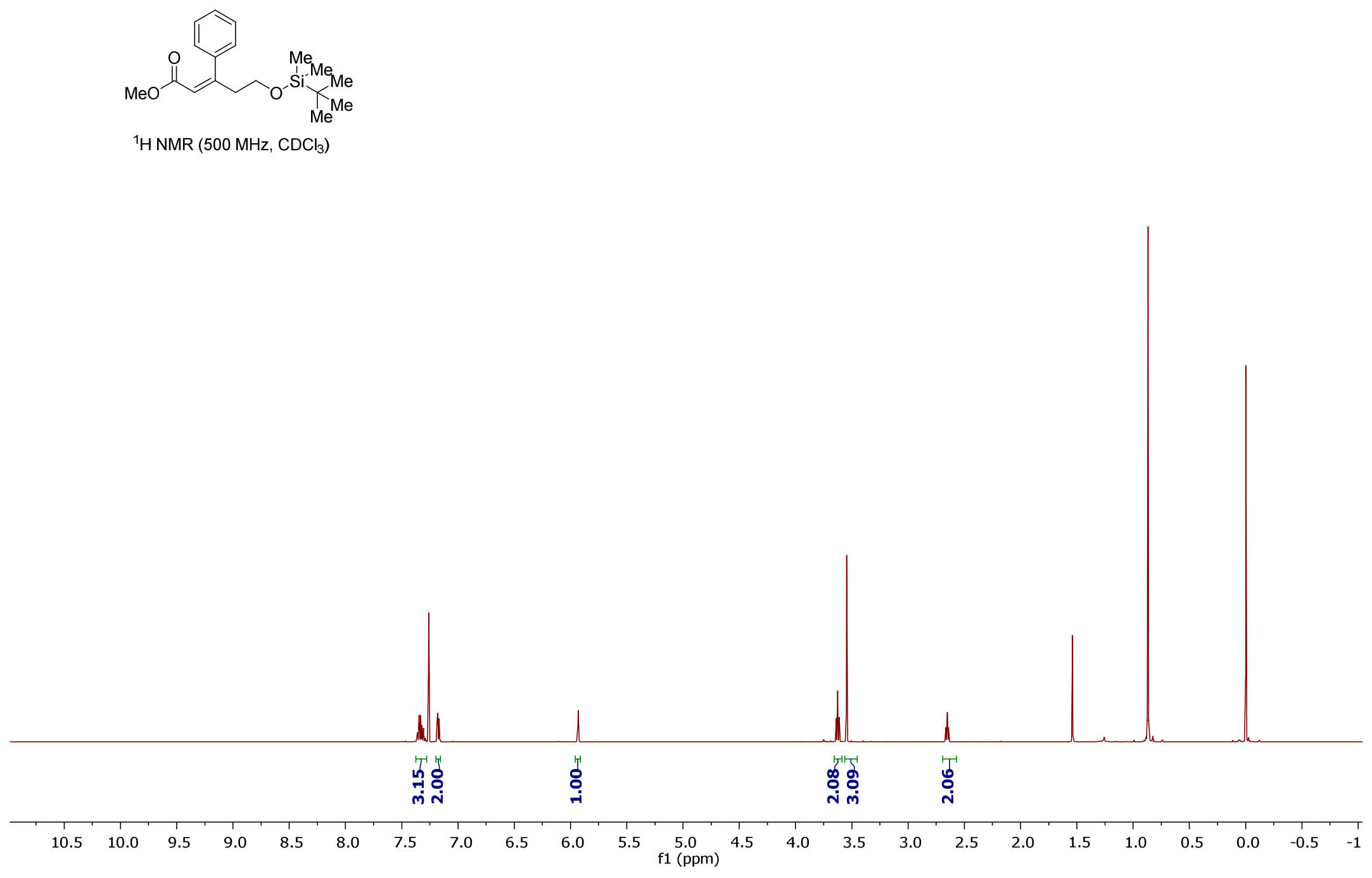

I I I

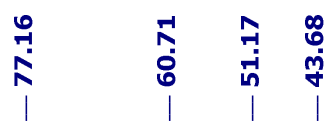
$\begin{array}{cc}-1 & \hat{m} \\ \dot{0} & \infty \\ \dot{0} & \stackrel{\infty}{-1} \\ 1 & \mid\end{array}$
$\underset{\substack{\text { Sִ } \\ i}}{i}$

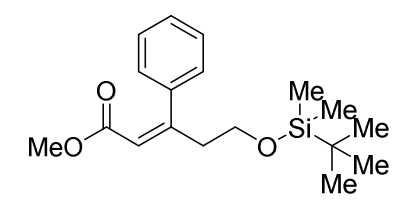

${ }^{13} \mathrm{C}\left\{{ }^{1} \mathrm{H}\right\} \mathrm{NMR}\left(126 \mathrm{MHz}, \mathrm{CDCl}_{3}\right)$

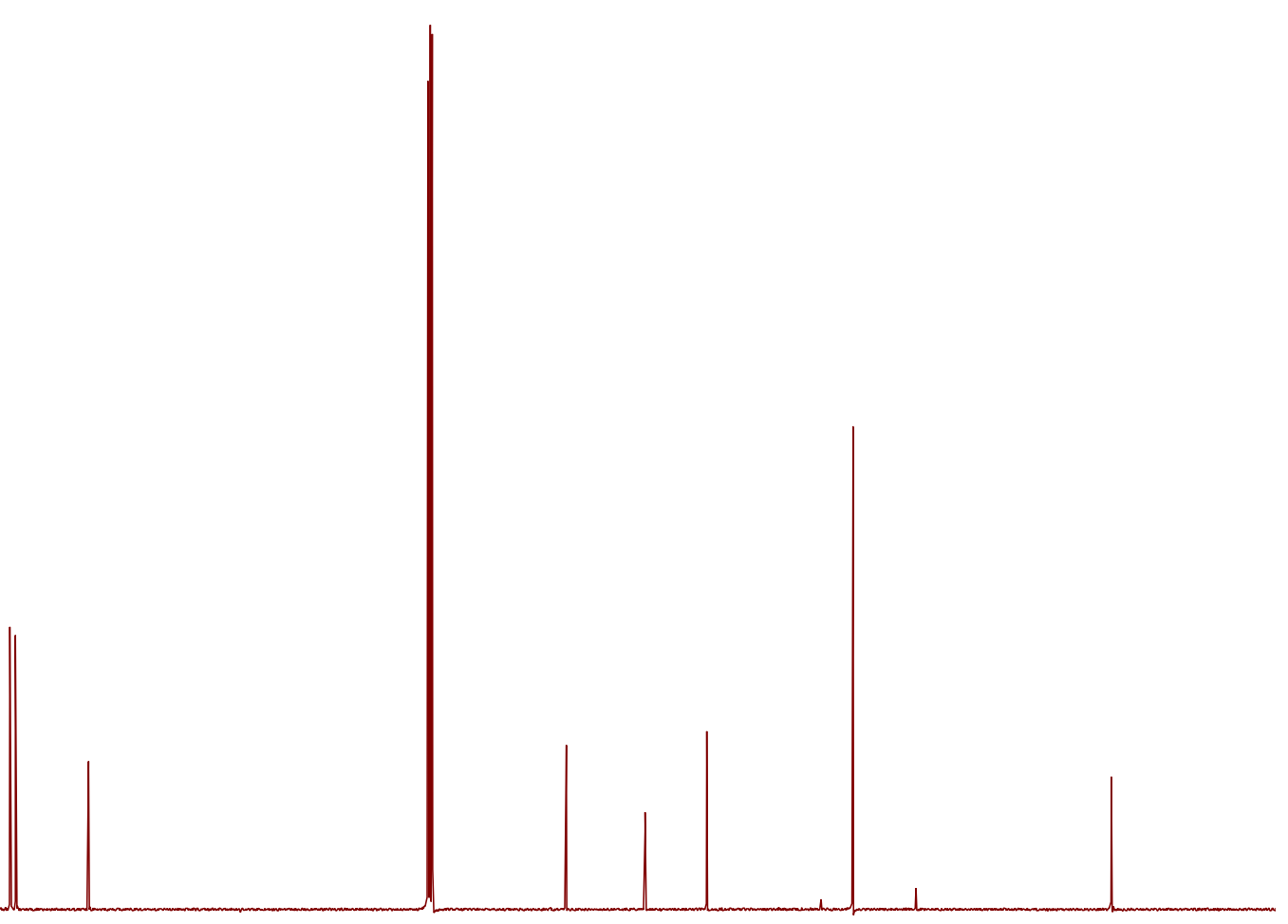

\begin{tabular}{llllllllllllllllllllllllllll}
\hline 20 & 210 & 200 & 190 & 180 & 170 & 160 & 150 & 140 & 130 & 120 & 110 & 100 & 100 & 80 & 70 & 60 & 50 & 40 & 30 & 20 & 10 & 0 & -10 & -20
\end{tabular} 


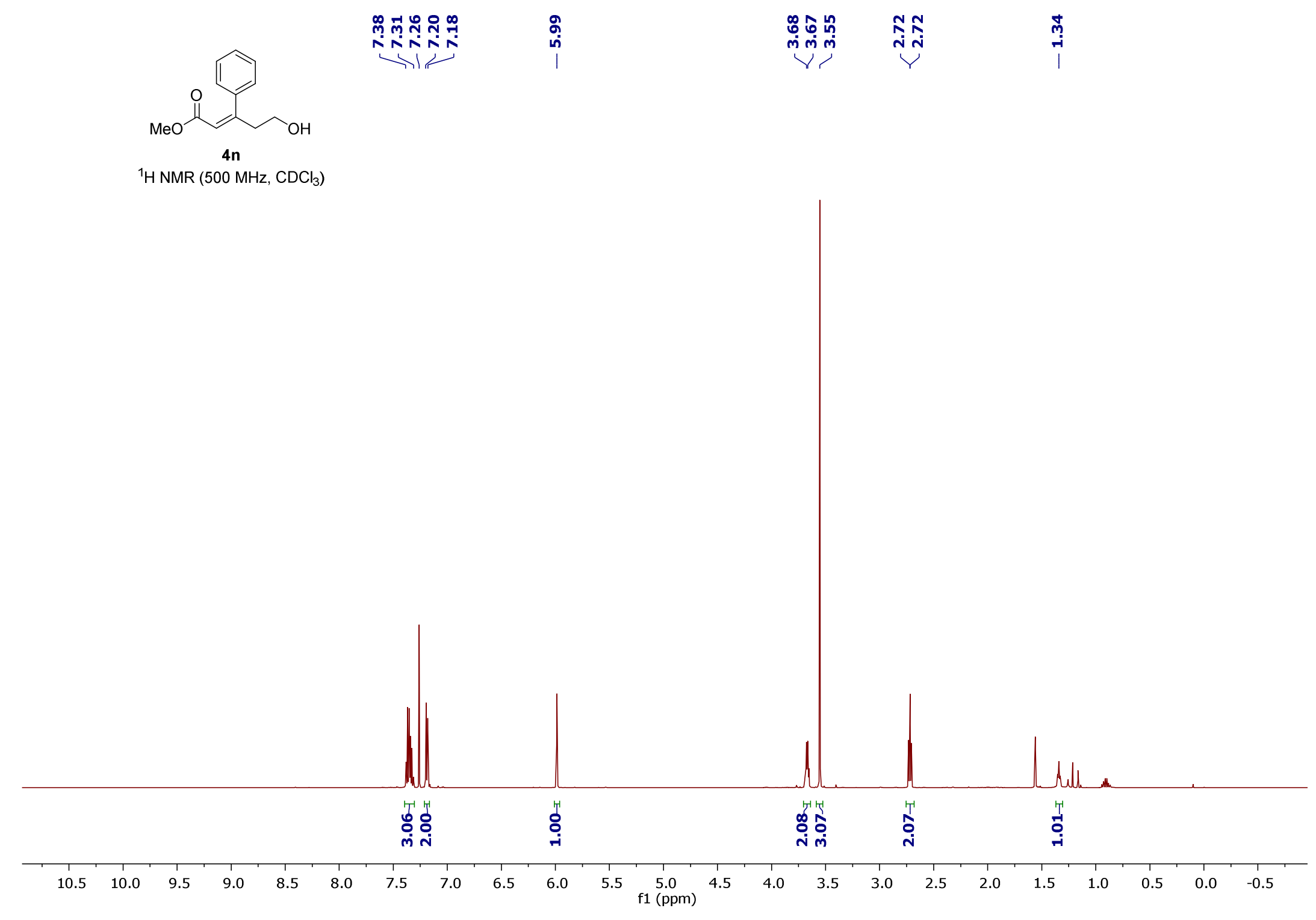




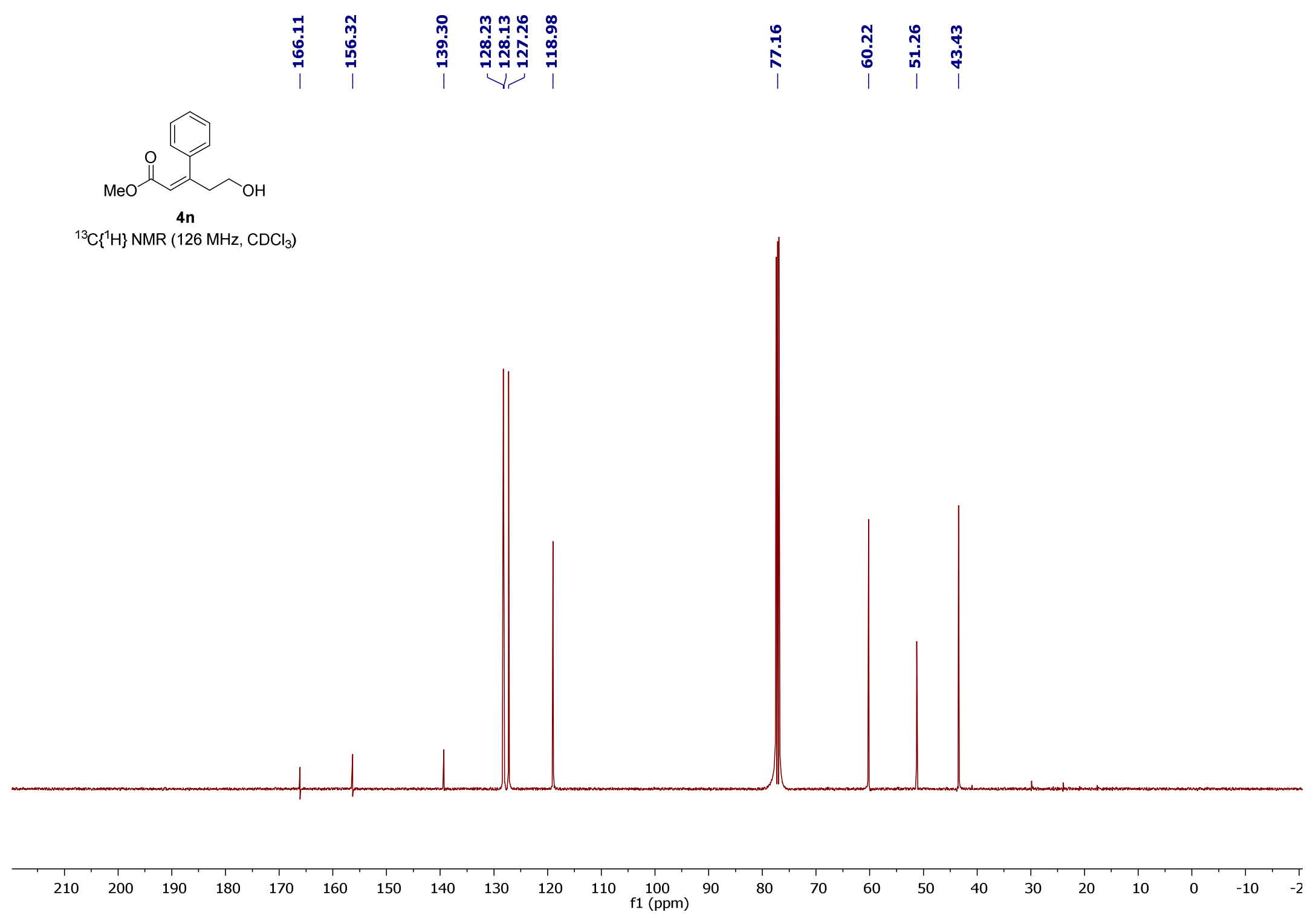



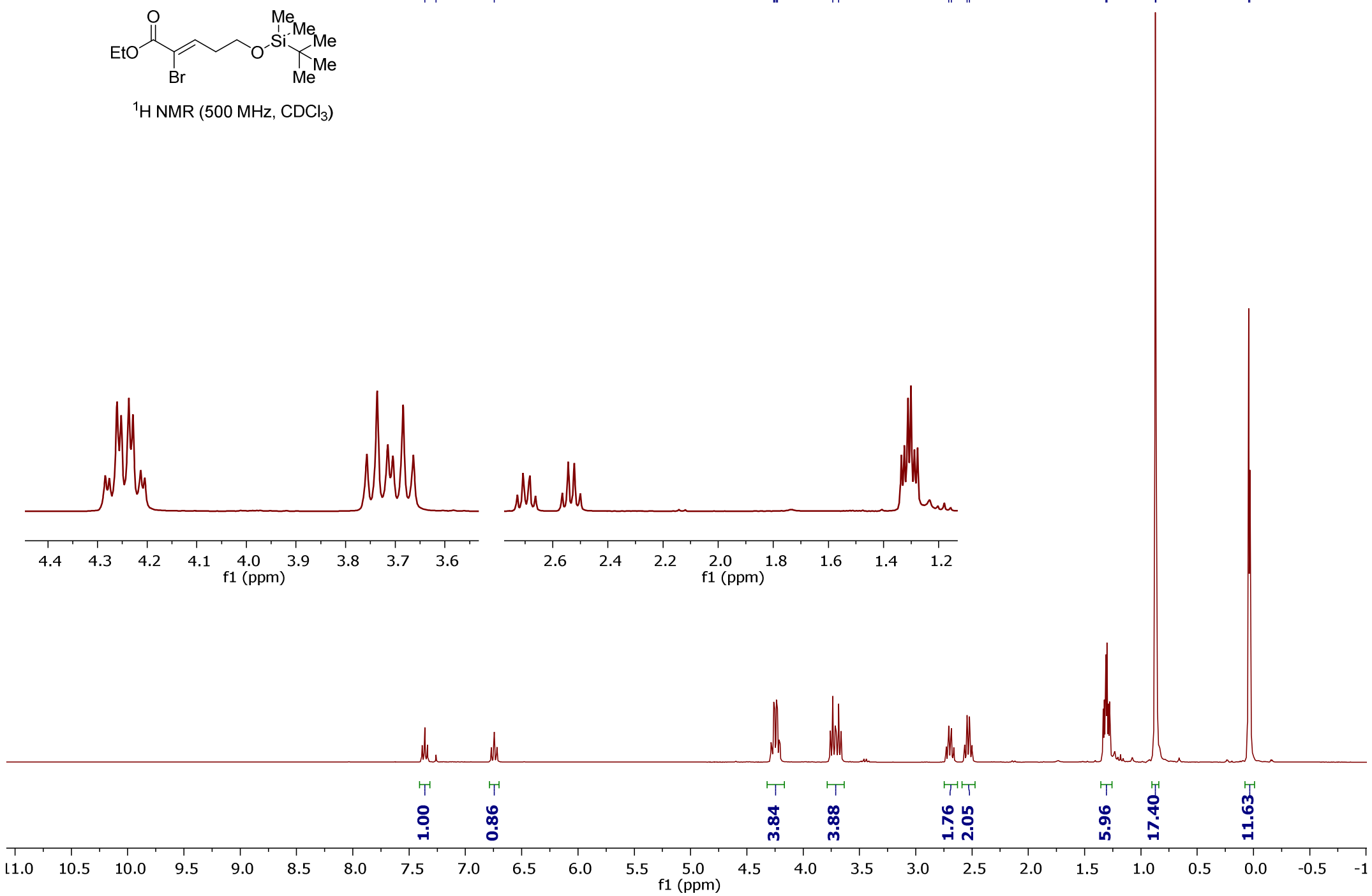
$\mathrm{Br}_{\mathrm{Br}}^{\mathrm{Me}}$

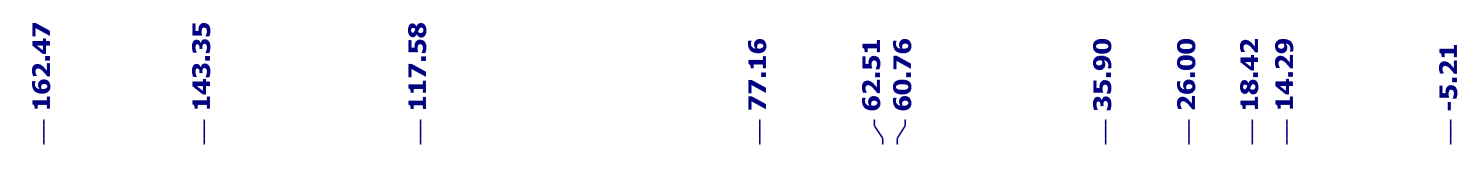

$\left.{ }^{13} \mathrm{C}^{1} \mathrm{H}\right\} \mathrm{NMR}\left(126 \mathrm{MHz}, \mathrm{CDCl}_{3}\right)$

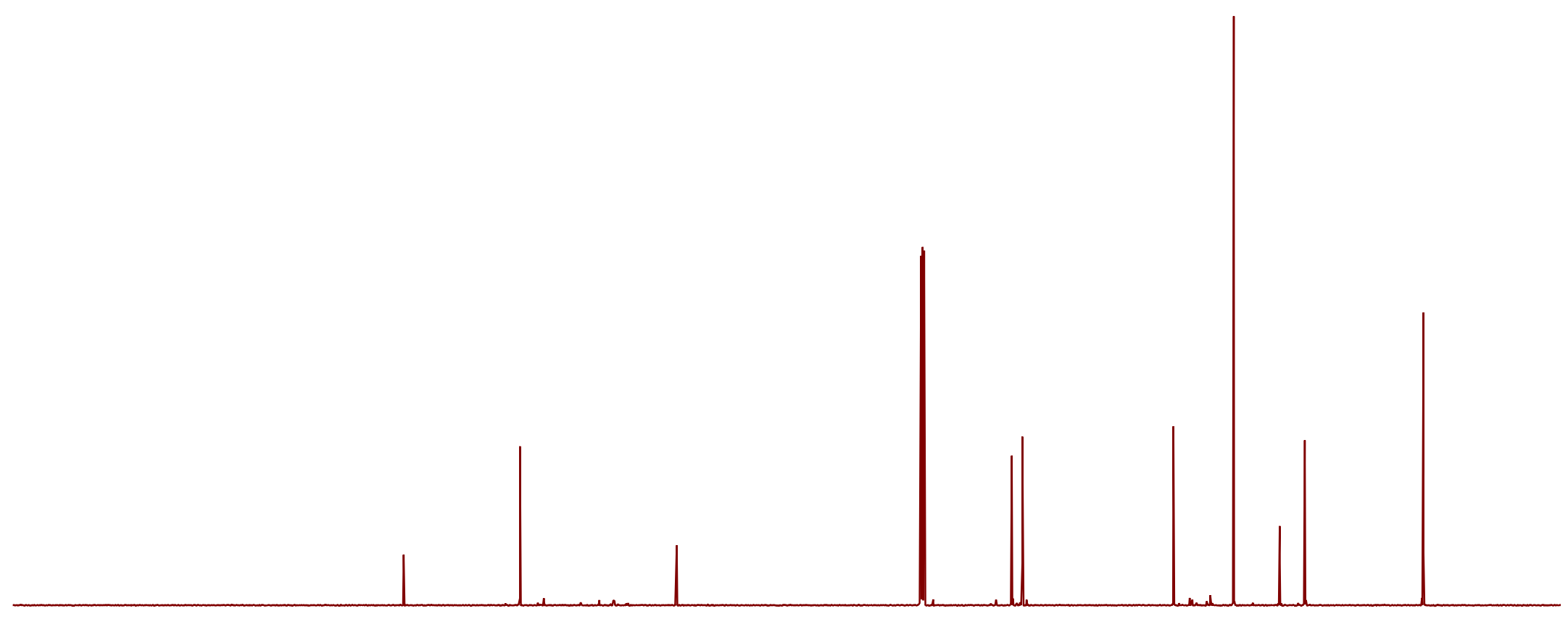

$\begin{array}{lllllllllllllllllllllllllllllllllll}1 & 220 & 210 & 200 & 190 & 180 & 170 & 160 & 150 & 140 & 130 & 120 & 110 & 100 & 90 & 80 & 70 & 60 & 50 & 40 & 30 & 20 & 10 & 0 & -10 & -20\end{array}$ 


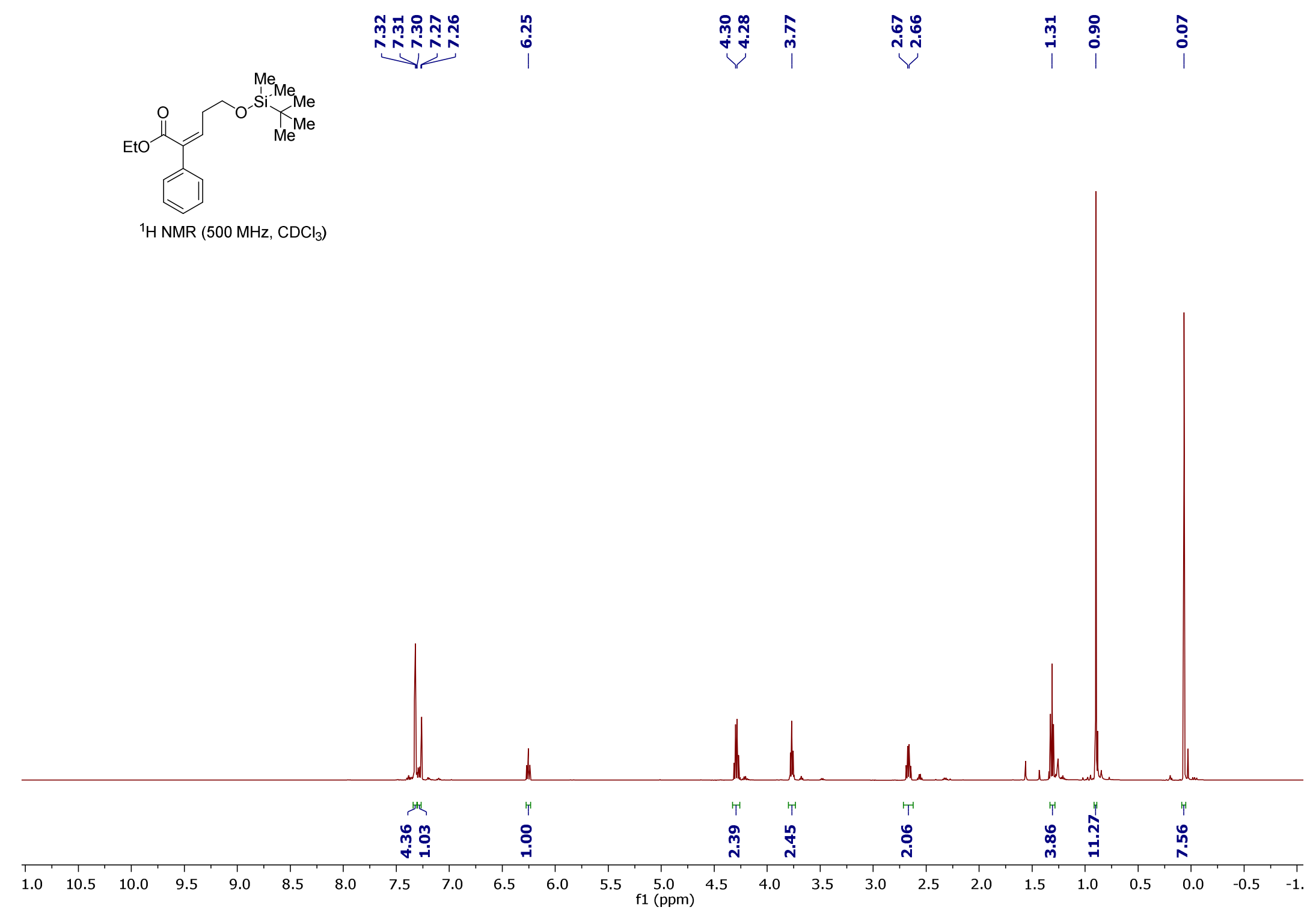




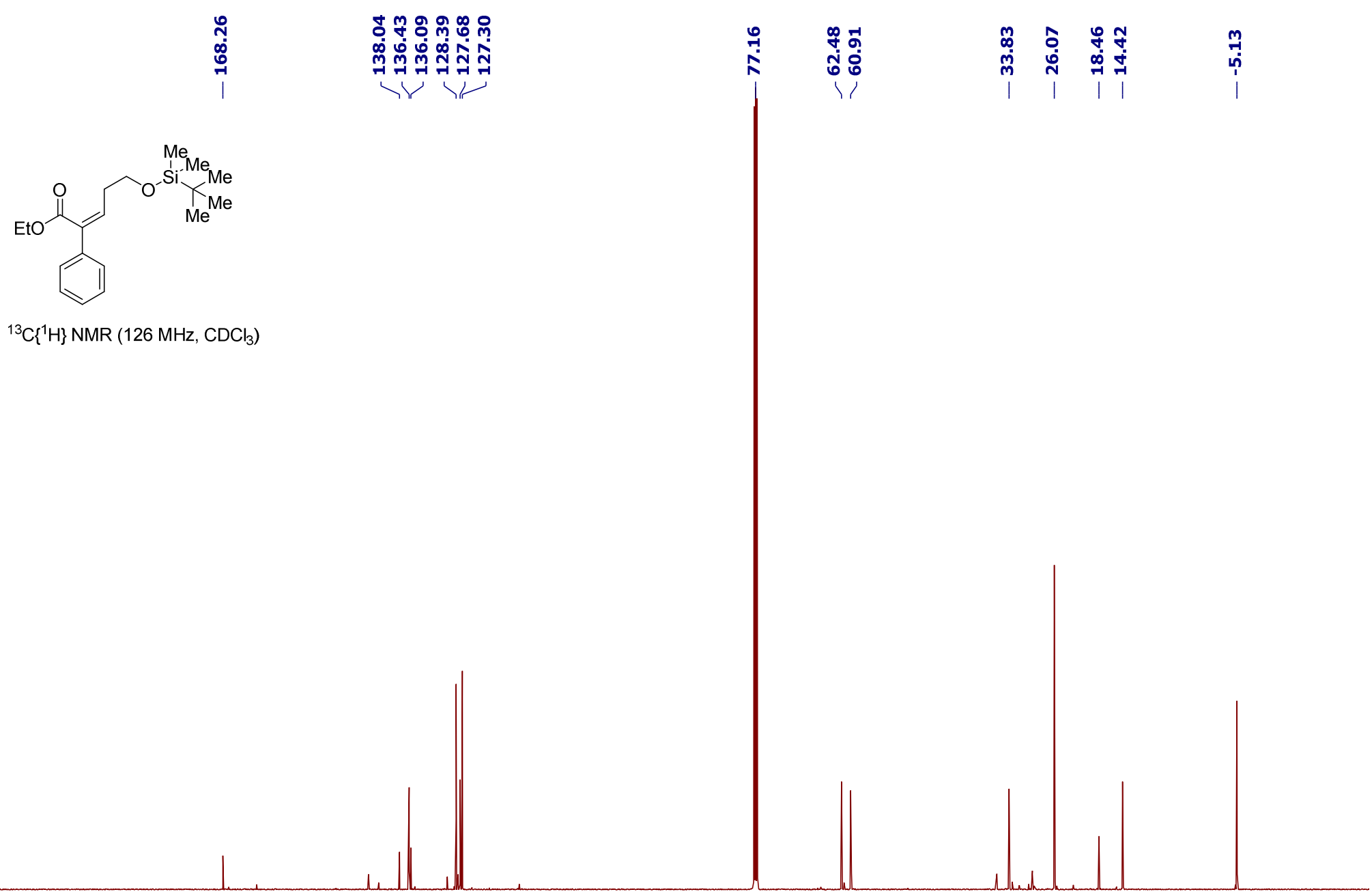

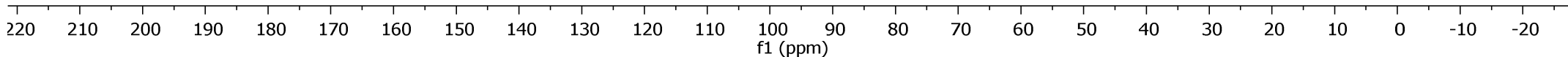




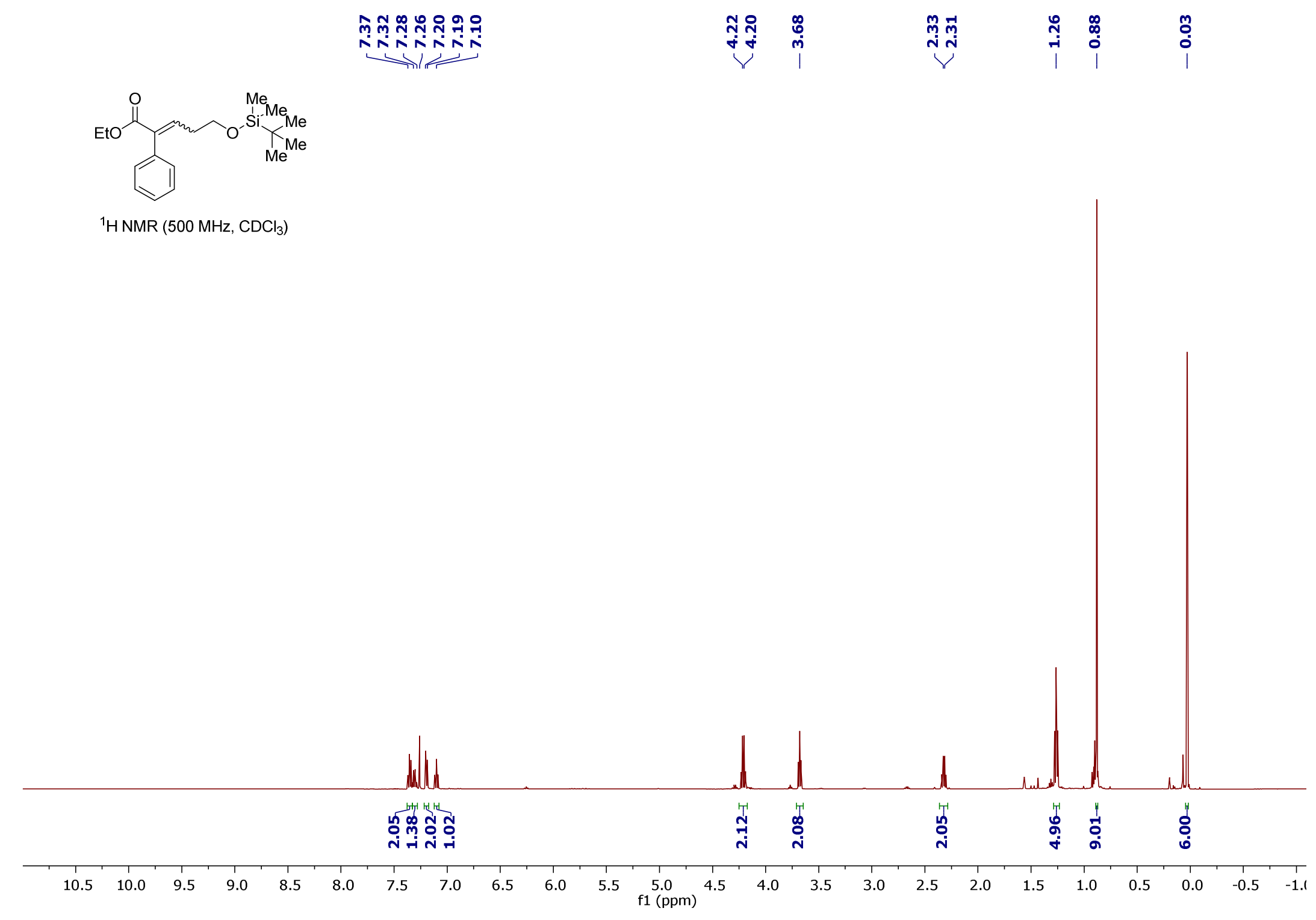




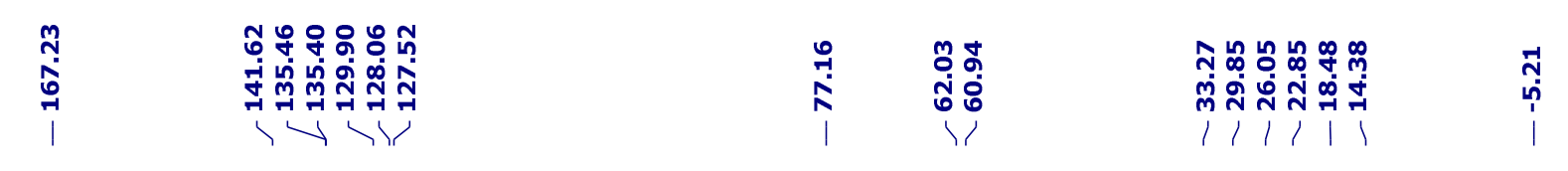<smiles>CCOC(=O)/C(=C/CCO[Si](C)(C)C(C)(C)C)c1ccccc1</smiles>

$\left.{ }^{13} \mathrm{C}^{1}{ }^{1} \mathrm{H}\right\} \mathrm{NMR}\left(126 \mathrm{MHz}, \mathrm{CDCl}_{3}\right)$

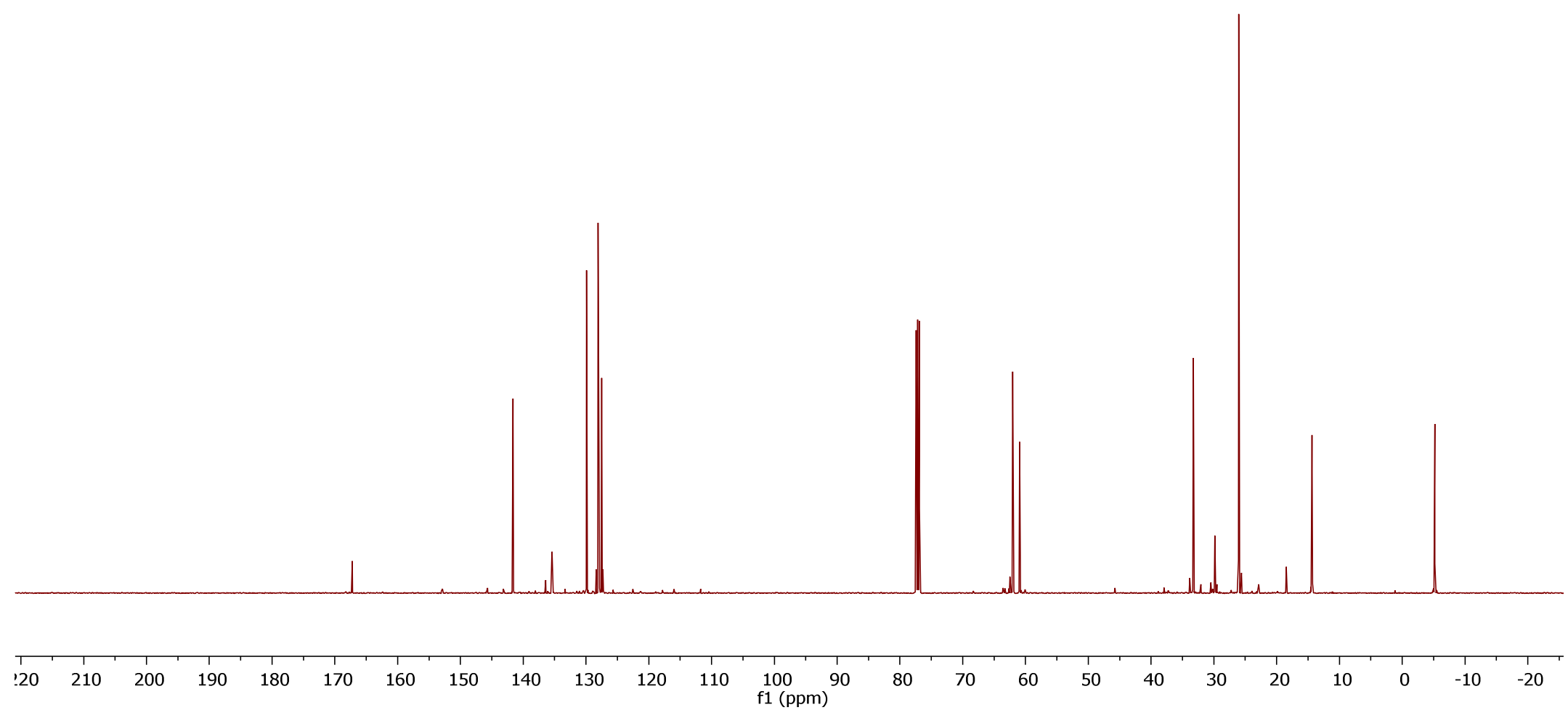




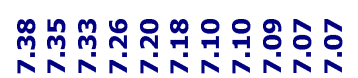

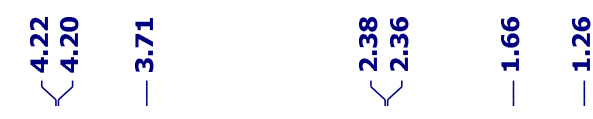

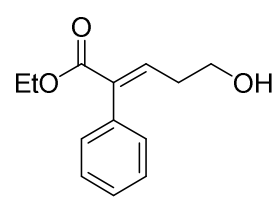

40

${ }^{1} \mathrm{H} \mathrm{NMR}\left(500 \mathrm{MHz}, \mathrm{CDCl}_{3}\right)$

NNnNהN

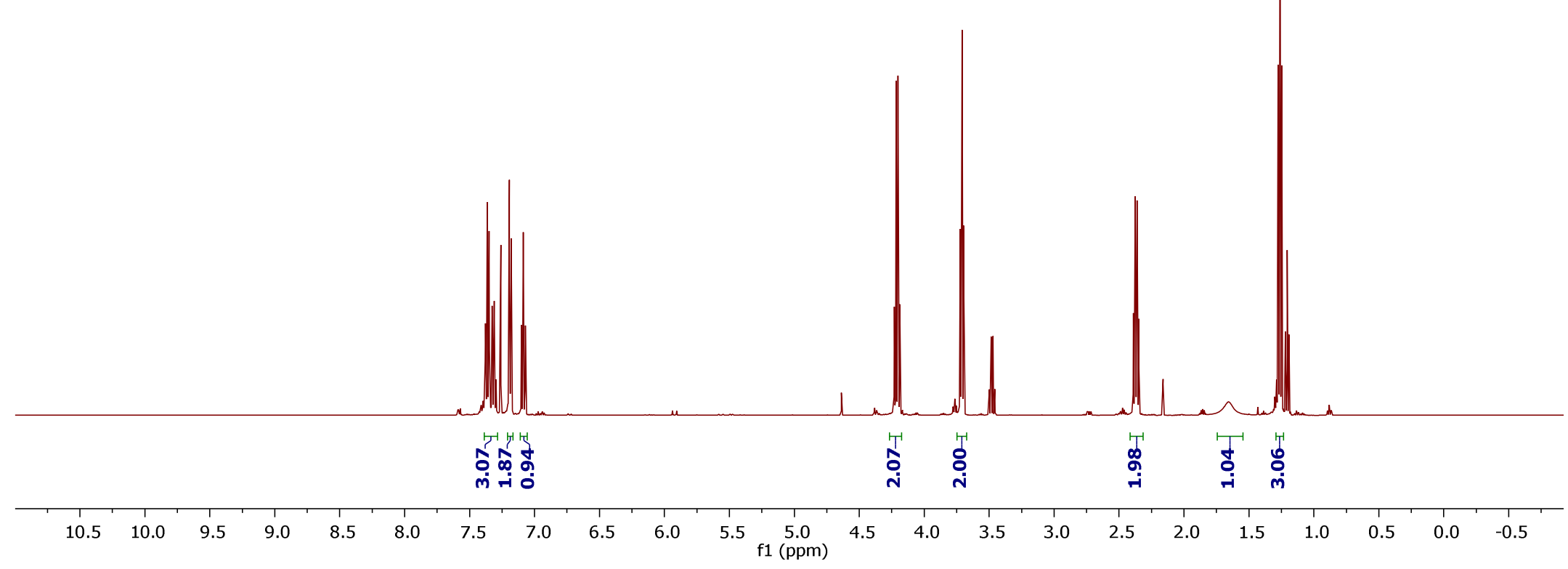




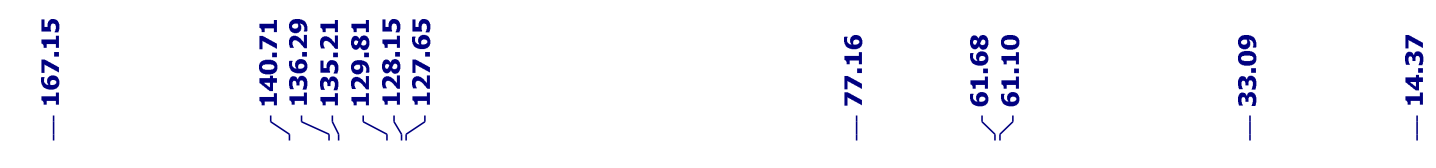

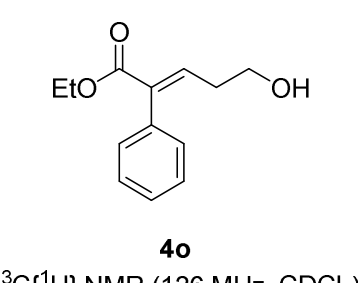

${ }^{13} \mathrm{C}\left\{{ }^{1} \mathrm{H}\right\} \mathrm{NMR}\left(126 \mathrm{MHz}, \mathrm{CDCl}_{3}\right)$

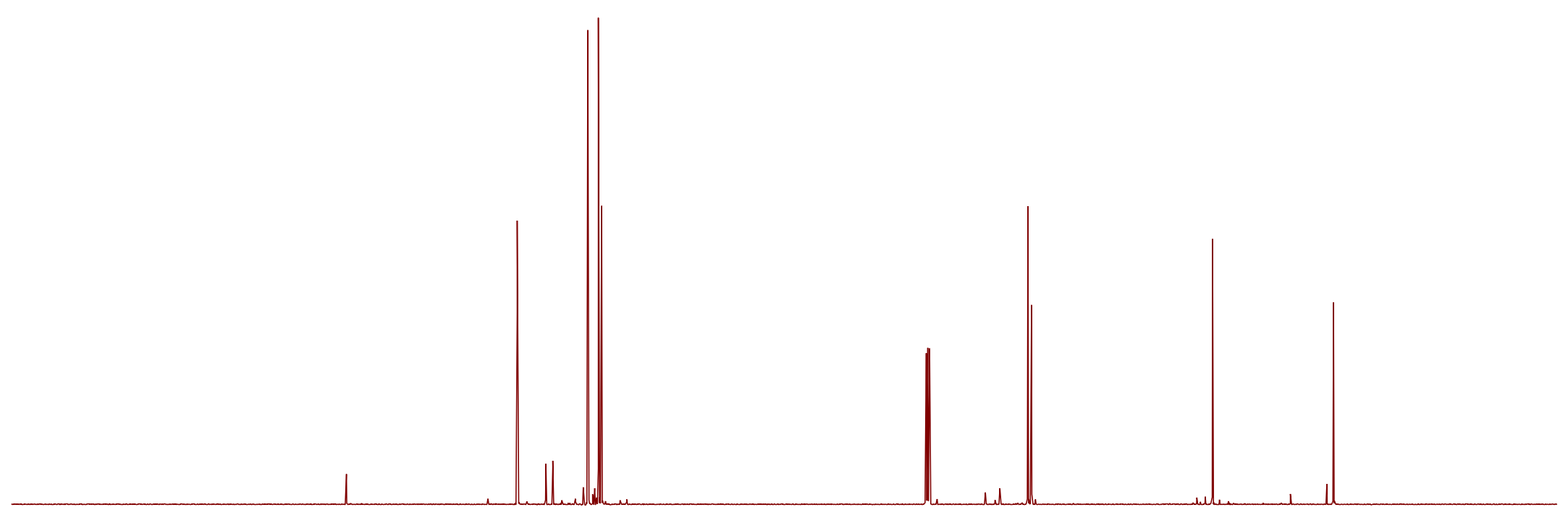

$\begin{array}{llllllllllllllllllllllllll}210 & 200 & 190 & 180 & 170 & 160 & 150 & 140 & 130 & 120 & 110 & \begin{array}{c}100 \\ \mathrm{f} 1(\mathrm{ppm})\end{array} & 90 & 80 & 70 & 60 & 50 & 40 & 30 & 20 & 10 & 0 & -10 & -i\end{array}$ 


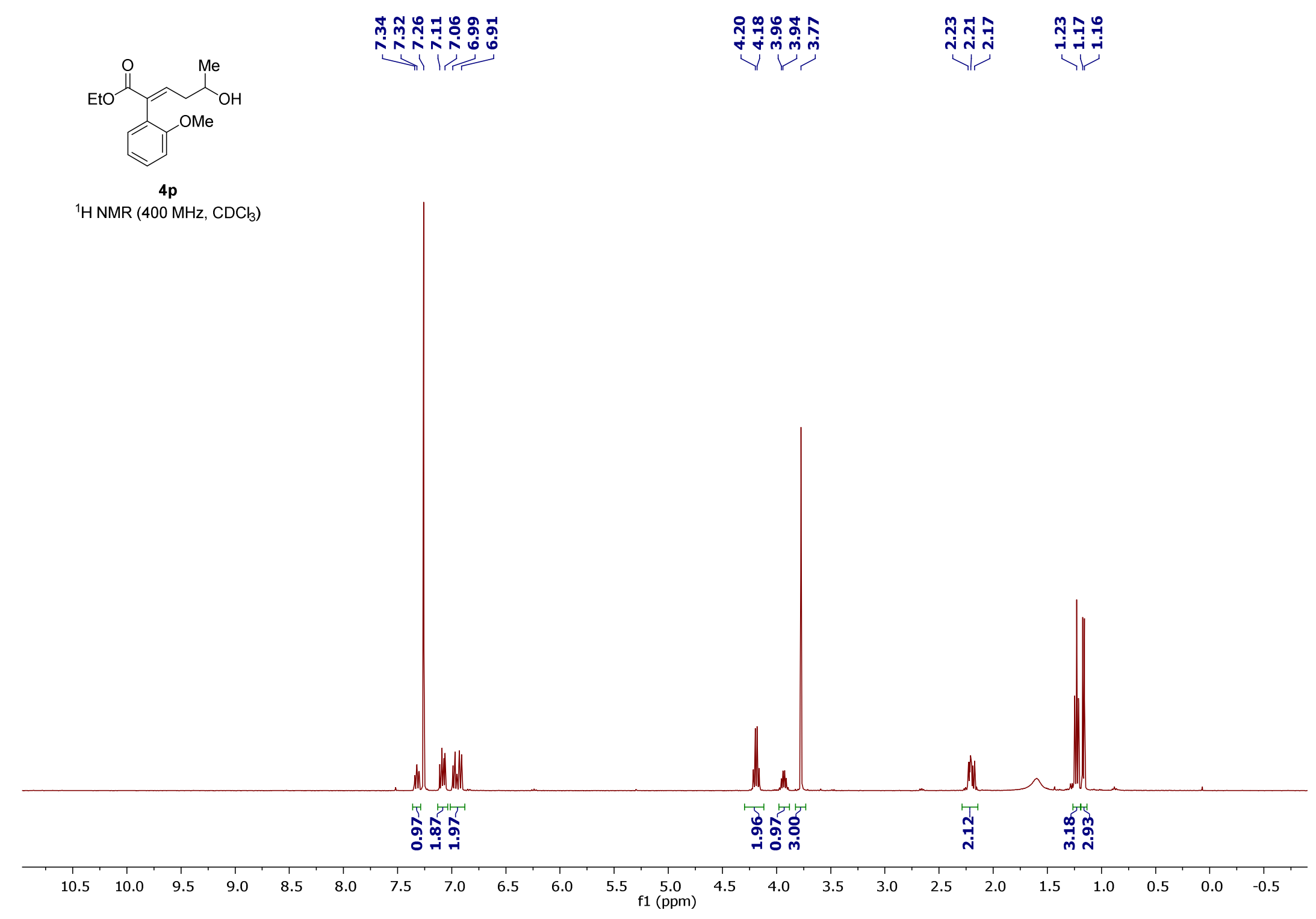




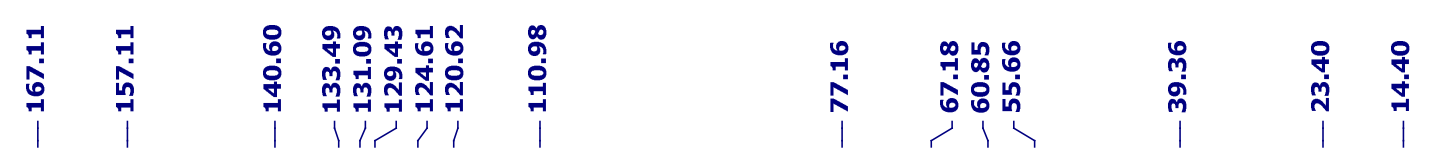

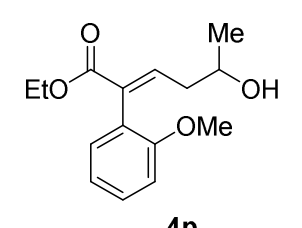

${ }^{13} \mathrm{C}\left\{{ }^{1} \mathrm{H}\right\}$ NMR $\left(101 \mathrm{MHz}, \mathrm{CDCl}_{3}\right)$

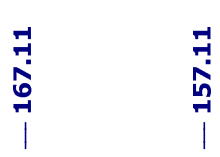

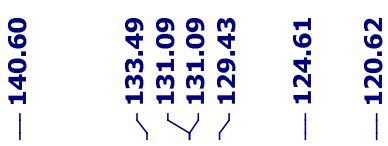

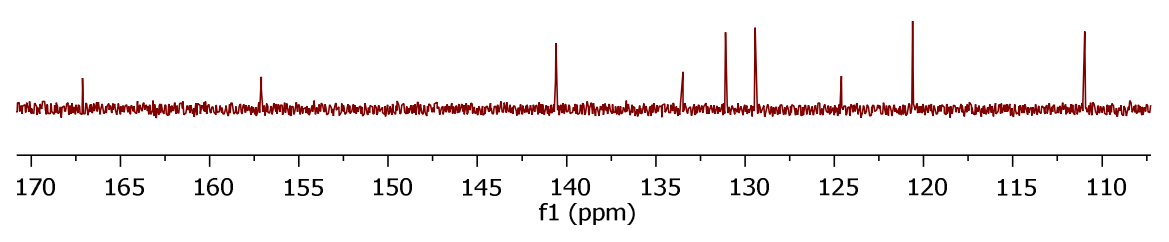

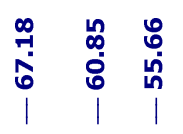

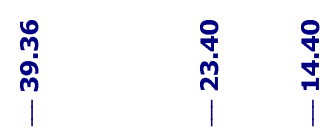

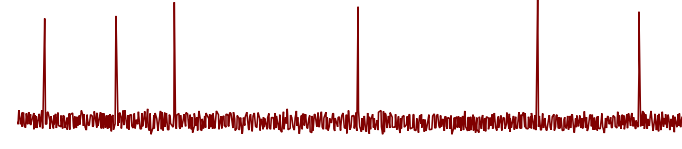

60

40
$\mathrm{f} 1(\mathrm{ppm})$

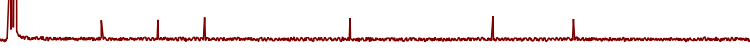

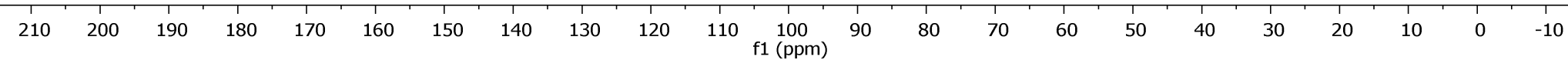



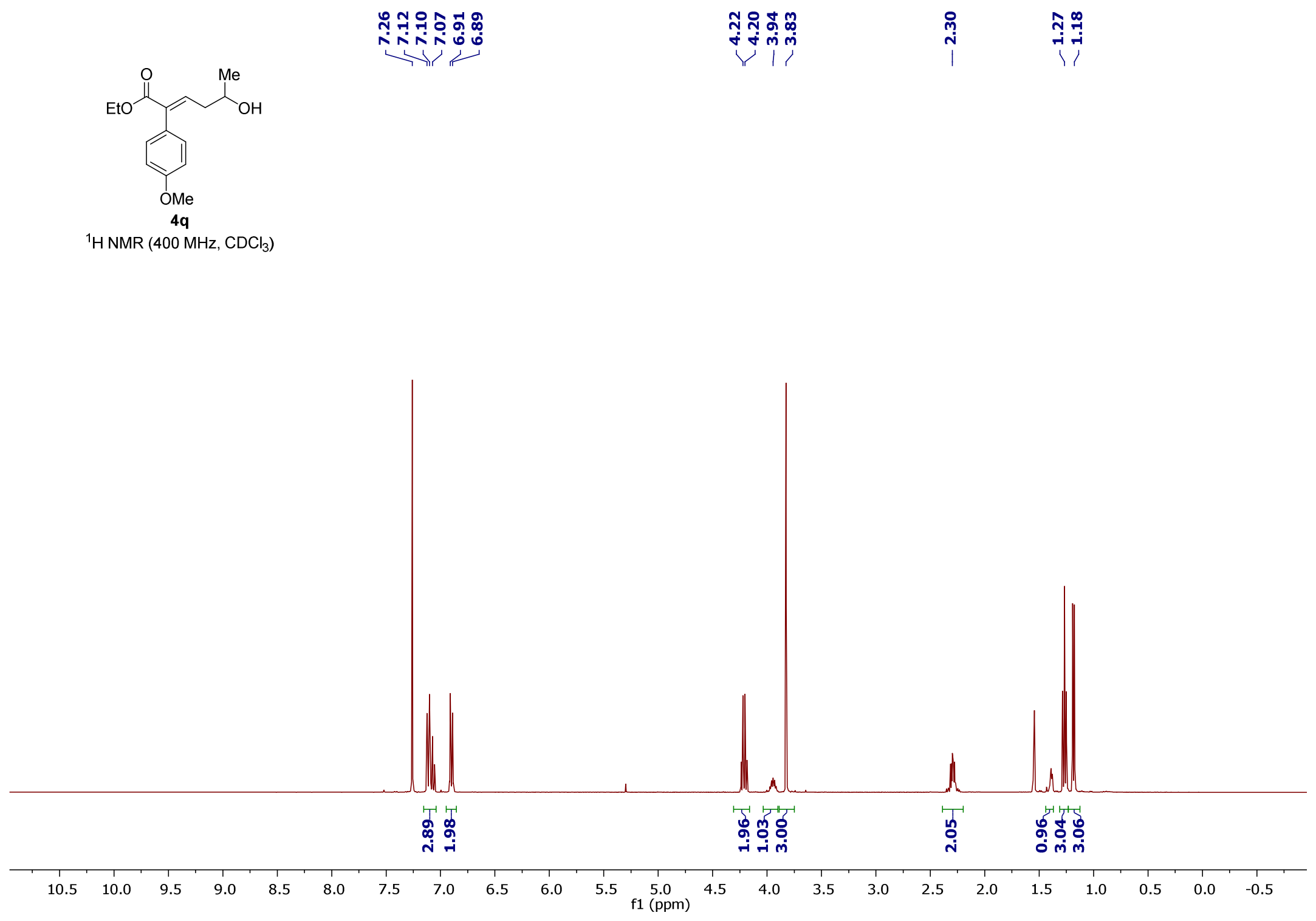


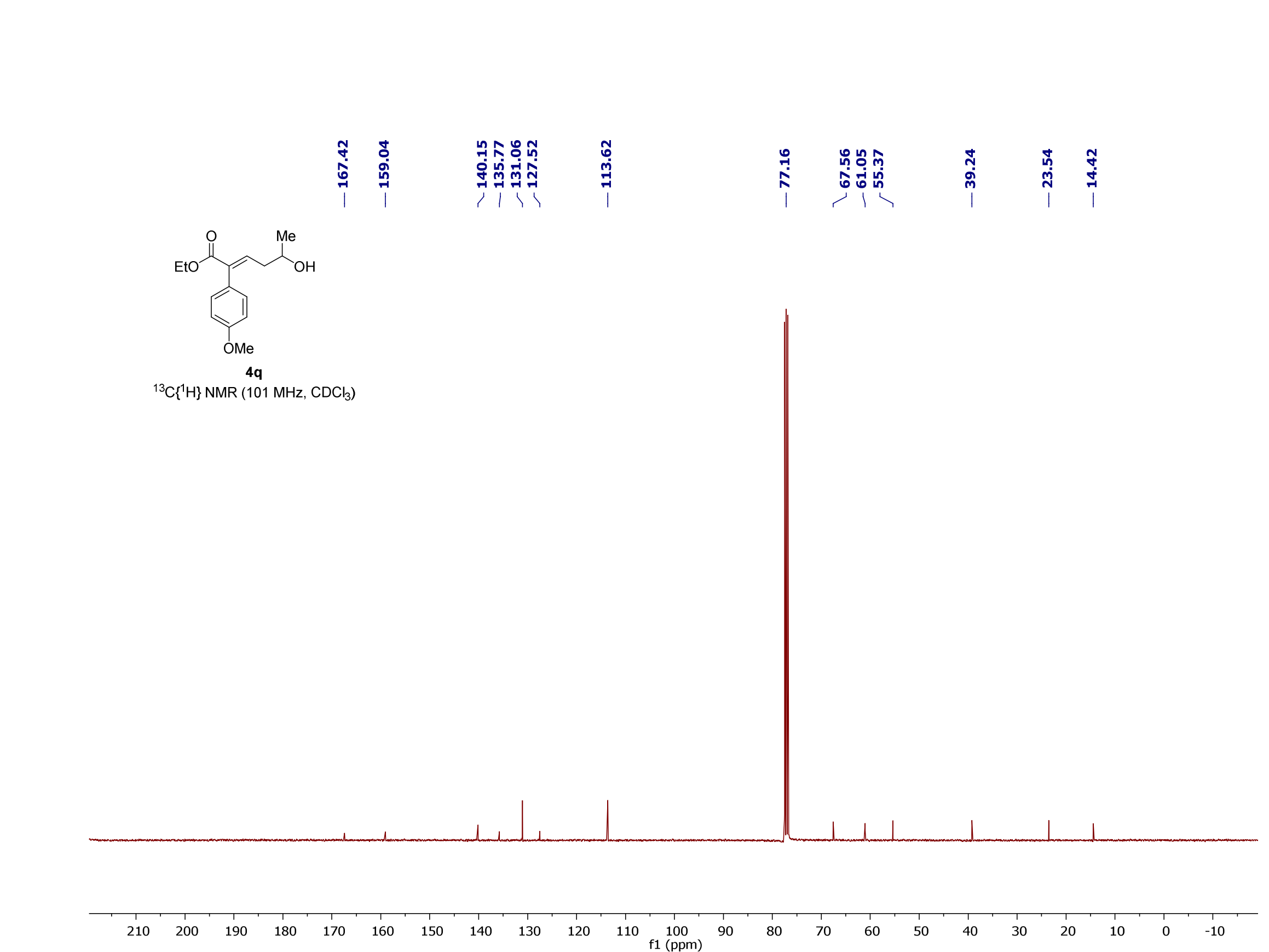




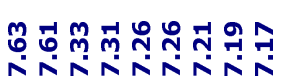

ññNañ

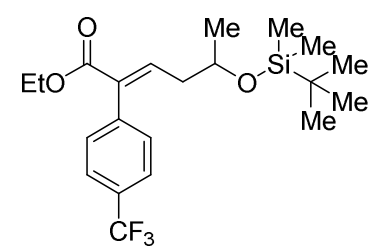

${ }^{1} \mathrm{H}$ NMR $\left(400 \mathrm{MHz}, \mathrm{CDCl}_{3}\right)$

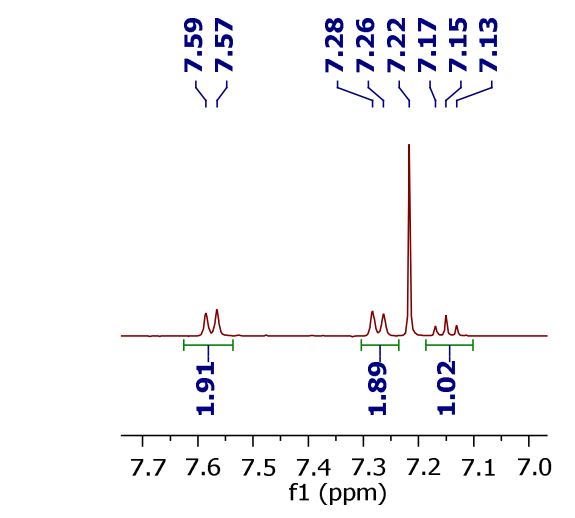

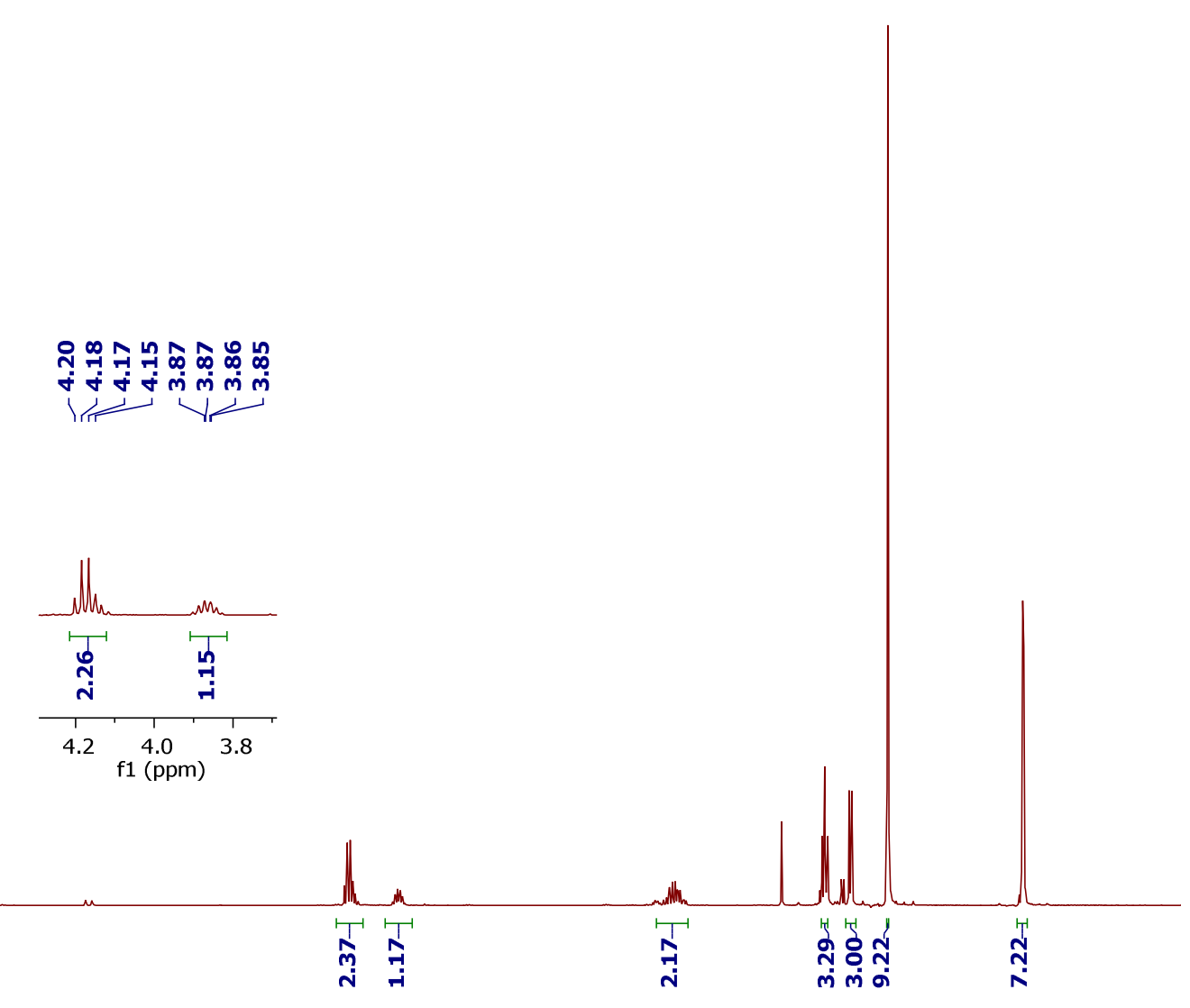

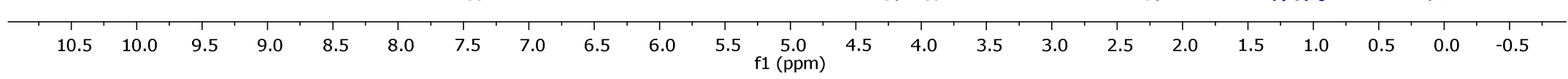




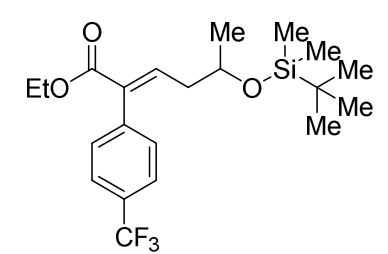

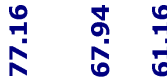

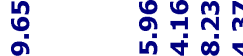

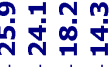

1) 1

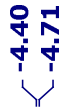

$\left.{ }^{13} \mathrm{C}^{1}{ }^{1} \mathrm{H}\right\} \mathrm{NMR}\left(101 \mathrm{MHz}, \mathrm{CDCl}_{3}\right)$

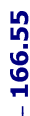

กิ

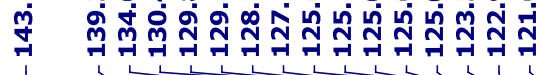

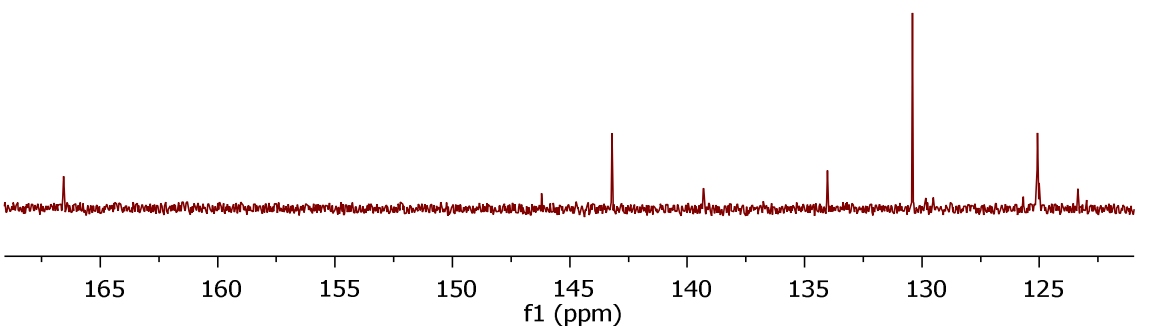

$\begin{array}{llll}210 & 200 & 190 & 180\end{array}$

$170 \quad 160$

$150 \quad 140$

$130 \quad 120$

$110 \quad 100$ 100
$\mathrm{f} 1(\mathrm{ppm})$

80

$60 \quad 50$

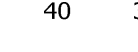

$\begin{array}{lll}10 & 0 & -10\end{array}$ 


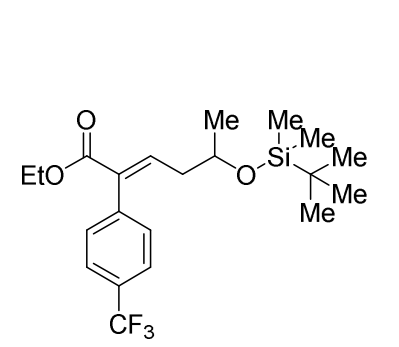

${ }^{19} \mathrm{~F}\left\{{ }^{1} \mathrm{H},{ }^{13} \mathrm{C}\right\}$ NMR $(283 \mathrm{MHz}, \mathrm{CDCl} 3)$

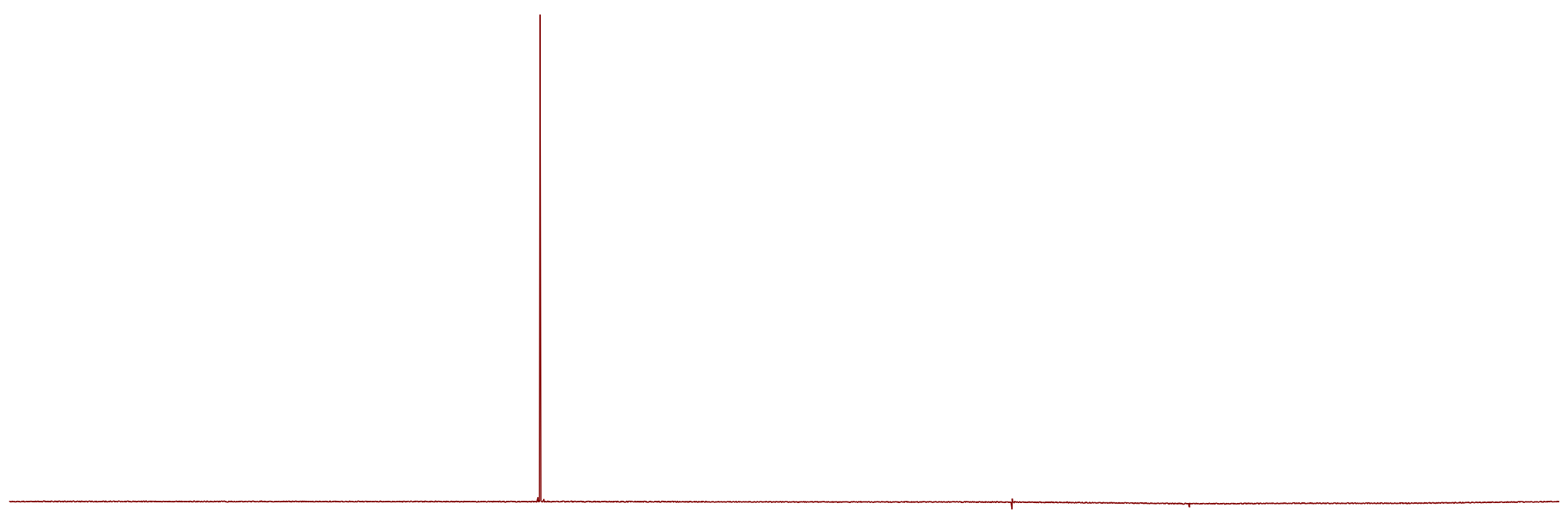




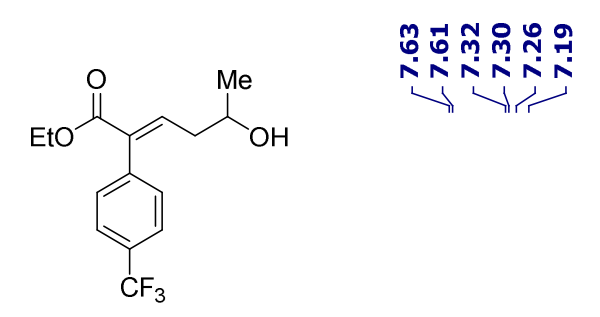

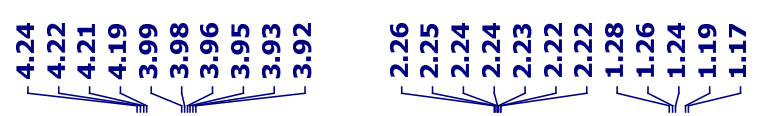

${ }^{1} \mathrm{HNMR}\left(400 \mathrm{MHz}, \mathrm{CDCl}_{3}\right)$

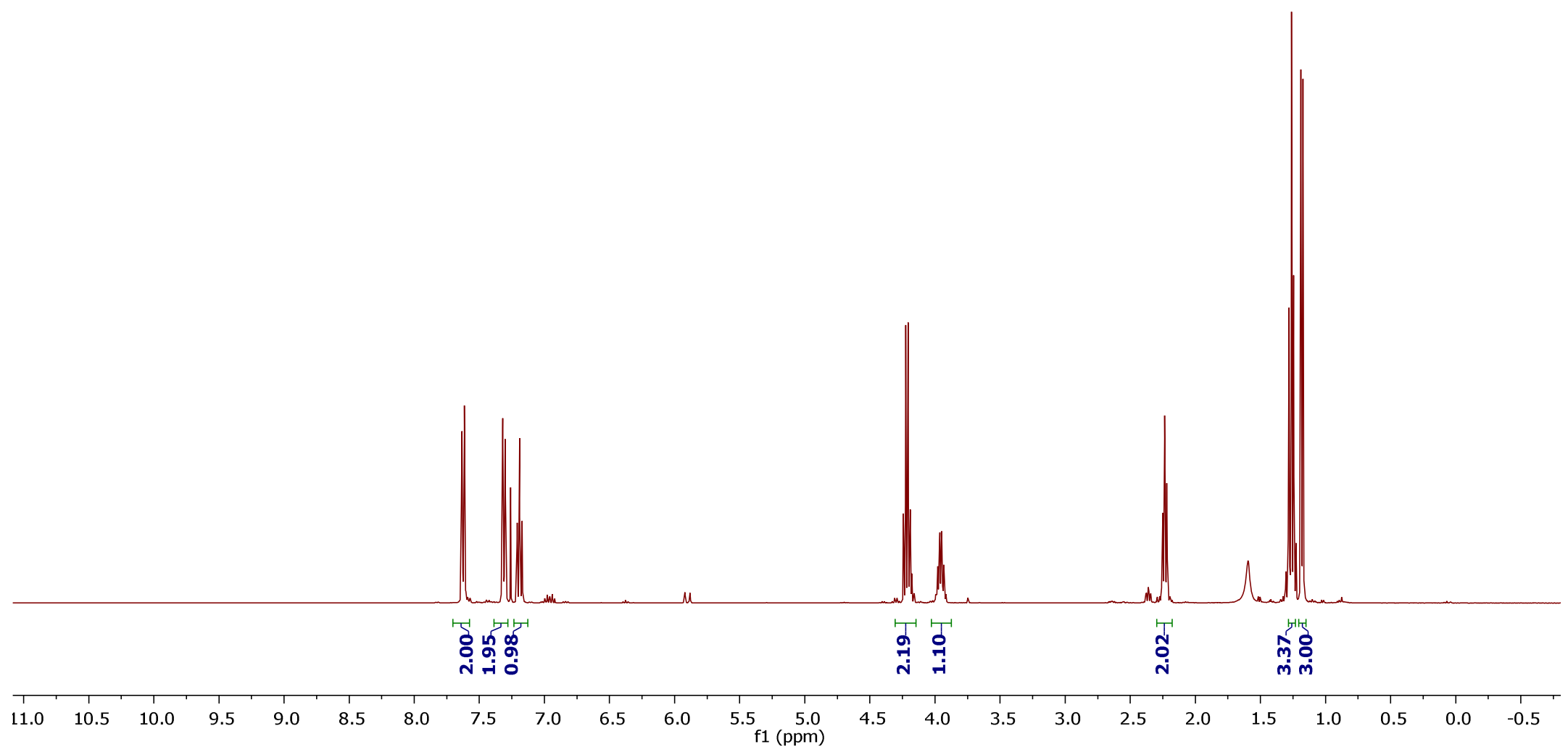




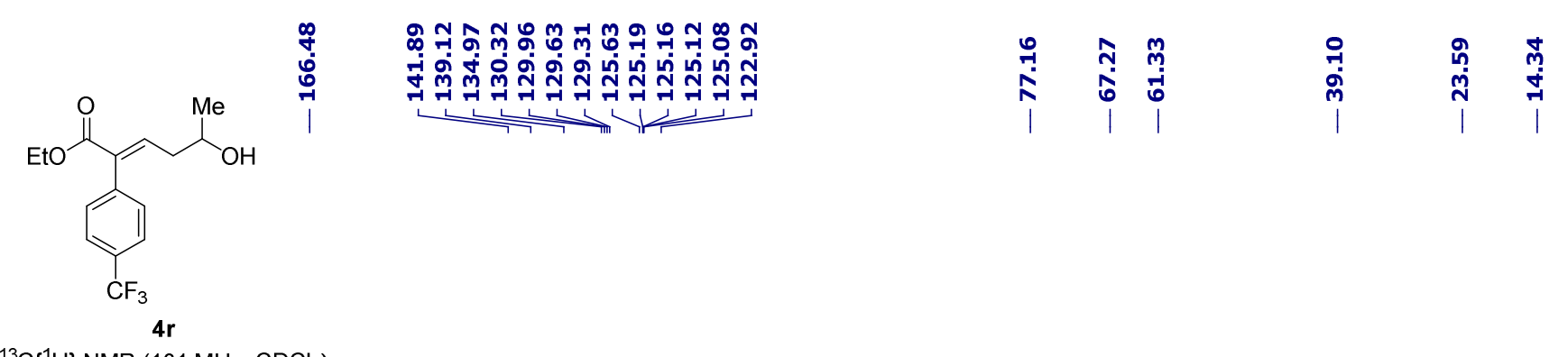

$\left.{ }^{13} \mathrm{C}^{1} \mathrm{H}\right\} \mathrm{NMR}\left(101 \mathrm{MHz}, \mathrm{CDCl}_{3}\right)$

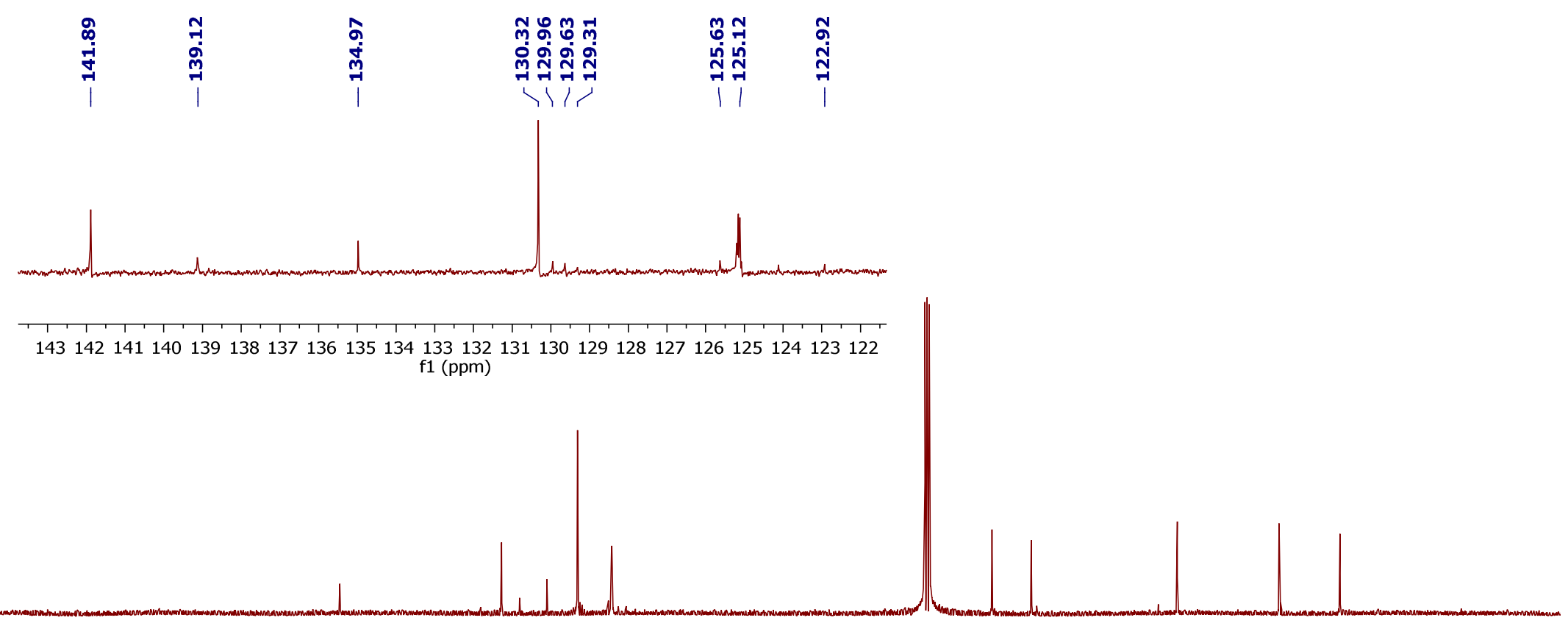

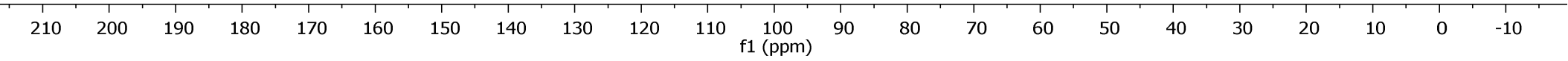


<smiles>CCOC(=O)/C(=C/CC(O)O)c1ccc(C(F)(F)F)cc1</smiles>

${ }^{19} \mathrm{~F}\left\{{ }^{1} \mathrm{H},{ }^{13} \mathrm{C}\right\}$ NMR $\left(283 \mathrm{MHz}, \mathrm{CDCl}_{3}\right)$

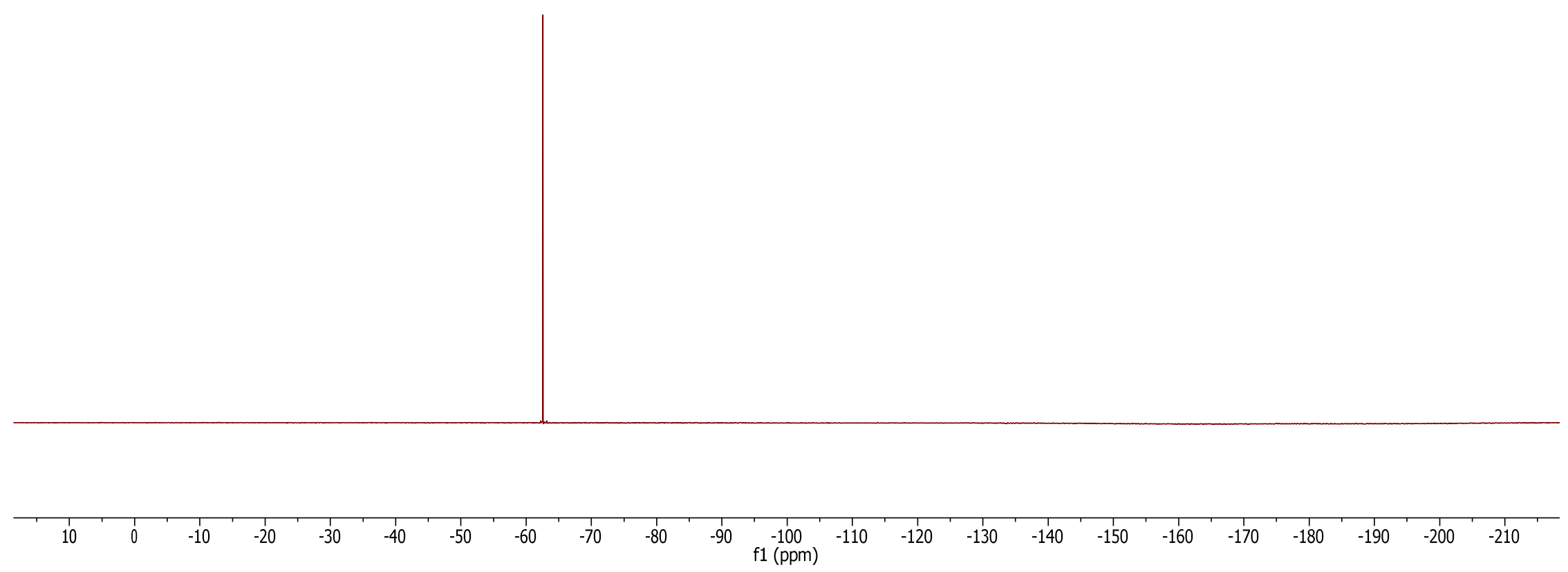




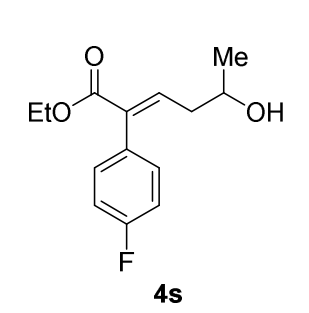

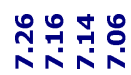

iny

กิรูำ

ㄱ)

กำ

${ }^{1} \mathrm{H} \mathrm{NMR}\left(400 \mathrm{MHz}, \mathrm{CDCl}_{3}\right)$

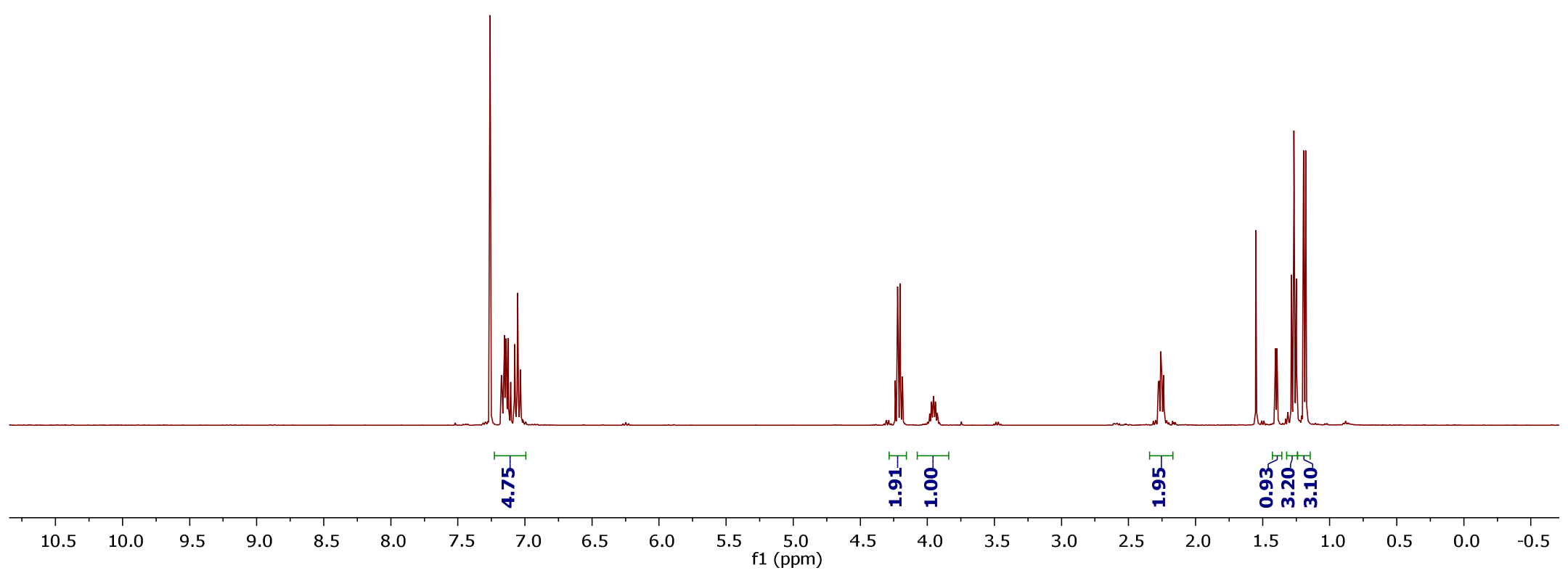




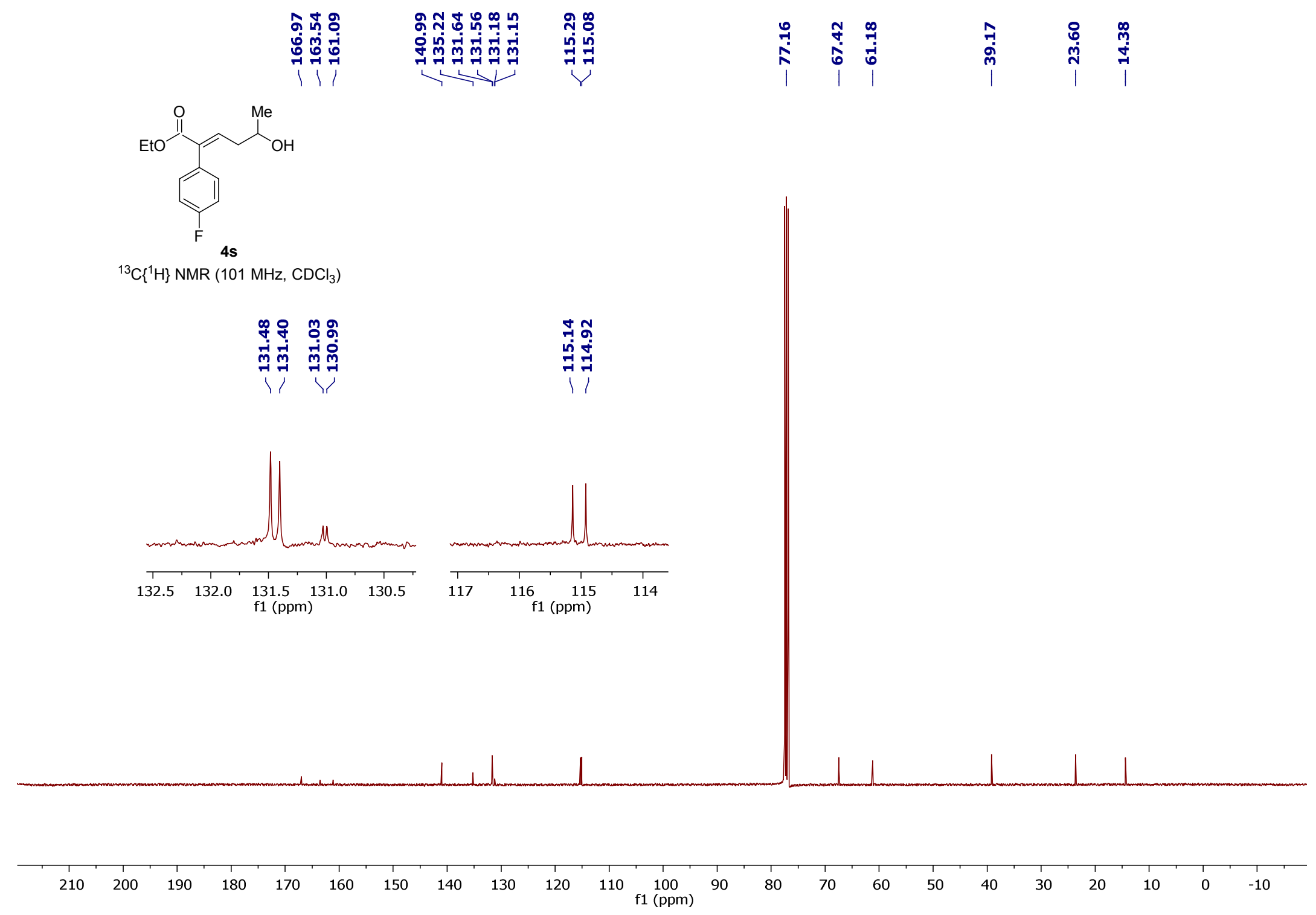




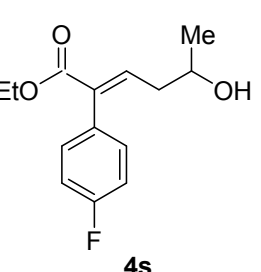

${ }^{19} \mathrm{~F}\left\{{ }^{1} \mathrm{H},{ }^{13} \mathrm{C}\right\}$ NMR $\left(283 \mathrm{MHz}, \mathrm{CDCl}_{3}\right)$

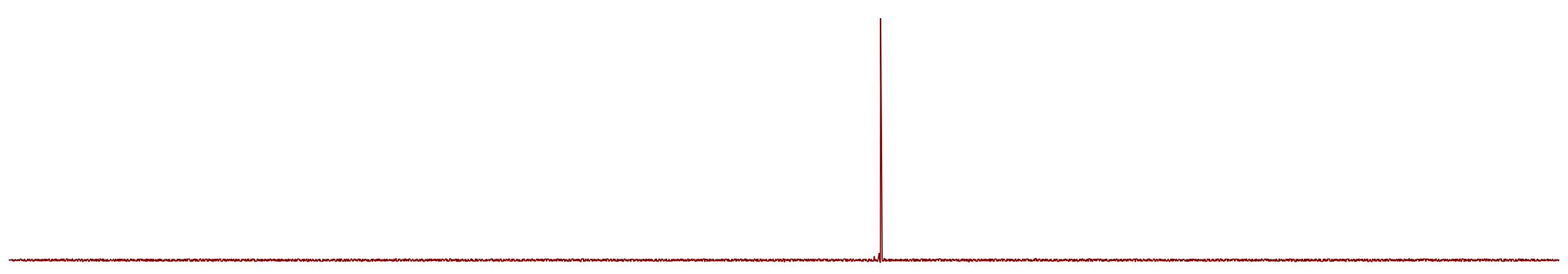

$\begin{array}{lllllllllllllllllllllllll}10 & 0 & -10 & -20 & -30 & -40 & -50 & -60 & -70 & -80 & -90 & -100 & -110 & -120 & -130 & -140 & -150 & -160 & -170 & -180 & -190 & -200 & -210 & \end{array}$ 


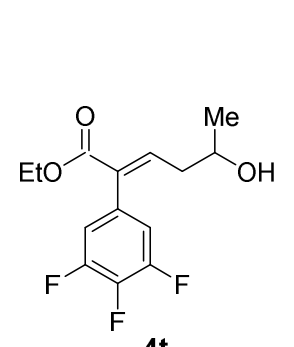

ํํำํํำ

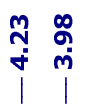

พู่

${ }^{1} \mathrm{H} \mathrm{NMR}\left(400 \mathrm{MHz}, \mathrm{CDCl}_{3}\right)$

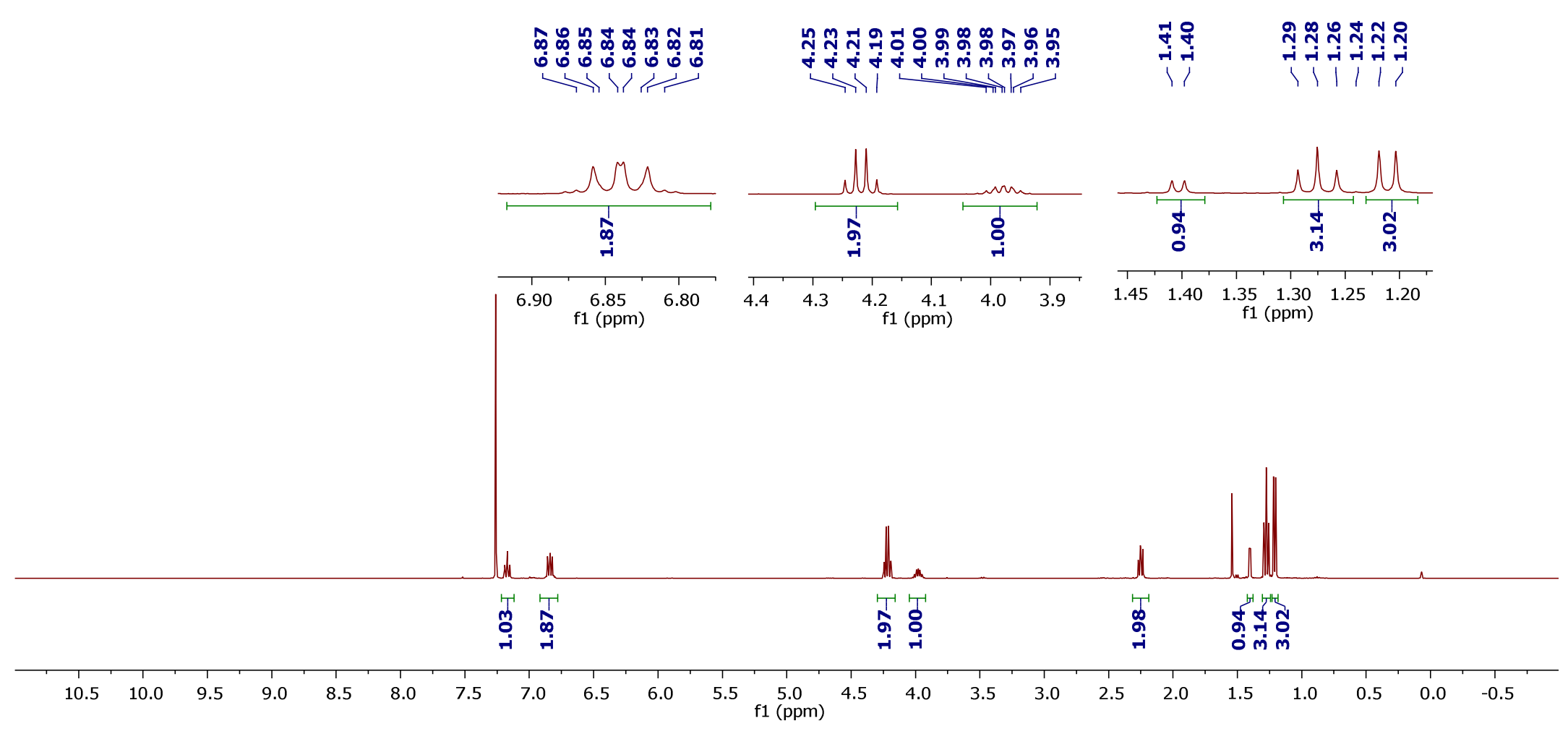




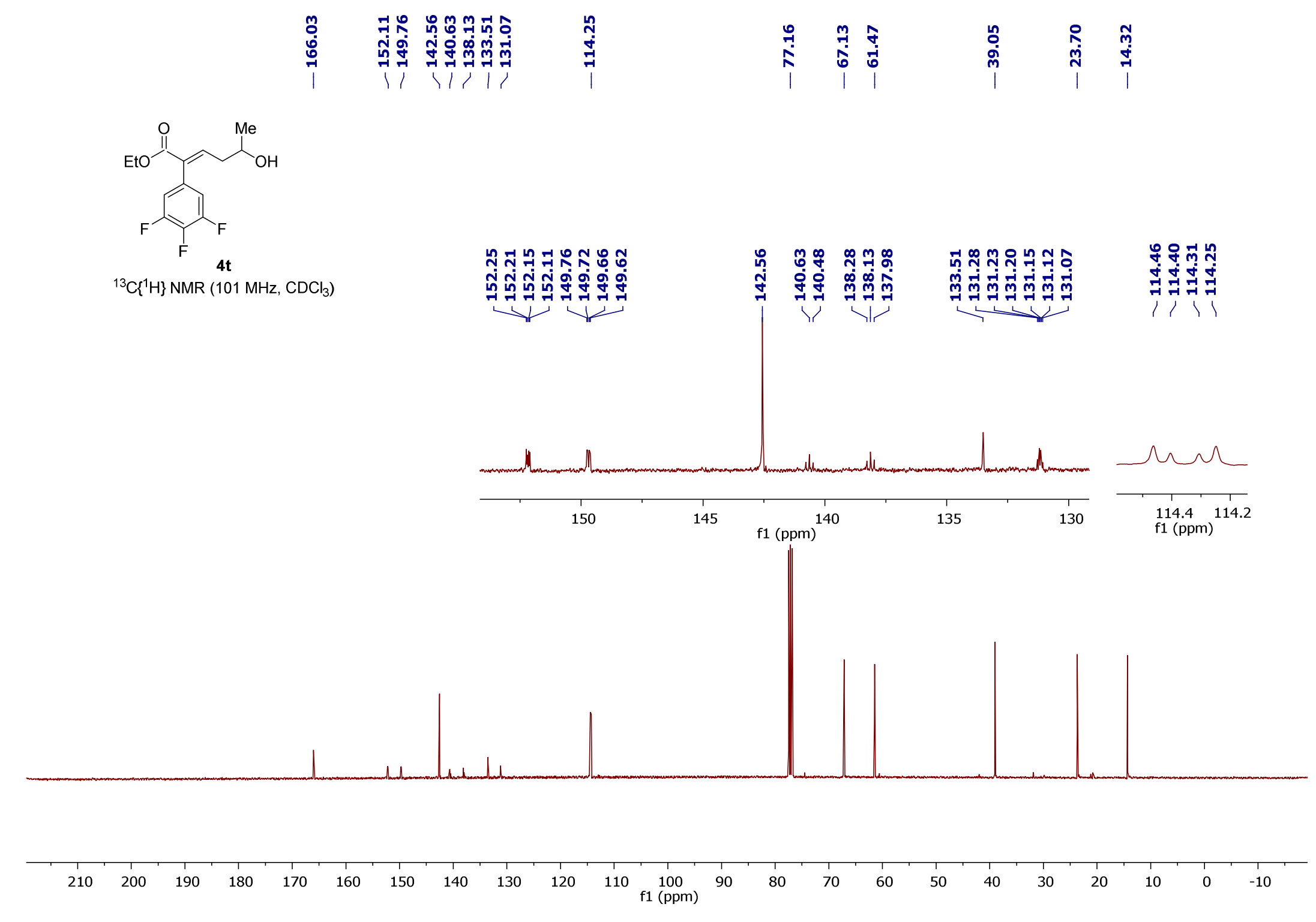




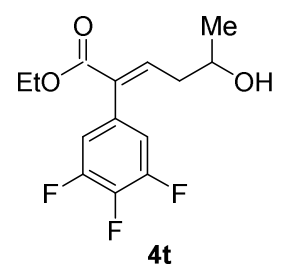

نَ

${ }^{19} \mathrm{~F}\left\{{ }^{1} \mathrm{H},{ }^{13} \mathrm{C}\right\} \mathrm{NMR}\left(283 \mathrm{MHz}, \mathrm{CDCl}_{3}\right)$

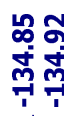

tion

فํㅓㅇ

il

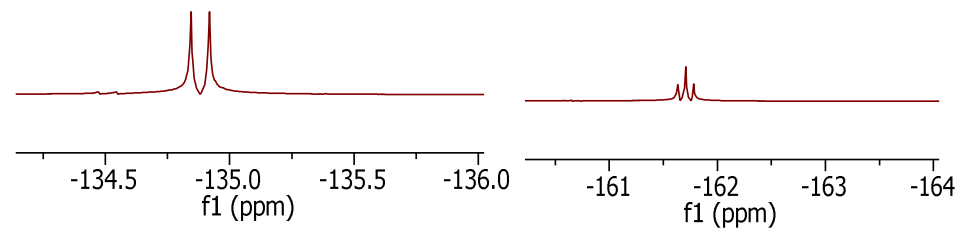

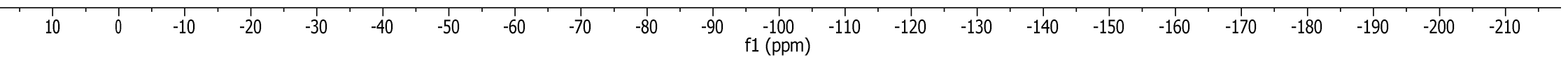




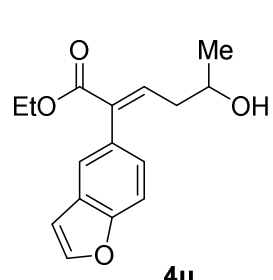

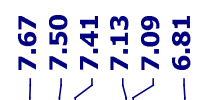

ֻิ

จุจุ

ปู่

${ }^{1} \mathrm{H}$ NMR $\left(400 \mathrm{MHz}, \mathrm{CD}_{2} \mathrm{Cl}_{2}\right)$

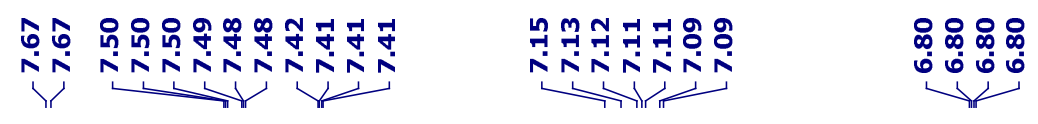
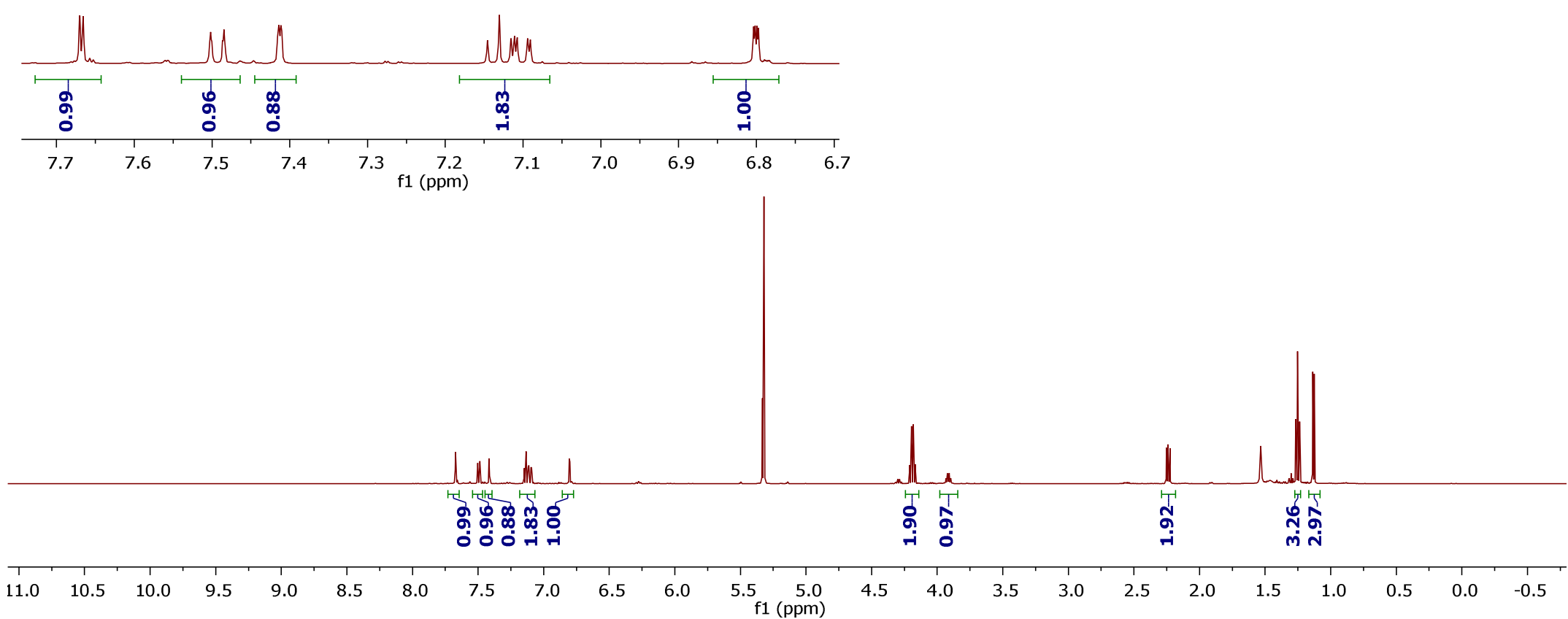


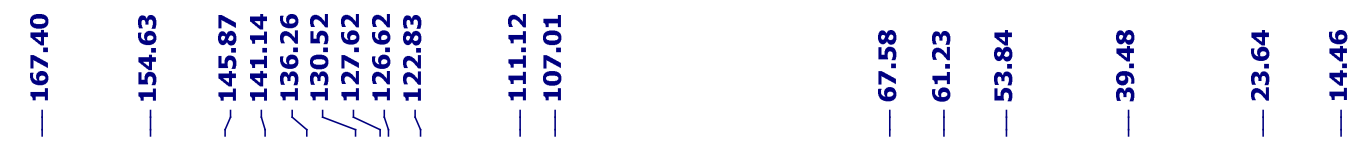

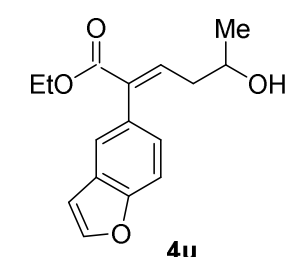

${ }^{13} \mathrm{C}\left\{{ }^{1} \mathrm{H}\right\}$ NMR $\left(101 \mathrm{MHz}, \mathrm{CD}_{2} \mathrm{Cl}_{2}\right)$

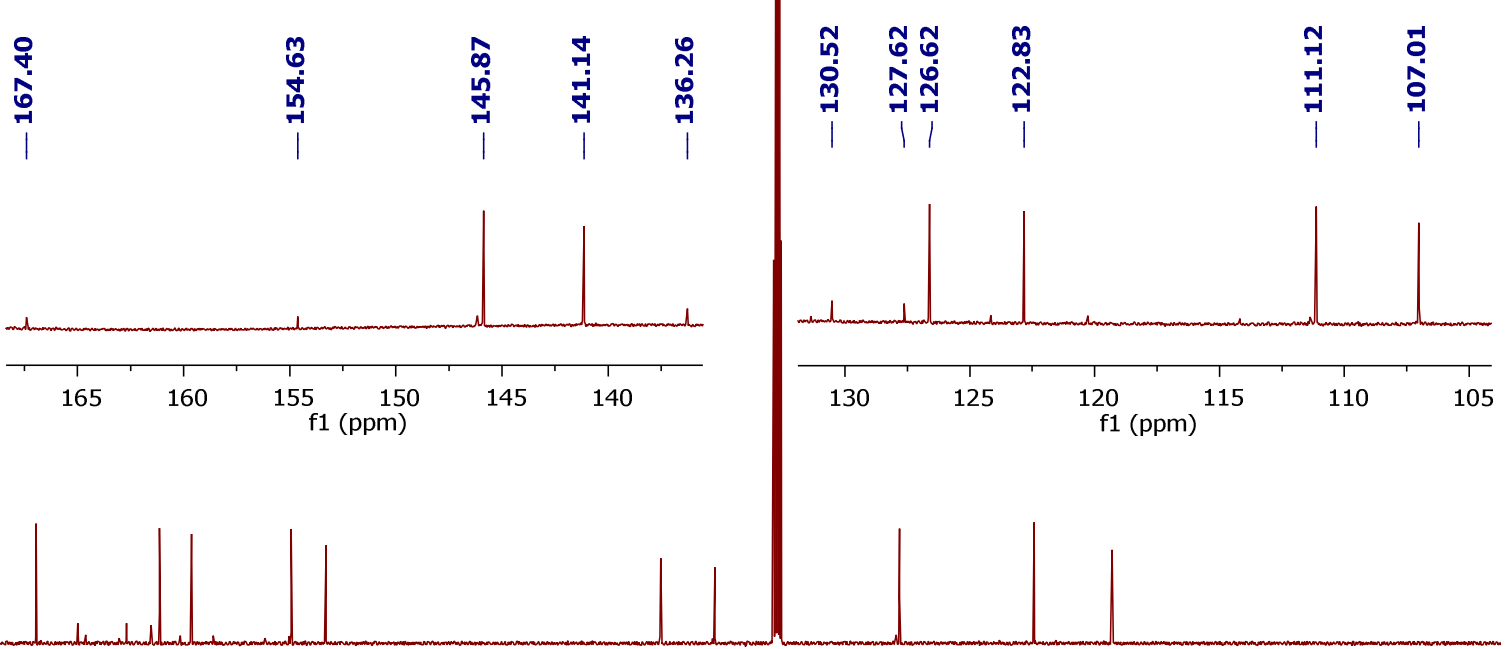

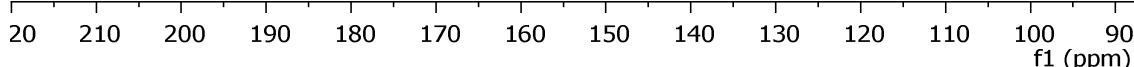

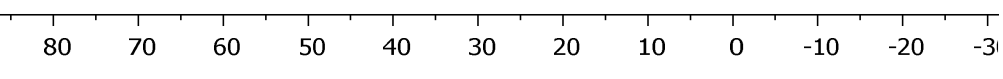




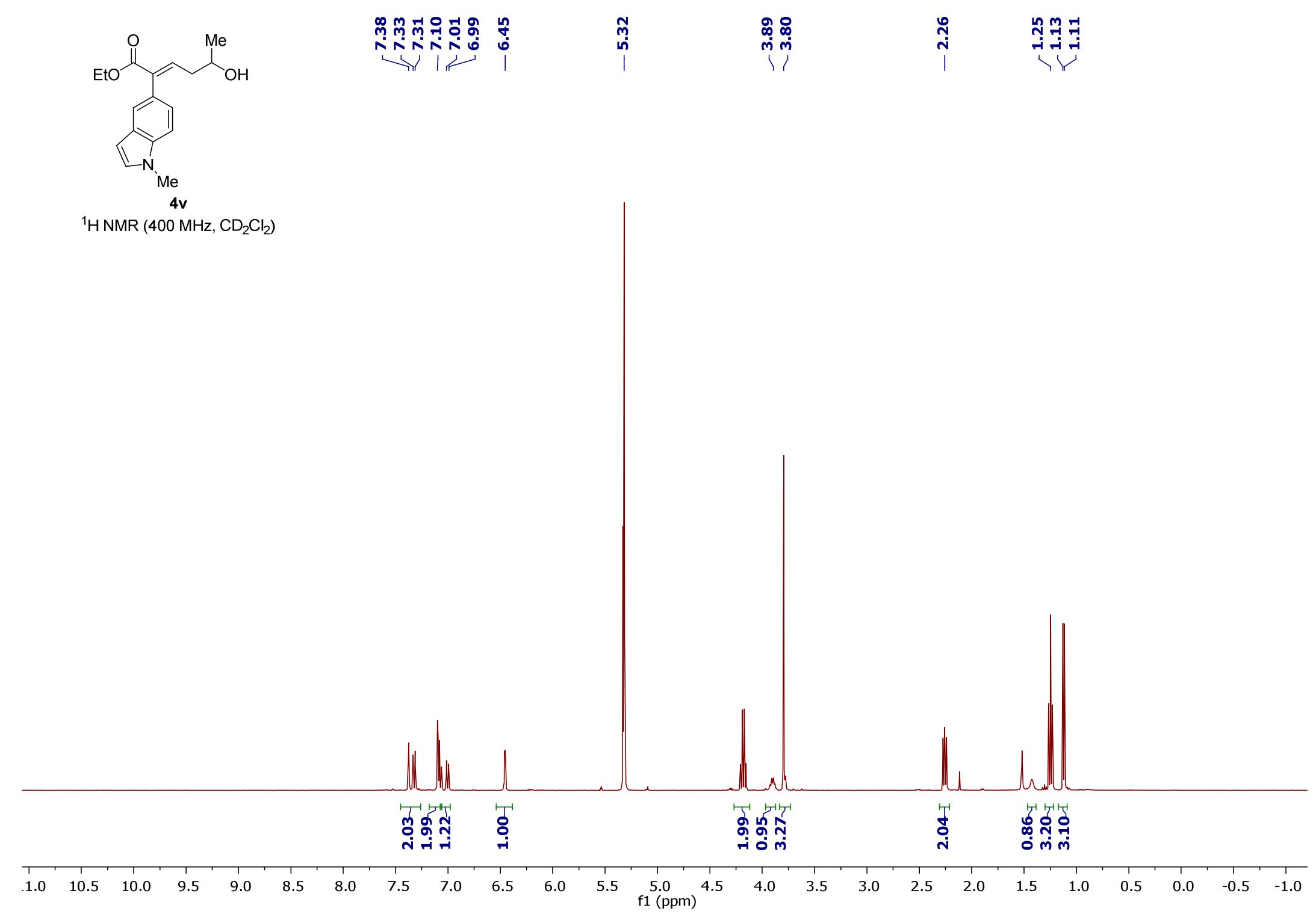




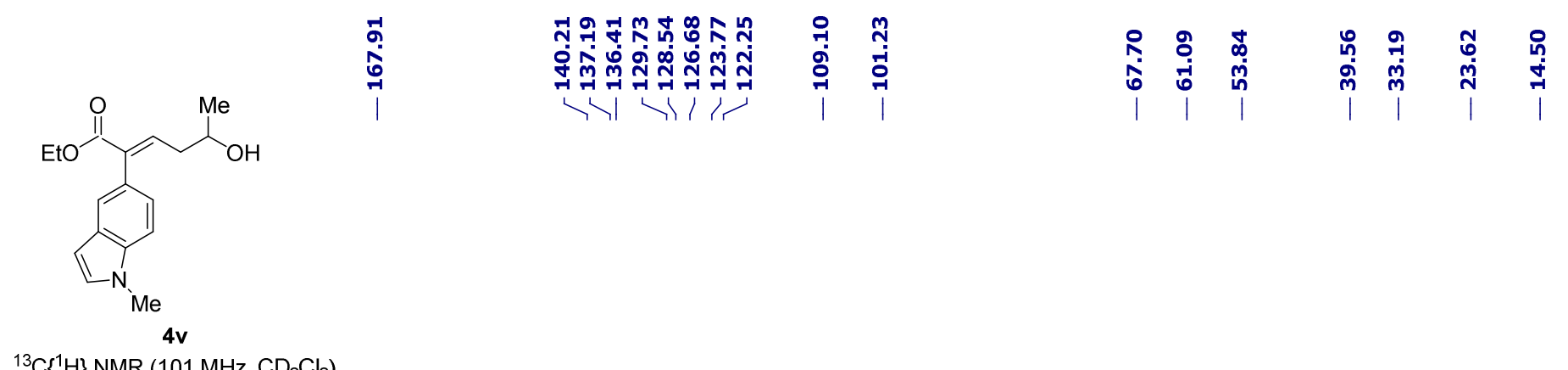

$\left.{ }^{13} \mathrm{C}^{1} \mathrm{H}\right\}$ NMR $\left(101 \mathrm{MHz}, \mathrm{CD}_{2} \mathrm{Cl}_{2}\right)$

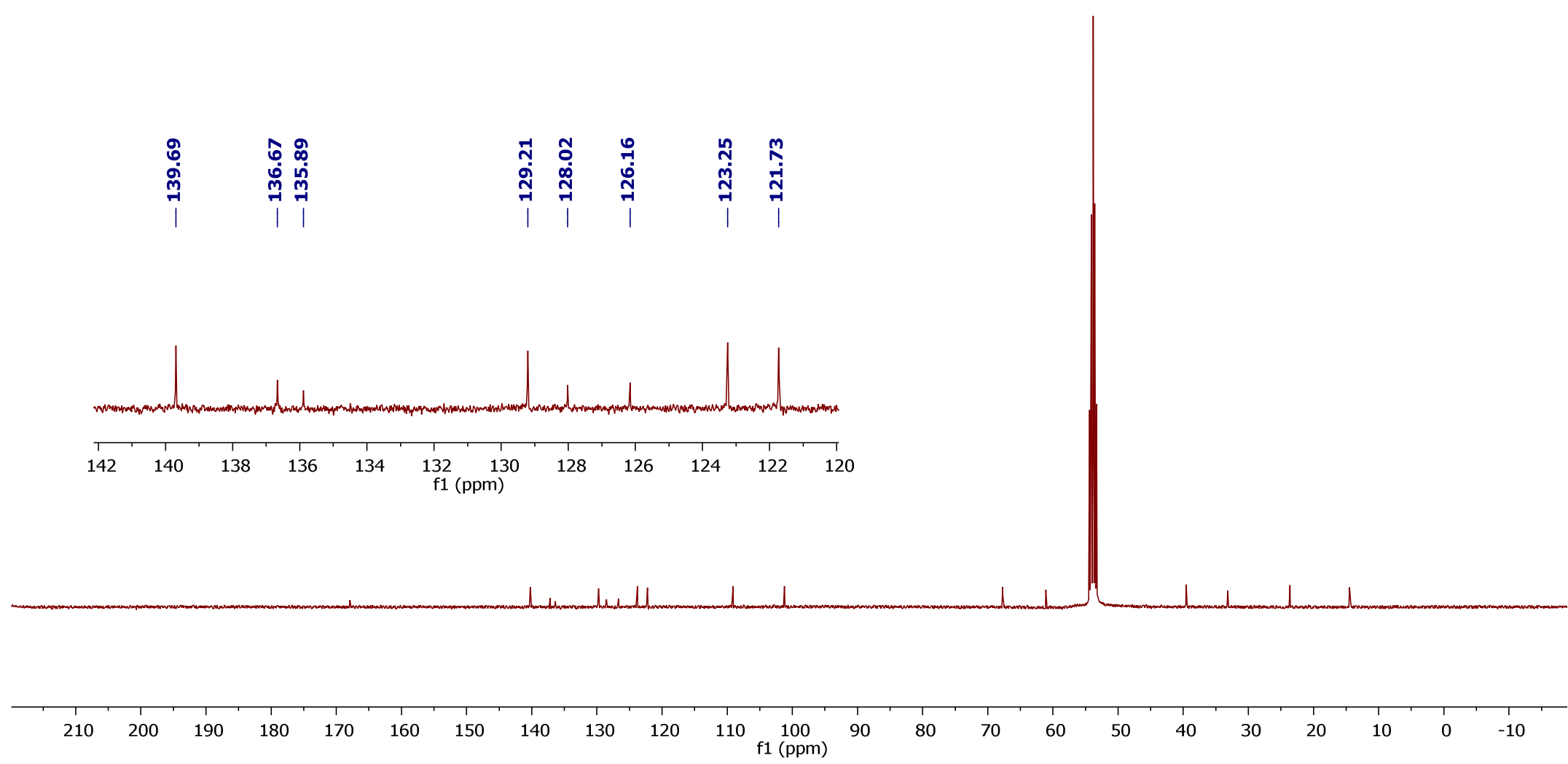




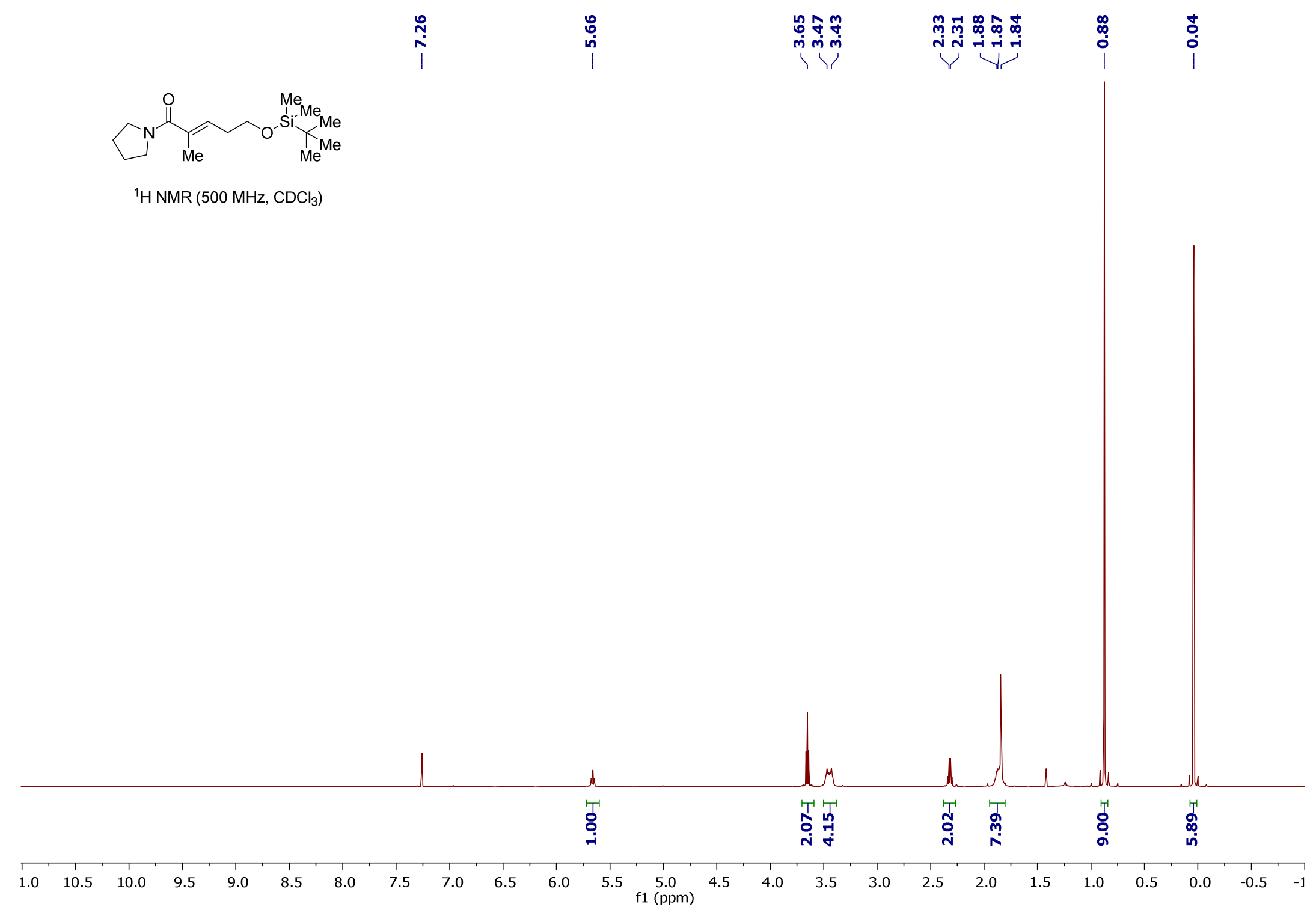



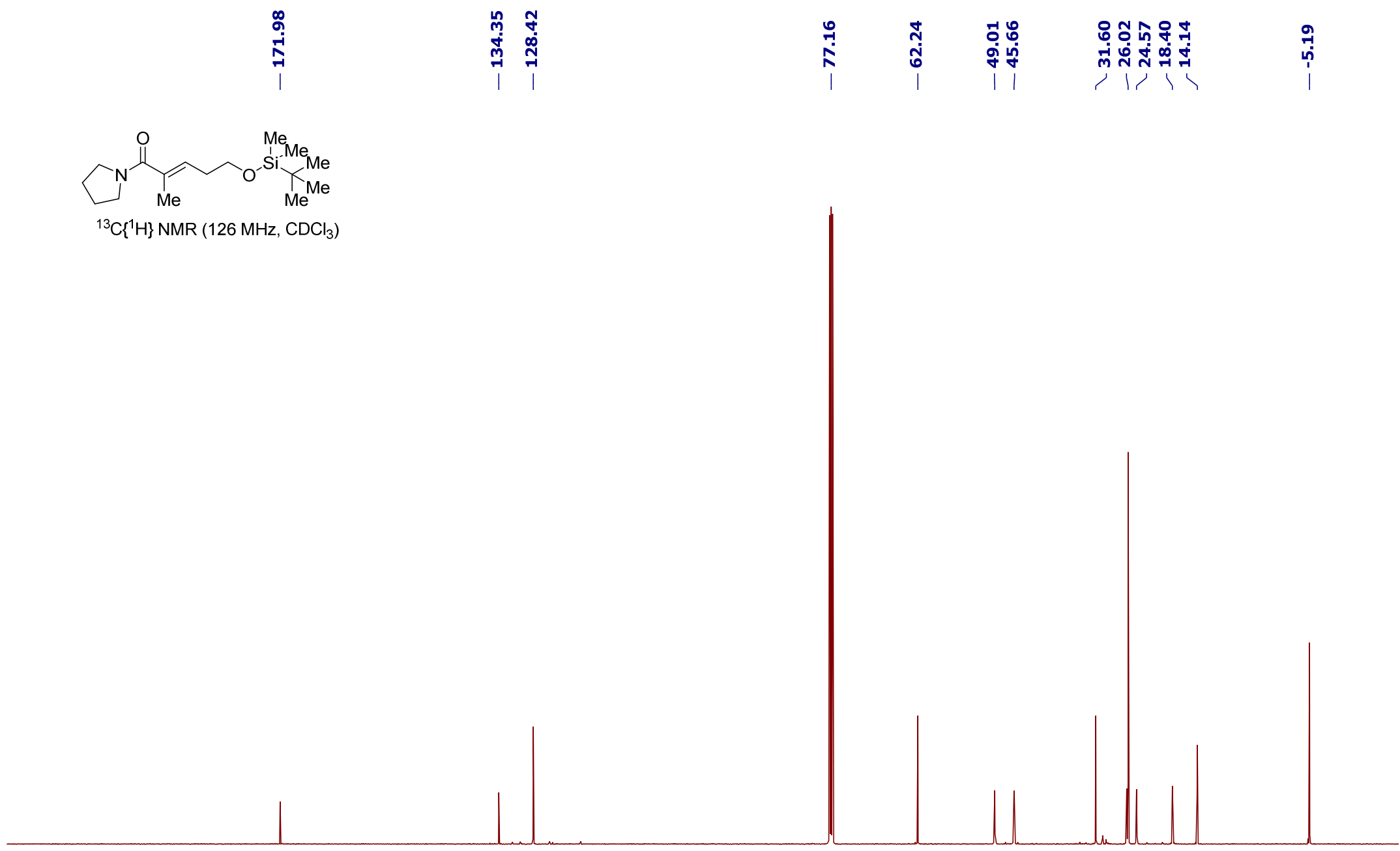

\begin{tabular}{llllllllllllllllllllllll}
\hline 210 & 200 & 190 & 180 & 170 & 160 & 150 & 140 & 130 & 120 & 110 & $\begin{array}{c}100 \\
\mathrm{f} 1(\mathrm{ppm})\end{array}$ & 90 & 80 & 70 & 60 & 50 & 40 & 30 & 20 & 10 & 0 & -10 & -2
\end{tabular} 


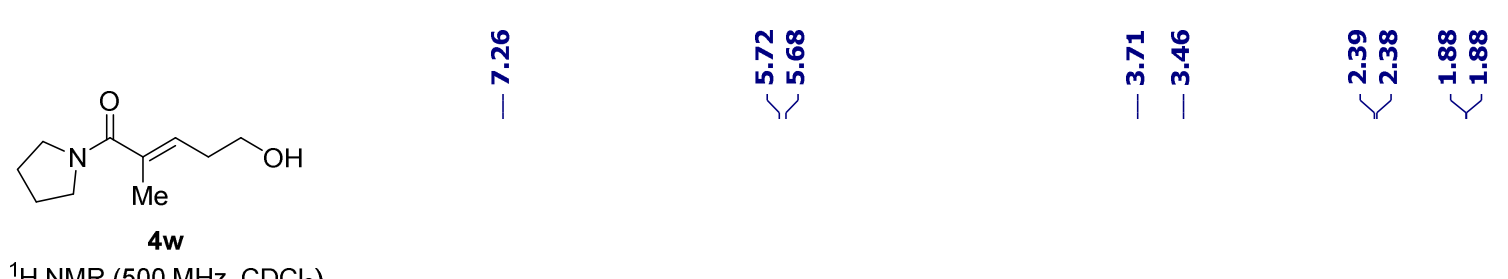

${ }^{1} \mathrm{H}$ NMR $\left(500 \mathrm{MHz}, \mathrm{CDCl}_{3}\right)$

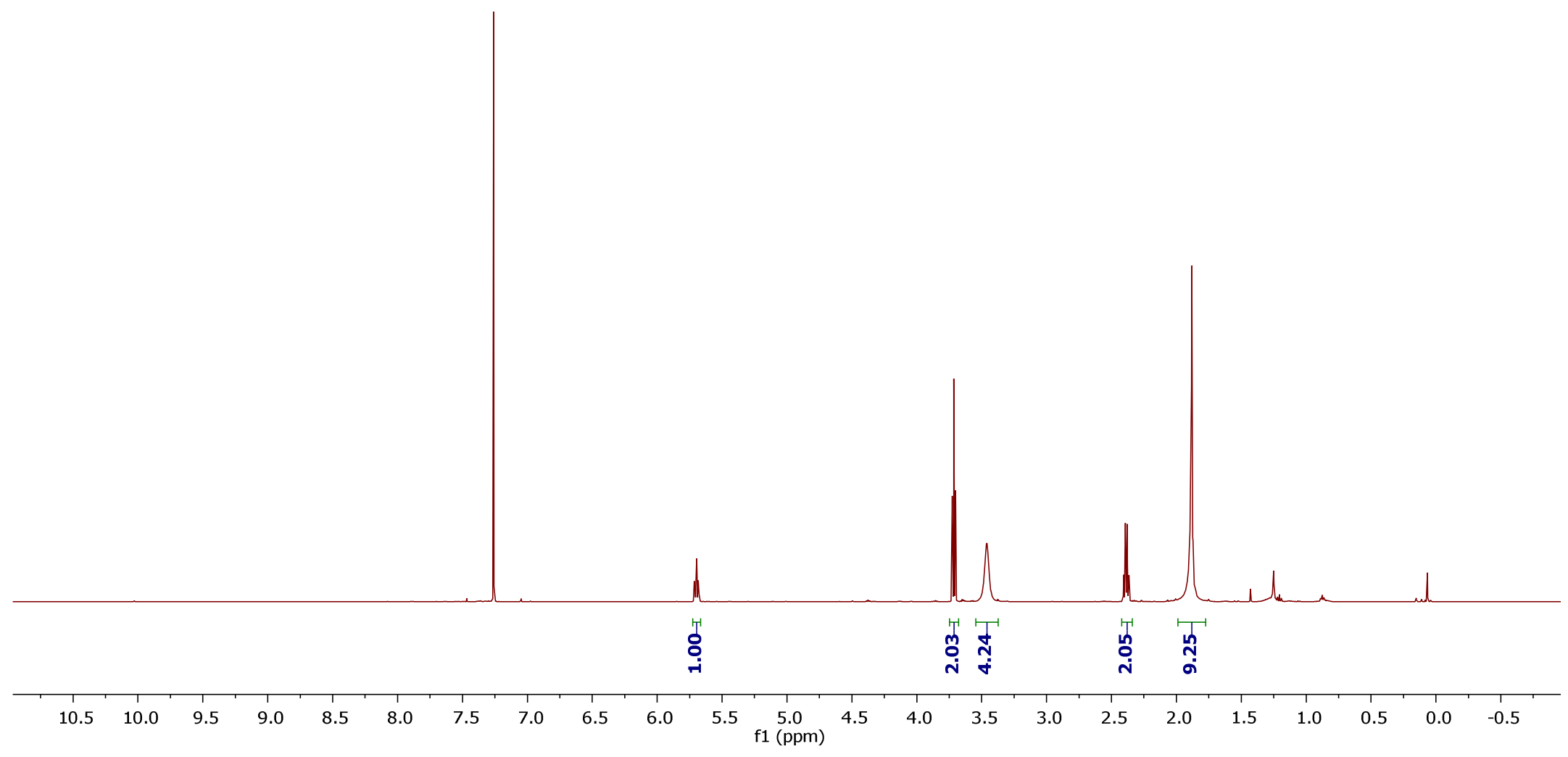




$$
\text { ..... }
$$




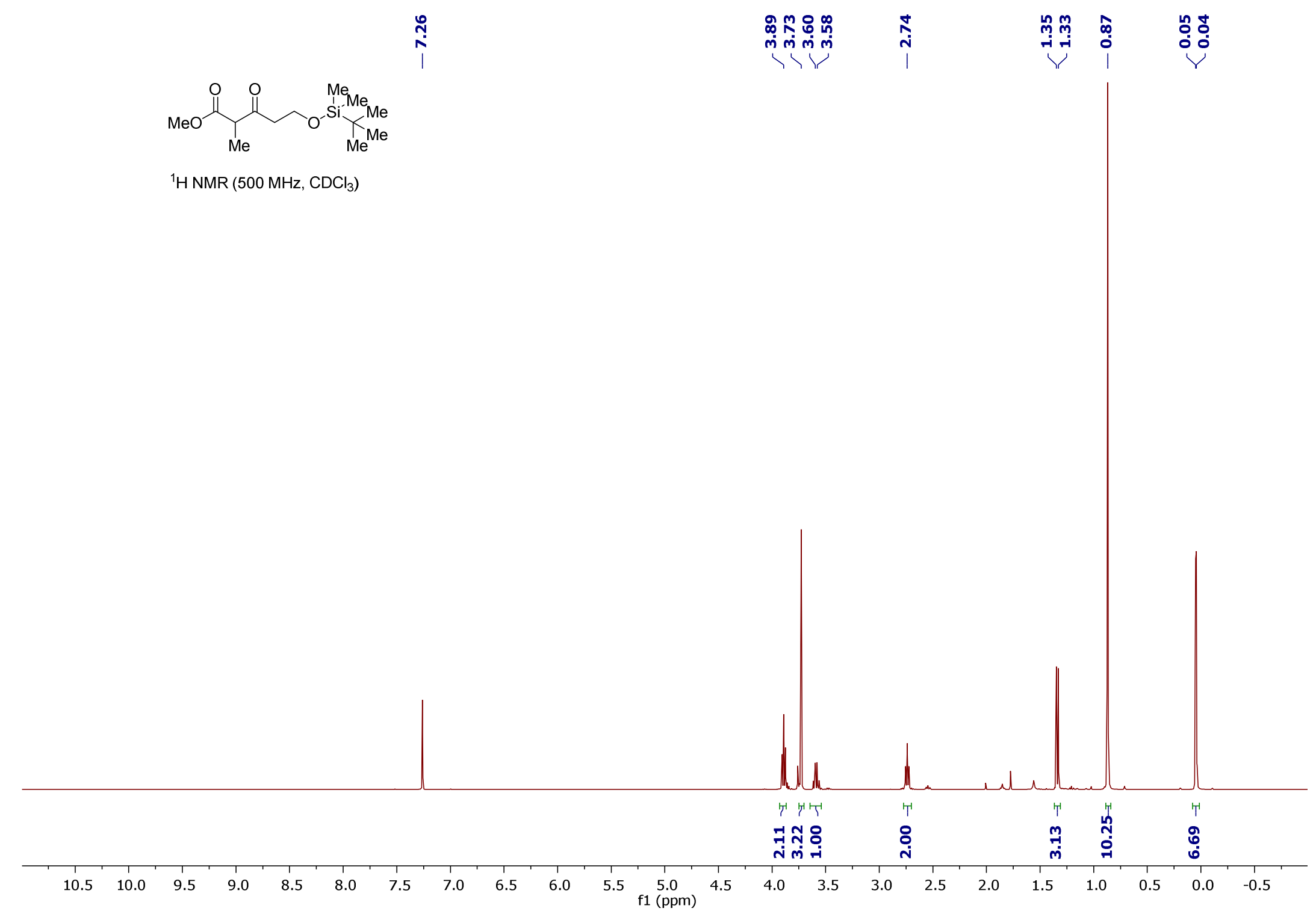




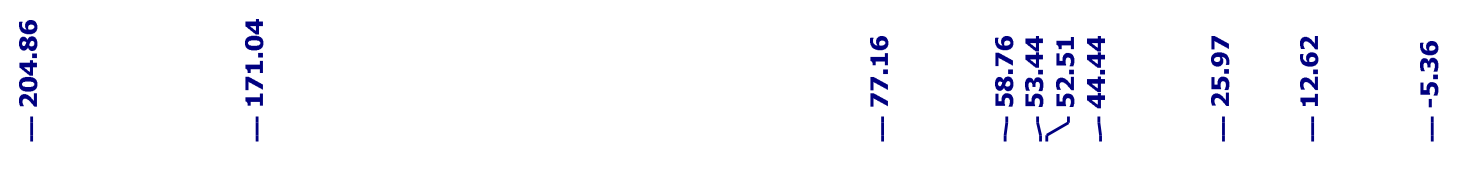

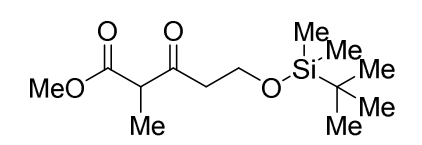

$\left.{ }^{13} \mathrm{C}^{1} \mathrm{H}\right\} \mathrm{NMR}\left(126 \mathrm{MHz}, \mathrm{CDCl}_{3}\right)$

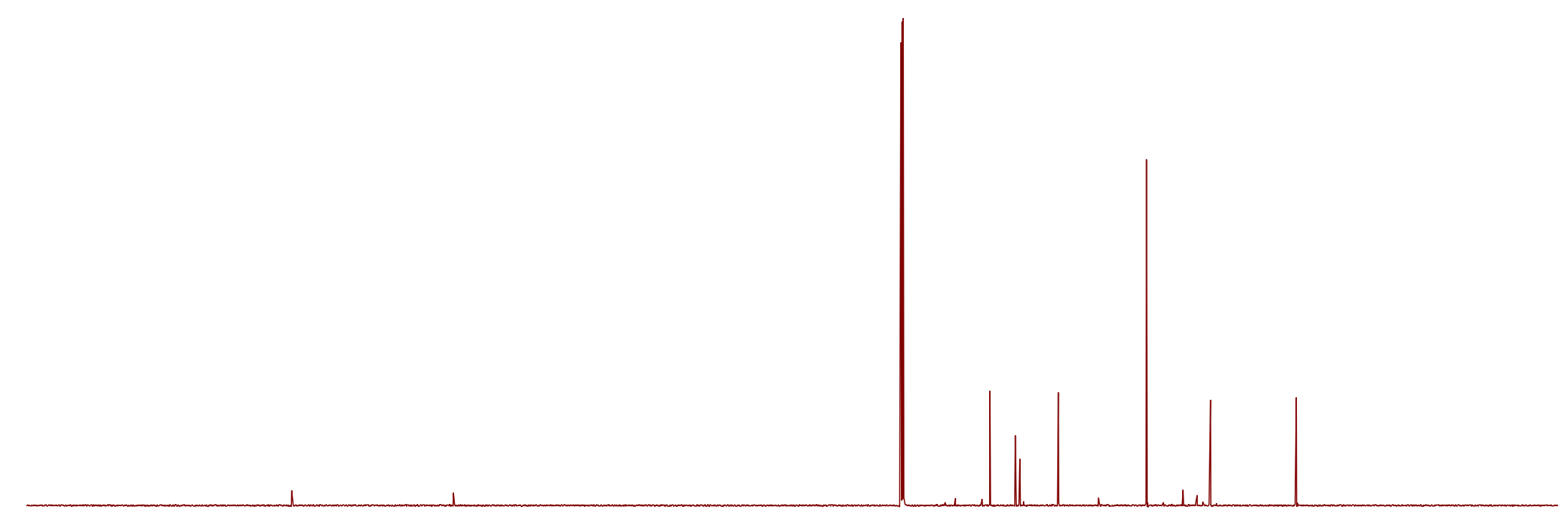

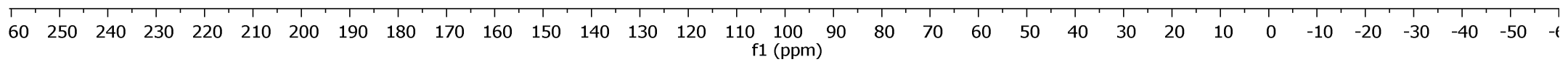




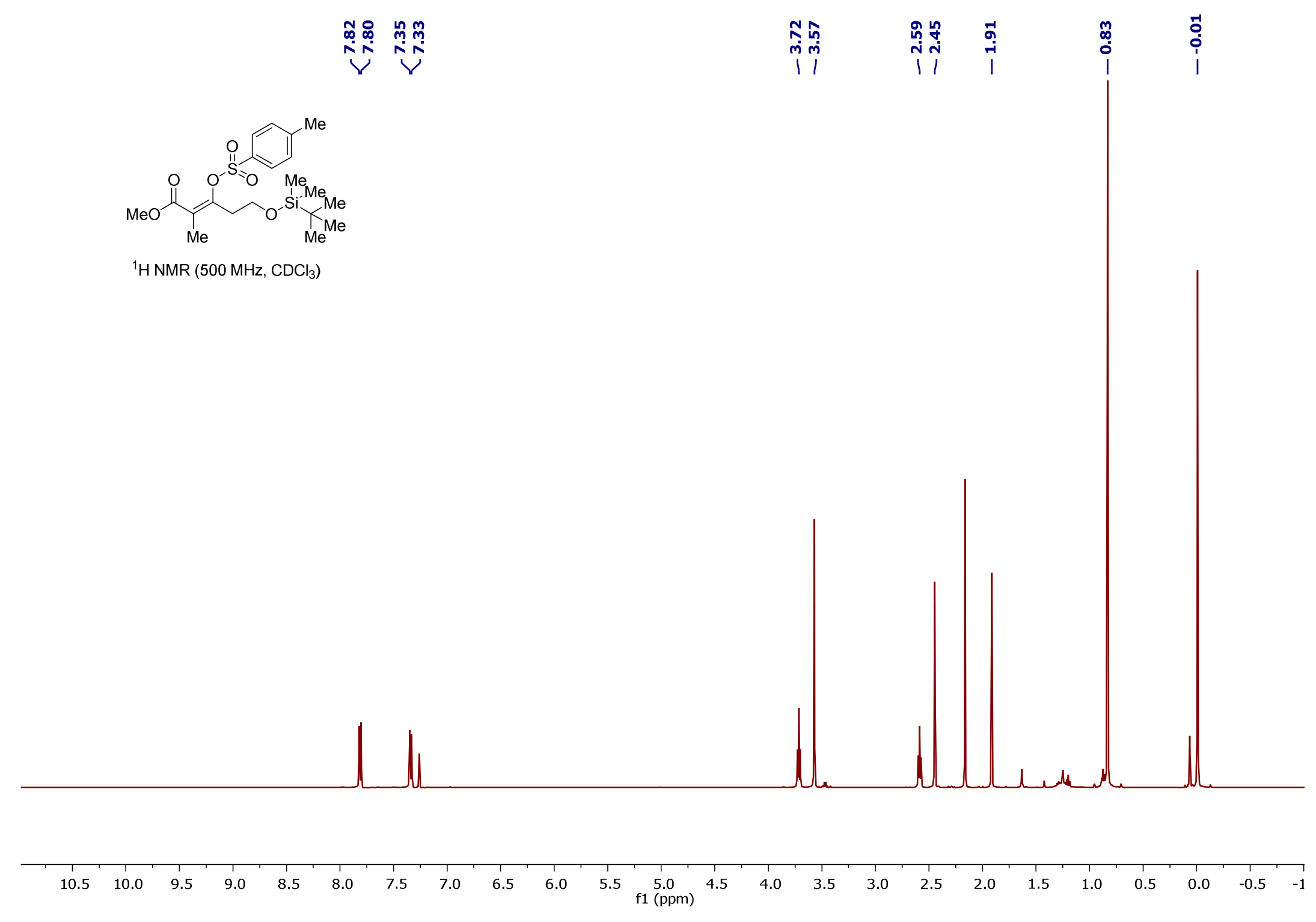



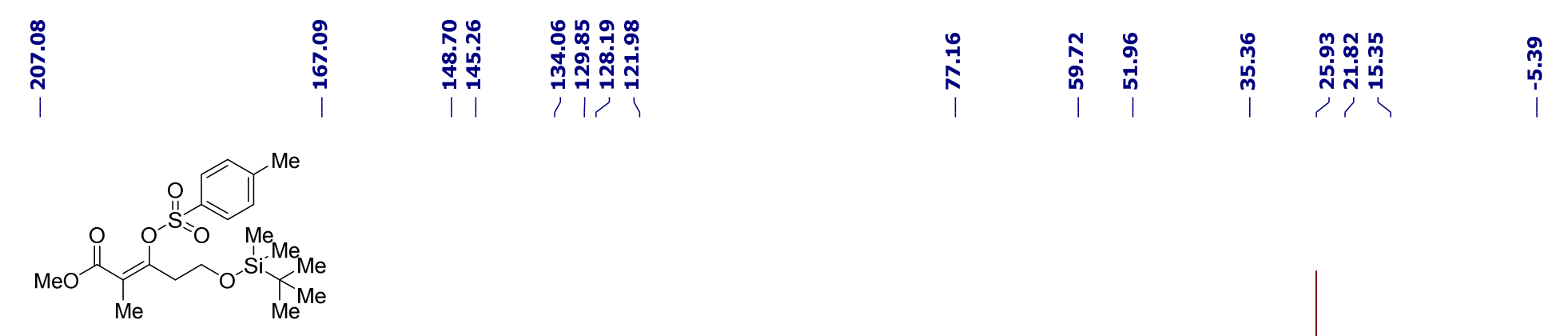

$\left.{ }^{13} \mathrm{C}^{1} \mathrm{H}\right\} \mathrm{NMR}\left(126 \mathrm{MHz}, \mathrm{CDCl}_{3}\right)$

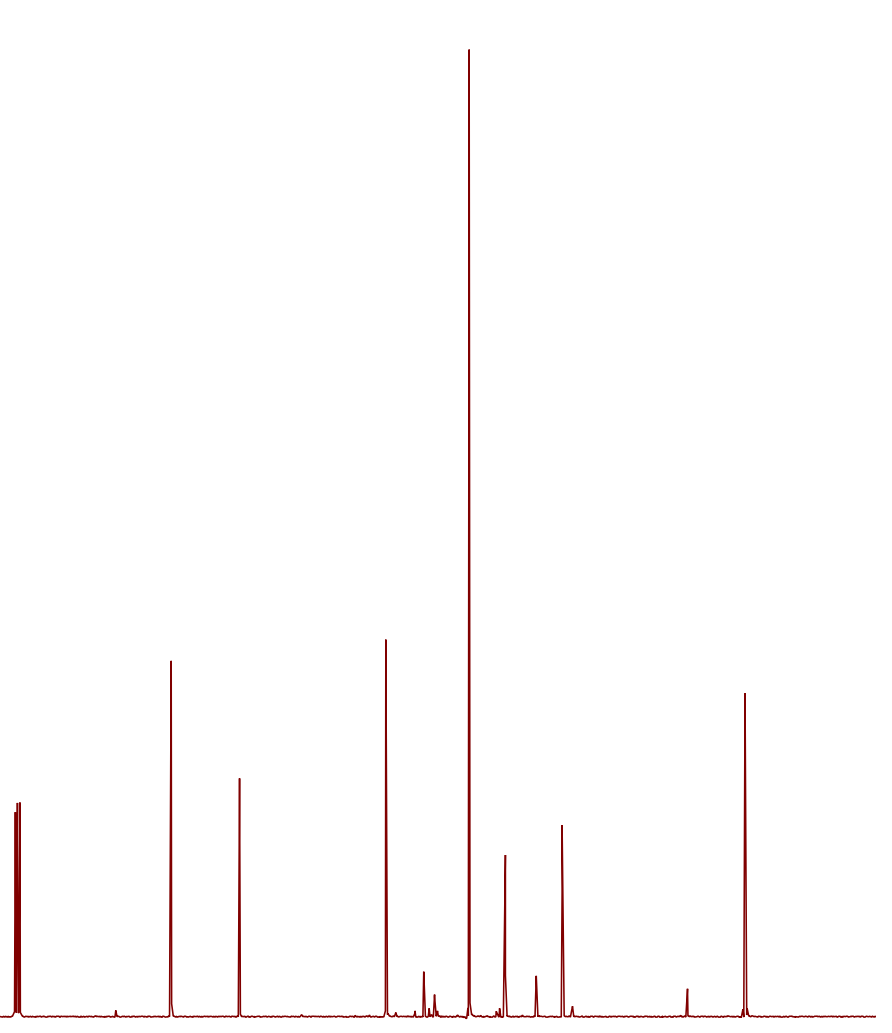

\begin{tabular}{rlllllllllllllllllllllllllll}
\hline 220 & 210 & 200 & 190 & 180 & 170 & 160 & 150 & 140 & 130 & 120 & 110 & 100 & 90 & 80 & 70 & 60 & 50 & 40 & 30 & 20 & 10 & 0 & -10 & --2 \\
\hline
\end{tabular} 


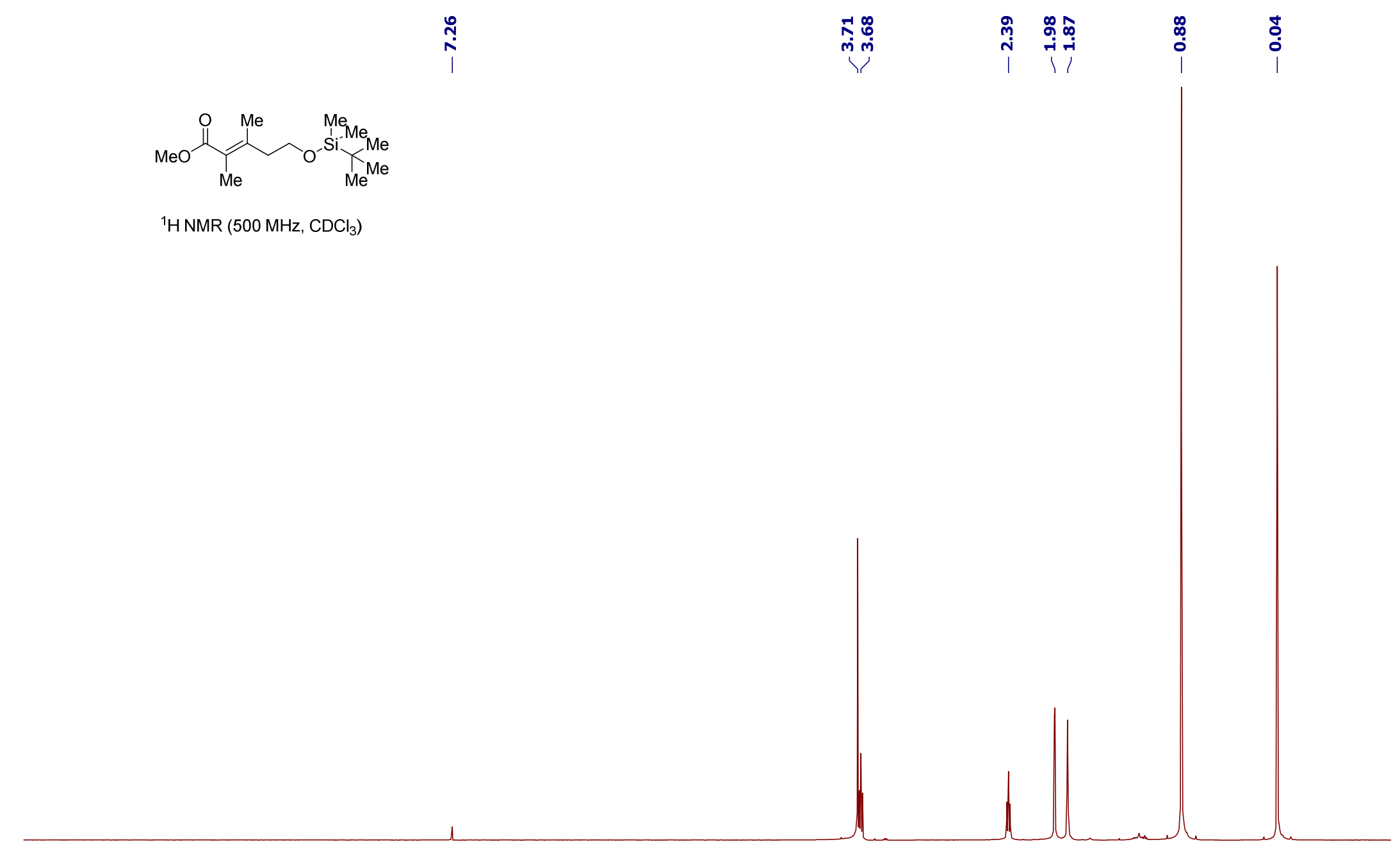

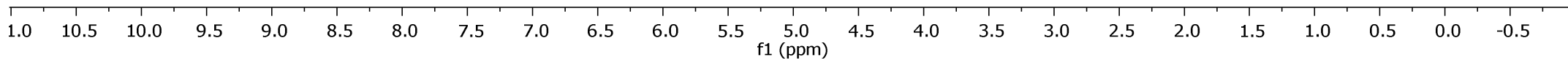




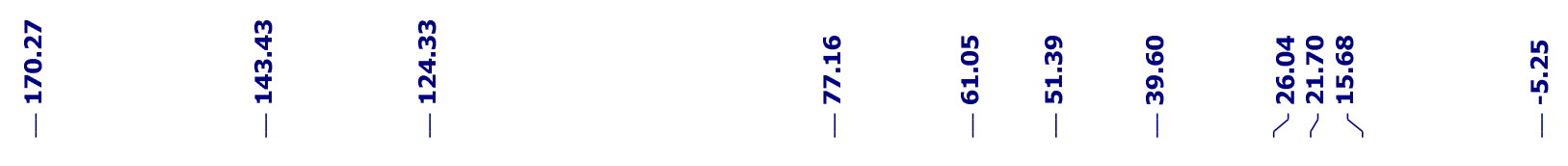

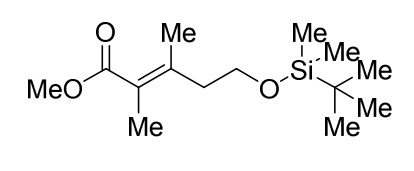

${ }^{13} \mathrm{C}\left\{{ }^{1} \mathrm{H}\right\} \mathrm{NMR}\left(126 \mathrm{MHz}, \mathrm{CDCl}_{3}\right)$

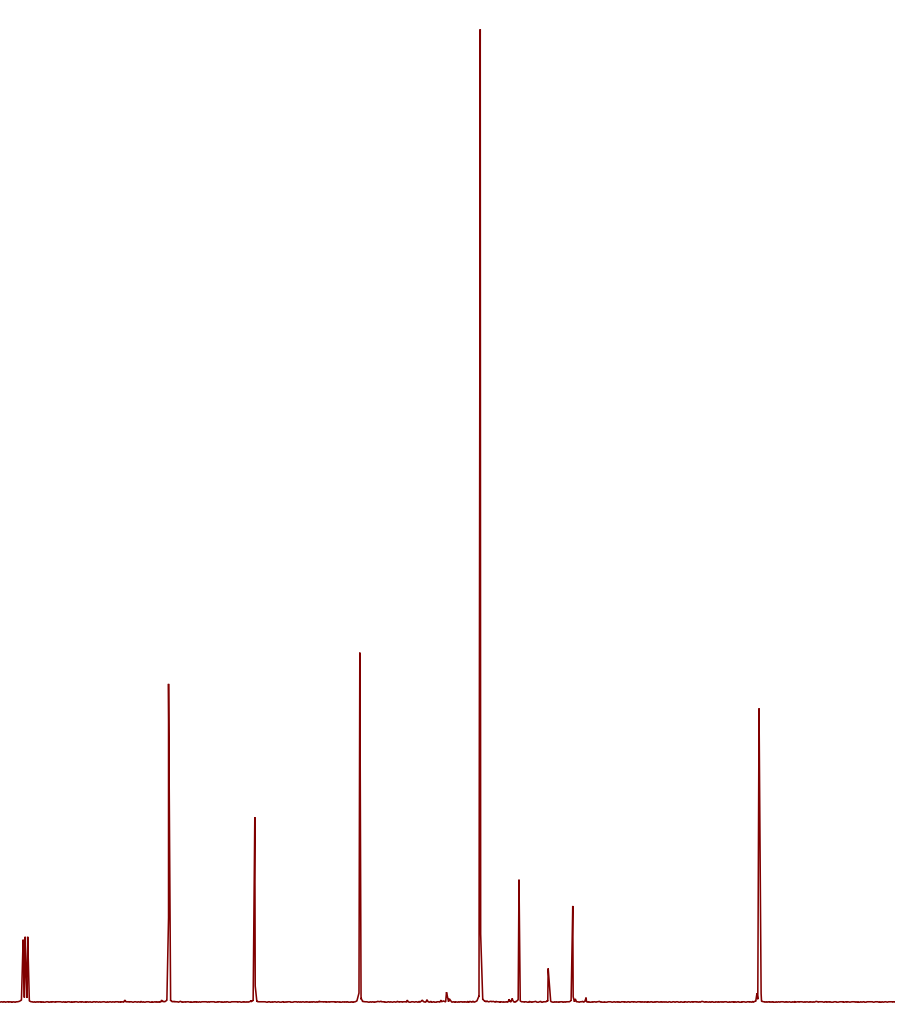

$\begin{array}{lllllllllllllllllllllllllll}1 & 1 \\ 210 & 200 & 190 & 180 & 170 & 160 & 150 & 140 & 130 & 120 & 110 & 100 & 90 & 80 & 70 & 60 & 50 & 40 & 30 & 20 & 10 & 0 & -10 & -2\end{array}$ 
$\overbrace{\mathrm{Me}}^{\mathrm{Me}}$

$4 \mathrm{x}$

${ }^{1} \mathrm{H} \mathrm{NMR}\left(500 \mathrm{MHz}, \mathrm{C}_{6} \mathrm{D}_{6}\right)$

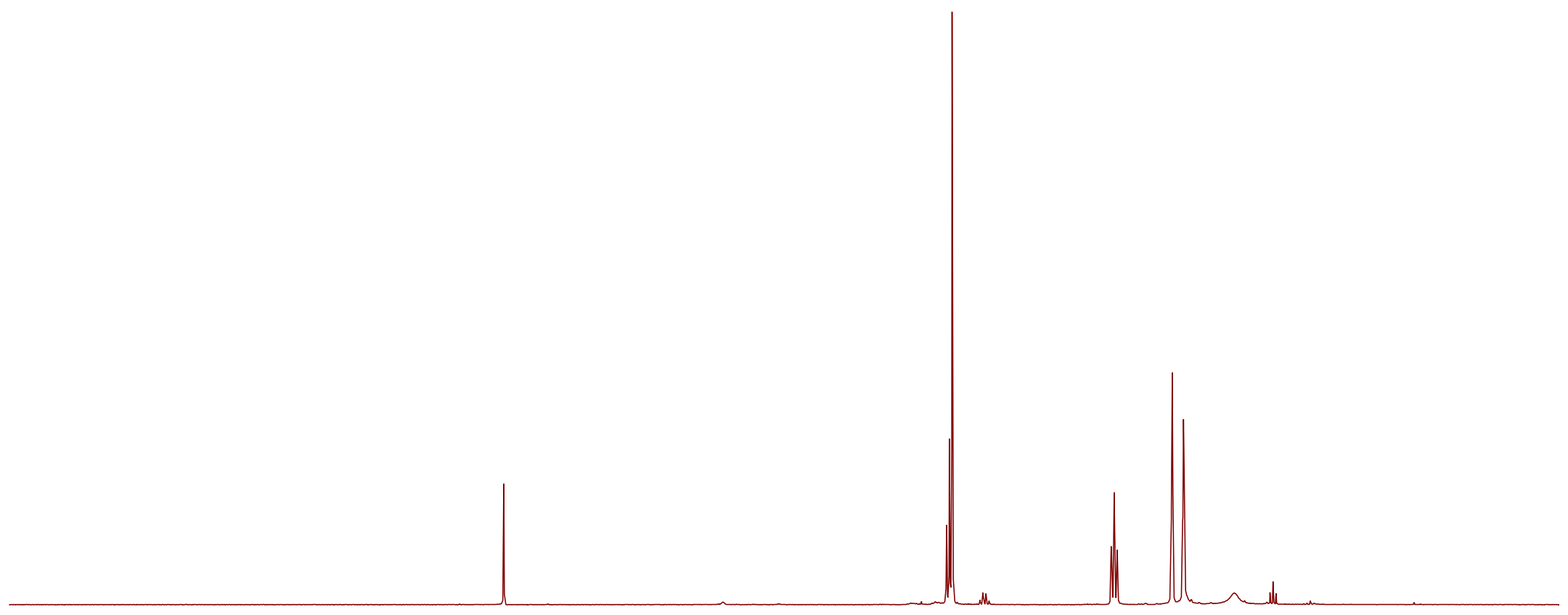

\begin{tabular}{llllllllllllllllllllllllllllllllll}
\hline 1.0 & 10.5 & 10.0 & 9.5 & 9.0 & 8.5 & 8.0 & 7.5 & 7.0 & 6.5 & 6.0 & 5.5 & $\begin{array}{c}5 \\
\mathrm{f} 1(\mathrm{ppm})\end{array}$ & 4.5 & 4.0 & 3.5 & 3.0 & 2.5 & 2.0 & 1.5 & 1.0 & 0.5 & 0.0 & -0.5 & -1.0
\end{tabular} 


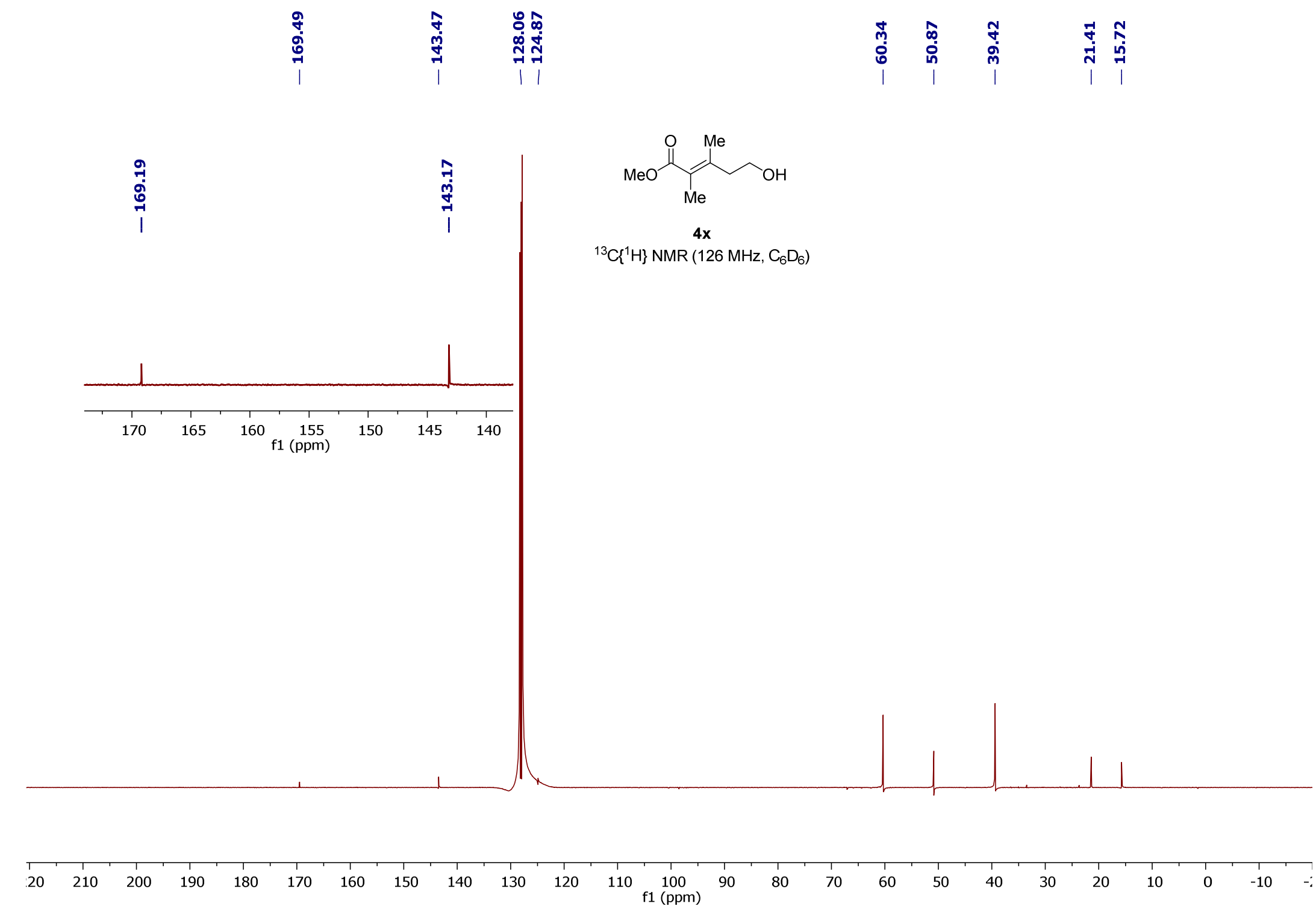




$$
\text { กู่ }
$$

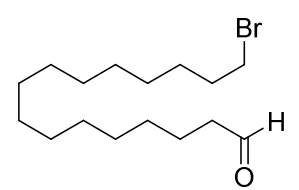

${ }^{1} \mathrm{H} \mathrm{NMR}\left(400 \mathrm{MHz}, \mathrm{CDCl}_{3}\right)$

$$
\text { 皇 }
$$

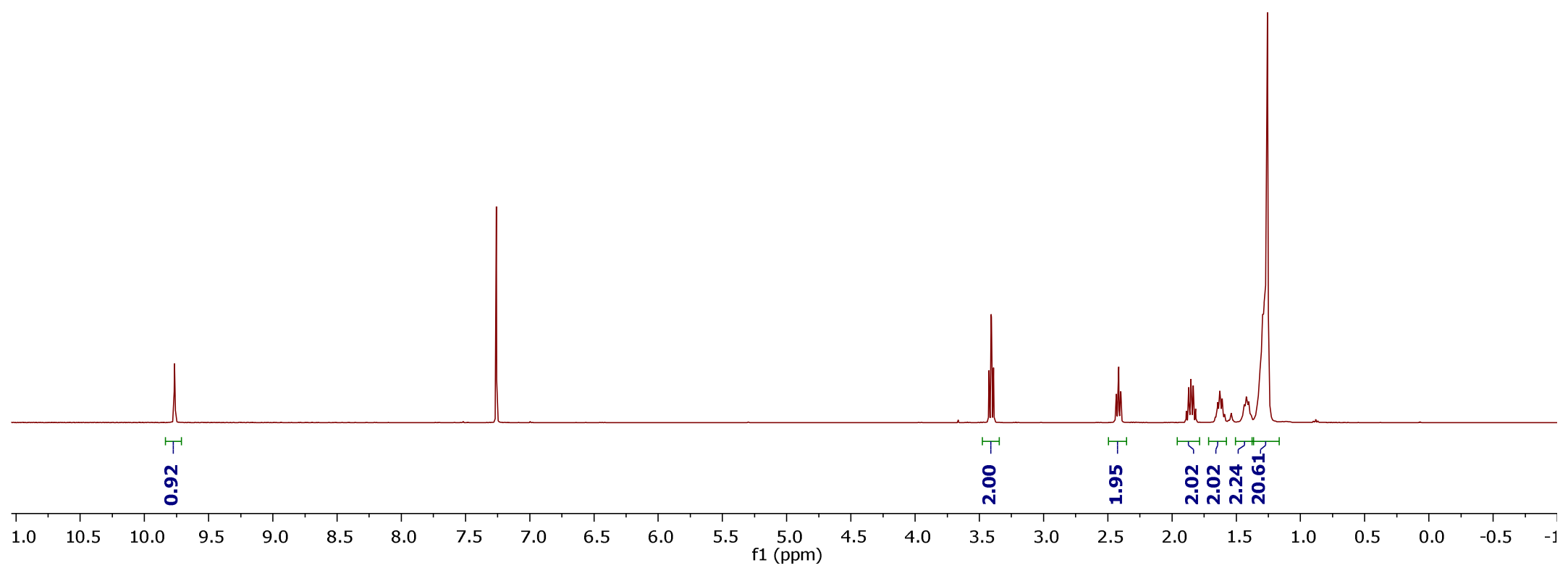




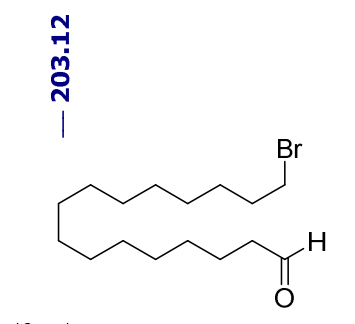

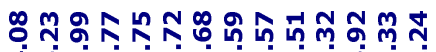

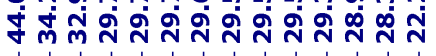

$\left.{ }^{13} \mathrm{C}^{1} \mathrm{H}\right\} \mathrm{NMR}\left(101 \mathrm{MHz}, \mathrm{CDCl}_{3}\right)$

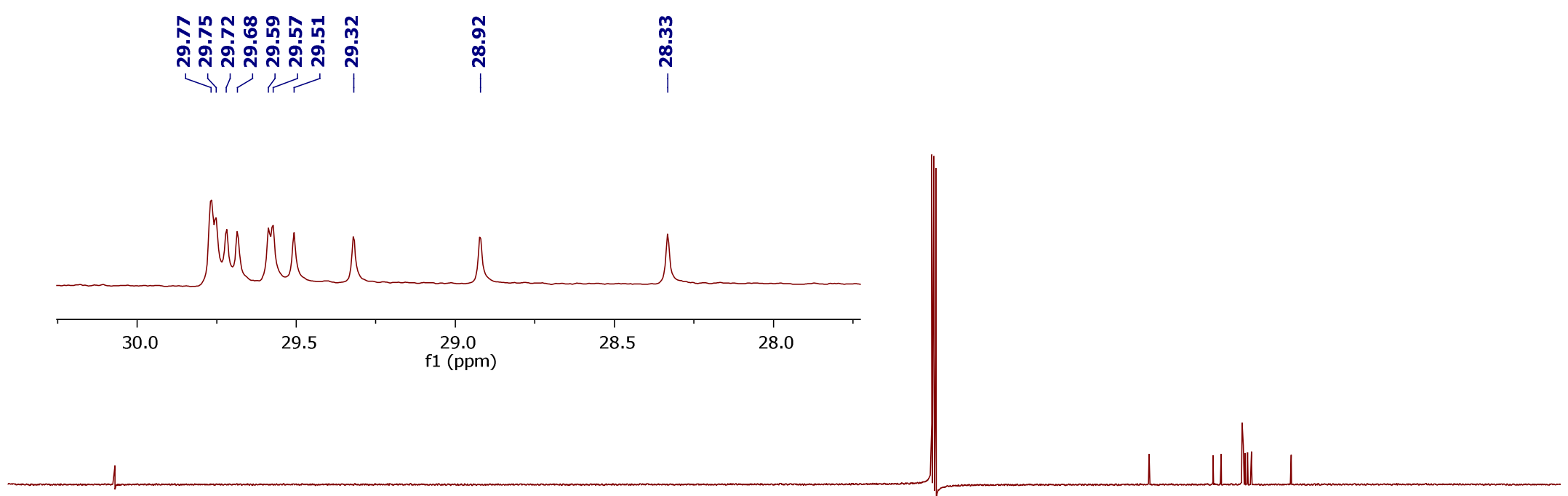

$\begin{array}{llllllllllll}210 & 200 & 190 & 180 & 170 & 160 & 150 & 140 & 130 & 120 & 110 & 100\end{array}$ 100
$\mathrm{f} 1(\mathrm{ppm})$ $80 \quad 70$ 


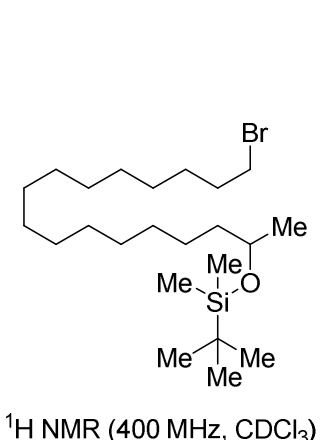

$\stackrel{\stackrel{p}{m}}{\stackrel{F}{\sim}}$

苟

$\left.{ }^{1} \mathrm{H} \mathrm{NMR} \mathrm{(400} \mathrm{MHz,} \mathrm{CDCl}_{3}\right)$

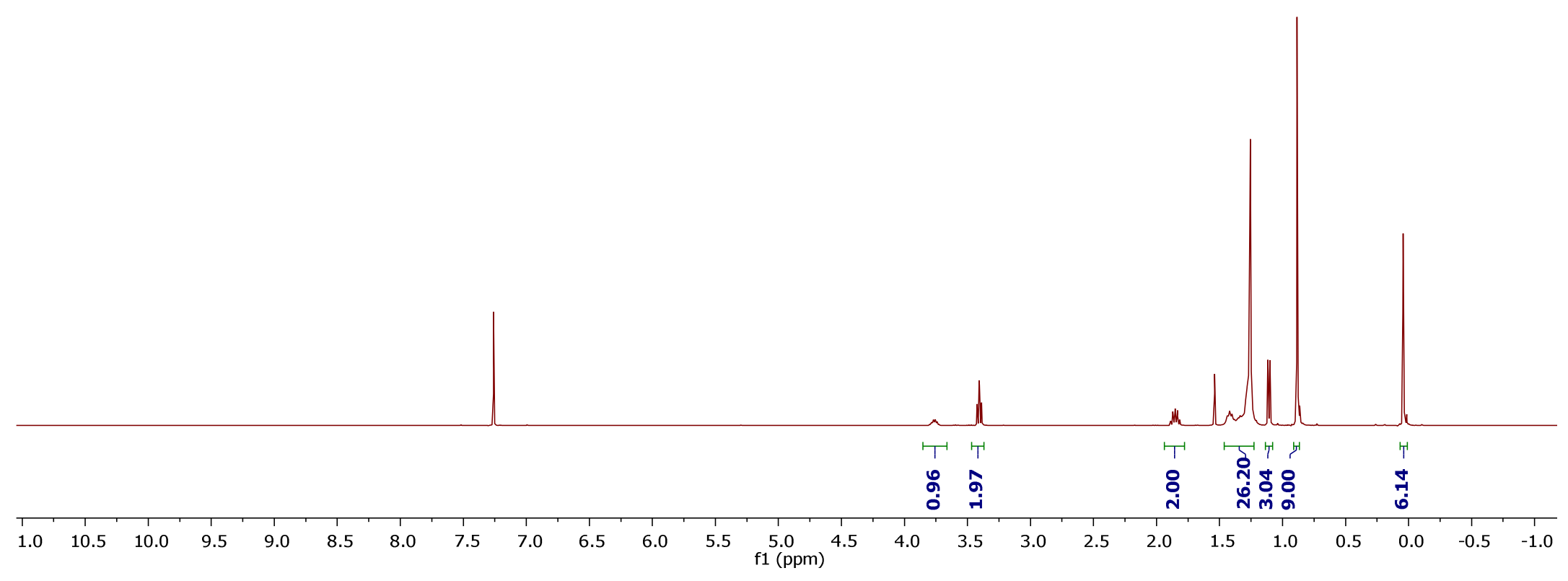




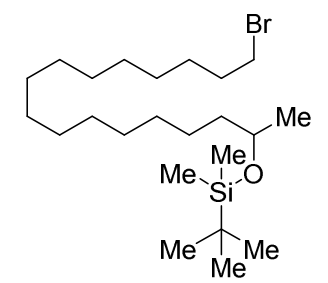

䓹

$\left.{ }^{13} \mathrm{C}^{1} \mathrm{H}\right\} \mathrm{NMR}\left(101 \mathrm{MHz}, \mathrm{CDCl}_{3}\right)$

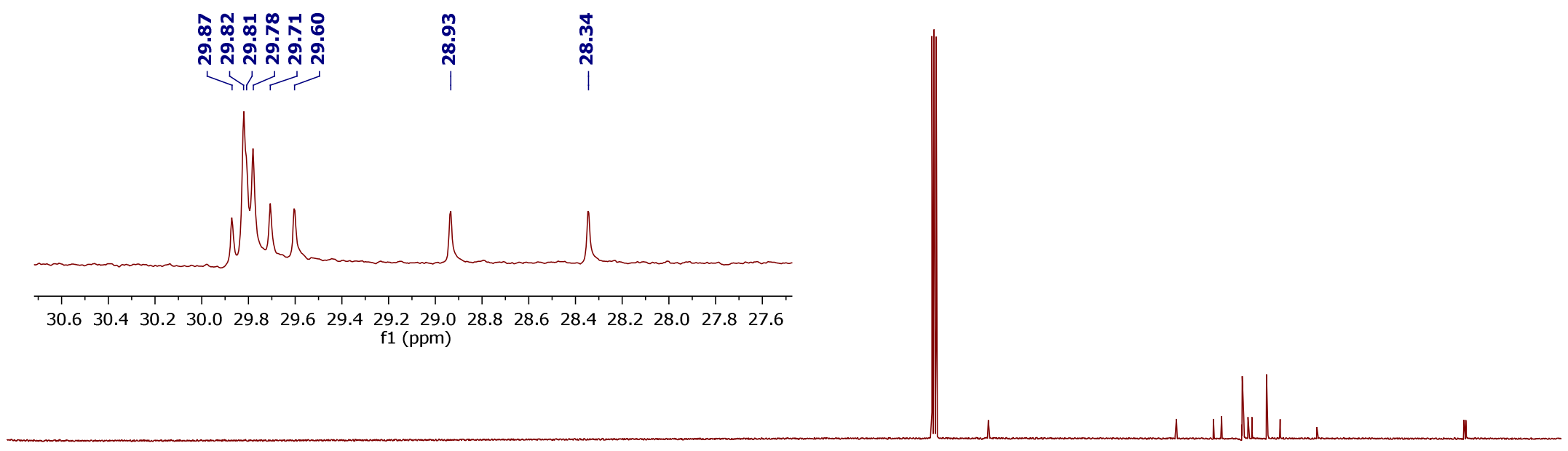

$\begin{array}{llllllllllll}210 & 200 & 190 & 180 & 170 & 160 & 150 & 140 & 130 & 120 & 110 & 100\end{array}$ f1 $(\mathrm{ppm})$ 8070 


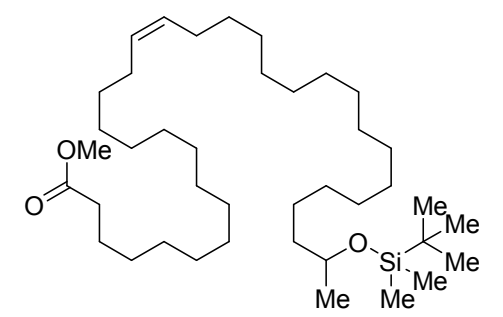

${ }^{1} \mathrm{H} \mathrm{NMR}\left(400 \mathrm{MHz}, \mathrm{CDCl}_{3}\right)$

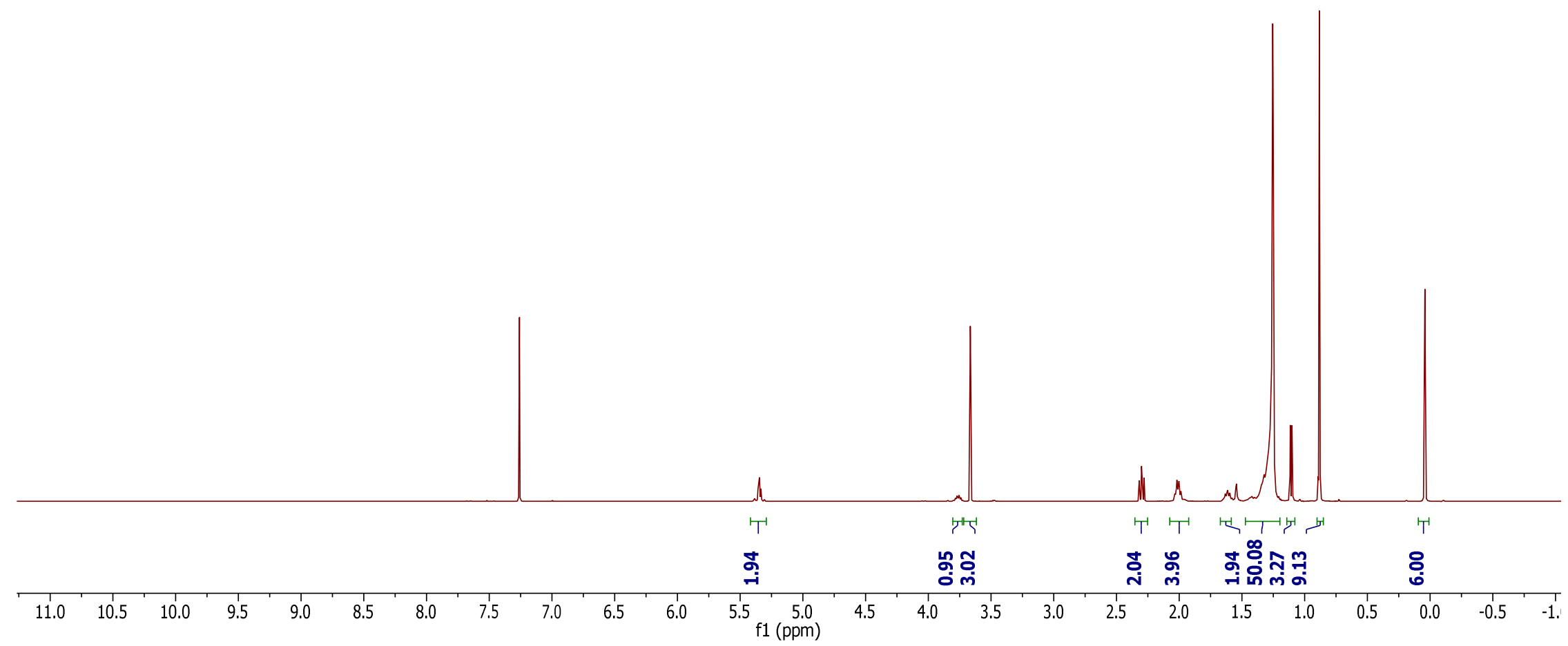




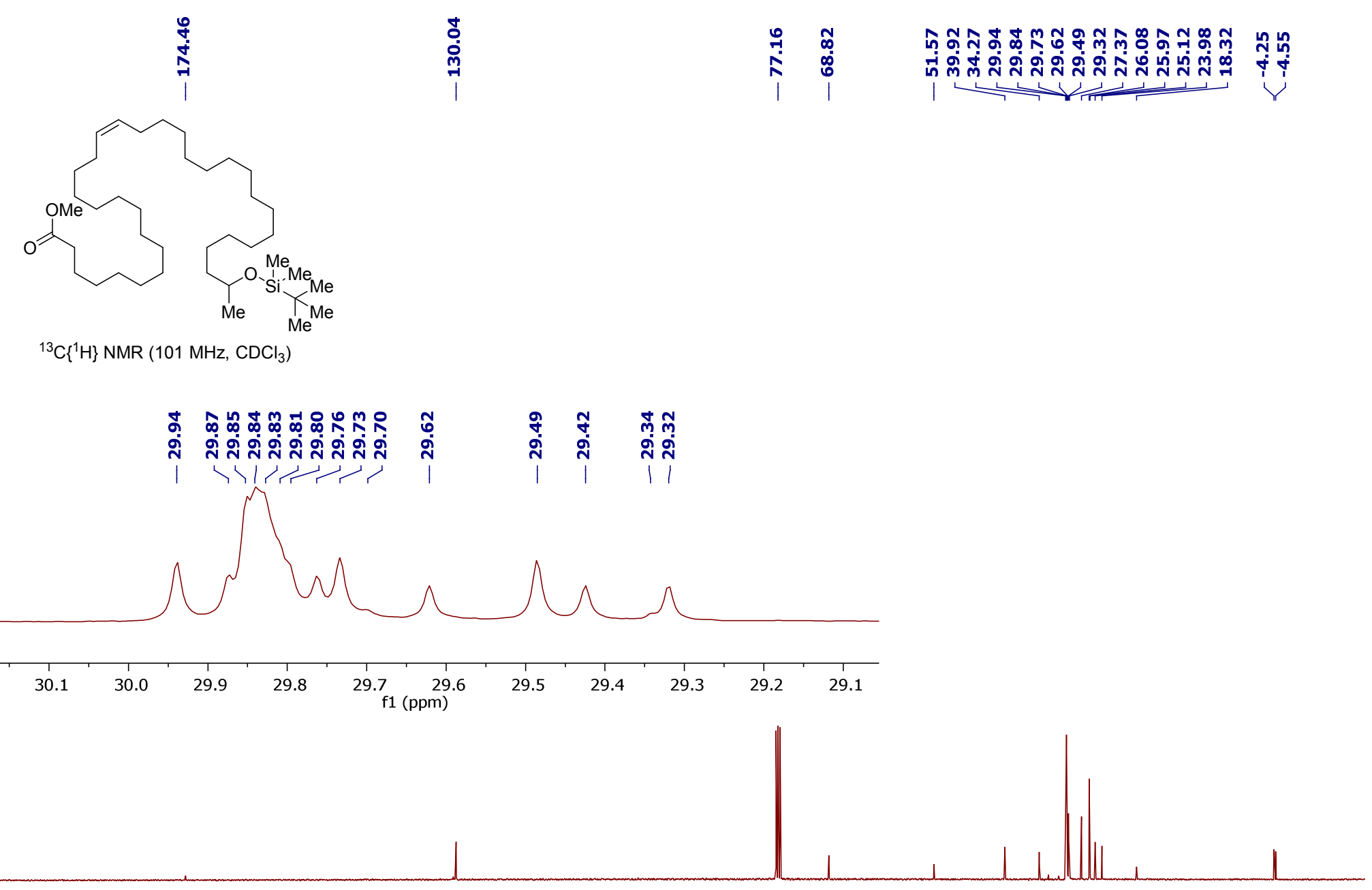

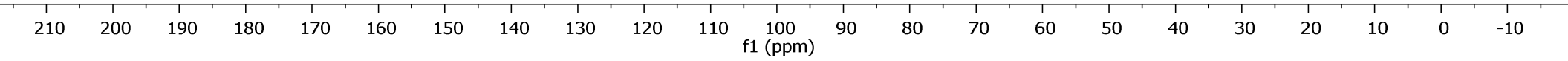




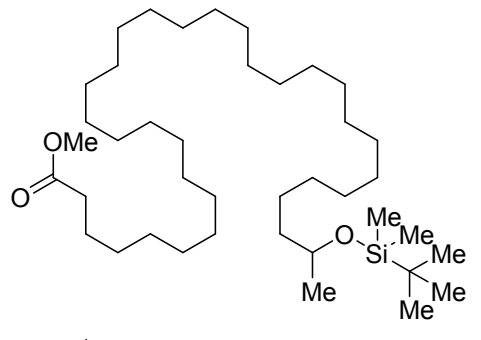

${ }^{1} \mathrm{H}$ NMR (400 MHz, $\mathrm{CDCl}_{3}$ )

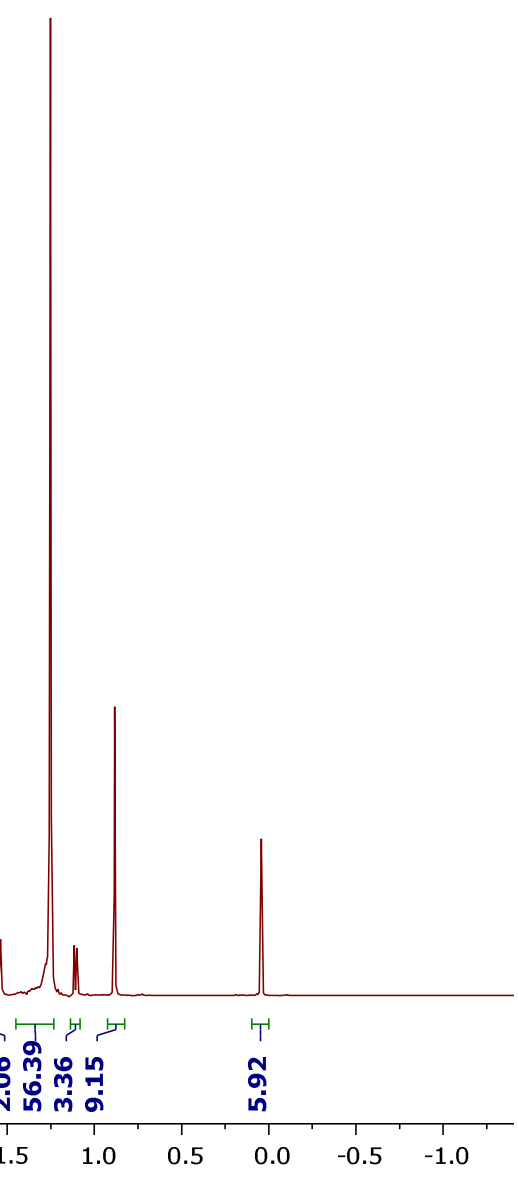

$\begin{array}{llllllllllll}10.5 & 10.0 & 9.5 & 9.0 & 8.5 & 8.0 & 7.5 & 7.0 & 6.5 & 6.0 & 5.5 & 5.0 \\ \mathrm{f} 1(\mathrm{ppm}) & 4.5\end{array}$ 


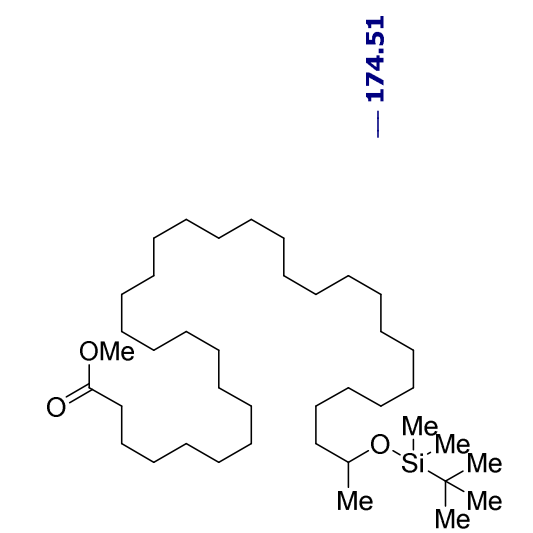

${ }^{13} \mathrm{C}\left\{{ }^{1} \mathrm{H}\right\} \mathrm{NMR}\left(101 \mathrm{MHz}, \mathrm{CDCl}_{3}\right)$

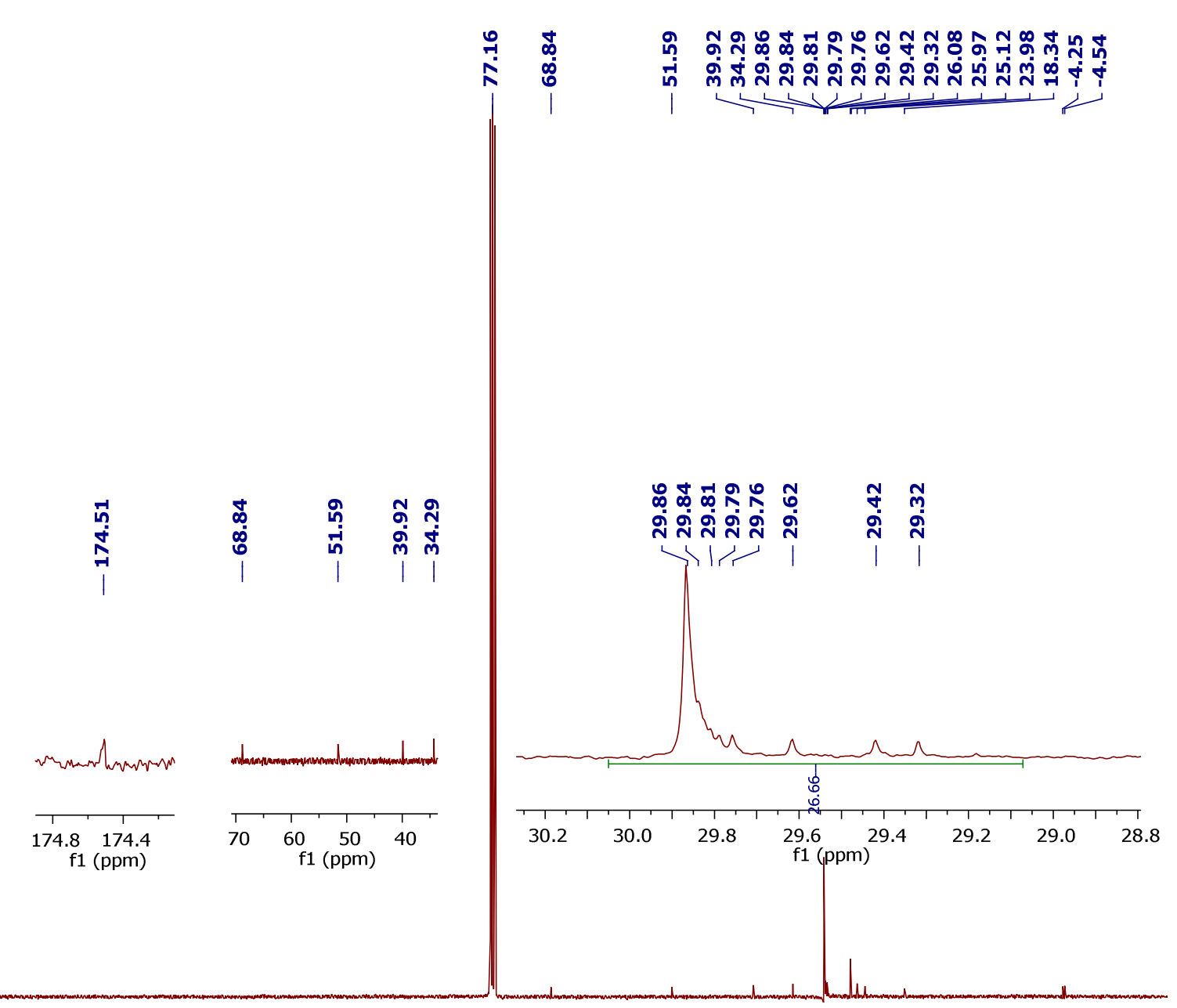

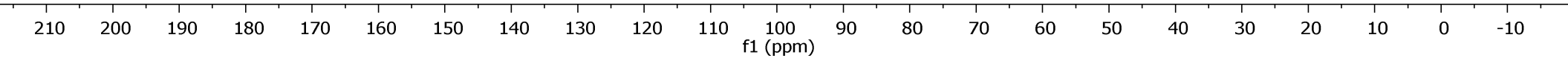




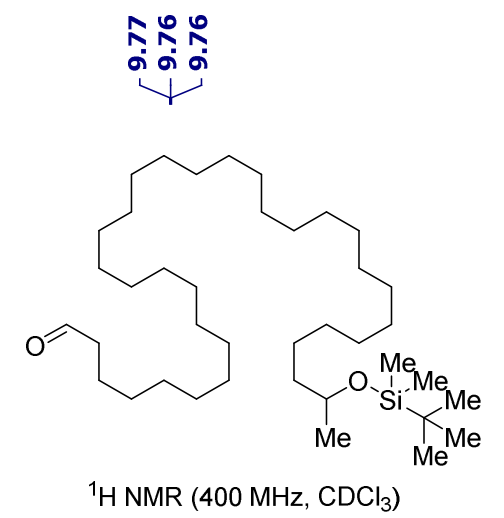

H NMR $\left(400 \mathrm{MHz}, \mathrm{CDCl}_{3}\right)$

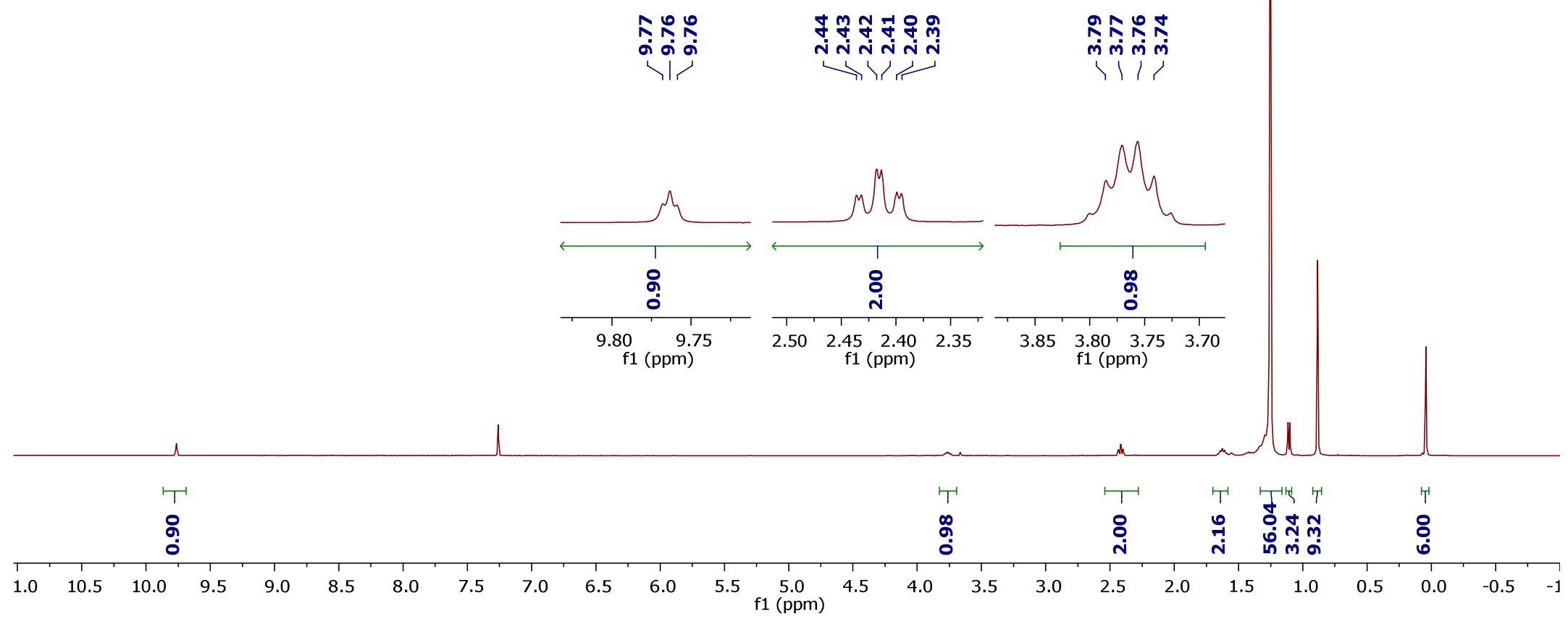




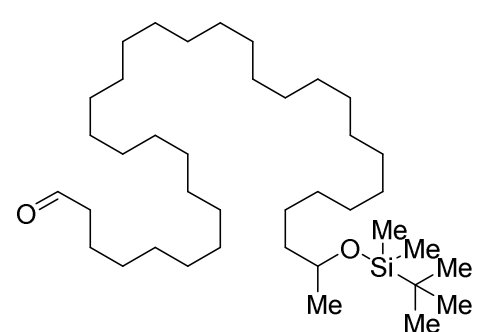

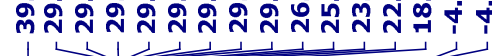

${ }^{13} \mathrm{C}\left\{{ }^{1} \mathrm{H}\right\} \mathrm{NMR}\left(101 \mathrm{MHz}, \mathrm{CDCl}_{3}\right)$

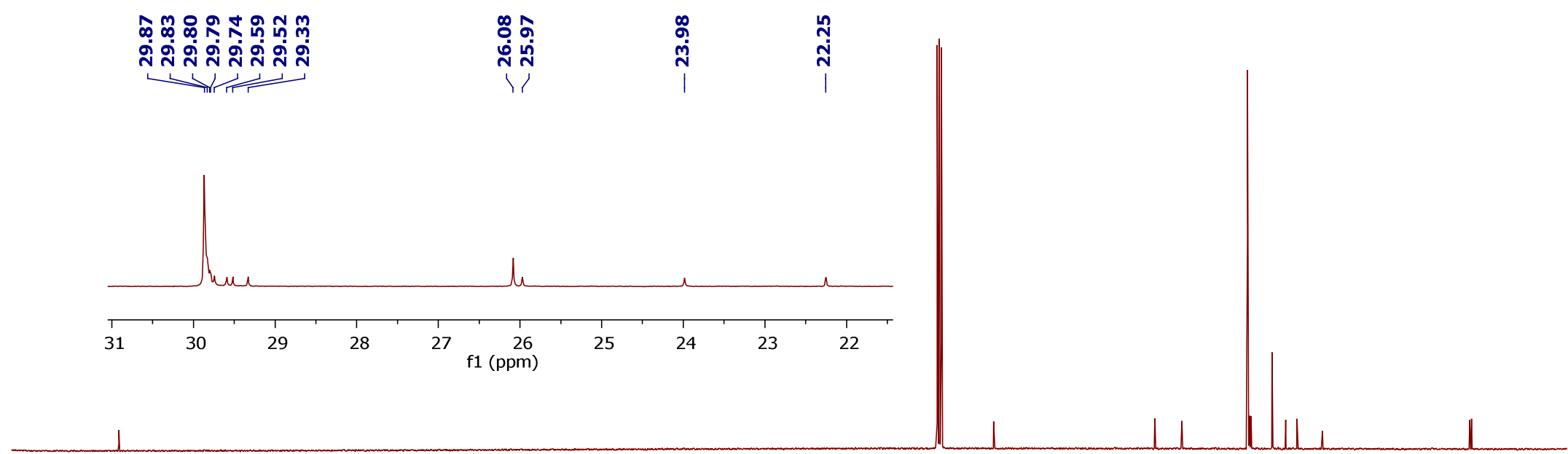

$\begin{array}{llllllllllll}210 & 200 & 190 & 180 & 170 & 160 & 150 & 140 & 130 & 120 & 110 & 100\end{array}$ 100
$\mathrm{f} 1(\mathrm{ppm})$ 8070 60 $50 \quad 40$ 


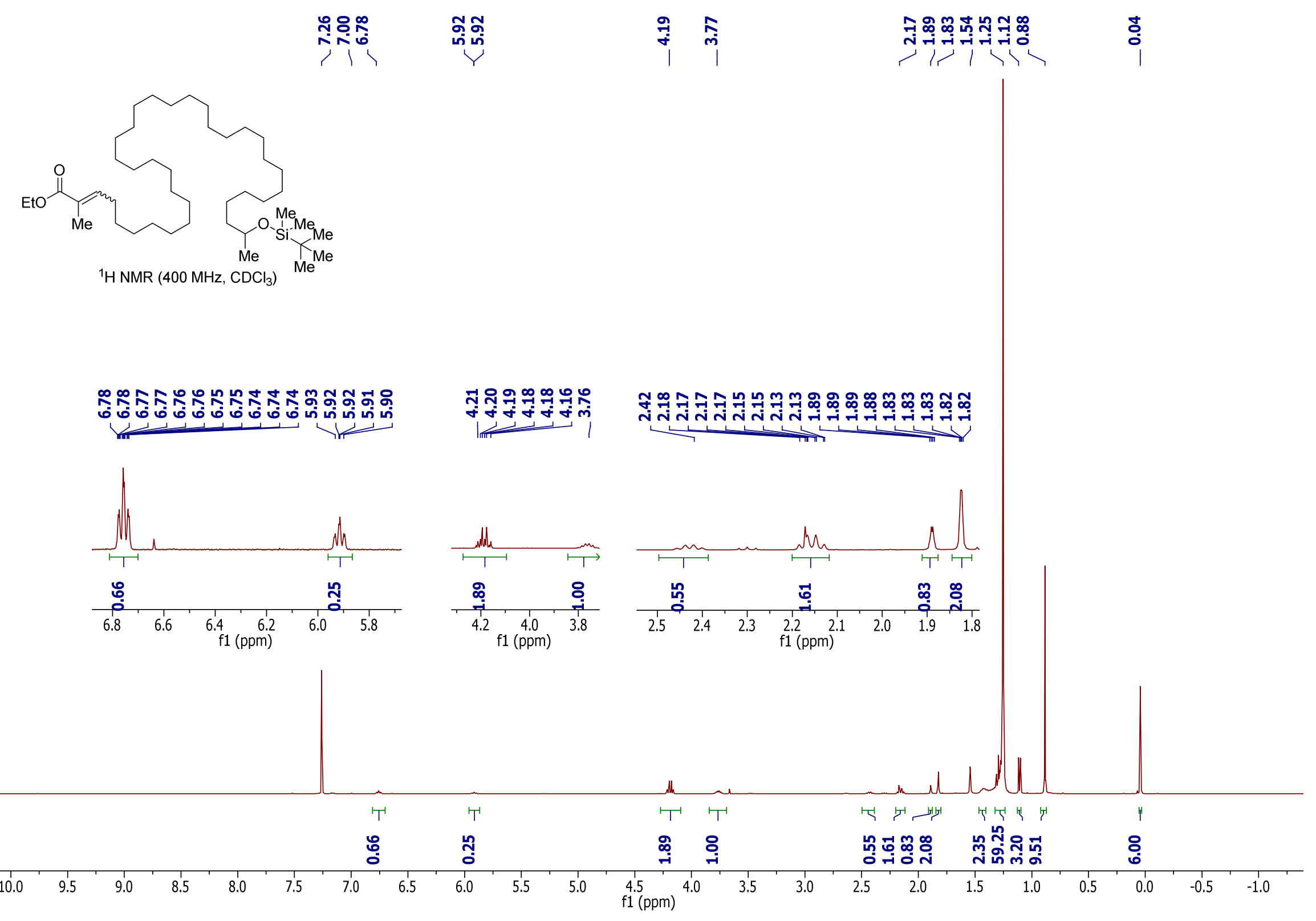




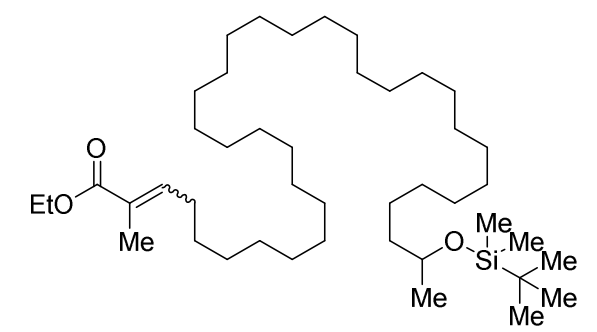

${ }^{13} \mathrm{C}\left\{{ }^{1} \mathrm{H}\right\}$ NMR (101 MHz, $\left.\mathrm{CDCl}_{3}\right)$

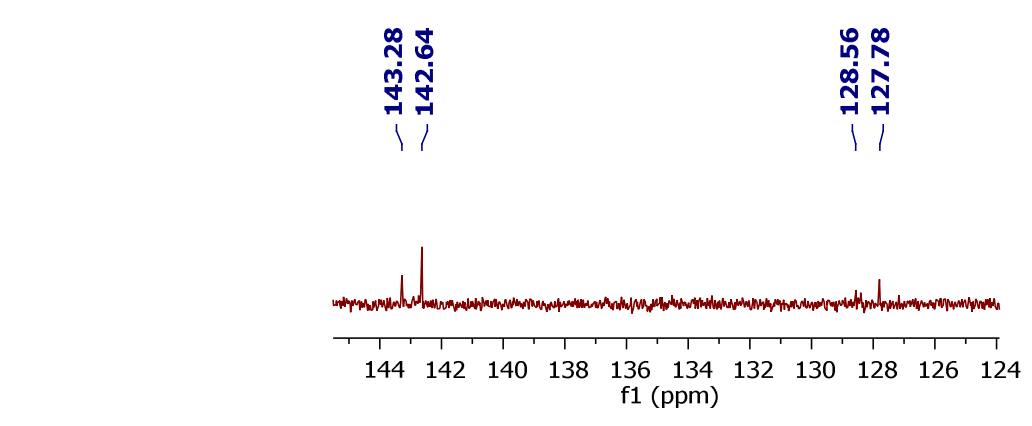




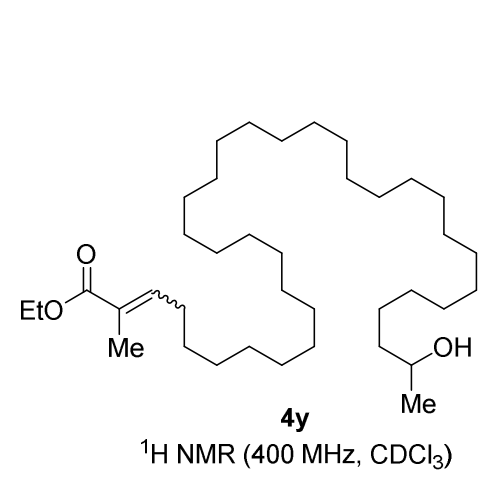

i̊n

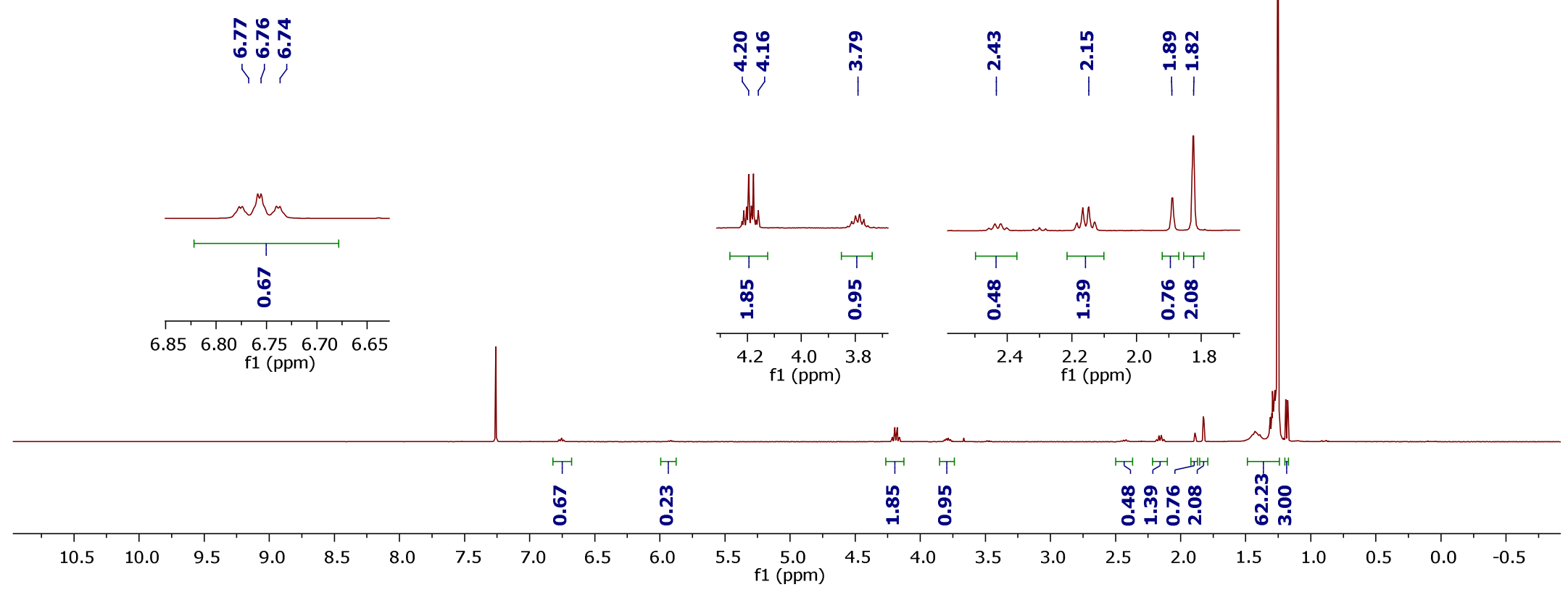




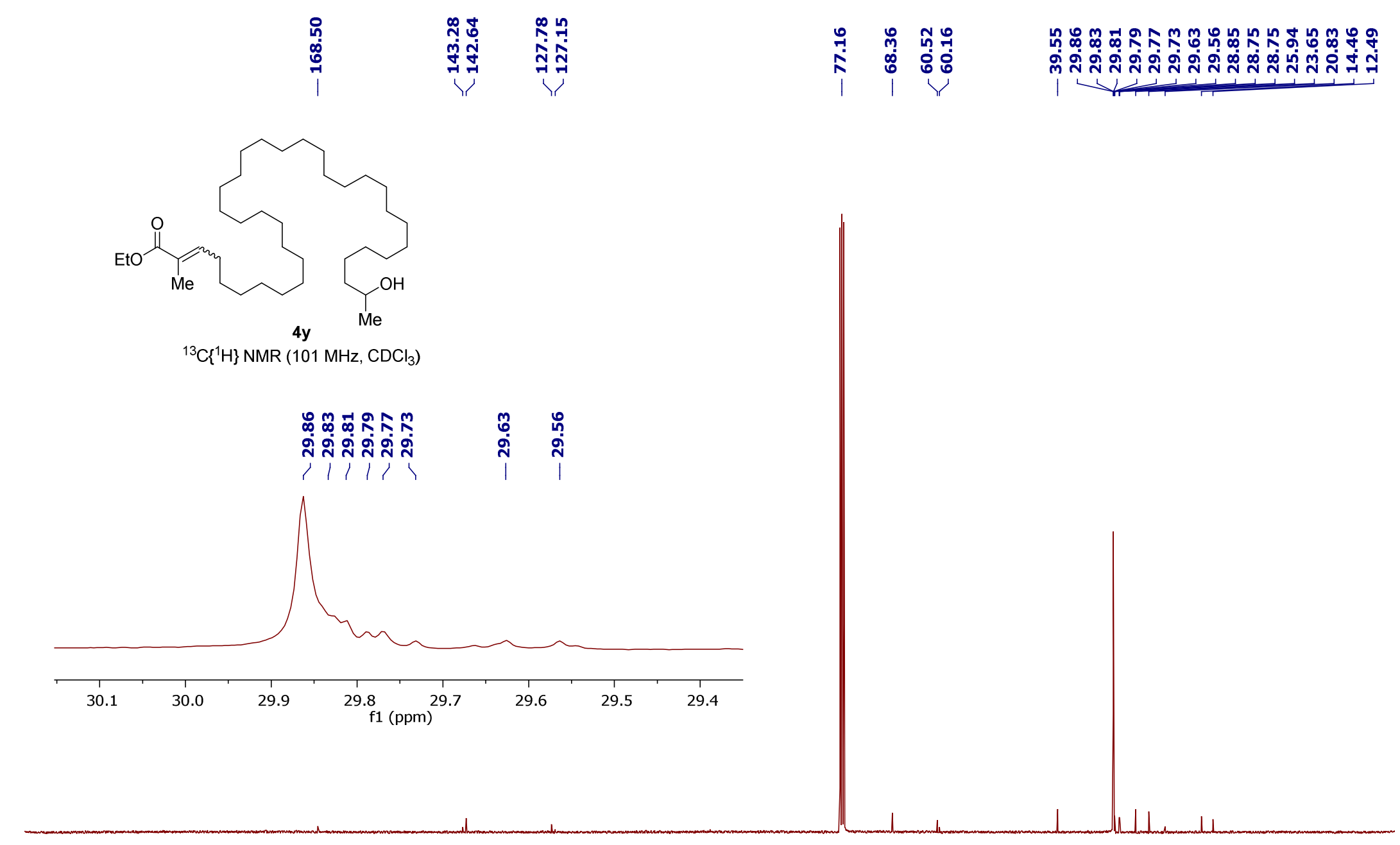

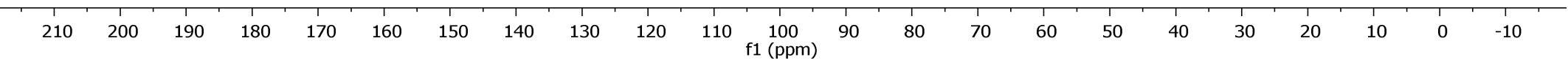


ตู่

ris

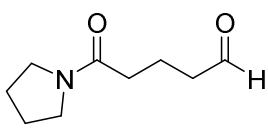

${ }^{1} \mathrm{H}$ NMR $\left(500 \mathrm{MHz}, \mathrm{CDCl}_{3}\right)$

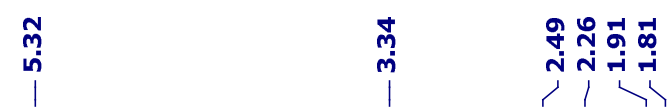

\section{\%}




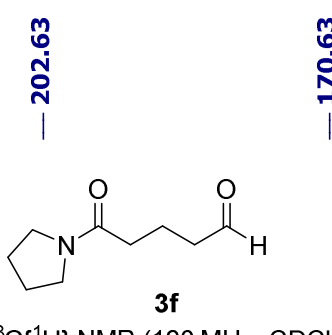

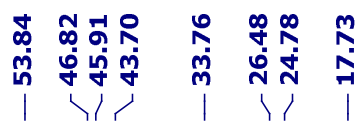

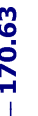

${ }^{13} \mathrm{C}\left\{{ }^{1} \mathrm{H}\right\} \mathrm{NMR}\left(126 \mathrm{MHz}, \mathrm{CDCl}_{3}\right)$

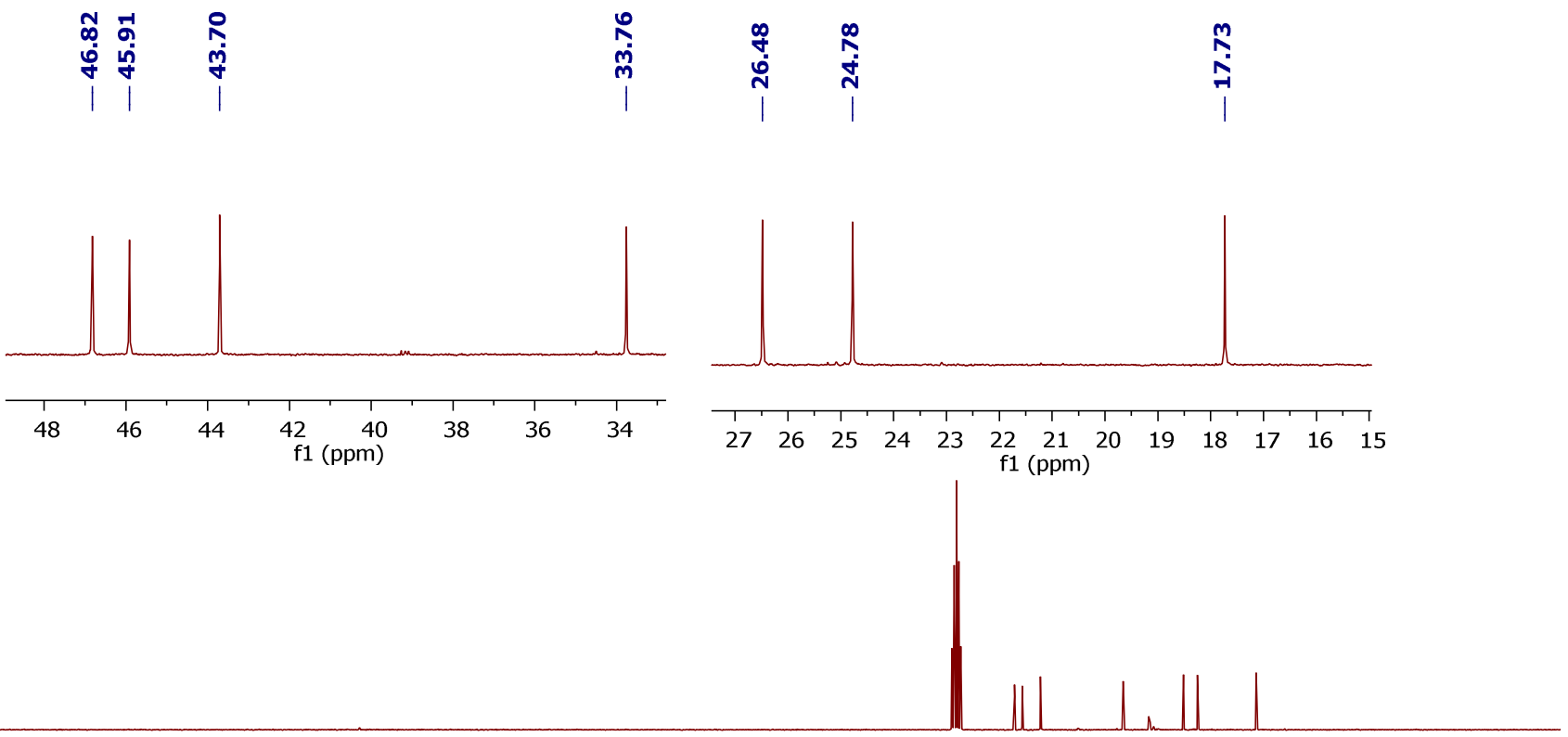

$\begin{array}{llllllllllllllllllllllllll}210 & 200 & 190 & 180 & 170 & 160 & 150 & 140 & 130 & 120 & 110 & 100 & 10 & 80 & 70 & 60 & 50 & 40 & 30 & 20 & 10 & 0 & -10\end{array}$ 

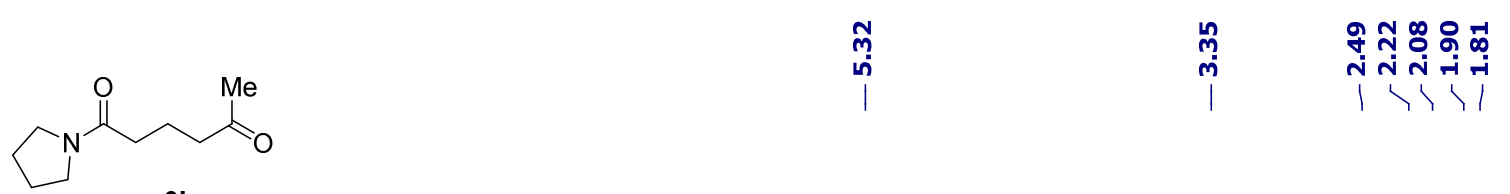

${ }^{1} \mathrm{H} \mathrm{NMR}\left(500 \mathrm{MHz}, \mathrm{CDCl}_{3}\right)$

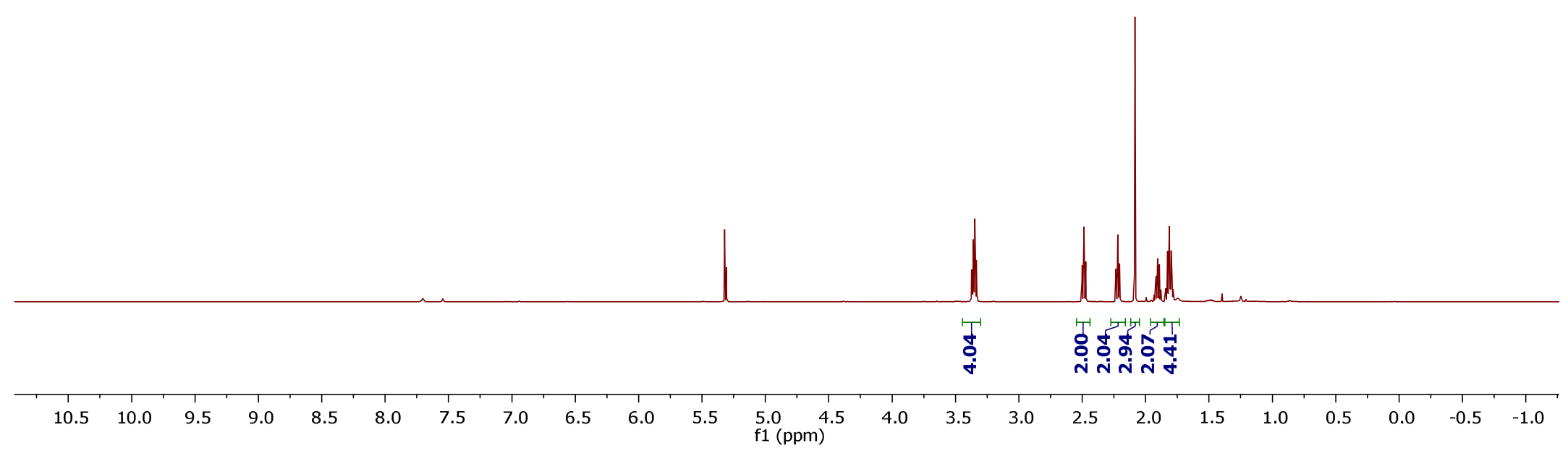




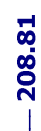

\section{క ది}

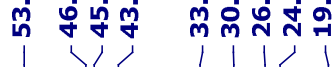

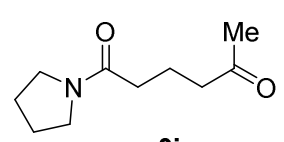

${ }^{13} \mathrm{C}\left\{{ }^{1} \mathrm{H}\right\} \mathrm{NMR}\left(126 \mathrm{MHz}, \mathrm{CDCl}_{3}\right)$
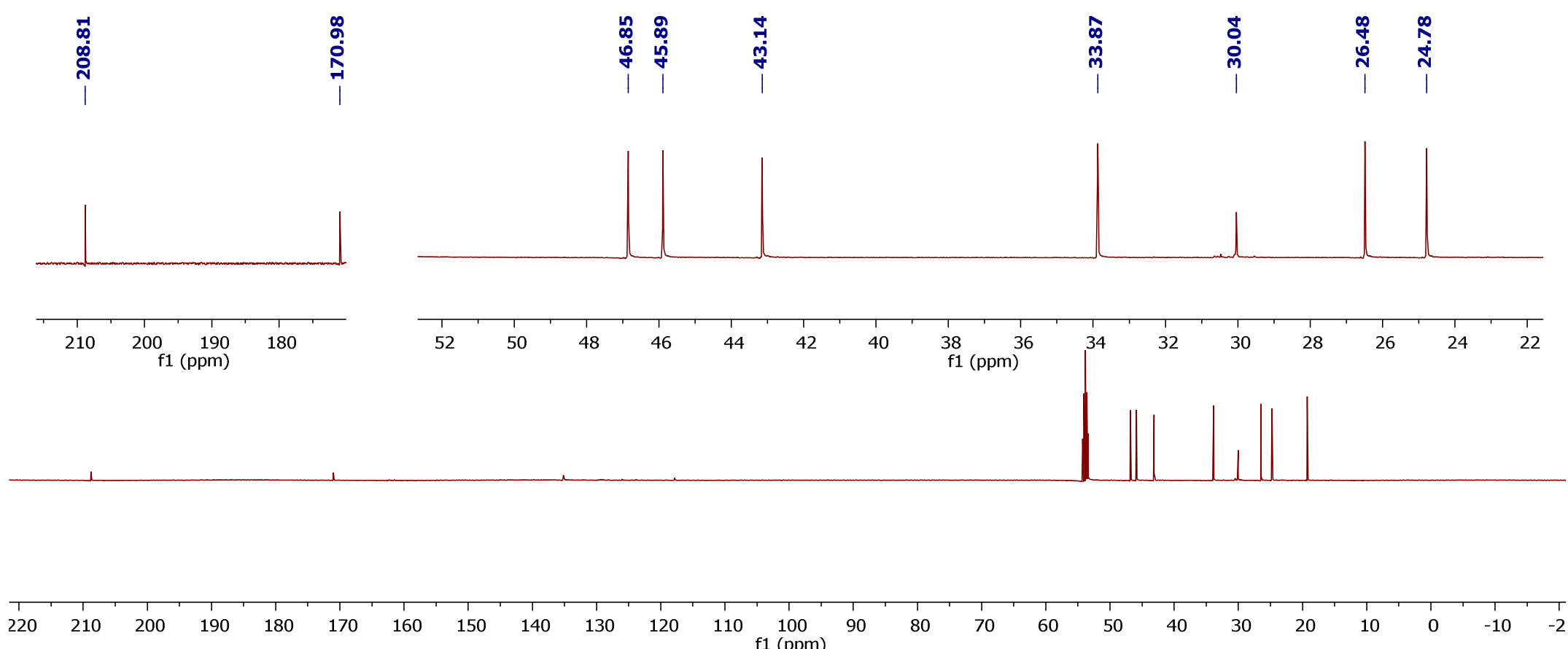

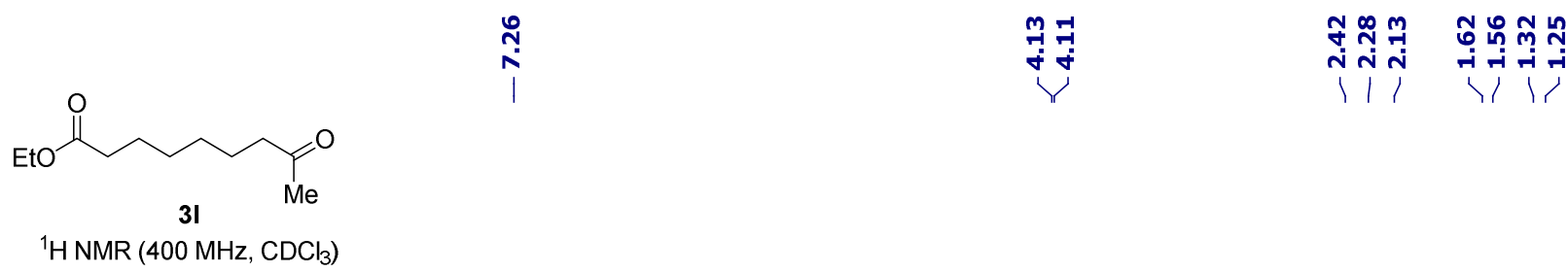

${ }^{1} \mathrm{H}$ NMR $\left(400 \mathrm{MHz}, \mathrm{CDCl}_{3}\right)$

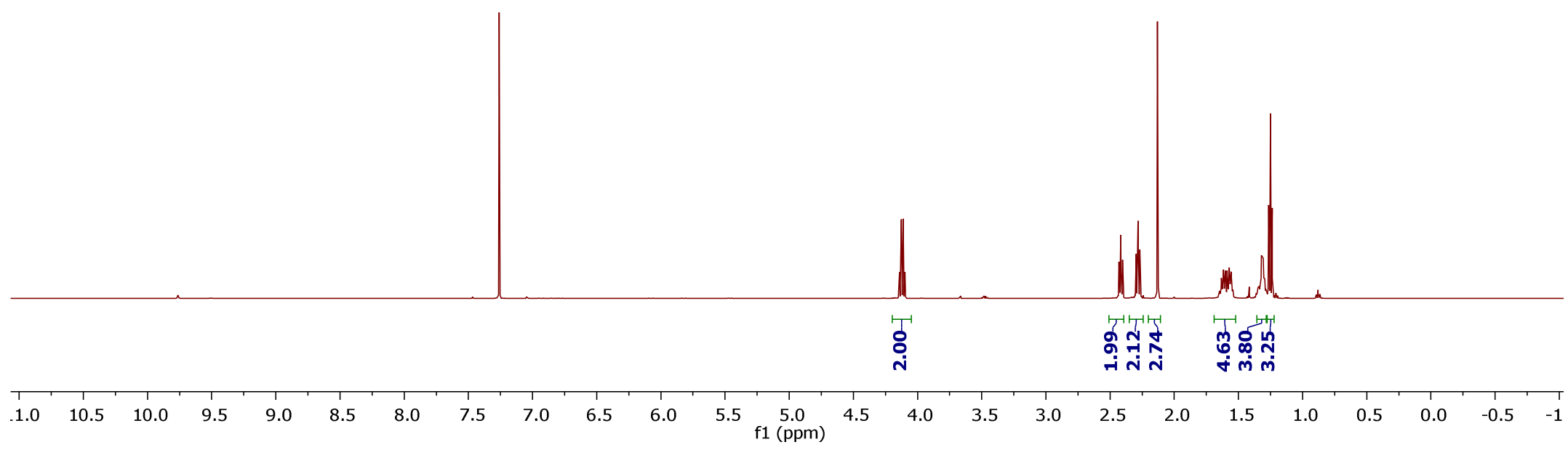



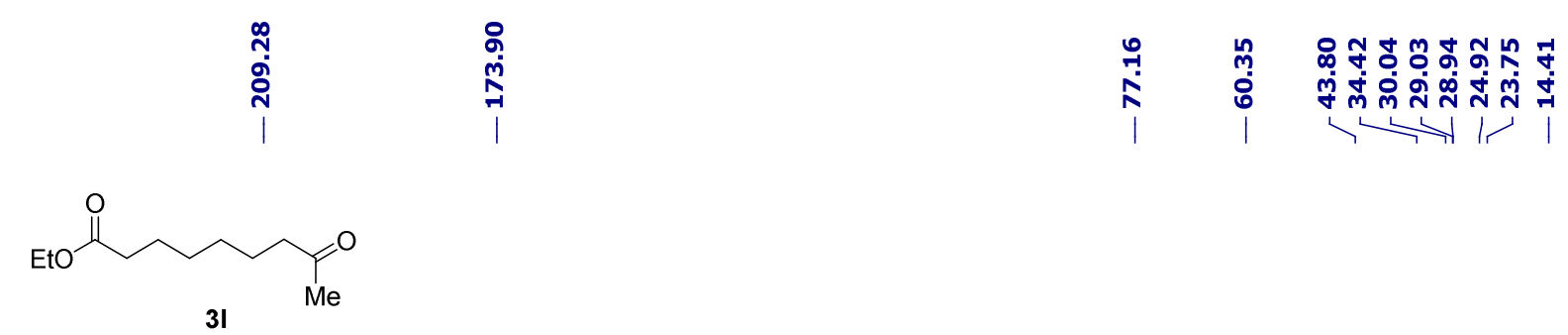

${ }^{13} \mathrm{C}\left\{{ }^{1} \mathrm{H}\right\} \mathrm{NMR}\left(101 \mathrm{MHz}, \mathrm{CDCl}_{3}\right)$

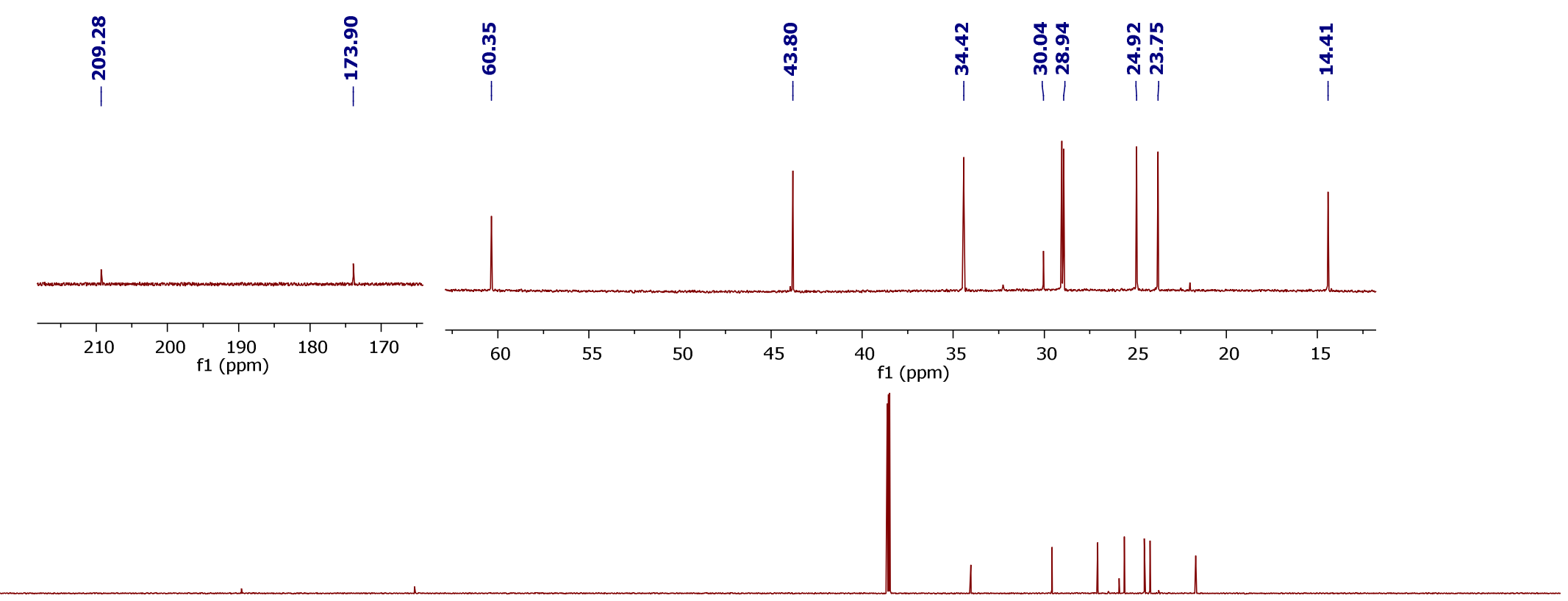

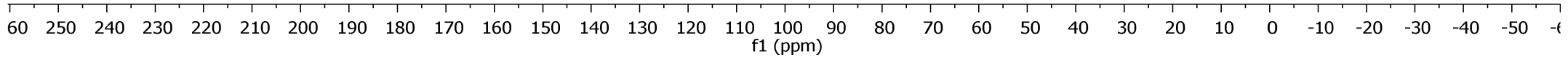




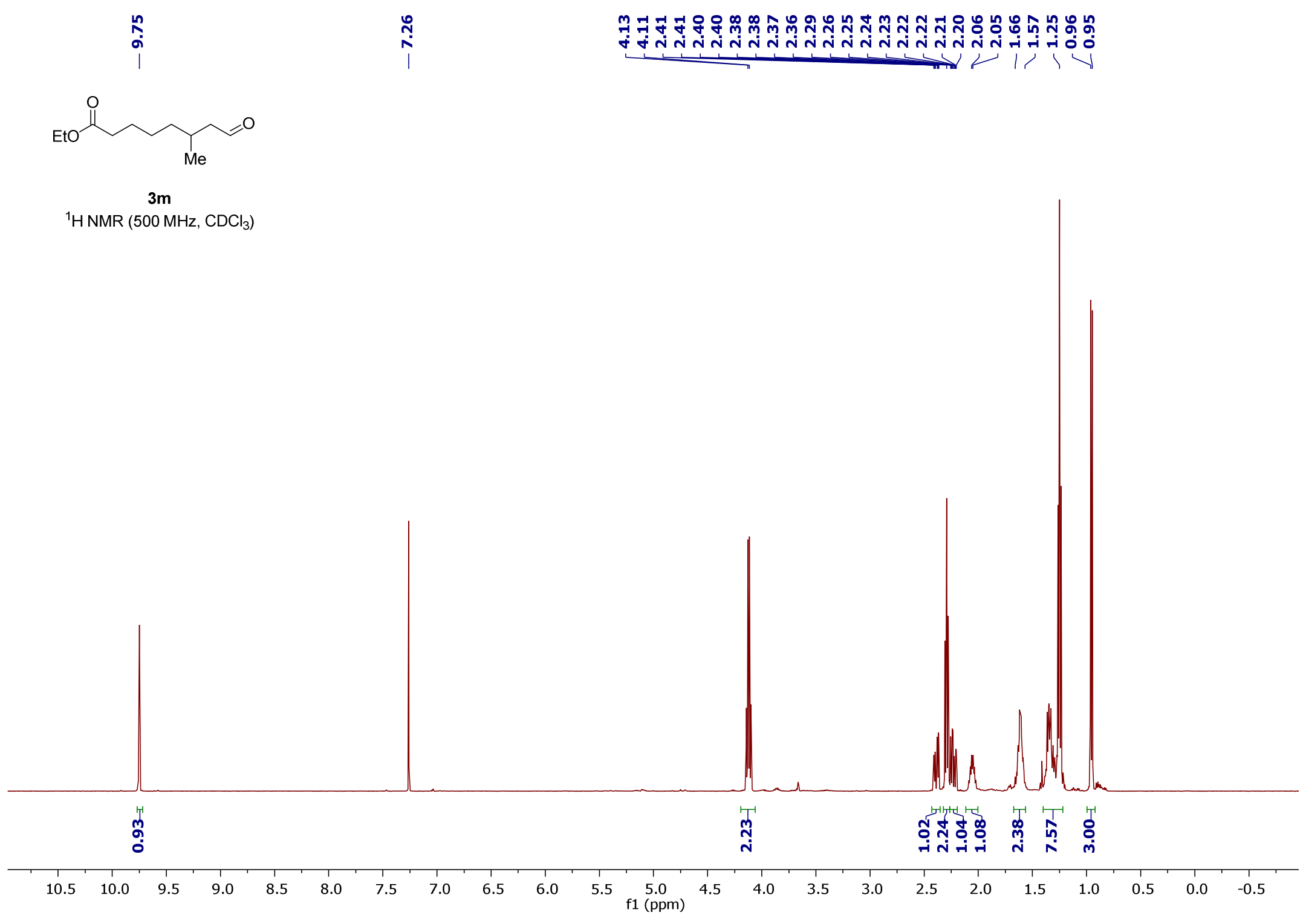




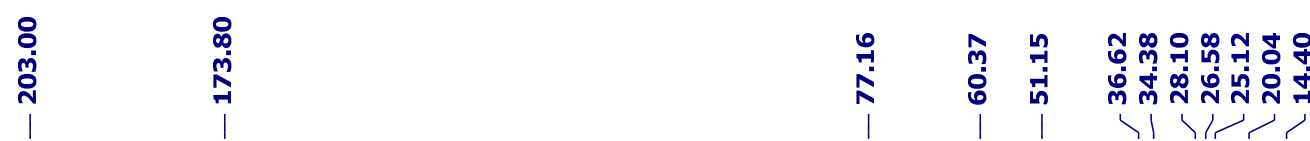

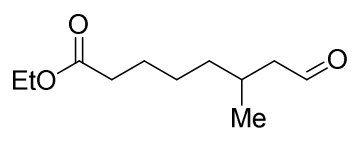

$3 \mathrm{~m}$

${ }^{13} \mathrm{C}\left\{{ }^{1} \mathrm{H}\right\} \mathrm{NMR}\left(126 \mathrm{MHz}, \mathrm{CDCl}_{3}\right)$

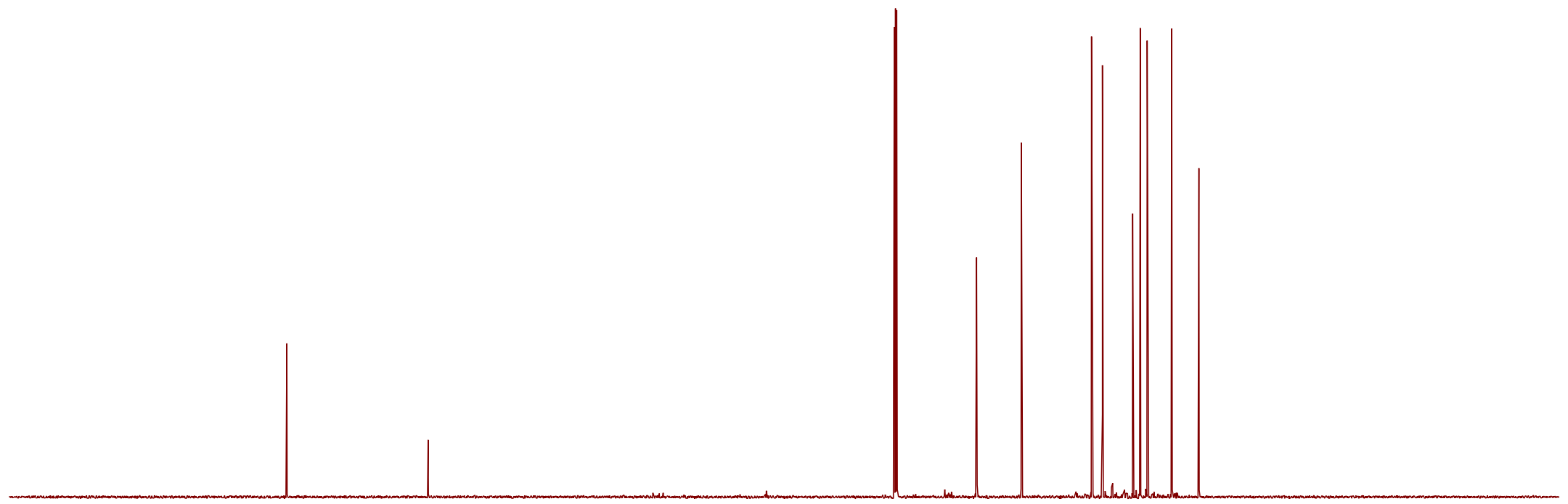

$\begin{array}{lllllllllllllllllllllllllllllllllllllllllll}60 & 250 & 240 & 230 & 220 & 210 & 200 & 190 & 180 & 170 & 160 & 150 & 140 & 130 & 120 & 110 & 100 & 90 & 80 & 70 & 60 & 50 & 40 & 30 & 20 & 10 & 0 & -10 & -20 & -30 & -40 & -50 & -4\end{array}$ 


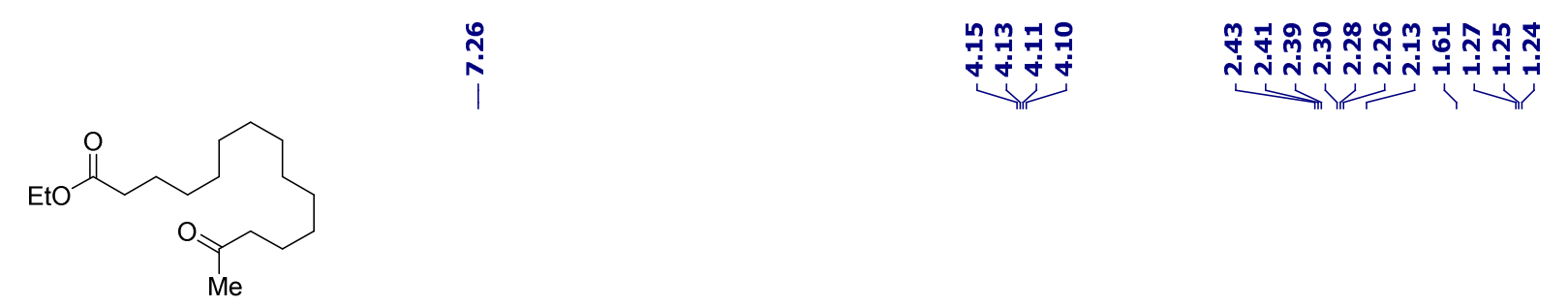

${ }^{1} \mathrm{H} \mathrm{NMR}\left(400 \mathrm{MHz}, \mathrm{CDCl}_{3}\right)$

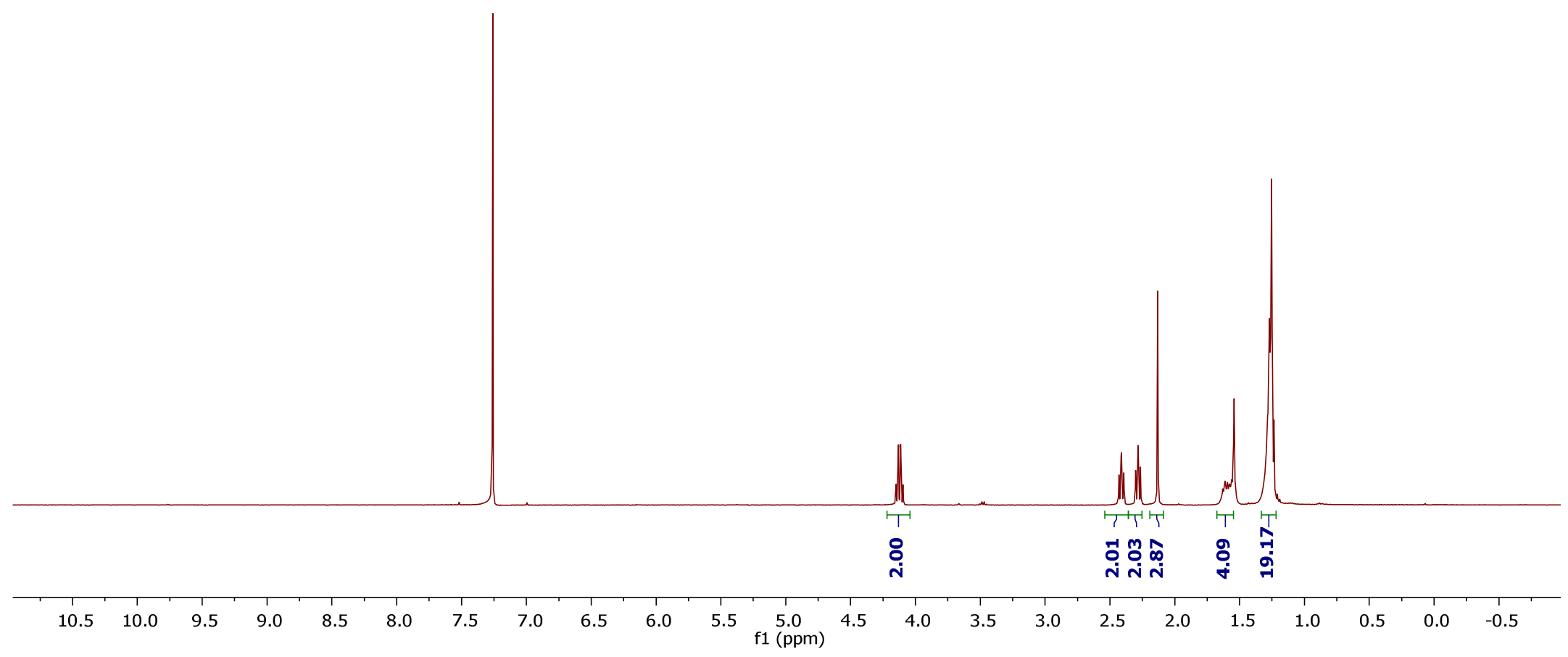




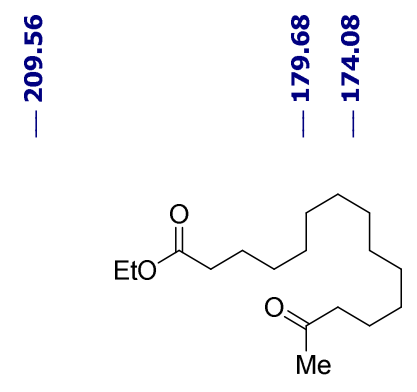

$\left.{ }^{13} \mathrm{C}\left\{{ }^{1} \mathrm{H}\right\} \mathrm{NMR}(101 \mathrm{MHz}, \mathrm{CDCl})_{3}\right)$
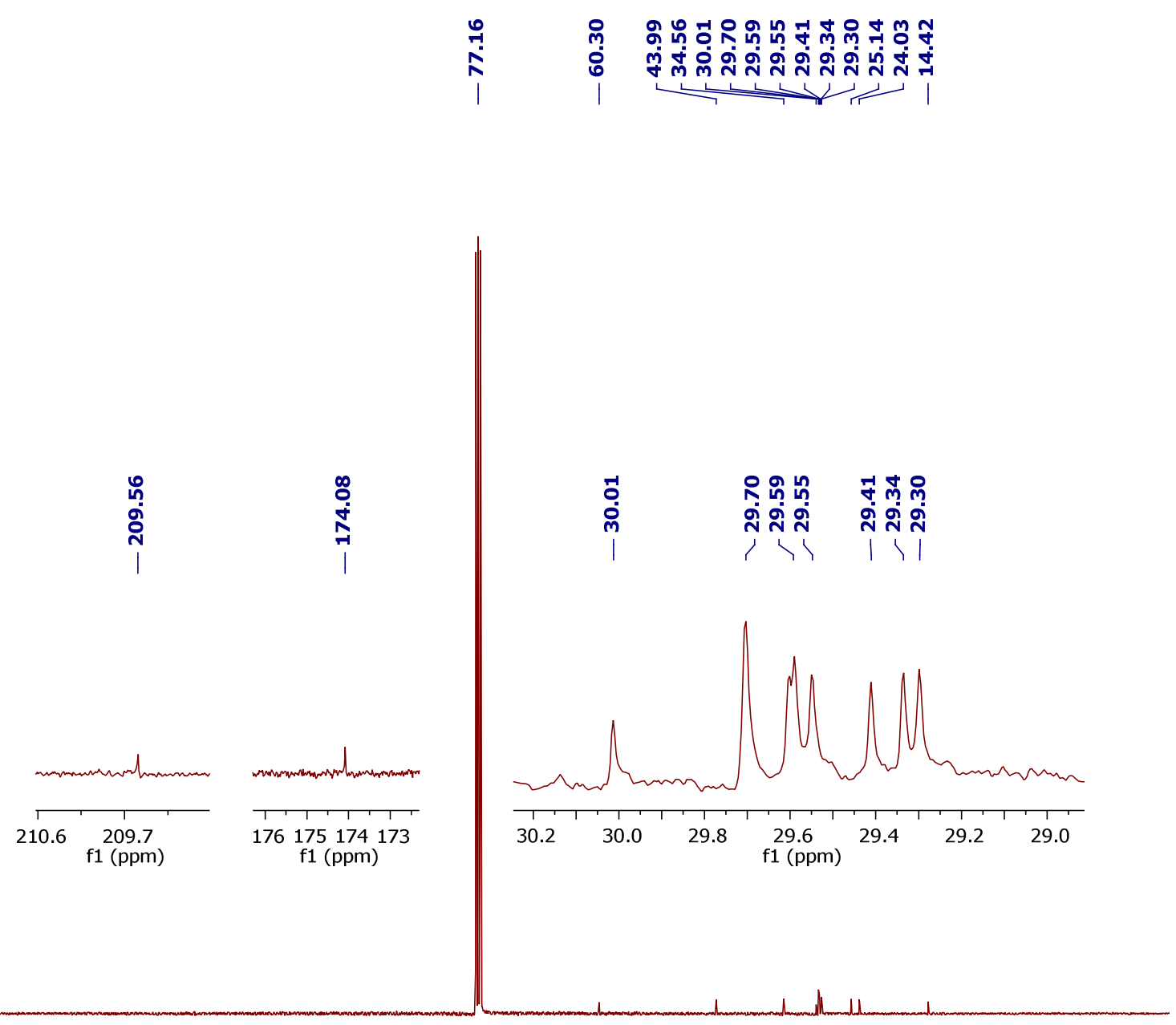

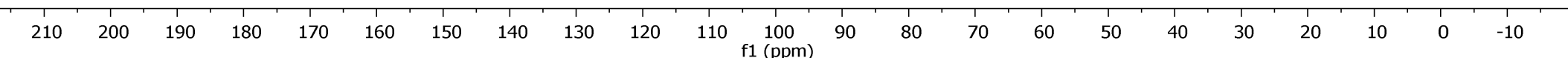




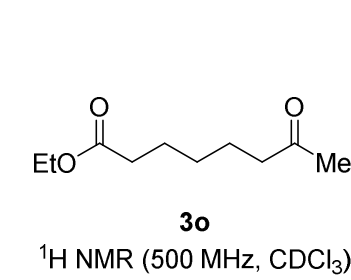

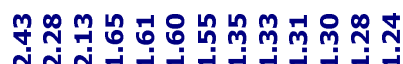

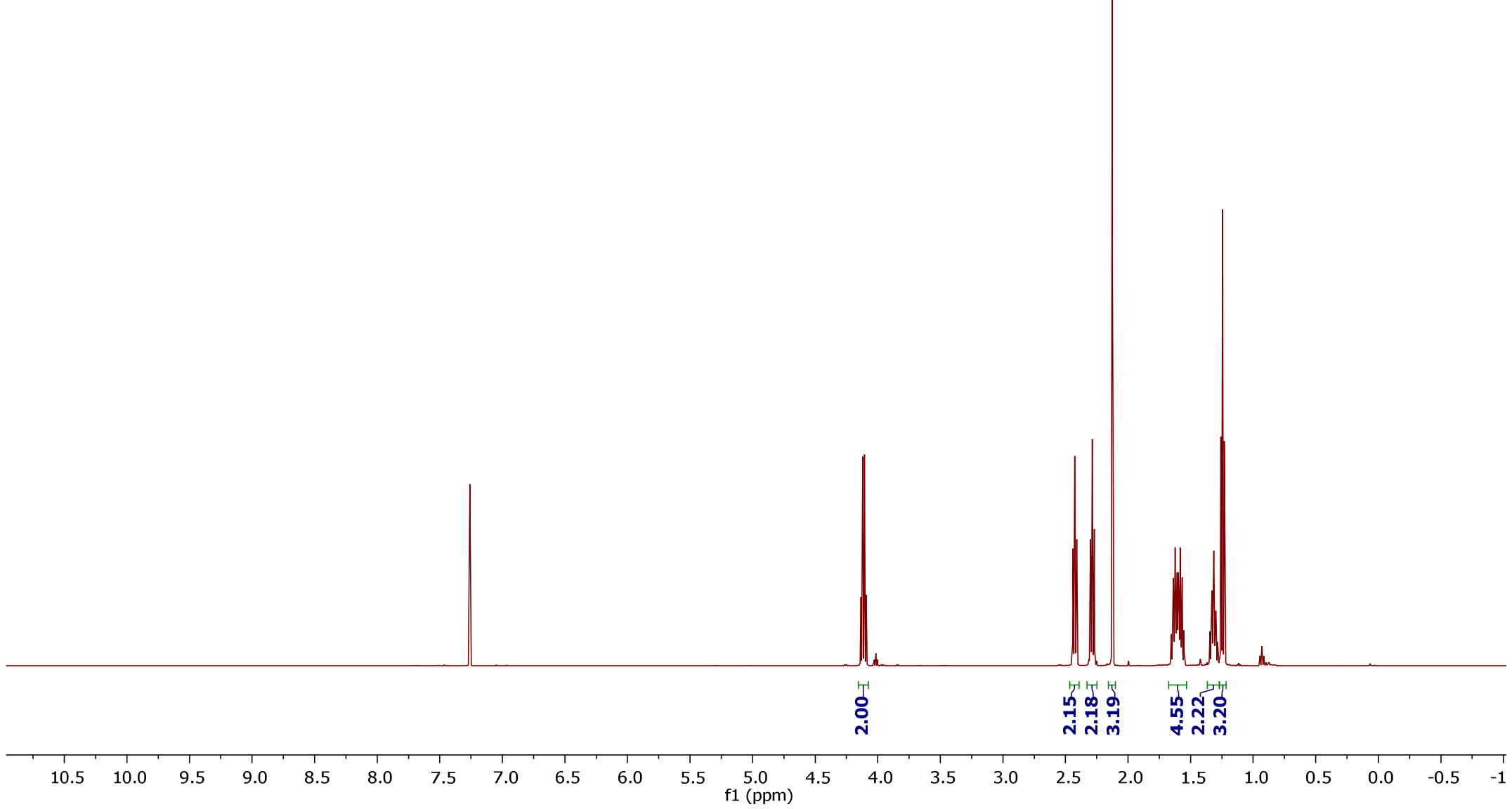




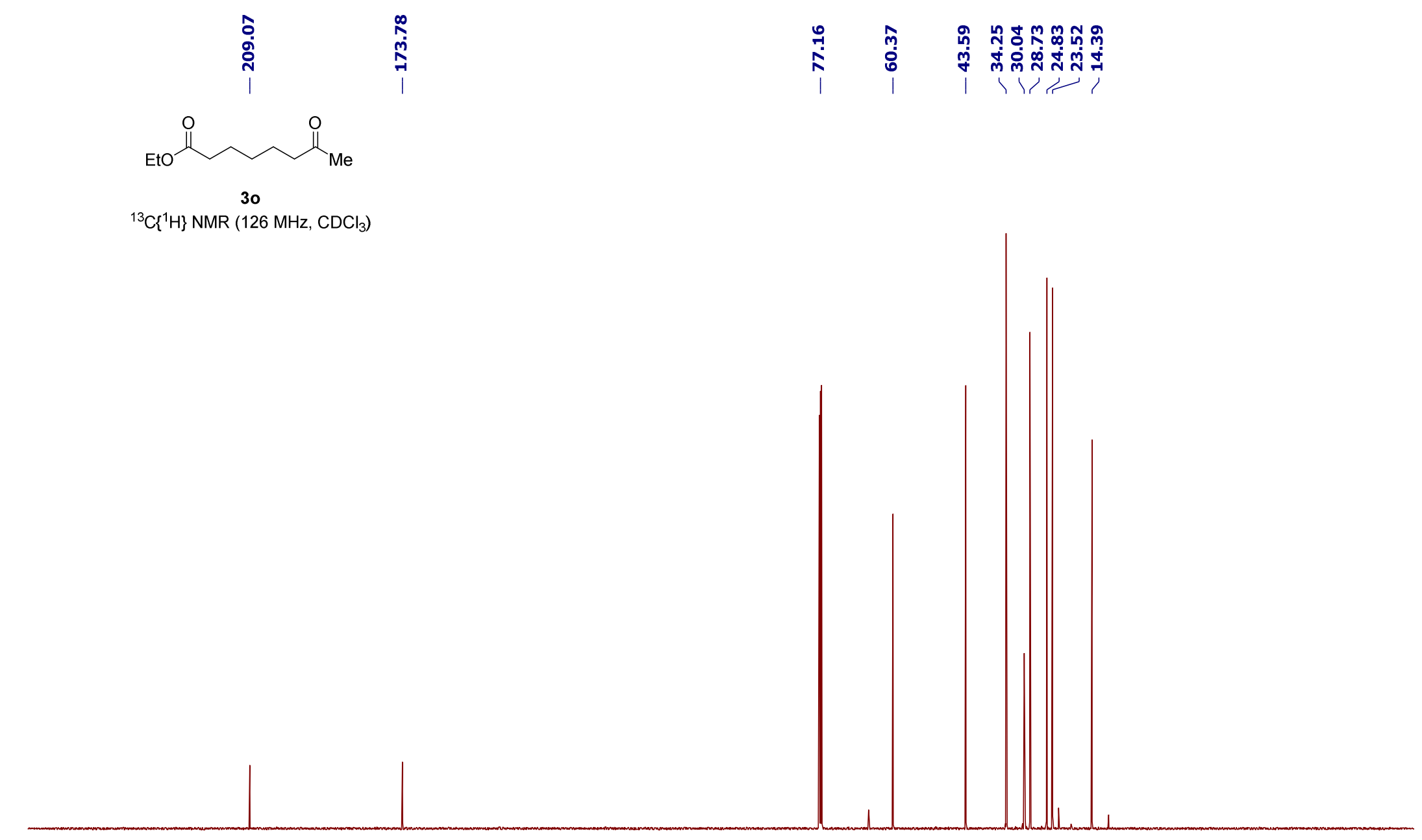

$\begin{array}{lllllllllllllllllllllllllllllllllllll}60 & 250 & 240 & 230 & 220 & 210 & 200 & 190 & 180 & 170 & 160 & 150 & 140 & 130 & 120 & 110 & 100 & 90 & 80 & 70 & 60 & 50 & 40 & 30 & 20 & 10 & 0 & -10 & -20 & -30 & -40 & -50 & -4\end{array}$ 

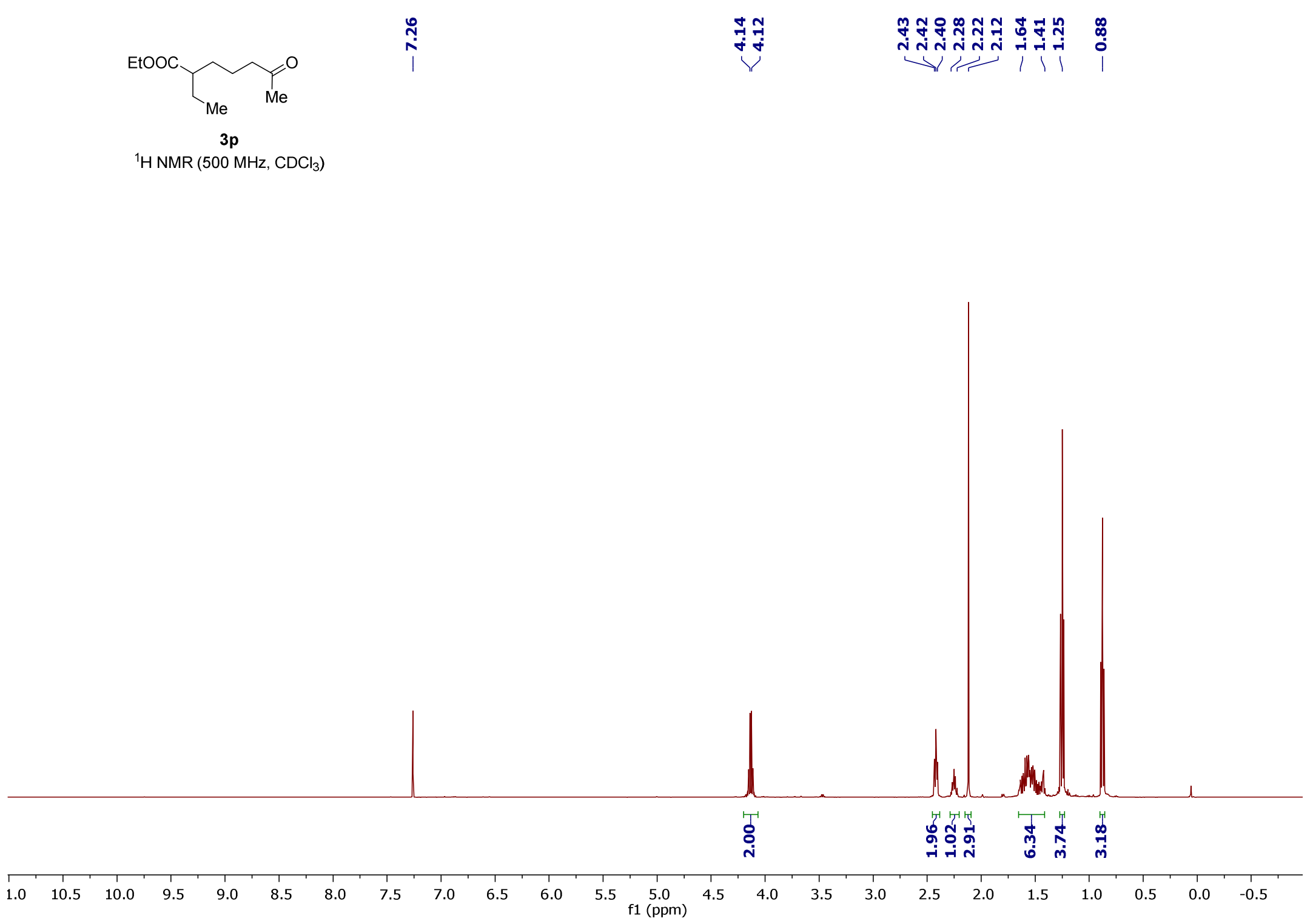

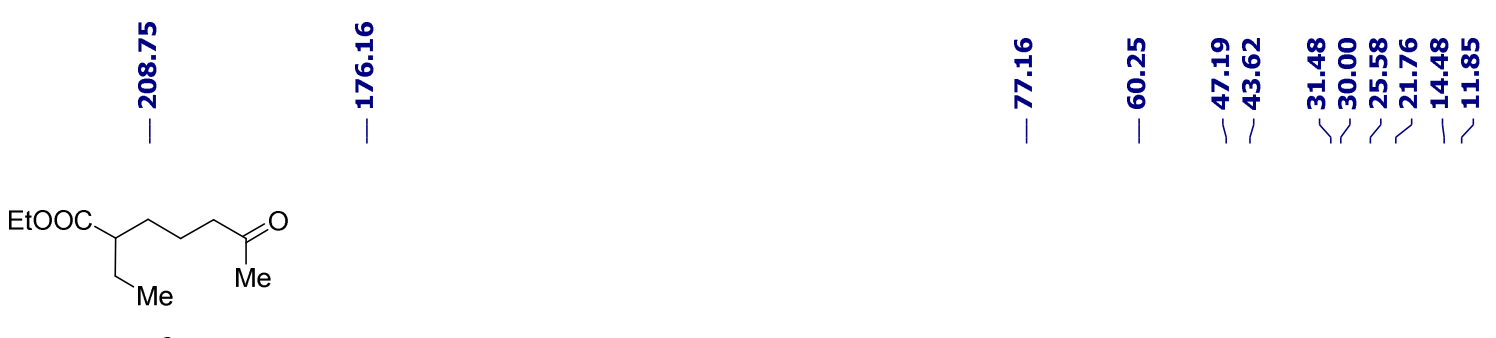

${ }^{13} \mathrm{C}\left\{{ }^{1} \mathrm{H}\right\} \mathrm{NMR}\left(126 \mathrm{MHz}, \mathrm{CDCl}_{3}\right)$

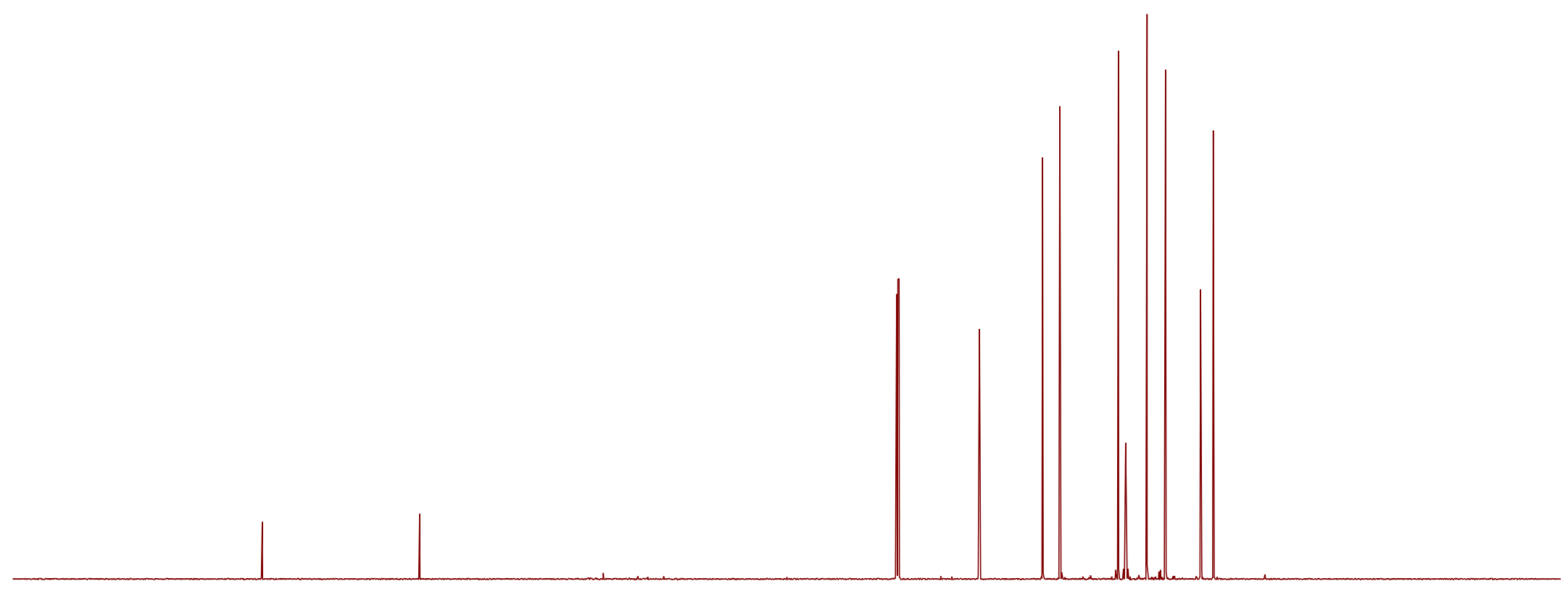

$\begin{array}{lllllllllllllllllllllllllllllllllllll}60 & 250 & 240 & 230 & 220 & 210 & 200 & 190 & 180 & 170 & 160 & 150 & 140 & 130 & 120 & 110 & 100 & 90 & 80 & 70 & 60 & 50 & 40 & 30 & 20 & 10 & 0 & -10 & -20 & -30 & -40 & -50 & -t\end{array}$ 


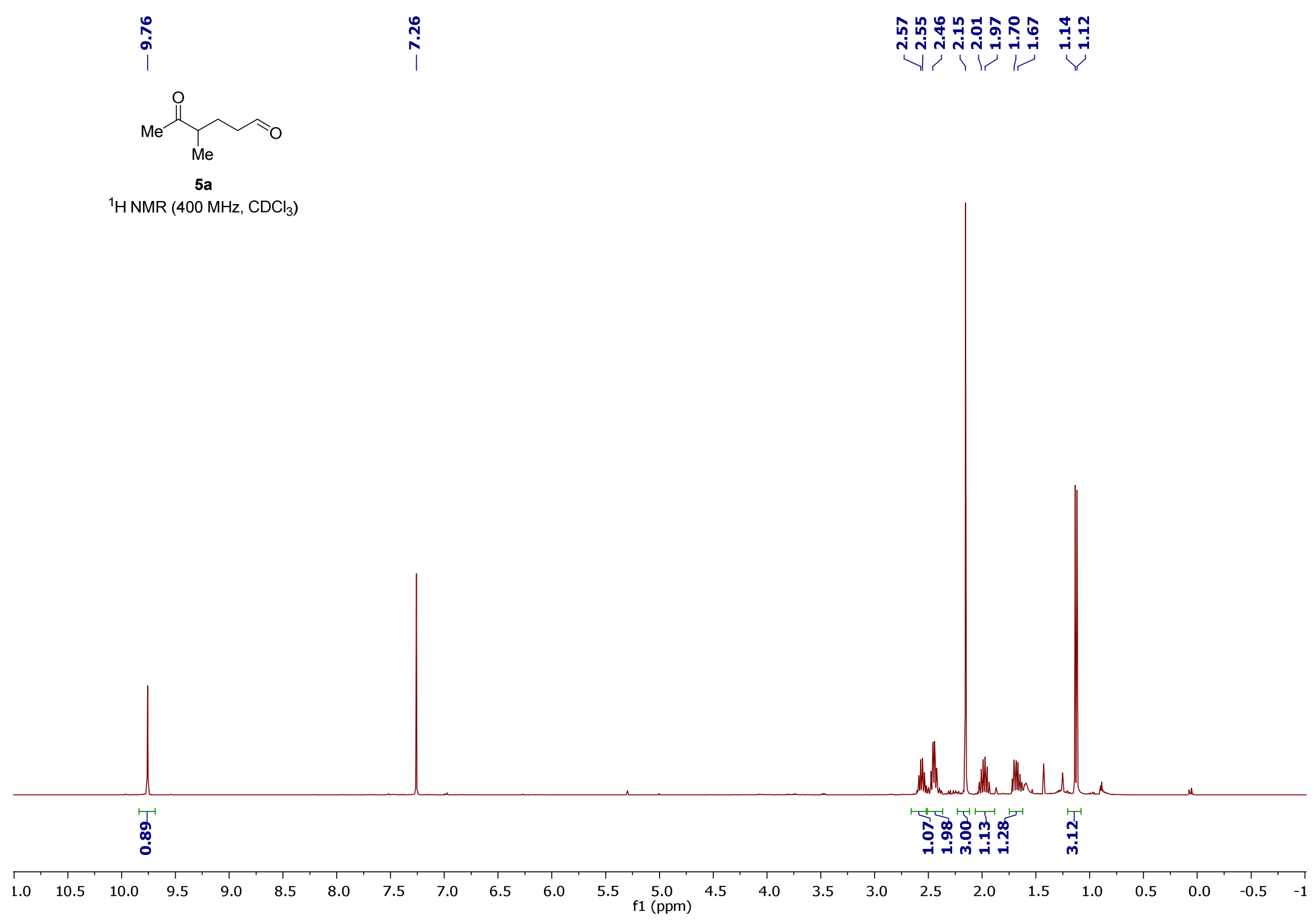




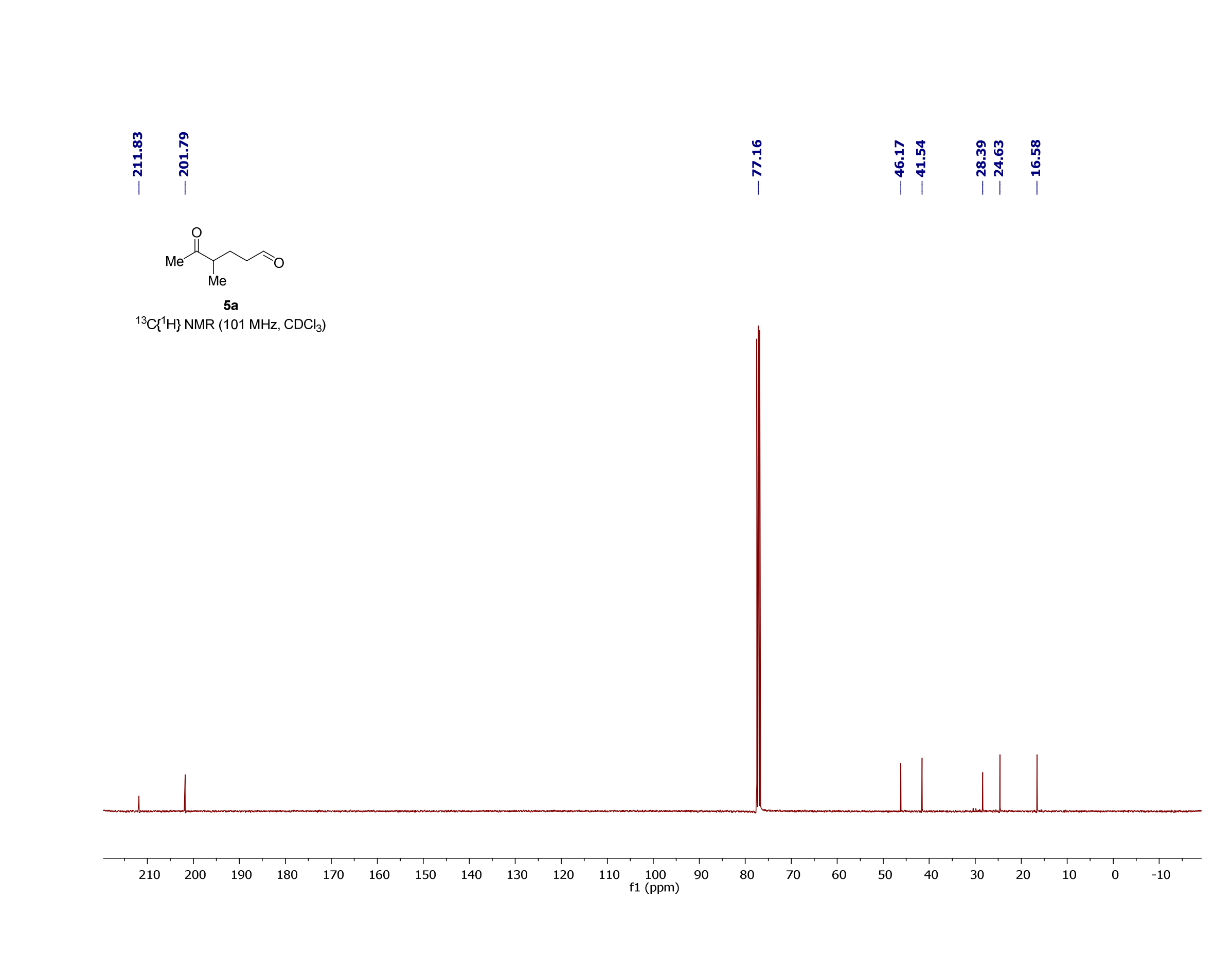




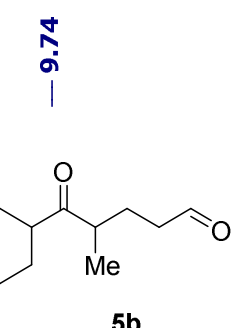

${ }^{1} \mathrm{H}$ NMR $\left(400 \mathrm{MHz}, \mathrm{CDCl}_{3}\right)$

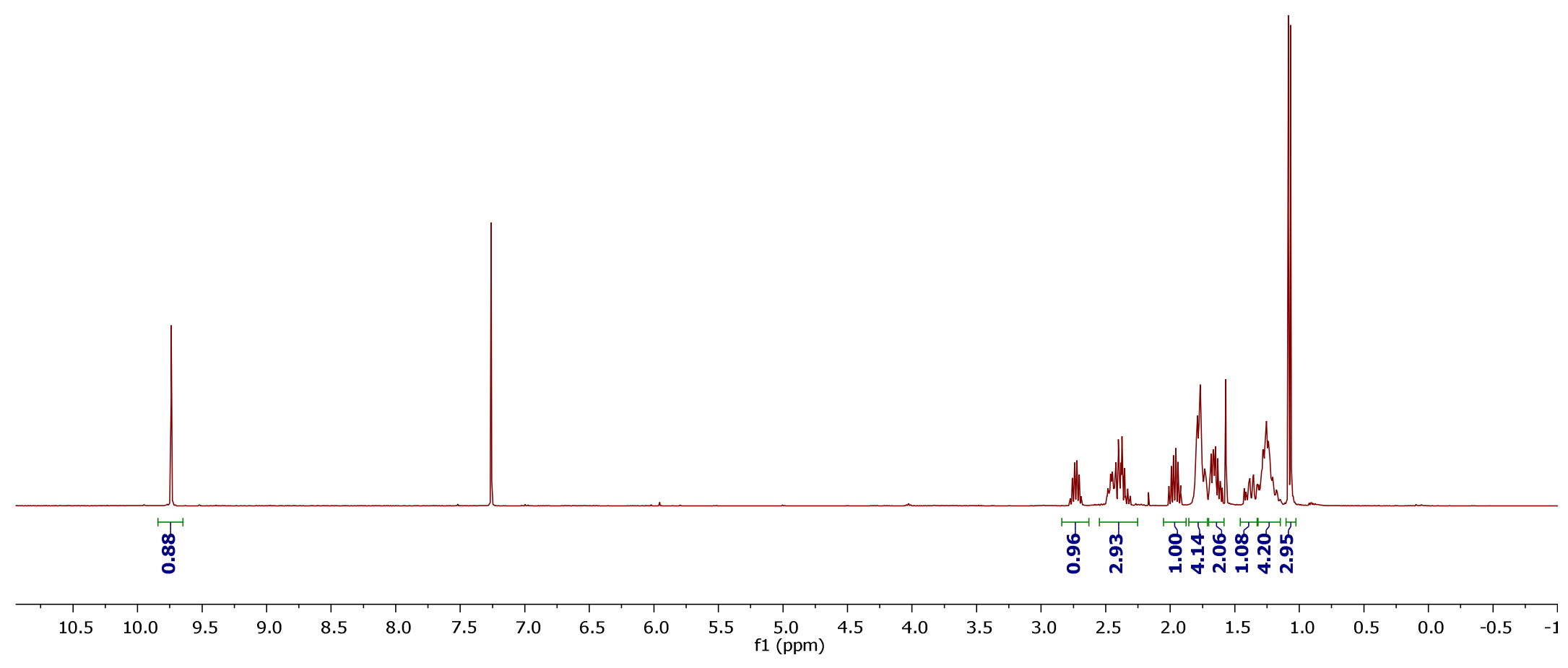




$$
\overline{1}
$$




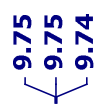<smiles>C=CCCC(C)C(=O)CCCC</smiles>

5c $\mathrm{Me}$

${ }^{1} \mathrm{H} \mathrm{NMR} \mathrm{(400} \mathrm{MHz,} \mathrm{CDCl}_{3}$ )

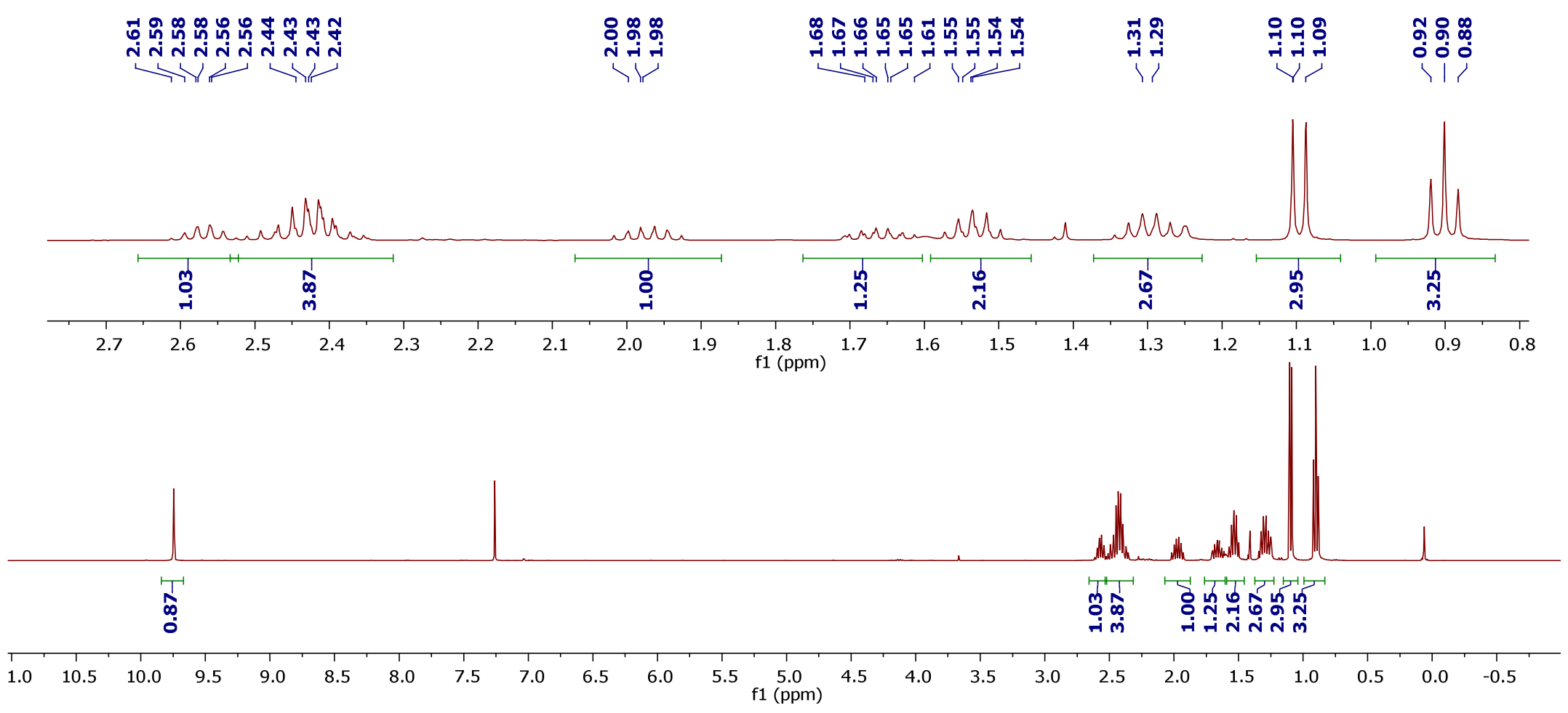

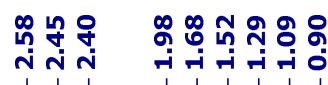

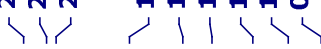



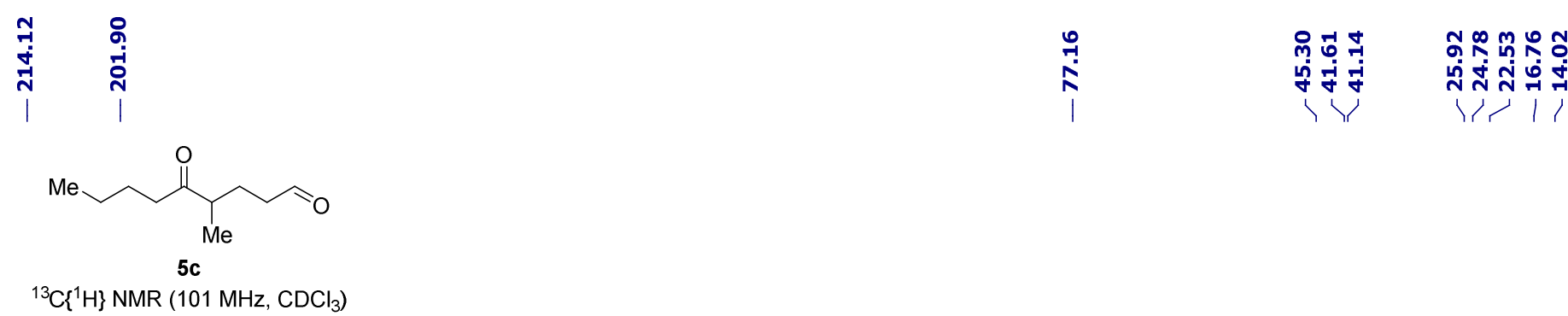

${ }^{13} \mathrm{C}\left\{{ }^{1} \mathrm{H}\right\}$ NMR $\left(101 \mathrm{MHz}, \mathrm{CDCl}_{3}\right)$

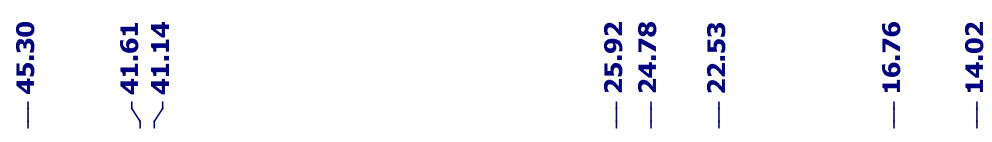

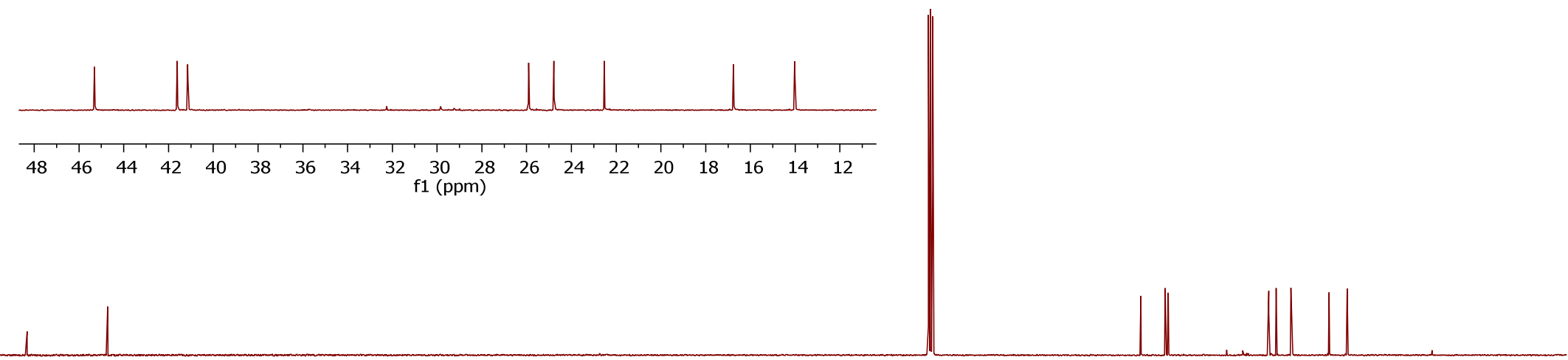

$\begin{array}{lllllllllllllllllllllllll}1 & 210 & 200 & 190 & 180 & 170 & 160 & 150 & 140 & 130 & 120 & 110 & 100 & 90 & 80 & 70 & 60 & 50 & 40 & 30 & 20 & 10 & 0 & -10\end{array}$ 


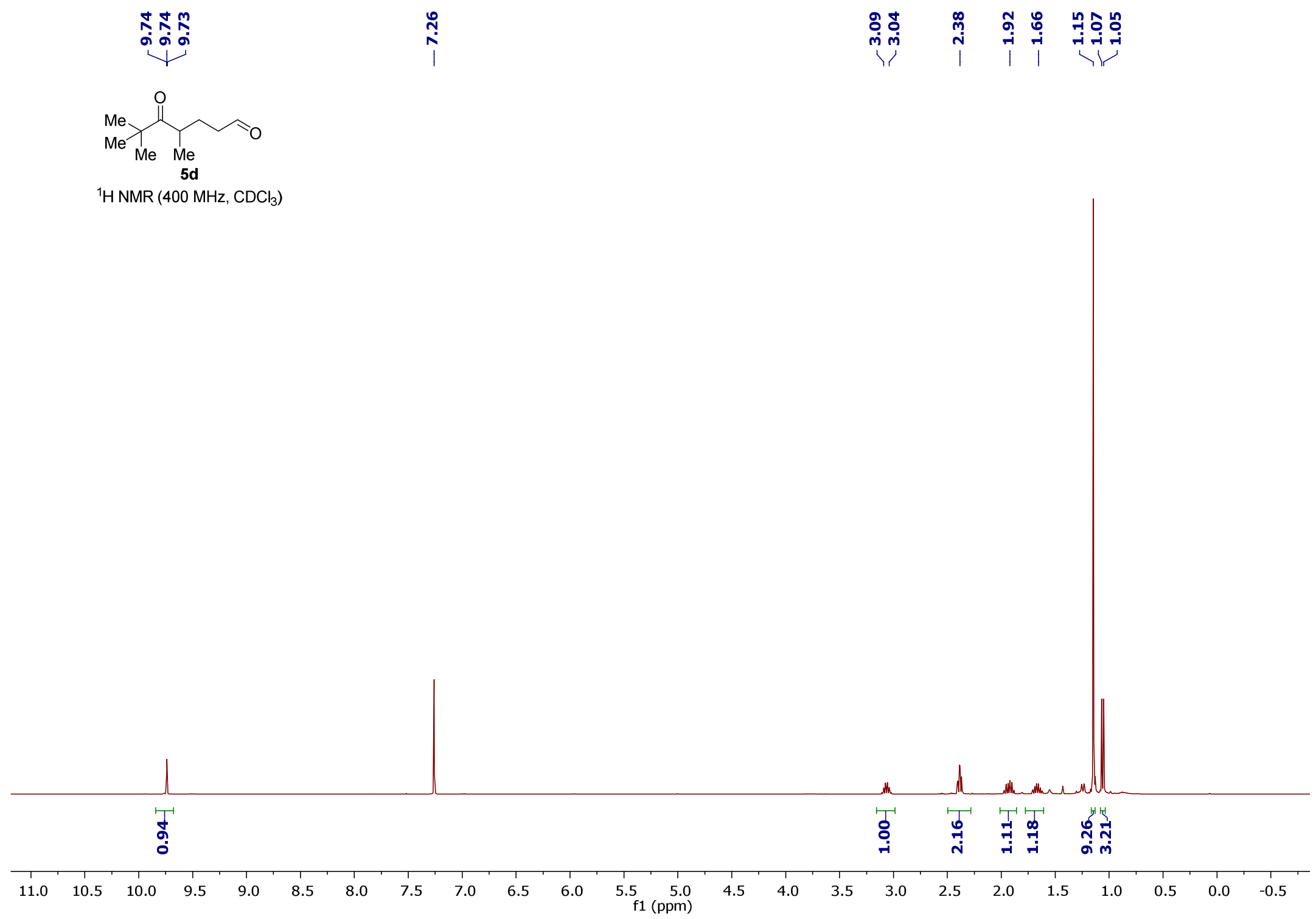




$$
\text { มั่ }
$$

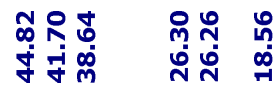

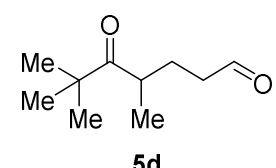

${ }^{13} \mathrm{C}\left\{{ }^{1} \mathrm{H}\right\} \mathrm{NMR}\left(101 \mathrm{MHz}, \mathrm{CDCl}_{3}\right)$

กิ

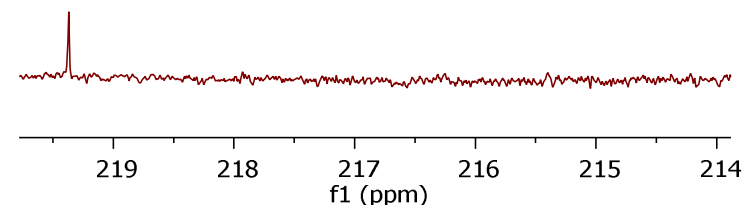

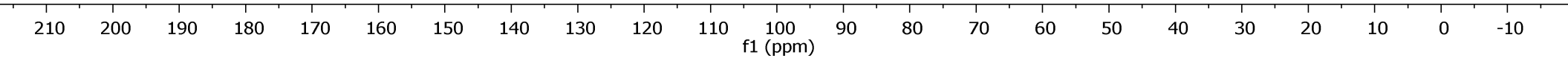




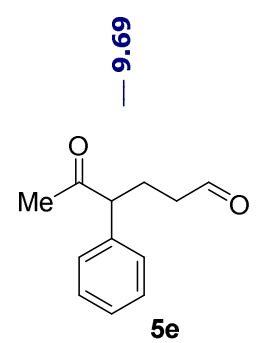

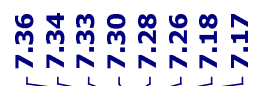

$\stackrel{\infty}{\circ}$

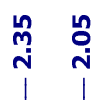

${ }^{1} \mathrm{H} \mathrm{NMR}\left(400 \mathrm{MHz}, \mathrm{CDCl}_{3}\right)$
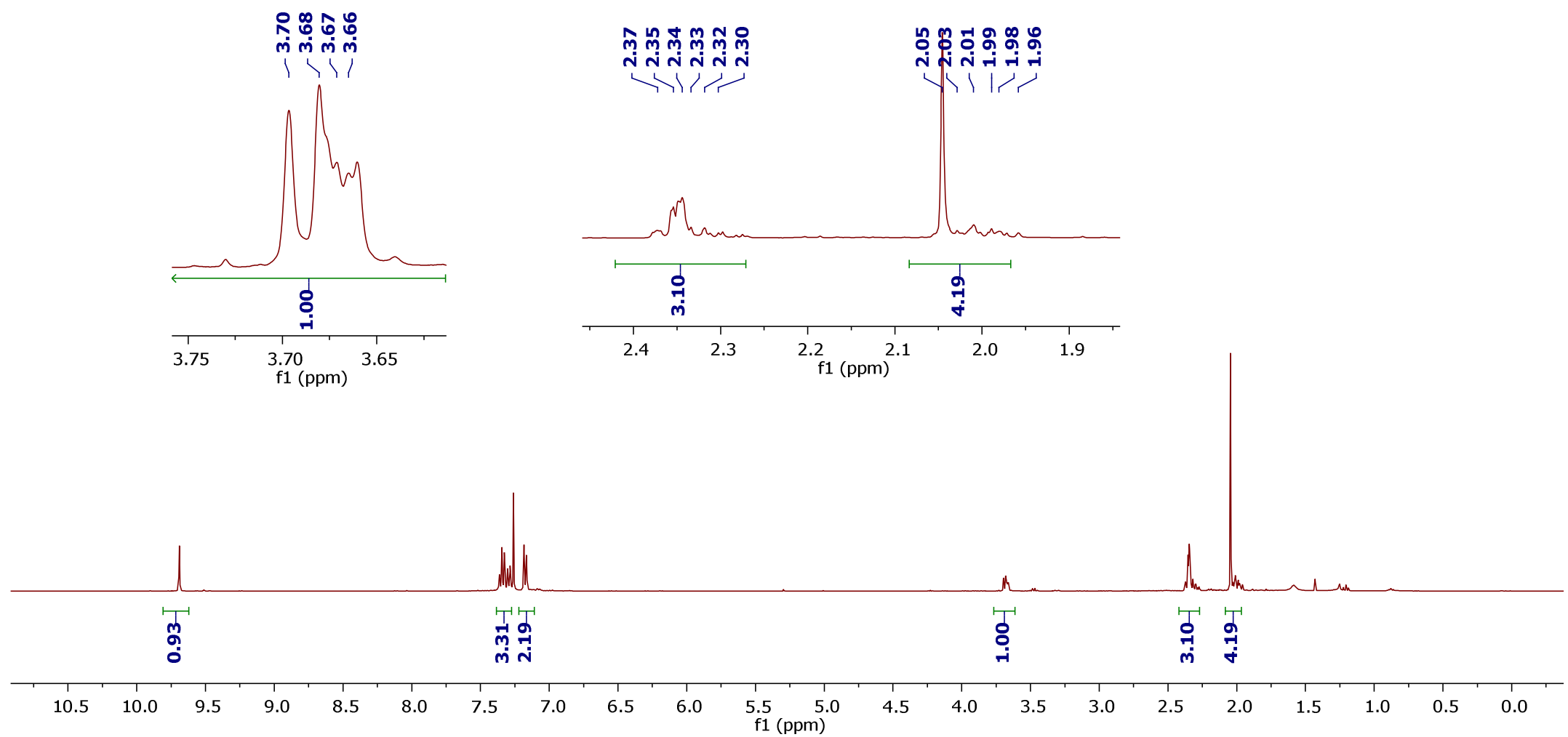


$$
\begin{array}{lll} 
\\
\hline
\end{array}
$$




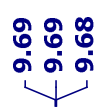

$\stackrel{2}{7}$

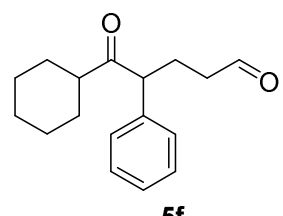

${ }^{1} \mathrm{H} \mathrm{NMR} \mathrm{(400} \mathrm{MHz,} \mathrm{CDCl}_{3}$ )

人रोi

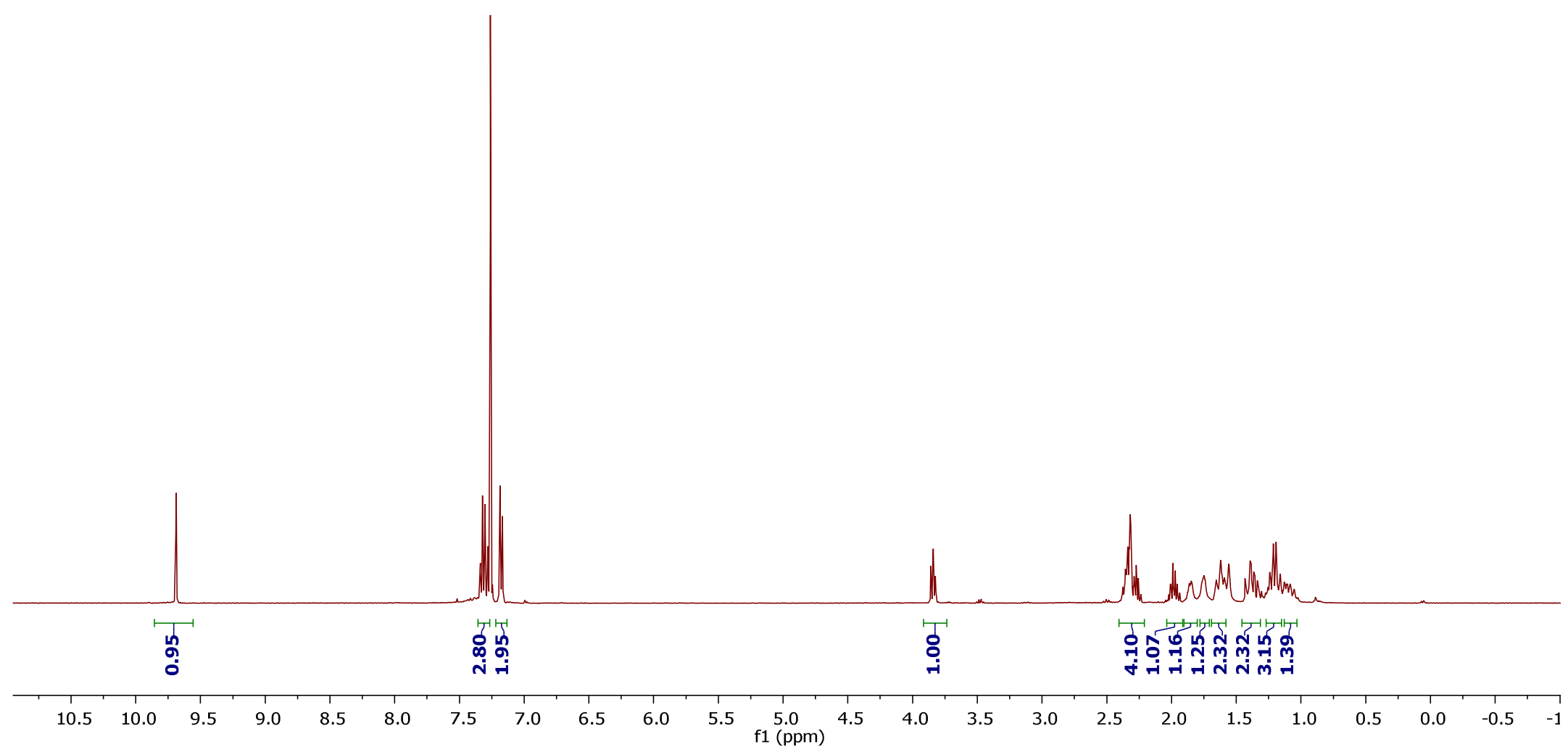




$$
4=1
$$




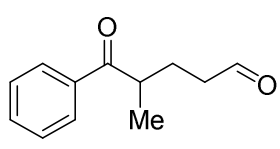

$5 g$

${ }^{1} \mathrm{H} \mathrm{NMR}\left(500 \mathrm{MHz}, \mathrm{CDCl}_{3}\right)$

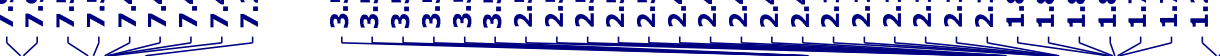

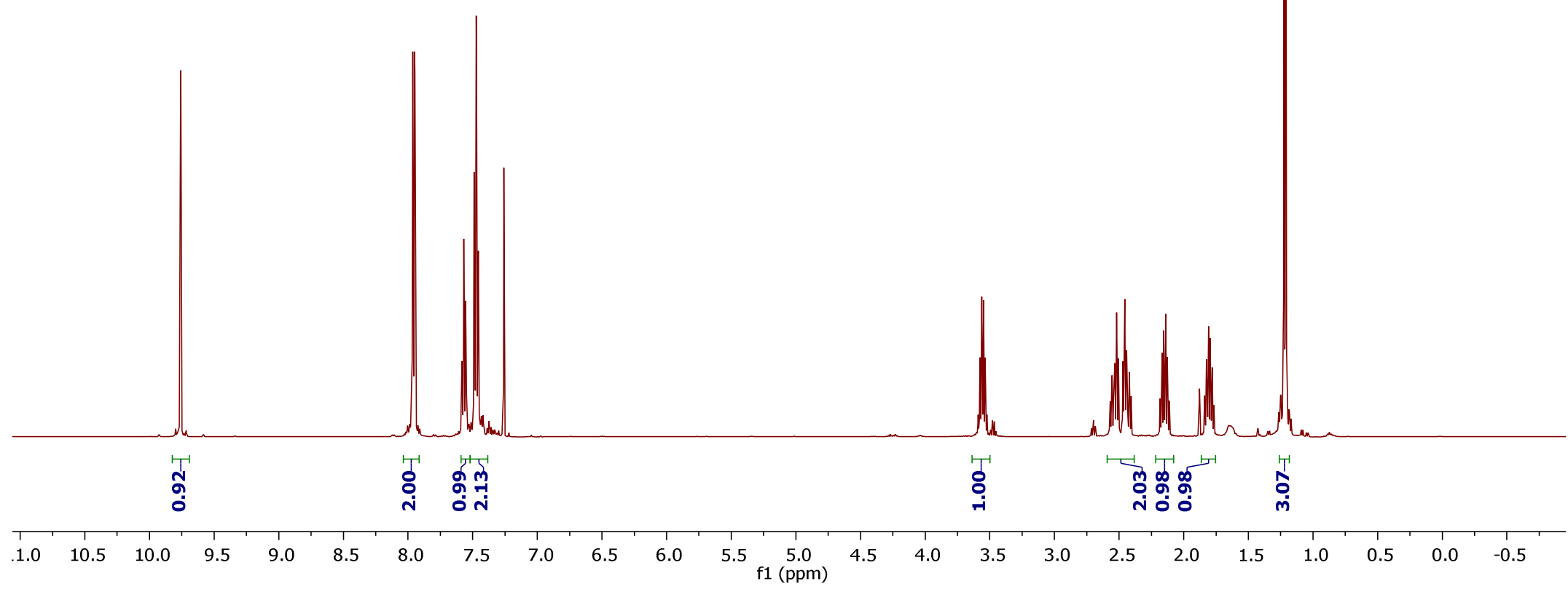


กุ๊

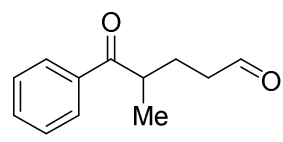

$5 \mathrm{~g}$

${ }^{13} \mathrm{C}\left\{{ }^{1} \mathrm{H}\right\} \mathrm{NMR}\left(126 \mathrm{MHz}, \mathrm{CDCl}_{3}\right)$

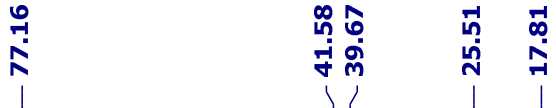
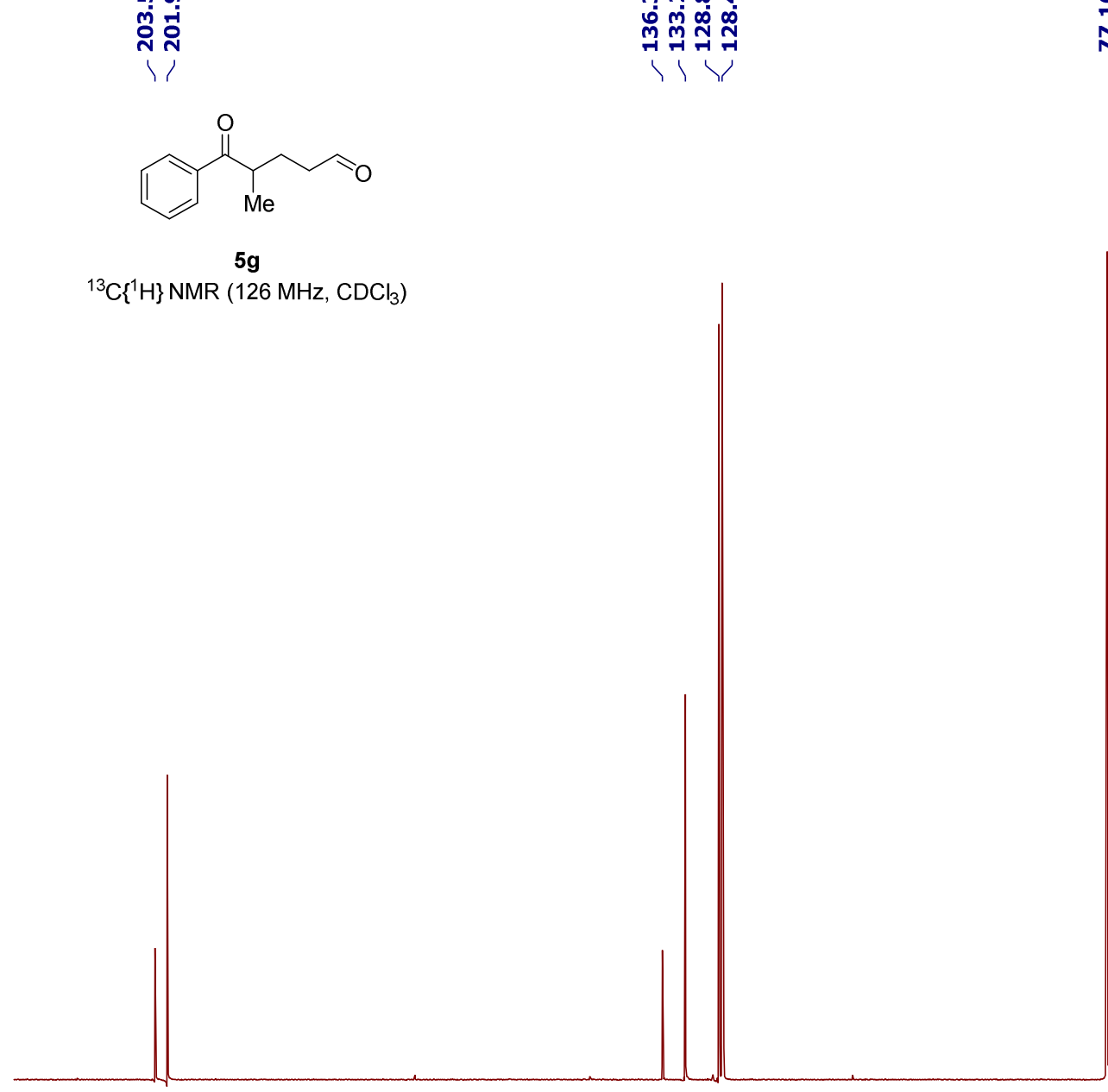

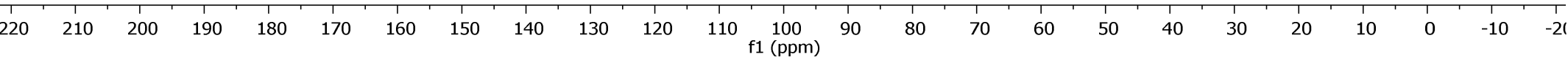




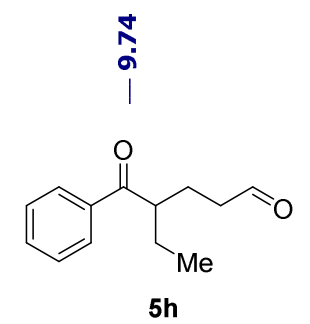

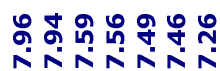

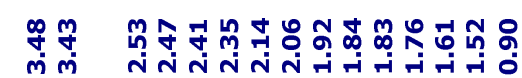

रnina

)

${ }^{1} \mathrm{HNMR}\left(500 \mathrm{MHz}, \mathrm{CDCl}_{3}\right)$

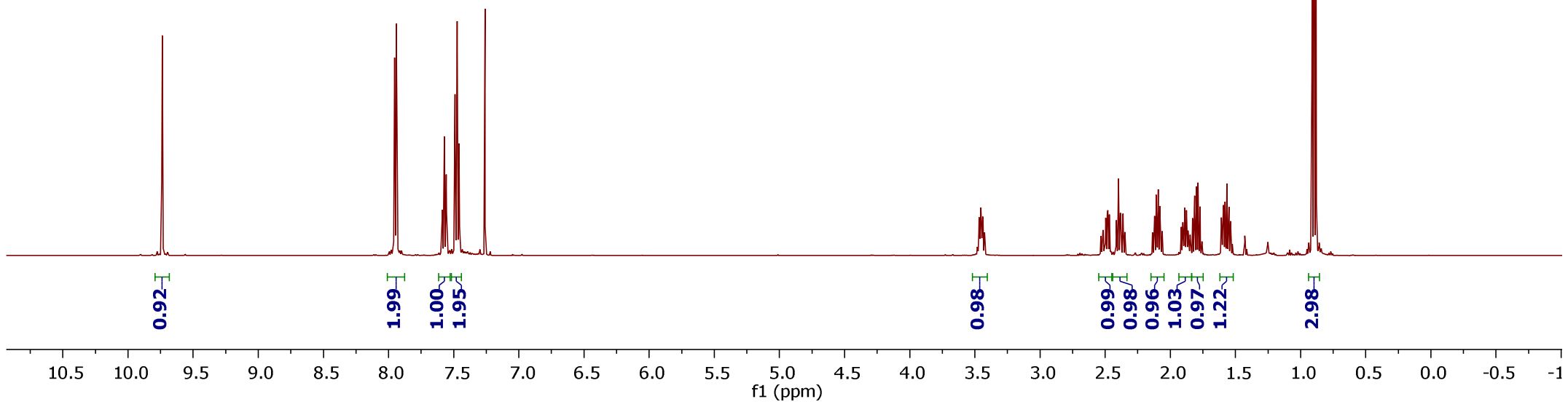



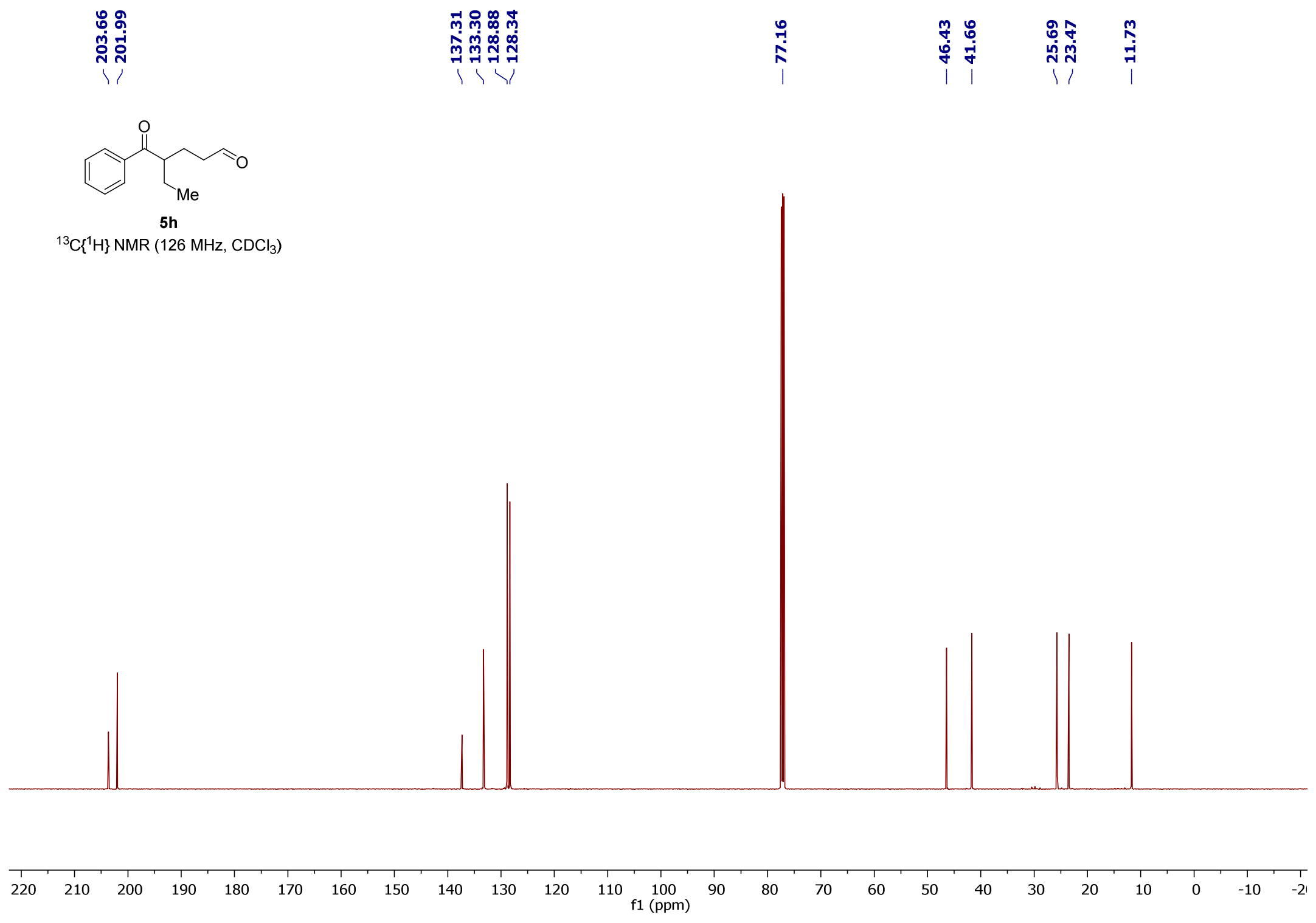


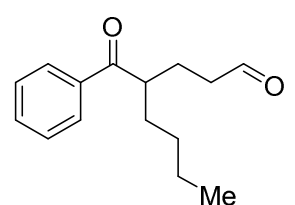

$5 \mathbf{i}$

${ }^{1} \mathrm{H} \mathrm{NMR}\left(500 \mathrm{MHz}, \mathrm{CDCl}_{3}\right)$

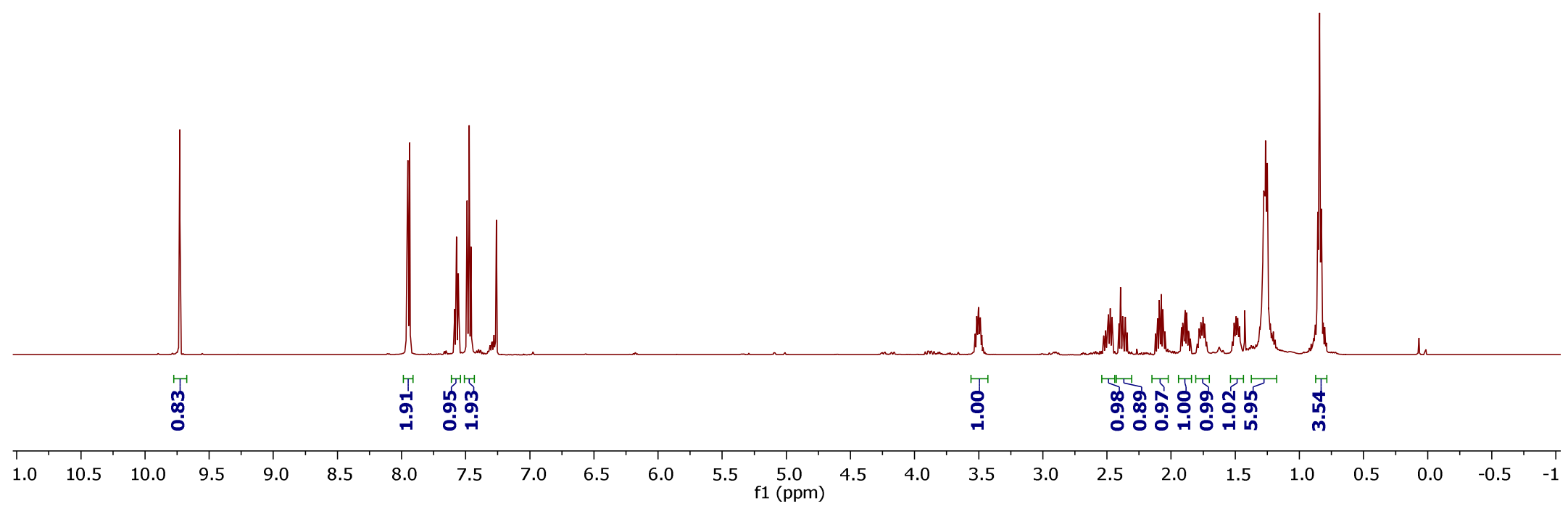




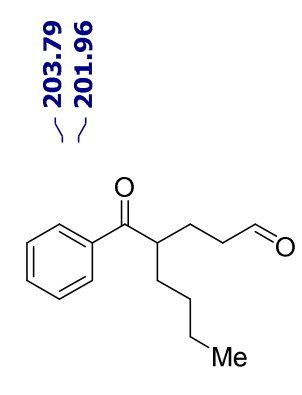

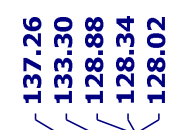

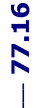

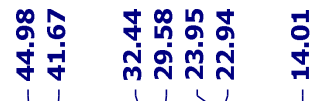

$\left.{ }^{13} \mathrm{C}^{1} \mathrm{H}\right\} \mathrm{NMR}\left(126 \mathrm{MHz}, \mathrm{CDCl}_{3}\right)$

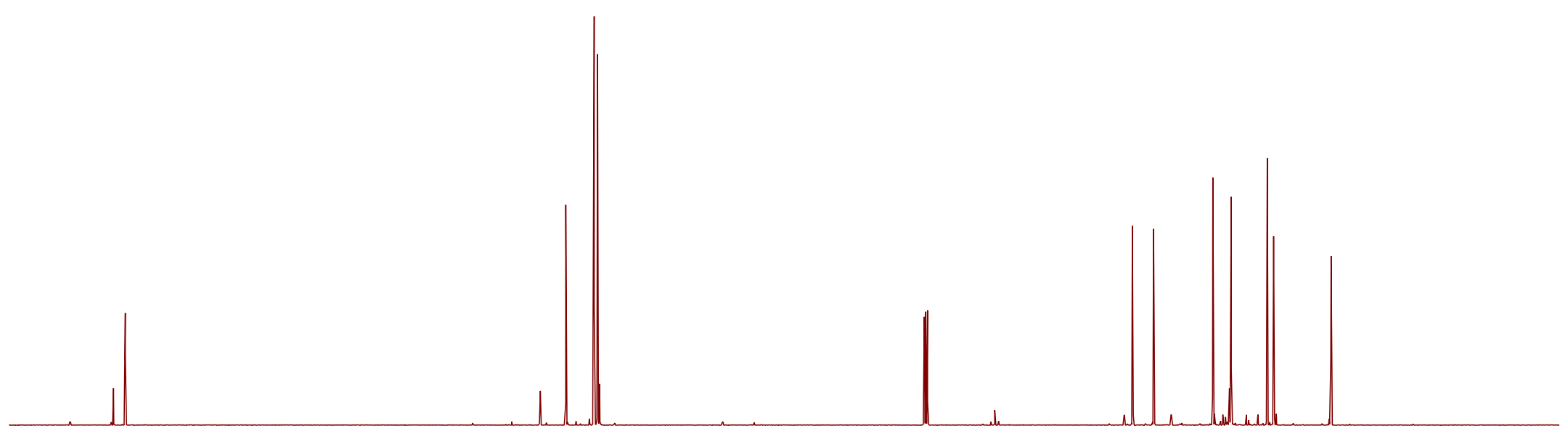

\begin{tabular}{|lllllllllllllllllllllllllllllllllllll}
\hline 20 & 210 & 200 & 190 & 180 & 170 & 160 & 150 & 140 & 130 & 120 & 110 & 100 & 90 & 80 & 70 & 60 & 50 & 40 & 30 & 20 & 10 & 0 & -10 & -21
\end{tabular} 


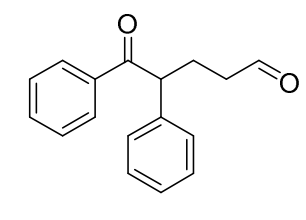

${ }^{1} \mathrm{H} \mathrm{NMR} \mathrm{(500} \mathrm{MHz,} \mathrm{CDCl}_{3}$ )

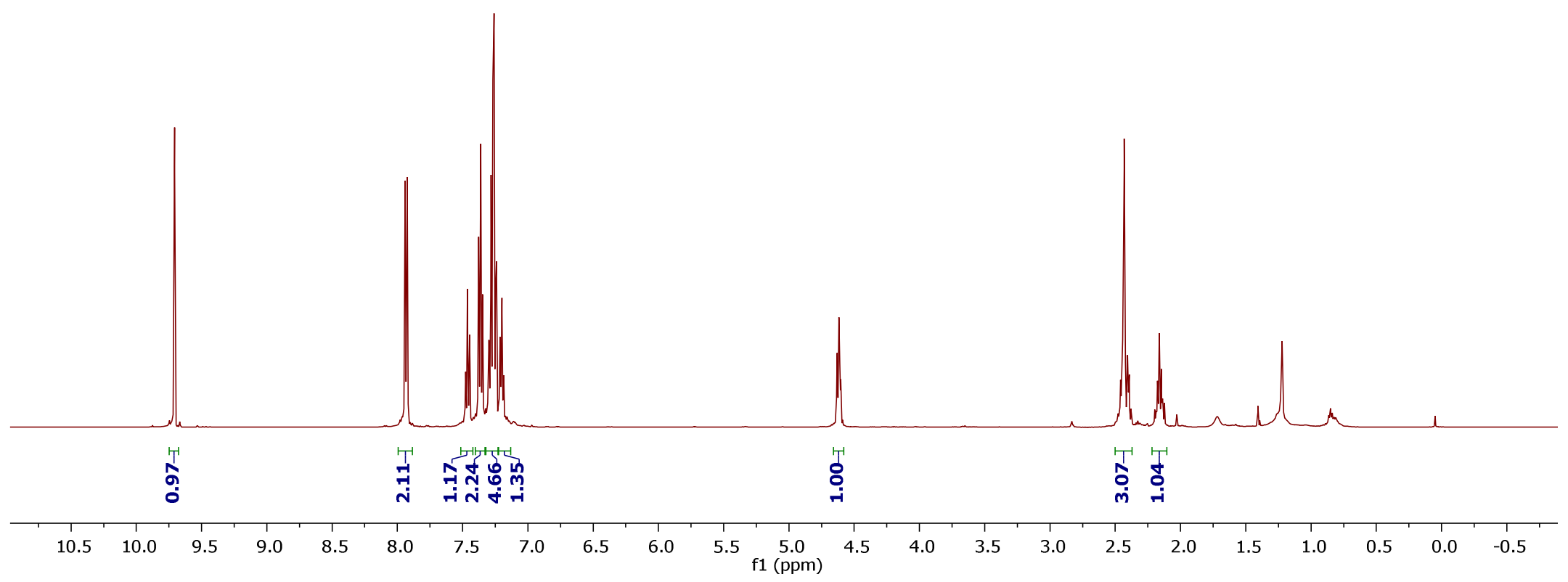




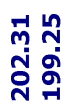

ตำำํํำำำ

所

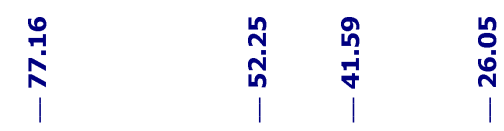

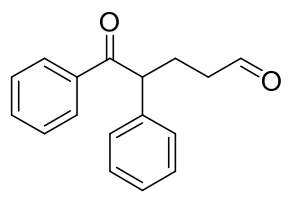

$\left.{ }^{13} \mathrm{C}^{-1} \mathrm{H}\right\} \mathrm{NMR}\left(126 \mathrm{MHz}, \mathrm{CDCl}_{3}\right)$

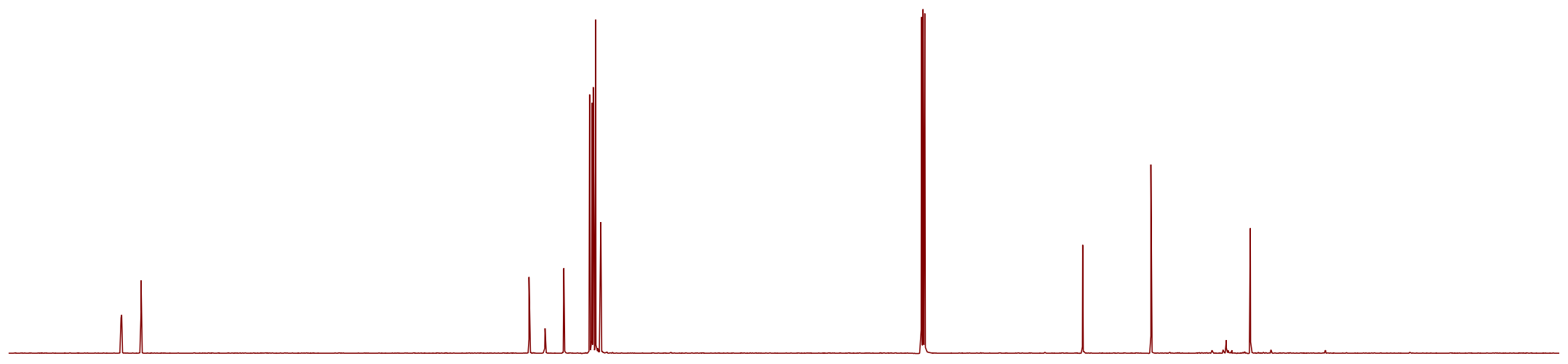

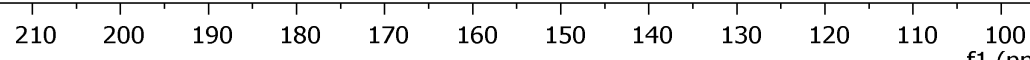

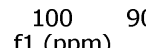
$80 \quad 70$ $60 \quad 50$ $40 \quad 30 \quad 20$ $10 \quad 0$ $\begin{array}{ll}-10 & -20\end{array}$ 
î.

$\mathrm{NtOOC}_{\mathrm{O}}$

Me

${ }^{1} \mathrm{H}$ NMR (400 MHz, $\mathrm{CDCl}_{3}$ )

(2)

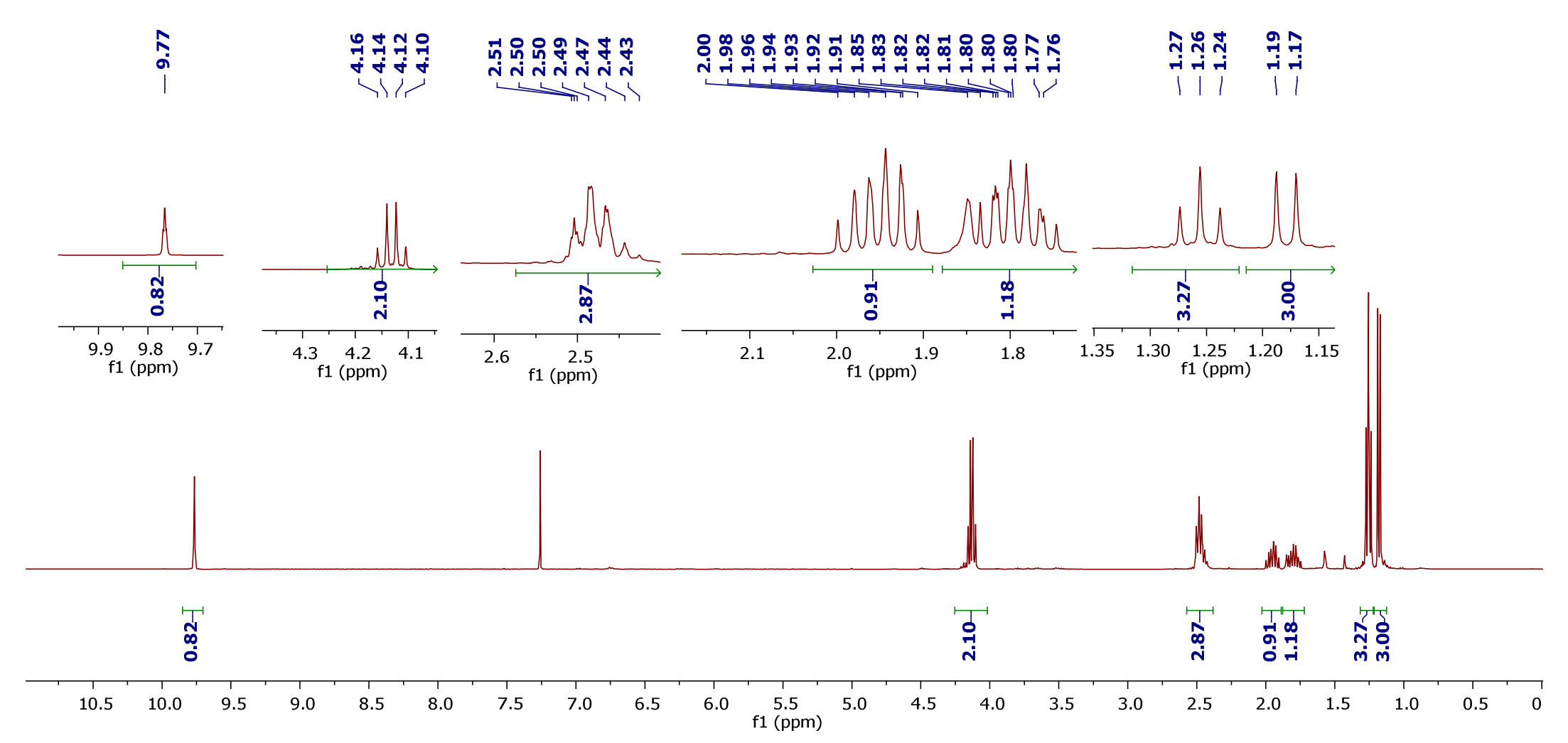




$$
\text { I. }
$$




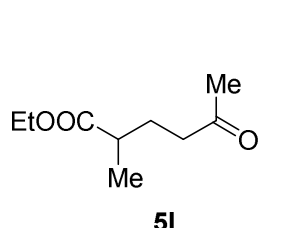

${ }^{1} \mathrm{HNMR}\left(400 \mathrm{MHz}, \mathrm{CDCl}_{3}\right.$ )

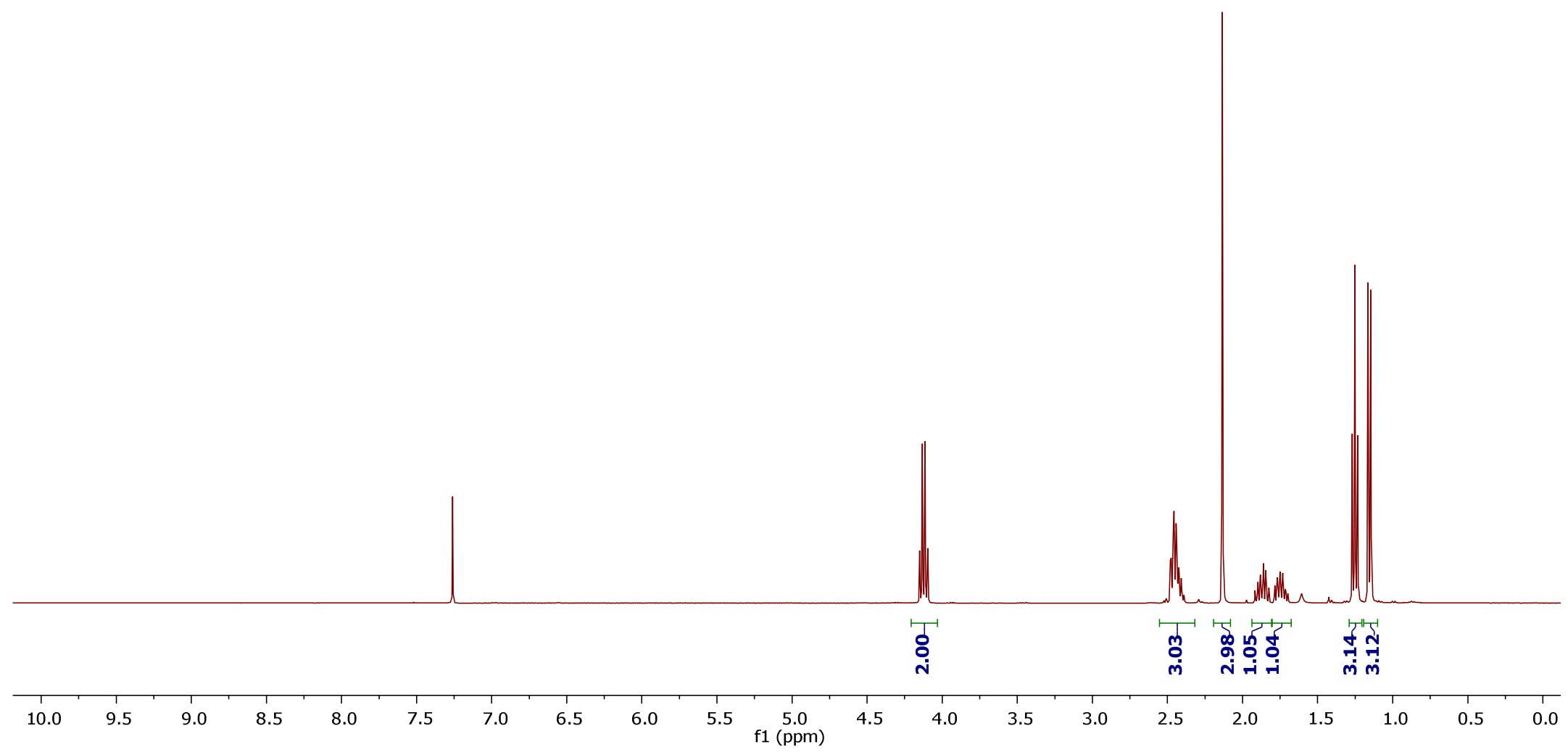




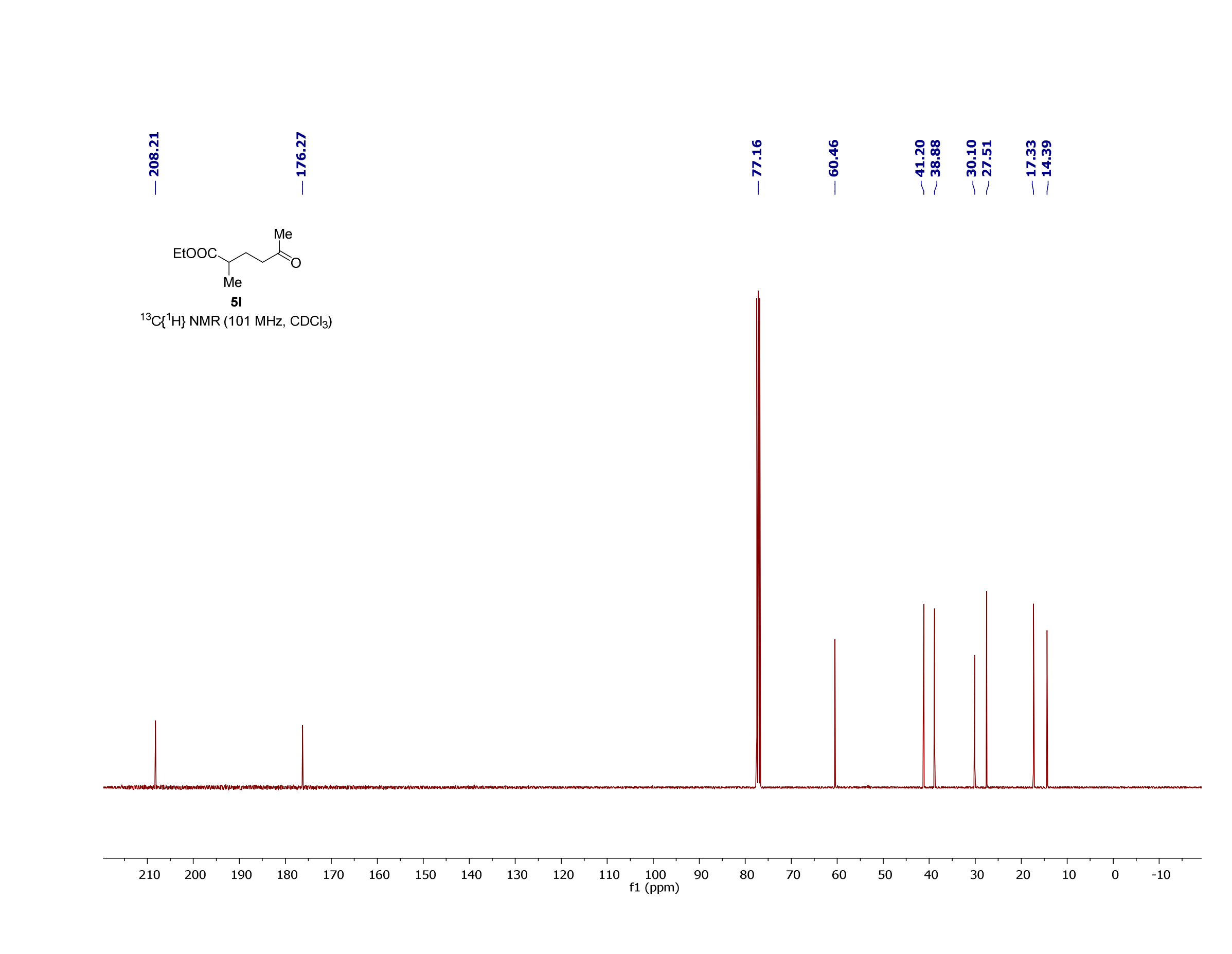




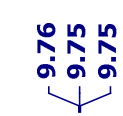

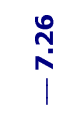

$\stackrel{7}{7}$

${ }_{1 \mathrm{HNMR}}\left(400 \mathrm{mHz}, \mathrm{CDCl}_{3}\right)$
นึำ

|

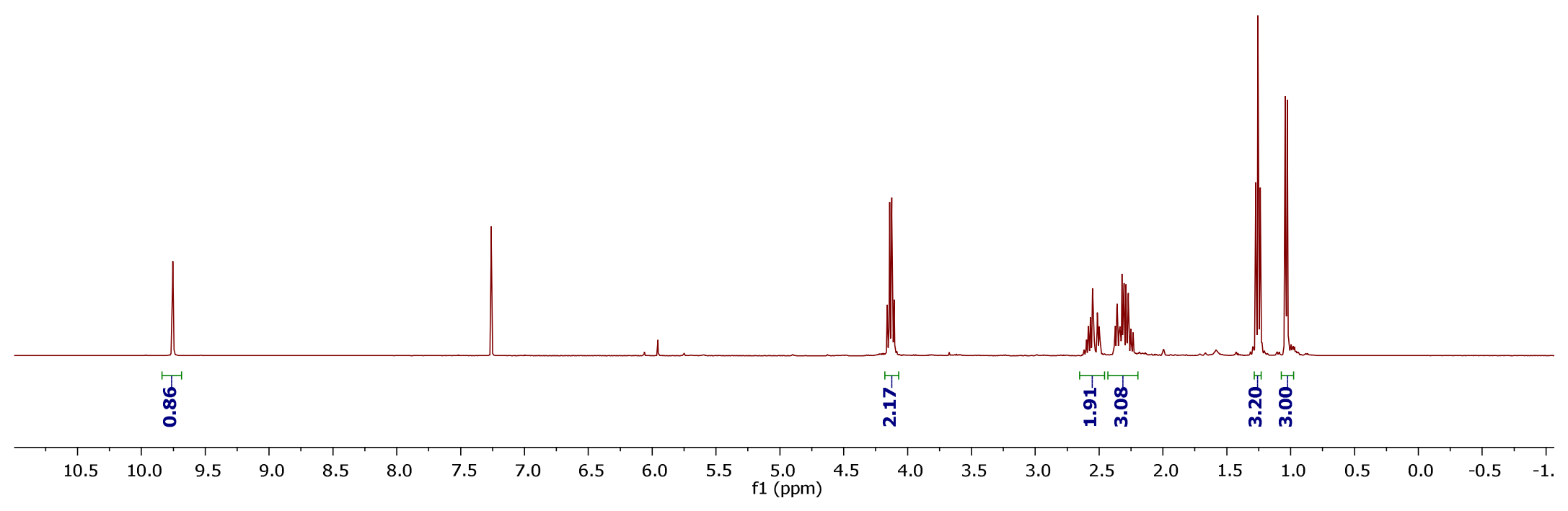




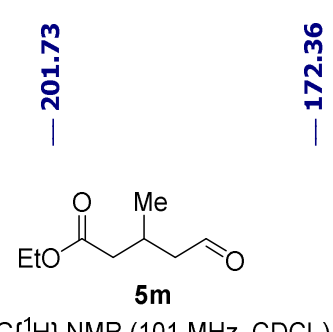

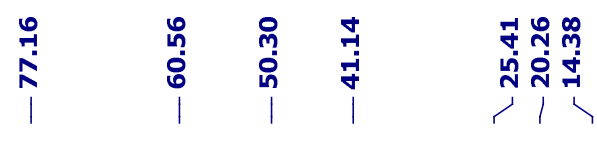

$\left.{ }^{13} \mathrm{C}_{\{}{ }^{1} \mathrm{H}\right\} \mathrm{NMR}\left(101 \mathrm{MHz}, \mathrm{CDCl}_{3}\right)$

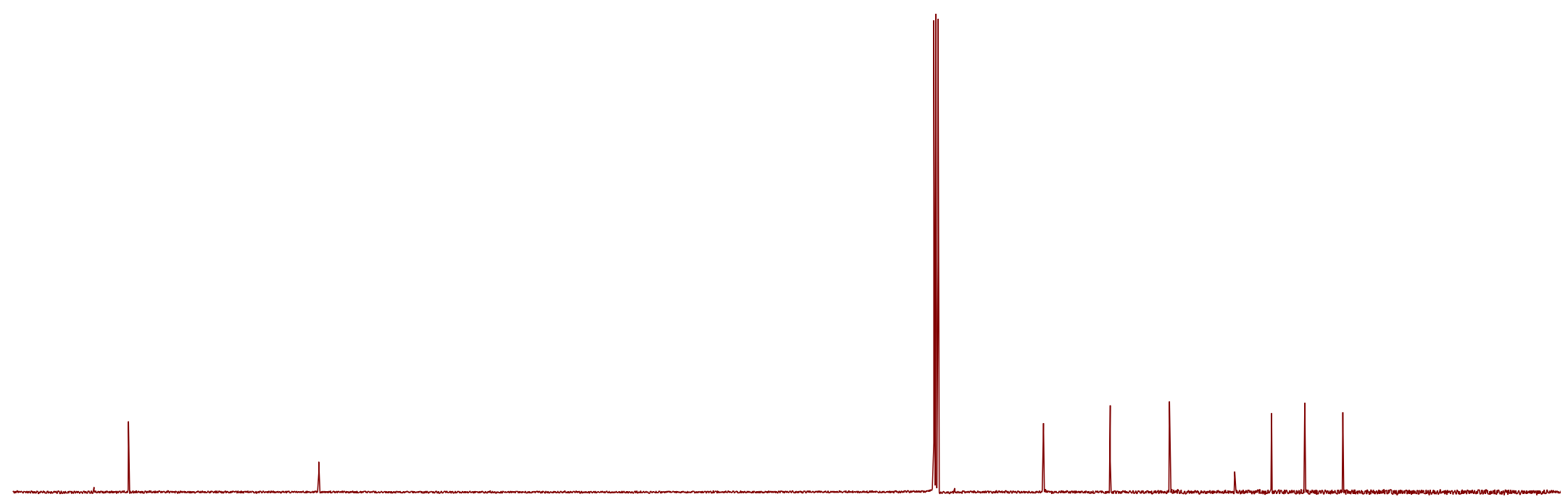

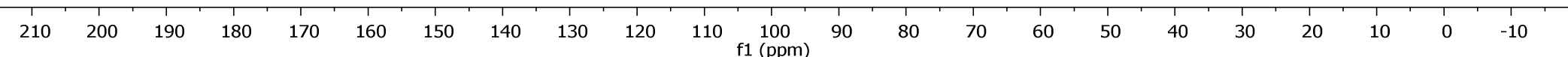


茴

Mำกำ ำ

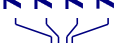

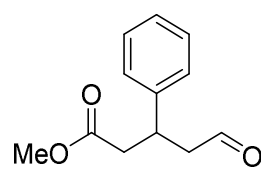

$5 n$

${ }^{1} \mathrm{HNMR}\left(500 \mathrm{MHz}, \mathrm{CD}_{2} \mathrm{Cl}_{2}\right)$

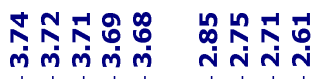

$\underbrace{2}$

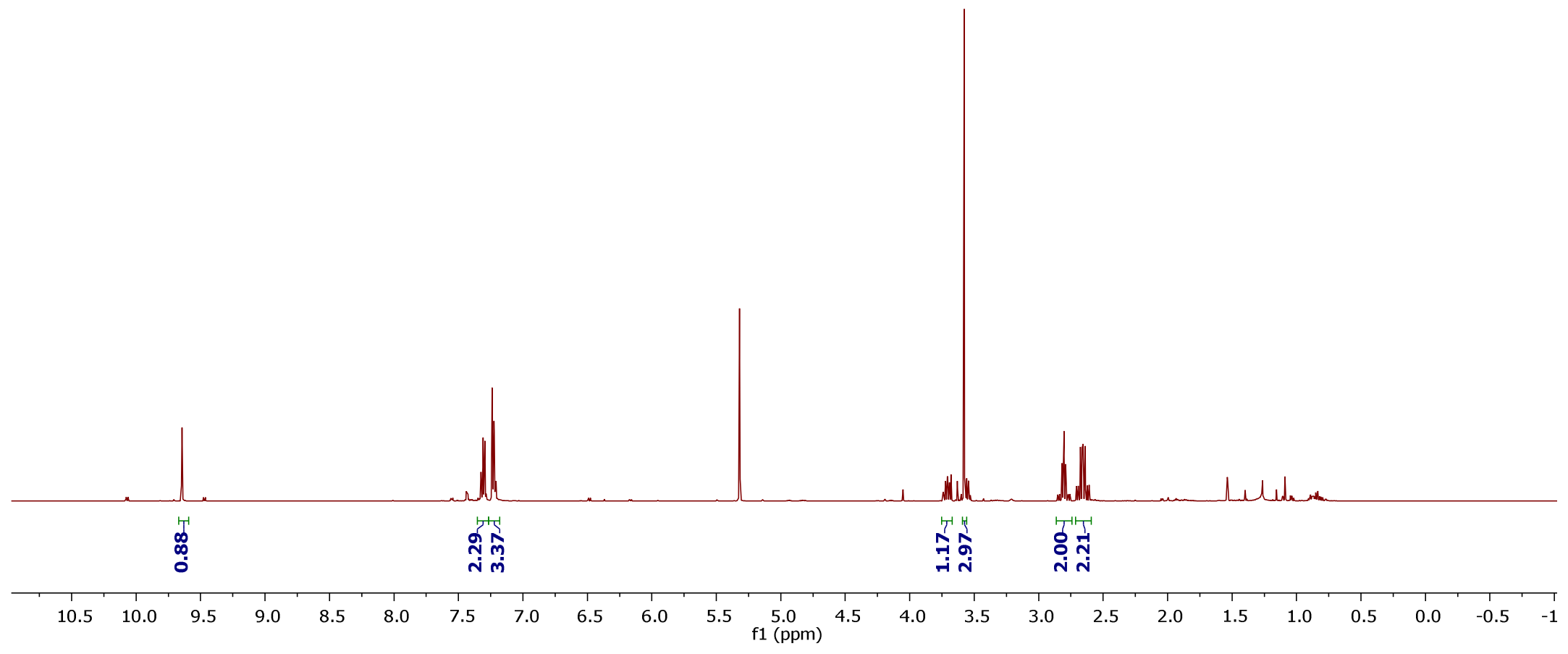



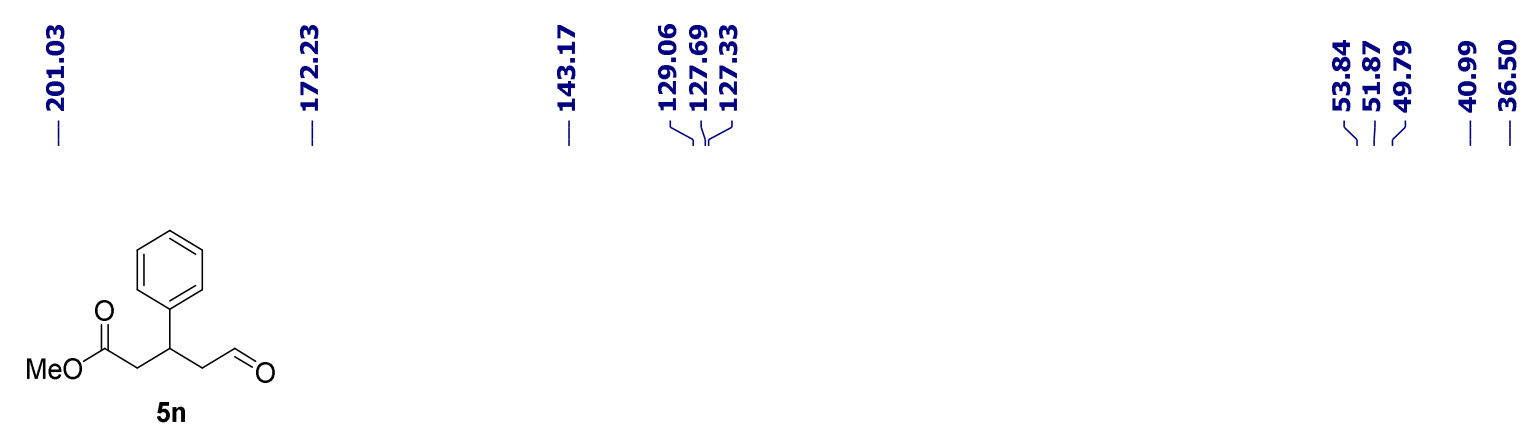

${ }^{13} \mathrm{C}\left\{{ }^{1} \mathrm{H}\right\} \mathrm{NMR}\left(126 \mathrm{MHz}, \mathrm{CD}_{2} \mathrm{Cl}_{2}\right)$

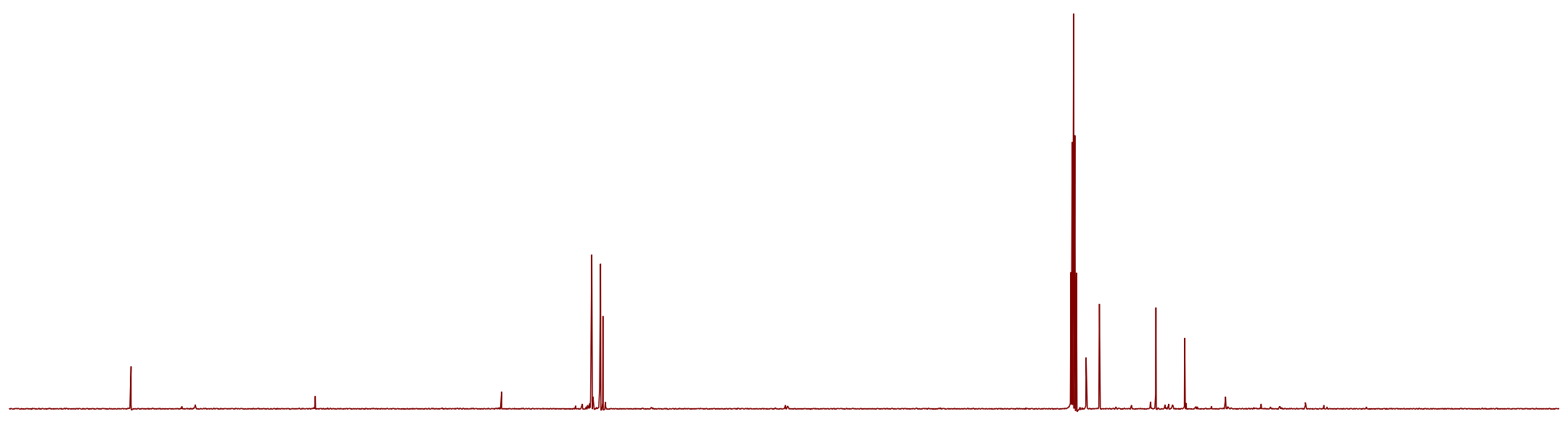

\begin{tabular}{|llllllllllllllllllllllllll}
\hline 20 & 210 & 200 & 190 & 180 & 170 & 160 & 150 & 140 & 130 & 120 & 110 & $\begin{array}{c}100 \\
\mathrm{f} 1(\mathrm{ppm})\end{array}$ & 90 & 80 & 70 & 60 & 50 & 40 & 30 & 20 & 10 & 0 & -10 & $-2 \mathrm{C}$
\end{tabular} 


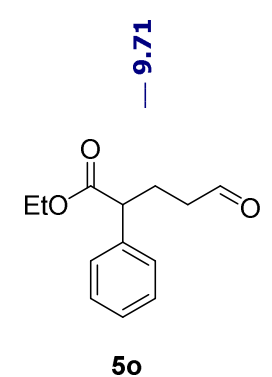

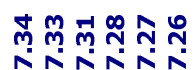

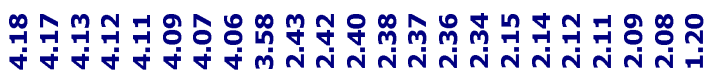

年

${ }^{1} \mathrm{H} \mathrm{NMR}\left(500 \mathrm{MHz}, \mathrm{CDCl}_{3}\right)$

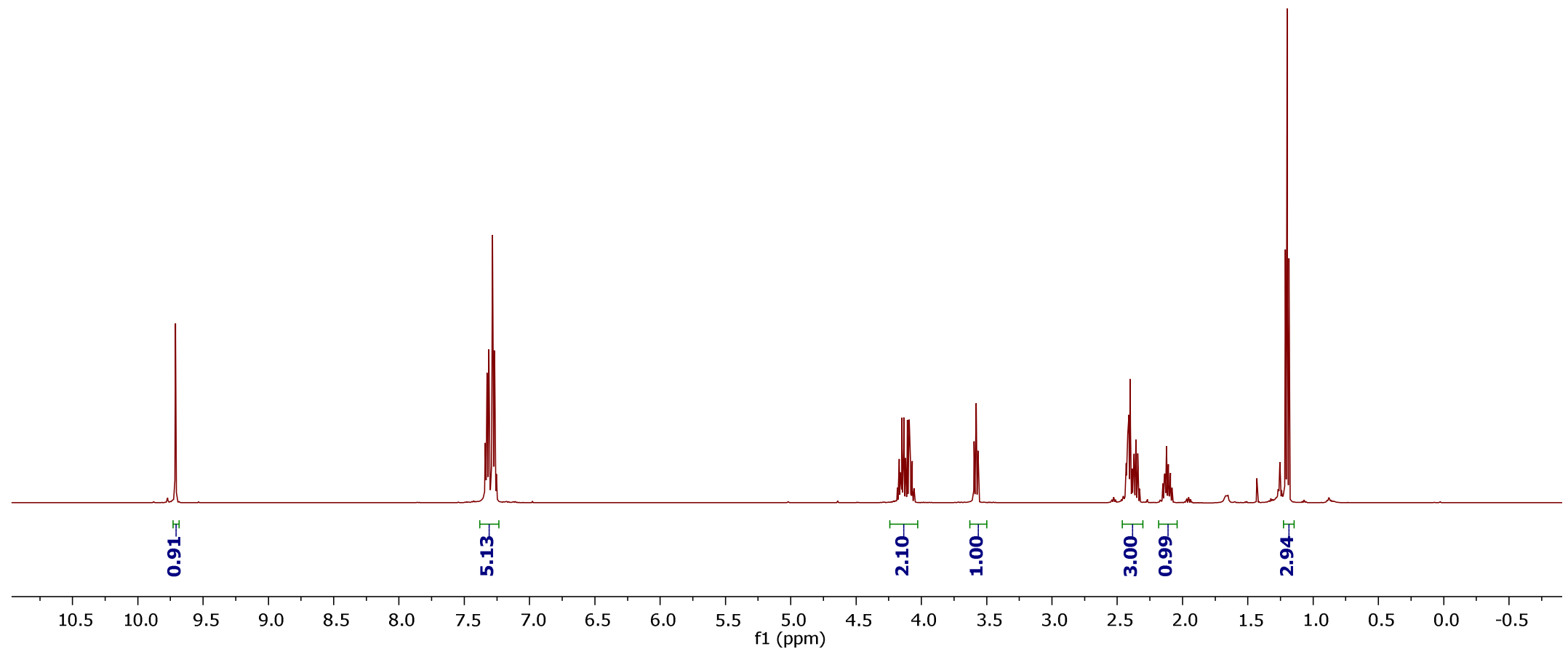




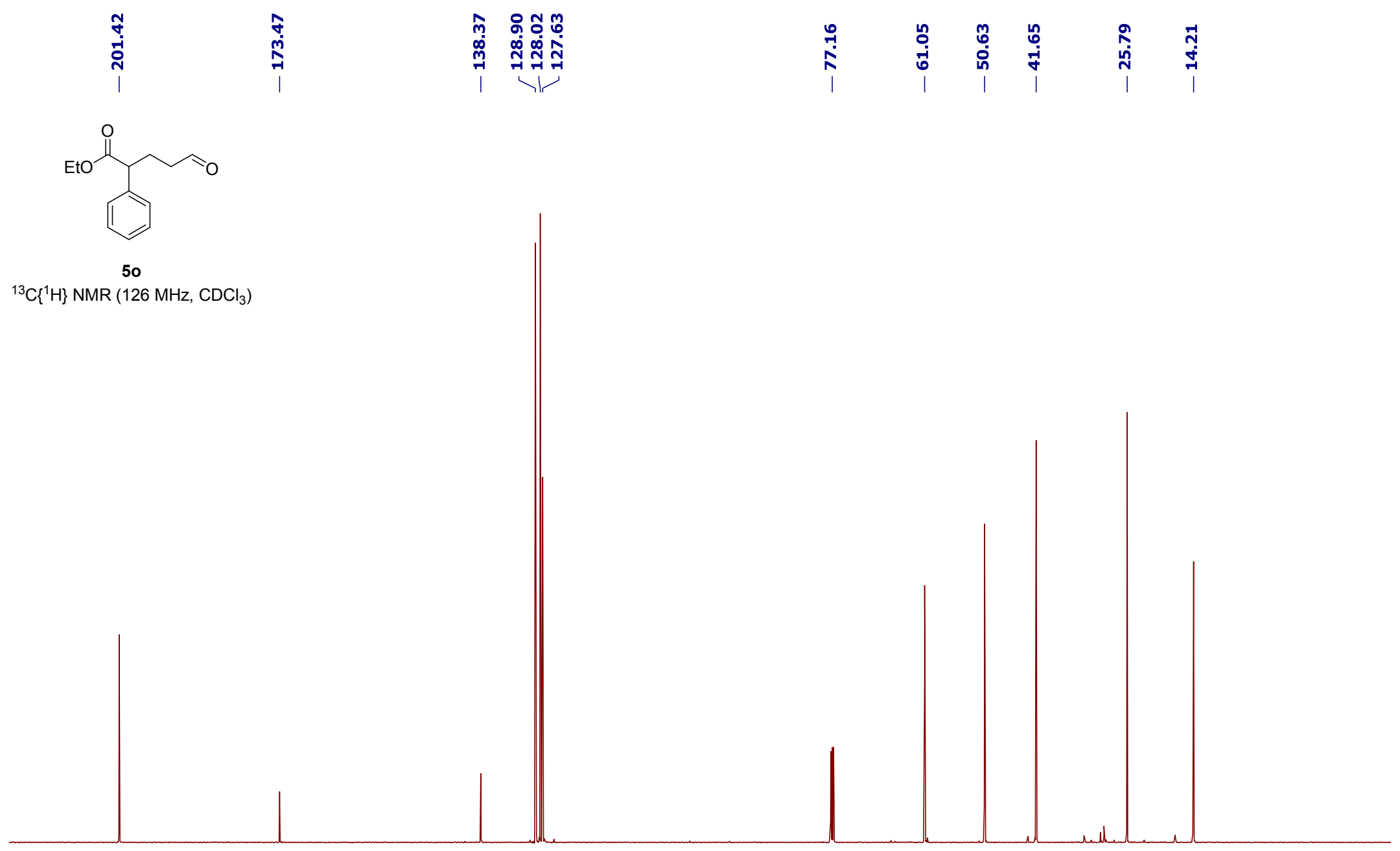

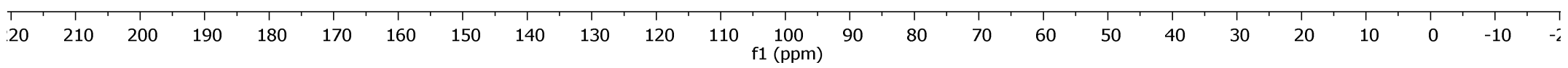




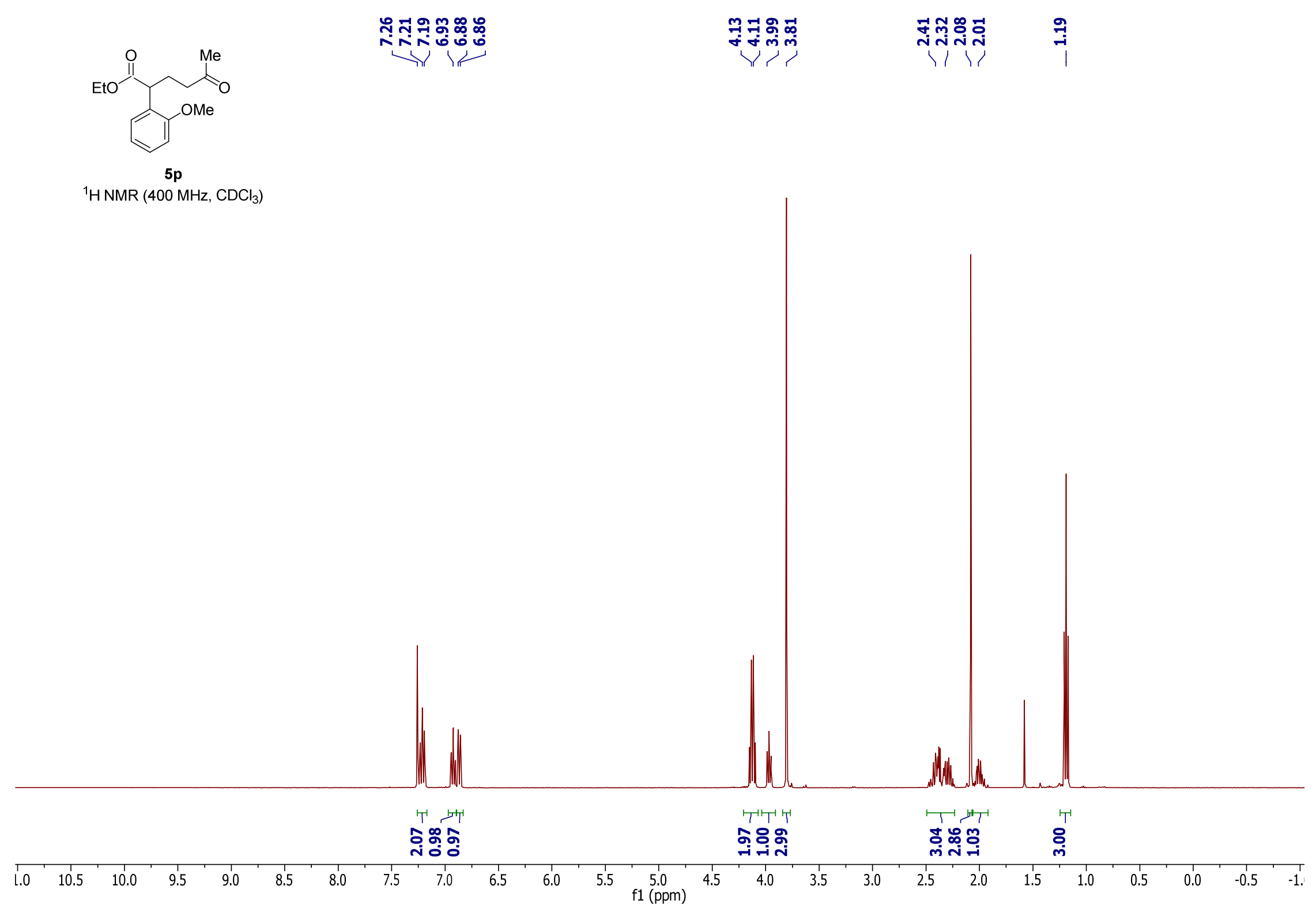




$$
\text { ten }
$$




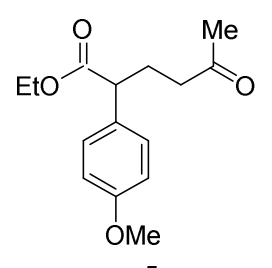

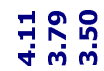

रो।

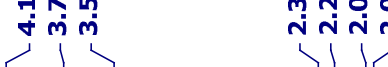

$\stackrel{9}{7}$

${ }^{1} \mathrm{H}$ NMR $\left(400 \mathrm{MHz}, \mathrm{CDCl}_{3}\right)$

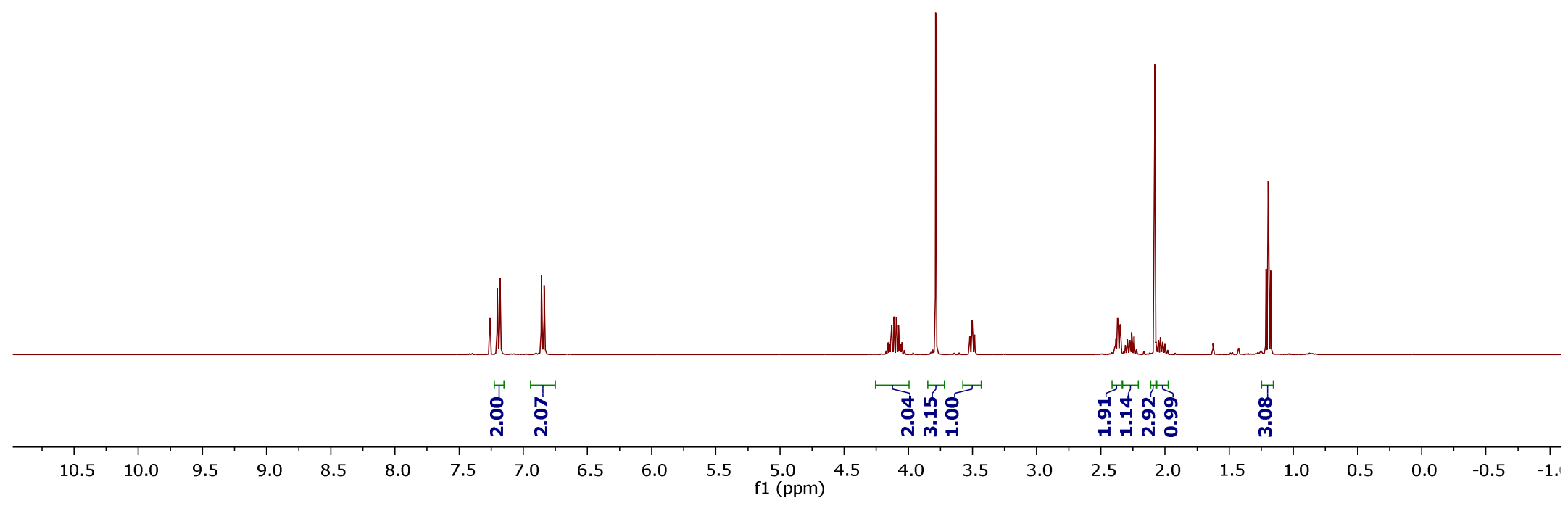




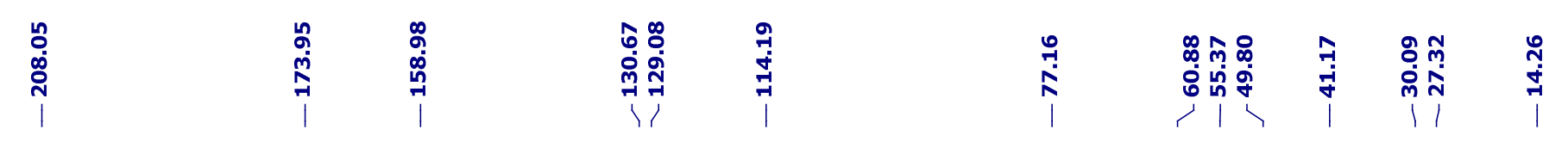

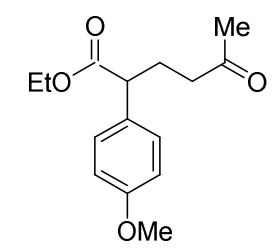

${ }^{13} \mathrm{C}\left\{{ }^{1} \mathrm{H}\right\} \mathrm{NMR}\left(101 \mathrm{MHz}, \mathrm{CDCl}_{3}\right)$

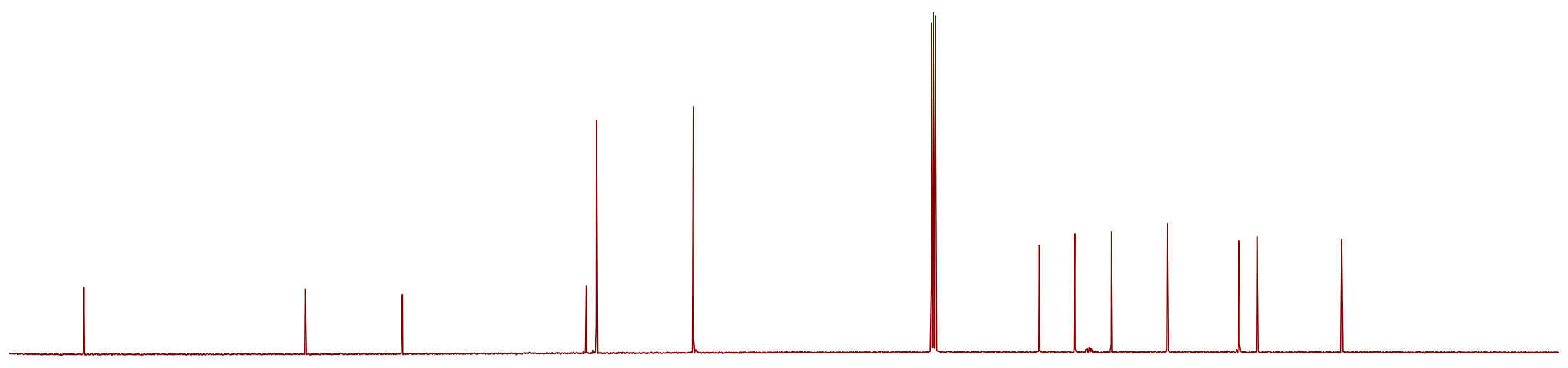

$\begin{array}{llllllllllllllllllllllllll}210 & 200 & 190 & 180 & 170 & 160 & 150 & 140 & 130 & 120 & 110 & 100 & 90 & 80 & 70 & 60 & 50 & 40 & 30 & 20 & 10 & 0 & -10\end{array}$ 


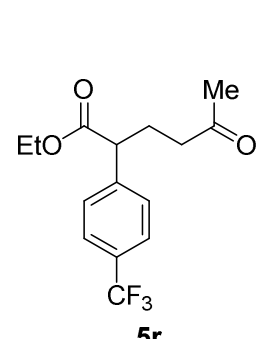

กิ่าำำ

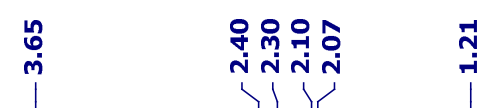

${ }^{1} \mathrm{HNMR}\left(400 \mathrm{MHz}, \mathrm{CDCl}_{3}\right)$

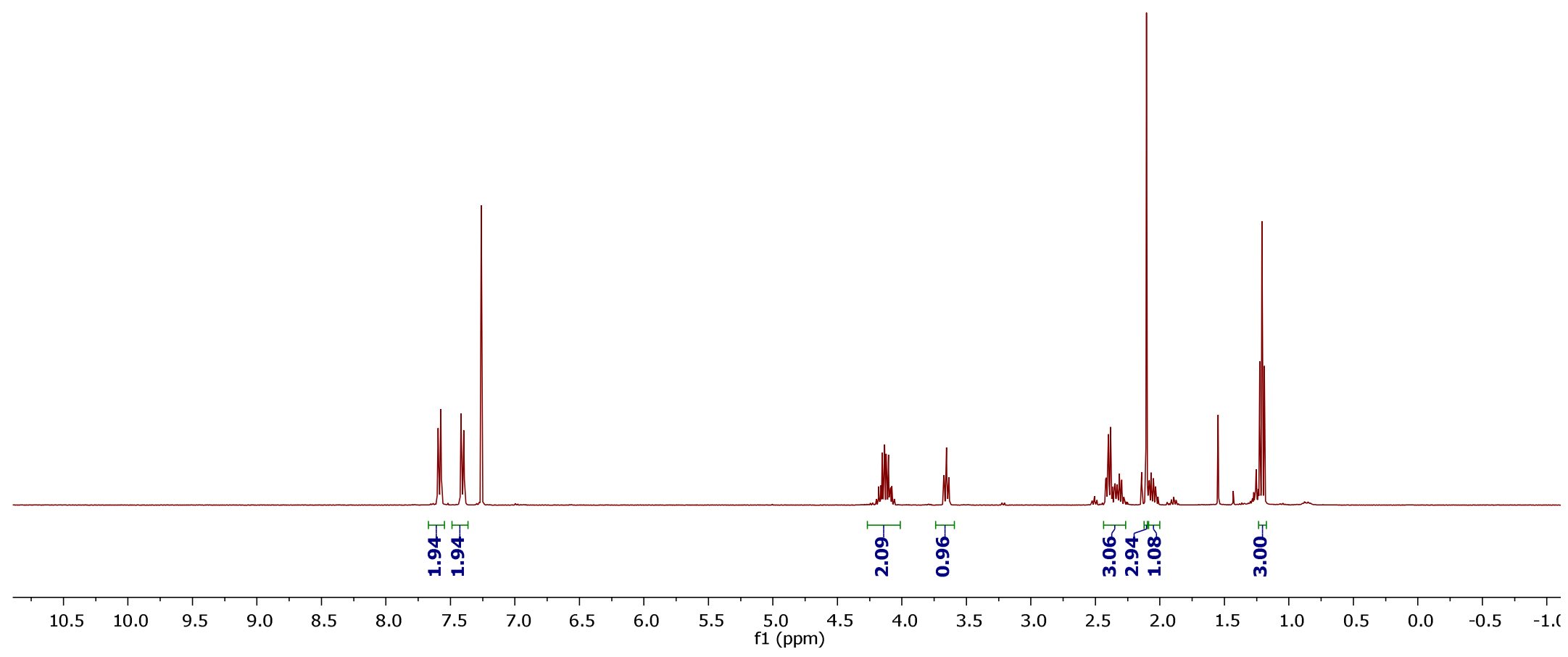




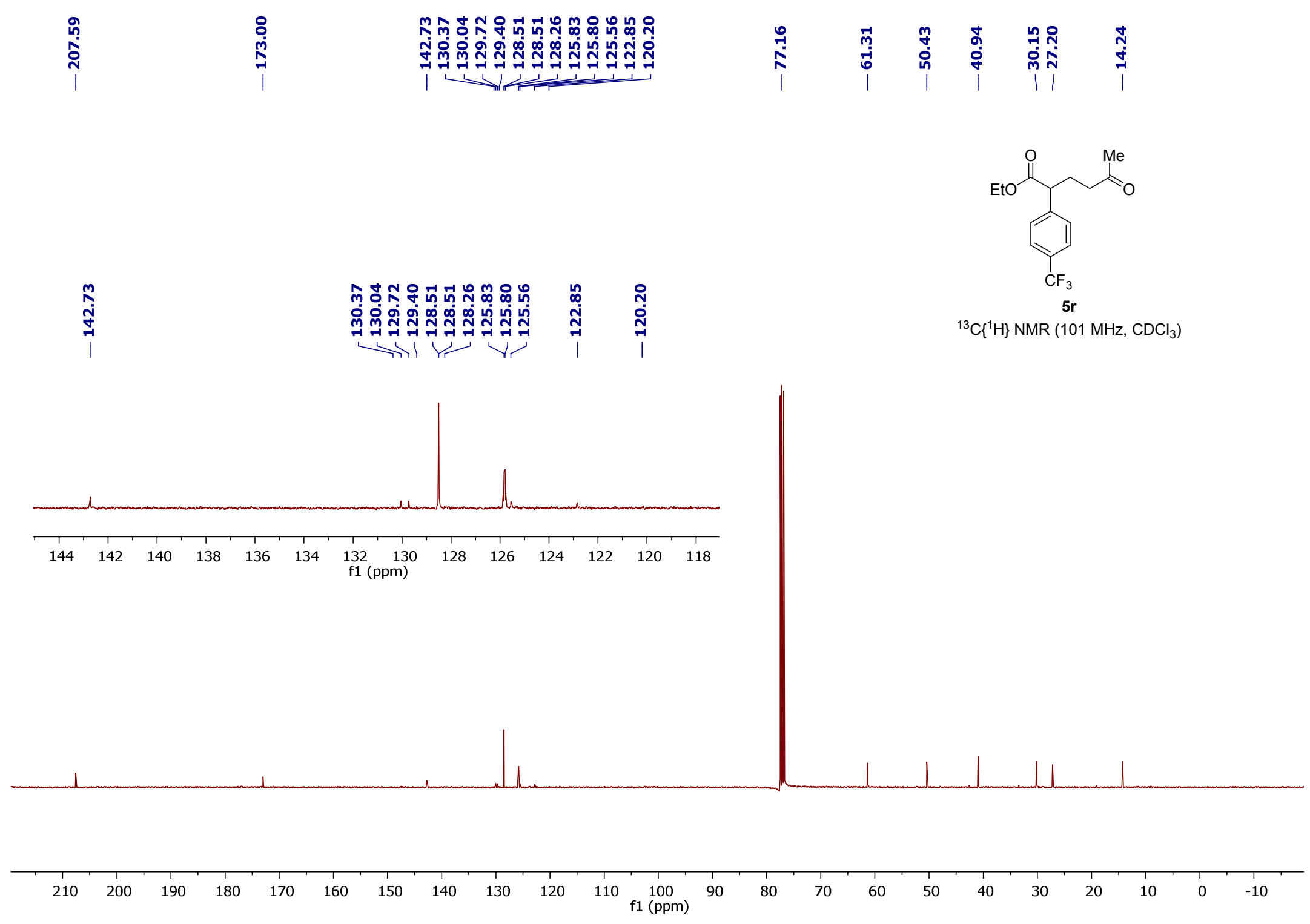




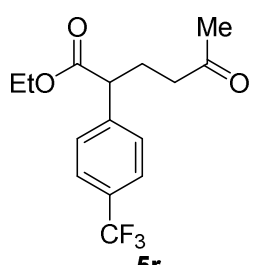

ถึํํ

${ }^{19} \mathrm{~F}\left\{{ }^{1} \mathrm{H},{ }^{13} \mathrm{C}\right\} \mathrm{NMR}\left(283 \mathrm{MHz}, \mathrm{CDCl}_{3}\right)$

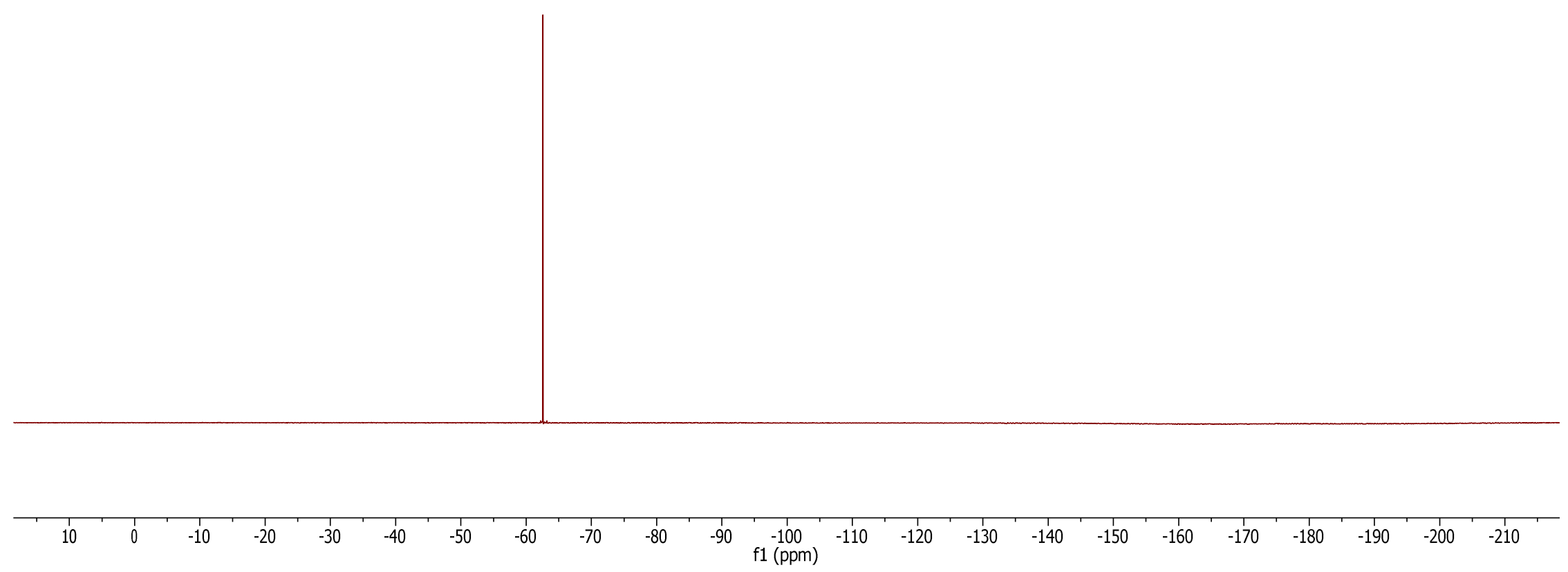




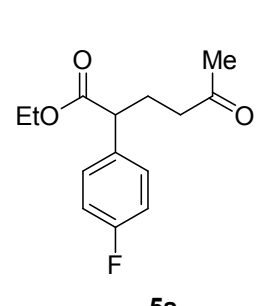

ํำ

iरi

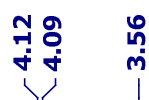

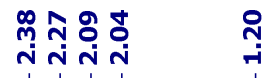

${ }^{1} \mathrm{H}$ NMR $\left(400 \mathrm{MHz}, \mathrm{CDCl}_{3}\right)$

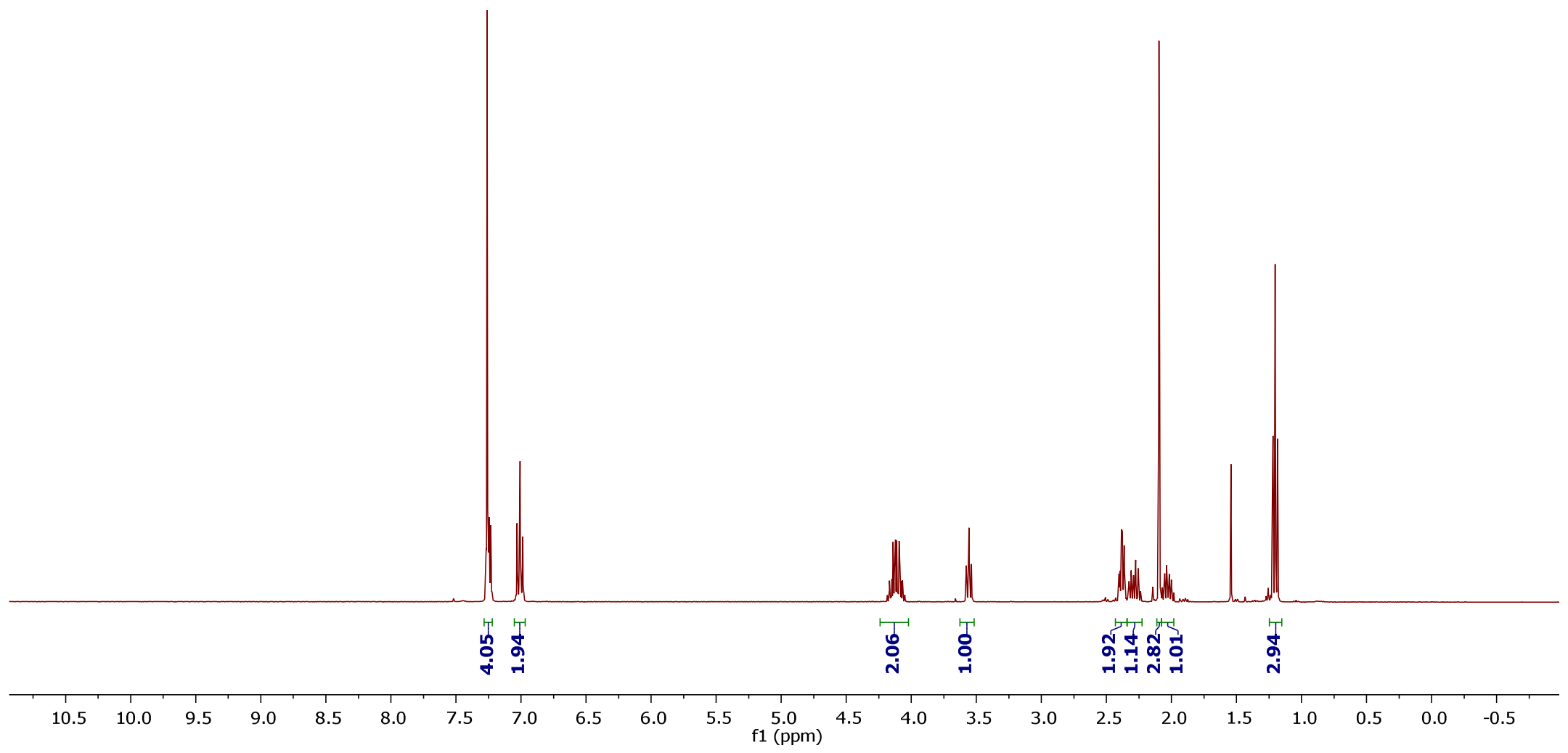




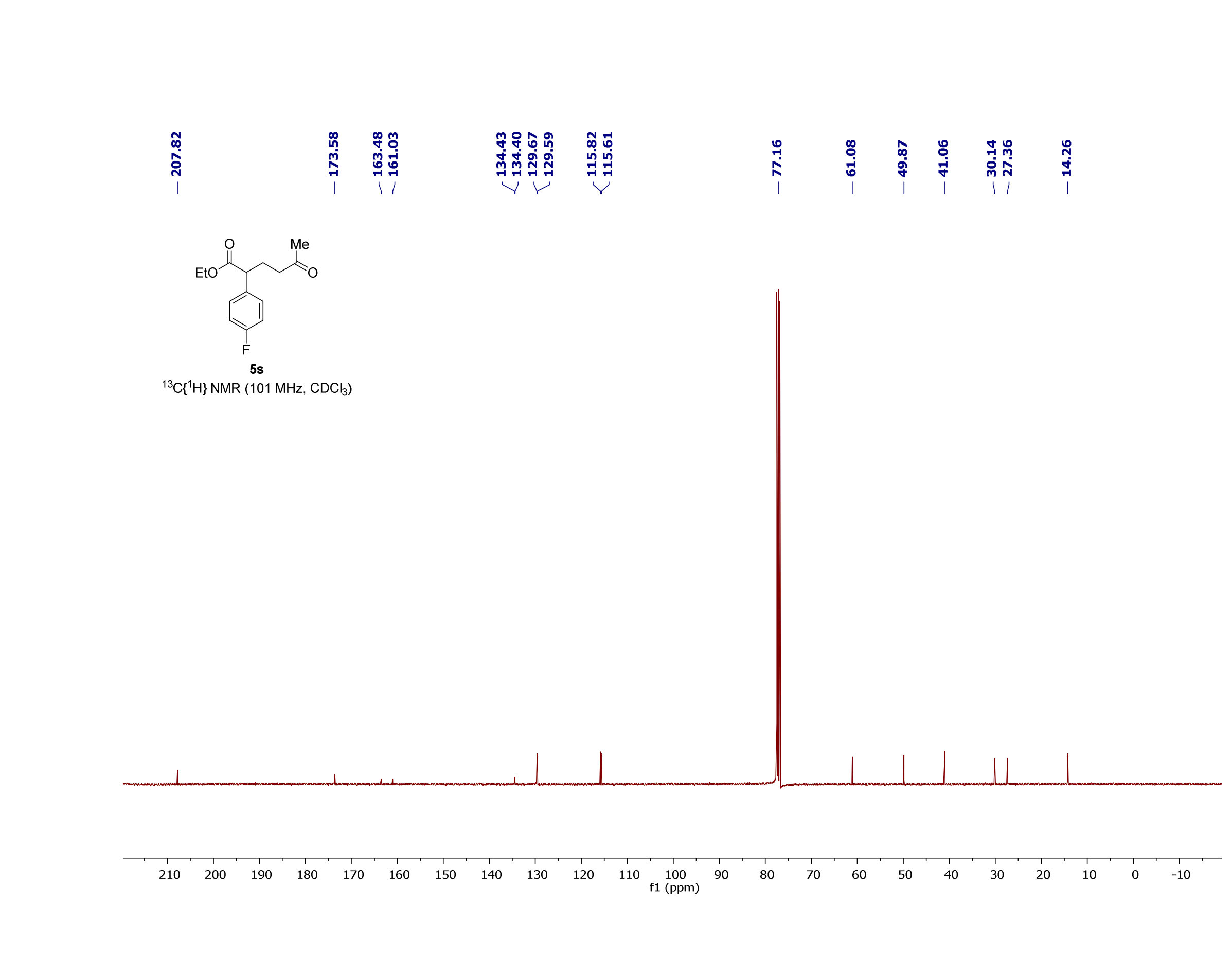


$\left.{ }^{19} \mathrm{~F}^{1}{ }^{1} \mathrm{H},{ }^{13} \mathrm{C}\right\} \mathrm{NMR}\left(283 \mathrm{MHz}, \mathrm{CDCl}_{3}\right)$

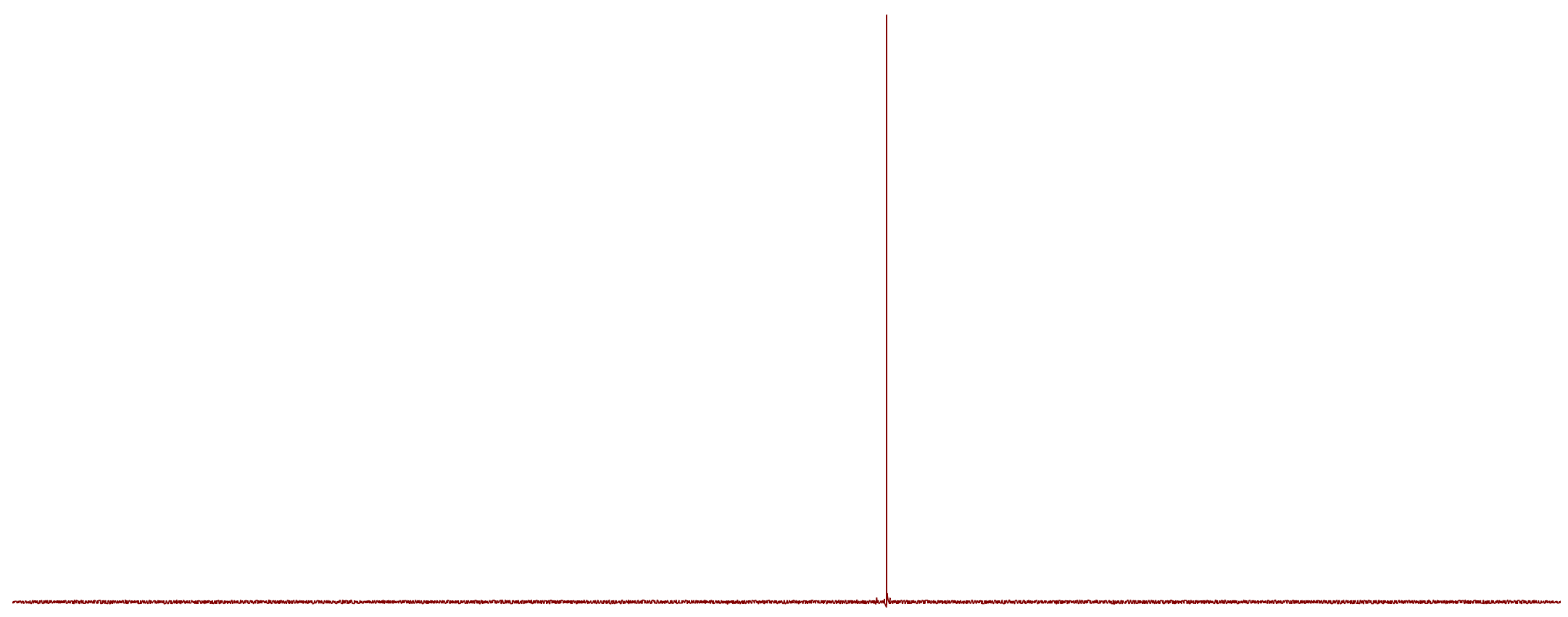




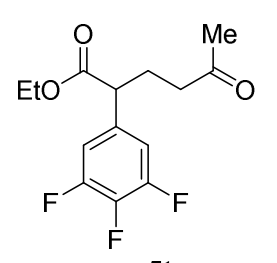

${ }^{1} \mathrm{H} \mathrm{NMR} \mathrm{(400} \mathrm{MHz,} \mathrm{CDCl}_{3}$ )

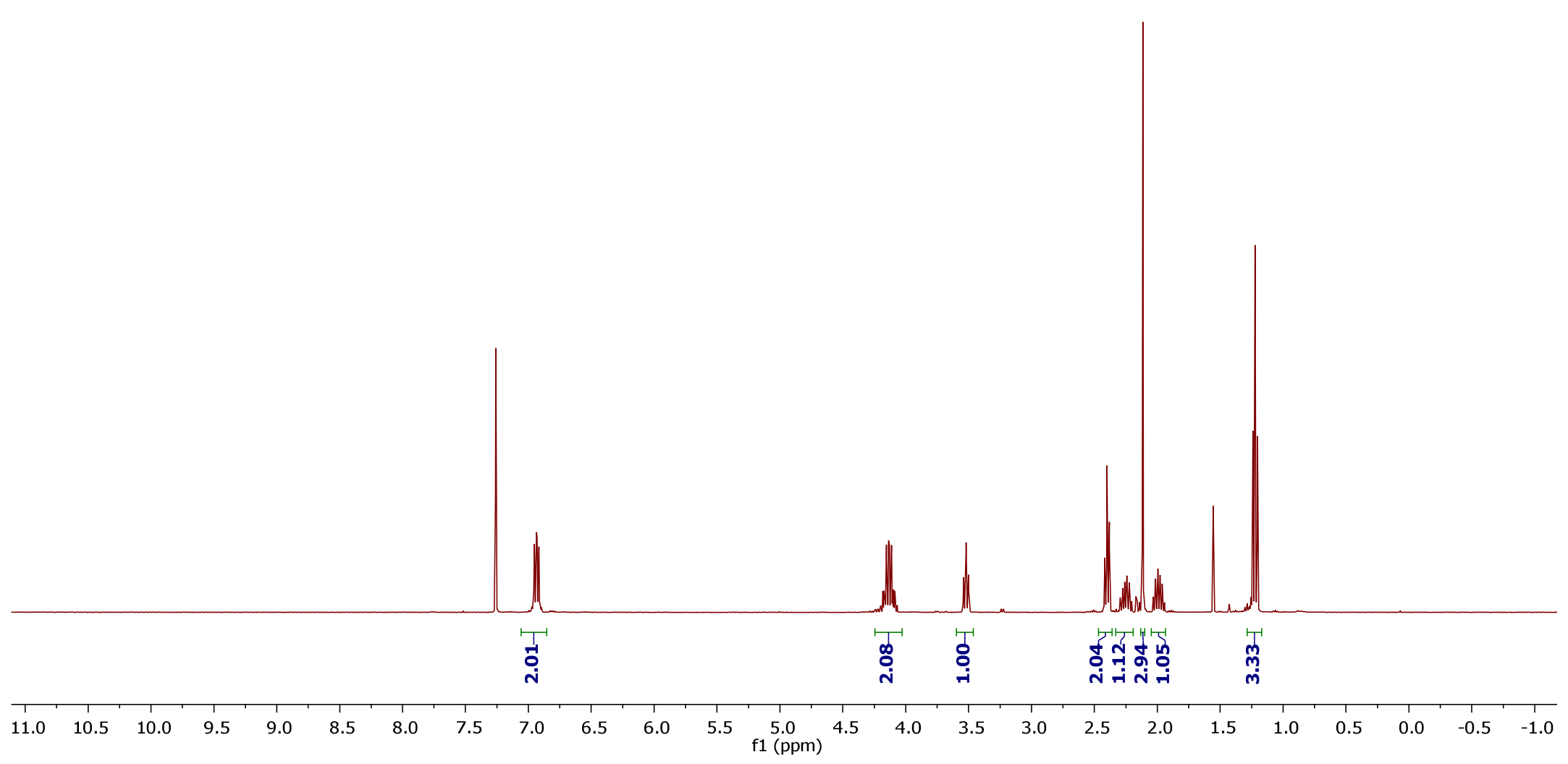




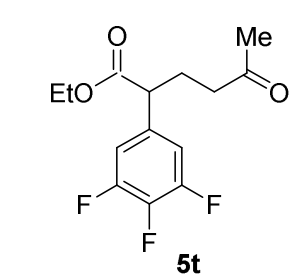

${ }^{13} \mathrm{C}\left\{{ }^{1} \mathrm{H}\right\}$ NMR $\left(101 \mathrm{MHz}, \mathrm{CDCl}_{3}\right)$

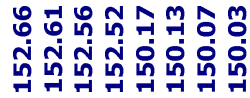

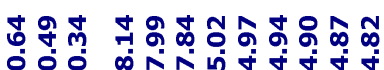

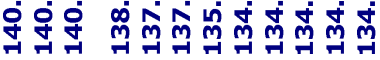

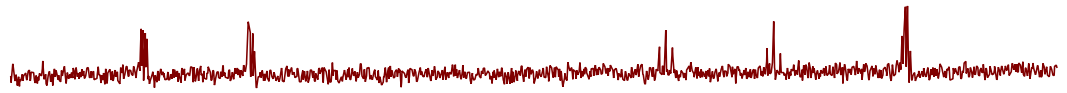

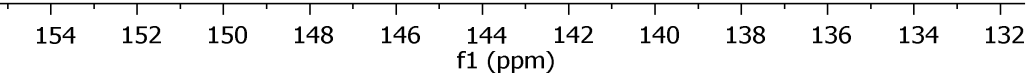

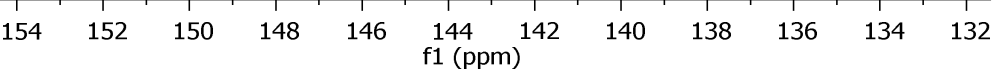

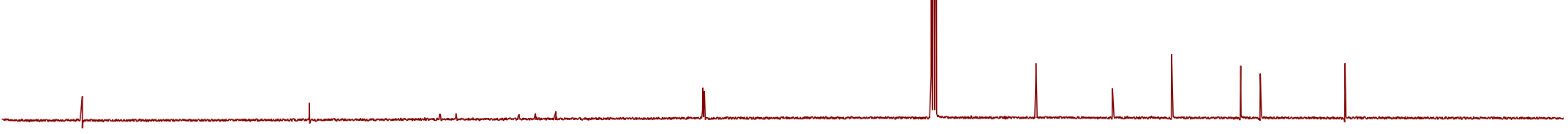

$\begin{array}{llll}210 & 200 & 190 & 180\end{array}$

$70 \quad 160$

$150 \quad 14$

$130 \quad 120$

$110 \quad 100$ 100
$\mathrm{f} 1(\mathrm{ppm})$

80

$70 \quad 60$

50

$40 \quad 30$

$\begin{array}{lll}0 & -10\end{array}$ 


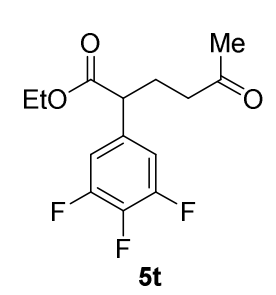

${ }^{19} \mathrm{~F}\left\{{ }^{1} \mathrm{H},{ }^{13} \mathrm{C}\right\}$ NMR $\left(283 \mathrm{MHz}, \mathrm{CDCl}_{3}\right)$

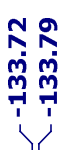

요

둔

iा

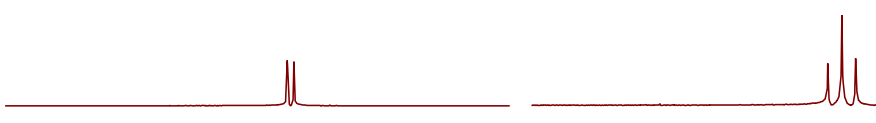

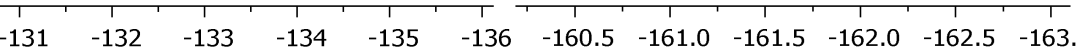

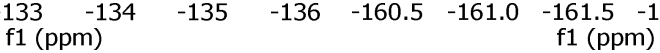

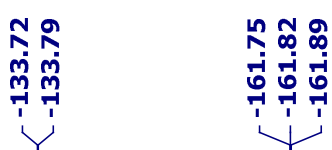

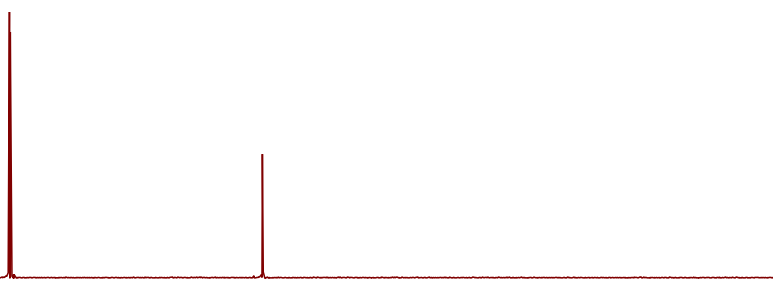

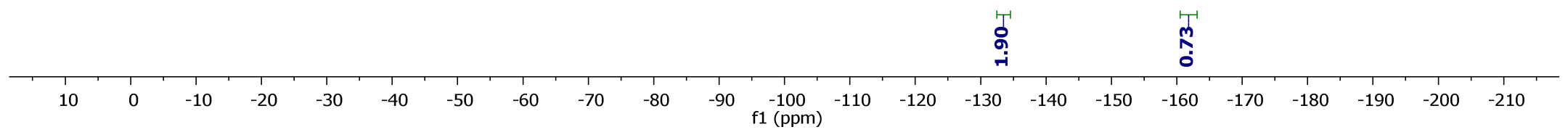




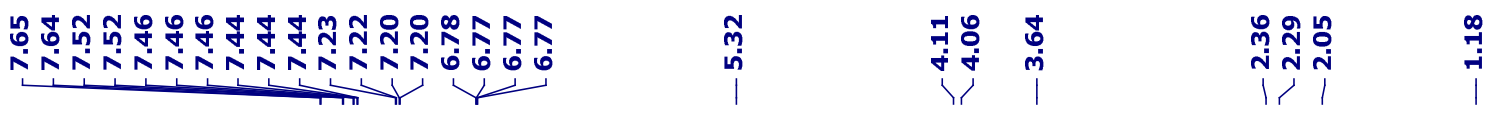

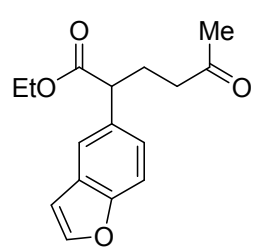

${ }^{1} \mathrm{H}$ NMR $\left(400 \mathrm{MHz}, \mathrm{CD}_{2} \mathrm{Cl}_{2}\right)$

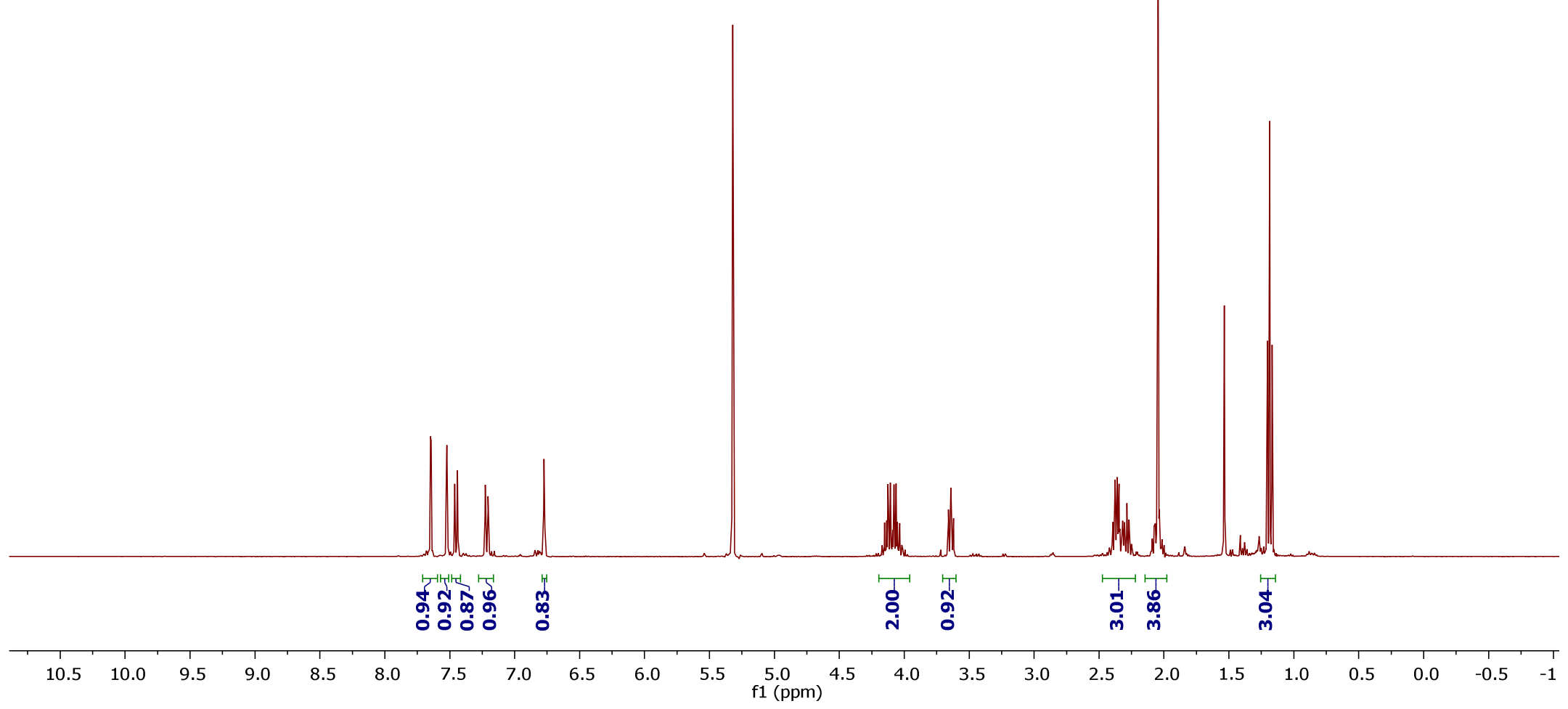



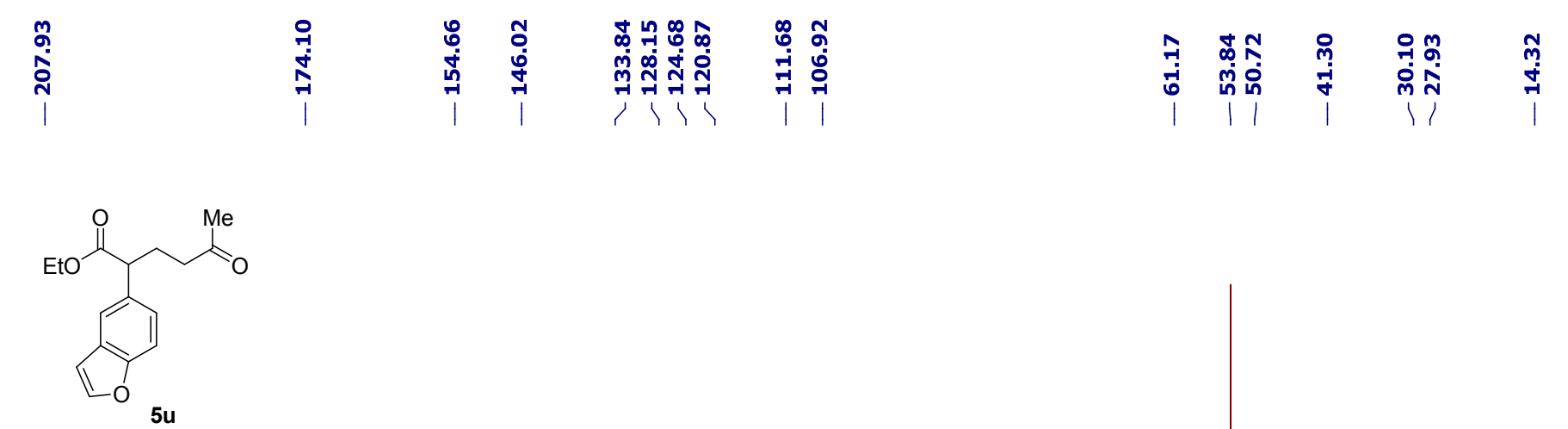

${ }^{13} \mathrm{C}\left\{{ }^{1} \mathrm{H}\right\}$ NMR $\left(101 \mathrm{MHz}, \mathrm{CD}_{2} \mathrm{Cl}_{2}\right)$

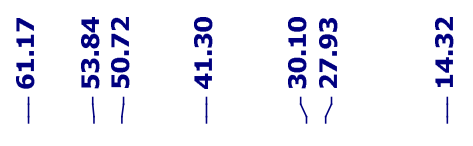

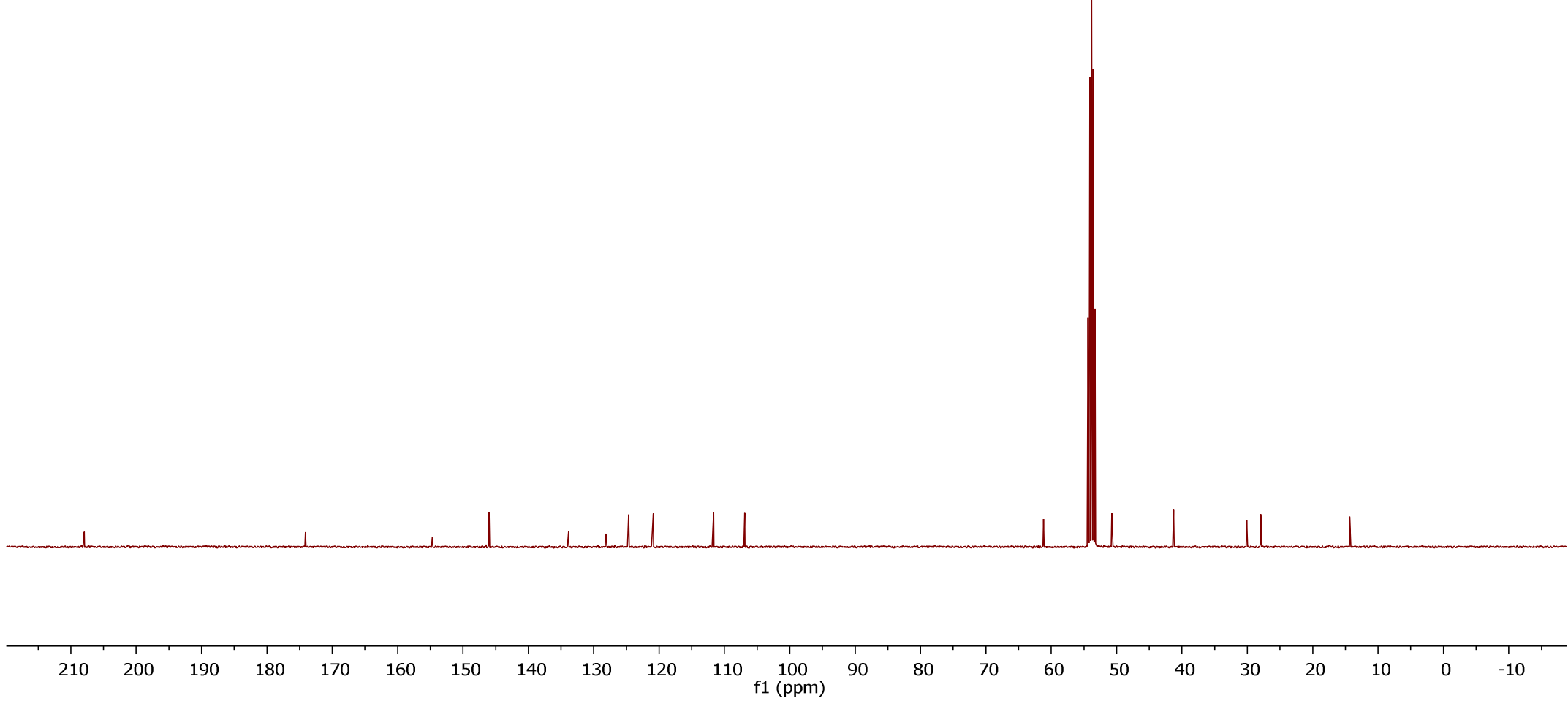




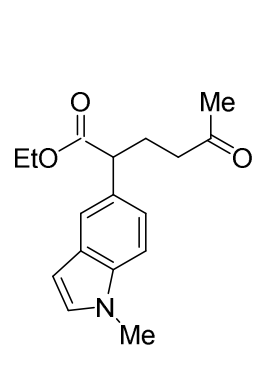

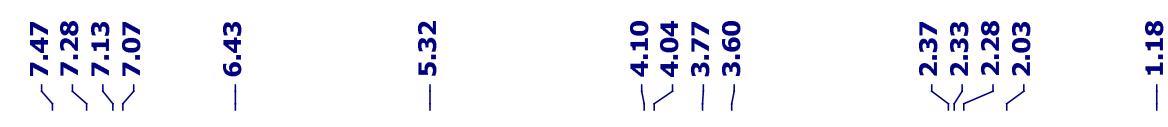

${ }^{1} \mathrm{H} \mathrm{NMR}\left(400 \mathrm{MHz}, \mathrm{CD}_{2} \mathrm{Cl}_{2}\right)$

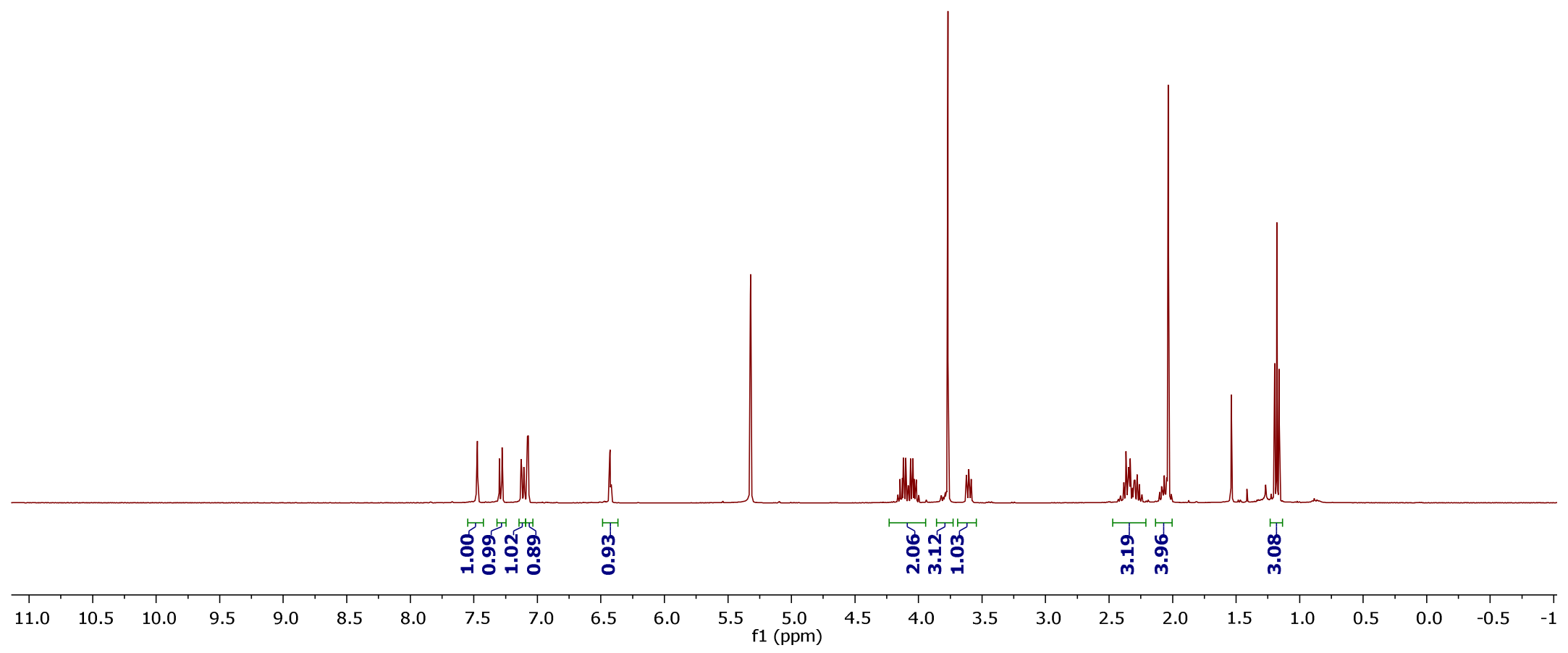



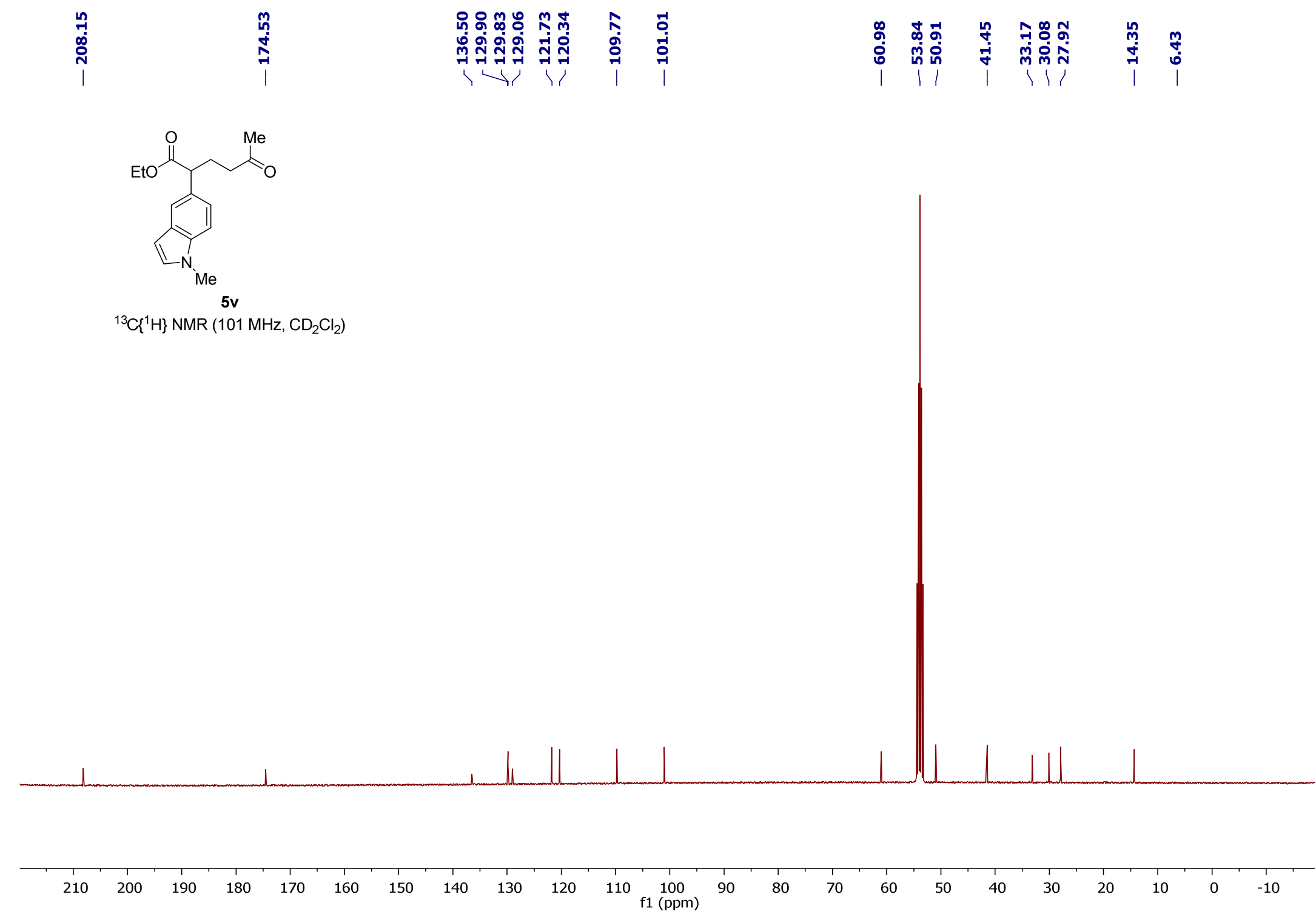

${ }^{13} \mathrm{C}\left\{{ }^{1} \mathrm{H}\right\}$ NMR $\left(101 \mathrm{MHz}, \mathrm{CD}_{2} \mathrm{Cl}_{2}\right)$

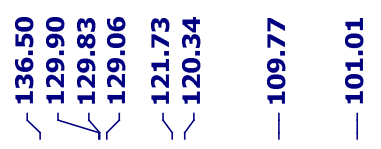

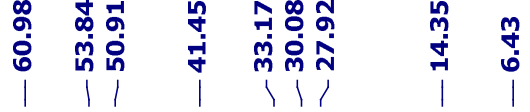




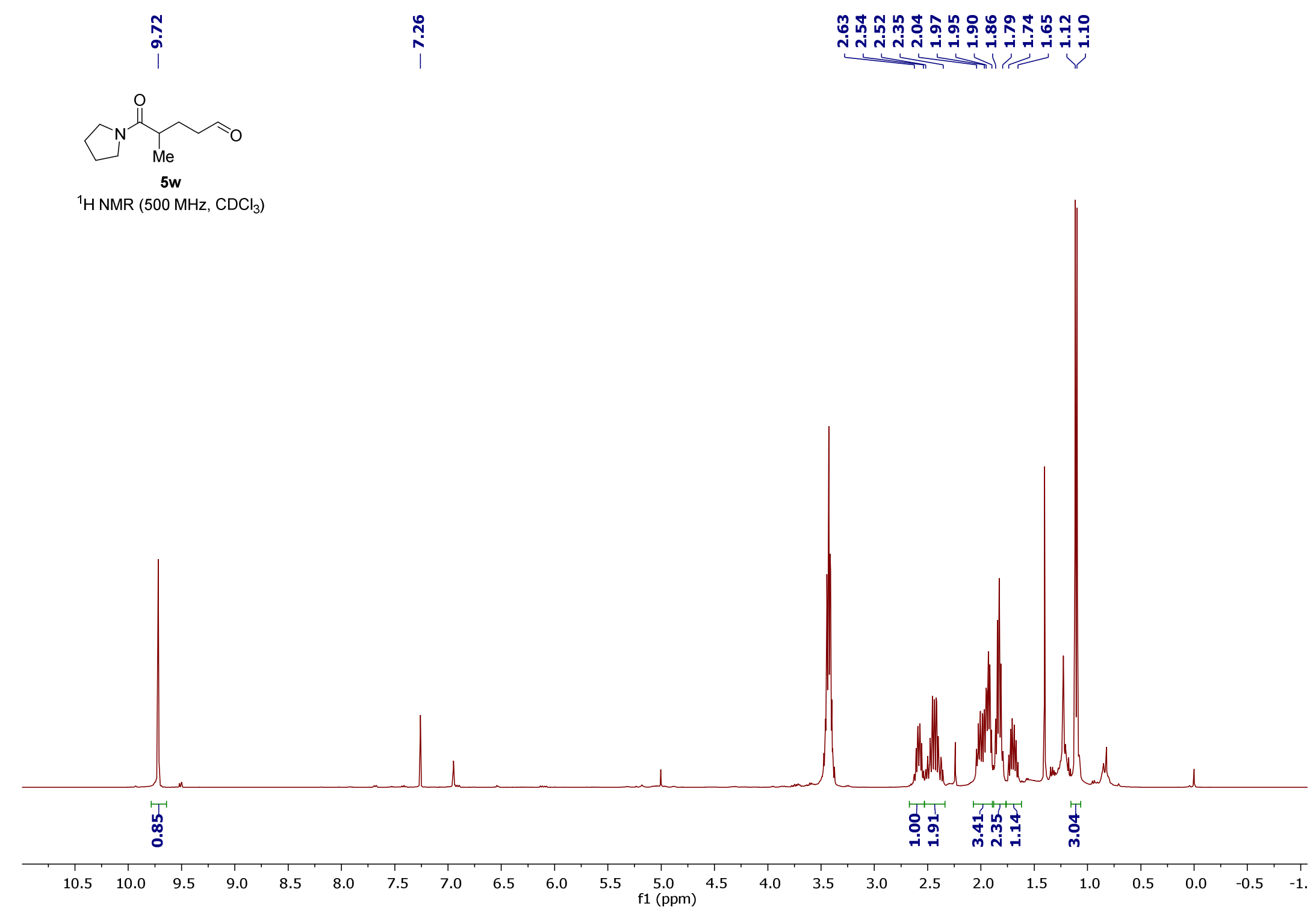




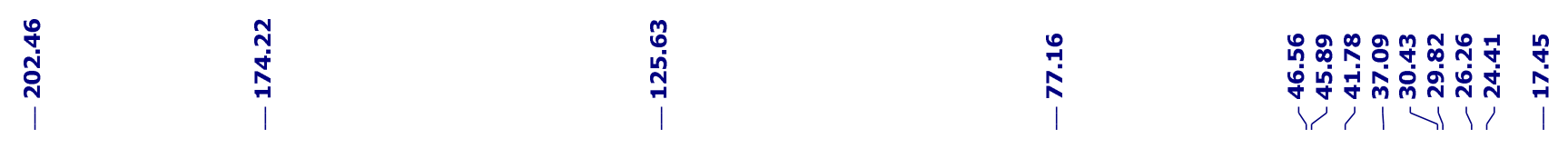

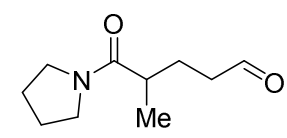

$5 \mathrm{w}$

$\left.{ }^{13} \mathrm{C}^{1} \mathrm{H}\right\}$ NMR $\left(126 \mathrm{MHz}, \mathrm{CDCl}_{3}\right)$

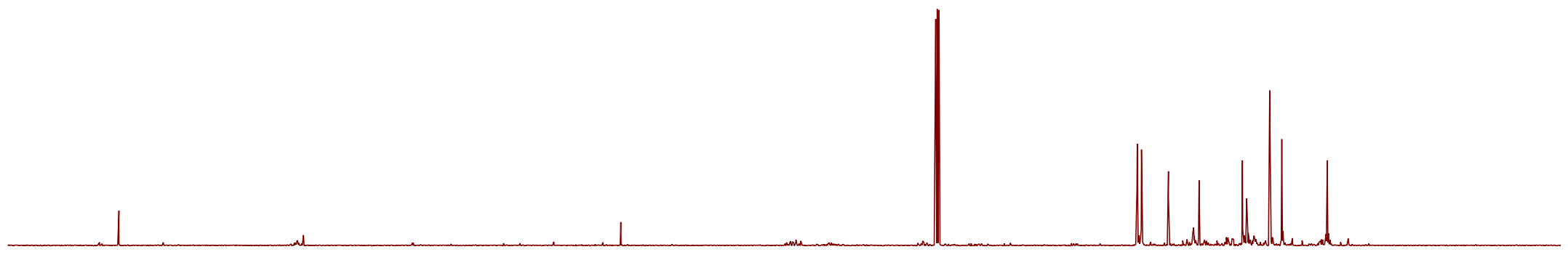
$\begin{array}{llllllllllll}210 & 200 & 190 & 180 & 170 & 160 & 150 & 140 & 130 & 120 & 110 & 100 \\ \mathrm{f} 1(\mathrm{ppm})\end{array}$ 

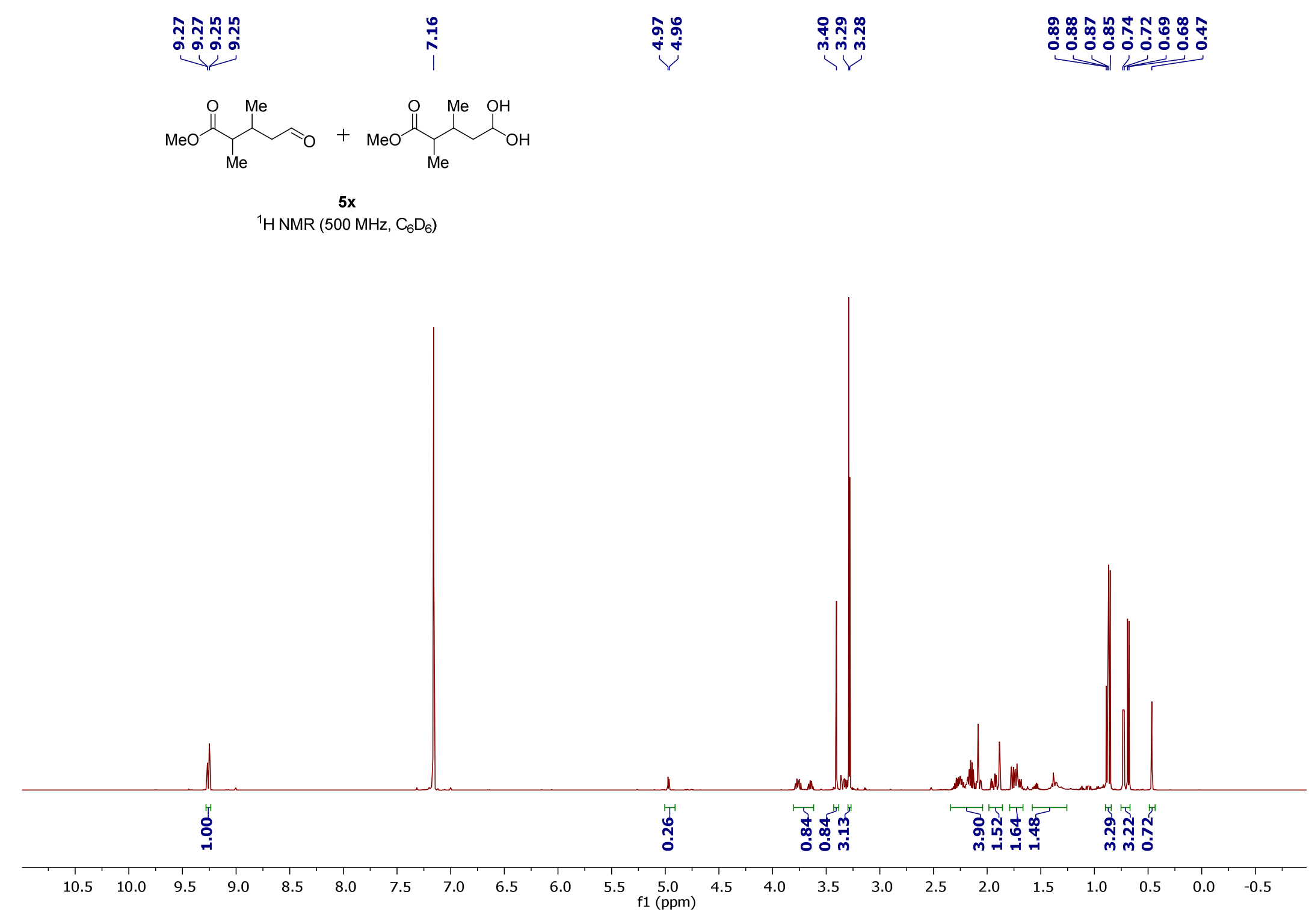


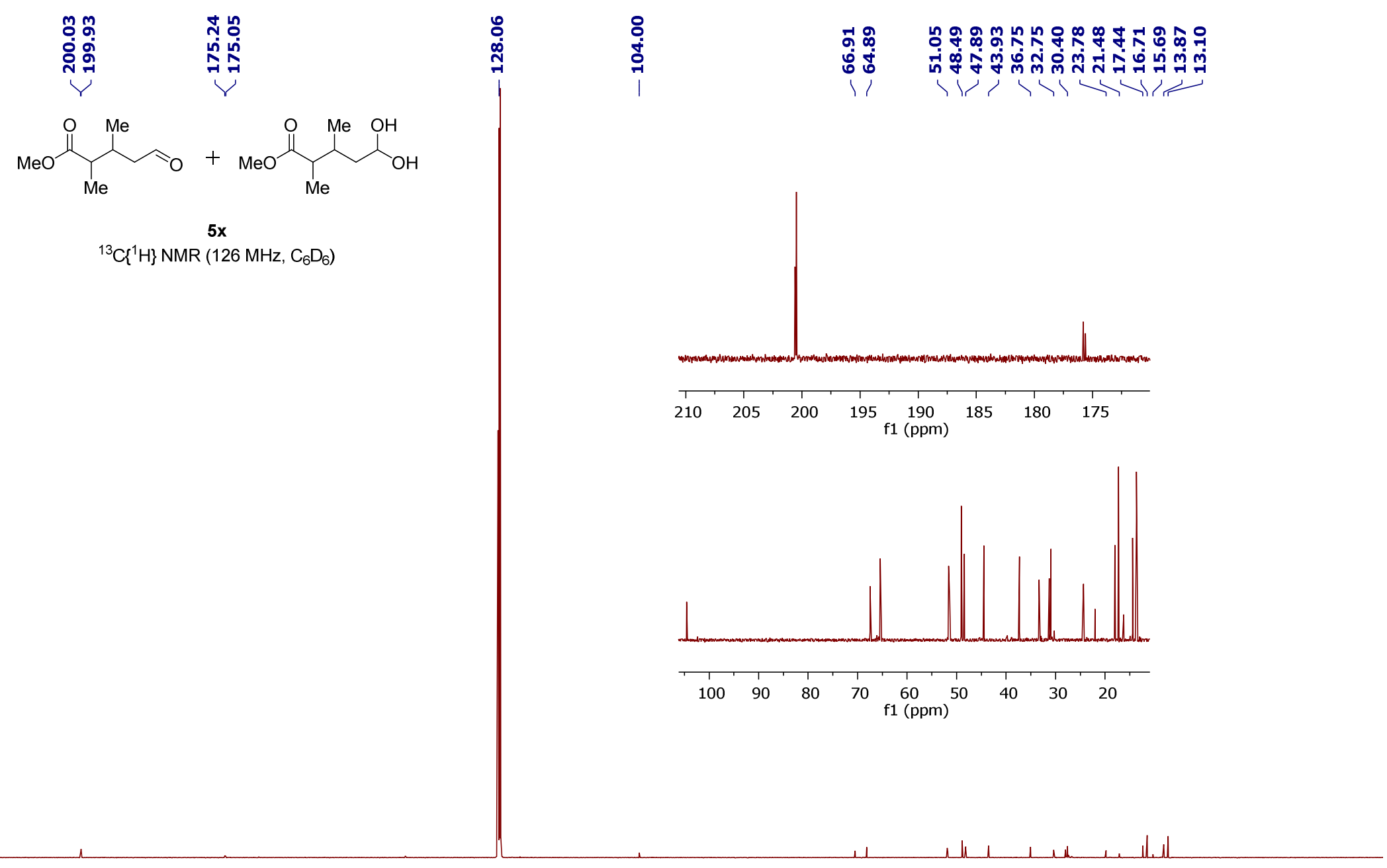

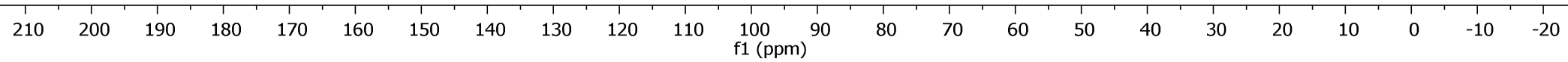




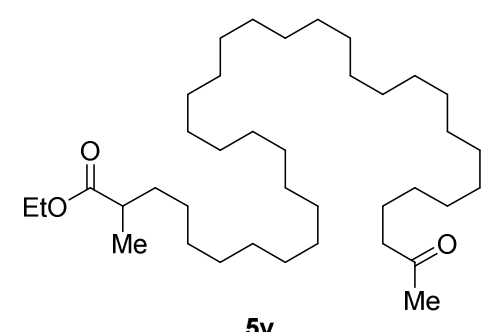

और

$\left.{ }^{1} \mathrm{H} \mathrm{NMR} \mathrm{(400} \mathrm{MHz,} \mathrm{CDCl}_{3}\right)$

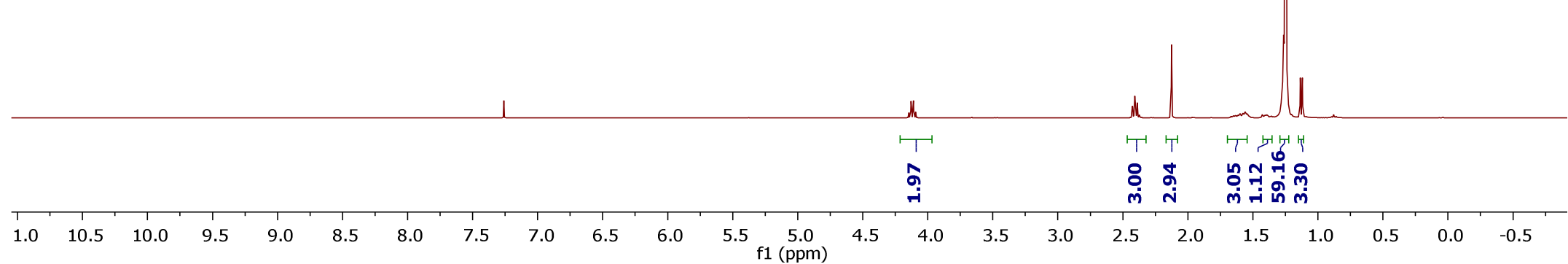




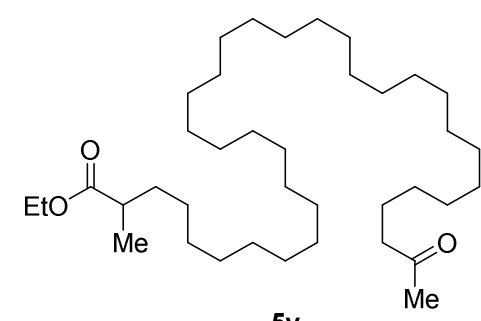

$\left.{ }^{13} \mathrm{C}^{1} \mathrm{H}\right\} \mathrm{NMR}\left(101 \mathrm{MHz}, \mathrm{CDCl}_{3}\right)$

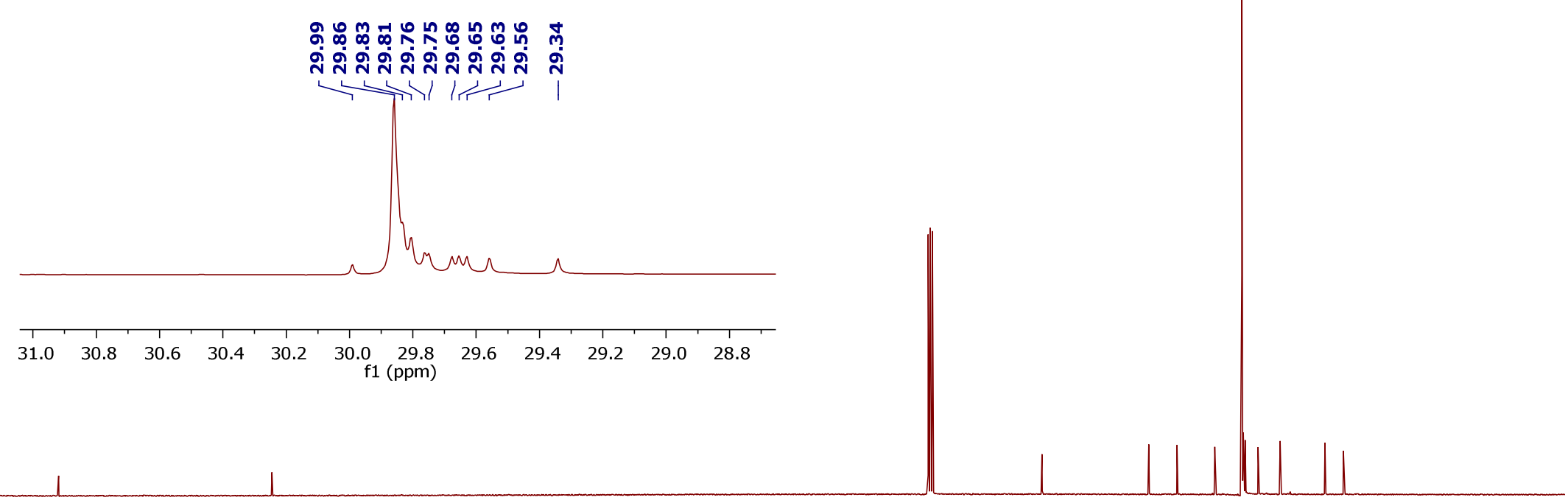

$\begin{array}{lllllllllllllllllllllll}210 & 200 & 190 & 180 & 170 & 160 & 150 & 140 & 130 & 120 & 110 & \begin{array}{c}100 \\ \mathrm{f} 1\end{array}(\mathrm{ppm}) & 90 & 80 & 70 & 60 & 50 & 40 & 30 & 20 & 10 & 0 & -10\end{array}$ 